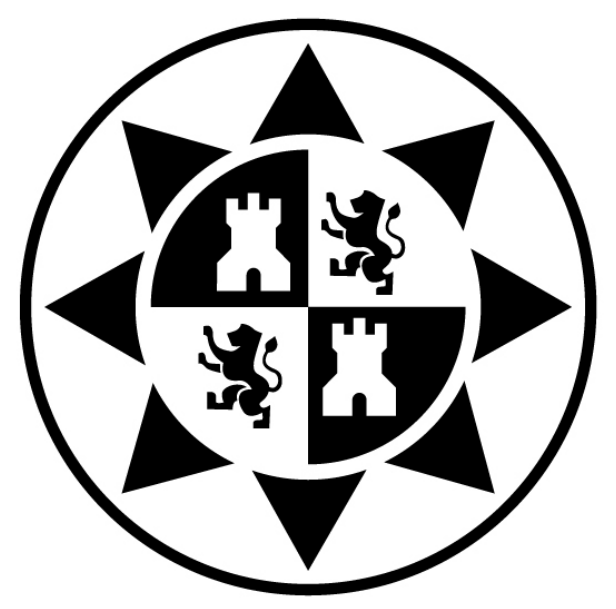

Universidad de Politécnica de Cartagena

Departamento de Ingeniería Térmica y Mecánica de Fluidos

\title{
HEAT TRANSFER AND ICE SLURRY PRODUCTION ANALYSIS IN SCRAPED SURFACE PLATE HEAT EXCHANGERS
}

\author{
David S. Martínez Hernández \\ Dirigida por: \\ Antonio Viedma Robles \\ Juan Pedro Solano Fernández
}

A dissertation submitted in partial satisfaction of the requirements
for the degree of Doctor of Philosophy in Industrial Technologies 



\section{Universidad}

Politécnica

de Cartagena

DT-16

\section{CONFORMIDAD DE SOLICITUD DEAUTORIZACIÓN DE DEPÓSITO DE TESIS DOCTORAL POR EL/LA DIRECTOR/A DE LA TESIS}

D. Antonio Viedma Robles Director de la Tesis "Transferencia de calor y generación de hielo líquido en intercambiadores de placas con rascadores rotativos"

\section{INFORMA:}

Que la referida Tesis Doctoral, ha sido realizada por D David Sebastián Martínez Hernández, dentro del programa de doctorado de Tecnologías Industriales, dando mi conformidad para que sea presentada ante la Comisión de Doctorado para ser autorizado su depósito.

La rama de conocimiento en la que esta tesis ha sido desarrollada es:

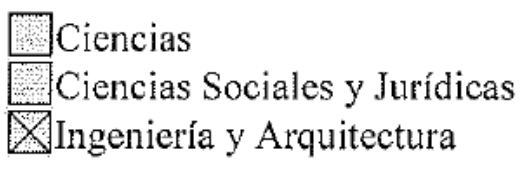

En Cartagena, a 8 de enero de 2016

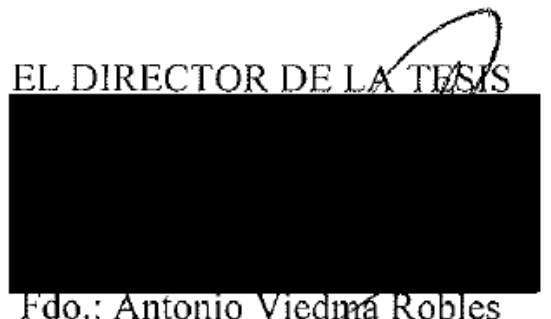

\section{COMISIÓN DE DOCTORADO}





\section{Universidad \\ Politécnica \\ de Cartagena}

DT-16

\section{CONFORMIDAD DE SOLICITUD DEAUTORIZACIÓN DE DEPÓSITO DE TESIS DOCTORAL POR EL/LA DIRECTOR/A DE LA TESIS}

D. Juan Pedro Solano Fernández Director de la Tesis "Transferencia de calor y generación de hielo líquido en intercambiadores de placas con rascadores rotativos"

\section{INFORMA:}

Que la referida Tesis Doctoral, ha sido realizada por D David Sebastián Martínez Hernández, dentro del programa de doctorado de Tecnologías Industriales, dando mi conformidad para que sea presentada ante la Comisión de Doctorado para ser autorizado su depósito.

La rama de conocimiento en la que esta tesis ha sido desarrollada es:

Ciencias
Ciencias Sociales y Jurídicas
Xngeniería y Arquitectura

En Cartagena, a $\gamma$ de euaro de 2016

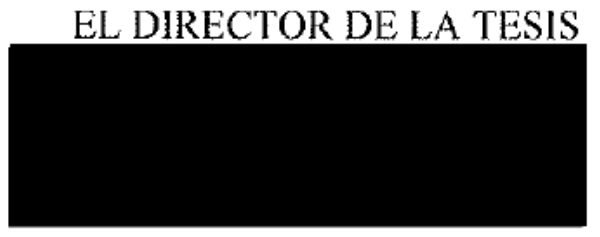

Fdo.: Juan Pedro Solano Fernández

\section{COMISIÓN DE DOCTORADO}





\section{Universidad \\ Politécnica \\ de Cartagena}

DT-17

\section{CONFORMIDAD DE DEPÓSITO DE TESIS DOCTORAL POR LA COMISIÓN ACADÉMICA DEL PROGRAMA}

D.JUAN SUARDíAZ MURO, Presidente de la Comisión Académica del Programa Tecnologias Industriales.

\section{INFORMA:}

Que la Tesis Doctoral titulada, "Transferencia de calor y generación de hielo en intercambiadores de placas con rascadores rotativos", ha sido realizada, dentro del mencionado programa de doctorado, por D David Sebastián Martínez Hernández, bajo Ia dirección y supervisión del Dr. Antonio Viedma Robles y el Dr. Juan Pedro Solano Fernández

En reunión de la Comisión Académica de fecha 07/01/2016 visto que en la misma se acreditan los indicios de calidad correspondientes y la autorización del Director de la misma, se acordó dar la conformidad, con la finalidad de que sea autorizado su depósito por la Comisión de Doctorado.

La Rama de conocimiento por la que esta tesis ha sido desarrollada es:

$\square$ Ciencias

$\square$ Ciencias Sociales y Jurídicas

区Ingeniería y Arquitectura

En Cartagena, a 07 de enero de 2016

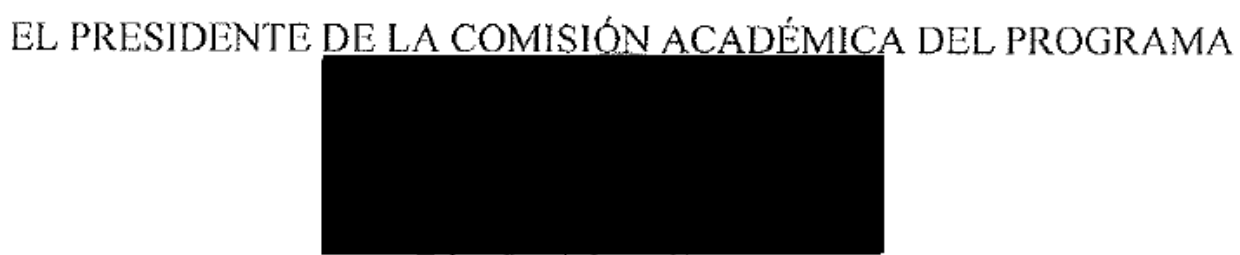

Fdo: Juan Suardíaz Muro

\section{COMISIÓN DE DOCTORADO}



To those who thought of everything else so that I could think only of this 



\section{Acknowledgements}

First and foremost I would like to thank my supervisors Dr. Antonio Viedma and Dr. Juan Pedro Solano for giving me the opportunity of this Ph.D thesis. Their continuous support, guidance and motivation helped me in all the time of research and writing of the thesis. They provided me the freedom to explore on my own and at the same time they were always present to guide my steps, being a source of knowledge and inspiration. They have not only been supervisors but also a reference to aim for.

Besides my supervisors, I would specially like to thank professor Fernando Illán for all the precious help and knowledge transferred in all involving refrigeration and ice slurry, crucial in the development of this Ph.D thesis.

My sincere thanks also goes to Dr. Pedro G. Vicente for his great support and advices during the visualization campaign at the University Miguel Hernández of Elche. Also to Dr. Damián Crespí for answering all my questions and sharing the lab hours.

I thank Dr. Alidad Amirfazli for hosting me in his research group, leading me working on an exciting project that enriched me personally and professionally.

I also want to thank Dr. Alberto García, a guiding light in my first steps in research and experimental work.

I gratefully acknowledge the FPU funding program from the Ministerio de Educación Cultura y Deporte of the Spanish Government, which made my Ph.D. work possible.

Last but not the least, I would like to thank all the department staff and in particular the technicians for their support in the laboratory. 



\section{Abstract}

The present Doctoral Thesis reports a detailed study of the ice slurry production in a scraped surface plate heat exchanger (SSPHE). The investigation work considers fundamental aspects as the heat transfer, the power consumption and the morphology of the produced ice crystals. The heat exchanger consists of a cylindrical tank with a scraped heat transfer surface at the bottom. The scraper system is composed of four rotating blades driven by a rotating shaft. Different sodium chloride brines (2.9 to 9.4 wt \%) are employed as the base solution for the generation of ice slurries in a batch process. The heat transfer surface is cooled underneath by a constant flow of calcium chloride solution in water, subsequently cooled by the expansion of a refrigerant in a compact evaporator. The action of the scrapers pull off the ice adhered to he plate surface, adding the microscopic ice crystals to the slurry. A wide range of operating conditions has been tested: scraping velocities from 0.1 to $0.8 \mathrm{~s}^{-1}$ and logarithmic temperature differences from 0.5 to $2.5{ }^{\circ} \mathrm{C}$. Heat and mass transfer is promoted due to the action of the scrapers, which not only guarantees the existence of nucleation directly on the subcooled surface but also induces macroscopic displacements of the flow.

Nusselt number results have been obtained through the accurate measurement of the precess temperatures and the slurry properties. Experimental results showed a dependence of the nucleation onset with the scraping velocity and the wall subcooling degree. The Nusselt number decreased for increasing wall subcooling degrees as a consequence of the ice layer scaling, with a low effect of the scraping velocity. The driving motor power consumption showed also a dependence with the presence of ice on the scraped surface, not being very influenced by other parameters.

Flow visualization with icelike polymer particles has performed to study the twophase flow and the eventual stratification. PIV technique has been employed to obtain the velocity field inside he SSPHE with a single phase flow for equivalent rotating Reynolds numbers. The main flow structure has been identified and related to the mixing mechanism of the flow.

Complementary to the experimental analysis, a numerical flow analysis was carried out employing the software package OpenFAOM. The numerical results proved their validity in reproducing the flow pattern observed experimentally, providing additional detail of the flow structure inside the SSPHE.

The obtained information extends the understanding of the mechanisms involved in the ice slurry production in the SSPHE, with an special attention to essential aspects like heat transfer and power consumption. Future designs of devices similar to the SSPHE can be benefited of this knowledge for achieving a better operating conditions. 



\section{Resumen}

En esta Tesis Doctoral se realiza un estudio de la producción de hielo líquido en un intercambiador de calor de placas de superficie rascada rotativo (SSPHE). La investigación considera aspectos fundamentales como la transferencia de calor, el consumo de potencia o la morfología de los cristales de hielo producidos. El intercambiador de calor consiste en un tanque cilíndrico con una superficie rascada en la base. El sistema de rascado se compone de cuatro palas rascadoras accionadas por un eje rotativo. Como base para producir el hielo líquido se usa una salmuera de cloruro de sodio con diferentes concentraciones (2.9 to 9.4 wt \%), produciéndose éste en modo discontinuo o "batch". La superficie rascada se enfría por el lado opuesto mediante un flujo constante de cloruro de sodio en agua, a su vez enfriado por la expansión de un refrigerante en un evaporador compacto. La acción de los rascadores arranca el hielo adherido a la placa base, añadiendo los cristales microscópicos de hielo a la mezcla. El rango de condiciones de trabajo ensayadas ha sido amplio: velocidades de rascado desde $0.1 \mathrm{a}$ $0.8 \mathrm{~s}^{-1}$ y diferencias logarítmicas de temperatura desde 0.5 a $2.5^{\circ} \mathrm{C}$. La acción de los rascadores promueve la transferencia de calor y masa, que no solo garantiza la ausencia de una gruesa capa de hielo sobre la superficie si no que también induce desplazamientos macroscópicos del flujo.

El número de Nusselt se ha obtenido mediante la medida precisa de las temperaturas del proceso y las propiedades del hielo líquido. Los resultados experimentales muestran una dependencia del inicio de la nucleación con la velocidad de rascado y el subenfriamento de la pared. Como consecuencia del crecimiento de la capa de hielo, el número de Nussel disminuye para valores crecientes de subenfriamiento, mientras que el efecto de la velocidad de rascado es bajo. Así mismo la potencia de accionamiento muestra una mayor dependencia con la presencia de hielo en la pared, y no con otros parámetros.

Se ha realizado una visualización del flujo empleando partículas similares al hielo para estudiar el flujo bifásico. Para estudiar el campo de velocidades con flujo monofásico se ha empleado la técnica de Velocimetría por Imagen de Partículas, analizando valores de número de Reynolds rotativo equivalentes. El patrón de flujo ha sido identificado y relacionado con los mecanismos de mezclado del flujo.

Complementariamente al análisis experimental se ha llevado a cabo un estudio numérico empleando el código OpenFOAM. Los resultados numéricos han sido parcialmente validados con los datos experimentales, proporcionando información acerca del flujo tridimensional en el SSPHE.

La información obtenida y analizada permite extender el conocimiento de los mecanismos implicados en la producción de hielo líquido en el SSPHE basándose en aspectos fundamentales como la transferencia de calor y la potencia de accionamiento. Así 
mismo, ésta puede ser de gran utilidad en el diseo de futuros dispositivos, ayudando a alcanzar mejores condiciones de trabajo. 




\section{Contents}

$\begin{array}{lr}\text { Abstract } & 13\end{array}$

$\begin{array}{ll}\text { Resumen } & 15\end{array}$

$\begin{array}{llr}1 & \text { Introduction } & \mathbf{3 7}\end{array}$

1.1 The ice slurry . . . . . . . . . . . . . . . . . . 37

1.1.1 Characteristics of the ice particles . . . . . . . . . . . 38

1.1.2 Rheological and thermophysical properties . . . . . . . . . . 39

1.1 .3 Fluid dynamics . . . . . . . . . . . . . . . . . . . 40

1.1.4 Applications . . . . . . . . . . . . . . . . . . 41

1.1.5 Production techniques . . . . . . . . . . . . . . . . 43

1.2 Research on the scraper surface ice slurry generation systems . . . . . . 48

1.3 Previous works on the research group . . . . . . . . . . . . . 53

1.4 Objectives of the work . . . . . . . . . . . . . . 53

1.5 Research methodology . . . . . . . . . . . . . . . . . . . 55

1.6 Thesis outline . . . . . . . . . . . . . . . . . . 55

2 Materials and methods I: ice slurry set-up $\quad 57$

2.1 Ice slurry experimental set-up . . . . . . . . . . . . . . . . . 57

2.1.1 Scraping system . . . . . . . . . . . . . . . . . 61

2.1 .2 Instrumentation . . . . . . . . . . . . . . . . . . 64

2.1.3 Wall temperature measurement system and calibration . . . . . . 67

2.1.4 Control system . . . . . . . . . . . . . . . . . . . . 69

2.1.5 Thermophysical properties and ice content. . . . . . . . . 69

2.1.6 Experimental procedure . . . . . . . . . . . . . . . 71

2.1.7 Working range . . . . . . . . . . . . . . . . . . 74

2.1.8 Uncertainty calculation . . . . . . . . . . . . . . . 75

3 Materials and methods II: flow visualization rig $\quad 81$

3.1 Visualization facility . . . . . . . . . . . . . . . . . . 81

3.2 Visualization of the particle motion . . . . . . . . . . . . . 82

3.2.1 Working fluid and icelike particles . . . . . . . . . . . 83

3.2.2 Image processing and particle motion . . . . . . . . . . . . . 86

3.3 Particle stratification tests . . . . . . . . . . . . . . . . . . 89

3.4 Particle Image Velocimetry _ . . . . . . . . . . . . . . . . . . . . . . 89

3.4.1 Fundamentals and technical considerations . . . . . . . . . . 89

3.4.2 Facility for PIV tests . . . . . . . . . . . . . . . . . . . . . 94 
3.4 .3 Image processing . . . . . . . . . . . . . . . . . . . . . . . . 98

3.4.4 Estimation of uncertainties . . . . . . . . . . . . . . 100

$\begin{array}{llr}4 & \text { Ice slurry production in the SSPHE } & 105\end{array}$

4.1 Thermal response . . . . . . . . . . . . . . . . . . . 105

4.2 Supercooling . . . . . . . . . . . . . . . . 106

4.3 Wall temperature distribution . . . . . . . . . . . . . . . 108

4.4 Heat transfer . . . . . . . . . . . . . . . . . . . . . 110

4.4 .1 Time resolved values . . . . . . . . . . . . . . . . . 110

4.4 .2 Time-averaged values . . . . . . . . . . . . . . . . . 113

4.4.3 Correlation for Nusselt number estimation . . . . . . . . . . . . . 116

4.5 Ice removal evaluation . . . . . . . . . . . . . . . . . . . . . . 119

4.6 Power consumption . . . . . . . . . . . . . . . . . . . . . 122

4.6.1 Power, torque and force . . . . . . . . . . . . . . . 123

4.6.2 Adaptable scraper system results . . . . . . . . . . . . . . 126

4.6.3 Rigid scraper system results . . . . . . . . . . . . . . . . . 132

4.7 Friction mechanism on the SSPHE . . . . . . . . . . . . . . 134

4.8 Ice crystals morphology . . . . . . . . . . . . . . . . . . . 135

4.9 Conclusions . . . . . . . . . . . . . . . . . . . 138

5 Performance evaluation for different $\mathrm{NaCl}$ concentrations $\quad 141$

5.1 Salt concentration and freezing point . . . . . . . . . . . . . 141

5.2 Heat transfer . . . . . . . . . . . . . . . . . . . . . 142

5.3 Power consumption . . . . . . . . . . . . . . . . . . . 145

5.4 Performance evaluation . . . . . . . . . . . . . . . . . . 147

5.4 .1 Local consideration: SSPHE . . . . . . . . . . . . . . . . . 147

5.4.2 Global consideration: SSPHE + refrigeration system . . . . . . . 149

5.5 Influence in the ice crystal morphology . . . . . . . . . . . . . . . . 153

5.6 Conclusions . . . . . . . . . . . . . . . . . . . . 157

$6 \quad$ Experimental flow pattern assesment 159

6.1 Particle motion . . . . . . . . . . . . . . . . . . . 160

6.2 Flow stratification . . . . . . . . . . . . . . . . 162

6.3 Particle Image Velocimetry results . . . . . . . . . . . . . . . . . 166

6.3.1 Previous considerations . . . . . . . . . . . . . 166

6.3.2 Phase-averaged velocity field . . . . . . . . . . . . . 170

6.3 .3 Instantaneous velocity field . . . . . . . . . . . . . . 183

6.4 Flow pattern influence in the heat transfer results . . . . . . . . . . . . 184

6.5 Conclusions . . . . . . . . . . . . . . . . . . . 188

$\begin{array}{llr}7 & \text { Numerical modelling } & 191\end{array}$

7.1 Previous considerations . . . . . . . . . . . . . . . . . . 191

7.2 Governing equations . . . . . . . . . . . . . . . . . 192

7.3 Turbulence modelling . . . . . . . . . . . . . . . . . . 192

7.3.1 Reynolds Averaging . . . . . . . . . . . . . . . . . 193

7.3 .2 LES modelling . . . . . . . . . . . . . . . . . . . . . . 195

7.4 Finite Volume Method . . . . . . . . . . . . . . . . . . . . 196 
7.4.1 Domain discretization . . . . . . . . . . . . . . . . . 197

7.4 .2 Discretised equations . . . . . . . . . . . . . . . . . . 197

7.4.3 Temporal discretisation . . . . . . . . . . . . . . . . . . 199

7.4.4 Pressure-Velocity coupling . . . . . . . . . . . . . . . 200

7.4.5 Boundary conditions . . . . . . . . . . . . . . 200

7.5 The two-dimensional model: lagrangian ice particles tracking . . . . . . 201

7.5.1 Translating reference frame . . . . . . . . . . . . . . 201

7.5.2 Computational domain and mesh . . . . . . . . . . . . 203

7.5.3 The lagrangian frame: injected particles . . . . . . . . . . . 203

7.5.4 Initial and boundary conditions . . . . . . . . . . . . . . . 205

7.6 The three-dimensional model . . . . . . . . . . . . . . . . 207

7.6.1 Rotating reference frame . . . . . . . . . . . . . . 207

7.6.2 Computational domain and mesh . . . . . . . . . . . . 208

7.6.3 Boundary conditions . . . . . . . . . . . . . . . . . 209

7.6 .4 Important remarks . . . . . . . . . . . . . . . . . . 209

8 Numerical flow pattern assesment 213

8.1 Results for the two-dimensional approach . . . . . . . . . . . . . 213

8.1 .1 The $k-\omega$ turbulence modelling . . . . . . . . . . . . . . . 213

8.1 .2 The LES turbulence modelling . . . . . . . . . . . . . . 225

8.2 Results for the three-dimensional approach . . . . . . . . . . . . . 229

8.3 Conclusions . . . . . . . . . . . . . . . . . . . . . . . 240

9 Conclusions and final remarks $\quad 243$

9.1 Experimental methodology . . . . . . . . . . . . . . . . . . 243

9.1 .1 Ice slurry production facility . . . . . . . . . . . . . . 243

9.1 .2 Flow visualization facility . . . . . . . . . . . . . . . . 244

9.2 Findings in the study and modelling of the ice slurry production in the SSHPE . . . . . . . . . . . . . . . . . . . . 244

9.3 Findings in the performance evaluation for different $\mathrm{NaCl}$ concentrations 245

9.4 Findings in the experimental flow pattern assessment . . . . . . . . . . . 246

9.5 Numerical methodology . . . . . . . . . . . . . . . . . . . . . . 247

9.6 Findings in the numerical flow pattern and particle tracking assessment 247

9.7 Publications . . . . . . . . . . . . . . . . . . . 248

$\begin{array}{ll}\text { A Adaptable scraping system desing } & 251\end{array}$

B Embedded thermocouples: calibration and uncertainty calculation 255

$\begin{array}{ll}\text { Bibliography } & \mathbf{2 6 0}\end{array}$ 


\section{List of Figures}

1.1 A microscopic photograph of the ice crystals in a slurry, showing that common sizes are from $200 \mu \mathrm{m}$ to $300 \mu \mathrm{m}$ to. . . . . . . . . 38

1.2 Comparison of coolant flow rate and storage tank volume for ice slurry and conventional chilled water $[3] \ldots \ldots \ldots \ldots$. . . . . . . . 43

1.3 Shell and tube scraped surface ice slurry generator (Votator type) [39]. . 44

1.4 Diagram of the orbital rod ice slurry generator [40]. . . . . . . . . . 45

1.5 Schematic diagram of the effect of fluidized solid particles [41]. . . . . . 46

1.6 Schematic diagram of a supercooling ice slurry generation system [42]. . 47

1.7 Schematic diagram of a direct contact heat exchanger [43] . . . . . . . . 47

1.8 Vacuum ice slurry generator used in thermal energy storage system [44]. 48

1.9 Tubular SSHE investigated by Lakhdar et al. [46] . . . . . . . . . . . . . . 50

1.10 SSTHE investigated by Qin et al. [47] . . . . . . . . . . . . . . 50

1.11 The CDCC investigated by Vaessen et al. [56] . . . . . . . . . . . 51

1.12 The SSPHE investigated by Qin et al. [65] . . . . . . . . . . . . 52

2.1 Schematic view of the experimental facility . . . . . . . . . . . . . 58

2.2 Images different SSPHE elements . . . . . . . . . . . . . . . . . . . . 60

2.3 Experimental facility, general view . . . . . . . . . . . . . . 61

2.4 Cross sectional view of the two different scrapers tested . . . . . . . . . 62

2.5 Scraper system view. . . . . . . . . . . . . . . . . . . . 62

2.6 Rigid scraper system . . . . . . . . . . . . . . . . . . . 63

2.7 Adaptable scraper system . . . . . . . . . . . . . . . . . . . 64

2.8 Data and control variables acquired on the experimental facility: (1) Wattmeter, (2) Hall-effect transducer + tachometer, (3) brine-slurry PTC probes, (4) SSPHE coolant inlet/outlet PTC probes, (5) Plate embedded thermocouples, (6) Coriolis flowmeter, (7) PTC probe, (8) coolant inlet/outlet HE-evaporator PTC probes, (9) Refrigerant inlet/outlet PTC probes + pressure transducers, (10) Refrigerant liquid line PTC probe + pressure transducer, (11) Refrigerant vapour line PTC probe + pressure transducer, (12) Microscope + CCD camera. . . . . . . . . 65

2.9 Assembly of the PT100 probe for SSPHE-coolant inlet/outlet flow. . . . 66

2.10 Window on the SSHPE top (left), camera emplacement and light sources (center) and camera view (right) . . . . . . . . . . . 67

2.11 Thermocouples location (up) and welding technique (down): sketch and

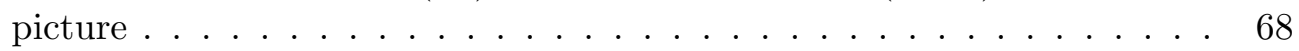

2.12 Detail of the welded thermocouple. . . . . . . . . . . . 69 
2.13 Specific heat measurements at different temperatures for a coolant sample. 71

2.14 Calculated heat fluxes for the coolant and ice slurry sides along time. . . 74

2.15 Planned and done tests: scraping velocities, initial $\mathrm{NaCl}$ concentration and averaged wall supercooling degree. . . . . . . . . . 76

2.16 Reynolds values for the initial $\mathrm{NaCl}$ concentrations and ice content experimented ranges. . . . . . . . . . . . . . . . 77

2.17 Heat transfer coefficient error for different values of $\Delta \mathrm{T}_{b, w}$ and $\Delta \mathrm{T}_{c}$. . . 79

3.1 Experimental facility overview . . . . . . . . . . . . . . . 82

3.2 Schematic view of the experimental facility adapted for the particle motion visualization tests . . . . . . . . . . . . . . . . 83

3.3 Sketch of the particles injection system. a) Cross sectional view. b) Side view. . . . . . . . . . . . . . . . . . . . 84

3.4 Microscopic image of the polyethylene particles used as ice crystal emulators. . . . . . . . . . . . . . . . . . . . . 85

3.5 Particles displacement estimation method: original frames (up) and background-subtracted (down). . . . . . . . . . . . . 87

3.6 Background subtraction and enhancing process: a) background clean image, b) particles image and c) sustracted and enhanced image. . . . .

3.7 Cross-correlation coefficient values after and before background subtraction process. a) With background, $\mathrm{PPR}=1.3$. b) Without background, $\mathrm{PPR}=2.1 \ldots \ldots \ldots \ldots \ldots \ldots$

3.8 Window sub-division process based on the initial displacement information (up) and new displacements (down) . . . . . . . . . . . . 90

3.9 Typical PIV arrangement $[80] \ldots \ldots$. . . . . . . . . . . . . . . 91

3.10 Image windowing (spots) and cross-correlation map [81] . . . . . . . . 92

3.11 Sub-pixel interpolation of correlation peak . . . . . . . . . . . . . 92

3.12 PIV imaging system . . . . . . . . . . . . . . . . . . . 94

3.13 Sketch of the PIV system arrangement . . . . . . . . . . . . . . 95

3.14 Trigger system between scrapers, camera and laser. . . . . . . . . . . 96

3.15 Typical image of one of the tests, where the illuminated area is on the right side of the scraper arm (center of the image) . . . . . . . . . . 97

3.16 Displacement vectors obtained after processing (a) pass 1 (b) pass 3. . . 99

3.17 Mean velocity $u_{\theta}$ and $v$ values evolution with the number of image pairs (samples). $\operatorname{Re}_{\mathrm{rot}}=3.9 \cdot 10^{4}$. . . . . . . . . . . . . . 100

3.18 Possible misalignments of the camera and the laser sheet. . . . . . . . . 101

3.19 Fractional displacement histogram representative of the processed PIV results . . . . . . . . . . . . . . . . . 103

4.1 Representative case of the evolution of the process temperature along time106

4.2 Supercooling amplitude $\zeta$ for different $R i$ numbers . . . . . . . . . . . 107

$4.3 \mathrm{R}$ and $\mathrm{L}$ coordinates for the plate thermocouple locations . . . . . . . 108

4.4 Temperature distribution in the plate along $\mathrm{R}$ and $\mathrm{L}$ for different test conditions . . . . . . . . . . . . . . . . . . 109

4.5 Heat fluxes on the subcooled plate along the test duration for different $\overline{\Delta T}_{\log \text { values } \ldots \ldots \ldots \ldots \ldots \ldots \ldots \ldots} \ldots \ldots \ldots \ldots \ldots$ 
4.6 Heat transfer coefficient on the subcooled plate along the test duration

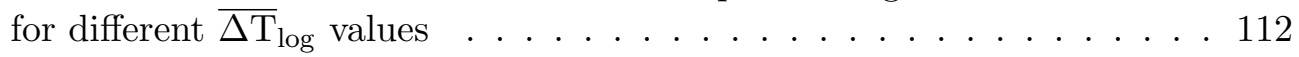

4.7 Influence of $\overline{\Delta T}_{\text {log }}$ on the heat flux averaged over the freezing period. Results coloured by scraping velocity . . . . . . . . . . . . . . . . 114

4.8 Influence of $\overline{\Delta T}_{\log }$ on the heat transfer coefficient averaged over the freezing period. Results coloured by the scraping velocity . . . . . . . . 114

4.9 Influence of the scraping velocity on the heat flux averaged over the freezing period for different scraper systems and $\overline{\Delta T}_{\log }$ values . . . . . 115

4.10 Influence of the scraping velocity on the heat transfer coefficient averaged over the freezing period for different scrapper systems and $\overline{\Delta T}_{\log }$ values

$4.11 \bar{\Delta}_{\log }$ vs. $\overline{\Delta \mathrm{T}}_{s}$ coloured by the heat transfer coefficient (adaptable scrapers) . . . . . . . . . . . . . . . . . . . . 117

4.12 Nusselt $(N u)$ number vs. rotating Reynolds $\left(R e_{r o t}\right)$ number for different experiments (adaptable scrapers) . . . . . . . . . . . . . . 118

4.13 Comparison between experimental and calculated Nusselt number values 119

4.14 Ice detection over the plate surface with the rigid scraping system; $\mathrm{N}=0.5$

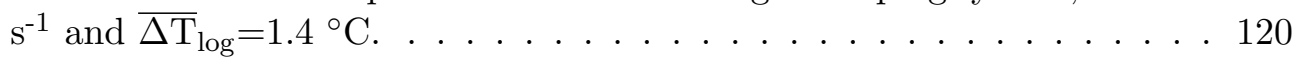

4.15 Scraper system view. . . . . . . . . . . . . . . . . . . . . 121

4.16 The friction force as a function of velocity for lubrication . . . . . . . 124

4.17 Friction regimes for ice, related to the liquid layer height [99] . . . . . 125

4.18 Scraping power consumption along time for different scraping velocities $\mathrm{N}=0.08$ and $0.8 \mathrm{~s}^{-1}$ (left to right) and mean logarithmic temperatures

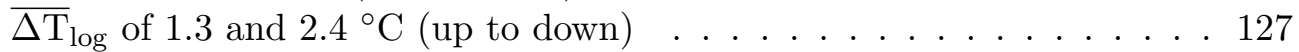

4.19 Scraping power comparative for $\Delta \mathrm{T}=1.3{ }^{\circ} \mathrm{C}$ and $\Delta \mathrm{T}=2.4{ }^{\circ} \mathrm{C} \ldots 128$

4.20 Averaged scraping power comparative for different scraping velocities

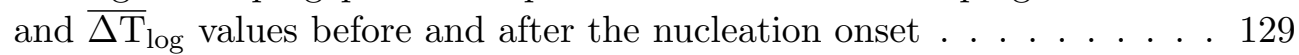

4.21 Averaged scraping power during the freezing period (ice content from 1 to $20 \%$ ) with their respective mean logarithmic temperatures for different scraping velocities . . . . . . . . . . . . . . . 130

4.23 Averaged torque comparative for $\Delta \mathrm{T}=1.1^{\circ} \mathrm{C}, \Delta \mathrm{T}=1.3{ }^{\circ} \mathrm{C}$ and $\Delta \mathrm{T}=2.4$ ${ }^{\circ} \mathrm{C}$ at different scraping velocities. The dashed line is plotted as a guide to the eye . . . . . . . . . . . . . . . . . 131

4.22 Averaged torque power comparative for different $\overline{\Delta \mathrm{T}}_{\log }$ and $\mathrm{N}$ values . . 131

4.24 Averaged power number vs. averaged $R e_{\text {rot }}$ number comparative for different values of $\overline{\Delta T}_{\text {log }}$ and ice concentrations. . . . . . . . . 133

4.25 Averaged scraping power during the freezing period (ice content from 1 to $20 \%$ ) for different scraping velocities and averaged mean logarithmic temperatures: rigid scraper system . . . . . . . . . . . . . 133

4.26 Sketch of the friction process in the SSHPE. a) Chilling b) Freezing at

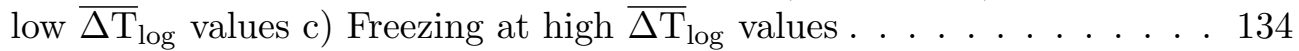

4.27 Ice crystals images taken with microscope at the end of each test (ice content $\approx 20 \%) \ldots \ldots \ldots \ldots \ldots \ldots$

4.28 Ice crystals images taken with microscope at different ice concentrations for $\overline{\Delta T}_{\log }=1.1^{\circ} \mathrm{C}$ and $\mathrm{N}=0.46 \mathrm{~s}^{-1} \ldots \ldots \ldots \ldots \ldots \ldots$

5.1 Observed vs. predicted freezing point for different $\omega_{0}$ tested values . . . 142 
5.2 Brine/slurry temperature evolution in time during tests for different $\omega_{0}$

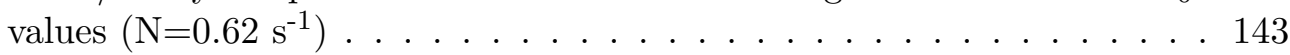

5.3 Heat flux for different $\omega_{0}$ values and scraping velocities at $\overline{\Delta \mathrm{T}}_{l o g} \approx 1.3{ }^{\circ} \mathrm{C} 143$

5.4 Heat transfer coefficient for different $\omega_{0}$ values and scraping velocities at $\overline{\Delta \mathrm{T}}_{l o g} \approx 1.3{ }^{\circ} \mathrm{C} \ldots \ldots \ldots \ldots \ldots \ldots \ldots \ldots$

5.5 Averaged, global heat flux (a) and Nusselt number (b) for all the performed experiments. Optimal operating conditions are highlighted in grey color. . . . . . . . . . . . . . . . . . . . . 146

5.6 Scraping power $S_{p}$ for different $\omega_{0}$ values and scraping velocities at $\overline{\Delta \mathrm{T}}_{l o g} \approx 1.3{ }^{\circ} \mathrm{C}$, averaged between $2 \%$ and $8 \%$ of ice content $\ldots \ldots . .147$

5.7 Power number $P$ o for different $\omega_{0}$ values and scraping velocities at $\overline{\Delta T}_{l o g} \approx 1.3$ ${ }^{\circ} \mathrm{C}$, averaged between $2 \%$ and $8 \%$ of ice content . . . . . . . . . . 148

5.8 Performance number $\eta$ (Eq. 5.1) for the tested values of scraping velocity, $\omega_{0}$ values and $\overline{\Delta T}_{\log }$ (averaged for a $2-8 \%$ ice content interval.) . . . . 149

5.9 Energy flux in the global process of ice slurry production . . . . . . . 150

5.10 Averaged evaporation temperature vs. wall subcooling $\overline{\Delta \mathrm{T}}_{s}\left(\omega_{0}=7.1 \%\right.$. $)$ coloured by the averaged heat flux (left) and COP of the condensing unit for different evaporation temperatures coloured by the ambient temperature (right). . . . . . . . . . . . . . . . . . . . 151

5.11 Coolant capacity $\mathrm{q}_{\mathrm{c}}$ of the condensing unit for different evaporation temperatures and coloured by the ambient temperature. . . . . . . . . 153

5.12 Ice crystals images taken with microscope at the end of each test (ice content $\approx 20 \%$ ): $\mathrm{N}=0.83 \mathrm{~s}^{-1}, \overline{\Delta \mathrm{T}}_{\log }=1.3{ }^{\circ} \mathrm{C} \ldots \ldots \ldots \ldots$

5.13 Ice crystals images taken with microscope at the end of each test (ice content $\approx 20 \%): \mathrm{N}=0.83 \mathrm{~s}^{-1}, \overline{\Delta \mathrm{T}}_{\log }=1.8^{\circ} \mathrm{C} \ldots \ldots \ldots \ldots$

6.1 Image sequence of the particle cloud (enhanced in red) at different time instants for $\mathrm{Fr}=0.42$ and $\mathrm{Re}_{\mathrm{rot}}=1.6 \cdot 10^{4}$. . . . . . . . . . . . . 161

6.2 Image sequence of the particle cloud (enhanced in red) at different time instants for $\mathrm{Fr}=0.68$ and $\mathrm{Re}_{\mathrm{rot}}=2.5 \cdot 10^{4}$. . . . . . . . . . . . . 161

6.3 Image sequence of the particle cloud (enhanced in red) at different time instants for $\mathrm{Fr}=1.3$ and $\mathrm{Re}_{\mathrm{rot}}=4.8 \cdot 10^{4} \ldots \ldots$. . . . . . . . . . 162

6.4 Relative displacement vectors for the injected particles cloud after $\Delta \mathrm{T}=33$ $\mathrm{ms}, \mathrm{Fr}=4.2 \cdot 10^{-1}$ and $\mathrm{Re}_{\mathrm{rot}}=1.6 \cdot 10^{4}$. Scale $\mathrm{x} 2 \ldots \ldots \ldots$

6.5 Relative displacement vectors for the injected particles cloud after $\Delta \mathrm{T}=24$ $\mathrm{ms}, \mathrm{Fr}=6.8 \cdot 10^{-1}$ and $\mathrm{Re}_{\mathrm{rot}}=2.5 \cdot 10^{4}$. Scale $\mathrm{x} 1 \ldots \ldots \ldots$

6.6 Relative displacement vectors for the injected particles cloud after $\Delta \mathrm{T}=15$ $\mathrm{ms}, \mathrm{Fr}=1.3$ and $\mathrm{Re}_{\mathrm{rot}}=4.8 \cdot 10^{4}$. Scale $\mathrm{x} 0.6 \ldots \ldots$. . . . . . . 164

6.7 Stratification test images for different Froude and rotating Reynolds numbers and $4 \%$ wt. particle content . . . . . . . . . . . . . 165

6.8 Velocity field composition process from different PIV studied regions under equivalent $\mathrm{Re}_{\mathrm{rot}} \ldots \ldots \ldots \ldots$. . . . . . . . . . 167

6.10 Velocity decomposition in the laser plane. . . . . . . . . . . . . . 168

6.9 Error in the measurement of in-plane displacements due to out-of-plane motion in the SSPHE . . . . . . . . . . . . . . . . . 169 
6.11 Velocity vectors and the corresponding magnitude coloured iso-contours from a stationary frame of reference (scraper moves from right to left).

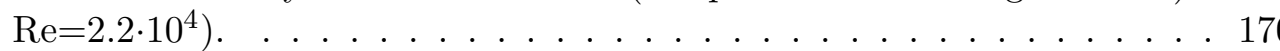

6.12 Normalized, relative (from a rotating frame of reference) velocity field, $\left.\hat{u} *_{\theta}, v * . \operatorname{Re}=2.2 \cdot 10^{4}\right) . \ldots \ldots \ldots \ldots \ldots 171$

6.13 Locations of the comparative velocity profiles. . . . . . . . . . . . 172

6.14 Tangential and axial velocity profiles at X1. a) $\left.\hat{u} *_{\theta}, \mathbf{b}\right) v * \ldots 174$

6.15 Tangential and axial velocity profiles at X2. a) $\left.\hat{u} *_{\theta}, \mathbf{b}\right) v * \ldots 175$

6.16 Tangential and axial velocity profiles at Y1. a) $\hat{u} *_{\theta}$, b) $v * \ldots 176$

6.17 Tangential and axial velocity profiles at Y2. a) $\left.\hat{u} *_{\theta}, \mathbf{b}\right) v * \ldots 176$

6.18 Streamlines computed from the velocity relative to the scraper in the laser plane for a) $\mathrm{Re}=2.2 \cdot 10^{4}$ and $\left.\mathbf{b}\right) \mathrm{Re}=1 \cdot 10^{5} \ldots \ldots \ldots \ldots$. . . . . 177

6.19 Roll up vortex for a) $\mathrm{Re}=2.2 \cdot 10^{4}$ and $\left.\mathbf{b}\right) \mathrm{Re}=3.9 \cdot 10^{4} \ldots \ldots \ldots$. . . . . . 178

6.20 Vortex dimensionless tangential velocity along its radius $\left(r_{v} / r_{l}\right)$ for the different $\mathrm{Re}_{\mathrm{rot}}$ tested. . . . . . . . . . . . . . . . . . . . 178

6.21 Vortex normalized swirl velocity for the different tested cases (a) compared with different classical solutions (b) from [121]. . . . . . . . . . 179

6.22 Sketch of flow pattern inside the SSPHE retrieved from PIV experiements. Radial components are not considered. . . . . . . . . . . . . . . 180

6.23 Dimensionless vorticity contours for a) $\operatorname{Re}=2.2 \cdot 10^{4}$, b) $\operatorname{Re}=6.5 \cdot 10^{4}$ and

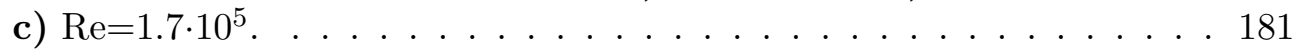

6.24 Dimensionless vorticity peak values $\Omega *$ variations for the tested rotative Reynolds number. . . . . . . . . . . . . . . . . . . . . 182

6.25 Dimensionless turbulent kinetic energy $k *$ contours in the investigated area for $R e=2.2 \cdot 10^{4} \ldots \ldots \ldots$. . . . . . . . . . . . . 183

6.26 Dimensionless turbulent kinetic energy $k *$ contours for a) $\operatorname{Re}=2.2 \cdot 10^{4}$, b) $\operatorname{Re}=6.5 \cdot 10^{4}$ and c) $\operatorname{Re}=1.7 \cdot 10^{5} \ldots \ldots \ldots \ldots \ldots$. . . . . . . . . . . . . . . .

6.27 Dimensionless turbulent kinetic energy $k *$ peak values for the different

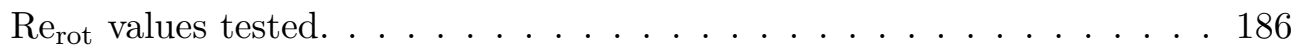

6.28 Different instantaneous velocity vector fields for $\mathrm{Re}_{\mathrm{rot}}=3.9 \cdot 10^{4}$. . . . 187

7.1 a) Bidimensional approach to the SSPHE problem b) periodic domain with absolute velocities c) periodic domains using the translating reference frame (relative velocities) . . . . . . . . . . . . . . 202

7.2 Mesh used for the particle tracking simulations with the $k$-Omega SST Low Reynolds model. . . . . . . . . . . . . . . . . . . . . . . 204

7.3 Distribution of the injected particles by their diameter, very close to a normal distribution. . . . . . . . . . . . . . . . . 205

7.4 Moving reference frame applied to the SSPHE. . . . . . . . . . . . 208

7.5 3D computational domain. . . . . . . . . . . . . . . . . 210

7.6 Fluid domain volume subdivision for meshing. . . . . . . . . . . . . . . 211

7.7 Mesh detail at different cross sections of the domain. . . . . . . . . . . . 212

8.1 Vector field and iso-contours for $U *$ using the $k-\omega$ turbulence modelling, $\operatorname{Re}_{\mathrm{rot}}=6.5 A \cdot 10^{4}$. Scraper moves from right to left. . . . . . . . . . . . . 214 
8.2 Comparison of dimensionless velocity profiles for the different simulated cases. Rotating Reynolds number are in correspondence with those tested in the visualization experiments (see chapter 6) . . . . . . . 216

8.3 Particles location for the different tested cases after 15 seconds of the injection. a) $\operatorname{Re}_{\mathrm{rot}}=2.2 \cdot 10^{4}$, b) $\left.\operatorname{Re}_{\mathrm{rot}}=3.9 \cdot 10^{4}, \mathbf{c}\right) \operatorname{Re}_{\mathrm{rot}}=6.5 \cdot 10^{4}$. Scraper motion from right to left. . . . . . . . . . . . . . .

8.3 Location of the particles for the different tested cases after 15 seconds of the injection. d) $\operatorname{Re}_{\text {rot }}=1.0 \cdot 10^{4}$, e) $\operatorname{Re}_{\text {rot }}=1.7 \cdot 10^{5}$. Scraper motion from right to left. . . . . . . . . . . . . . . . . 218

8.4 Cumulative distribution of particles (\%) along the vertical distance. . . 219

8.5 Concentration of particles $C_{P}$ [particles $\left./ \mathrm{cm}\right]$ for the different cases simulated. . . . . . . . . . . . . . . . . . . . . 220

8.6 Vertical location of the particles after 15 seconds of the injection as a function of the operating regime $\left(\operatorname{Re}_{\mathrm{rot}}\right)$ and particle diameter $\left(\mathrm{d}_{\mathrm{p}}\right) \ldots \ldots 221$

8.7 Trajectories of the different particles for eeach one of the cases $\left(\operatorname{Re}_{\text {rot }}\right)$ simulated. a) $\left.d_{p}=100 \mu \mathrm{m} \mathrm{b}\right) d_{p}=250 \mu \mathrm{m}$ and c) $d_{p}=460 \mu \mathrm{m} . \ldots 223$

8.8 Location of the particle for the different tested cases $\left(\operatorname{Re}_{\text {rot }}\right)$ after 15 seconds of its injection. a) $d_{p}=100 \mu \mathrm{m}$, b) $d_{p}=250 \mu \mathrm{m} \ldots \ldots . . .224$

8.8 Location of the particle for the different tested cases $\left(\operatorname{Re}_{\text {rot }}\right)$ after 15 seconds of its injection. c) $d_{p}=460 \mu \mathrm{m}$ (continued) . . . . . . . . . 225

8.9 Normalized particle residence time vs. number of turn of the particle over the domain. a) $d_{p}=100 \mu \mathrm{m}$, b) $d_{p}=460 \mu \mathrm{m} . \ldots \ldots . \ldots 226$

8.10 Time averaged iso-contours and streamlines for $U *$ using LES turbulence modelling, $\mathrm{Re}_{\mathrm{rot}}=1.7 \cdot 10^{5}$. Scraper moves from right to left. . . . . . . 227

8.11 Time averaged iso-contours and streamlines for $U *$ using LES turbulence modelling. Scraper move from right to left. a) $\operatorname{Re}_{\mathrm{rot}}=3.9 \cdot 10^{4} \mathbf{b}$ )

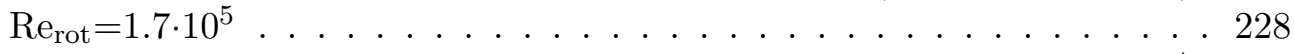

8.12 Particle trajectories for each one of the cases simulated. a) $\operatorname{Re}_{\mathrm{rot}}=3.9 \cdot 10^{4}$, b) $\operatorname{Re}_{\mathrm{rot}}=1.7 \cdot 10^{5} \ldots \ldots \ldots \ldots$. . . . . . . . . . . . . . . 229

8.13 Iso-contours and vector field for $\mathrm{U}^{*}$ obtained by the 3D numerical model at the location corresponding with the laser sheet of the PIV experiments $\left(\operatorname{Re}_{\mathrm{rot}}=6.5 \cdot 10^{4}\right)$. a) plane location sketch and velocities . . . . . . . 231

8.13 Iso-contours and vector field for $\mathrm{U}^{*}$ obtained by the 3D numerical model at the location corresponding with the laser sheet of the PIV experiments $\left.\left(\operatorname{Re}_{\mathrm{rot}}=6.5 \cdot 10^{4}\right) . \mathbf{b}\right)$ detail of extracted velocities (continued) . . . . . 232

8.14 Three-dimensional vector field for $\mathrm{U}^{*}$ in a tangential plane $r=\mathrm{R} / 2\left(\mathrm{Re}_{\mathrm{rot}}=6.5 \cdot 10^{4}\right) .233$

8.15 Iso-contours and vector field for $\mathrm{U}^{*}$ in different radial planes $\left(\operatorname{Re}_{\mathrm{rot}}=6.5 \cdot 10^{4}\right)$ :

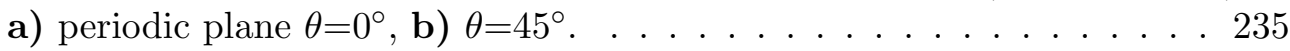

8.15 Iso-contours and vector field for $\mathrm{U}^{*}$ in different radial planes $\left(\operatorname{Re}_{\mathrm{rot}}=6.5 \cdot 10^{4}\right)$ :

c) scraper plane $\theta=90^{\circ}$, d) $\theta=135^{\circ}$ (continued). . . . . . . . . 236

8.16 Iso-contours and vector field for $\mathrm{U}^{*}$ in different tangential planes planes $\left(\mathrm{Re}_{\mathrm{rot}}=6.5 \cdot 10^{4}\right)$ : a) $r / \mathrm{R}=0.4$, b) $r / \mathrm{R}=0.8 \ldots \ldots \ldots \ldots \ldots \ldots$

8.17 Stream lines rake (origin is indicated in the figure) colored by the $\mathrm{U}^{*}$ values $\left(\operatorname{Re}_{\mathrm{rot}}=6.5 \cdot 10^{4}\right)$. a) top view b) front view. . . . . . . . . 238

8.18 Different views of the stream lines coloured by the type of flow encountered in the SPHEE $\left(\operatorname{Re}_{\mathrm{rot}}=6.5 \cdot 10^{4}\right)$ : radial flow (vortex) $\mathbf{a r c u l a r}$ flow (tangential) . . . . . . . . . . . . . . . . . . 239 
A.1 Torsion spring. From left to right: spring dimensions (mm), scraperpush tail and working position . . . . . . . . . . . 251

A.3 Working angle (left) and maximum scraper blade vertical displacement (right), corresponding for the maximum spring torsion . . . . . . . 252

A.2 Force diagram acting on the spring. . . . . . . . . . . . . . . 252

A.4 Scraper blades misalignment for two consecutive arms. $\mathrm{S}=7 \mathrm{~mm}$. . . . 253

B.1 Time evolution of the temperature measured by thermocouples and reference probes inside the freezing cabinet; detail of the stable region from where calibration point was averaged (left), calibration curve with uncertainty intervals for one of the thermocouples (right) . . . . . . . . 256

B.2 Uncertainty curves for all the thermocouples (left) and uncertainty histogram at $-11.5{ }^{\circ} \mathrm{C}$ (right). . . . . . . . . . . . . . . 259 


\section{List of Tables}

1.1 Rheological behaviour of ice slurries according to different authors, taken from $[14] \ldots \ldots \ldots \ldots$

2.1 Dimensions and operating conditions for the heat transfer characterization 75

2.2 Accuracy on the directly measured variables . . . . . . . . . . . 75

2.3 Accuracy on the thermophysical properties of the ice slurry . . . . . . 78

3.1 Total uncertainty result for the PIV experiments . . . . . . . . . . . 102

7.1 Velocity and pressure boundary condition for the two-dimensional model 206

7.2 Turbulence variables boundary conditions for RANS . . . . . . . . . . 207

7.2 Turbulence variables boundary conditions for RANS (continuation) . . . 207 


\title{
Nomenclature
}

\author{
$A \quad$ Heat exchange area $\left(\mathrm{m}^{2}\right)$ \\ $C$ Constant term $\left(\mathrm{W} \mathrm{m}^{-2} \mathrm{~K}^{-1}\right)$ \\ $C_{f} \quad$ Correlation factor \\ $D \quad$ SSPHE Internal diameter $(\mathrm{m})$ \\ E Error \\ $F \quad$ Force $(\mathrm{N})$ \\ $F_{F} \quad$ Total force in freezing conditions (N) \\ $F_{S} \quad$ Scraping force $(\mathrm{N})$ \\ $H \quad$ SSPHE internal height $(\mathrm{m})$ \\ $H_{S} \quad$ Scraper height $(\mathrm{m})$ \\ $I \quad$ Digital image \\ $K$ Correlation constant \\ $L_{f} \quad$ Ice specific latent heat of fusion at $0{ }^{\circ} \mathrm{C}(\mathrm{J})$ \\ $M \quad$ Magnification factor \\ $M_{T}$ Torque $(m \cdot N)$ \\ $N \quad$ Rotational speed $\left(\mathrm{s}^{-1}\right)$ \\ $P \quad$ Power (W) \\ $Q \quad$ Heat flux $\left(\mathrm{W} / \mathrm{m}^{2}\right)$ \\ $S_{P} \quad$ Scraping power $(\mathrm{W})$ \\ $T$ Temperature $\left({ }^{\circ} \mathrm{C}\right)$ \\ TI Turbulence intensity \\ $U \quad$ Fluid flow velocity $(\mathrm{m} / \mathrm{s})$ \\ $U_{p} \quad$ Velocity of the tracer particle $\left(\mathrm{m} \mathrm{s}^{-1}\right)$ \\ $W \quad$ Digital image window \\ $\dot{m}$ Coolant mass flow $\left(\mathrm{kg} \mathrm{s}^{-1}\right)$ \\ $\hat{c}_{p} \quad$ Apparent specific heat $\left(\mathrm{J} \mathrm{kg}^{-1} \mathrm{~K}^{-1}\right)$ \\ a Correlation constant \\ $c_{p} \quad$ Specific heat $\left(\mathrm{J} \mathrm{kg}^{-1} \mathrm{~K}^{-1}\right)$ \\ $d_{i} \quad$ Image distance $(\mathrm{m})$ \\ $d_{o} \quad$ Object distance $(\mathrm{m})$ \\ $d_{p} \quad$ Diameter of the particle $(\mu \mathrm{m})$ \\ $e \quad$ Ice layer thickness $(\mathrm{m})$ \\ $f \quad$ Focal length $(\mathrm{m})$ \\ $f \#$ Aperture number \\ $g \quad$ Gravity acceleration $\left(\mathrm{m} \mathrm{s}^{-2}\right)$ \\ $h \quad$ Heat transfer coefficient $\left(\mathrm{W} \mathrm{m}^{-2} \mathrm{~K}^{-1}\right)$ \\ $i \quad$ Specific enthalpy $\left(\mathrm{J} \mathrm{kg}^{-1} \mathrm{~K}^{-1}\right)$
}


$k \quad$ Turbulent kinetic energy $\left(\mathrm{m}^{2} \mathrm{~s}^{-2}\right)$

$k_{t} \quad$ Thermal conductivity $\left(\mathrm{W} \mathrm{m}^{-1} \mathrm{~K}^{-1}\right)$

$m_{p} \quad$ Mass of the tracer particle

$n \quad$ Number of scrapers

$q \quad$ Transferred heat (W)

$r_{l} \quad$ Radial coordinate of the PIV investigated plane $(\mathrm{m})$

$r_{v} \quad$ Vortex local radial coordinate $(\mathrm{m})$

$s \quad$ Number of scrapers

$t$ Time $(\mathrm{s})$

$t_{n i} \quad$ Nucleation induction time (s)

$u^{\prime} \quad$ Velocity fluctuating component

$v \quad$ Coolant velocity $\left(\mathrm{m} / \mathrm{s}^{-1}\right)$

$v_{c} \quad$ Particle settling velocity $(\mathrm{m} / \mathrm{s})$

\section{Dimensionless numbers}

Fr Froude number $(\Omega(D / 2))^{2} / H g\left(1-\rho_{i} / \rho_{b}\right)$

$N u \quad$ Nusselt number $h D / k_{t}$

Po $\quad$ Power number $\left(\frac{P}{\rho N^{3} D^{5}}\right)$

$\operatorname{Pr} \quad$ Prandtl number $\mu c_{p} / k_{t}$

Re Reynolds number $\rho v D / \mu$

$R e_{\text {rot }}$ Rotating Reynolds number $\rho N D^{2} / \mu$

Ri Richardson number $g \beta \Delta T_{b, w}(H / 2) /(\Omega(D / 2))^{2}$

\section{Greek symbols}

$\beta \quad$ Thermal expansion coefficient $\left({ }^{\circ} \mathrm{C}^{-1}\right)$

$\chi \quad$ Factor proportional to the plate surface covered by ice $\in[0,1]$

$\Delta \mathrm{T}_{\log }$ Logarithmic temperature difference $\left({ }^{\circ} \mathrm{C}\right)$

$\Delta \mathrm{T}_{\mathrm{s}}$ Wall supercooling degree $T_{f}-T_{w}\left({ }^{\circ} \mathrm{C}\right)$

$\delta_{v} \quad r_{v}$ value for the vortex maximum velocity location $(\mathrm{m})$

$\epsilon \quad$ Perspective error

$\Gamma \quad$ Spatial resolution $(\mathrm{nm})$

$\gamma \quad$ Friction coefficient (-)

$\lambda \quad$ Wavelength (nm)

$\mu \quad$ Dynamic viscosity $\left(\mathrm{kg} \mathrm{m}^{-1} \mathrm{~s}^{-1}\right)$

$\nu \quad$ Kinematic viscosity $\left(\mathrm{m}^{2} \mathrm{~s}^{-1}\right)$

$\Omega \quad$ Vorticity

$\omega \quad$ Specific dissipation rate $\left(\mathrm{s}^{-1}\right)$

$\omega_{0}$ Global mass fraction of solute; mass of $\mathrm{NaCl} /$ mass of solution and ice

$\Phi \quad$ Viscous dissipation term (W)

$\phi \quad$ Cross-correlation function

$\rho \quad$ Density $\left(\mathrm{kg} \mathrm{m}^{-} 3\right)$

$\sigma_{u} \quad$ Velocity standard deviation $\left(\mathrm{m} \mathrm{s}^{-1}\right)$

$\Theta \quad$ Rotor angular velocity $\left(\mathrm{rad} \mathrm{s}^{-1}\right)$

$\varphi_{m} \quad$ Ice mass fraction

$\varphi_{v} \quad$ Ice volume fraction

$\zeta \quad$ Supercooling amplitude $\left({ }^{\circ} \mathrm{C}\right)$ 


\section{Subscripts}

$\theta \quad$ Tangential component

$b \quad$ Brine

C Chilling

c Coolant

calc Calculated

cond Condensation

$e \quad$ Inlet

evap Evaporation

exp Experimental

$F \quad$ Final

$f \quad$ Freezing

fr Friction

$h \quad$ Hydrodynamic

$i \quad$ Ice

$\max$ Maximum

$n \quad \mathrm{n}^{\text {th }}$ Element

$o$ Outlet

$p \quad$ Particle

ps Process side

$s \quad$ Subcooling

st Plate stainless steel

$w \quad$ Wall

$x, y, z$ Cartesian components

0 Initial

B Buoyancy

D Drag

T Total

\section{Superscripts}

* Normalized value

- Relative value

- $\quad$ Time averaged value 


\section{Chapter 1}

\section{Introduction}

For more than twenty years ice slurry has been considered as a promising technology in the refrigeration industry. The employment of ice slurry as secondary refrigerant can reduce the large quantity of primary refrigerants used in common expansion systems. Notwithstanding CFCs deplete the ozone layer, and their most employed substitutes, the HFCs, have an important global warming potential. On the contrary, ice slurry is environment friendly and does not represent any danger for the human safety in case of leakage.

Compared with single-phase refrigerants, it is justified by the high cooling capacity given by the latent heat of phase change, representing a very efficient solution. Moreover, the ice slurry can be stored, opening the door to different energy management strategies and increasing its possibilities in refrigeration. Moreover, the interest on the ice slurry goes beyond the classical refrigeration applications, being used also in a wide range of situations that goes from the food preserving to the medical/surgical operations.

Nevertheless, the production of ice slurries is still one of the aspects that requires more research. To sustain the promising aspects of the ice slurry, an efficient, reliable and economic generation is required, adaptable to the different ice slurry applications. From all the available techniques, the generation in scraped surface devices is one of the most promising ones, combining its simplicity with an effective way to control the product characteristics and increasing the heat transfer.

\section{$1.1 \quad$ The ice slurry}

Ice slurry can be defined as an homogeneous mixture of ice particles in an aqueous solutions, which works as a carrier liquid [1]. This carrier liquid, which eventually can be fresh water, is commonly a solution consisting on water and a freezing point depressant. The most common freezing depressants are sodium chloride, ethanol, ethylene glycol and propylene glycol [2].

Both the homogeneity of the mixture and the particle's size distribution can vary, ranging roughly from 50 microns to $1 \mathrm{~mm}$, with typical average values around 200 microns. The particle size distribution, however, has to be more or less uniform to be considered an ice slurry.

The high energy storage density of the ice slurry is based on the latent heat of fusion 
of its ice crystals. The large heat transfer surface created by its numerous crystals also confers to it a fast cooling rate. Compare to liquid, and due to the phase change process, slurry maintains a constant low temperature level during the cooling process, providing also a higher heat transfer coefficient.

A remarkable benefit of the ice slurries is that -for some applications- it can be produced when the demand is low or non-existent, being stored for later use. The high energy storage density together with the ability to be pumped to the desired location once it is required allows to reduce the size of the equipments, with a better energy management [3-5].

The morphology of the ice crystals, their concentration and degree of homogeneity in the slurry play an important role in the ability of the ice slurry to by pumped. Moreover, each ice slurry application may require a mixture with different characteristics.

\subsubsection{Characteristics of the ice particles}

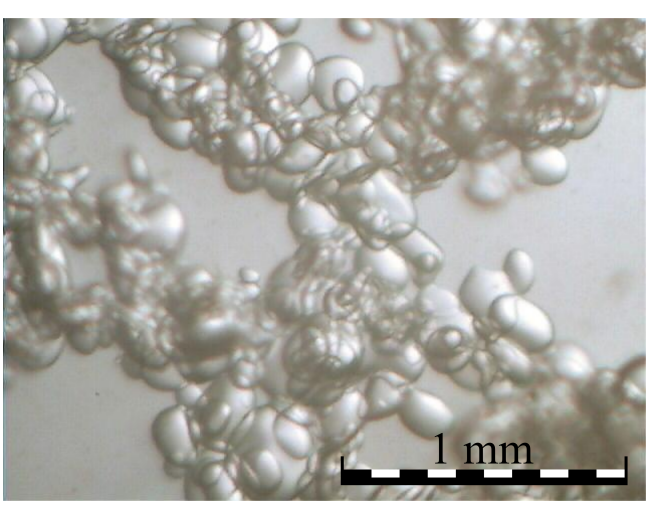

Figure 1.1: A microscopic photograph of the ice crystals in a slurry, showing that common sizes are from $200 \mu \mathrm{m}$ to $300 \mu \mathrm{m}$ to.

The morphological aspects of the ice particles present in the slurries have been studied by different authors. There can be found different reviews of the work in the topic $[2,6]$.

Some of the earliest microscopic pictures of ice slurry particles were due to Kauffeld et al., corresponding to an ice slurry produced with a $3 \%$ wt. $\mathrm{MgCl}_{2}$ additive [7] and by Fukusako et al. for an ethylene-glycol aqueous solution [8]. Different authors like Kasza et al. [9] have assessed that the ice particles in the slurries must be in the micro-scale for a proper performance.

The ice crystals morphology was classified later by Bel [10], who found them to have an hexagonal structure. However, other authors like Sari et al. showed approximately ellipsoidal particles. Indeed in the present work almost all the particles observed responded to the latter pattern (see Fig. 1.1). Kasza and Hayashi [9] identified two type of ice particles that can make a bad ice slurry: dendritic (rough and elongated, which form large entangled clusters decreasing the pumpability) and globular (with rough surfaces that entangle to a lesser degree but still are far from optimum). The quality of the particles can be improved adding a freezing point depressor to the liquid ( $\mathrm{NaCl}$ in the present work), which leads to smoother ice particles that increase the fluidity of the slurry. Decreasing the particles surface roughness allows the ice particles to slip past one another without tangling or agglomerating. In addition, the smoothing also allows the ice slurry to be pumped out of the container much easier and flow through delivery conduits at much higher loadings without plugging.

Aside from the effect of a freezing point depressor, the observed differences in the morphologies can be related to different things. One of them is due to the number of collisions among particles and particles and walls, which may erode them or coalesce, leading to bigger particles. Another explanation comes from something called the 
time behaviour [11]: the mean diameter of the ice crystals growths as a function of time [12]. Other authors like Okawa et al. also observed a time-behaviour during the investigations of the permeability of ice water mixtures [13], but in his case the ice particles exceed the $1 \mathrm{~mm}$ diameter level to be considered ice slurry.

The growth of the particles and their morphological evolution to this process is still not completely clear and further research is needed. Furthermore, the particle growing may also depend on the type of flow that particles are immersed, as well as many other aspects, in which the ice slurry generation technique may play an important role.

What is clear is that as particles size grows, the physical properties of the slurry become time-dependant, as it happens with an increasing number of particles. This should be taken carefully in the slurry storage, which if not well performed leads to a decrease of the rheological parameters (the viscosity and the critical shear stress) of up to $60 \%$. [6]

\subsubsection{Rheological and thermophysical properties}

There are different models describing the rheology and thermal properties of the ice slurries, i.e., density, viscosity, specific enthalpy and ice mass fraction. Very detailed revisions on the matter can be found in $[2,6,14,15]$ and in particular in the Handbook on Ice Slurry - Fundamentals and engineering, which was published by the International Institute of refrigeration.

The measurement of the rheological properties in slurries is always complex. To the difficulties inherent to this type of studies it is added the fact that the ice particles "evolve" with time, temperature, flow shear and many other parameters. Moreover, particles tend to stratify. Hence, existing models may differ considerably from one author to another one. In general, all the models take into account both the effect of the ice particles suspension and the properties of the carrier fluid -including the one of freezing point suppressor. Liquid-solid mixtures are often considered as nonNewtonian, where different models exist all based in yield stress below which no flow is observed. This value depends strongly on the solid particle fraction .

The carrying fluid in ice slurries is generally considered as Newtonian, as can slurries themselves if the ice fraction remains low. The meaning of "low" is something diffuse, but most of the authors agree that the ice mass fraction limit separating the Newtonian and non-Newtonian behaviour is somewhere around $6 \%$ and $15 \%$ [14]. As will be seen in chapter 2, Eq. 2.8 was proposed as a correlation for the apparent viscosity by Christensen and Kauffeld [16]. A summary of the Newtonian to non-Newtonian transition in ice slurries, together with the non-Newtonian model proposed by each author is included in table 1.1 from [14]

Nevertheless, the rheological model measurement depends strongly on the measurement technique -for example, the viscometer type- and the application of the models is very constricted to certain operating situations, as flow in ducts.

The direct measurement of the ice concentration is in rather complicated. Some authors evaluated it from the statistical analysis of microscopic pictures [17]. Later on, Melinder published data on physical properties of numerous aqueous solutions [18] that were very useful for ice slurry applications. These data can be applied to -or combined with- the theoretical models that describe the behaviour of various ice slurries, providing more accurate models. Melinder $[19,20]$ also published later a paper specifically 
Table 1.1: Rheological behaviour of ice slurries according to different authors, taken from $[14]$

\begin{tabular}{|c|c|c|c|}
\hline & $\begin{array}{l}\text { Descriptions of the } \\
\text { slurries }\end{array}$ & Viscosimeters & Results \\
\hline Bel and Lallemand (1996) & $\begin{array}{l}\mathrm{x}_{\mathrm{s}}<33 \% \\
\mathrm{~d}<400 \mu \mathrm{m}\end{array}$ & Bob-and-cup & $\begin{array}{l}\text { Newtonian fluid for } \mathrm{x}_{\mathrm{s}}<12 \% \\
\text { (for } \mathrm{x}_{\mathrm{s}}=12 \%, \mu=28 \mu \mathrm{Pa} \mathrm{s} \text { ) }\end{array}$ \\
\hline $\begin{array}{l}\text { Ben Lakdhar and Lallemand } \\
\text { (1998) }\end{array}$ & $\begin{array}{l}\mathrm{x}_{\mathrm{s}}<28 \% \\
\mathrm{~d}<400 \mu \mathrm{m}\end{array}$ & $\begin{array}{l}\text { Ostwald rheometer } \\
\text { (horizontal tube: } \\
\mathrm{L}=2.15 \mathrm{~m} \text {, } \\
\mathrm{D}_{\mathrm{i}}=14.7 \mathrm{~mm}\end{array}$ & $\begin{array}{l}\text { Newtonian fluid for } \mathrm{x}_{\mathrm{s}}<6 \% \\
\text { non Newtonian fluid for } \mathrm{x}_{\mathrm{s}}=\geq 6 \% \\
\text { When } \mathrm{x}_{\mathrm{s}}>13 \%, \mu_{\text {app }} \text { becomes } \\
\text { dependent on the slurry behaves then } \\
\text { like a pseudoplastic fluid }\end{array}$ \\
\hline $\begin{array}{l}\text { Christensen and Kauffeld } \\
\text { (1997) }\end{array}$ & $\begin{array}{l}\mathrm{x}_{\mathrm{s}}<35 \% \\
\mathrm{~d}<100 \mu \mathrm{m}\end{array}$ & Ostwald rheometer & $\begin{array}{l}\text { Newtonian fluid for } \mathrm{xs}<15 \% \\
\text { (in streamline flow) } \\
\text { Bingham fluid (or pseudoplastic) } \\
\text { for } \mathrm{xs}>15 \%, \mu_{\text {s }}>\mu_{f l u i d}\end{array}$ \\
\hline Jensen et al. (2000) & $\begin{array}{l}\mathrm{x}_{\mathrm{s}}<30 \% \\
\mathrm{~d}<200 \mu \mathrm{m}\end{array}$ & $\begin{array}{l}\text { (horizontal tube: } \\
12.8<\mathrm{D}_{\mathrm{i}}<20.7 \mathrm{~mm} \text {, } \\
\mathrm{L}=2 \times 12 \mathrm{~m}\end{array}$ & $\begin{array}{l}\text { Newtonian fluid for } \mathrm{x}_{\mathrm{s}}<0.15 \% \\
\text { Bingham fluid for } \mathrm{x}_{\mathrm{s}}>0.15 \%\end{array}$ \\
\hline Doetsch (2001) & $\mathrm{x}_{\mathrm{s}}<45 \%$ & $\begin{array}{l}\text { Ostwald rheometer } \\
\text { (horizontal tube: } \\
\mathrm{D}_{\mathrm{i}}=10-22-38 \mathrm{~mm}\end{array}$ & $\begin{array}{l}\text { Newtonian fluid for } x_{s}<10 \% \\
\text { Casson fluid for } x_{s}>20 \%\end{array}$ \\
\hline Royon (1998) & $\begin{array}{l}\text { Slurry stabilized } \\
\mathrm{x}_{\mathrm{s}}<45 \% \\
\mathrm{~d}<300 \mu \mathrm{m}\end{array}$ & $\begin{array}{l}\text { Bob-in-cup, with board } \\
\text { air-gap, and vertical } \\
\text { grooves }\end{array}$ & $\begin{array}{l}\text { Newtonian fluid whose viscosity } \\
\text { depends on the temperature. }\end{array}$ \\
\hline
\end{tabular}

for ice slurry calculation and modelling, including data on the specific enthalpy. One of the most important properties of the ice slurry due to its applications in refrigeration is the apparent specific heat capacity. It can be obtained by taking the derivative of the specific enthalpy as a function of the temperature.

A detailed description of the calculation of ice slurry properties calculation in the present work can be found in the chapter 2, section 2.1.5.

\subsubsection{Fluid dynamics}

The majority of studies on the fluid dynamics of ice slurries are focused on pipes $[14,15,21-23]$ or heat exchangers [24-26]. Because of the high viscosity of ice slurries, they tipically flow in laminar regime with non-Newtonian behaviour, which make the flow very dependent on the geometry. Ayel et al. [14] reported and extensive review of the works on the pressure drop in cylindrical tubes, with a wide variety of solid concentration and ice particle sizes.

The considerations about the flow regime -laminar or turbulent- and flow type -Newtonian or non-Newtonian models- are only valid when the ice particles are homogeneously distributed. In practice this may not be true, especially at low velocities and for some geometries. In those cases the ice particles rise by buoyancy to the top of the tube/device, and stratification (a non-uniform ice concentration profile) occurs. There are different works about the flow types and the corresponding flow phase diagrams (Kitanovski and Poredos [27]) but as they depend on many parameters they are very difficult to determine.

Moreover, when dealing with ice slurry production, the ice content during the initial 
stages is low or non-existent, and it will increase until the desired concentration. This situation, combined with certain types of ice slurry generators as the scraped surface heat exchanger may lead to turbulent or transitional flows, where the flow regime and its rheological properties changes as the ice content increases -specially in a batch production system.

The flow stratification is certainly one of the main concern in the flow or storage of ice slurries. The buoyant force over an ice particle can be significant, specially in low velocity flows or in the stagnant regions of high velocity flows. As explained in [14] the flow stratification depends mainly on the particle size, shape, its weight and the particles concentration (as they interact between them and can slow down the flow or agglomerate). Avoiding flow stratification is very important, as an stratified fluid will increase the pressure drop of the flow, and at the same time can agglomerate, limiting its fluidity. On the other hand, if the flow rate is very high the risk of stratification will be avoided, but it will turn to very high pressure drops. In order to partially simplify the complexity of the stratification study, some authors employed polymer particles ins substitutions of ice -with a similar density- avoiding the effect of the phase change and the temperature dependants [28].

Regarding heat transfer, ice slurries are capable of high heat transfer coefficients, approximately $3000 \mathrm{~W} / \mathrm{m}^{2} \mathrm{~K}$ for laminar and $4500 \mathrm{~W} / \mathrm{m}^{2} \mathrm{~K}$ for turbulent flows [24, 25, $29,30]$. The heat transfer in forced convection showed to depend on the Prandtl and Reynolds and Hedstrom numbers. Stamatiou et al. [31] and Bellas [25] performed heat transfer measurements with plate heat exchangers. Sari et al. [see in ayel 31] found when the flow is laminar the heat transfer in tubes occurs only at the boundary layer level, as the thermal diffusivity is low. The later opens the door to the employment of heat transfer enhancing techniques either passive or active, well explained in Webb [32].

\subsubsection{Applications}

It is without doubt that the main and more promising application of the ice slurry is as secondary refrigerant. However many other applications exists and other new arises continuously. The ability of being pumped and stored and its high apparent specific heat are the main reason of it. The Handbook on Ice Slurry - Fundamentals and engineering [33] provides a complete review of applications until 2005. Other complete and extended reviews on the topic were published by Kauffeld et al [2], Zhang et al [34], Illan [26], Egolf et al [6] and Bellas et al. [35] among others. A brief summary will be done here. The readers are referred to the previous references for more information.

Early usages of the ice slurry include indirect contact cooling applications from comfort cooling of buildings and gold mines to process cooling of breweries. However, new uses have been gradually introduced, including direct contact cooling applications, the fish processing and supermarket display cases. Over the last five years there have been a large number of installations completed in over 40 countries for direct contact cooling of various food products [14]. Together with the applications as a secondary refrigerants, ice slurries have found a place, for example, in the food processing industry, produce packing, fishery, as well as its new emerging application for protectively cooling organs during medical emergencies and surgery.

The main applications can be classified in three different blocks: ice slurry used 
through heat exchangers, ice slurry used directly and ice slurry used in medical cooling:

\section{Applications with heat exchangers}

Ice has been a common way of energy storage, as it could be produced during off-peak demand periods. However ice was not pumped out of the tank. Instead, it was melted and chilled water was piped to the load points. The pumped transmission of ice slurry to the loads reduces the size of piping, pumps and heat exchangers, as less amount of mass is required for transporting the same amount of cooling capacity. The system miniaturization by using ice slurry is clearly observed in Fig 1.2: depending on the ice content the storage tank can be reduced by a factor of 10 compared to chilled water, and the new pipe system by a factor of $1 / 3$. The main drawback is to avoid the ice stratification, specially in the storage tank, which can lead to particles agglomeration and excessive crystal growing.

This combination of an ice slurry production-storage-transport system with the use of heat exchangers [24] is used successfully for building cooling. The ice slurry can be used only as an energy storage medium, or being also pumped to the loads. This field is without doubt one of the more promising applications for the ice slurries. In big buildings it can reduce considerably the amount of primary refrigerant needed, still being able to be pumped at very low temperatures directly to the loads.

Being able to produce ice slurry with high ice fractions $(\approx 50 \%)$, store it, and then extract it for distribution in a piping network to the loads has been still elusive and represents a frontier for this technology. However, new studies appear continuously and continue making progresses on the field [36-38].

Other applications of the ice slurries used in heat exchangers are related with the food industry -as a refrigerant in breweries, large kitchens- and in trucks and railway cooling.

\section{Direct contact food cooling applications}

Direct contact is used mainly to cool down or keep cold products. For instance, in the baker industry the temperature control during dough mixing is essential in any operation. As there is quite a lot of heat released during dough preparation, providing ice slurry instead of cool water can absorb the excess of heat. Hence, ice slurry technology offers an effective solution for dough cooling.

Ice slurry is also used in product packing. For many food products as vegetables, a rapid cooling immediately after the harvest is essential for preserving its quality. Ice slurry is a very effective cooling system for all the kind of vegetables: its liquid consistency allows it to fill any gap between them, with a fast temperature decrease to the phase change. A widely used approach is to inject ice slurry into palletized product cases manually through the hand openings.

Another important application, if not the main one, using ice slurry in direct contact with the food product is the fishery. Fish begins to deteriorate rapidly as soon as it dies, and this deterioration process is accelerated by elevated temperatures. For a good preserving it is essential an immediate chilling to a temperature above the freezing point, keeping it constant through the cold chain. As with the vegetables, ice slurry maximizes the chilling velocity and therefore it has been investigated and used for this 


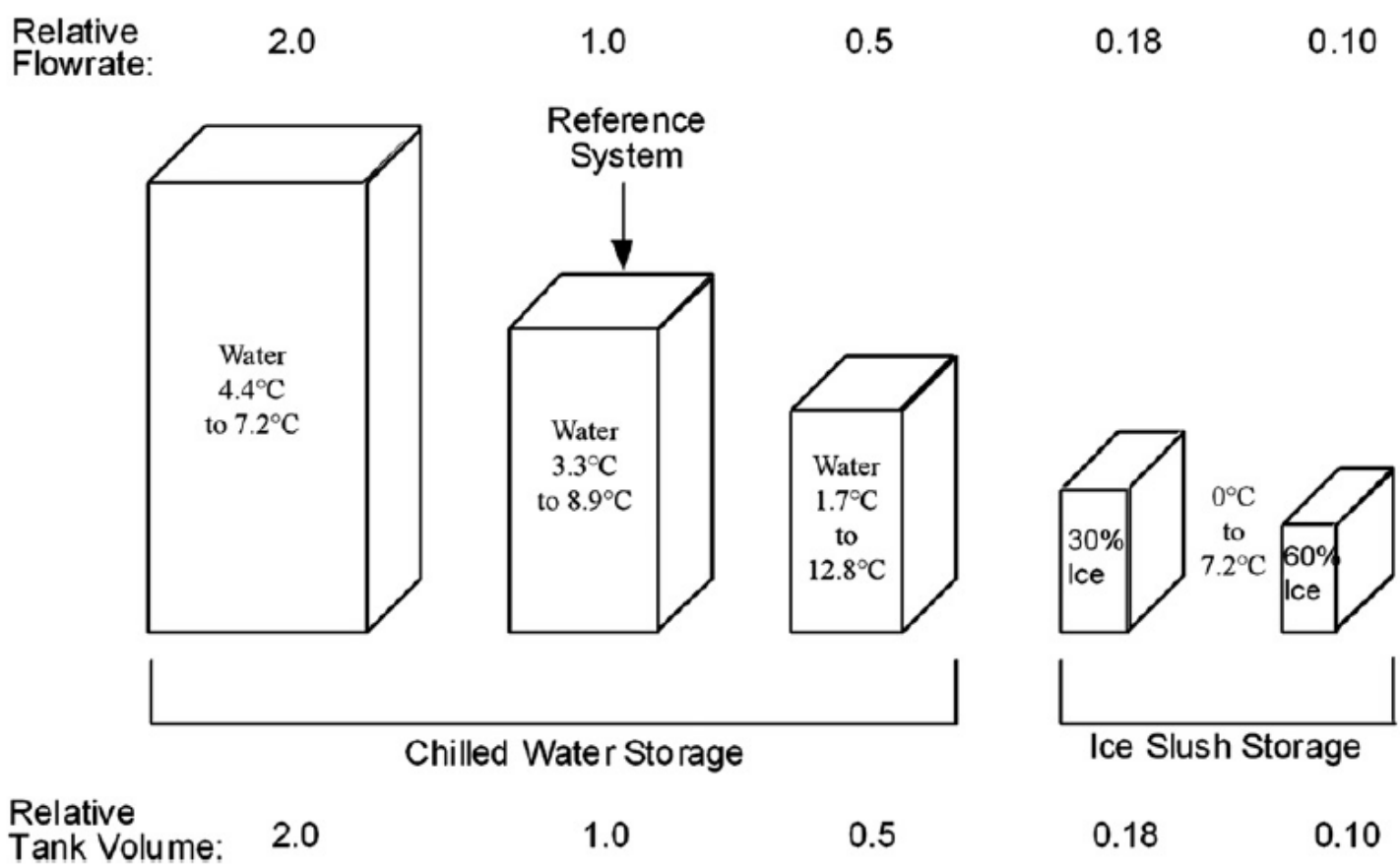

Figure 1.2: Comparison of coolant flow rate and storage tank volume for ice slurry and conventional chilled water [3]

purpose for almost 30 years. Nowadays there are over 700 ice slurry systems installed in the fishery industry, being one of the largest market for this technology. On the other hand, it is also the industry that presents more challenges. In particular, any ice slurry production system is subjected to mobility, as it it is required as soon as the fish is caught. Therefore in most of the cases the generation system has to be mounted on board. The latter brings out two other requirements: a compact and automatic system.

\section{Applications in medical cooling}

Ice slurry in medical applications has to do with inducting protective hypothermia in critical organs. In short, when an organ is cooled down rapidly -about 4-15 K below its normal temperature- it reduces the amount of oxygen it needs before cell death occurs, providing more time for medical treatments. Global cooling frequently fails to protect a specific organ, and is here where ice slurry plays an important role, surrounding directly the target and increasing the cooling velocity. Ice slurry for this application has to be highly controlled both in the composition -saline solutions are usually employed- and in the morphology and size of the particles -must be under $0.1 \mathrm{~mm}$ of diameter.

\subsubsection{Production techniques}

Ice slurry generation is still the most important aspect of the technology, being not yet clear which is the best method. There is a considerable number of ice slurry generation techniques. Whereas some of them are well know and and had been in use for several years, others are still at the initial stage of research. However, even for the older ones 
there is still a lot to do in terms of efficiency and product quality. A complete review of ice slurry generation techniques can be found in $[6,26,33,34]$. Since here they will only briefly introduced, the readers are refereed to the given publications for a more detailed information.

In general, the device selected will depend on the ice slurry application because each technique produces a different type of ice particles. Some types produce dendritic particles whereas other produce more sphere-like particles. The ice slurry generation techniques used and/or investigated until now are enlisted hereafter.

\section{Scraped surface generator}

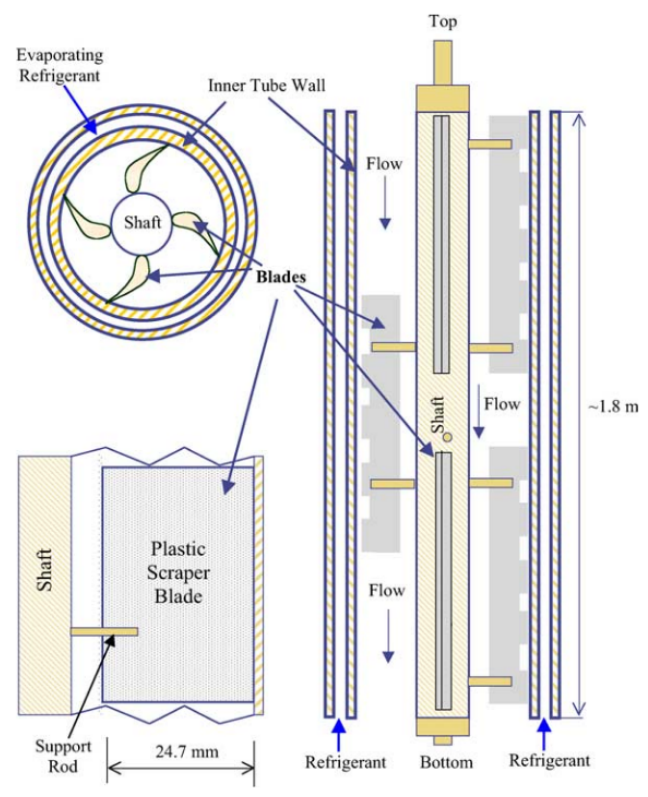

Figure 1.3: Shell and tube scraped surface ice slurry generator (Votator type) [39].
The ice slurry scraped surface generation is one of the more used and investigated techniques over the world, applied in a variety of scales. The most common uses a shell-an-tube heat exchanger in which the evaporating refrigerant flows through the shell side while the ice crystals are generated in the tube side. A rotating set of scraper blades is mounted over a shaft in the center of the inner tube, scraping the crystals from the surface and adding them to the slurry (see Fig. 1.3). As one of the first and most employed techniques, there exist a wide variety of sub-types based on the same principle. Thus, the scrapers can be either plastic or brush type, and the concept has been translated to plate heat exchangers. Has the system studied in this thesis belongs to this group, a more detailed explanation has been included in section 1.2

\section{Orbital rod ice slurry generator}

The orbital rod ice slurry generator also involves moving parts (see Fig. 1.4). It consist of a -usually- vertical shell-and-tube heat exchanger with an evaporating refrigerant flowing from the bottom to the top in the shell side, whereas inside the shell there is a tube bundle formed by many thin tubes. Each of the tubes has a rotating whip rod inside, which does not rotate in the tube center but around the tube circumference. The rotating rods push part of the upper income liquid flowing around the tube circumference to form a falling film. This enhances the film coefficient and the flow turbulence and therefore the heat transfer coefficient between the film and the refrigerant. It also prevents the generated ice crystals from sticking to the heat transfer surface, as the scrapers do in the scraped surface ice slurry generators. The rotation of the whip rod is usually twice the one of the scraper, acting the falling film as the lubricant and minimizing the motor's power consumption.

Even if this type of generator posses a slightly lower cooling capacity, it is also widely used due to its stability and the homogeneity of the produced ice particles. 


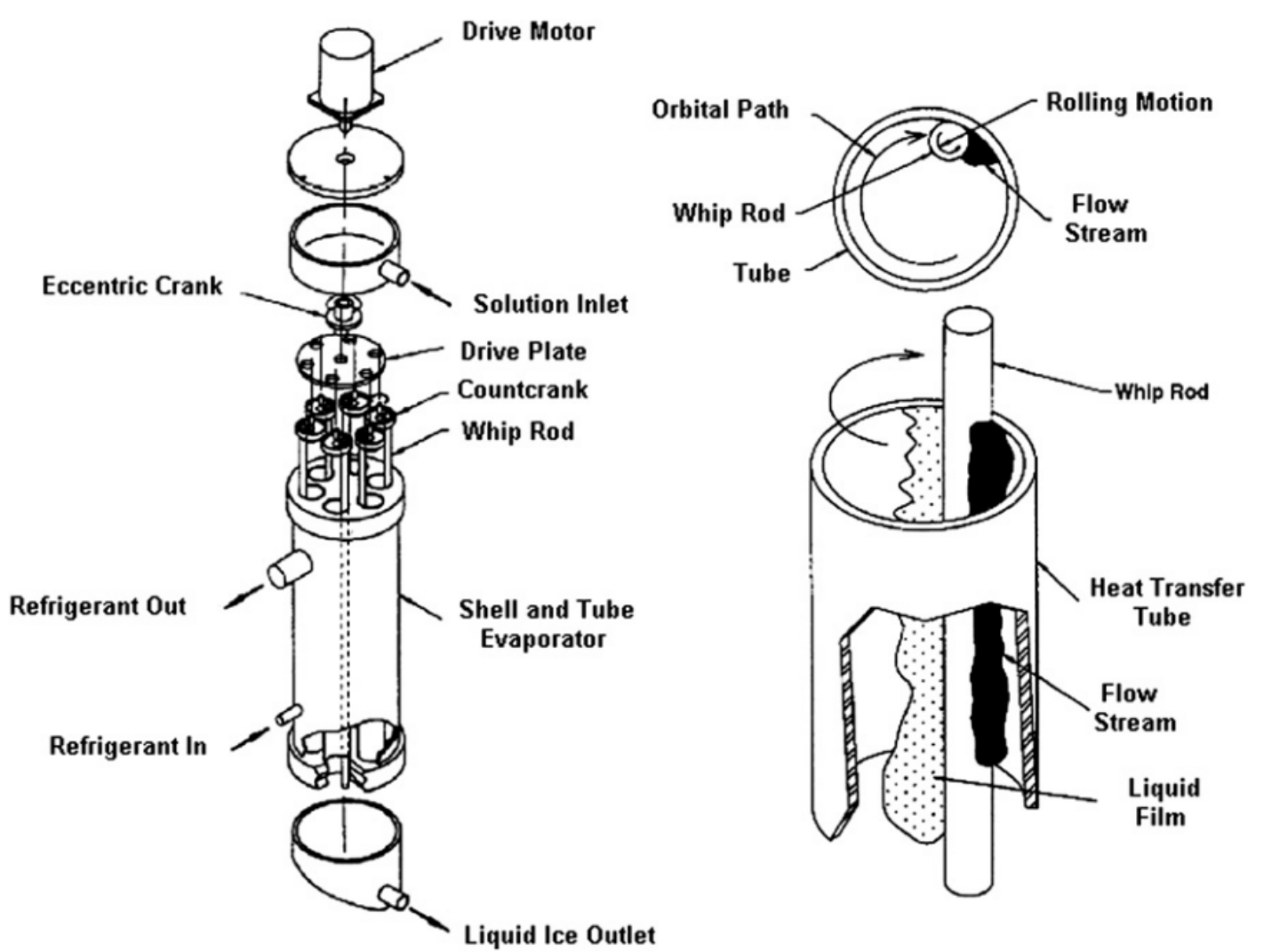

Figure 1.4: Diagram of the orbital rod ice slurry generator [40].

\section{Fluidized bed generator}

In a fluidized bed heat exchanger small solid particles are inserted into the tubes in a shell-and-tube heat exchanger to detach the adherence on the tube walls. The inserted particles are usually spherically shaped stainless steel or globular glass balls. The solid particles are continuously impacting the walls, since the flow is going from down to top. The solid crystals formed in the wall are therefore broken off and added to the fluid. This technique, as the other scraped surface ones, increases the transfer with but has one additional mechanism for it. Not only the boundary layer is broken, but also the solid particles transport the heat from the wall to the fluid. Therefore, the heat transfer coefficient is determined by both the flow velocity of the fluid and also the bed voidage (the ratio of the space that is not occupied by the solid particles to the entire space).

The advantages of the fluidized bed generation include the simplicity of the system with a low operation cost and the homogeneity of ice particles generated due to the violence of the solid particle impacts on the wall. On the contrary, the main disadvantage is the existence of a maximum allowable temperature difference between the fluid inside the tube and the refrigerant (subcooled wall), below which ice slurry can be generated stably with a nearly constant heat transfer coefficient. Higher temperature differences increase excessively the amount of ice adhered to the wall and the impacts of the solid particles are not strong enough to remove it. Additionally, this ice layer 


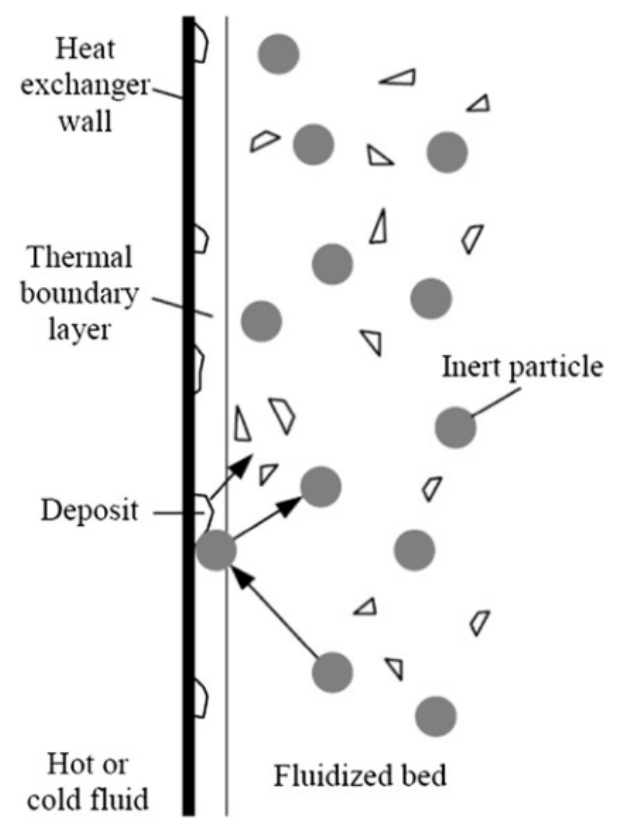

Figure 1.5: Schematic diagram of the effect of fluidized solid particles [41].

decreases the heat transfer coefficient.

\section{Supercooling method}

In the supercooling method the water -or aqueous solution- is cooled in an evaporator to a few degrees below its freezing temperature without crystallization. Then, the supercooled liquid is forced to flow through a supercooling releaser, releasing the supercooling state: ice crystals appear and the liquid turns into ice slurry. The ice crystals are suddenly generated in the liquid, which turns to a solidliquid ice slurry. This method is simple, with low operating costs and without any complex apparatus or mechanical devices. However, it has to be carefully designed to operate in a stable point. Crystals cannot appear inside the heat exchanger, because they will cover the walls, reducing the heat transfer coefficient and finally blocking completely the flow.

As the crystallization has a strong stochastic character, a hard control of the ice generation is required, the evaporation temperature must be controlled very accurately. The influence of the supercooling degree, refrigerant temperature and fluid flow rate on the ice generation has been already investigated, and different models for predicting the fluid temperature variation have been proposed [33, 34], always trying to avoid the ice generation inside the supercooling heat exchanger.

The supercooling releaser is also important to the effective crystal generation, and several releasing approaches have been investigated and utilized. Figure 1.6 shows one of them, where the collision of the supercooled fluid is with liquid bulk in the tank. Other modes use collision with solid surfaces, or with the generated slurry itself. The collision provides the momentum increase to release the supercooling state, where the probability of freezing mainly depends on the collision force. There exist also variants that use the ultrasonic vibration to promote the transition, an extra supercooling of 


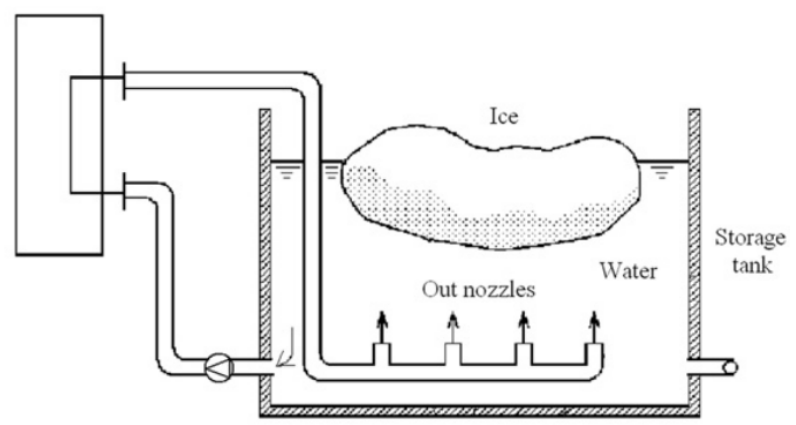

Figure 1.6: Schematic diagram of a supercooling ice slurry generation system [42].

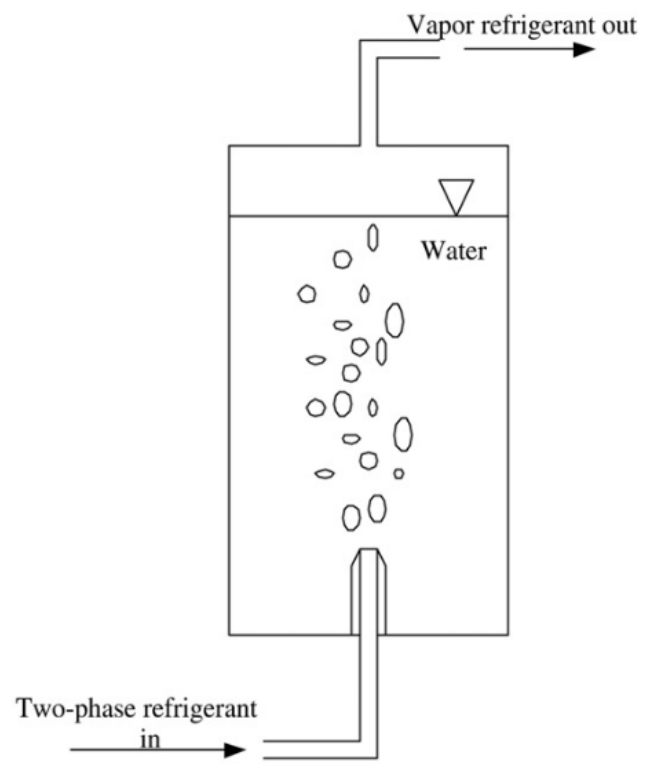

Figure 1.7: Schematic diagram of a direct contact heat exchanger [43].

part of the liquid or even electric charges. The supercooling generation method is, indeed, one of the techniques with more potential and therefore is receiving a significant research attention nowadays.

\section{Direct contact heat exchanger}

This technique uses the direct contact heat transfer between the water-or aqueous solution- with an immiscible cool refrigerant, which includes primary and other secondary refrigerants. Ice slurry can be then generated directly inside the storage tank, as it is shown in Fig. 1.7. On it, primary refrigerant is used, and during the its evaporation dispersed ice particles are generated. The refrigerant can be easily separated as later it turns to vapour. The main drawback is the high pressure needed by the heat exchanger to work, which increases the complexity of the system.

A variant of this system uses refrigerants with lower freezing temperature and usually larger density than water, which make it sink to bottom of the tank after the heat exchange and then returning to the pump and chiller for another cycle. In all 


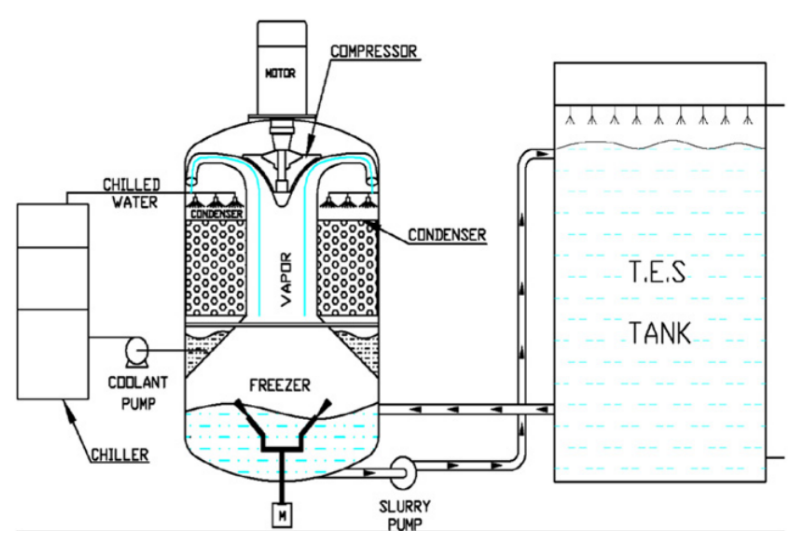

Figure 1.8: Vacuum ice slurry generator used in thermal energy storage system [44].

the variants the direct contact of the slurry with the primary refrigerant leads to the contamination of the first one by the compressor oil, and needs of additional complex solutions solutions to avoid it. Finally, the generated ice crystals are usually in the shape of thin and long flake with 1-4 mm length, which is more larger than those obtained by other methods.

Compared to other ice slurry generators, the utilization of direct heat exchange avoids the sticking of ice crystals on the heat transfer surface and strongly enhances the heat transfer performance. The disadvantages of this method are on the other hand the high requirements of the design and the contamination of the slurry with the refrigerant, which will be later released to the environment.

\section{Vacuum ice slurry generator}

The ice slurry is generated here in a deep vacuum freezer. The water-or aqueous solution- inside the freezer is kept at deep vacuum, close to the triple-point. Part of the water becomes therefore vapour, absorbing the latent heat of vaporization from the rest of the water, which is frozen generating ice particles and the ice-water mixture.

Figure 1.8 shows an schematic view of an ice generator vacuum system. On it, a high velocity centrifugal compressor driven by a motor is used to generate a deep vacuum condition. The generated ice slurry contains ice crystals of $0.5-1.0 \mathrm{~mm}$, and is continuously pumped to the storage tank, where the slurry fraction can reach the $50 \%$.

One of the inconvenients of these systems is the large size of the generator due to large specific volume of water vapour under deep vacuum conditions. Other options are to use lithium bromide vapour absorption machine to generate the vacuum conditions, which is more compact in size. Other variants of this system can be found in [33, 34].

\subsection{Research on the scraper surface ice slurry generation systems}

As mentioned above, the scraped surface systems are one of the most common in the ice slurry generation [39]. Compared with other techniques the scraped surface ones are relatively simpler. But far from being an inconvenience, its simplicity is equivalent 
of more compact and robust systems, with a high level of reliability. They can work at ambient pressure, without any risk of contamination from the refrigerant and they don't require an excessive control system as others like the supercooling technique does. Moreover, the scraping action allows to control the crystals size, and their ability to work in continuous or batch modes makes them able to reach high ice content values.

The scraper surface systems posses an additional advantage, which they share with the scraped surface heat exchangers: the continuous removal of the boundary layer by the scrapers increases the mixing and enhances considerable the heat transfer [45]. This heat enhancement is much more significant when not only the boundary layer but also a solid phase is removed, allowing a constant nucleation on the sub cooled surface.

Among the disadvantages, it is worth to mention that the production units have a relatively high capital cost [33], as the evaporator design has to be made according to the unit, avoiding the use of commercial available ones. The minimum concentration of freezing point depressant is also another reason of the high cost: scraped surface systems require higher concentrations than other systems, because if the concentration is not high enough the scrapers action is not enough to prevent the ice layer scaling on the walls. The later will also require lower freezing temperatures. The abrasion of the blades, which need to be replaced from time by time, increases also the maintenance costs.

These systems can be mainly divided in two types: tubular and plate devices. The first ones have received more attention from researchers, and several commercial models exists since several years, partially promoted by the knowledge accumulated in the tubular scraped surface heat exchangers $[32,45]$. The research works studying the second type are more scarce, and even though several works have been published in the last years, the different phenomena occurring inside have been not deeply investigated yet.

\section{Tubular SSHE production systems}

As mentioned before, these type of devices have been quite more investigated for ice slurry production than any other one of the scraped surface techniques. Indeed, most of the commercialism systems belongs to this type. A good review of them can be found in the Handbook on Ice Slurry by the International Institute of refrigeration [33].

The knowledge on these ice generation systems is a natural extension of the one in the single-phase heat exchangers, where the scraping action prevents fouling and enhances the heat transfer. Heat transfer in tubular SSHEs, mostly regarding horizontal models -where an inner cylindrical surface is continuously scraped by means of rotating blades- have been therefore widely investigated [48]. One of the most extensively used models for describing heat transfer in SSHEs uses the penetration theory. Although several researchers have questioned its validity in the transition and turbulent region $[49,50]$, other studies confirmed that liquids having low viscosity could be adequately described using the penetration theory [51], and it has been subsequently modified to include the effects of parameters such as rotating velocity and number of blades [52].

There are different studies on the ice slurry production considering tubular geometries, mostly with rotating blades -even if the linear, alternative movement of scraper devices inside the tube has been also employed [26]. Stamatiou et al. [39] summarized the available Nusselt number correlations for this type of systems. Goede [53] 


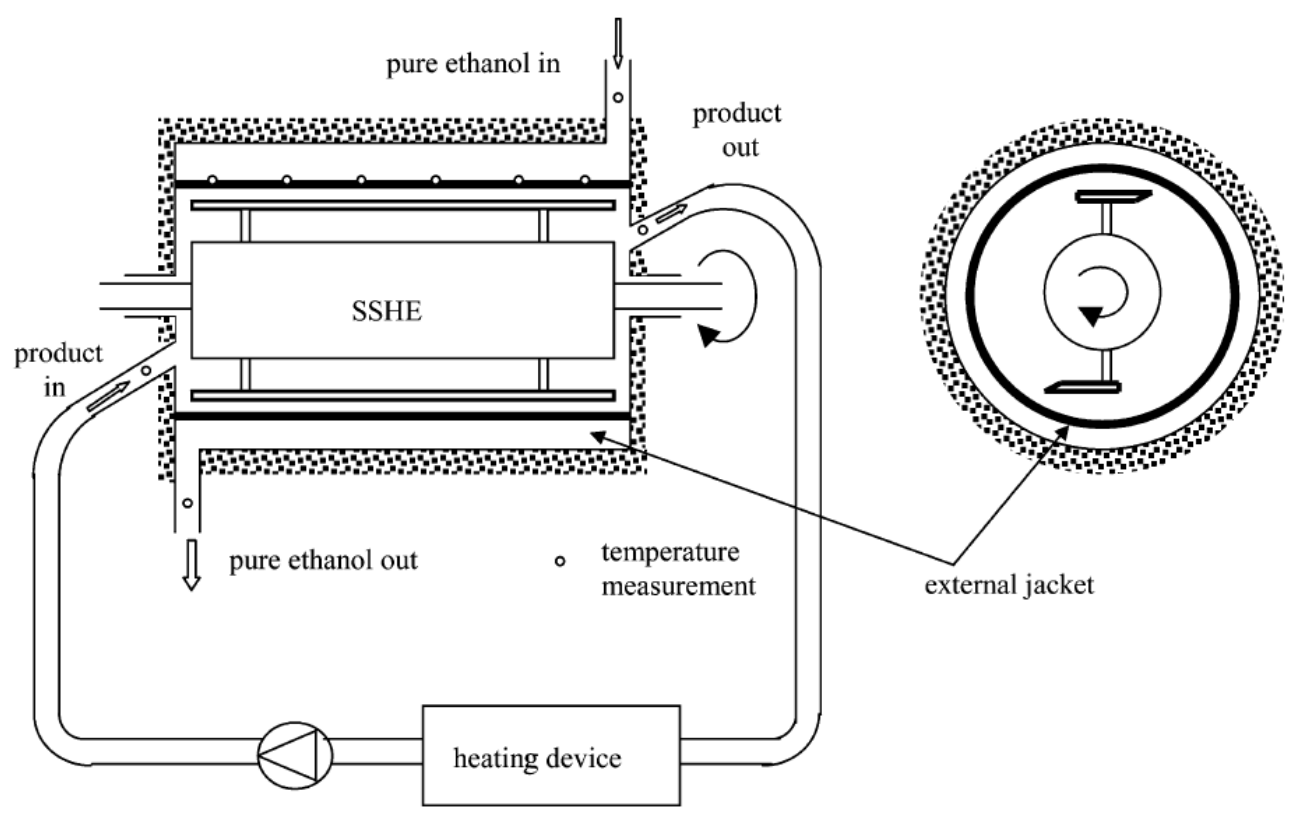

Figure 1.9: Tubular SSHE investigated by Lakhdar et al. [46]

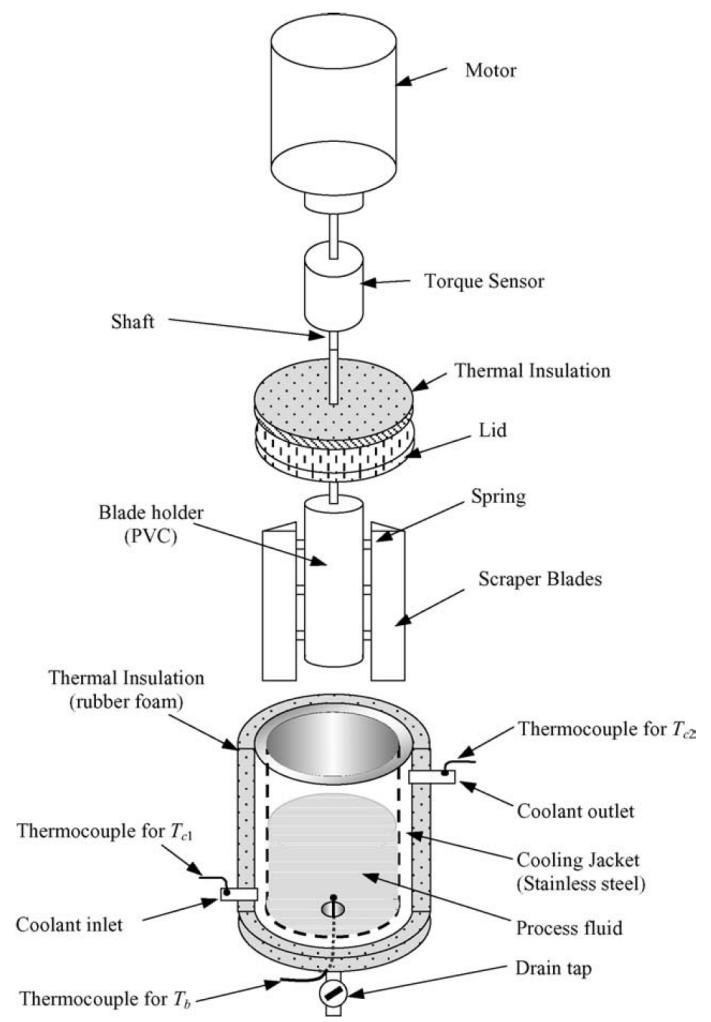

Figure 1.10: SSTHE investigated by Qin et al. [47] 
was one of the firsts to study the use of tubular SSHE in crystallization processes, including the heat transfer in the the analysis with a comparison with the predictions of the penetration theory. Time later, Lakhdar et al. [46] studied the continuous ice slurry production in a tubular, horizontal SSHE from a sucrose aqueous solution. The authors measured the slurry and coolant bulk temperatures, detecting also the supercooling in the solution prior to the onset of nucleation. However, the wall temperature was measured only in the coolant side.

Qin et al. $[47,54]$ studied a vertical configuration of a tubular SSHE producing also ice slurry from a water-sucrose solution. However, they performed batch experiments, arriving to ice contents of around $60 \%$. The authors also reported data of the shaft torque, relating it with the production stages, and finding that as it increases the ice content increases. However, and as reported in [46] the scraped wall temperature was not measured, and hence the heat transfer coefficient on that surface could only be retrieved based in the global heat transfer coefficient and the coefficient in the coolant side. Regarding the flow pattern study, there exist remarkable works [55] in terms of the flow pattern study) using the particle image velocimetry technique, but where unfortunately the effect of the solid phase could not be included.

\section{Plate SSHE production systems}

Several investigations have been reported in the open literature about crystallization in scraped surface plate heat exchangers (SSPHEs). One of the earliest works was done by van der Ham et al. [57, 58], where a column disk crystallizer was investigated for water purification and phase separation. The works focused more on the phase separation and did not report meaningful data on the heat transfer. The same research group continued investigating the same device and later on Vaessen et al. published dif-

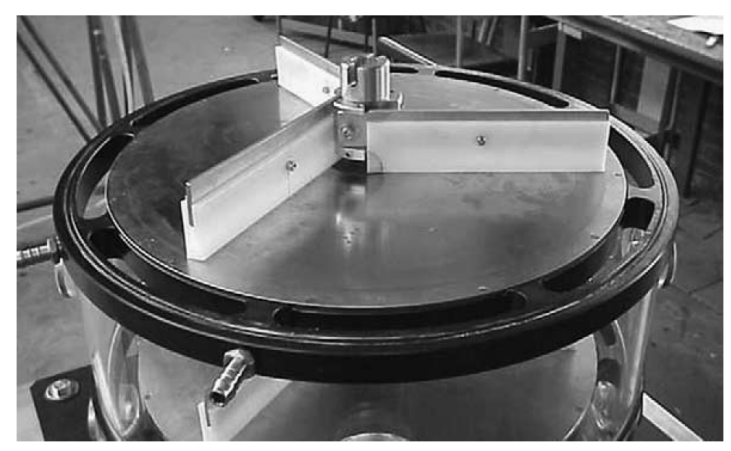

Figure 1.11: The CDCC investigated by Vaessen et al. [56] ferent works with an special attention to the heat transfer $[56,59,60]$. The investigated scraped surface plate heat exchanger, called by them cooled disc column crystallizer (CDCC) (Fig. 1.11) consisted in a cylindrical volume where a disc was refrigerated underneath through a coiled circuit by the flow of a coolant.

The authors studied the effect of the electrolyte concentration and the scraping velocity on the heat transfer from the cooled wall surface to the process liquid (without phase change). The results could be accurately described by the penetration theory, which assessed also its applicability to the phase change situation. However they did not calculate the thermophysical properties of the slurry and therefore not direct comparisons with the penetration theory were presented during the phase change. As a major conclusion, the authors observed that the increase of ice content in the crystallizer probably dominates over the increase of the scraping velocity, decreasing the process-side heat transfer with higher temperature differences and scraping rates.

According to the authors the behaviour of the SSHEs when used for crystallization 
processes can be characterized by the induction time, defined as the maximum interim value between two ice removal actions of the scraper. Vaessen et al. found that higher supercooling degrees led to lower induction times, whereas the opposite was found regarding the electrolyte solute content.

The effect of the temperature difference between wall and coolant in the ice layer scaling was reported by Pronk et al. [61]. However they did not directly measured the wall temperature but the slurry bulk temperature and the coolant inlet and outlet temperature. Rodriguez-Pascual et al. [62] measured the wall temperature by Liquid Crystal Thermometry (LCT), and reported also the flow pattern in radial planes through PIV. They observed the influence of the wall temperature in the ice layer scaling, and assessed the importance of an uniform surface cooling to avoid local ice scaling points. However, the LCT technique cannot provide valid data as the fluid becomes opaque due to the ice crystals in suspensions.

Further investigations from the same group (Rodríguez-Pascual et al. [63, 64]) focused on the flow pattern and particle motion, including LES numerical simulations. Their experiments provided a good understanding of the effect of the scrapers geometry on the particles distribution after being scraped. The stereoscopiv PIV experiments also revealed the strong radial component in the flow pattern induced by the scrapers. Nonetheless, the particles employed were more dense than the liquid phase, and were released with a non-developed flow. Hence, the effect of buoyancy that occurs with the ice particles was not reproduced experimentally.

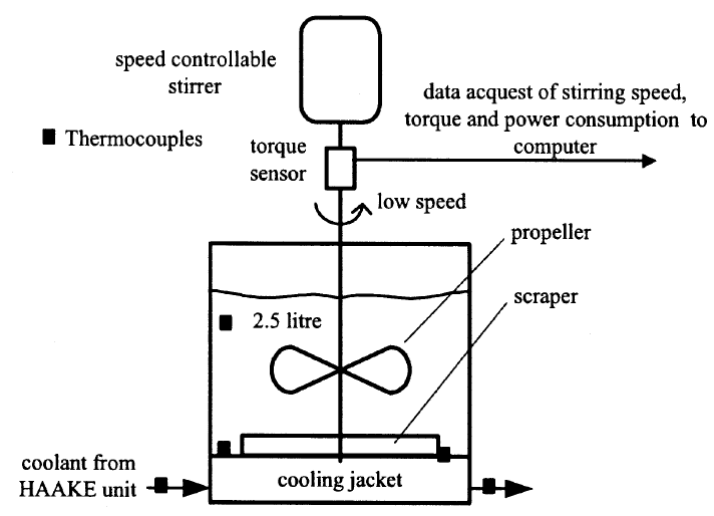

Figure 1.12: The SSPHE investigated by Qin et al. [65]

In parallel, a different research group carried out several investigations on a similar horizontal scraper surface plate heat exchanger. The device, shown in Fig. 1.12 included a propeller at the middle heigh of the tank to promote the slurry mixing. Qin et al. [65] performed a more in depth study of the heat transfer process with an special focusing on the nucleation onset, describing the three stages in which the formation of the ice slurry occurs: chilling, nucleation and crystallization. The authors observed increased heat transfer coefficients for the stage with crystallization, arguing that although crystal formation on the cooling surface is considered disadvantageous to heat transfer, liberation of latent heat of crystallization on the cooling surface actually increased the heat transfer.

The same authors reported afterwards a study on the heat transfer and power consumption in a SSHE while freezing aqueous solutions [66] and on the ice fouling on a subcooled metal surface [67]. They found that the reduction of the cooler surface supercooling degree may delay the ice fouling and prolong the induction time or even perhaps avoid it. However they did not measure the temperatures at the subcooled wall. Qin et al [68] obtained a similar conclusion as Vaessen [59], finding that the fouling induction time is mainly determined by the degree of wall supercooling. Therefore, a constant presence of a thin ice layer can be assumed, due to the inefficacy of the 
scrapers to remove it completely, whereas the scale formation is only avoidable by a real mechanical removal, entailing a sudden decrease on heat transfer.

In spite of the work done, the complex fluid mechanical and heat transfer phenomena occurring in such systems is the responsible of the lack of any meaningful heat transfer correlations based on the physical phenomena during the forced-convective crystallization involving phase change and agitation. The design of SSHEs for ice slurry generation does not attend to general guides, depending mostly on the operating conditions. Moreover, very little information is available regarding the ice crystallisation process in the scraped surface. Most of the theories depend on punctual evidence, somewhat speculative.

\subsection{Previous works on the research group}

The present doctoral thesis is framed in the research line of the group regarding scraped surface heat exchangers and heat transfer enhancement. Different doctoral thesis have preceded this, acting as a support and being the base of the knowledge in many concepts used here.

Illan [26] first worked in the ice slurry topic on his thesis. He used a tubular scraped surface heat exchanger with alternating linear scraper devices to produce the ice slurry, which was after stored in an agitated tank. The experimental work focused more more on the ice slurry performance working as a secondary refrigerant in different tube geometries and heat exchangers. The author proposed several correlations to predict the heat transfer, based on the ice content of the slurry and other governing parameters as the Reynolds number based in the slurry thermophysical properties.

Solano [69] carried out an experimental study on tubular scraped surface heat exchangers where the scrapers consisted in alternative linear movement devices. The scope of the work included different scraper geometries working under different forced flow rates and scraping velocities. Heat transfer and pressure drop experiments provided a wide range of data pertinently reduced through the Reynolds, Prandtl and Nusselt numbers and the velocity ratio of the fluid and scrapers velocity. The author ended with several correlations that were able to predict the heat transfer as a function of the governing parameters. Moreover, he did perform also visualization experiments, establishing the flow pattern and relating it with the heat transfer.

Crespí [70] continued the work of Solano extending the experiments to non-Newtonian flows. A cellulose based powder was used as additive to prepare aqueous solutions with a power-law -mainly- behaviour. The author performed experiments to determine both the pressure drop and the heat transfer, reducing the data with modified expressions of the Reynolds and Nusslet numbers that included the non-Newtonian effect through a modified viscosity model. Different correlations were also presented.

\subsection{Objectives of the work}

As the existent works on the literature show, ice slurry production in scraped surface heat exchangers depends strongly on the device and operating parameters. The available data on research in tubular devices is therefore practically useless -at least in a direct manner- when plate heat exchangers generation systems have to be designed. 
On the other hand, the studies focused on this type of devices are scarce. Due to the inherent technical difficulties and to the complex physical phenomena involved in the process, practically there are not experimental data on such systems operating under really ice slurry production conditions considering the different number of parameters that may play a role.

Heat transfer is an important part of the process and has to be characterized accurately. The measurement of variables like the scraped wall temperature or the heat flux transferred through that wall becomes then essential to understand the problem. At the same time, the flow pattern has a crucial part in the production, as it is not only the responsible of the convective heat transfer on the wall but also of the product mixing and evolution till the ice content level reaches the desired level. Finally, the obtained product characteristics, in terms of the morphology of the ice particles, must also be considered as the fluidity of the slurry and its heat transfer properties will depend on them.

The aim of the present work is then to perform a study on the ice slurry production in a scraped surface plate heat exchanger working in batch mode taking into account the different aspects of the problem which are relevant to it: heat transfer, flow pattern, power consumption and product characteristics. The operating conditions that have to be considered are the scraping velocity, the wall subcooling and the initial freezing point depressant concentration. The objectives that must be reached in each aspect can be defined as follows:

1. The heat transfer has to be characterized through the governing parameters of the physical problem, which must include variables like the thermophysical properties of the aqueous solution or the ice content in the slurry. For that, the temperature on the scraped wall has to be measured properly in order to study not only the heat transfer coefficient, but also the effect of the phase change on the heat transfer process. One or several correlations must be proposed relating the heat transfer with the other governing parameters.

2. The flow pattern has to be studied including the single phase and two-phase flow problems. The single phase flow, characterized by the rotating Reynolds number, represents the situation where the ice content is very low, or the slurry is completely homogeneous, behaving as a Newtonian fluid. It has to be defined then for which rotational velocities is the flow homogeneous or not, and what is the effect of the scraping velocity on the ice particles distribution.

3. The power consumption must be related with the production process as it is one of the main design parameters. The mechanical poser coming by the shaft torque is one of the main concerns as intervenes directly in the problem, but the global performance of the system including the refrigeration unit must be also considered.

4. The product characteristic, regarding the ice crystals morphology is of great importance for the latter applications of the ice slurry. Size and shape of the particles have to be measured and related with the operating conditions during their production. 


\subsection{Research methodology}

The proposed research methodology was developed to accomplish all the above described objectives. Based on that two different approaches were established, one experimental and one numerical. The experimental approach is in turn composed by two sub-approaches: one for the heat transfer, power consumption and product characteristics and the second one for the flow pattern study.

In the first experimental sub-approach, an experimental facility was designed and built for the analysis of the phase-change heat transfer process that occurs during the ice slurry generation, using sodium chloride $(\mathrm{NaCl})$ as a freezing point depressant. The effect of the wall subcooling degree -the difference between the wall temperature and the freezing point temperature of the aqueous solution-, the rotating velocity and the initial $\mathrm{NaCl}$ were investigated through a proper measurement of the problem variables as the rotating velocity, thermopysical properties, scraped wall temperature and heat flux across the surface. The experimental data were reduced to the governing dimensionless parameters to characterize the different operating conditions. The power consumed by the shaft driving motor was also measured, whereas the ice particles morphology was studied by microscopic images. In the second experimental sub-approach, a visualization facility was built with the same dimensions of the heat transfer rig. The facility allowed to do particle image velocimetry experiments on a single phase fluid, obtaining the flow velocity field. In addition, medium density polyethylene -with a density similar to the one of ice- particles were used to observe the two-phase flow and estimate the particle motions after being scraped from the surface.

Alternatively, numerical simulations were performed to complete the information on the flow pattern and particle motion provided by the experimental results. Ice particle motion was studied through a 2D, lagrangian model considering two different turbulence modelling approaches: RANS and LES. The main purpose of these simulations was to stablish the limit on the scraping velocity that ensures no stratification for the solid particles. On the other hand, the single-phase flow inside the scraped surface heat exchanger was studied through a 3D, steady model using the rotating reference frame methodology. The expectable complexity of the flow, with high velocity and pressure gradients, and the associated mesh requirements reduced the simulations to single-phase flow, with RANS turbulence modelling.

\subsection{Thesis outline}

The dissertation consists on nine chapters, which describe the work and results of the doctoral thesis:

Chapter 2 This chapter presents the experimental facility for the ice slurry production tests and the planning of the experiment cases. A detailed description of the different elements that compose the facility is made, including the design parameters of the scrapers. The calculation procedure of the different slurry thermophysical properties is also included here. The different measurement techniques employed are enlisted with the determination of the corresponding uncertainties. 
Chapter 3 This chapter describes the flow visualization experimental facility. A brief introduction to the particle-cloud tracking techniques, and in particular to particle image velocimetry (PIV) technique is included. The uncertainty estimations are finally reported.

Chapter 4 This chapter reports the results for the ice slurry production experiments, performed with a single initial $\mathrm{NaCl}$ concentration equal to $7 \%$. The effect of the rotating velocity of the scrapers, wall subcooling degree and ice content in the slurry is studied. The data is reduced through the rotating Reynolds number, the Nusselt number and the Froude number. The chapter also presents microscopic images of the obtained ice particles for a $20 \%$ of ice content.

Chapter 5 This chapter extends the results of the ice slurry production experiments to different initial $\mathrm{NaCl}$ concentration values. The chapter also includes the results of the driving motor power consumption, pertinently non-dimensionalized by the Power number. A global performance analysis is also performed considering the $\mathrm{COP}$ of the refrigeration unit to determine what is the optimal operating point.

Chapter 6 This chapter analyses the flow visualization results. A first part of the chapter presents the particle motion results performed with the polyethylene particles. A second part of the chapter presents the PIV results and describes the encountered flow pattern. The flow field velocities are expressed in terms of relative velocities to the scraper, and non-dimensionalized by them.

Chapter 7 This chapter introduces the numerical modelling procedure. A brief description of the Finite Volume Method is done first, explaining after the different approached to model turbulence. The two different models are explained: 2D, lagrangian particle tracking and 3D fluid flow simulations, including their respective computational domain, boundary conditions and meshes.

Chapter 8 This chapter report the results obtained from the numerical simulations. In the first part of the chapter the evolution of the ice particles is presented for the different scraping velocities and turbulence model approaches. The second part of the chapter includes the results for the 3D simulations, describing the flow pattern and relating it with the flow visualization experimental results.

Chapter 9 This final chapter summarizes the results of this work and outlines the main conclusions. 


\section{Chapter 2}

\section{Materials and methods I: Ice slurry experimental set-up}

The characterization of the scraped surface heat exchangers in terms of heat transfer and product quality is of great importance from an industrial perspective. The requirement of considering simultaneously -as they do interact- all the phenomena above mentioned discard most of the available approaches. Computational methods have proved their success in fluid dynamics and heat transfer research and design. However, being a good approach for particular aspects -study of the fluid flow in the SSPHE- they fail in combining phenomena like ice adhesion and breakage with the phase change and forced convection. Using them for a global consideration of the problem would result either impracticable or excessively inaccurate. Moreover, the singular geometry of the SSPHE makes the problem particular. The available analytical or empirical models are therefore not useful beyond giving approximate values. An experimental approach considering the whole process is then imperative for a complete characterization of the SSPHE.

In the present work two different aspects are considered: the analysis of the heat transfer during the slurry production and the study of flow inside. According to that, two different facilities are employed. The one located in the Technical University of Cartagena produces ice slurry in batch mode, allowing the measurement of the different process temperatures, mass flow and power consumption for different scraping velocity and brine types. Moreover, the final slurry product can also be analysed through microscopy. The second facility is used for flow visualization. The two-phase flow was emulated by using Polypropilene particles emulating ice, whereas the single phase flow was analysed by the Particle Image Velocimetry (PIV) technique. The description of the first facility together with the explanation of the experimental procedure is detailed in this chapter.

\subsection{Ice slurry experimental set-up}

The ice slurry experimental set-up is located at the Technical University of Cartagena. Conceived specifically for the heat transfer analysis in the SSHPE during ice slurry production, it was designed to test different operating conditions. Thus, experiments with different rotating Reynolds number, initial brine freezing temperature and wall 


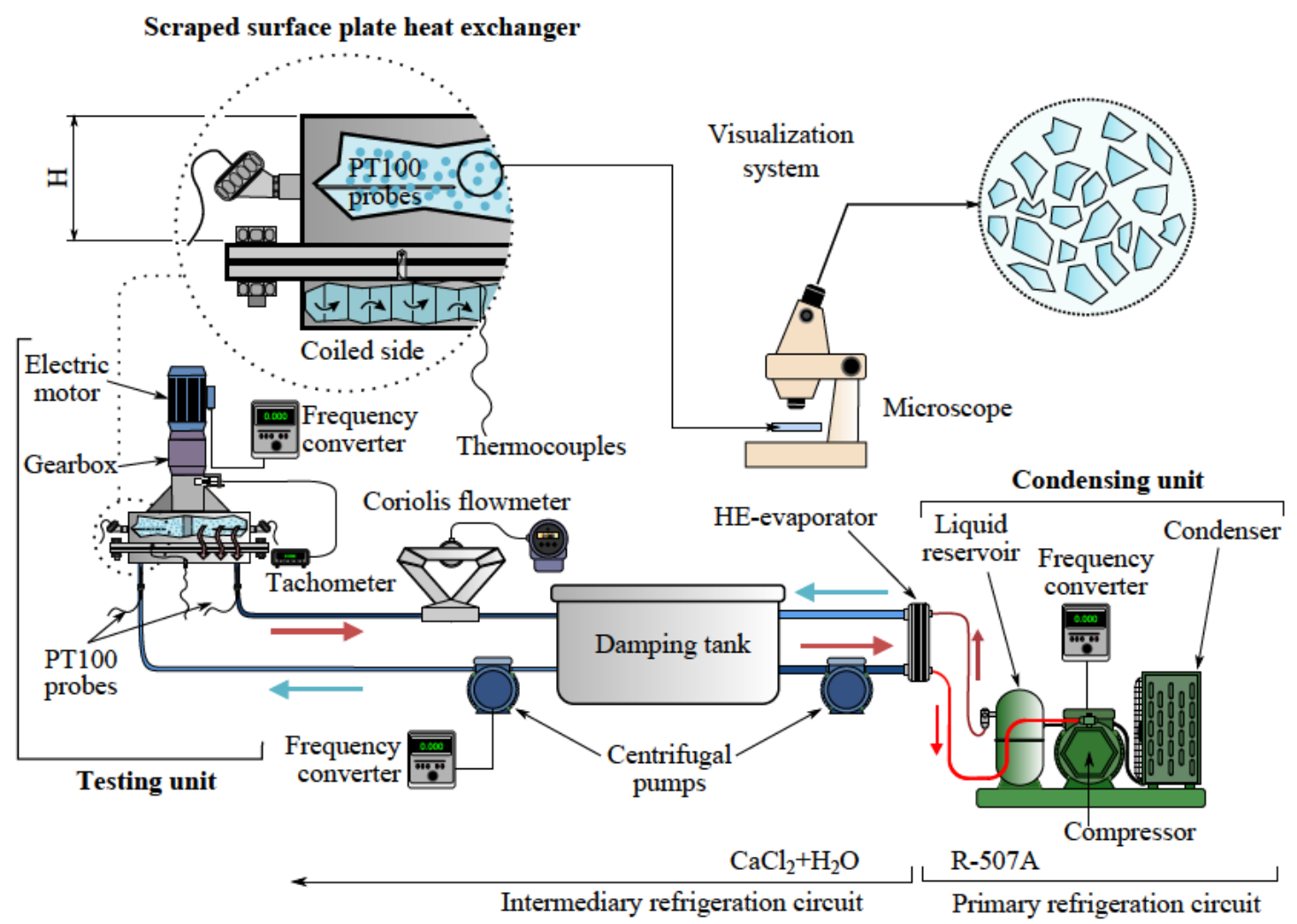

Figure 2.1: Schematic view of the experimental facility

supercooling degree. Additional, it provides information about the characteristics of the obtained product (e.g, ice crystals morphology) and the scrapers effectiveness. The main capabilities of the facility are described hereafter:

- Providing of heat transfer data during both the chilling and freezing stages, including different ice content values

- Testing of different operating conditions to study the effect of the rotating Reynolds number, initial brine freezing point and wall supercooling degree (controlled through the coolant temperature)

- Testing of different scraping conditions by means of different scraper models. Each scraper provided different contact degree and pressure over the plate

- Providing of data regarding the scraping motor power consumption for each testing condition

- Providing of data regarding the ice slurry at different testing times, in terms of ice content and crystals morphology

- Providing of qualitative information regarding the ice scaling in the scraped surface

A schematic diagram of the experimental set-up is presented in Fig. 2.1. It is composed by two circuits aimed for refrigeration (primary and intermediary) and one 
for the brine loading and slurry unloading of the SSPHE The heat exchanger (ice slurry production unit, or testing unit) consists of a $28 \cdot 10^{-3} \mathrm{~m}^{3}$ AISI 316 tank of $0.6 \mathrm{~m}$ diameter and $0.1 \mathrm{~m}$ height with a scraped heat transfer surface at the bottom. Different concentrations of sodium chloride brine (2.9\%-9.4\%), accurately measured by a conductivity meter, is employed as the base solution for the generation of ice slurries in a batch process (see sec. 2.1.5).

The heat transfer surface is cooled underneath by a constant flow of calcium chloride solution in water $\left(25 \%\right.$ wt., $-9{ }^{\circ} \mathrm{C}<T_{c}<-4{ }^{\circ} \mathrm{C}$ ), which flows across a coiled circuit (Fig. 2.4a). To minimize the heat transfer resistance in the bottom side of the SSPHE, the coolant flow was the maximum possible provided by the centrifugal pump ( $\dot{m}=1.5$ $\mathrm{kg} \mathrm{s}^{-1}$ ), kept constant during all the test duration. By this way a turbulent flow was ensured in the coiled circuit $\left(R e \sim 10^{4}\right)$. The coolant is stored in a damping tank of 0.3 $\mathrm{m}^{-3}$-used to avoid instabilities in the coolant temperature- and subsequently cooled in a compact heat exchanger-evaporator, conforming the intermediary refrigeration circuit (Fig. 2.1). The coolant inlet and outlet locations for the SSPHE and the HE-evaporator circuits, as indicated in Fig. 2.1 are specifically located to avoid any stratification in the tank enhancing the mixing inside.

The HE-evaporator is a plate heat exchanger (Swep V25THX with 20 plates of AISI 316 stainless steel), where the expansion of a R507 refrigerant flow is used to cool down the calcium chloride solution. The circuit for the refrigerant (primary refrigeration circuit in Fig. 2.1) consists of an expansion valve and a commercial condensing unit Bizter LH32/2HC-1.2Y, with compressor, condenser and liquid tank, and a maximum cooling power at the evaporator of $2.98 \mathrm{~kW}$. The compressor operating regime is settled by a frequency converter regulated by a PID controller, providing a constant coolant temperature during the running of the experiment.

Regularly distributed thermocouples are embedded in the rear side of the heat transfer plate (see Sec. 2.1.3) with an accuracy of $\pm 0.3{ }^{\circ} \mathrm{C}$. They provide an indirect measurement of the wall temperature in the scraped surface, while four immersed PT100 sensors placed $90^{\circ}$ apart circumferentially at $50 \mathrm{~mm}$ of height retrieve the bulk fluid temperature of the $\mathrm{NaCl}$ brine in the SSPHE upper side. The heat transfer to the cooling circuit is accounted for with PT-100 sensors which measure the inlet and outlet temperature of the coolant flow, whereas a Coriolis flow meter is used for obtaining the mass flow rate of coolant. The heat exchanger and the coolant tubes are fully insulated by an elastomeric thermal insulation material of $30 \mathrm{~mm}$ thickness and thermal conductivity $0.04 \mathrm{~W} /(\mathrm{m} \cdot \mathrm{K})$, in order to minimize heat losses to the ambient. Wall temperature measurements under the most unfavourable conditions reported maximum losses of $4 \%$ respect to the heat exchanged inside the SSPHE.

The coolant mass flow proved to be high enough to minimize the temperature differences across the heat transfer plate, were the maximum found standard deviation among them was of $\approx 0.2{ }^{\circ} \mathrm{C}$.

The scraper system is composed of four rotating blades $-90^{\circ}$ apart circumferentiallydriven by a rotating shaft. A frequency controlled electrical motor $(750 \mathrm{~W}, 1405 \mathrm{rpm})$ is connected to the shaft through a gearbox (ratio 26:1). The power consumed by the motor is measured by a wattmeter, placed between it and the frequency converter. Each blade is made of a steel arm where the actual scrapers are mounted. The scraper are made of PEEK, a polymer with high resistance to the erosion. As detailed in Sec. 2.1.1, two different configurations of scrapers were tested: rigid and adaptable. The 


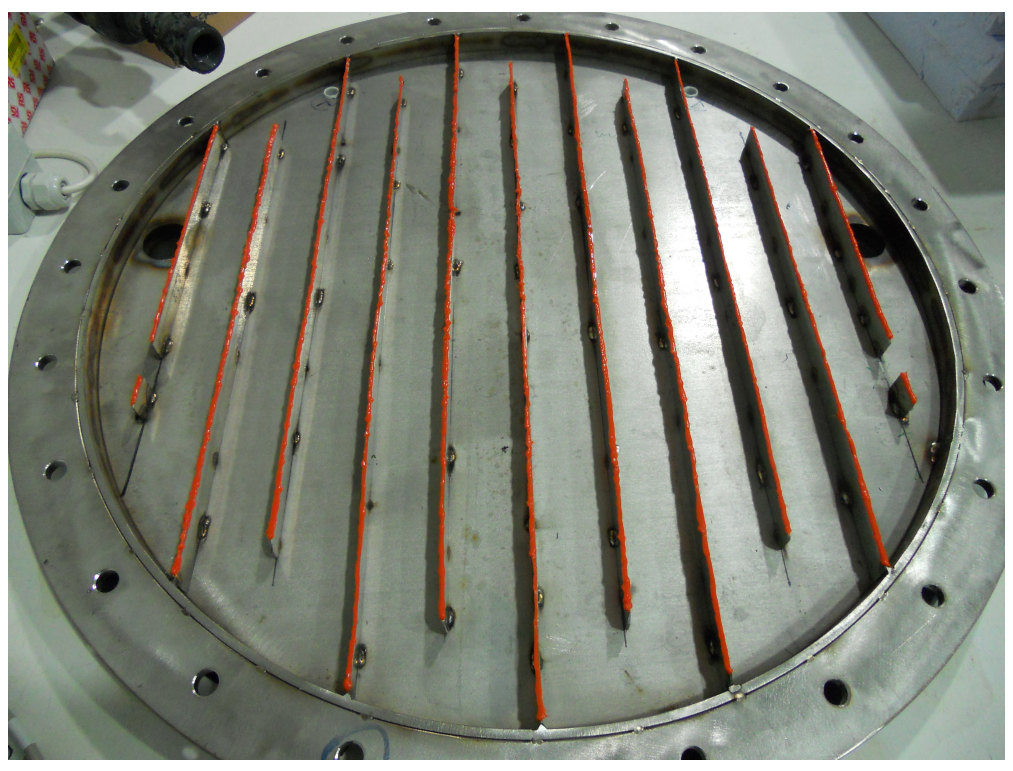

(a) Coolant channels under the subcooled plate

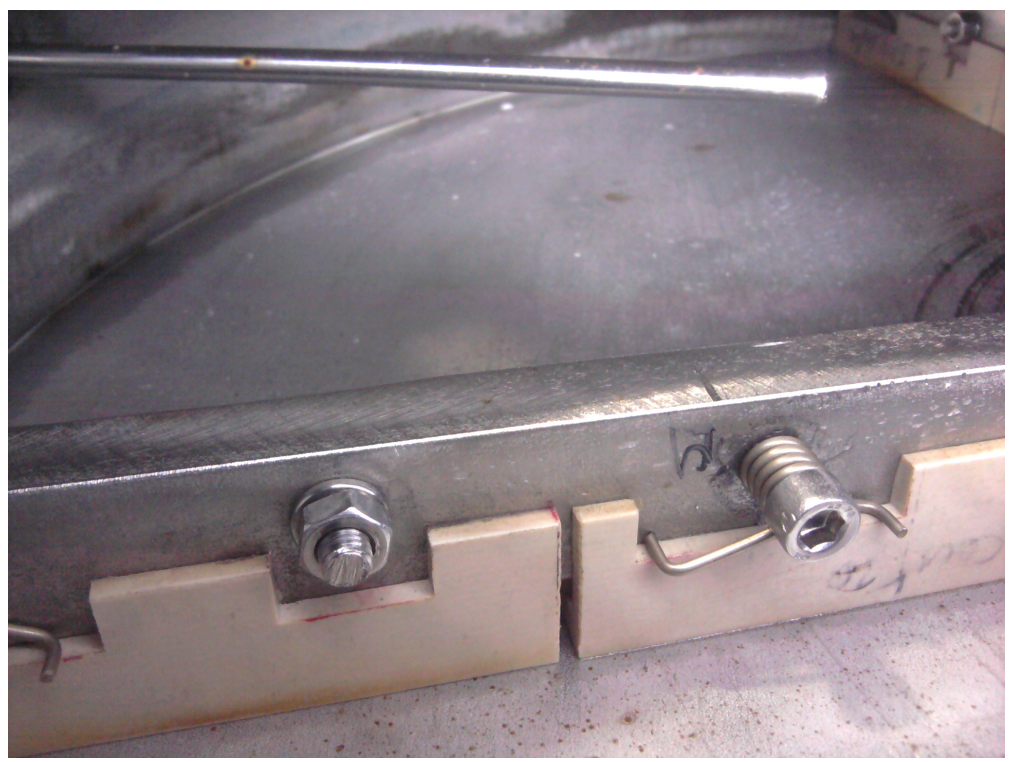

(b) Adaptable scrapers

Figure 2.2: Images different SSPHE elements 


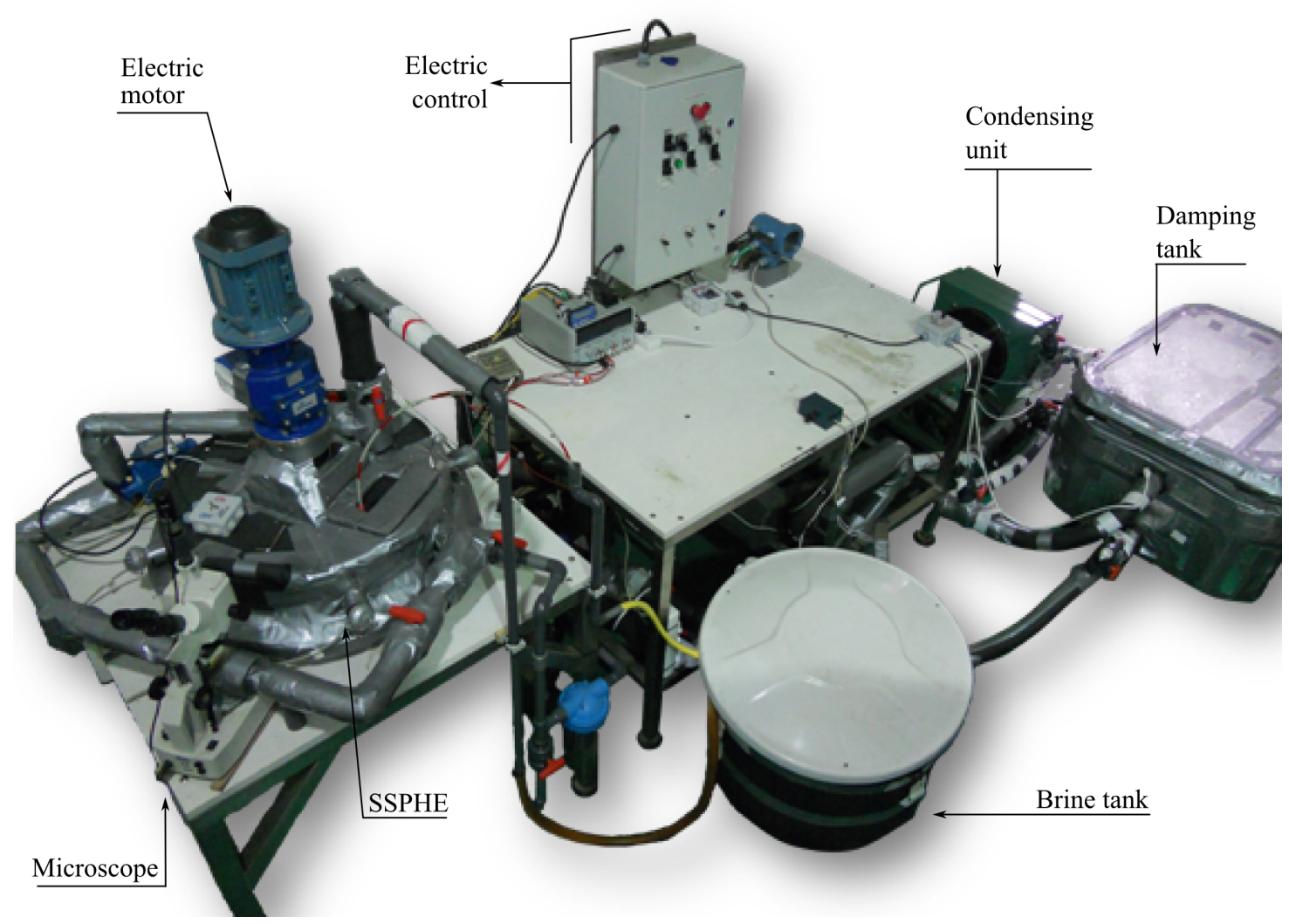

Figure 2.3: Experimental facility, general view

first one consisted on single piece scrapers fixed to each steel arm, where the movement between the arm and the scraper was restricted. The second system employed three independent scrapers mounted on each arm. Each scraper device was connected to the arm by means of two torsion springs, which maximizes the contact between the scraper and the plate even if the latter loses its planar shape. The independent motion of the different scrapers, together with the force applied by the torsion springs, provide a high adaptability to the plate surface. The most representative geometrical details of the experimental facility are summarized in Table 2.1.

All the data acquisition is done by a modular data logger system Agilent HP34970 A, which is connected to a computer through a RS-232 serial port. The data logger software also allows the data monitoring in real time.

Figure 2.3 shows an overview picture of the facility schematically represented in Fig. 2.1. It may be noticed that the tubes corresponding to the primary and intermediary circuits are placed under the supporting bases.

\subsubsection{Scraping system}

As explained in the previous section, the scraping is done by a four arms system, connected to a rotating shaft. Each arm is a 318 AISI stainless steel with L shape, which provides a higher resistance to bending stresses. The PEEK blades -the ice removal elements in contact with the surface--are mounted on these arms. In order to investigate 


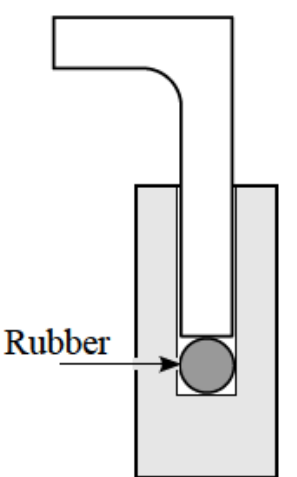

(a) Rigid

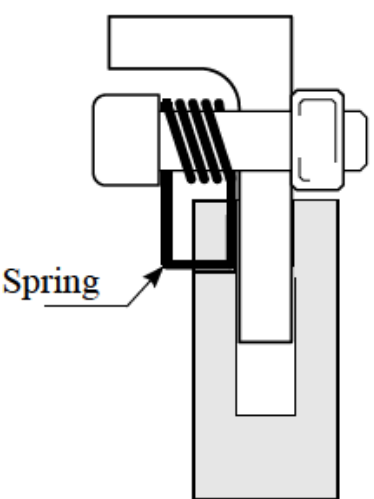

(b) Adaptable

Figure 2.4: Cross sectional view of the two different scrapers tested

the consequences of the scraping effectiveness, two different scraper mounting systems are tested. In the first one, called here rigid, a single piece of PEEK is mounted in each arm. The contact between scraper and surface is only provided by the pressure exerted on the shaft and transmitted to the arms. The second -adaptable- system was conceived as an improvement of the first one, splitting each piece of PEEK on three independent scrapers. Moreover the pressure of each of them was increased through torsional springs. A schematic view of each of them is shown in Fig. 2.4. Both systems are detailed below.

\section{Rigid scraping system}

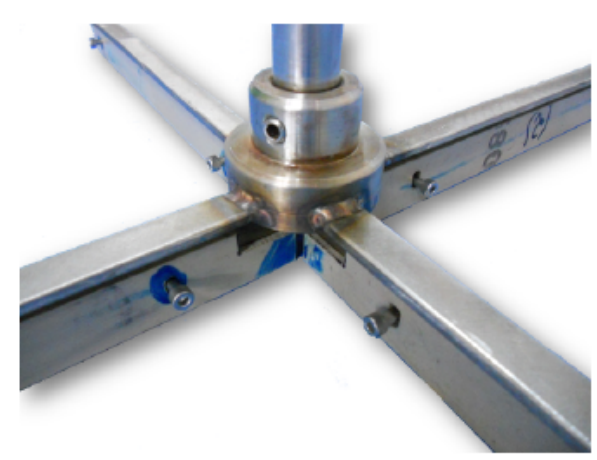

Figure 2.5: Scraper system view.

The rigid system was the system originally conceived for the SSPHE. It is shown in Fig. 4.15. Four U shape PEEK elements are mounted on the L shape stainless steel arms as indicated in Fig. 2.4a. Both the steel arm and the PEEK scraper have the same length, conforming a single scraper. The scraping action is make by the flat, bottom of the PEEK element. A rubber cord is placed between arm and PEEK all along them. The function of the cord is to absorb the force over the scrapers, avoiding them to deform the arms and ensuring the contact between scraper and surface.

Each scraper is fixed to the scraper arms by two screws placed on the sides. A small vertical movement of the scraper along the arm (which will deform the rubber) is allowed by using rail holes on the scraper as depicted on Fig. 2.6.

While the rigid system is simple, it provides contact in all the surface area only if the surface is completely flat. Any surface curvature due to, for example, thermal stresses, or the coolant pressure in the bottom side will affect to the scraping. Moreover the rubber crew has a low deformation capacity, and therefore it may lead to high momentum efforts in the rod-arm junction if ice scaling occurs. If, as a consequence, the arms are bended at the shaft junction, the scraping action will be reduced significantly. 


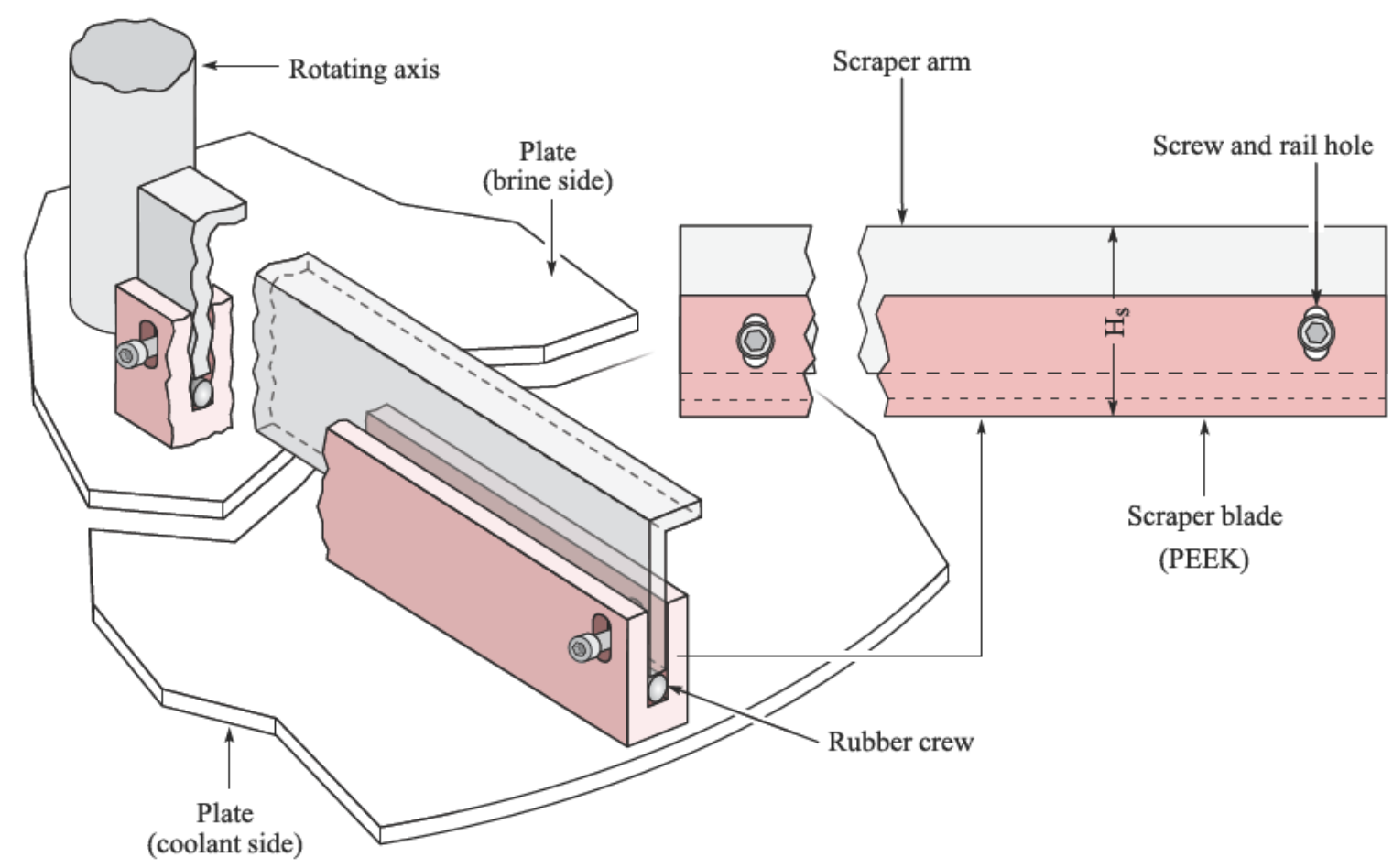

Figure 2.6: Rigid scraper system

\section{Adaptable scraping system}

The adaptable system is aimed at improve the scraping effectiveness by two different ways: increasing the pressure of the scrapers over the plate and increasing their adaptability to possible surface curvatures. The augmentation of pressure can be achieved increasing the pressure exerted by the shaft. However, any of these systems should also include a damping mechanism to avoid transmitting extra stresses to the arm-shaft junction or to the gearbox. On the other hand there is still the problem associated to the difficulties of the scrapers to adapt to possible surface curvatures.

To sort out these two problems a modified design of the previous rigid scrapers was made. First, the adaptability of the scrapers to possible curvatures was improved by splitting each scraper into three. Secondly, each of the new scraper blades was dotted of extra pressure, independently of each other, by adding torsional springs between them and the arms. An schematic view of the system can be observed in Fig. 2.7. The torsion springs are fixed by a $5 \mathrm{~mm}$ diameter screw threaded onto the arm, with a lock nut. The screw keeps the position while allowing the torsion. The spring tails, $90^{\circ}$ bended towards each other, act as a pusher craft of the scrapers. Therefore the scrapers are continuously pushed down by the springs, increasing the pressure over the plate. A detailed explanation about the torsion springs design can be found in A. 


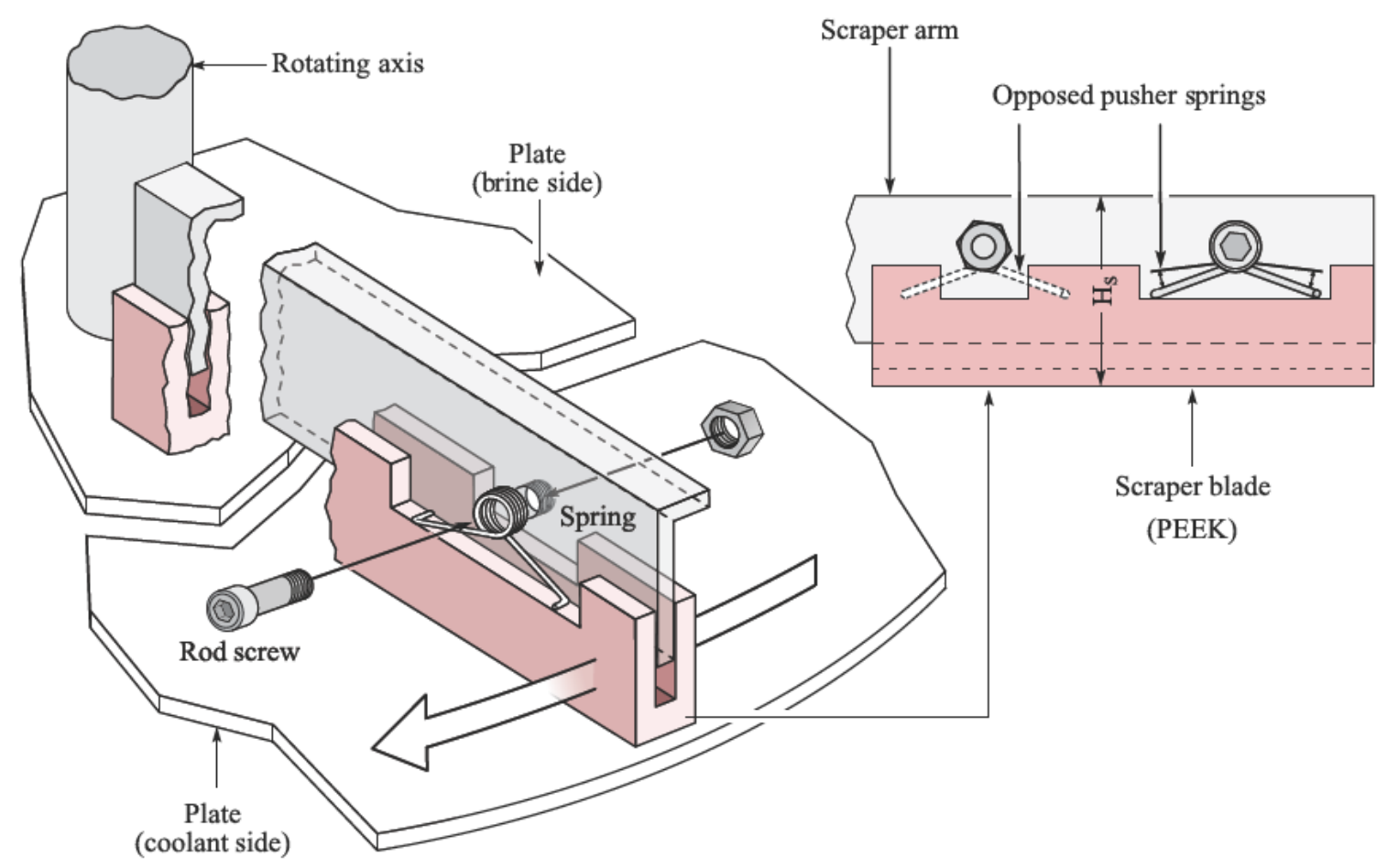

Figure 2.7: Adaptable scraper system

\subsubsection{Instrumentation}

The different variables which are acquired can be classified into data variables and control variables. The control variables are those ones indicative of different technical aspects of the facility, but they do not take part in the ice slurry production process. As their name indicates, they only are used to control the installation. The data variables are those ones scientifically significant, taking part in the slurry formation process. They are processed either independently or combined.

Figure 2.8 shows an sketch of the acquired variables and the instrumentations system. All the sensors and transducers employed in the experimental facility are connected to a data logger system Hewlett-Packard HP-34970A. The data logger is controlled from a computer with the software Agilent BencLink Data Logger, connected trough a RS-232 port. The data logger is equipped with three modules HP-34971A, each one with 20 independent channels adaptable to the signal type.

Power consumption The power consumption in the electric motor which drives the scrapers is measured by an active-power, three-wires wattmeter (1), connected to the frequency controller output. The data is transmitted to the data logger through a 2-core shielded wire in a $0 \div 10 \mathrm{~V}$ analog signal. The signal is directly proportional to the measured power where $0 \mathrm{~V}$ corresponds to $0 \mathrm{~W}$ and $10 \mathrm{~V}$ to $800 \mathrm{~W}$. The accuracy is of $0.45 \%$ of reading $+0.05 \%$ of full scale. To avoid the noise due to the frequency controller, a low pass RC filter was used, with a cut-off frequency of $f_{c}=100 \mathrm{~Hz}$. 


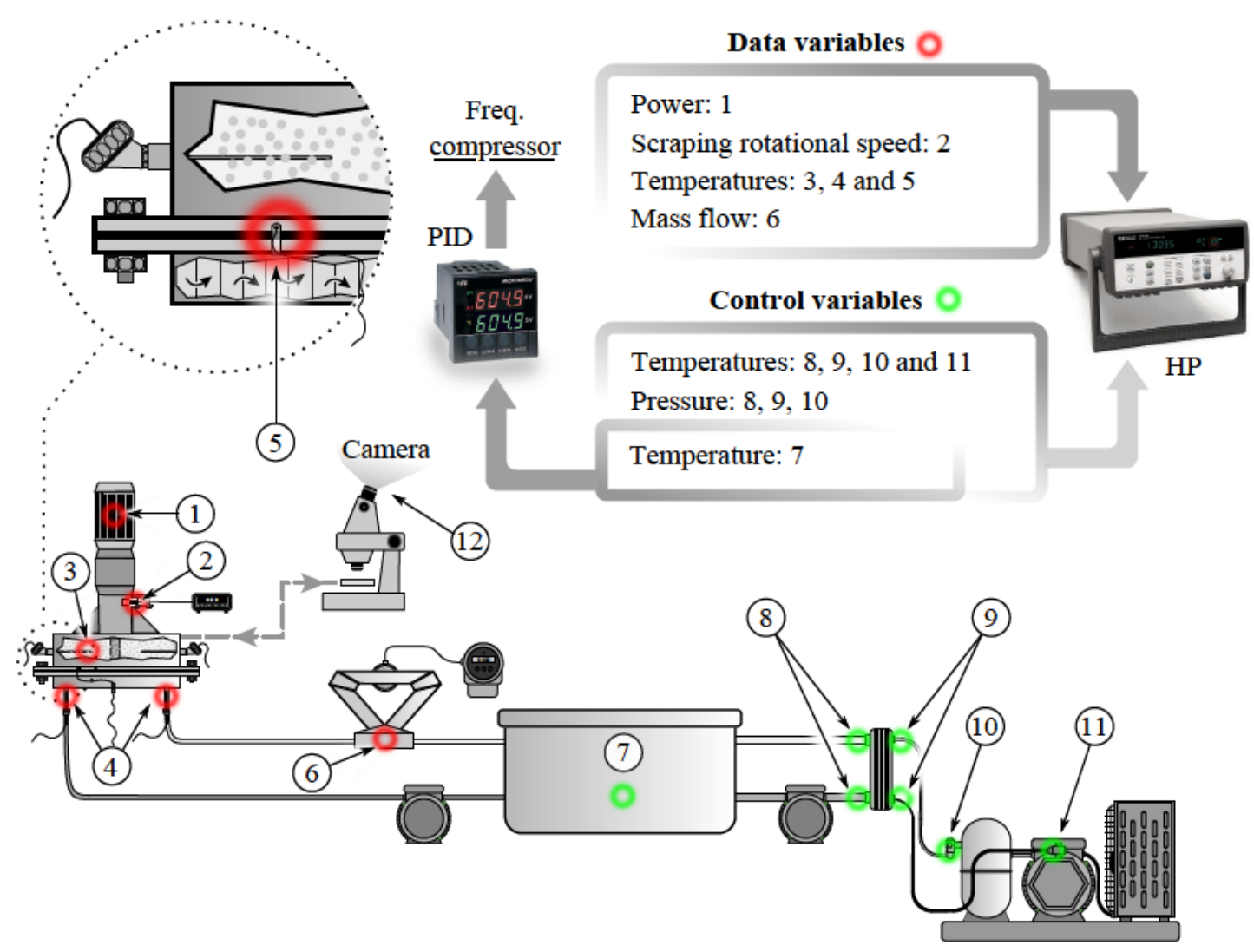

Figure 2.8: Data and control variables acquired on the experimental facility: (1) Wattmeter, (2) Hall-effect transducer + tachometer, (3) brine-slurry PTC probes, (4) SSPHE coolant inlet/outlet PTC probes, (5) Plate embedded thermocouples, (6) Coriolis flowmeter, (7) PTC probe, (8) coolant inlet/outlet HE-evaporator PTC probes, (9) Refrigerant inlet/outlet PTC probes + pressure transducers, (10) Refrigerant liquid line PTC probe + pressure transducer, (11) Refrigerant vapour line PTC probe + pressure transducer, (12) Microscope + CCD camera. 
Mass flow The coolant mass flow is measured by means of a Coriolis flowmeter (6) Micro Motion CMF100M. It has a multi variable transmitter Micro Motion Coriolis Transmitter 2700. Both are interconnected through a 4-core shielded cable, providing power supply the transmitter to the transducer and transmitting the output electric signal from to the transducer to the transmitter. The accuracy is of $\pm 0.1 \%$ of the mass flow for $\dot{m}>1360 \mathrm{~kg} / \mathrm{h}$. The communication between the transmitter and the data is done through a $4 \div 20 \mathrm{~mA}$ signal, where the 4 and the $20 \mathrm{~mA}$ values can be assigned to any measure given by the flowmeter.

Scraping velocity The rotating velocity of the driving shaft is measured by using a Hall effect transducer. The detecting point is a ferromagnet placed in an accessible point of the driving shaft. The transducer is connected to a digital tachometer which provided also the voltage supply to the transducer. The tachometer thus is connected to the data logger through a 2-core shielded wire, which transmits an analog $0 \div 10 \mathrm{~V}$ signal. The rotating velocity values corresponding to the 0 and $10 \mathrm{~V}$ where adjusted manually according to the required range. The uncertainty on the tachometer is of $\pm 0.005 \%$ whereas the uncertainty on the output signal is of $\pm 0.1 \%$ of full scale.

Brine temperature The temperature in the brine/ice slurry side of the SSHPE is measured by four RTD probes (3). In particular, 4-wire PT100 immersion probes of class $1 / 10$-DIN according to IEC-751. The picture of Fig. 2.4b shows, in the top part, one of the probes. They all consist of a $6 \mathrm{~mm}$ diameter sheath made of stainless steel, which contains and protects the sensor, placed at the end of the sheath. The probes are threaded to the SSHPE vertical wall $90^{\circ}$ apart, being the sheaths aligned with the SSPHE radius. Their vertical location corresponds with the half of the SSHPE height, $\mathrm{H} / 2$. The length of the probe sheaths varies: two of them are $60 \mathrm{~mm}$ length whereas the other two are $160 \mathrm{~mm}$. Equal length probes are placed one in front of the other. In that way, different radial locations are measured.

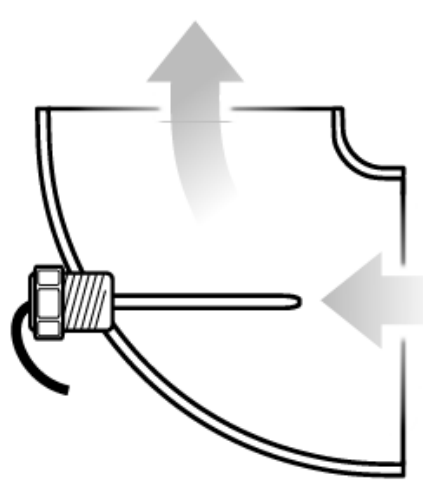

Figure 2.9: Assembly of the PT100 probe for SSPHEcoolant inlet/outlet flow.
Coolant temperatures The measurement of the coolant inlet and outlet temperatures to the SSPHE is done also by RTD, 4-wire PT100 probes (4). They are also $1 / 10$-DIN immersion probes, in this case with a 3 $\mathrm{mm}$ diameter stainless steel sheath of length $66 \mathrm{~mm}$. The coolant inlet/outlet to the SSPHE coil circuit is done intentionally through two elbows, which allow to mount the probes parallel and towards the incoming flow (Fig. 2.9). The same type of probes was used for the HE-evaporator inlet/outlet coolant temperatures (8) and for refrigerant temperature measurement, either in the liquid or vapour lines $(9,10$ and 11$)$. In all the cases the mounting system indicated in Fig. 2.9 was used.

The damping tank temperature (7) was measured with a RTD 4-wire PT100 probe of class B-1/3, according to IEC-751. It consisted of a $6 \mathrm{~mm}$ diamter, $200 \mathrm{~mm}$ length stainless steel sheath located at the bottom of the 


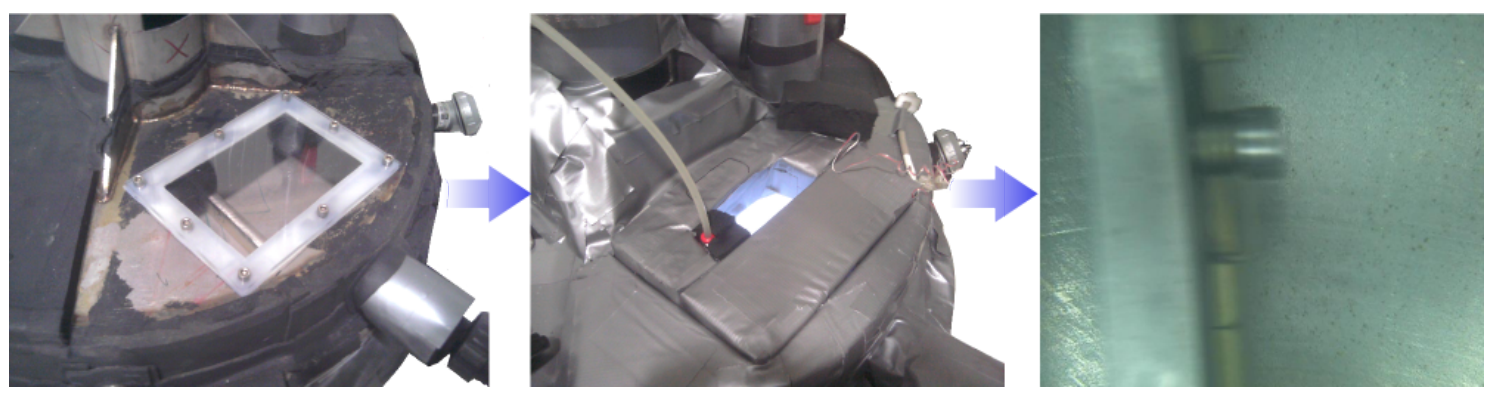

Figure 2.10: Window on the SSHPE top (left), camera emplacement and light sources (center) and camera view (right)

tank. The active mixing produced by the disposition of the inlet/outlet tubes for the HE-evaporator and SSPHE avoid thermal stratification.

Wall temperature The wall temperature measurement (5) was done by 26 embedded thermocouples all along the plate. Thermocouples were previously calibrated for the temperature working range. Section 2.1.3 contains a detailed explanation of the welding procedure, the calibration and the obtained uncertainties.

Refrigerant pressures The pressure in the liquid and vapour refrigerant lines $(9$, 10 and 11) is measured by absolute pressure piezo-resistive transducers General Electric UNIK 5000. The output signal is an analog $0 \div 10 \mathrm{~V}$ transmitted through a 2-core shielded wire. The transducers calibrated at room constant temperature, with an accuracy of $\pm 0.04 \%$ full scale.

Microscopic imaging The images of the ice slurry are obtained by CCD camera coupled to an stereoscopic microscope Zuzi 235-B (12). The measurement of the ice crystal size are done by previous calibration of the images by using known patterns.

$\mathrm{NaCl}$ concentration $\mathrm{The} \mathrm{NaCl}$ initial concentration in the brine $(\mathrm{gr} / \mathrm{l})$ was measured by a portable conductivity meter CRISON CM35+ with an uncertainty of $\pm 0.1 \%$ full scale .

Process visualization To observe the process inside the SSHPE a rectangular window was made on the top of it, as it is shown in Fig. 2.10. Continuum cold-LED light sources where used to illuminate the interior whereas a valve was used to purge any possible bubbles under the screen glass. A CMOS camera able to take pictures ( 8 Mpixel resolution) and videos (HD 720p at $30 \mathrm{fps}$ ) was used to capture the different situations inside. In particular, the images aided to identify a correct or defective ice removal from the surface in the early stages of the process, as well as to detect static ice blocks on the top as the test progresses.

\subsubsection{Wall temperature measurement system and calibration}

The measurement of the wall temperature (the temperature on the scraped surface) is of great importance for the determination of the heat flux through it. Therefore the 


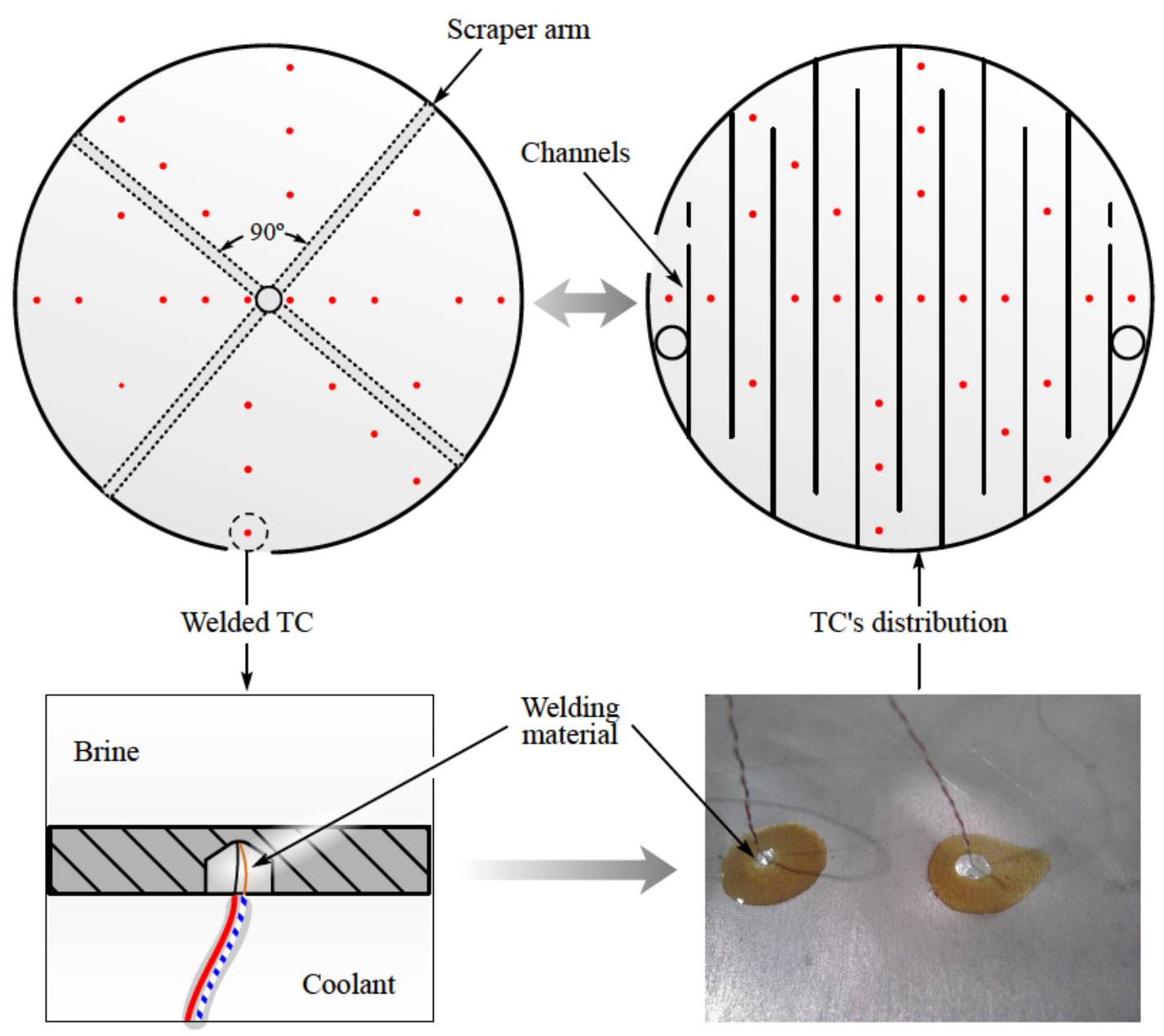

Figure 2.11: Thermocouples location (up) and welding technique (down): sketch and picture

measurement point has to be as close to the surface as possible, but obviously cannot be over it. The usage of accurate RTD probes was initially discarded. Any attempt to introduce them into the wall would lead to the interruption of the material continuity, modifying also the heat flux. If the RTDs -or any other probe- are placed below the plate and in contact with it, the measured temperatures would not correspond with the ones on the scraped side.

The adopted solution consisted in embedding thermocouples inside the plate. For that, the plate was drilled from the bottom, arriving at $\approx 1.5 \mathrm{~mm}$ from the top side. The hole was then filled with the welding material, containing the thermocouple wires and acting also as the thermocouple junction material. An sketch of the technique is shown in Fig. 2.11, together with a picture of the thermocouples just after welding. The welding material was the commercial metal bare brazing CRONATRON 53, selected by its good characteristics when using for welding stainless steels with a variety of metals (copper, brass, stainless, zinc, etc...). As both the welding material and the plate are metals, the disturbance of the temperature field on the plate is lower than using 
any other compound adhesive and a prefabricated thermocouple. Thermocouples were welded from a $\mathrm{T}$ type thermocouple wire (copper-constantan), suited for measurements in the 200 to $350{ }^{\circ} \mathrm{C}$ range with a sensitivity of about $43 \mu \mathrm{V} /{ }^{\circ} \mathrm{C}$ [71]. A total of 26 thermocouples were welded into the plate, distributed all over it -their location is depicted in Fig. 2.11. The thermocouples distribution accounts not only with possible radial temperature differences, but also with the effect that the channels and the coolant flow through them could have on the wall temperature. To protect the thermocouple wire from the stresses that the coolant flow may cause, the contact with the wall was reinforced by a composite adhesive 2.12 .

Once welded, the thermocouples were calibrated

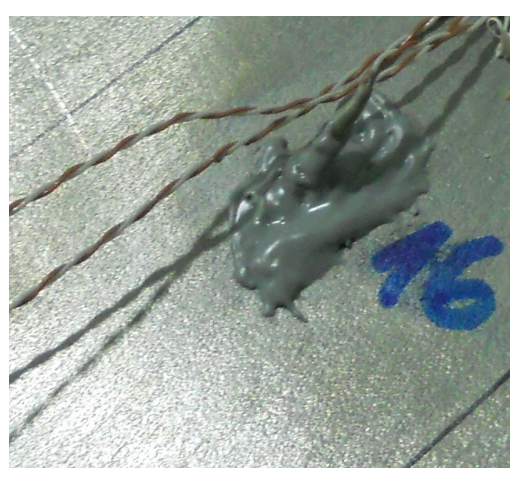

Figure 2.12: Detail of the welded thermocouple. before mounting the plate into the SSHPE. The reader is referred to the Apendix $\mathrm{B}$, where a detailed description of the calibration procedure, together with an analysis of the uncertainty of the measurements is presented.

\subsubsection{Control system}

The coolant temperature was controlled automatically by means of a PID (proportional band) temperature controller, in order to be constant during all the test duration. The PID (OMEGA CN77353) received as an input the temperature signal coming from the PT100 probe of the damping tank ((7) on Fig. 2.8) and provided a $0 \div 10 \mathrm{~V}$ analog signal which drive the frequency converter connected to the compressor in the condensing unit. The accuracy in the reading is of \pm 0.9 full scale. As the minimum operating frequency at which the compressor should work was of 35 $\mathrm{Hz}$, the $0 \mathrm{~V}$ corresponds to $35 \mathrm{~Hz}$ whereas the $10 \mathrm{~V}$ does for $50 \mathrm{~Hz}$.

\subsubsection{Thermophysical properties and ice content}

\section{Brine and ice slurry}

The thermophysical properties of the brine - density, viscosity, specific heat, thermal conductivity and freezing point temperature- were calculated using the relations recommended by Melinder [72]. The thus obtained properties, including the effect of the ice crystals in the slurry, are later used in the dimensionless numbers evaluation.

Since, excepting the supercooling, thermal equilibrium can be assumed (with no solute in the ice crystals), the $\mathrm{NaCl}$ mass fraction can be determined by the temperature of the mixture from the liquidus curve,

$$
\omega\left(T_{b}\right)=-0.004473 T_{b}^{2}-1.6022 T_{b}+0.041643 ;
$$

where, in equilibrium, the slurry temperature is equal to the freezing point temperature $T_{b}=T_{f}(\omega) \Leftrightarrow \omega=\omega_{f}\left(T_{b}\right)$.

The global mass fraction of solute in the slurry $\omega_{0}$ (kg solute $/ \mathrm{kg}$ solution+ice)remains constant and equal to the value of $\omega$ before freezing. Since the initial $\mathrm{NaCl}$ mass fraction is known, the ice mass fraction in the slurry ( $\mathrm{kg}$ ice $/ \mathrm{kg}$ slurry) is calculated as, 


$$
\varphi_{m}=1-\frac{\omega_{0}}{\omega\left(T_{b}\right)}
$$

The slurry density is obtained by addition of ice and brine volume,

$$
\frac{1}{\rho}=\frac{\varphi_{m}}{\rho_{i}}+\frac{1-\varphi_{m}}{\rho_{b}}
$$

The volume mass fraction of the ice slurry is then,

$$
\varphi_{v}=\varphi_{m} \frac{\rho}{\rho_{i}}
$$

The thermal conductivity is obtained by the Maxwell lower bound relation [73] for dilute solid/ liquid suspensions,

$$
k_{t}=k_{t, b}\left[\frac{2 k_{t, b}+k_{t, i}-2 \varphi_{v}\left(k_{t, b}-k_{t, i}\right)}{2 k_{t, b}+k_{t, i}+\varphi_{v}\left(k_{t, b}-k_{t, i}\right)}\right]
$$

where $k_{t, b}$ and $k_{t, i}$ are the thermal conductivities of the brine and ice and $\varphi_{v}$ can be obtained from the slurry temperature.

The specific enthalpy of the slurry is obtained by those of ice and brine

$$
i=\varphi_{m}\left(-L_{f}+\int_{0^{\circ} C}^{T_{F}} c_{p, i}(T) d T\right)+\left(1-\varphi_{m}\right) \int_{0{ }^{\circ} C}^{T_{F}} c_{p, b}(T) d T
$$

Since $c_{p}$ is a function of the temperature as it is $\varphi_{m}$ (through Eq. 2.1), the specific enthalpy will depend only on the temperature for a given solute mass fraction $\omega_{0}$. An apparent specific heat can then be defined for the slurry (needed energy to increase by $1{ }^{\circ} \mathrm{C}, 1 \mathrm{~kg}$ of slurry following the liquidus curve). This specific heat is hereafter used to calculate the Prandtl number.

$$
\hat{c}_{p}=\frac{d i}{d T}
$$

The ice slurry behaviour depends strongly on the ice content, and most of the authors agree that the limit between Newtonian and non-Newtonian behaviour is between 6 and 15\% [14, 15]. The relation proposed by Thomas [74] for solid/liquid suspensions was used to calculate the dynamic viscosity as a function of the brine viscosity and the ice particle concentration

$$
\mu=\mu_{b}\left(1+2.5 \varphi_{v}+10.05 \varphi_{v}^{2}+0.00273 e^{16.6 \varphi_{v}}\right)
$$

The above equation considers homogeneous and non Newtonian flow, and is commonly valid for solid phase concentrations up to $62.5 \%$ and for all particle sizes between 0.099 and $435 \mu \mathrm{m}$. However, for the particular case of ice slurries, the equation is limited to ice concentrations below $15 \%$. Higher concentration values lead to viscosity values greater than the experimentally obtained.

\section{Coolant}

The properties of the coolant $\left(\mathrm{CaCl}_{2}\right)$, as the properties of the $\mathrm{NaCl}$ brine, were calculated according to [72]. In particular, an accurate measurement of the specific heat 


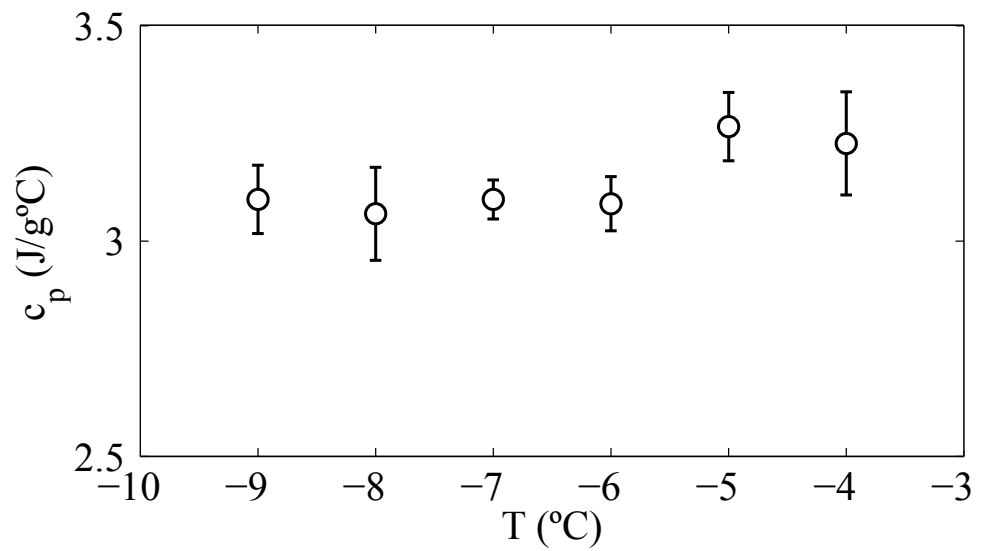

Figure 2.13: Specific heat measurements at different temperatures for a coolant sample.

was of great importance as it is involved in SSHPE energy balance evaluation (see Sec. 2.1.6). Since the $\mathrm{CaCl}_{2}$ concentration was not know precisely, the specific heat capacity was measured by using a differential scanning calorimeter Mettler Toledo DSC822e. Different samples were taking in time and at each coolant solution renewal, correlating them with the corresponding test in which the coolant was used. The values of $c_{p}$ were measured also at different temperatures, interpolating after each coolant $c_{p}$ value for its corresponding temperature. Figure 2.13

\subsubsection{Experimental procedure}

The experimental procedure described here was followed carefully for all the heat transfer/ice slurry production tests. The base brine was prepared in a separated tank, with a higher capacity than the SSPHE upper side. $\mathrm{NaCl}$ was added gradually to the water in the tank while it was continuously recirculated between it and the SSPHE upper side, ensuring an homogeneous $\mathrm{NaCl}$ concentration in all the fluid.

Once the $\mathrm{NaCl}$ reached the desired content the SSPHE upper side was closed. The cooling system was used then to decrease the brine temperature to the initial test temperature. Prior to the beginning of the experiment, the coolant was cooled down to its corresponding temperature. Once the scraping velocity was fixed, the pumping of the coolant to the SSPHE bottom side set the beginning of the test. Scraping velocity, coolant mass flow and coolant temperature were kept constant during all the experiment. Each test took the necessary time to reach a $20 \%$ of ice content if the conditions inside allowed it (i.e. no ice scaling).

Once the test arrived to its end the slurry in the SSPHE upper side was replaced by a new volume of brine, starting again the process for the next experiment. The $\mathrm{NaCl}$ concentration was only changed once the test corresponding to that content were done, where the $\%$ wt. value was measured at the beginning and end of each test. Samples of the coolant $\mathrm{CaCl}_{2}$ were taken regularly to determine its specific heat capacity.

Data was acquired to the data logger each 5 seconds. The magnitudes directly measured during the test were later employed for the calculation of indirect variables (i.e. heat flux or heat transfer coefficient) or dimensionless numbers. In particular, the rotating Reynolds number 


$$
R e_{\mathrm{rot}}=\frac{\rho N D^{2}}{\mu},
$$

was used to characterize the flow regime inside the SSHPE. The values of $\rho$ and $\mu$ where those calculated for the corresponding ice slurry concentration (see Sec. 2.1.5) and $N$ was the value accquired by the tachometer, in rev $\cdot \mathrm{s}^{-1}$.

The Richardson number, the ratio between natural and forced convection, was also evaluated as a representative parameter of possible flow stratifications. It is defined as,

$$
R i=\frac{g \beta \Delta \mathrm{T}_{b, w}(H / D)}{\Omega(D / 2)^{2}}
$$

where $g$ is the gravitational acceleration, $\beta$ is the thermal expansion coefficient, $\Delta \mathrm{T}_{b, w}$ is the difference between bulk fluid and wall temperatures, $H / D$ is the location heigh of the temperature probes inside the SSPHE and $\Omega$ is the rotating velocity of the scrapers in $\mathrm{rad} \cdot \mathrm{s}^{-1}$. The thermal expansion coefficient $\beta$ of the slurry was calculated from those of the pure ice $\beta_{i}$ and of the $\mathrm{NaCl}$ solution $\beta_{b}$

$$
\frac{1}{\beta}=\frac{\varphi_{m}}{\beta_{i}}+\frac{1-\varphi_{m}}{\beta_{b}}
$$

The heat flux calculation procedure, together with the calculation of the corresponding dimensionless number is detailed in the following section.

\section{Heat flux measurement}

The heat flux exchanged through the surface was estimated by a heat balance in the heat exchanger. Viscous dissipation effects were neglected. To minimize the heat losses to the ambient the entire SSPHE together with the coolant pipes were thermally isolated with an elastomeric $4 \mathrm{~cm}$-thick insulating material $(k=0.040 \mathrm{~W} / \mathrm{m} \mathrm{K})$. The insulating material thickness was chosen on the basis of the expected slurry, coolant and ambient (surrounding) temperatures, applying all of them in the existent correlation for natural convection in vertical and horizontal surfaces [75]. The insulation thickness ensured heat losses lower than $5 \mathrm{~W}$ for the most unfavourable conditions i.e., lowest slurry and coolant temperatures and highest ambient temperature, which represented a $4 \%$ of the heat flux measured inside the SSHPE. Moreover the ambient and SSPHE outer wall temperatures were measured confirming the expected low heat fluxes to the ambient.

With the the previous considerations, the heat lost by the slurry is gained by the coolant flow. Since inlet and outlet coolant temperatures together with its mass flow are known, then

$$
q=\dot{m}_{c} c_{p, c}\left(T_{c, o}-T_{c, e}\right)
$$

The coolant mass flow $\dot{m}_{c}$ was kept high enough to have a fully turbulent flow $\left(R e \sim 10^{4}\right)$ with elevated heat transfer coefficient values in order to avoid any limiting heat transfer resistance in the coolant-side of the heat exchanger (bottom side).

The total amount of heat transferred is directly related to the temperature difference between the coolant and the ice slurry. Since the temperature of the coolant increases during its transport through the coiled side, the driving of the temperature difference 
is best expressed by the logarithmic mean temperature difference

$$
\Delta \mathrm{T}_{\log }=\frac{T_{c, o}-T_{c, e}}{\ln \left(\frac{T_{c, o}-T_{b}}{T_{c, e}-T_{b}}\right)}
$$

where during the freezing $T_{b}=T_{f}(\omega)$ (Eq. 2.1). The logarithmic temperature has been chosen as a characteristic parameter for each experiment, averaged along the test time.

The heat flux given by eq. 2.12 corresponds predominantly to the sum of the latent heat of the ice formation and the sensible heat of ice and brine. Furthermore, there is friction between scrapers and plate, and the induced flow gives rise to viscous dissipation. These two contributions represent a power input in the system, noted as $\Phi$. Considering a time interval $\Delta t$ during the ice formation process, then

$$
q=\rho_{b, 0} V\left(\frac{\left.L_{f} \Delta \varphi_{m}\right|_{t} ^{t+\Delta t}}{\Delta t}+\frac{\left.\left(\left(1-\varphi_{m}\right) c_{p i}+\varphi_{m} c_{p b}\right) \Delta \mathrm{T}_{b}\right|_{t} ^{t+\Delta t}}{\Delta t}\right)+\Phi
$$

where $V$ is the initial brine volume in the SSPHE upper side and $\rho_{b, 0}$ the brine density. Whereas the first part of eq. 2.14 is easily calculable from the measured temperatures, the value of $\Phi$ has not an evident definition. It can be approached assuming that the power consumed by the driving motor is all transformed into heat by viscous dissipation and friction between materials. That is obviously not true, since there are thermal and mechanical losses in the both motor and the gear box. However, for the sake of simplicity it was assumed that $\Phi$ was equal to the power measured by the wattmeter. Figure 2.14 shows the obtained values of $q$ calculated from eqs. 2.12 and 2.14. The fact that $\Phi$ is overestimated can explain the slightly higher values given by eq. 2.14.

The heat transfer coefficient is determined from the heat flux given by Eq. 2.12 and the difference between the averaged temperature of the embedded thermocouples $T_{w}=\bar{T}_{w, n}$ and the immersed PT100 probes $T_{b}=\bar{T}_{b, n}$

$$
h=\frac{Q}{A\left(T_{b}-T_{w}\right)}=\frac{q}{\left(T_{b}-T_{w}\right)}
$$

Once the freezing starts, the temperature difference in the above expression $T_{b}-T_{w}$ becomes equal to the wall supercooling degree $\Delta \mathrm{T}_{s}$, since $T_{b}=T_{f}$.

The heat transfer coefficient can be expressed in a dimensionless way through the Nusselt number, defined as

$$
N u=h \frac{L}{k_{t}},
$$

where $L$ is a the characteristic length of the heat transfer problem. As in the SSHPE the heat transfer takes place perpendicularly to the plate, the top side high $H$ has been chosen as characteristic length, and therefore

$$
N u=h \frac{H}{k_{t}} .
$$




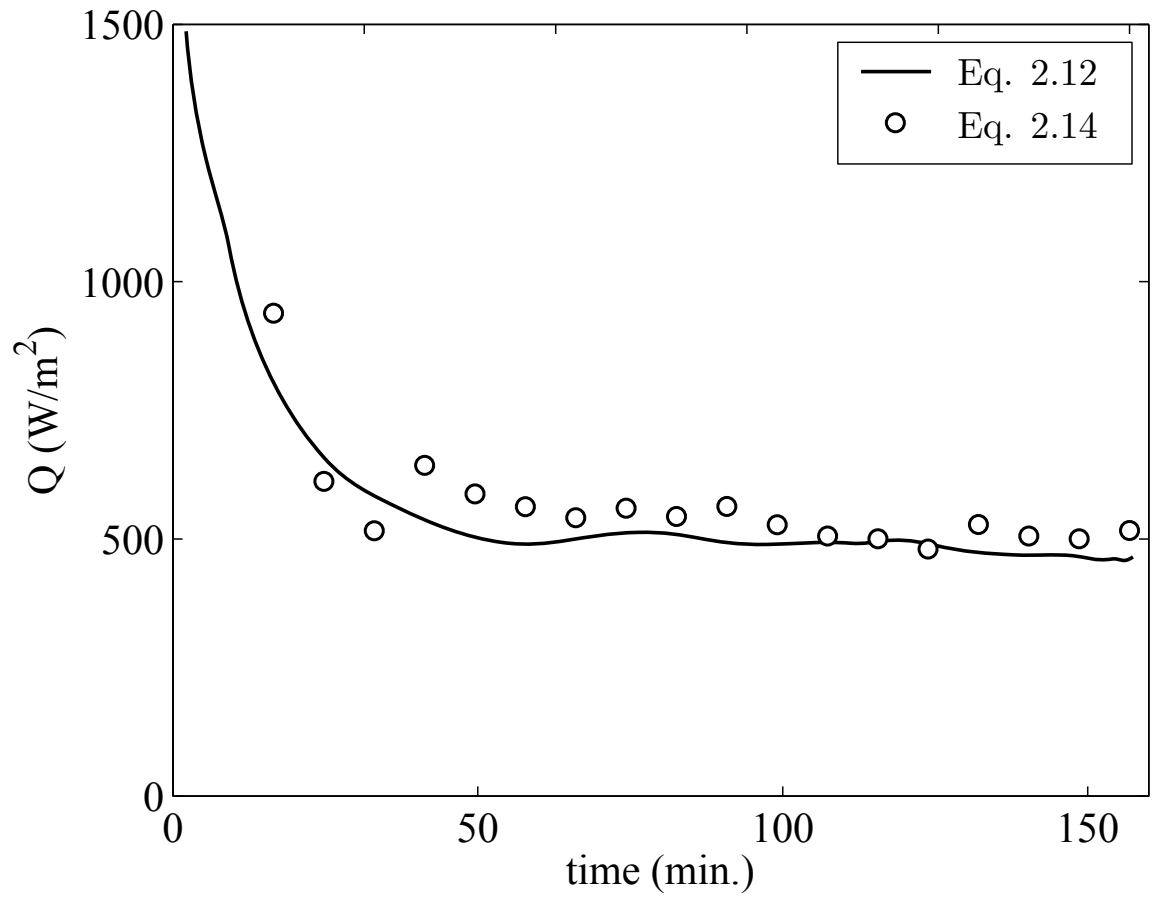

Figure 2.14: Calculated heat fluxes for the coolant and ice slurry sides along time.

\subsubsection{Working range}

In the search of the optimal working conditions for the SSHPE the present investigation covered a wide range of situations by changing the three control variables: the scraping rotating velocity, the initial $\mathrm{NaCl}$ concentration in the brine and the wall temperature -wall supercooling degree. The ice concentration, which is supposed to have also an effect over the process will be a consequence of the previous three variables and the time. Whereas the rotating velocity and the initial $\mathrm{NaCl}$ concentration in the Brine are of direct control and easy to stablish accurately, the wall supercooling degree can be only controlled indirectly through the coolant temperature.

The scraping rotating velocity tested values were repeated for each $\mathrm{NaCl}$ concentration, where the velocity was constant during all the test duration. The wall supercooling degree instead changed in time during the test, as the temperature of the slurry decreased with the increasing ice content. As a reference, for each test it was considered the difference of temperatures $\overline{\Delta \mathrm{T}}_{s, R}$ between the initial freezing point of the brine -for the initial concentration of $\mathrm{NaCl}-$ and the averaged wall temperature for all the test.

The range covered for each one of the variables is

- Scraping rotating velocity $\left.0.08 \leq N \mathrm{~s}^{-1}\right) \leq 0.8$

- Initial $\mathrm{NaCl}$ concentration $2.9 \leq$ wt. $\% \leq 9.4$

- Wall supercooling degree $1.4 \leq \overline{\Delta T}_{s, R} \leq 3.9$

- Ice content from 0 to $20 \%$ (test end). 
Table 2.1: Dimensions and operating conditions for the heat transfer characterization

\begin{tabular}{llll}
\hline Parameter & Value & Parameter & Value \\
\hline \hline$H$ & $0.1 \mathrm{~m}$ & $\omega_{0}$ & $7 \mathrm{wt.} \%$ \\
$H_{S}$ & $3.9 \mathrm{~cm}$ & $N$ & $0.1-0.8 \mathrm{~s}^{-1}$ \\
$D$ & $0.610 \mathrm{~m}$ & $\Delta \mathrm{T}_{\text {log }}$ & $0.5-2.5{ }^{\circ} \mathrm{C}$ \\
$A$ & $0.29 \mathrm{~m}^{2}$ & $R e_{c}$ & $\approx 7000$ \\
$n$ & 4 & $R e_{\text {rot }}$ & $350-1.4 \cdot 10^{5}$ \\
\hline
\end{tabular}

While the values of $N$ were equally tested for al the $\mathrm{NaCl}$ concentrations, the values of $\overline{\Delta T}_{s, R}$ revealed to be critical in the ice scaling over the plate, and therefore some of them were not possible to be tested. Figure 2.15 shows the initially plated tests together with those finally done. The $\mathrm{NaCl}$ concentrations of 2.9 and $9.4 \mathrm{wt} \%$ are, as the two extremes, the most critical of the five tested. The one of $2.9 \mathrm{wt}$. \% leaded to a fast ice scaling and a consequent scrapers blockage. With a considerable risk for the scraper system integrity, only few test were successfully done. On the other hand the 9.4 wt. \% concentration and its low freezing point required the lowest coolant temperatures, being out of the range of the condensing unit capabilities.

The rotating Reynolds number $R e_{\text {rot }}$-the main dimensionless number which characterizes the flow inside the SSHEP- defined by Eq. 2.9) was calculated with the physical properties corresponding to those of the ice slurry (see Sec. 2.1.5). The tested $R e_{\text {rot }}$ values, corresponding to the working range indicated above are shown in Fig. 2.16 , where the density and viscosity of the ice slurry for the different ice content concentrations are calculated according with the exposed in Sec. 2.1.5.

\subsubsection{Uncertainty calculation}

The total uncertainties of the different derived variables, as $R e_{\text {rot }}$ or $N u$ comes from the uncertainties of the different variables involved in their calculation. Those uncertainties in thus come from the accuracy of the different measuring probes and instruments (see Table 2.2). The uncertainty of the thermopysical properties was estimated by introducing the uncertainty in the temperature measurement into the equations given by [72]. The obtained uncertainty values are included in the Table 2.3 for the different $\mathrm{NaCl}$ concentration tested. In that way the uncertainties were estimated for the different

Table 2.2: Accuracy on the directly measured variables

\begin{tabular}{ll}
\hline Measurement & Accuracy \\
\hline \hline Coolant mass flow $\left(\dot{m}_{c}\right)$ & $\pm 0.1 \%$ full scale \\
Wall temperature $\left(T_{w}\right)$ & $\leq \pm 0.26^{\circ} \mathrm{C}$ \\
Bulk temperature $\left(T_{b}\right)$ & $\pm 0.03^{\circ} \mathrm{C}$ \\
Coolant inlet temperature $\left(T_{c, e}\right)$ & $\pm 0.03^{\circ} \mathrm{C}$ \\
Coolant outlet temperature $\left(T_{c, o}\right)$ & $\pm 0.03^{\circ} \mathrm{C}$ \\
Rotating velocity $(N)$ & $\pm 0.005 \%$ singal $+ \pm 0.1 \%$ full scale \\
\hline
\end{tabular}



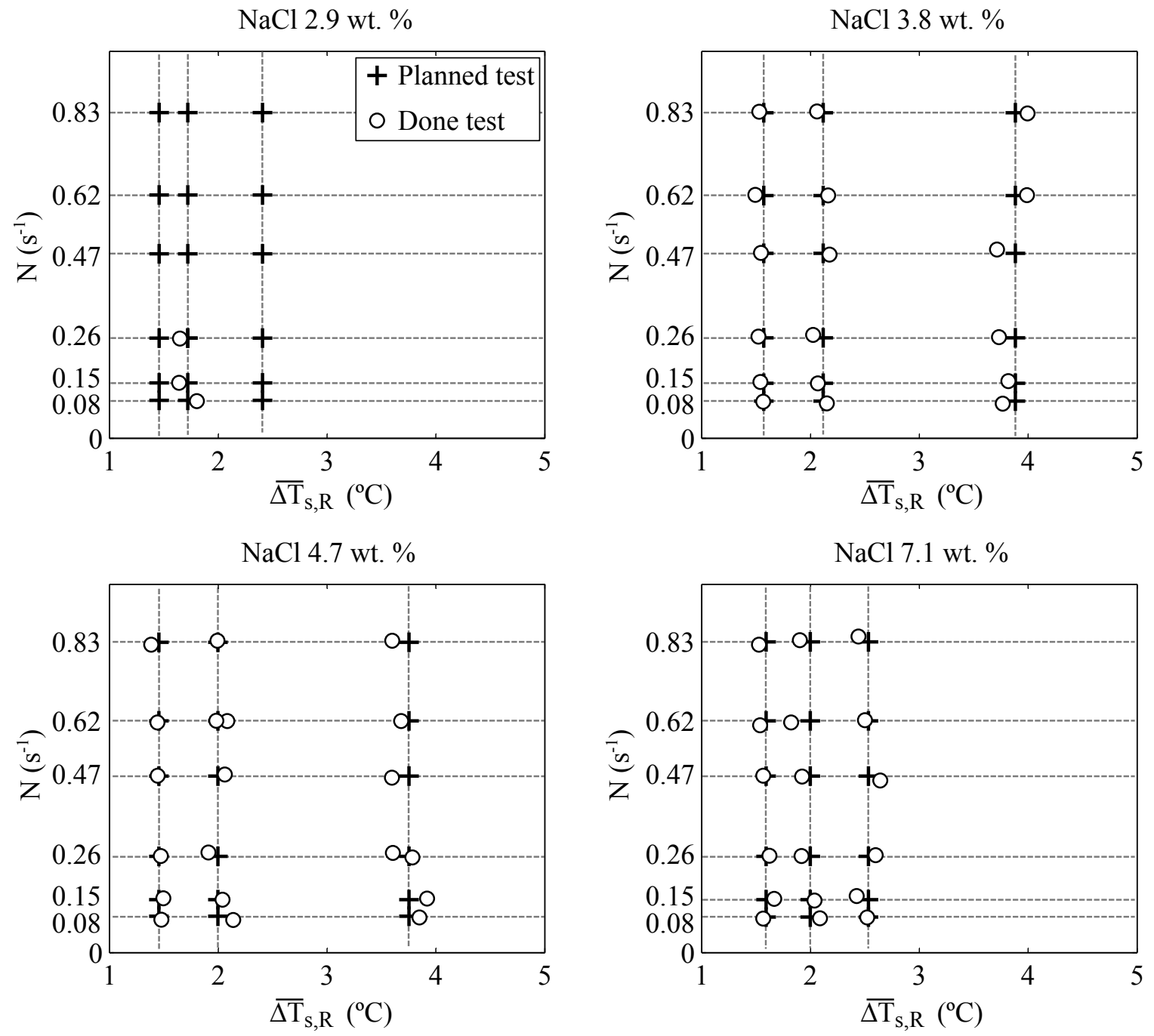

$\mathrm{NaCl} 9.4$ wt. \%

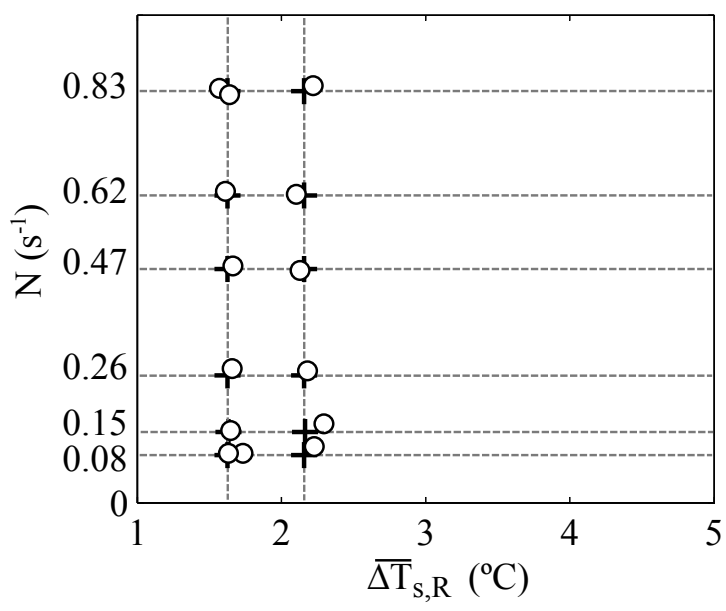

Figure 2.15: Planned and done tests: scraping velocities, initial $\mathrm{NaCl}$ concentration and averaged wall supercooling degree. 

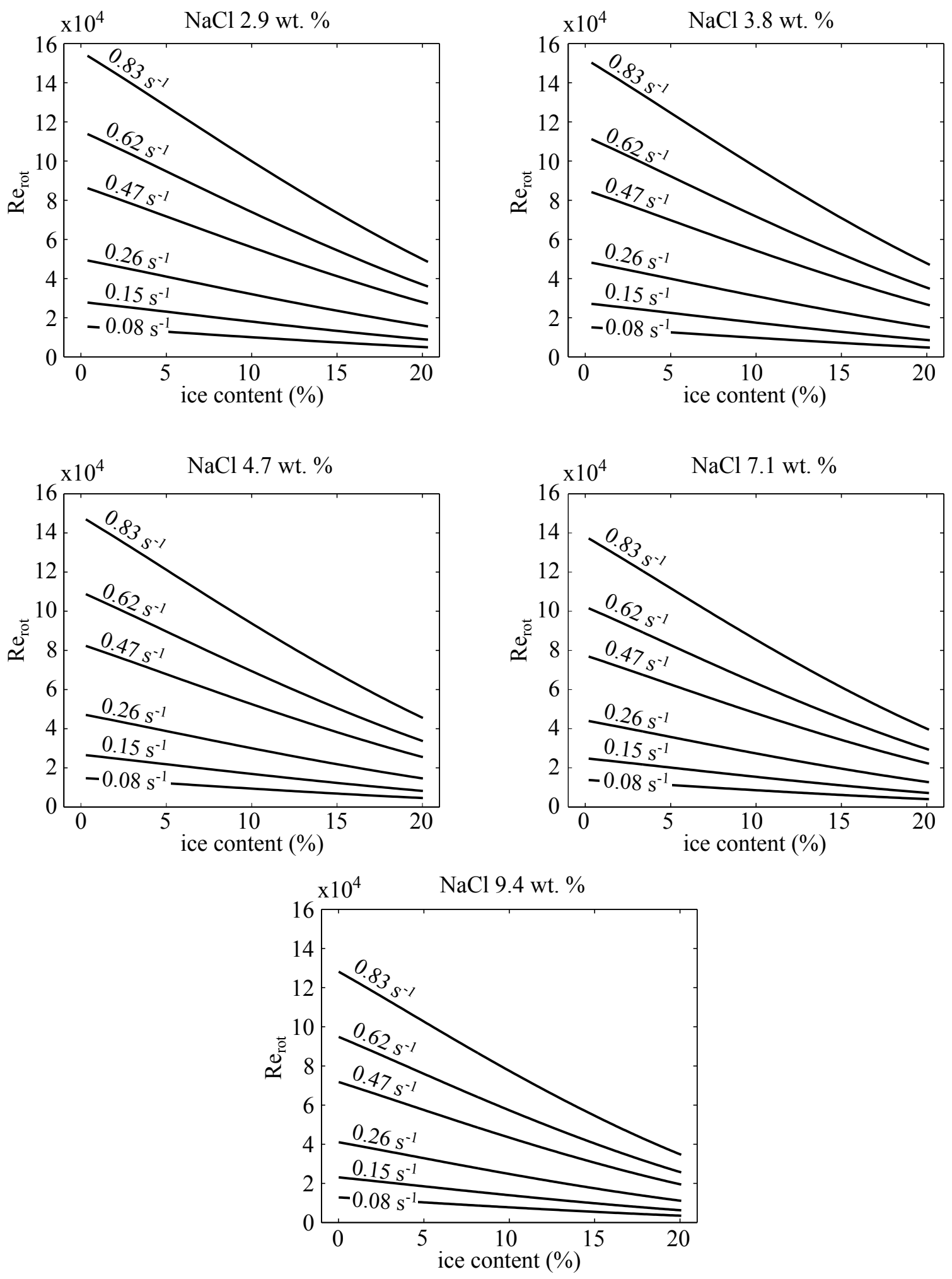

Figure 2.16: Reynolds values for the initial $\mathrm{NaCl}$ concentrations and ice content experimented ranges. 
Table 2.3: Accuracy on the thermophysical properties of the ice slurry

\begin{tabular}{clllll}
\hline \multirow{2}{*}{$\mathrm{NaCl}_{0}(\%)$} & \multicolumn{5}{c}{$E(\%)$} \\
\cline { 2 - 6 } & $\mathrm{NaCl}$ & $\rho$ & $k$ & $\mu$ & $\phi_{m}$ \\
\hline \hline 2.9 & 0.06 & 0.004 & 0.07 & 0.4 & 0.3 \\
3.8 & 0.04 & 0.002 & 0.04 & 0.2 & 0.2 \\
4.7 & 0.03 & 0.002 & 0.03 & 0.2 & 0.1 \\
7.1 & 0.01 & 0.001 & 0.01 & 0.1 & 0.1 \\
9.4 & 0.01 & 0.001 & 0.01 & 0.1 & 0.1 \\
\hline
\end{tabular}

$\mathrm{NaCl}$ concentration tested. The uncertainty in the SSHPE scraped surface area $A$ was calculated as $0.3 \%$. The total uncertainties were calculated according to [76] and based on a $95 \%$ confidence level, in agreement with the methodology recommended in ISO [77].

\section{Heat transfer coefficient}

The heat transfer coefficient is calculated by introducing the heat flux expression (Eq. 2.12) in to Eq. 2.15.

$$
h=\frac{\dot{m}_{c} c_{p, c} \Delta \mathrm{T}_{b, w}}{A \Delta \mathrm{T}_{c}}
$$

being $\Delta \mathrm{T}_{c}$ is the temperature difference between coolant inlet and outlet and $\Delta \mathrm{T}_{b, w}$ is the temperature difference between wall and bulk $T_{b}-T_{w}$. The uncertainty on $h, E(h)$ can be then obtained as

$$
\begin{array}{r}
E(h)^{2}=\left(\frac{\partial h}{\partial \dot{m}_{c}}\right)^{2} E(\dot{m})^{2}+\left(\frac{\partial h}{\partial c_{p, c}}\right)^{2} E\left(c_{p, c}\right)^{2}+\left(\frac{\partial h}{\partial A}\right)^{2} E(A)^{2} \\
+\left(\frac{\partial h}{\partial \Delta \mathrm{T}_{b, w}}\right)^{2} E \Delta\left(T_{b, w}\right)^{2}+\left(\frac{\partial h}{\partial \Delta \mathrm{T}_{c}}\right)^{2} E\left(\Delta \mathrm{T}_{c}\right)^{2}
\end{array}
$$

where $E\left(\Delta \mathrm{T}_{c}\right)^{2}=E\left(T_{c, e}\right)^{2}+E\left(T_{c, o}\right)^{2}$ and $E\left(\Delta \mathrm{T}_{b, w}\right)^{2}=E\left(T_{w}\right)^{2}+E\left(T_{b}\right)^{2}$. Applying 2.19 to 2.18 :

$$
\begin{array}{r}
E(h)^{2}=\left(\frac{c_{p, c} \Delta \mathrm{T}_{c}}{A \Delta T_{b, w}}\right)^{2} E\left(\dot{m}_{c}\right)^{2}+\left(\frac{\dot{m}_{c} c_{p, c}}{A \Delta T_{b, w}}\right)^{2} E\left(\Delta \mathrm{T}_{c}\right)^{2} \\
+\left(\frac{\dot{m}_{c} c_{p, c} \Delta \mathrm{T}_{c}}{A \Delta T_{b, w}^{2}}\right)^{2} E\left(\Delta \mathrm{T}_{b, w}\right)^{2}+\left(\frac{\dot{m}_{c} \Delta \mathrm{T}_{c}}{A \Delta T_{b, w}}\right)^{2} E\left(c_{p, c}\right)^{2} \\
+\left(\frac{\dot{m}_{c} c_{p, c} \Delta \mathrm{T}_{c}}{\Delta T_{b, w} A^{2}}\right)^{2} E(A)^{2}
\end{array}
$$

Since $c_{p, c}$ and $\dot{m}$ are constant during the test and almost independent of the tem- 


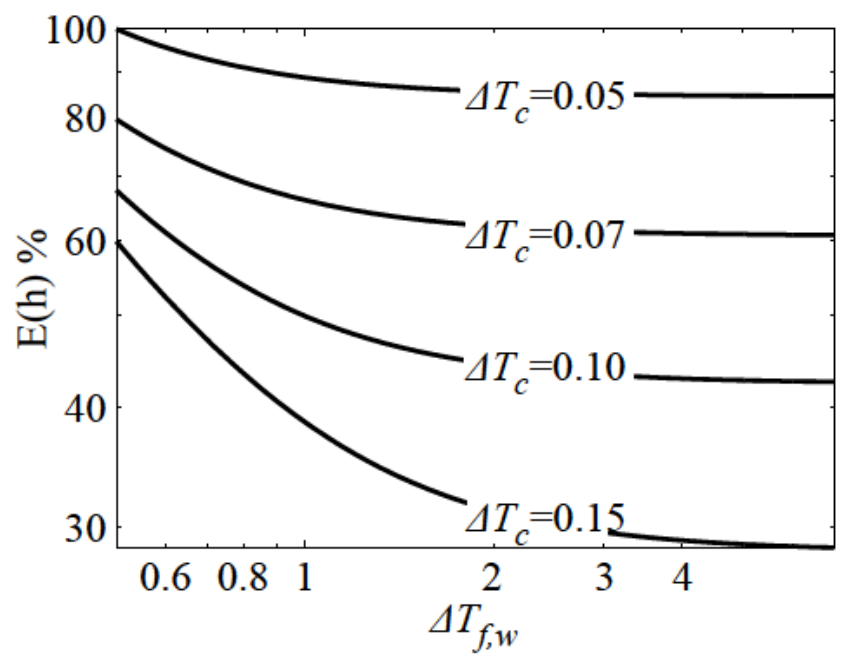

Figure 2.17: Heat transfer coefficient error for different values of $\Delta \mathrm{T}_{b, w}$ and $\Delta \mathrm{T}_{c}$.

perature, the error in the heat transfer coefficient changes mostly with $\Delta \mathrm{T}_{b, w}$ and $\Delta \mathrm{T}_{c}$. Figure 2.17 shows the uncertainty in the heat transfer coefficient (\%) for different values of $\Delta \mathrm{T}_{b, w}$ and $\Delta \mathrm{T}_{c}$. As it is possible to see, $E(h)$ increases dramatically with $\Delta \mathrm{T}_{c}$. In the majority of the experiments the values of $\Delta \mathrm{T}_{c}$ ranged from 0.10 to $0.14{ }^{\circ} \mathrm{C}$, which means errors on $h$ of around $\pm 23 \%$. This high uncertainty is the price to pay for having low $\Delta \mathrm{T}_{c}$ values which in turn guarantees low differences among the temperatures on the wall: higher $\Delta \mathrm{T}_{c}$ values would mean higher differences between the coolant temperatures along the coiled circuit and therefore significant wall temperature differences.

\section{Nusselt number}

The error in the Nusselt number can be obtained by differentiation of Eq. 2.17 respect to the depending variables,

$$
E(N u)^{2}=\left(\frac{\partial N u}{\partial h}\right)^{2} E(h)^{2}+\left(\frac{\partial N u}{\partial H}\right)^{2} E(H)^{2}+\left(\frac{\partial N u}{\partial k_{t}}\right)^{2} E\left(k_{t}\right)^{2}
$$

The differentiation of Eq. 2.17 leads to

$$
E(N u)^{2}=\left(\frac{H}{k_{t}}\right)^{2} E(h)^{2}+\left(\frac{h}{k_{t}}\right)^{2} E(H)^{2}+\left(\frac{h H}{k_{t}^{2}}\right)^{2} E\left(k_{t}\right)^{2}
$$

which in thus depends on the uncertainties on $h$ and $k_{t}$-are already know- and $H$, the height of the SSHPE upper side with an uncertainty of $\pm 0.5 \mathrm{~mm}$. The uncertainty values obtained in Eq. 2.22 are very similar to those obtained by h, as the uncertainties in $H$ and $k_{t}$ are very low compared with the one of $h$. Therefore an uncertainty of $\pm 23 \%$ can be also assumed for $\mathrm{Nu}$. 


\section{Reynolds number}

By the same procedure followed for $h$ and $N u$, the uncertainty in the rotating Reynodls number (2.9) can be obtained from

$$
\begin{aligned}
E\left(R e_{\mathrm{rot}}\right)^{2} & =\left(\frac{\partial R e_{\mathrm{rot}}}{\partial \rho}\right)^{2} E(\rho)^{2}+\left(\frac{\partial R e_{\mathrm{rot}}}{\partial N}\right)^{2} E(N)^{2} \\
& +\left(\frac{\partial R e_{\mathrm{rot}}}{\partial D}\right)^{2} E(D)^{2}+\left(\frac{\partial R e_{\mathrm{rot}}}{\partial \mu}\right)^{2} E(\mu)^{2}
\end{aligned}
$$

Substituting then we have

$$
\begin{gathered}
E\left(R e_{\mathrm{rot}}\right)^{2}=\left(\frac{N D^{2}}{\mu}\right)^{2} E(\rho)^{2}+\left(\frac{\rho D^{2}}{\mu}\right)^{2} E(N)^{2} \\
+\left(\frac{2 \rho N D}{\mu}\right)^{2} E(D)^{2}+\left(\frac{\rho N D^{2}}{\mu^{2}}\right)^{2} E(\mu)^{2} .
\end{gathered}
$$

The obtained errors on $R e_{\text {rot }}$ revealed a maximum value of $2 \%$ for the most unfavourable situation.

\section{Richardson number}

The uncertainty in the Richardson number is given by

$$
\begin{gathered}
E(R i)^{2}=\left(\frac{\partial R i}{\partial \beta}\right)^{2} E(\beta)^{2}+\left(\frac{\partial R i}{\partial \Delta \mathrm{T}_{b, w}}\right)^{2} E\left(\Delta \mathrm{T}_{b, w}\right)^{2} \\
+\left(\frac{\partial R i}{\partial H}\right)^{2} E(H)^{2}+\left(\frac{\partial R i}{\partial D}\right)^{2} E(D)^{2}+\left(\frac{\partial R i}{\partial \Omega}\right)^{2} E(\Omega)^{2}
\end{gathered}
$$

and applying the above equation to Eq 2.10

$$
\begin{array}{r}
E(R i)^{2}=\left(\frac{g \Delta \mathrm{T}_{b, w}(H / 2)}{\Omega\left(D / 2^{2}\right)}\right)^{2} E(\beta)^{2}+\left(\frac{\left.g \beta \Delta \mathrm{T}_{b, w}\right)}{2 \Omega\left(D / 2^{2}\right)}\right)^{2} E(L)^{2} \\
+\left(\frac{g \beta(H / 2)}{\Omega(D / 2)}\right)^{2} E\left(\Delta \mathrm{T}_{b, w}\right)^{2}+\left(\frac{g \beta \Delta \mathrm{T}_{b, w}(H / 2)}{\Omega^{2}(D / 2)^{2}}\right)^{2} E(\Omega)^{2} \\
+\left(\frac{g \beta \Delta \mathrm{T}_{b, w}(H / 2)}{\Omega(D / 2)^{4}}\right)^{2} E(D)^{2}
\end{array}
$$

As with the heat transfer coefficient, the effect of the low $\Delta \mathrm{T}_{b, w}$ is dramatic and takes off the uncertainty values for the Richardson number, with $\pm 25 \%$. 


\section{Chapter 3}

\section{Materials and methods II: Flow visualization rig}

The heat transfer performance in scraped surface heat exchangers is directly related with the flow structure induce by their geometry and operating conditions. Low velocity or stagnant regions, the presence of recirculations or even stratified flows are responsible of poor mixing and in general should be avoided. In the case of crystallization processes, the mixing and interaction between solid and liquid phases is determinant due to two reasons: a) the final product quality depends to a certain level of degree of mixing. If the flow is not adequately homogenized, it may lead to stratification and later pump blockage. b) the impact of the ice particles driven by the flow on the scraped surface may induce additional fouling, reducing the heat transfer. Hence, not only the flow pattern but also the solid-liquid interaction results of great interest in the experimental characterization of the SSPHE.

The simultaneous combination of visualization and heat transfer experiments involves many difficulties. Ice crystals are in general not a good tracer to study the fluid flow. In addition, any introduced seeding particles could affect the nucleation process and are therefore not viable. Moreover, the thermal isolation of the facility hampers any visual access or illumination. An alternative facility, able to match the ice slurry production conditions in therms of flow pattern, is then required. With that aim, a multi-purpose visualization facility was designed to carry out both PIV experiments for single phase test and flow visualization for multiphase solid-liquid flow, providing also valuable information for the validation of further numerical models. This chapter explains in detail the setup and the performed experiments.

\subsection{Visualization facility}

An overview picture of the visualization rig is shown Figure 3.1. It consists of an external bounding box made of PVC (total volume) which contains a methacrylate band disposed in a circular way, leading to an inner volume (SSPHE) and an outer volume. The external box contains methacrylate window through which a CMOS camera records the inside of the SSHPE. The two-volume configuration avoids optical distortions while acquiring images. The top side of the box is closed by a PVC tap which includes a window above the visualized region. This window allows the light 


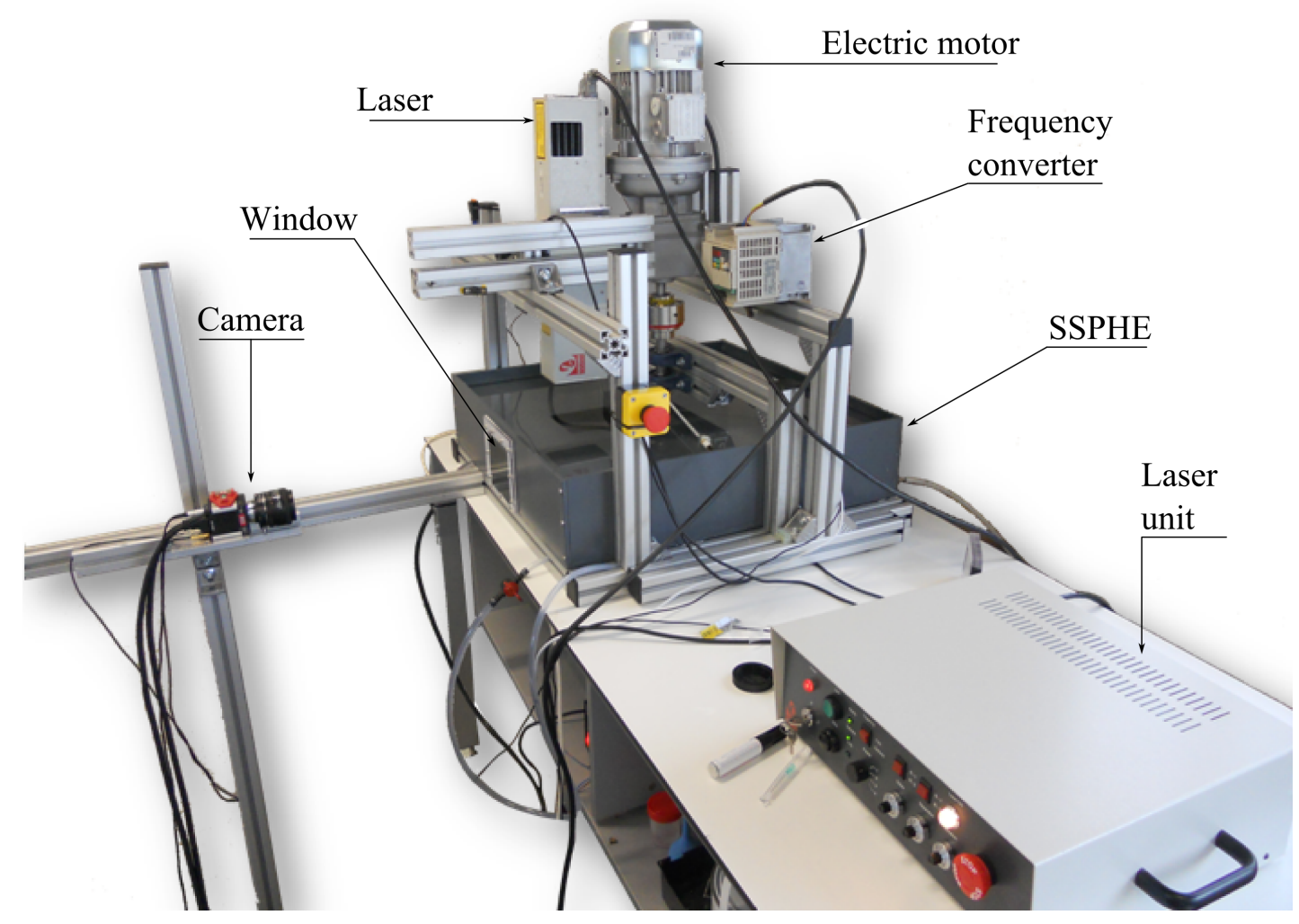

Figure 3.1: Experimental facility overview

source - either continuous light or laser beam- go inside the volume.

As in the ice slurry production facility, the scraper blades are formed by four stainless steel arms $90^{\circ}$ separated and covered by PEEK. The four blades are fixed to a rotating shaft. The shaft is actuated by a gear box driven by an electric motor. The rotating velocity of the electric motor and therefore of the scraper blades is also here accurately setted by a frequency controller. The driving motor is capable of wider rotating range compared to the ice slurry production setup.

\subsection{Visualization of the particle motion}

A sketch of the visualization facility adapted for the particle motion visualization tests is show in 3.2. The aim here is to emulate the solid-liquid multiphase flow that occurs in the SSHPE. Ice particles are substituted by a polymer with similar size and density of the particles but with temperature-independent properties. Two continuous light sources pointing to the top window were used to illuminate the SSPHE inside. A light diffuser is placed in the window, which ensures a uniform illumination of the visualized region.

In order to simulate the inclusion of the particles to the flow as it occurs the SSPHE by the scraping action, they were injected through a hole in the bottom surface. A slurry made of water/propylene-glycol mixture as working fluid and high particle content was prepared and pumped by means of a manual syringe in the instant that the scraper 


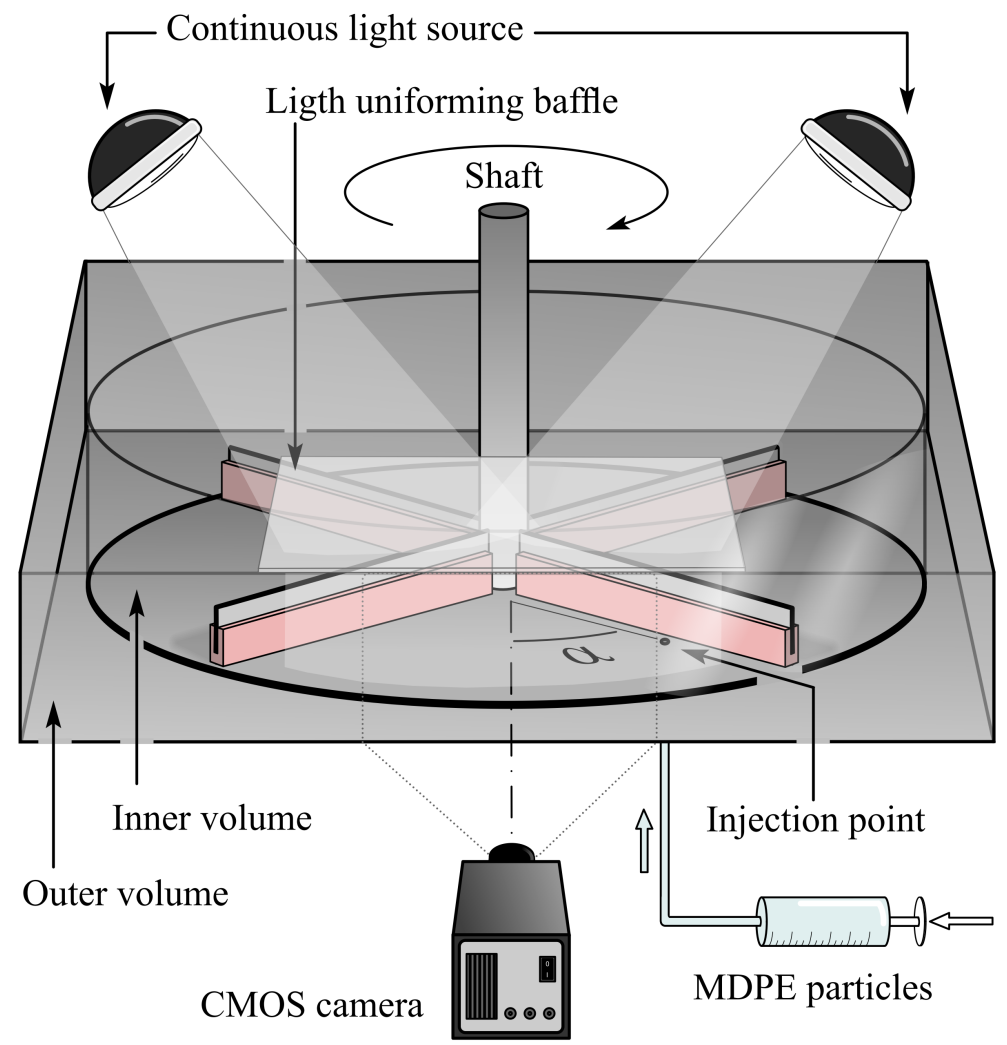

Figure 3.2: Schematic view of the experimental facility adapted for the particle motion visualization tests

passed over the injection hole The hole is placed at a distance of $14 \mathrm{~cm}$ from the shaft and at an angle $\alpha=40^{\circ}$ upstream the camera visual axis (see Fig. 3.1).

The injection system is depicted in Fig. 3.3. To avoid any vertical velocity on the injected particles that could represent a perturbation of the flow in the SSPHE, particles were deflected by a flexible sheet (see Fig. 3.3a). Nevertheless, injection were done at very low velocities, avoiding any perturbation of the flow. Particles were homogeneously distributed in the syringe slurry and injections corresponded to equal displacements of the plunger, ensuring an approximate equal amount of particles released for each injection. The developed injection system allowed to add the particles with a developed flow in the heat exchanger.

Images where continuously recorded at $30 \mathrm{~Hz}$ by a CMOS camera placed in front of the visualization window.

\subsubsection{Working fluid and icelike particles}

In order to have equivalent and representative results, the governing non dimensional numbers in the visualization tests should match those of the ice production experiments. In absence of heat transfer and neglecting any other thermal effect, the problem is characterized only by the rotating Reynolds number and the Froude number: 


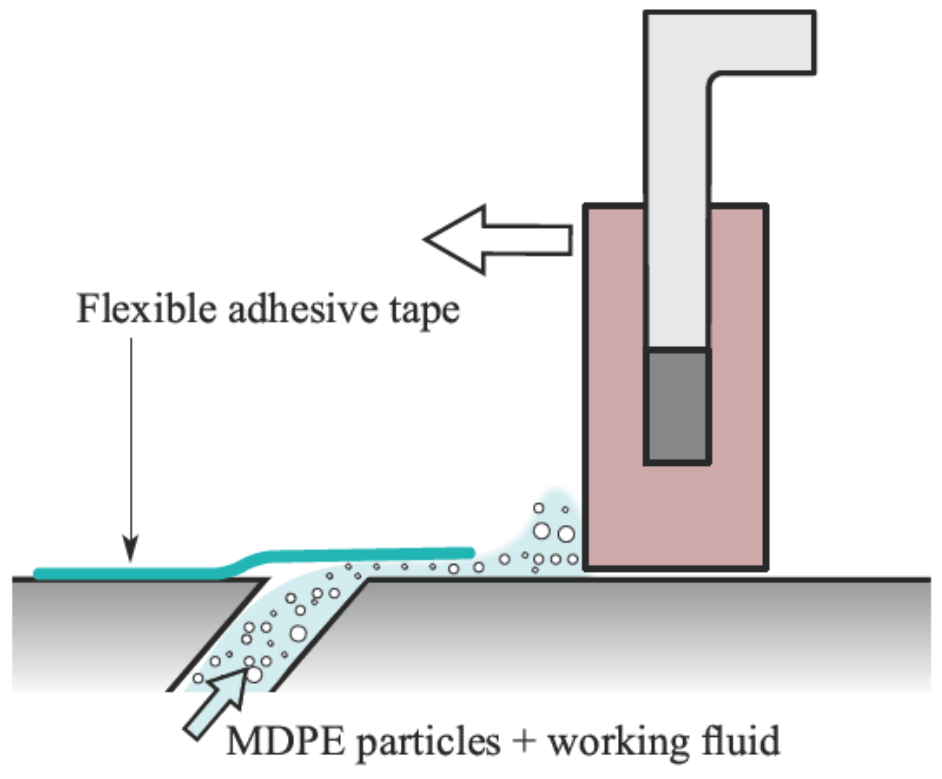

(a)

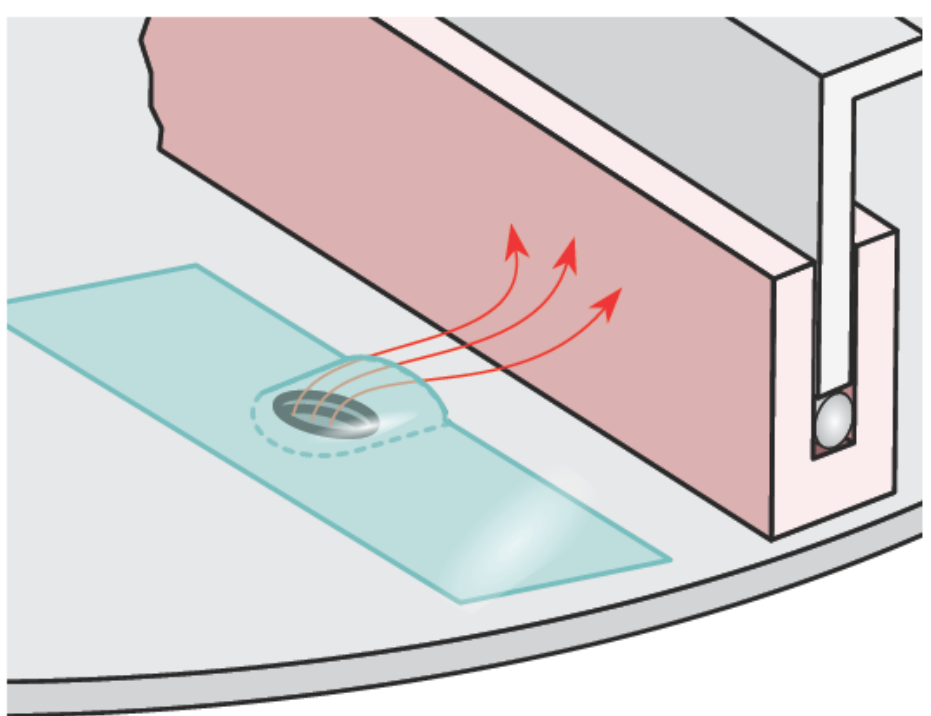

(b)

Figure 3.3: Sketch of the particles injection system. a) Cross sectional view. b) Side view. 


$$
\begin{gathered}
R e_{\text {rot }}=\frac{\rho_{l} N D^{2}}{\mu} \\
F r=\frac{(\Omega(D / 2))^{2}}{H g\left(1-\frac{\rho_{p}}{\rho_{l}}\right)}
\end{gathered}
$$

Whereas the rotating Reynolds number is the ratio between inertia and viscous forces in the flow, the Froude number represents here the ratio between the forced flow induced by the scraper arms and the buoyancy force of the particles. High Froude number values indicates then a predominant effect of the forced flow, where the solid particles are expected to be well distributed in the fluid. If the Froude number is low the buoyant force will be predominant and the particles will tend to accumulate in the top.

The experiments were designed to match the conditions where the content of ice in the slurry

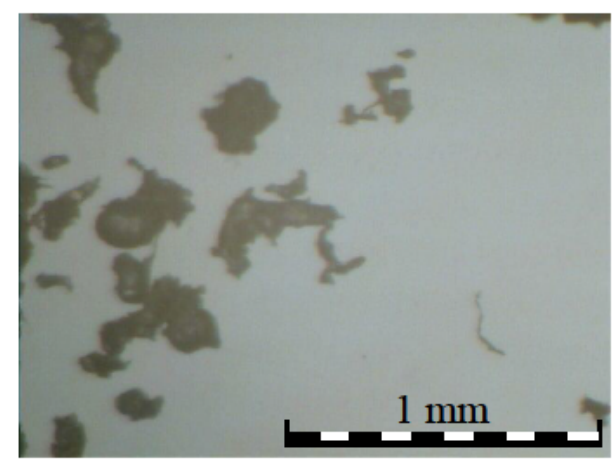

Figure 3.4: Microscopic image of the polyethylene particles used as ice crystal emulators. is very low and therefore its effect in the viscosity and density is negligible. To match $R e_{\text {rot }}$ then it is only required to make equal the rotating velocity $\mathrm{N}$ and the viscosity of the fluid. The value $\mathrm{N}$ was adjusted to the same values of the ice slurry production tests. To consider the effect of the $\mathrm{NaCl}$ in the viscosity, a mixture of propylene-glycol and water was used as a working fluid, adjusting the content of propylene-glycol to have the same viscosity values. Therefore we have that

$$
\left.\frac{\rho N D^{2}}{\mu}\right|_{w t-P G}=\left.\frac{\rho N D^{2}}{\mu}\right|_{w t-N a C l}
$$

For a $4 \% \mathrm{NaCl}$ aqueous solution it results in about $20.3 \%$ of propylene-glycol.

Medium density polyethylene (MDPE) particles were employed, with an average diameter of $350 \mu \mathrm{m}$ and density $\rho_{p}=938 \mathrm{~kg} / \mathrm{m}^{3}$. The chosen particles approaches in size and density the ice particles generated in the SSHPE (see chapter 44.8 ), with averages diameter and density of $300 \mu \mathrm{m}$ and $\rho_{h}=918 \mathrm{~kg} / \mathrm{m}^{3}$ respectively. The difference between ice and MDPE densities is $2 \%$, which even being such a low value does not represent a problem, as the values to match are the governing dimensionless numbers, Reynolds and Froude

A microscopic image of the polyethylene particles is shown in Fig. 3.4. The averaged diameter was measured in $\approx 200 \mu \mathrm{m}$, and the density in $938 \mathrm{~kg} / \mathrm{m}^{3}$-the ice density at, for instance, $-3{ }^{\circ} \mathrm{C}$ is $918 \mathrm{~kg} / \mathrm{m}^{3}$.

A maximum difference of $5 \%$ was found between Froude numbers when comparing the ice slurry and the MDPE-PG mixtures. 


\subsubsection{Image processing and particle motion}

The acquired images were analysed to determine the displacement of the particles. For this purpose, an optical flow technique was adopted -after the conversion of them to black and white color format- based on the comparison of successive images.

Optical flow basis Among all the different ways of measuring the difference or similarity between images, the correlation-based methods are based on the analysis of the gray level pattern around the point of interest and on the search for the most similar pattern in the successive image. Basically, having defined a window $W(\vec{x})$ around the point $\vec{x}$, similar windows are then considered $W^{\prime}(x+i \overrightarrow{, y}+j)$ shifted by the possible integer values in pixels in a search space $\mathrm{S}$ composed by the $i, j$ such as $-\Delta<i<\Delta$ and $-\Delta<j<\Delta$. The optical flow, i.e. the estimated image displacement is taken as the shift corresponding to the minimum of a distance function (or maximum correlation measure) between the intensity pattern in the two corresponding windows $f\left(W, W^{\prime}(i, j)\right)[78]$.

The basic implicit assumptions are that the gray level pattern is approximately constant between successive frames (no perspective effects) and that local texture contains sufficient unambiguous informations. Optical flow estimators based on correlation are less sensitive to noise than the derivative-based ones, with better performances if the texture is not relevant and in case of large inter-frame displacements.

The process can then be summarized in, having a square or rectangular window $-N x N$ or $N x M-$, computing the motion between a window centred in $(x, y)$ in the frame $I_{1}$ and a window shifter by $(i, j)$ in the following frame, $I_{2}$.

Since the method is based in the maximization of a correlation measure, the normalized cross-correlation (NCC) should be used; it is less sensitive to the noise and to changes in lighting conditions than the standard one [79]. The NCC is defined in Eq. 3.4 , where $i$ goes from $-N / 2$ to $N / 2$ and $j$ goes from $-M / 2$ to $M / 2$.

$$
C_{f}(\vec{x}, \vec{d})=\sum_{i, j} \frac{I_{1}(x+i, y+j) I_{2}\left(x+i+d_{x}, y+j+d_{y}\right)}{\sqrt{\sum_{i, j} I_{1}(x+i, y+j)^{2} \sum_{i, j} I_{2}\left(x+i+d_{x}, y+j+d_{y}\right)^{2}}}
$$

The window size has to be carefully chosen: if small windows are used, the amount of information inside the window is small and the estimate is not reliable, whereas if the windows are too large the hypothesis of negligible deformations of the pattern inside the window fails and the estimate can be wrong. Furthermore, the computational complexity is greatly increased. The ideal size depends on the texture inside.

Particle motion estimation The particle motion determination process for an initial window size (frame A) and interrogation area (frame B) is decomposed into the following steps:

a) Background removal with a clean image

b) Image enhancing

c) Image division and comparative through cross correlation algorithm 


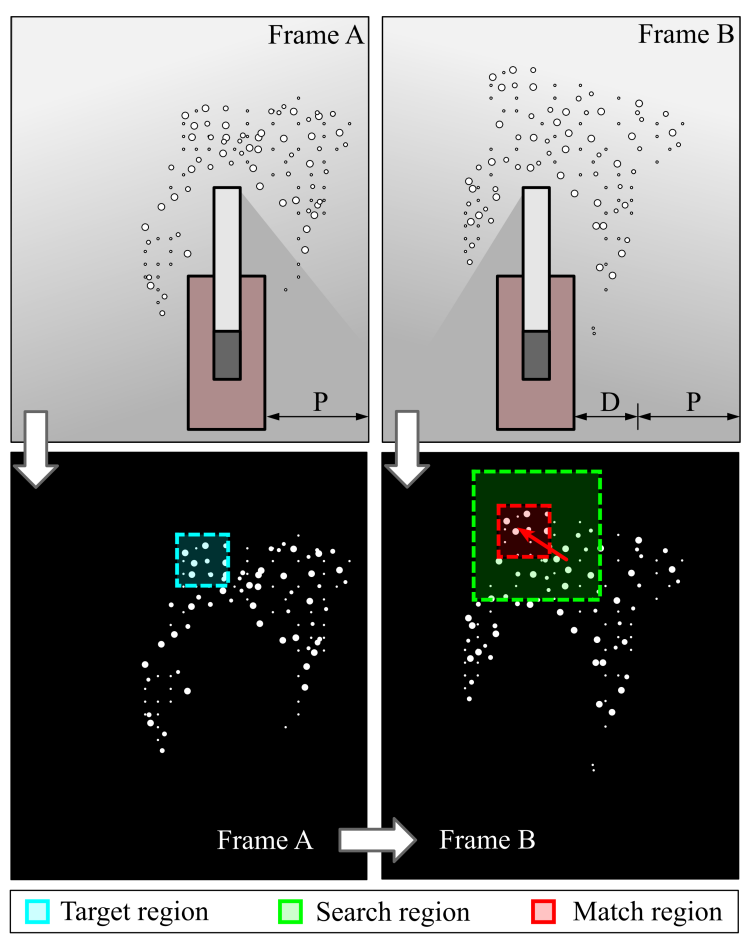

Figure 3.5: Particles displacement estimation method: original frames (up) and background-subtracted (down).

d) Determination of the displacements

The above process is expressed graphically in Fig. 3.5. Two consecutive frames are shown on the top. A displacement of the scraper equal to D occurs between them. Once the background is removed and the resulting images enhanced, a window (search region) in frame $\mathrm{A}$ is searched for in a region of interest of frame B. The peak given by the normalized cross correlation determines then which is the most probable displacement of the group of particles. A further step will comprise the sub-division of the window into four windows, whose search in the next frame will be based in the location of the initial found window. The subdivision process is explained more in detail further in this section.

An example of steps a)-b) is shown in Fig. 3.6. The background removal, i.e. the subtraction from the particles image of an identical (clean) image -without particlesremoves unnecessary information not related with the particles displacement. Moreover, it also corrects partially the non-uniform lighting conditions. The clean images were acquired before the particles injection under identical conditions of light and scraping velocity. The resultant image is then enhanced amplifying the particles signal in 3 or 4 times (contrast adjustment). The amplification level is a key-point: low levels will result in images without information about the particles, whereas high levels will result in the absence of texture in the particle cloud.

The background removal and image enhancing represent an increase in the peak detection. As a prove of this, Fig. 3.7 shows the obtained normalized cross-correlation planes obtained respectively for background and background-removed images. The 


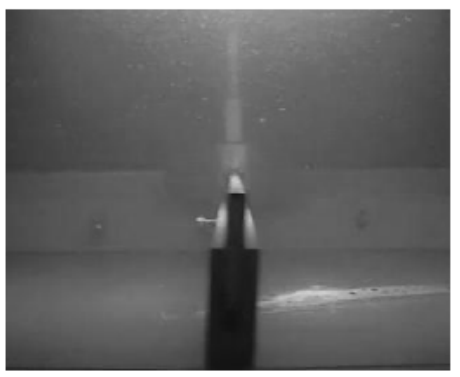

(a)

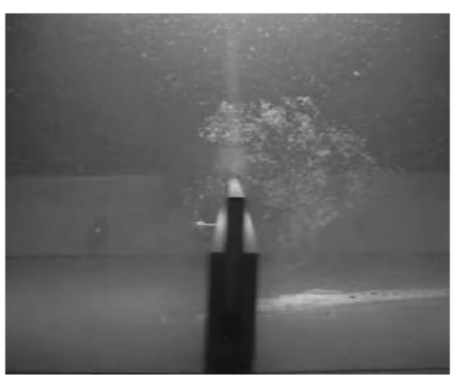

(b)

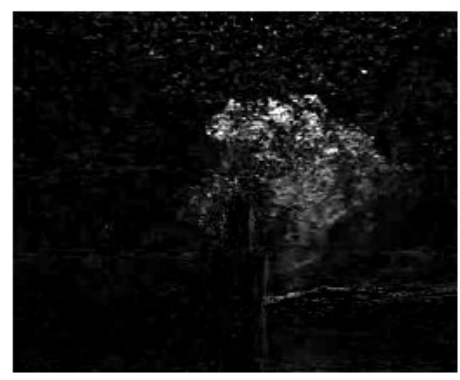

(c)

Figure 3.6: Background subtraction and enhancing process: a) background clean image, b) particles image and c) sustracted and enhanced image.

random correlation peaks distributed along the correlation plane correspond to correlations between distinct sub-image pairs. A valid displacement measurement is achieved when the highest detectable peak in the plane represents the true displacement. As a result, the strength (detectability) of the primary peak with respect to surrounding peaks represents the signal to noise ratio of the correlation plane. The peak-to-peak ratio (PPR) is taken as a quantitative measurement of the detectability of the primary peak, defined as,

$$
P P R=\frac{C_{\max }}{C_{2}}
$$

as it is indicated in Fig. 3.7. The background-removal and enhancing process represents therefore a $60 \%$ of increase in the PPR. The NCC was computed according to Eq. 3.4 and following the general procedure:

1. Calculate cross-correlation in the spatial or the frequency domain, depending on size of images.

2. Calculate local sums by pre-computing running sums.

3. Use local sums to normalize the cross-correlation to get correlation coefficients.

The window size (i.e., the group of particles to look for in the next image) varies depending on the particles group size, shape or even in the illumination conditions; a window size or interrogation area (where to look for the particles in the next frame) that resulted valid for one image/region could be not for another one. Therefore, the window size and the interrogation area were iteratively adjusted until a value of $P P R \geq 1.5$ was obtained.

The process consisted first in the division of the image into a set $10 \mathrm{x} 6$ sub-images grid. Each sub-image containing particles was searched in an interrogation area of the next frame, $2.5 \times 2.5$ times bigger than the window size. If the PPR value was lower than 1.5 then the area of the initial window was changed to one 1.25 times bigger and one 1.25 times smaller. If one of the changed windows retrieved a PPR value between the one obtained previously and 1.5 , then a further window size reduction/augmentation was performed. If the value of PPR was still lower than 1.5 the area was discarded, 


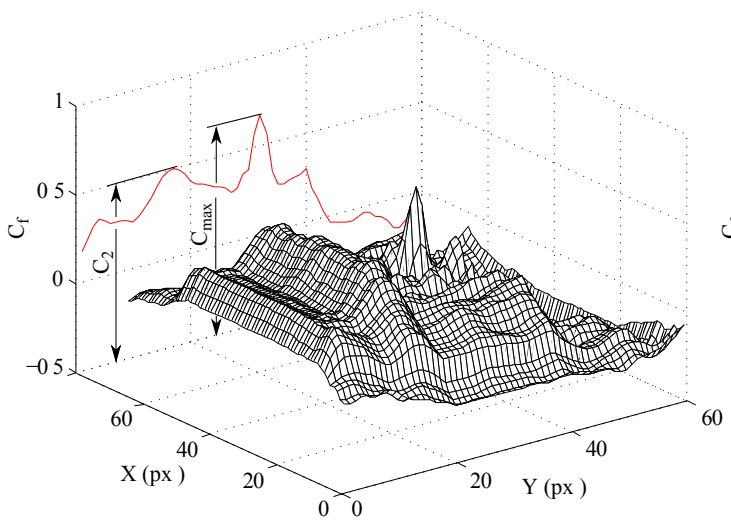

(a)

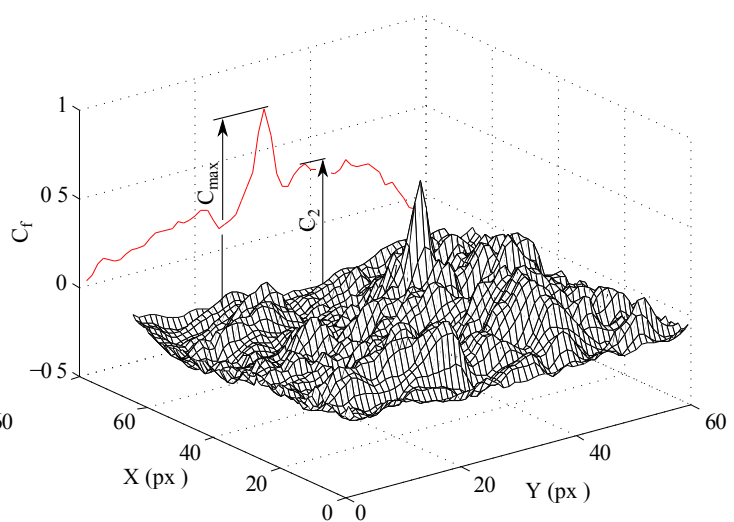

(b)

Figure 3.7: Cross-correlation coefficient values after and before background subtraction process. a) With background, $\mathrm{PPR}=1.3$. b) Without background, $\mathrm{PPR}=2.1$

and the algorithm passed to proceed with the next sub-image of the $10 \mathrm{x} 6$ grid. If in any moment a value of $\mathrm{PPR} \geq 1.5$ was reached then the corresponding displacement was calculated.

For all the valid displacement calculated (PPR $\geq 1.5)$, each window in the first frame was then divided into four equal windows. The interrogation area in the second frame was then taken as 1.5 times the fourth of the area where the particles where found, centred in the corresponding quadrant. This subdivision step is graphically explained in Fig. 3.8.

\subsection{Particle stratification tests}

To study the flow pattern with high ice concentrations, a water/propylene-glycol solution with $4 \%$ in weight of MDPE particles were added to the liquid. The velocity of the scrapers was then increased observing the degree of mixing until it was high enough to impede the light to cross through. To have light even when particles were stratified on the top, continuous light sources were places immersed on the fluid, in the space between the bounding box and the SSPHE plexiglass walls.

\subsection{Particle Image Velocimetry}

\subsubsection{Fundamentals and technical considerations}

Particle image velocimetry (PIV) technique provides instantaneous velocity fields over global domains by recording the position over time of small tracer particles introduced into the flow. The principle of the PIV technique is similar to the one exposed in the previous section for the particle motion technique. The difference lies in the fact that, while the particle motion algorithm intends to retrieve only the motion of the solid particles, PIV aims to extract the fluid velocity. For this purpose particles have to be real tracers of the flow, i.e., the effect of the buoyancy and inertia over them has to be negligible. Moreover, the time interval between frames has to be short enough 


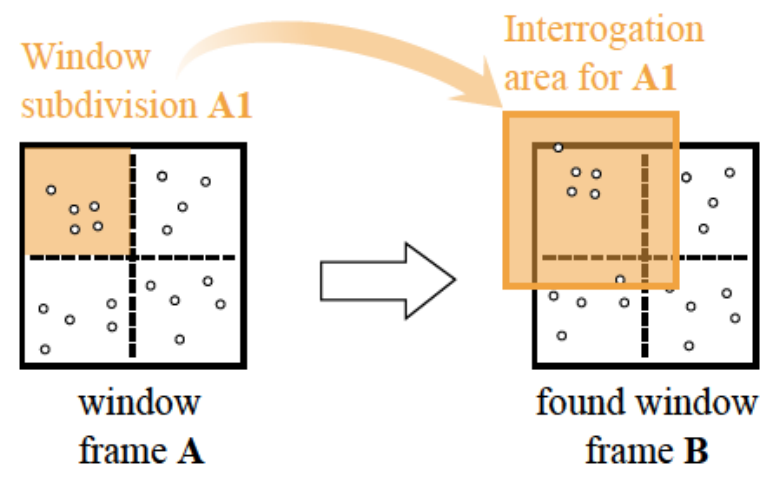

Initial and sub-division

obtained dusplacements

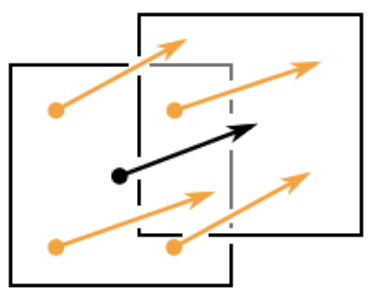

Figure 3.8: Window sub-division process based on the initial displacement information (up) and new displacements (down)

to catch equally fast particle displacements in correspondence with the flow velocity, which requires specific high speed cameras and illumination sources (usually double cavity lasers). Particles should also be well distributed among the fluid in order to have uniform information about the flow, and not only in the regions where particles are present. The post processing process is also more complex, able to perform multiple subdivision steps and even window deformation.

As a result, PIV can provide instantaneous velocity measurements over global (2D or $3 \mathrm{D}$ ) domains with high accuracy. A PIV system requires, at least, four basic components (Fig. 3.9):

- An optically transparent test-section containing the flow seeded with tracer particles

- A light source (commonly laser) to illuminate the region of interest (plane or volume)

- Recording hardware consisting of a high speed camera

- A computer with suitable software to process the recorded images and extract the velocity information from the tracer particle positions.

The sequence commonly followed in the image acquisitions is as follows: first the shutter on the sensor opens to admit the first pulse; the image is then stored in a buffer; the shutter opens again to admit the second pulse; finally both frames are transferred to the memory of the computer. Obviously, the laser, camera and computer must be triggered in the correct sequence. The buffer string is performed very rapidly, such that 


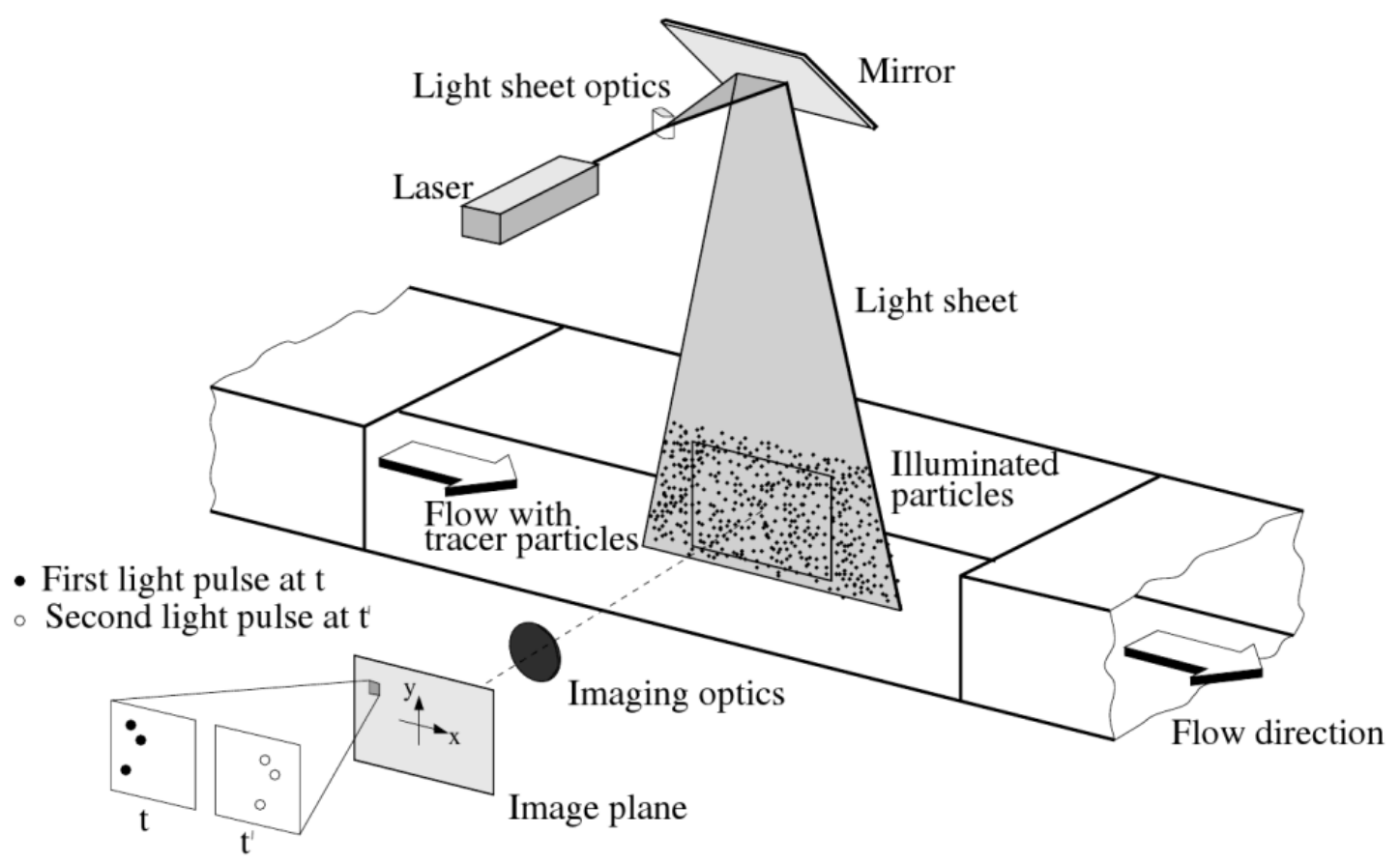

Figure 3.9: Typical PIV arrangement [80]

the pulse separation may be shortened to allow the measurement of even high-speed aerodynamic flows.

In contrast with particle tracking techniques, where image searching is individuated to single particles, PIV determines the average motion of small groups of particles contained within small regions known as interrogations spots. Essentially, the overall frame is divided into interrogation spots, and the correlation function is computed sequentially over all spots providing one displacement vector per spot (Fig. 3.10).

Typically, interrogation spots are square-shaped and hence the velocity map obtained from PIV presents vectors arranged on a uniform grid. Furthermore, the process of averaging over multiple particle pairs within an interrogation spot makes the technique remarkably noise-tolerant and robust in comparison to particle tracking.

The determination of the average particle displacement is accomplished by computing the spatial cross-correlation of the particle images:

$$
\phi(m, n)=\frac{\sum_{i, j=1}^{I, J} I(i, j) \cdot I^{\prime}(i-l, j-m)}{\sqrt{\operatorname{stdev}(I) \cdot \operatorname{stdev}\left(I^{\prime}\right)}}
$$

where $I$ and $I^{\prime}$ have been previously subtracted of their respective mean value. The denominator is a normalizing term ensuring that $\varphi$ varies between -1 and +1 . This process returns the discrete cross-correlation map (a two dimensional array), where the peak position relative to the origin indicates the average particle images displacement. The cross-correlation function is usually computed via a two dimensional Fast Fourier Transform (FFT) of the digitized intensity pattern, reducing in a considerably amount the number of operations. 


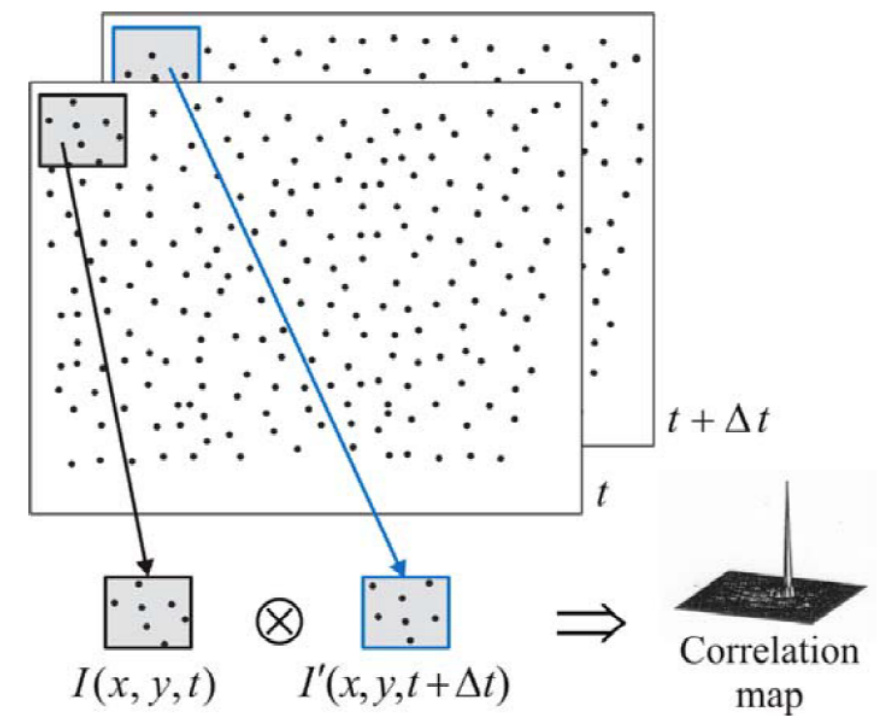

Figure 3.10: Image windowing (spots) and cross-correlation map [81]

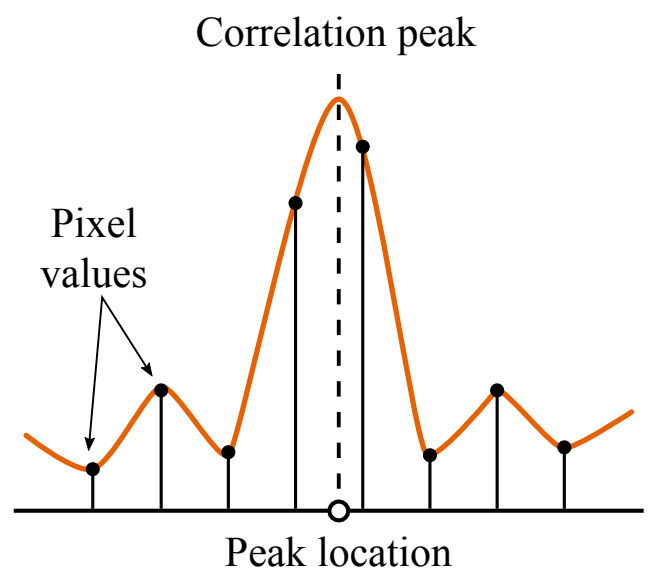

Figure 3.11: Sub-pixel interpolation of correlation peak

The peak position can be more accurately determined by performing a peak correlation, which returns the peak position with sub-pixel precision as shown in Fig. 3.11. This result indicates the particle motion in terms of pixel shift between the corresponding windows. The velocity is obtained dividing it by the known time separation between laser pulses, multiplying by the size of the pixel and dividing by the imaging magnification.

For obtaining a representative and accurate result of the flow, a certain number of rules must be followed regarding the tracer particles, illumination and imaging. They are described below.

Flow seeding Typically microscopic particles are employed to track fluid flows with PIV - with the appropriate amount they can be considered as not affecting the flow properties. The seeding tracers concentration $C$ typically ranges between $10^{9}$ and $10^{12}$ 
particles $/ \mathrm{m}^{3}$ [81]. Higher concentrations can affect the flow properties. Moreover, the tracer particles must be randomly distributed in the flow medium and convected along the local flow trajectory keeping unaltered their properties. The tracer ability can be evaluated through the slip velocity, i.e. the difference of velocities between the surrounding fluid and the particle, $U$ and $U_{p}$ respectively. For very small particles the Stokes drag is dominant and the difference can be expressed as [81]:

$$
U_{p}-U=\frac{d_{p}^{2}\left(\rho_{p}-\rho_{f}\right)}{18 \mu} \frac{d U_{p}}{d t}
$$

The condition of neutral buoyancy $\rho_{f} \approx \rho_{p}$ allows to obtain tracers that accurate follow the flow. This condition is easy to reach in the present case, where the working fluid is liquid. For instance, glass spheres, a common particle tracer for water, has a density of $10^{3} \mathrm{~kg} / \mathrm{m}^{3}$

On the other hand, the illuminated particle tracers must be clearly detected through imaging devices, requiring the particles to scatter light efficiently. The scattering efficiency is quantified by the scattering cross section: such quantity depends first upon the ratio of refractive index, then on the wavelength of light $\lambda^{-4}$ and finally on the particle diameter $d_{p}$. However, the easiest way to improve the efficiency is increasing the particle diameter, as far as the previous $V-U$ difference is small enough.

As $d_{p}$ affects in opposite ways to the scatter and tracer ability of the particles, the requirements of accurate flow tracking and sufficient light scattering are therefore in conflict with each other, requiring a compromise solution to get an optimum.

Illumination Particle tracers need to be illuminated and observed twice within the time separation $\Delta t$, and particle images should not appear as streaks but rather as circular dots. For the latter to be true $\delta t=d_{\tau} / V M$, where $d_{\tau}$ is the size of the particle in the image (see below).

Laser light sources are commonly used for PIV because of their excellent properties in terms of beam collimation and ease to shape into a thin light sheet. Pulsed light sources are most used because of the high temporal definition of the illumination pulse (in the order of nanoseconds). The Neodymium:YAG laser (semiconductor laser) provides an intensity of illumination independent of the flow velocity and they provide enough pulse energy to illuminate regions extending up to $0.5 \mathrm{~m}^{2}$. Two pulses are independently delivered by two separated lasers. The two beams are then combined by a mirror and a polarizing beam combiner.

Imaging A schematic of the PIV optical configuration is shown in Fig. 3.12. It is characterized by its focal length $f$, aperture number $f \#$ (given by the focal length divided by the aperture diameter $D$ ) and image magnification $M$ defined as the ratio of the image distance $d_{i}$ and object distance $d_{o}$, as described by the lens equations

$$
\frac{1}{f}=\frac{1}{d_{i}}+\frac{1}{d_{o}} ; \quad M=\frac{d_{i}}{d_{o}} .
$$

The magnification factor can also be evaluated as the ratio between the image size (sensor size) and the imaged object size. An acceptable approximation of the resulting particle image diameter is given by: 


$$
d_{\tau}=\sqrt{\left(M d_{p}\right)^{2}+(2.44 \lambda(1+M) f \#)^{2}}
$$

A suitable time separation $(\Delta t)$ between exposures for a given experiment can be approximated by knowing (roughly) the expected flow velocity in the object plane, the recording magnification, and $\delta_{\tau}$. A general guideline can be proposed for the autocorrelation technique:

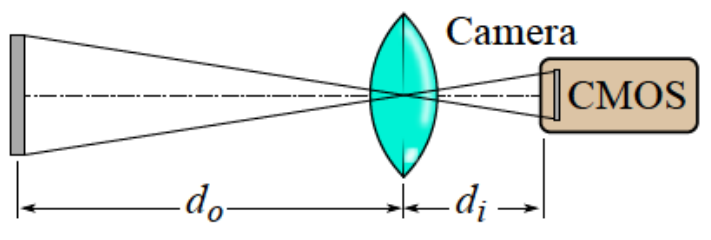

Figure 3.12: PIV imaging system

$$
\Delta t \leq \frac{d_{\tau}}{M U} \approx 0.2 \frac{l_{s}}{M U}
$$

where $l_{s}$ is the size of the interrogation spot size in the image plane.

\subsubsection{Facility for PIV tests}

A picture of the visualization facility adapted for the PIV tests is shown in Fig. 3.1. In addition to the components of the visualization facility, a high speed camera and a laser are used as image capturer and light source respectively. A sketch of the PIV arrangement is depicted in Fig. 3.13

The employed laser was a pulsed-diode OXFORD LASER HSI 5000, counting with the following characteristics:

- Laser diode light source

- Class IV

- Wavelength: $\lambda=808 \mathrm{~nm}$ (near-infrared)

- Peak power: $200 \mathrm{~W}$

- Single pulse width 1 to $80 \mu \mathrm{s}$

- Pulse energy 0.15 to $15 \mathrm{~mJ}$

It is a single cavity laser, arriving to maximum pulse frequencies of $1 \mathrm{kHz}$. The cylindrical and spherical lenses that shape the circular cross section beam delivered by the light source are mounted inside the laser body. This provides higher portability to the laser and reduces the required space, while makes the optics re-alignment more difficult if required.

The image recording was made by a high speed CMOS camera model Motion Scope M3. The camera provides a maximum resolution of $1280 \times 1024$ pixels for acquisition rates of 10-1000 fps. Higher rates imply a vertical resolution loose, with a maximum rate of $31000 \mathrm{fps}$ with a resolution of $1280 \times 10$ pixels. The camera allows external triggering. 


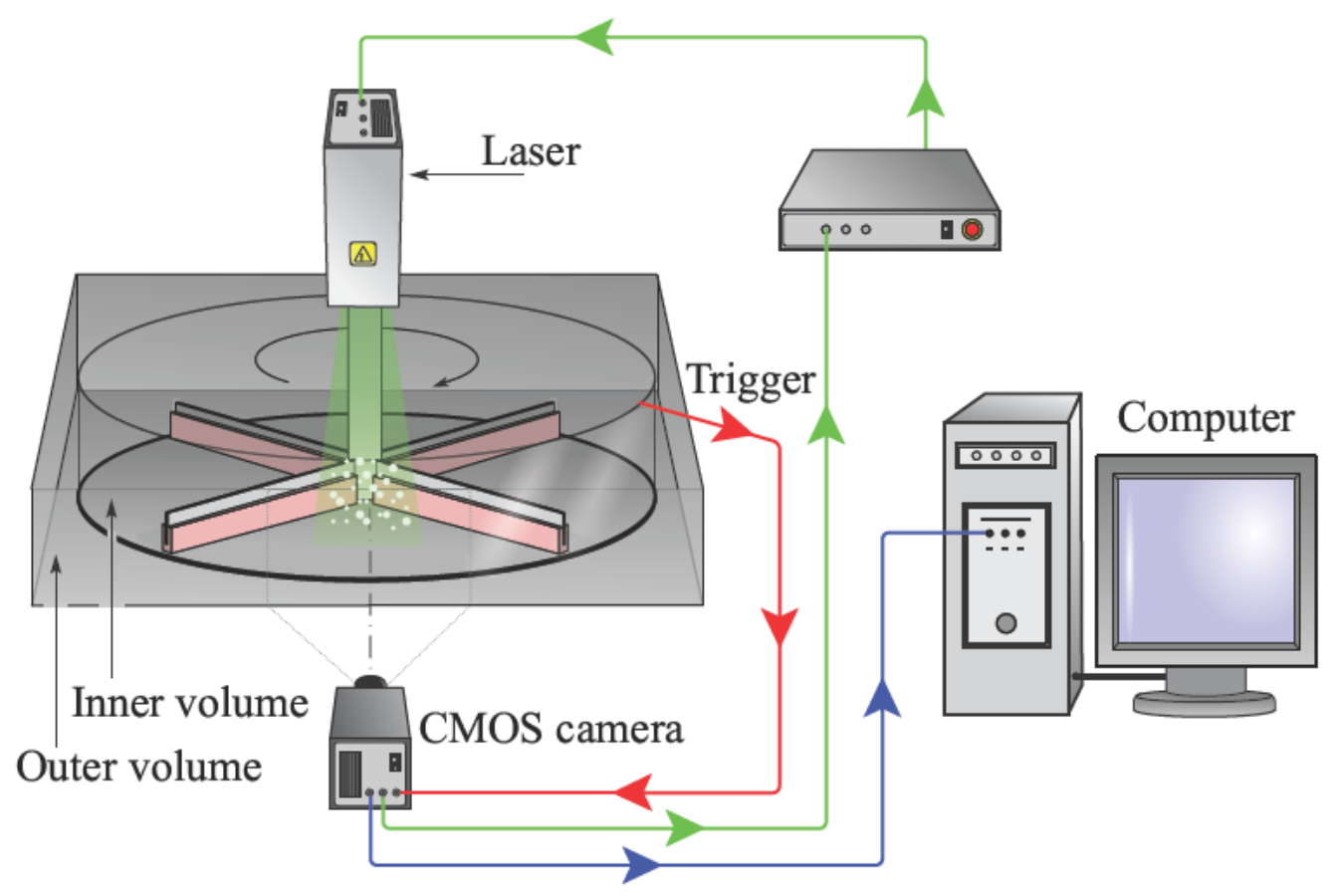

Figure 3.13: Sketch of the PIV system arrangement

Measurement procedure The acquired region was a plane perpendicular to the scraper arm at $r=0.6 R$. Images consisted in $1140 \times 928$ pixels, equivalent to $134 \times 109$ $\mathrm{mm}$ after performing spatial calibration with a spatial resolution of $\Gamma=118 \mu \mathrm{m} /$ pixel.

The level of accuracy of the obtained velocity field increases while increasing the number of image pairs and averaging the results. Increasing the acquired images also allows to a better evaluation of the turbulence characteristics of the flow. On the other hand, the flow is steady from an inertial reference system where the $z$ axis coincides with the driving shaft, rotating with it. As the camera is fixed, the captured flow will depend on the time instant -i.e. the location of the scrapers.

A common solution in situations with oscillating flows as in this case is to use the so called phase averaging [82]: images are acquired repeatedly at the same point of the cyclic flow. In that way the pair of images will always capture the same flow pattern, and the number of them can be as large as possible. This technique requires the employment of a trigger systems that shoots camera and laser at the exact time instant when in every shaft turn the scraper is in the same location.

The trigger system used here consists of a photoelectric sensor model E3JM-R4M4T$\mathrm{G}$ detecting the passage of a stick fixed to the rotating shaft as indicated in Fig. 3.14. Both the stick and the sensor are placed above the SSPHE top wall, not affecting the flow inside. The detection of the stick by the photo sensor delivered a $5 \mathrm{~V}$ voltage pulse that activates the camera, which in turn triggers the laser system. The location of the stick referred to the desired scraper (angle $\alpha$ ) could be modified in order the capture different regions. Moreover, a more accurate control could be performed through the camera software, which allowed the introduction of time delays between the input trigger signal and the camera-laser shooting. 

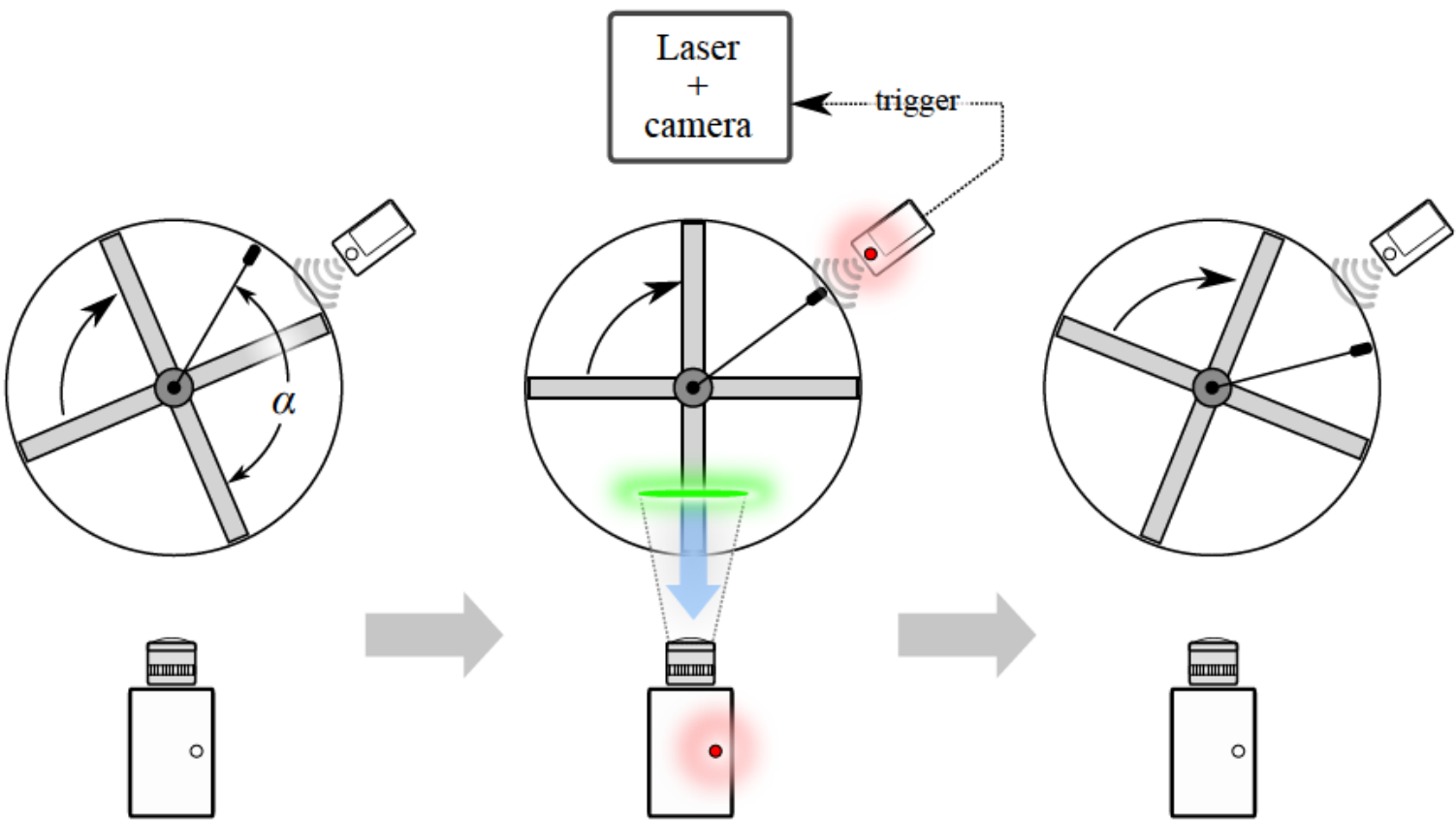

Figure 3.14: Trigger system between scrapers, camera and laser.

In order to validate future numerical simulations, different locations were investigated aside from the one of Fig. 3.14 for one of the scraping velocities tested. In particular, and if the $\alpha$ angle is the one that triggers the camera when the scraper is parallel to it (see Fig. 3.14), the locations of $\alpha+30^{\circ},+40^{\circ},+60^{\circ}$ and $+80^{\circ}$ where also investigated.

The acquiring frequencies ranged from 100 to $400 \mathrm{~Hz}$, with an exposure time of 70 $\mu \mathrm{s}$, and always taking a minimum of 1000 pair of images. The frequencies $(1 / \Delta t)$ were selected in order to have a particle displacement between successive frames of $\approx 8$ pixels. This corresponds to a non written rule that establishes the ideal particle displacement in $1 / 4$ of the window size. It is worth to mention here that the laser sheet plane is not a symmetry plane and therefore the particles will have always a component out of it. Particles will then "escape" from the plane which may result in a wrong performing of the cross-correlation. As a consequence camera frame rates have to be high enough to catch the same particles in both frames, before they leave the laser sheet, which could be in conflict with the time required for the minimum displacement of the particles between the two frames.

Working fluid Tap water was used for the PIV experiments. As a difference from the particle motion test, the rotative Reynolds number is the only one to be respected here. Therefore, the rotating velocity of the scrapers $\mathrm{N}$ can be adjusted to match the $R e_{\text {rot }}$ values between PIV and thermal experiments. If concentrations of ice until $10 \%$ are considered, the $R e_{\text {rot }}$ ranges from $\sim 10^{4}$ to $\sim 1.6 \cdot 10^{4}$ (including all the $\mathrm{NaCl}$ concentration and scraping velocities). Five cases were studied for the PIV studies including the values of $R e_{\text {rot }} 2.22 \cdot 10^{4}, 3.92 \cdot 10^{4}, 6.44 \cdot 10^{4}, 1.01 \cdot 10^{5}, 1.68 \cdot 10^{5}$. The tested cases aim to describe the flow pattern inside the SSPHE but, even more important, serve as validation test cases for numerical models, which can provide more detailed 


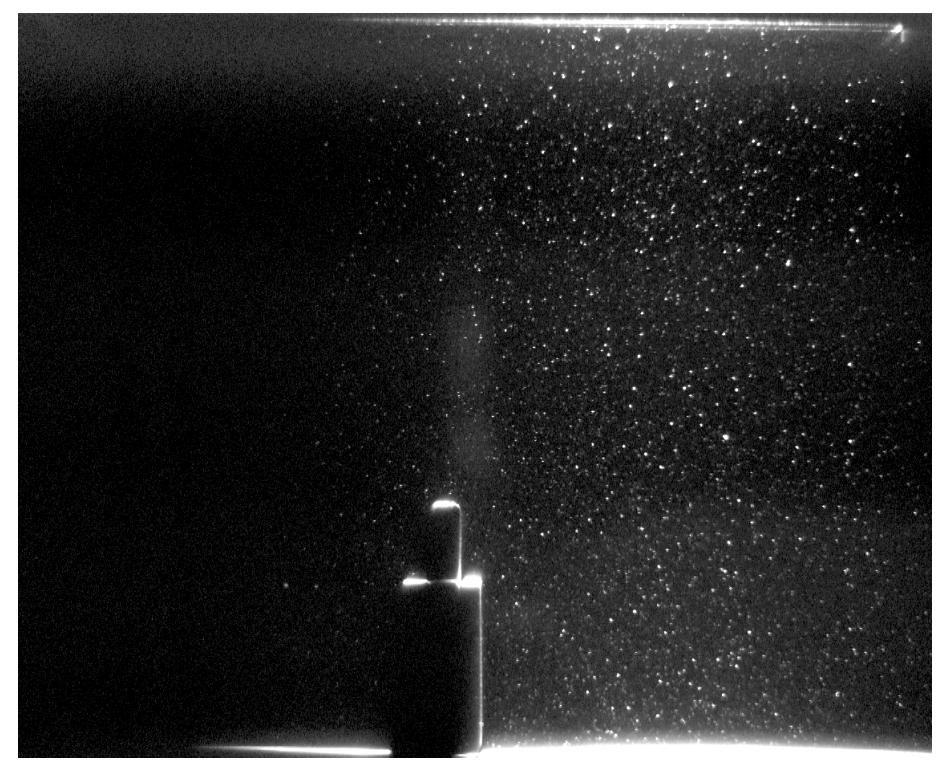

Figure 3.15: Typical image of one of the tests, where the illuminated area is on the right side of the scraper arm (center of the image)

and exhaustive information about the flow.

Tracer particles The employed tracer particles are polyamide spheres (in practice they have a round but not exactly spherical shape) with an average diameter of $d_{p}=50$ $\mu \mathrm{m}$, microporous and strongly recommended for water flow applications, as $\rho_{p}=1016$ $\mathrm{kg} / \mathrm{m}^{3}$. Figure 3.15 shows an image of the particles for one of the tests. The slip velocity $V-U$ of Eq. 3.7 can be evaluated according to the values of $d_{p}$ and $\rho_{p}$ and $\mu$ in $\approx 10^{-6}(d V / d t)$. The latter means that the slip velocity will be six order of magnitude lower that the acceleration experienced by the flow due to the scrapers, which makes any slip velocity negligible. For instance, in the case of buoyancy of the particles $d V / d t=g$, the gravitational acceleration $\left(g=9.81 \mathrm{~m} / \mathrm{s}^{2}\right)$. The slip velocity for that case is $\approx 0.02$ $\mathrm{mm} / \mathrm{s}$, a very low value compared with the linear velocity of the scrapers $(0.14-1.15$ $\mathrm{m} / \mathrm{s})$.

The concentration of the tracer particles was based on the recommendation of $10^{9}$ and $10^{12}$ particles $/ \mathrm{m}^{3}$, but later modified until reaching an optimum. The given recommendation is general and does not have into account the type of geometry or the flow. In this particular case the fluid volume that the laser has to illuminate is high compared with other cases where the region of interest are of few centimetres. Moreover the distance between the laser sheet and the window is also large, which makes the light absorbed by the particles contained in the fluid. Therefore, while higher particle concentration could be desirable, this two facts make them technically inaccessible.

The diameter of the particles in the image can be calculated according to Eq. 3.9. The magnification factor $M$ can be calculated as the ratio between the size of the image (sensor size) and the one of the captured image.

$$
M=\frac{L_{\text {image }}}{L_{\text {sensor }}}
$$


The sensor size is $15.4 \times 12.3 \mathrm{~mm}$, which gives a magnification factor of $M \approx 8.7$. The aperture was set to $f \#=2.8$, which leads to $d_{\tau}=0.44 \mathrm{~mm}$, higher than the size of a pixel $(\approx 0.12 \mathrm{~mm})$. Thus a typical particle in the image takes about four pixels. This result can be accepted as valid, avoiding the pixel-locking problem [80] that could lead into wrong displacement values after the cross correlation process for the lack of precision in the particle location inside a pixel.

\subsubsection{Image processing}

PIVlab [83] was used for the image processing. It is a GUI-based open-source tool programmed in MATLAB. It uses the FFTW [84], is a C subroutine library for computing the discrete Fourier transform in images of arbitrary input size.

A parametric study was done to determine the optimal image processing parameters. It was based in the ratio between non-valid and valid vectors after applying a data validation process: a velocity thresholds was determined semi-automatically by comparing each velocity component with a lower threshold and an upper threshold $\left(t_{\text {lower }}\right.$ and $\left.t_{\text {upper }}\right)$ :

$$
\begin{aligned}
& t_{\text {lower }}=\bar{u}-n \cdot \sigma_{u} \\
& t_{\text {upper }}=\bar{u}+n \cdot \sigma_{u}
\end{aligned}
$$

where $\bar{u}$ is the mean velocity, $\sigma_{u}$ is standard deviation of $\bar{u}$ and $n=9$ is the strictness of the filter. This filter works very well in practice, as it adapts to some extent to the nature of the flow. Moreover, the normalized median test was used [83], which evaluates the velocity fluctuation with respect to the median in a $3 \times 3$ neighbourhood around a central vector. The median of this fluctuation is then used as normalization for a more classical median test.

As a result, a contrast-limited adaptive histogram equalization was chosen as the best image pre-processing filter. A four passes analysis proved to provide the best results, starting with a big interrogation square are of 128 pixels and then decreasing gradually to 64,32 and 8 .

The first pass uses relatively large interrogation areas to calculate a reliable displacement. Larger interrogation areas lead to better signal-to-noise ratio (more robust cross correlation). The displacement information of the first pass is used to offset the interrogation areas in the second pass and so on. The interrogation areas of later passes are not only displaced, but they are also deformed. An example of the displacement vectors obtained after each pass is shown in Fig. 3.16

As left and right sides of the scraper were investigated separately, a mask was used to cover the non illuminated region plus the scraper (see Fig. 3.15). The obtained velocity fields were after combined into a single vector map.

The final velocity field was the result of the 1000 pairs ensemble average.

The standard deviation can be related with the turbulence intensity:

$$
T I=\frac{\overline{u^{\prime}}}{\bar{u}}=\frac{\overline{u^{\prime}}}{\bar{U}}
$$

where $u^{\prime}$ is the root-mean-square (RMS) or standard deviation value of the turbulence velocity fluctuations. 


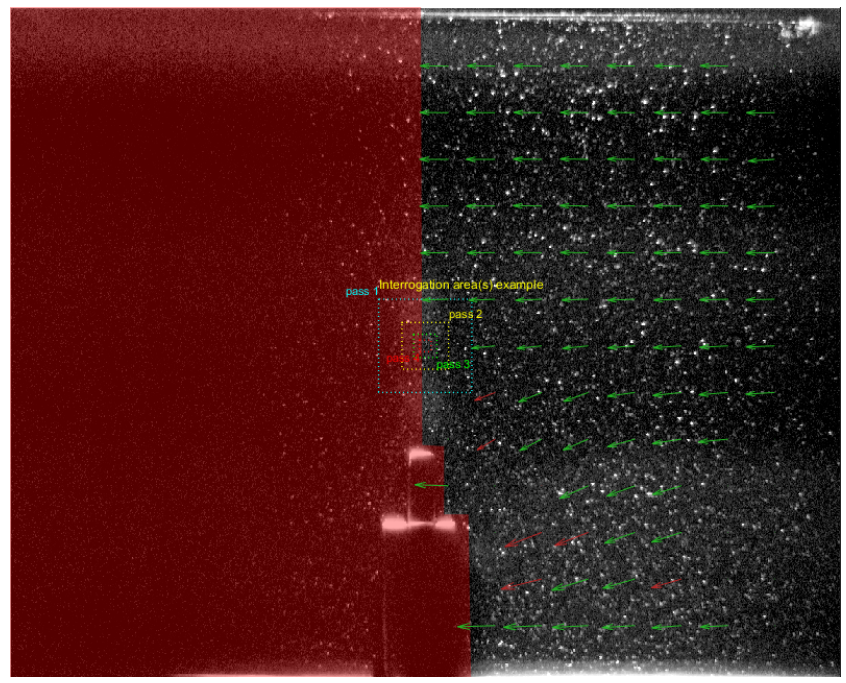

(a)

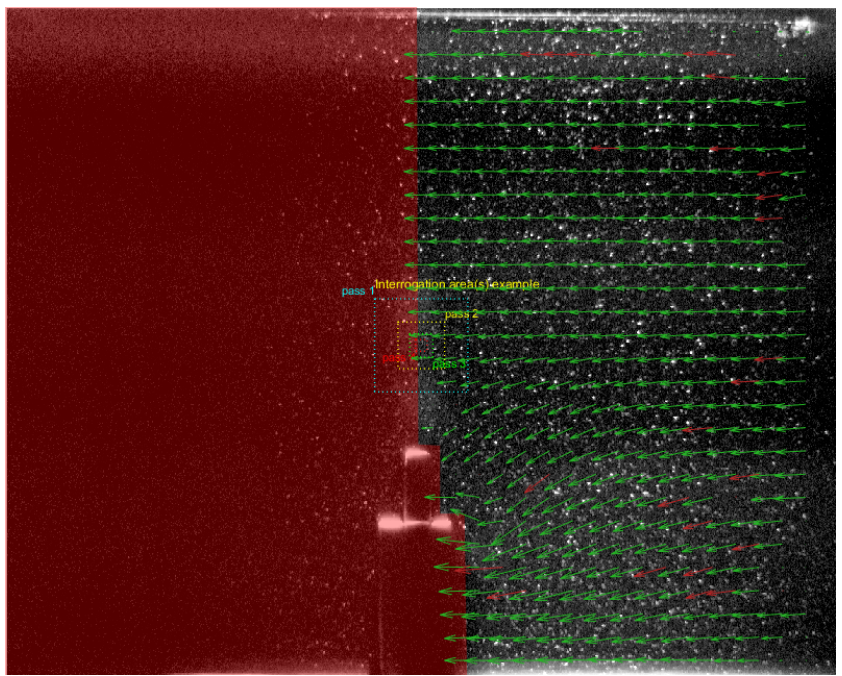

(b)

Figure 3.16: Displacement vectors obtained after processing (a) pass 1 (b) pass 3 . 

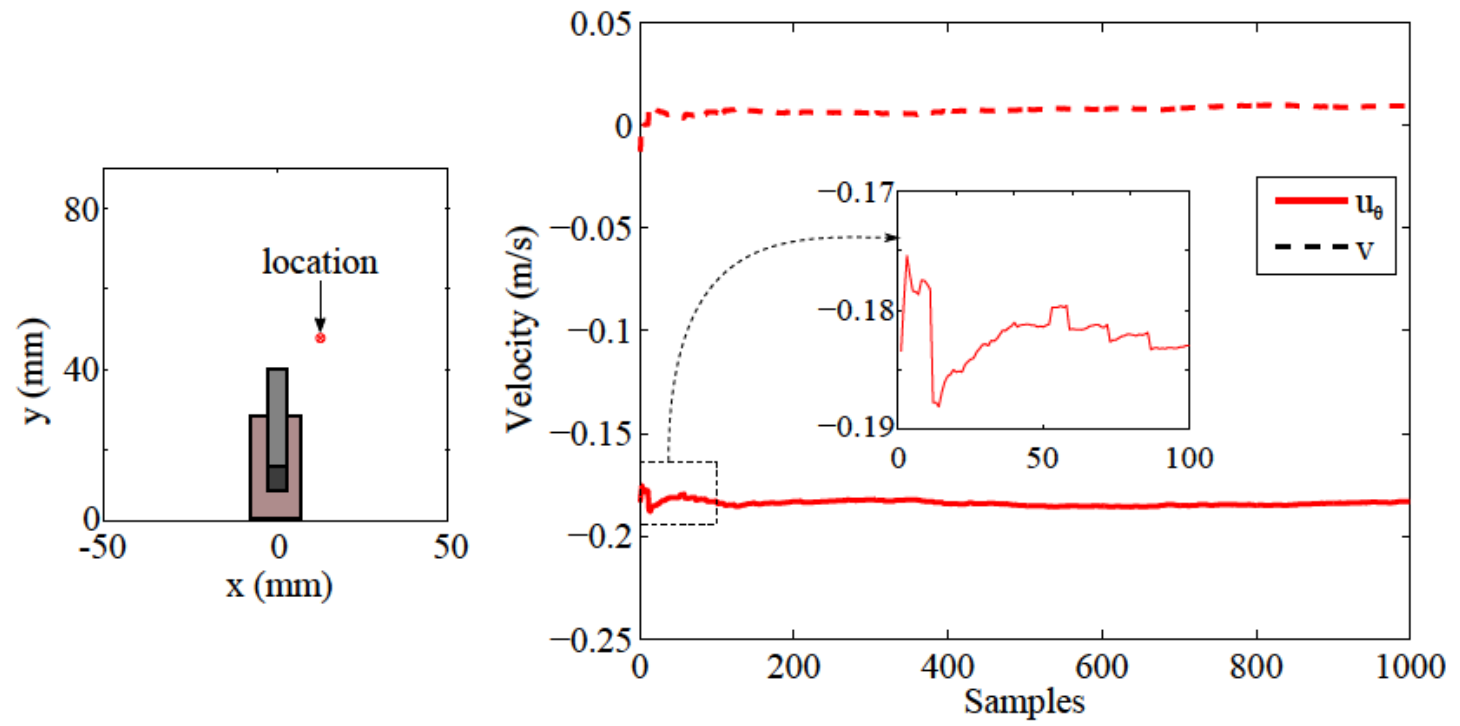

Figure 3.17: Mean velocity $u_{\theta}$ and $v$ values evolution with the number of image pairs (samples). $\operatorname{Re}_{\text {rot }}=3 \cdot 9 \cdot 10^{4}$.

$$
u=\bar{u}+u^{\prime}=U+u^{\prime}
$$

\subsubsection{Estimation of uncertainties}

There are different possible uncertainty sources that can affect the results on different steps of the measurement process: background noise, characteristics of light scattering, fluctuation of laser power, sampling and digitalize, correlations calculation, distortion of CMOS, image length, illumination uniformity, lens distortion, refraction at window, path curvature and pulse timing... . Wherever they take place, the uncertainties propagate to the final result through the data flow.

To determine the global uncertainty, an analysis according with [85] was performed. The uncertainty is considered as a sum of two components, one due to the systematic error and the other to the random error. Systematic errors are mainly due to the experimental set up. Random errors are related to the statistical error inherent to the measurement of the velocity.

Systematic errors The velocity associated to a seeding particle is given by:

$$
u=\Gamma \frac{\Delta x}{\Delta t} \pm E(u)
$$

where $\Delta x$ is the particle pixel displacement and $\Gamma$ is the spatial resolution. The term $E(u)$ keeps into account the uncertainties due to the calibration (spatial resolution), lag of tracers due to strong velocity gradients and projection from 3D physical space to $2 \mathrm{D}$ image plane.

Considering the following sources of error in the order they take place (1) flow seeding (2) image acquisition and (3) data processing, the uncertainty associated to the measurement of $u$ can then be obtained as: 


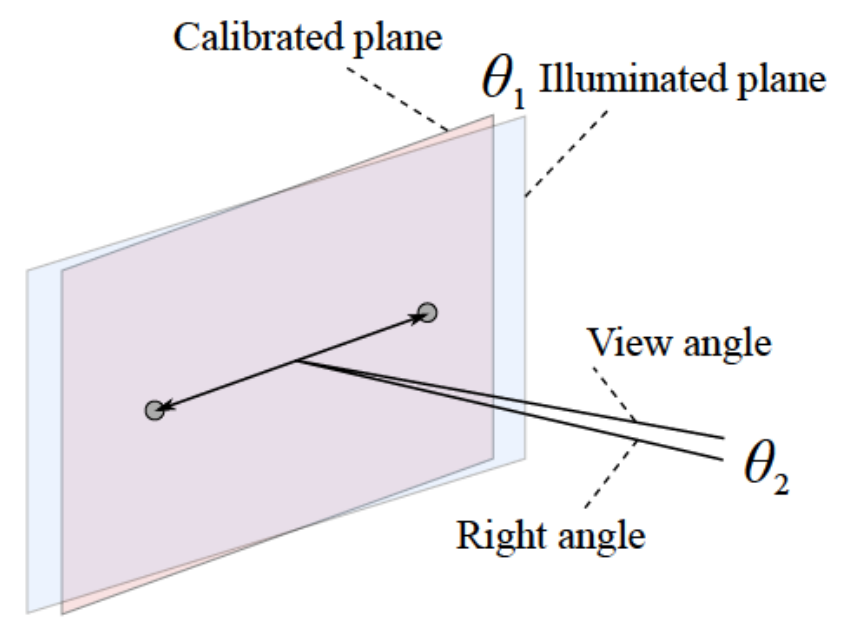

Figure 3.18: Possible misalignments of the camera and the laser sheet.

$$
E(u)=\left[\left(\frac{\partial u}{\partial \Gamma} E(\Gamma)\right)^{2}+\left(\frac{\partial u}{\partial \Delta x} E(\Delta x)\right)^{2}+\left(\frac{\partial u}{\partial \Delta t} E(\Delta t)\right)^{2}\right]^{1 / 2},
$$

which yields:

$$
\frac{E(u)}{u}=\left[\left(\frac{E(\Gamma)}{\Gamma}\right)^{2}+\left(\frac{E(\Delta x)}{\Delta x}\right)^{2}+\left(\frac{E(\Delta t)}{\Delta t}\right)^{2}\right]^{1 / 2}
$$

The estimation of each value in Eq. 3.17 is as follows:

Spatial resolution $\Gamma$ The uncertainty in the spatial resolution can be derived from its definition,

$$
\Gamma=\frac{l_{\text {ref }} \cdot \cos \left(\theta_{1}\right)}{l_{\text {image }}}
$$

where $l_{\text {ref }}(\mathrm{mm})$ is the reference length used for the calibration, $l_{\text {image }}$ is the pixel length of $l_{\text {ref }}$ in the image and $\theta_{1}$ is the angle between the calibration board and the laser sheet (see Fig. 3.18), assumed to be $\leq 2^{\circ}$. The uncertainty on $\Gamma$ is then:

$$
E(\Gamma)=\left[\left(\frac{\partial \Gamma}{\partial l_{\text {ref }}} E\left(l_{\text {ref }}\right)\right)^{2}+\left(\frac{\partial \Gamma}{\partial l_{\text {image }}} E\left(l_{\text {image }}\right)\right)^{2}+\left(\frac{\partial \Gamma}{\partial \theta_{1}} E\left(\theta_{1}\right)\right)^{2}\right]^{1 / 2}
$$

Also the optical system may affect the spatial resolution, mainly due to the aberration of lenses. The distortion of the image is expected to be $<0.5 \%$ of the measured length [86]. 
Table 3.1: Total uncertainty result for the PIV experiments

\begin{tabular}{|c|c|c|c|}
\hline Parameter & $E(\ldots)$ & $(\partial u / \partial \ldots)$ & $E(u)$ \\
\hline$\Gamma$ & $2 \cdot 10^{-3} \mathrm{~mm} /$ pixel & $2.4 \cdot 10^{3} \mathrm{pixel} / \mathrm{s}$ & $5 \mathrm{~mm} / \mathrm{s}$ \\
\hline$\Delta x$ & $2.2 \cdot 10^{-1}$ pixel & $35 \mathrm{~mm} /$ pixel $\cdot \mathrm{s}$ & $7 \mathrm{~mm} / \mathrm{s}$ \\
\hline$\Delta t$ & $2.12 \cdot 10^{-9} \mathrm{~s}$ & $1.98 \cdot 10^{7} \mathrm{~mm} / \mathrm{s}^{2}$ & $0.04 \mathrm{~mm} / \mathrm{s}$ \\
\hline$\delta u$ & $3.02 \mathrm{~mm} / \mathrm{s}$ & - & $3 \mathrm{~mm} / \mathrm{s}$ \\
\hline Total & & & $10 \mathrm{~mm} / \mathrm{s}$ \\
\hline
\end{tabular}

Particle displacement $\Delta x$ Laser power fluctuation and normal view angle $\theta_{2}$ are related to the particle image displacement $\Delta x$.

A maximum uncertainty on the laser power fluctuation of $25 \mu \mathrm{m}$ (half of the particle diameter) has been assumed, while the sensitivity factor (partial derivative) is the inverse of the spatial resolution $1 / \Gamma[86]$.

The angle $\theta_{2}$ between the camera axis and the laser sheet plane could be estimated as $\theta_{2} \leq 4^{\circ} \approx 0.07 \mathrm{rad}$. For an 8 pixels displacement the error is $\approx 0.02$ pixels.

On the other hand, during data processing mismatching errors and sub-pixel analysis could result in a source of uncertainty. This mismatching errors were estimated to be $\approx 0.2$ pixels, while the uncertainties of sub-pixel analysis were estimated to be $\approx 0.03$ pixels $([85])$.

Separation time $\Delta t$ The possible fluctuation for $\Delta t$ from the user manual is $1.5 \mathrm{~ns}$. On the other hand, the pulse laser itself has an uncertainty for the pulse timing, which was considered to be also of $1.5 \mathrm{~ns}$. Both sensitivity factors for the measurement time are 1.

Traced velocity $\delta u$ Velocity slip was neglected according to the value obtained for Eq. 3.7. According to [85], for three dimensional effects on perspective of velocity, error may be estimated as a function of the main velocity $\left(\leq 2 \pi \mathrm{N}_{\max } 0.6 \mathrm{R}=0.9 \mathrm{~m} / \mathrm{s}\right)$, uncertainty of the out of plane component of velocity (assumed 1\%) and perspective angle $\theta_{2}$ by the following equation $u \cdot 1 \% \tan \cdot \theta_{2}$.

Table 3.1 shows the total uncertainty results. The total systematic uncertainty has been estimated in $9.2 \mathrm{~mm} / \mathrm{s}$. If we consider an average flow velocity of $\approx 0.1$ to $1 \mathrm{~m} / \mathrm{s}$ (according with the scraper tangent velocity on the laser sheet area), it represents a relative systematic error of 9 to $0.9 \%$, an acceptable value.

Peak locking The peak locking effect is in practice a bias error. It comes from the curve fitting when performing the sub-pixel interpolation. Such a curve fit causes a bias towards discrete values of displacement [80]. Whether the peak locking was present in the results or not was investigated through the histogram of the fractional part of the displacement in pixel units: if the integer part of the displacement is truncated, only the fractional part between -0.5 and +0.5 pixel units remains. Figure 3.19 shows the histogram for one of the studied cases. The level of peak locking can be evaluated by [87]: 


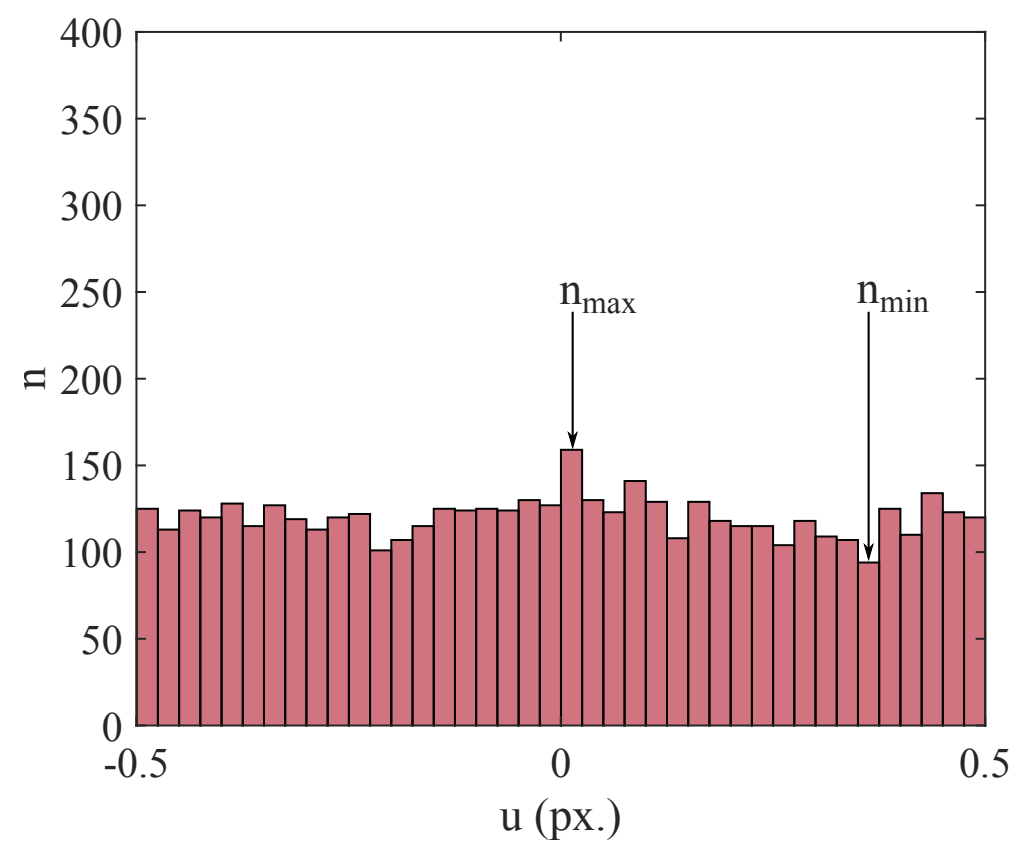

Figure 3.19: Fractional displacement histogram representative of the processed PIV results

$$
c=1-\frac{n_{\min }}{n_{\max }}
$$

being equal to 0.4 in the case of Fig 3.19. Such a value of $c$ can be considered as an acceptable, "mild" peak locking.

Statistical random errors Statistical errors disappear with the averaging of large samples

Random errors are related to the statistical error inherent to the measurement of the velocity. The statistical error is strongly dependent on the number of measurements performed to obtain the mean velocity. Considering a confidence level of $95 \%$, the statistical random error associated to a set of $n$ successive pair of images is given by:

$$
E(u)_{r}=2 \cdot \frac{T I}{\sqrt{n}}
$$

A value of turbulence intensity around $15-20 \%$ is common in mixers and stirrers [88]. The statistical random error is for that case, considering $\mathrm{N}=1000,0.01 \mathrm{~m} / \mathrm{s}$. However such a level of turbulence will be hardly reached in the SSPHE, as the aspect ratio is small and the rotative Reynolds number is low compared with most of the stirrers. 


\section{Chapter 4}

\section{Ice slurry production in the SSPHE: physical phenomena and modelling}

The aim of this chapter is to describe the basics of the ice slurry formation, identifying and studying the underlying physical phenomena. A brine with medium $\mathrm{NaCl}$ concentration was chosen as working fluid. The study of the process is based on the three different aspects: heat transfer, power consumption and morphology of the ice crystals.

The first part of the chapter (section 4.4) is devoted to the analysis of the thermal process and the heat transfer under batch operating mode. Section 4.4.1 presents the evolution of instantaneous magnitudes like the heat flux or the heat transfer coefficient for different scraping velocities and wall subcooling values whereas section 4.4.2 presents a comparative for the time averaged values. The rotating regime is conveniently nondimensionalized through the rotating Reynolds number, which includes the effects of the ice particles in the slurry through its density and viscosity. The heat transfer coefficient is non-dimensionalized through the Nusselt number. The same methodology is followed for the study of the two different scrapers tested. Finally, section 4.4.3 presents a correlation for the Nusselt number as a function of the different governing parameters and the ice slurry concentration.

The second part of the chapter (section 4.6) analyses the power consumption of the driving motor, relating the data with the different stages of the batch process. The power number, characteristic of stirrers, was used here to non- the values.

The last part of the chapter (section 4.8) is devoted to analysis of the the morphology of the ice crystals by using microscopical images, and relating it with the process variables

\subsection{Thermal response}

Figure 4.1 presents a typical evolution of the process temperatures during a batch experiment in the SSPHE, from 0 to $20 \%$ of ice content. The process is divided in two parts: chilling and freezing. The first part, from the beginning of the experiment until 


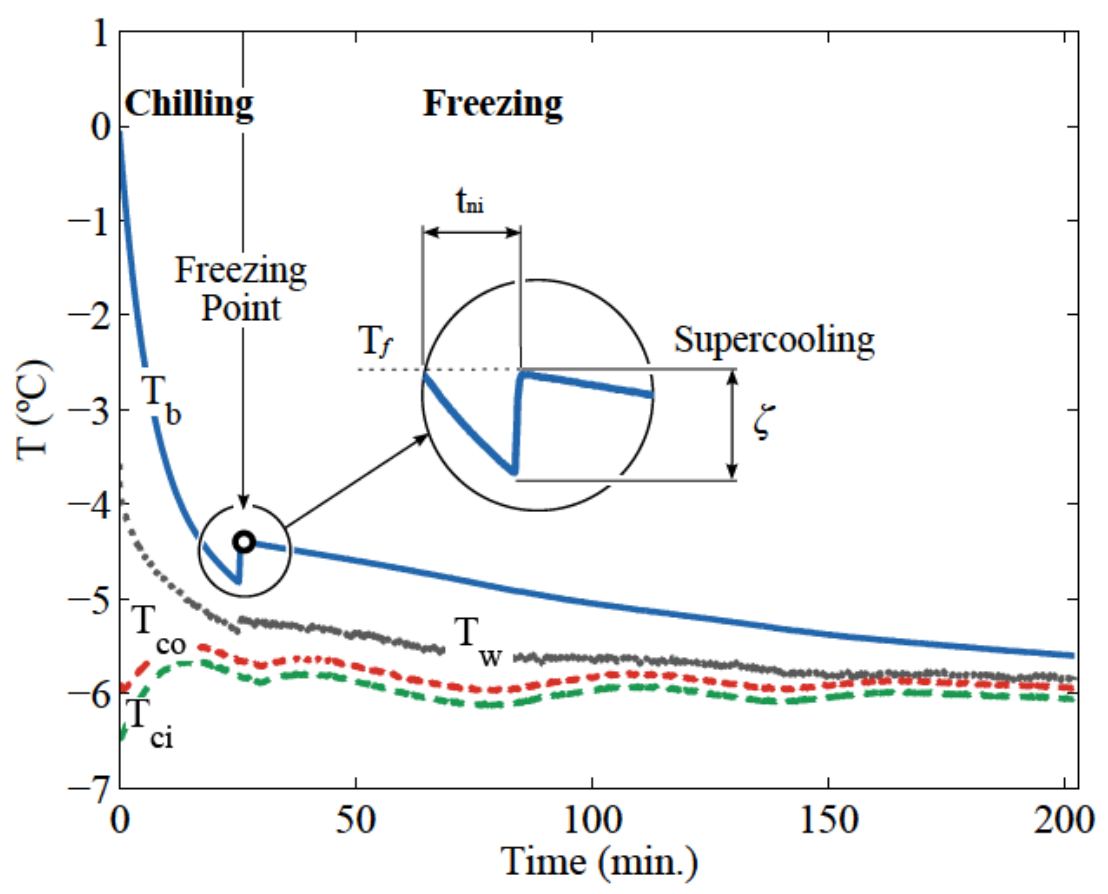

Figure 4.1: Representative case of the evolution of the process temperature along time

the onset of nucleation, corresponds to the chilling. In this stage, only sensible heat is exchanged and the brine remains liquid, with a fast decrease on the temperature $T_{b}$ as a consequence. The end of this period is not only limited by the brine temperature $T_{b}$, since it reaches values lower than the freezing point. The period when the brine temperature is lower than the freezing point $\left(T_{b}<T_{f}\right)$ is the so called supercooled state, where the brine is not in thermal equilibrium. The brine temperature continues decreasing during the supercooling until the nucleation induction time $t_{n i}$ is reached and the nucleation appears. The critical degree of supercooling and the nucleation induction time for the spontaneous nucleation on a subcooled surface is, nevertheless, unpredictable [67]. The temperature rises then abruptly arriving to the freezing temperature $\left(T_{b}=T_{f}\right)$. The sensible heat accumulated during the supercooling is transformed into latent heat by the formation of multitude of ice crystals. This release of heat during the phase change is the responsible of the sudden temperature increase. After that, the production of ice starts, and thus the so-called freezing stage. The predominance of latent heat over the sensible heat is the responsible of the low velocity in the temperature decrease, only due to the increase of $\mathrm{NaCl}$ in the remaining brine.

\subsection{Supercooling}

The temperature pattern of the supercooling phenomenon appeared to be different from case to case, being even not reproduced by the immersed probes for some of them. This fact has been characterized by a new parameter, the supercooling amplitude $\zeta$, defined as the amplitude of the temperature rise once the nucleation appeared (see Fig. 4.1).

The supercooling amplitude was found to vary directly with the scraping velocity and inversely with the temperature difference between the brine and the wall $\overline{\Delta \mathrm{T}}_{b, w}=$ 


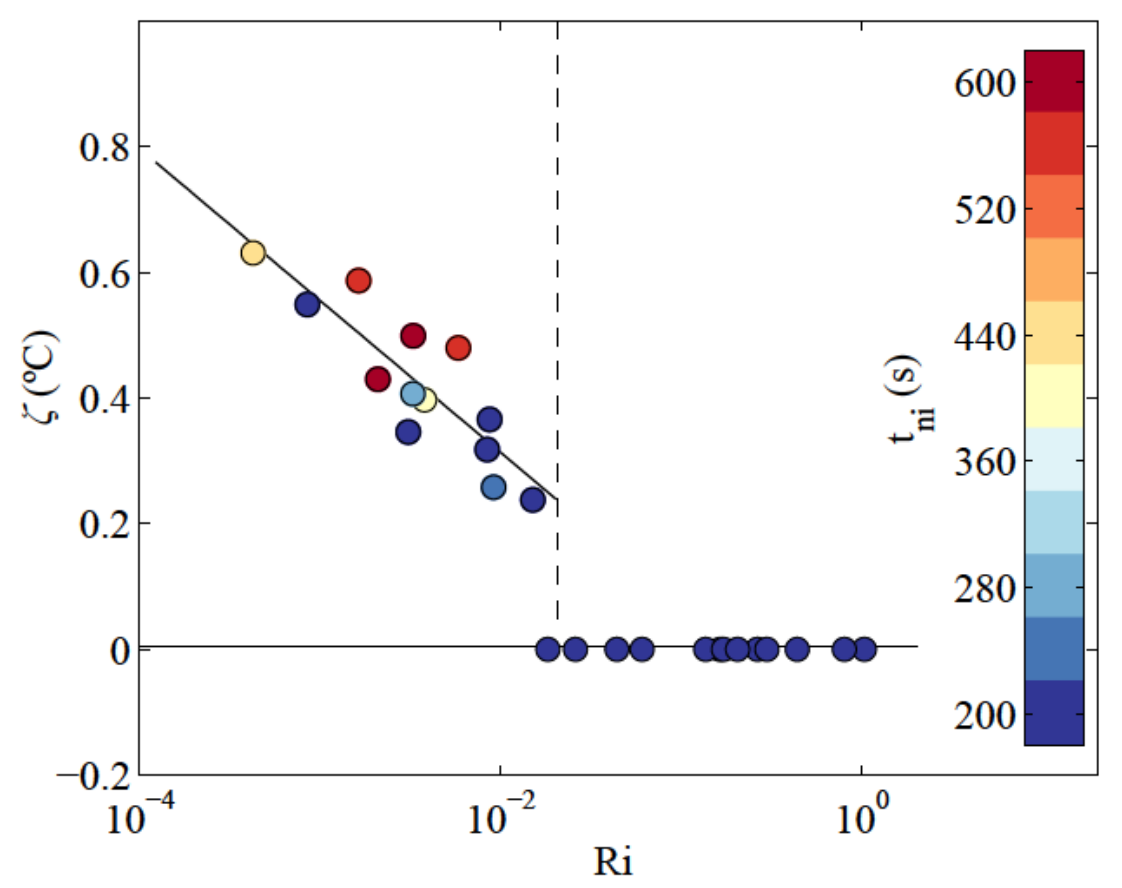

Figure 4.2: Supercooling amplitude $\zeta$ for different $R i$ numbers

$T_{b}-T_{w}$. The combination of these two factors suggests the presence of an stratified fluid layer in the bottom of the tank. In the presence of high $\overline{\Delta T}_{b, w}$ or low scraping velocities the fluid layer thickness increases, leading to large temperature gradients in the SSPHE fluid. As a consequence the fluid in contact with the bottom plate would reach the maximum $\zeta$ appearing the nucleation, whereas the fluid in the upper layers (e.g. around the immersed probes) would not be yet at the freezing temperature. Moreover, large $\overline{\Delta \mathrm{T}}_{w, b}$ values imply also large wall supercooling degrees $\overline{\Delta \mathrm{T}}_{s}$, which advances the nucleation. In those cases nucleation appears first on the plate and not all around the volume. The localized nucleation triggers the freezing once $T_{b}=T_{f}$, and therefore no supercooling occurs around the probes.

The experimental evidence relates the supercooling amplitude $\zeta$ with the Richardson number, which represents the ratio between natural and forced convection. Forced convection is due to the flow generated by the scrapers, playing in favour of higher $\zeta$ values. Natural convection acts against $\zeta$, keeping the colder and therefore heavier fluid in the bottom. This phenomenon hinders the mixing and as a consequence the appearance of the nucleation in all the fluid volume. Figure 4.2 shows the $\zeta$ values as a function of their correspondent Richardson numbers, averaged from $T=T_{f}$ until the onset of nucleation, $T=T_{f}-\zeta$. Supercooling phenomenon was detected only for $R i<0.02$, with increasing values of $\zeta$ as $R i$ decreases. The detection of the supercooling and its amplitude $\zeta$ are therefore an indicator of the mixing level in the SSPHE.

The symbols in Figure 4.2 are coloured according with the nucleation induction time, defined in Fig. 4.1. The lack of dependence between $t_{n i}$ and $R i$ can be associated with the unpredictable character of the nucleation onset. On the other hand, $t_{n i}$ is related with $\zeta$ through the heat exchanged between the supercooled brine and the wall. If the heat flux is assumed to be time-constant, and applying eq. 2.15 , then 


$$
t_{n i} \propto \zeta \frac{\rho_{b} V c_{p, b}}{h A \overline{\mathrm{T}}_{b, w}}
$$

where $\overline{\Delta T}_{b, w}=T_{b}-T_{w}$ and $T_{b}<T_{f}$.

\subsection{Wall temperature distribution}

An average wall temperature was used as a characteristic of the wall temperature variable $\bar{T}_{w}=\sum_{n=1}^{26} T_{w, n} / 26$. This is possible only if the wall temperature is uniform, with low dispersion values. To ensure a uniform wall temperature in the scraped surface, the coolant mass flow was stated to ensure low temperature increments between inlet and outlet. In that way the usage of an average wall temperature value in the heat transfer coefficient calculation does not represent a source of error. To validate this assumption the temperature distribution was checked in two ways: considering a radial distribution of the thermocouples and a linear distribution according to the channel flow in the bottom part of the SSPHE. Figure 4.3 shows the channel and thermocouples distribution, where the lasts have been noted by red dots and the coolant path through channels is represented by a red line.

The temperature distribution should not change significantly along radius or channel length dimensions. Figure 4.4 shows different temperature values averaged during 5 minutes for different experiments. Maximum standard deviations of $0.18{ }^{\circ} \mathrm{C}$ were found among the different thermocouple readings for the lowest scraping velocity $\left(\mathrm{N}=0.13 \mathrm{~s}^{-1}\right)$. This deviation decreases as the scraping velocity increases. The dispersion in the wall temperature measurements represents a variation in the heat transfer coefficient of around $10 \%$, which is lower than the calculated uncertainty. According to these values and to the lack of pat-

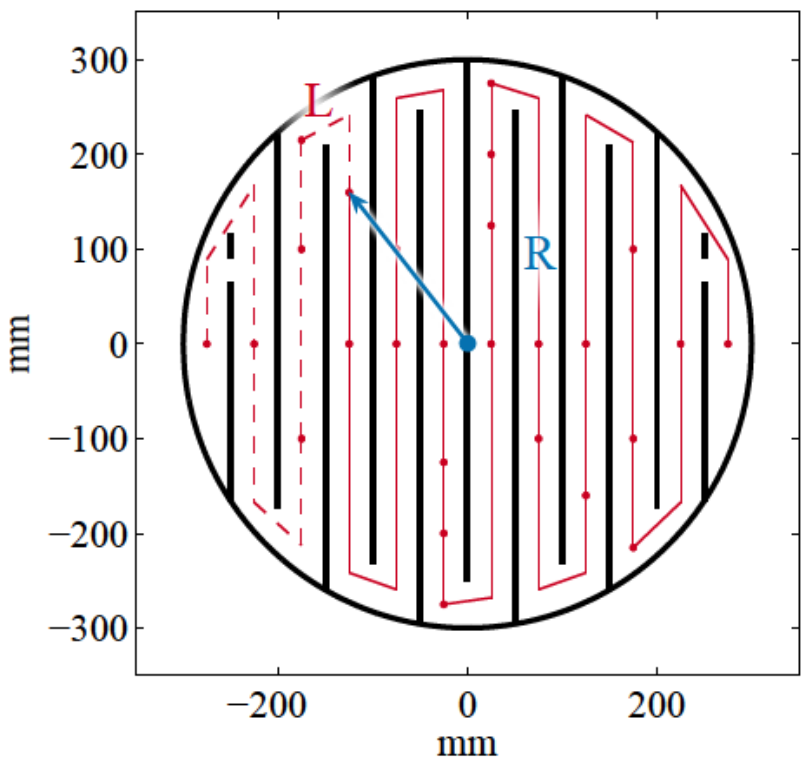

Figure 4.3: R and L coordinates for the plate thermocouple locations tern of the measured wall temperatures, the influence of any local heat transfer coefficient distribution was not taken into consideration.

It has then been proved that the wall temperature is uniform in practical terms. Therefore, the problem becomes axisymmetric, and any variation of the fluid temperature across the different immersed probes shall not take place (all of them are placed at the same height). Actually, the temperature differences between them were always lower than $1^{\circ} \mathrm{C}$. 

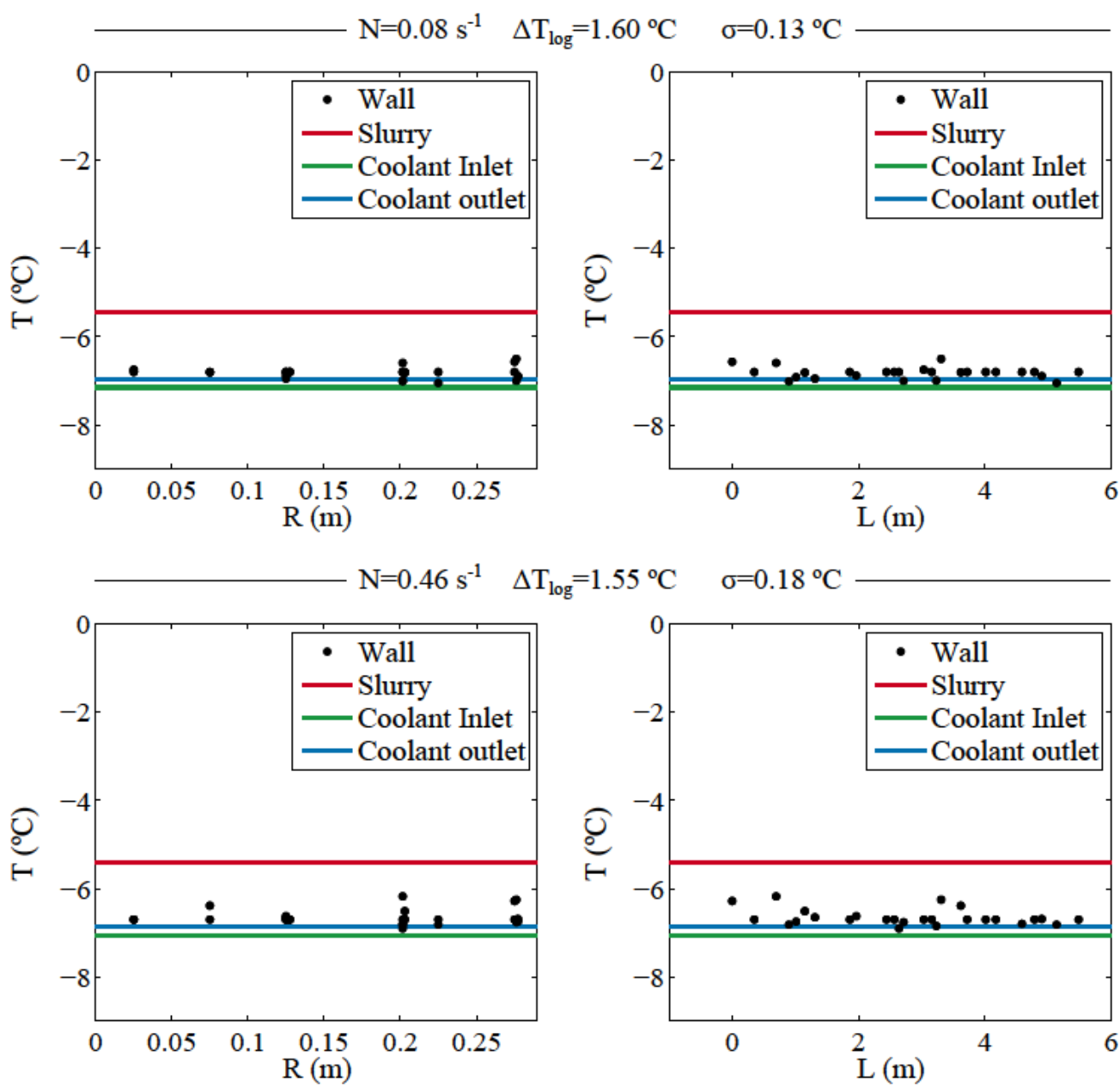

$\mathrm{T}_{\log }=1.55^{\circ} \mathrm{C} \quad \sigma=0.18^{\circ} \mathrm{C}$
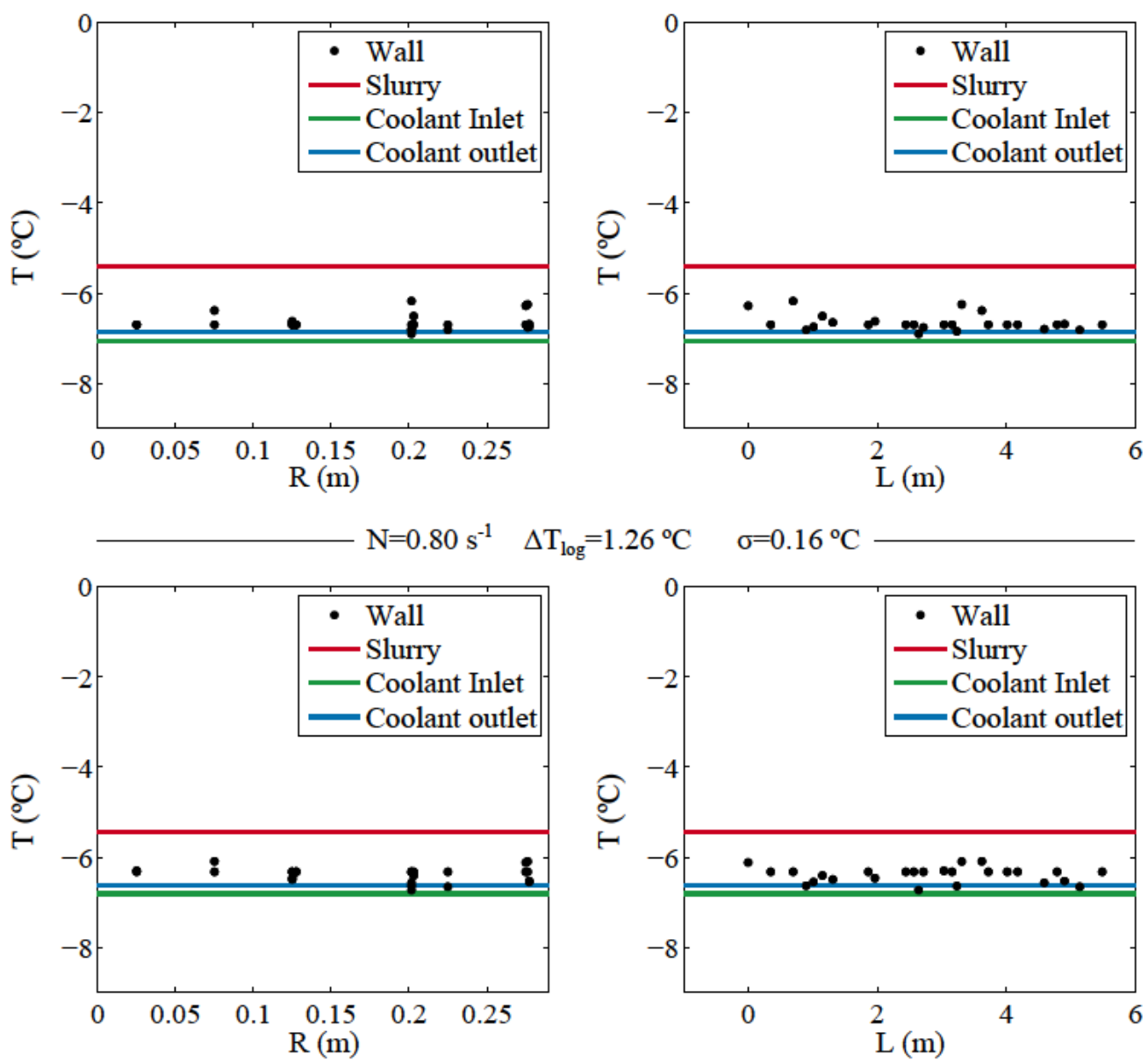

Figure 4.4: Temperature distribution in the plate along $\mathrm{R}$ and $\mathrm{L}$ for different test conditions 


\subsection{Heat transfer}

The study of the heat transfer inside de SSPHE provide important information about the production rate -heat extracted can be translated into ice production values.

\subsubsection{Time resolved values}

The evolution of the heat fluxes during the production of the ice slurry is depicted in Fig. 4.5 for different operating conditions (type of scraper, scraping velocity and logarithmic mean temperature difference). Figures $4.5 \mathrm{a}$ and $4.5 \mathrm{~b}$ correspond to two different values of $\overline{\Delta T}_{\text {log }}$, averaged during the freezing stage of the experiment, from the nucleation start until a $20 \%$ of ice content is reached. The effects of different scraper systems and scraping velocities are compared in each figure. The starting of the freezing stage can be recognized distinctively by the stabilization of the heat flux, which is a consequence of the stabilization of the process temperature. The heat transfer coefficients corresponding to the heat fluxes shown in figures $4.5 \mathrm{a}$ and $4.5 \mathrm{~b}$, calculated according to Eq. 2.12, are represented respectively in figures $4.6 \mathrm{a}$ and $4.6 \mathrm{~b}$.

- Effect of the scraper type

The effect of the adaptable scrapers yields a heat flux augmentation of around two times compared with the rigid scrapers, showing a slight dependency with $\overline{\Delta T}_{\text {log }}$ values: larger $\overline{\Delta T}_{\log }$ values lead to slightly higher heat fluxes (see Fig. 4.5).

Regarding the heat transfer coefficient (Fig. 4.6), adaptable scrapers retrieve again two-fold augmentations of $h$ compared to the rigid system. As opposed to the heat flux trend, heat transfer coefficients are in general slightly higher for the lowest values of $\overline{\Delta T}_{\text {log }}$. This can be explained considering that higher supercooling degrees $\left(\overline{\Delta T}_{s}\right)$ lead to faster growth rates, resulting in thicker ice layers on the plate surface [68].

- Effect of the scraping velocity

In general, higher scraping velocities increase the heat flux, whereas this effect is small compared with the scraper system or the $\overline{\Delta T}_{\text {log }}$ effect. The influence of the scraping velocity during the freezing period is more evident on the heat transfer coefficient, which undergoes maximum increases of around $40 \%$ when $N$ increases from 0.1 to 0.8 $\mathrm{s}^{-1}$. Moreover, these two bounding velocities lead to two different trends, particularly noticeable with the adaptable scrapers. The $h$ values for $N=0.1 \mathrm{~s}^{-1}$ reach a maximum when nucleation appears, after which $h$ remains almost constant. On the other hand, for $N=0.8 \mathrm{~s}^{-1}$ the onset of nucleation entails a sharp decrease on $h$, which reaches a maximum an instant before during the supercooling.

In spite of the similar heat fluxes observed during the freezing period, the time required to reach the $20 \%$ of ice content changed significantly with the scraping velocity (see Fig. 4.5 and 4.6).These differences are attributed to the stretch of the experiments prior to the nucleation onset: faster scraping velocities provided higher heat flux values as shown in Fig. 4.5, advancing the freezing start respect to the low scraping velocity cases.

Moreover, the higher scraping velocities ensured the presence of the supercooling phenomenon in all the brine volume. The heat released by the supercooled brine is suddenly recovered during the nucleation, where the amount of ice initially formed can be quantified by $\rho_{b} V c_{p, b} \zeta / L_{f}$. Considering a supercooling amplitude of $\zeta \approx 0.5^{\circ} \mathrm{C}$, the 


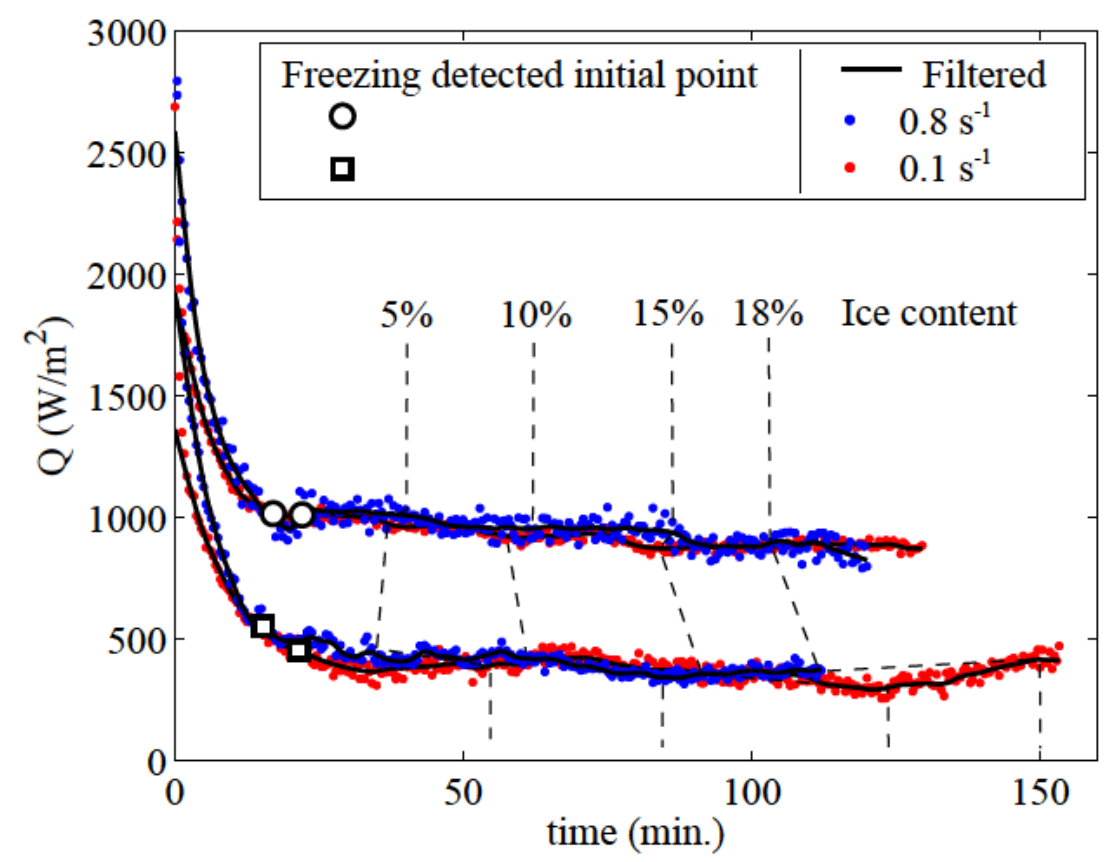

(a) $\overline{\Delta \mathrm{T}}_{\log }=1.3^{\circ} \mathrm{C}$

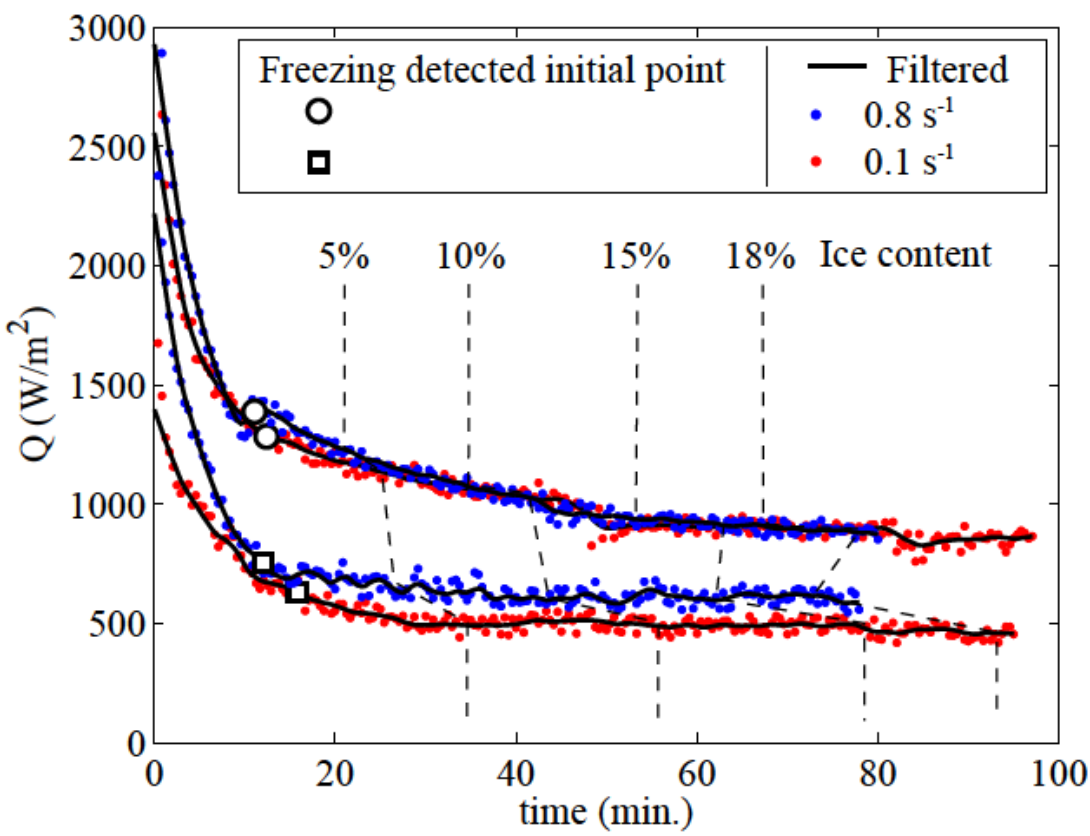

(b) $\overline{\Delta \mathrm{T}}_{\log }=2.4^{\circ} \mathrm{C}$

Figure 4.5: Heat fluxes on the subcooled plate along the test duration for different $\overline{\Delta \mathrm{T}}_{\log }$ values 


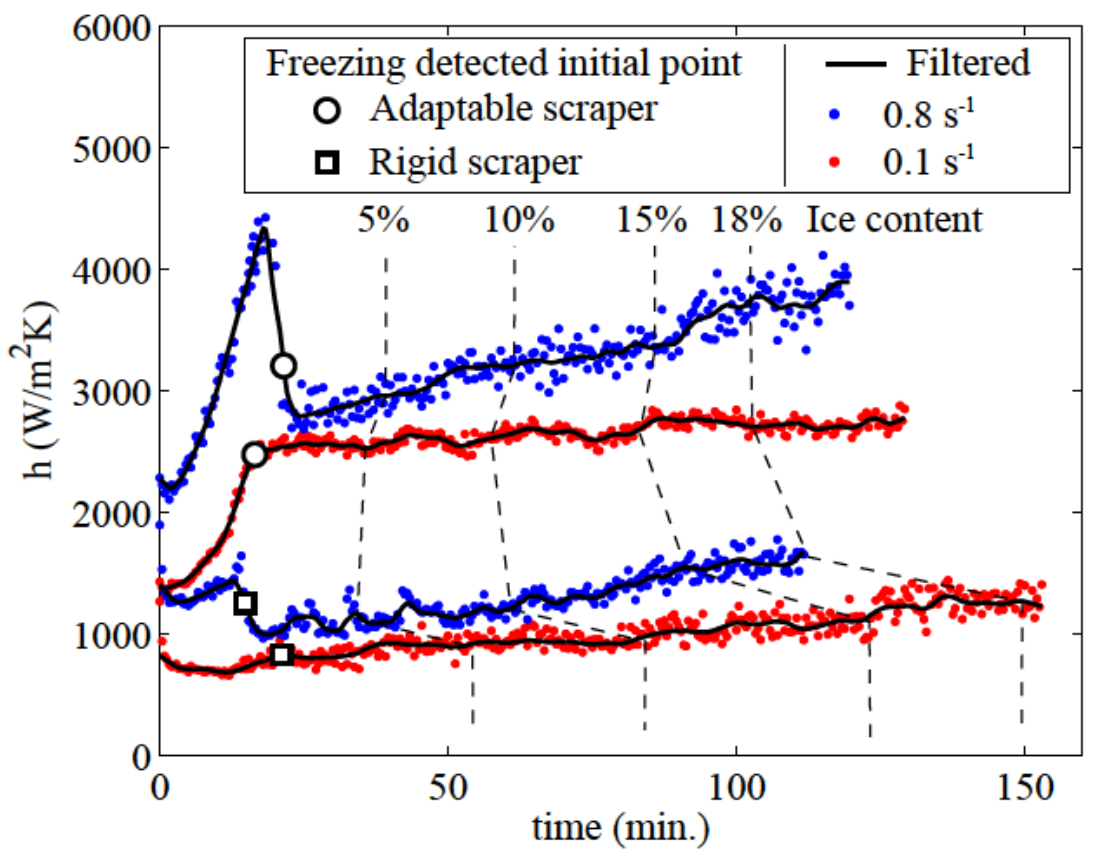

(a) $\overline{\Delta \mathrm{T}}_{\log }=1.3{ }^{\circ} \mathrm{C}$

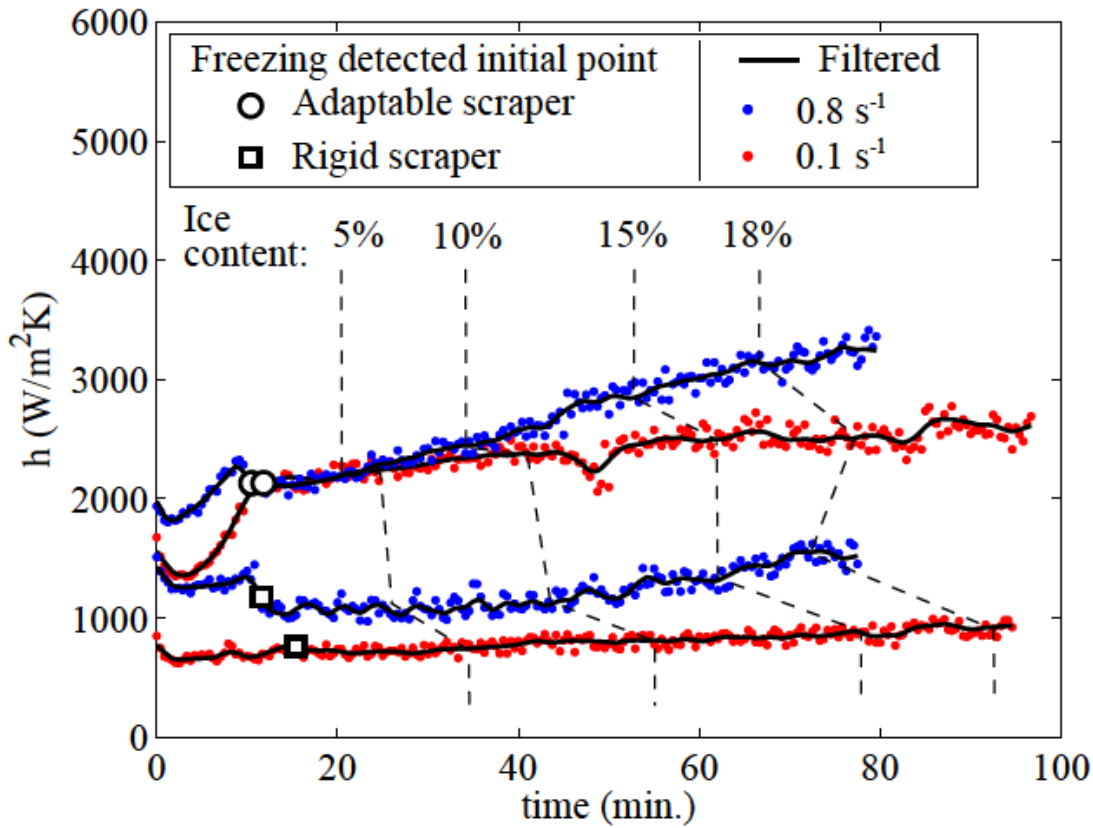

(b) $\overline{\Delta T}_{\log }=2.4^{\circ} \mathrm{C}$

Figure 4.6: Heat transfer coefficient on the subcooled plate along the test duration for different $\overline{\Delta \mathrm{T}}_{\log }$ values 
former expression gives values of around $2 \%$ of ice content. On the other hand, the lower scraping velocities do not benefit from the initially elevated heat flux values, and the nucleation onset does not represent significant values of ice content.

- Effect of the coolant temperature

The coolant temperature, which is the responsible of the wall supercooling degree, intensifies the different trends mentioned before. Smaller $\overline{\Delta T}_{\text {log }}$ values lead to more favourable mixing situations with lower $R i$ and higher supercooling amplitudes $\zeta$ (see Fig. 4.2). Supercooling occurring all around the SSHPE volume means an immediate and uniform appearance of the nucleation, which in turn implies the absence of an ice layer on the plate before that instant. This also explains the higher $h$ values obtained in these cases. Conversely, the absence of $\zeta$ indicates local nucleation on the plate, generating an ice layer that produces lower $h$ values.

Although $\overline{\Delta T}_{\text {log }}$ is computed as a time-averaged value, it is not constant during the experiment. The increasing $\mathrm{NaCl}$ content of the brine due to the ice formation leads to lower freezing temperatures, and therefore the wall supercooling degree $\overline{\Delta T}_{s}$ undergoes a continuous decrease since the coolant temperature is kept constant. The ice formation and growth rate over the subcooled surface is then lower, being more easily removed by the scrapers. As a consequence it can be observed that the heat transfer coefficient increases with time in Fig. 4.6b, especially for high scraping velocities and adaptable scrapers, where the scraping action is more intense.

\subsubsection{Time-averaged values}

For a better comparison between experiments, heat fluxes and heat transfer coefficients were averaged during the freezing period as defined in Fig. 4.1. Figure 4.7 shows the time-averaged heat flux over the freezing period for different scraping velocities $N$ and driving temperatures $\overline{\Delta \mathrm{T}}_{\log }$ whereas figure 4.8 does for the time-averaged heat transfer coefficient $\bar{h}$.

- Effect of the scraper type

Adaptable scrapers provide a two-fold augmentation of the global heat flux compared to the rigid scrapers (Fig. 4.7). The difference between the two scraping systems is mainly due to the poor scraper action of the rigid scrapers. Since the scrapers are not able to follow the surface (later explained in sec. 4.5), a scaled ice layer is developed. If the presence of such an ice layer is assumed, the obtained $\bar{h}$ values will correspond to the global heat transfer coefficient $\bar{h}_{T}$, which includes the heat conduction through an ice layer of thickness $e_{i}$. The process side heat transfer coefficient $\bar{h}_{p s}$ can be obtained then from,

$$
\frac{1}{\bar{h}_{p s}}=\frac{1}{\bar{h}_{T}}-\frac{e_{i}}{k_{t, i}}
$$

If the $\bar{h}$ values of the rigid scrapers are introduced into equation 4.2 , the resulting $\bar{h}_{p s}$ are comparable to the $\bar{h}$ values obtained for the adaptable scrapers (see Fig. 4.8).

- Effect of the scraping velocity

The scraping velocity appears to have a clear but small effect on the heat transfer, that mainly depends on the logarithmic mean temperature difference. Higher scraping velocities leads to higher heat flux values. The tests where supercooling was not 


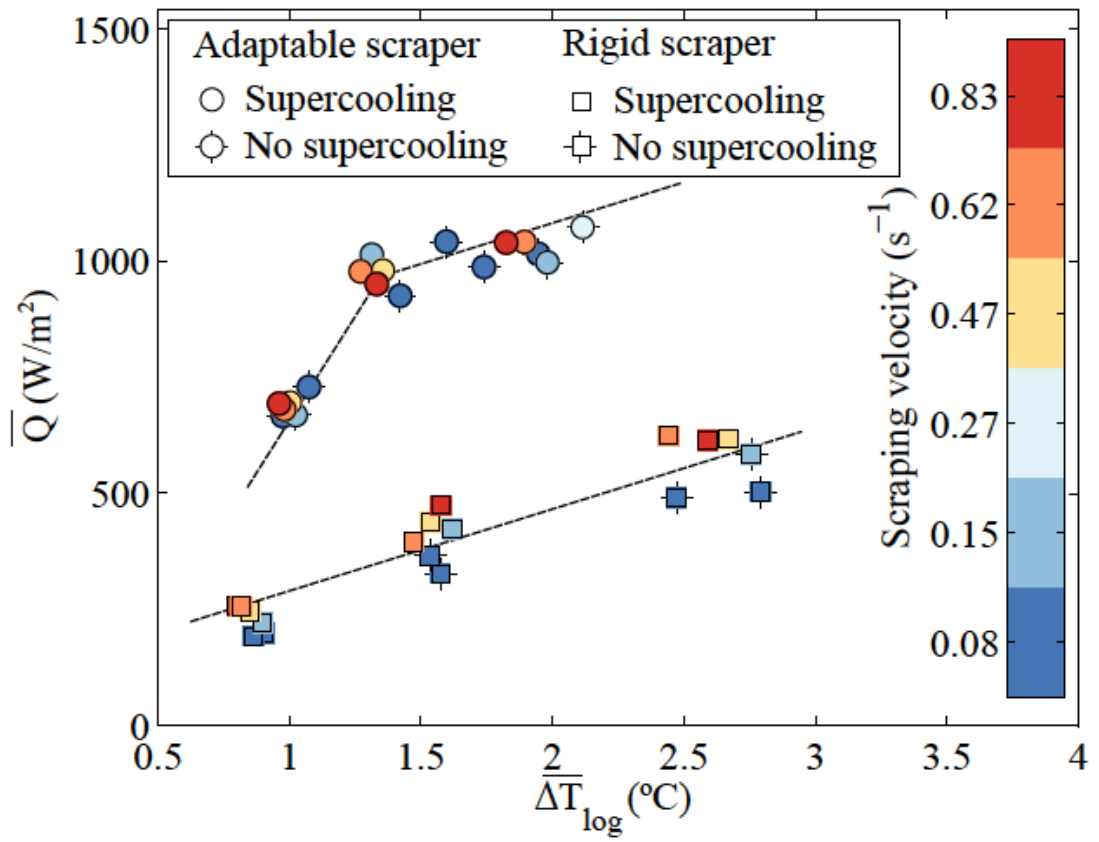

Figure 4.7: Influence of $\overline{\Delta T}_{\log }$ on the heat flux averaged over the freezing period. Results coloured by scraping velocity

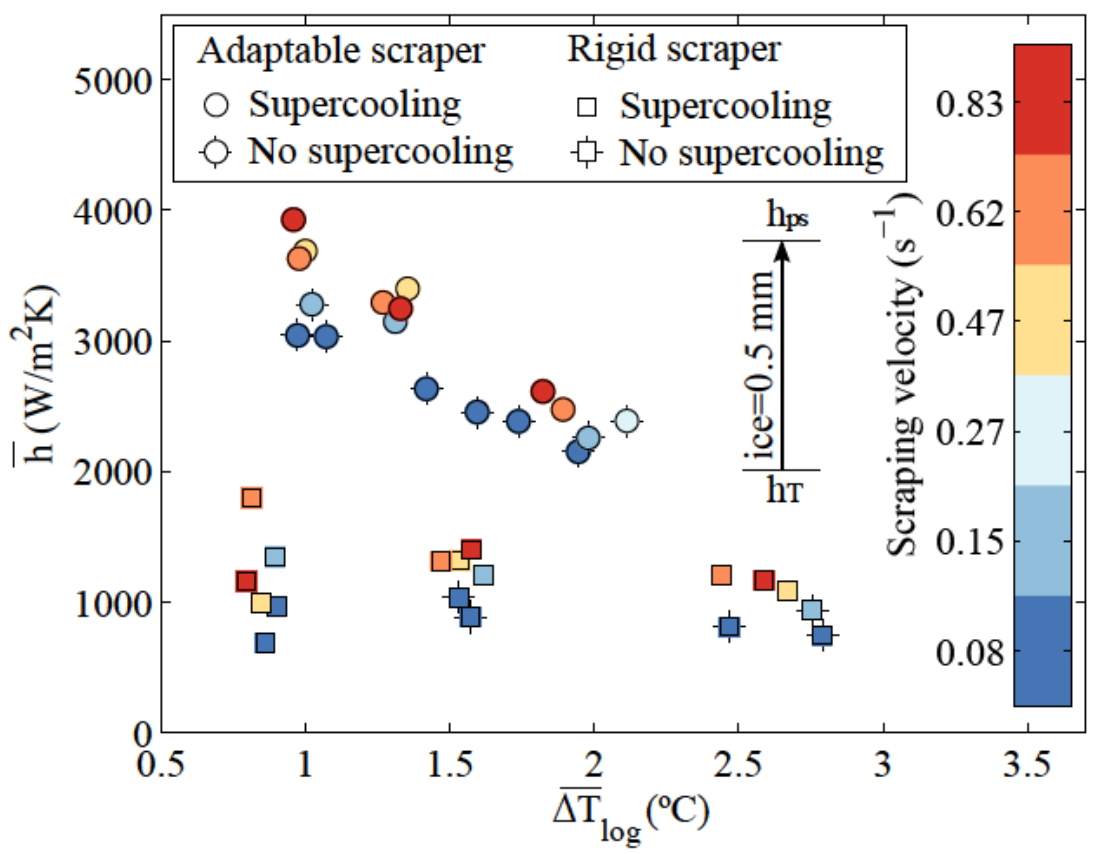

Figure 4.8: Influence of $\overline{\Delta \mathrm{T}}_{\log }$ on the heat transfer coefficient averaged over the freezing period. Results coloured by the scraping velocity 


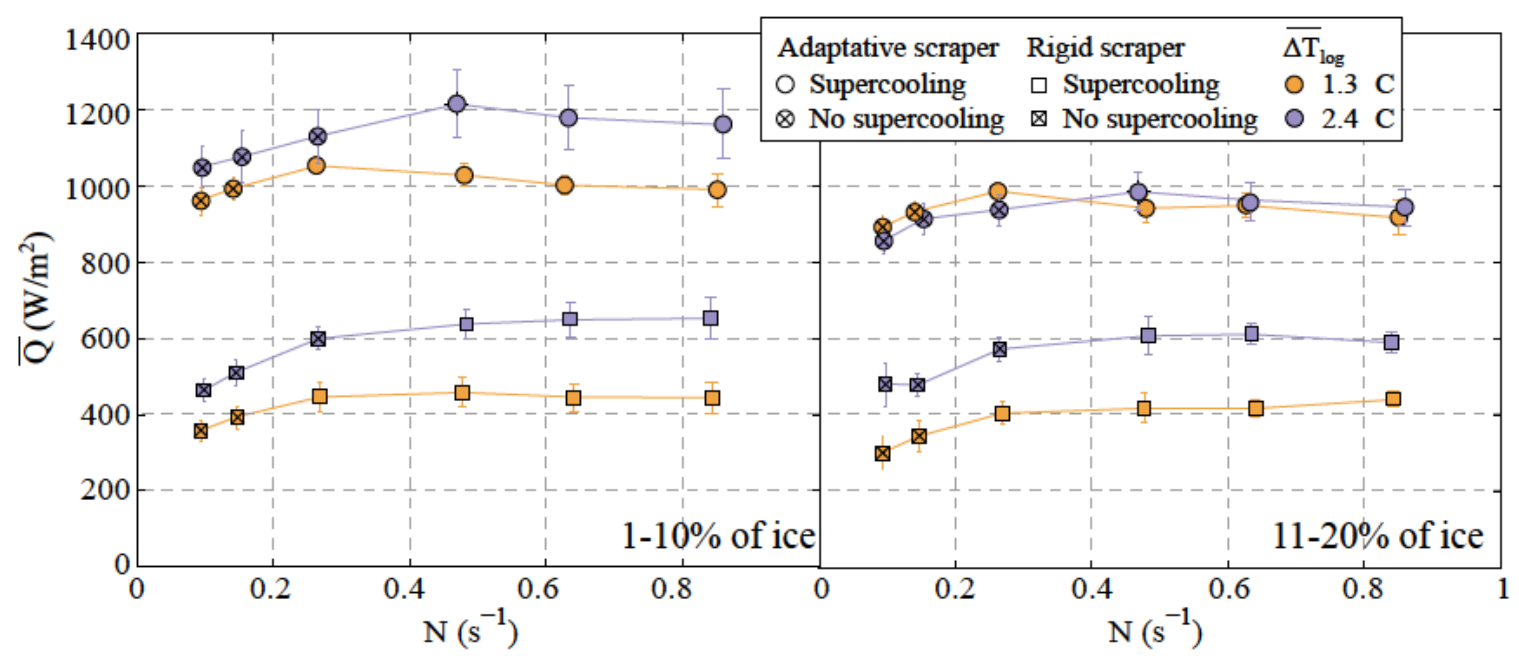

Figure 4.9: Influence of the scraping velocity on the heat flux averaged over the freezing period for different scraper systems and $\overline{\Delta T}_{\log }$ values

detected at the beginning of the freezing period (distinctively marked) correspond to low scraping velocities and indicate a poor mixing with an accumulation of ice over the subcooled surface; consequently the resulting heat fluxes are lower.

The net influence of the scraping velocity on the heat flux and the heat transfer coefficient is represented respectively in Figs. 4.9 and 4.10 for two different $\overline{\Delta T}_{\log }$ values. It is clear that the $\overline{\Delta T}_{\text {log }}$ effect (which is closely related with the plate subcooling $\overline{\Delta T}_{s}$ ) appears to be higher than the scraping velocity effect. Whereas the increase of $N$ from 0.1 to $0.8 \mathrm{~s}^{-1}$ results in an augmentation of $\bar{h}$ of around $20 \%$, the transition form $\overline{\Delta \mathrm{T}}_{\log }=2.4{ }^{\circ} \mathrm{C}$ to $1.3{ }^{\circ} \mathrm{C}$ leads to a maximum augmentation of $40 \%$ for $N=0.5 \mathrm{~s}^{-1}$. Both the heat flux and the heat transfer coefficient reaches a maximum for $\mathrm{N} \approx 0.45$ $\mathrm{s}^{-1}$. It can then be deduced from the figures that going for higher velocities increases the ice particles mixing and therefore their impact over the plate, increasing also the ice fouling [67].

The effect of $\mathrm{N}$ is therefore not independent and interacts with $\overline{\Delta \mathrm{T}}_{\mathrm{log}}$. For instance, at $\overline{\Delta \mathrm{T}}_{\log }=2.4^{\circ} \mathrm{C} h$ still experiences slight increases with $N$, whereas it remains almost constant or even decreases in other cases. The higher supercooling degree of the surface makes the surface more suitable to develop an ice layer and therefore the scrapers action may still be helpful for the heat transfer, with small augmentations of $\bar{h}$. For $\overline{\Delta T}_{\log }=1.3$ ${ }^{\circ} \mathrm{C} h$ decreases after $N=0.5 \mathrm{~s}^{-1}$ as the scraping velocity is fast enough to ensure a clean surface.

- Coolant temperature effect

Heat flux is directly proportional to augmentations on the logarithmic mean temperature difference. However, depending on $\overline{\Delta T}_{\log }$ the icing conditions of the surface can alter this dependency. From figure 4.7 it is possible to see that the heat flux increases as $\overline{\Delta T}_{\log }$ increases. For the adaptable scrapers, the leap from $\overline{\Delta \mathrm{T}}_{\log } \approx 1{ }^{\circ} \mathrm{C}$ to $\overline{\Delta \mathrm{T}}_{\log } \approx 1.5^{\circ} \mathrm{C}$ yields a significant augmentation compared to the further evolution, more similar to the one observed for the rigid scrapers. This similarity points to the presence of an ice layer, that even if removed by the scraper, is formed continuously: the 


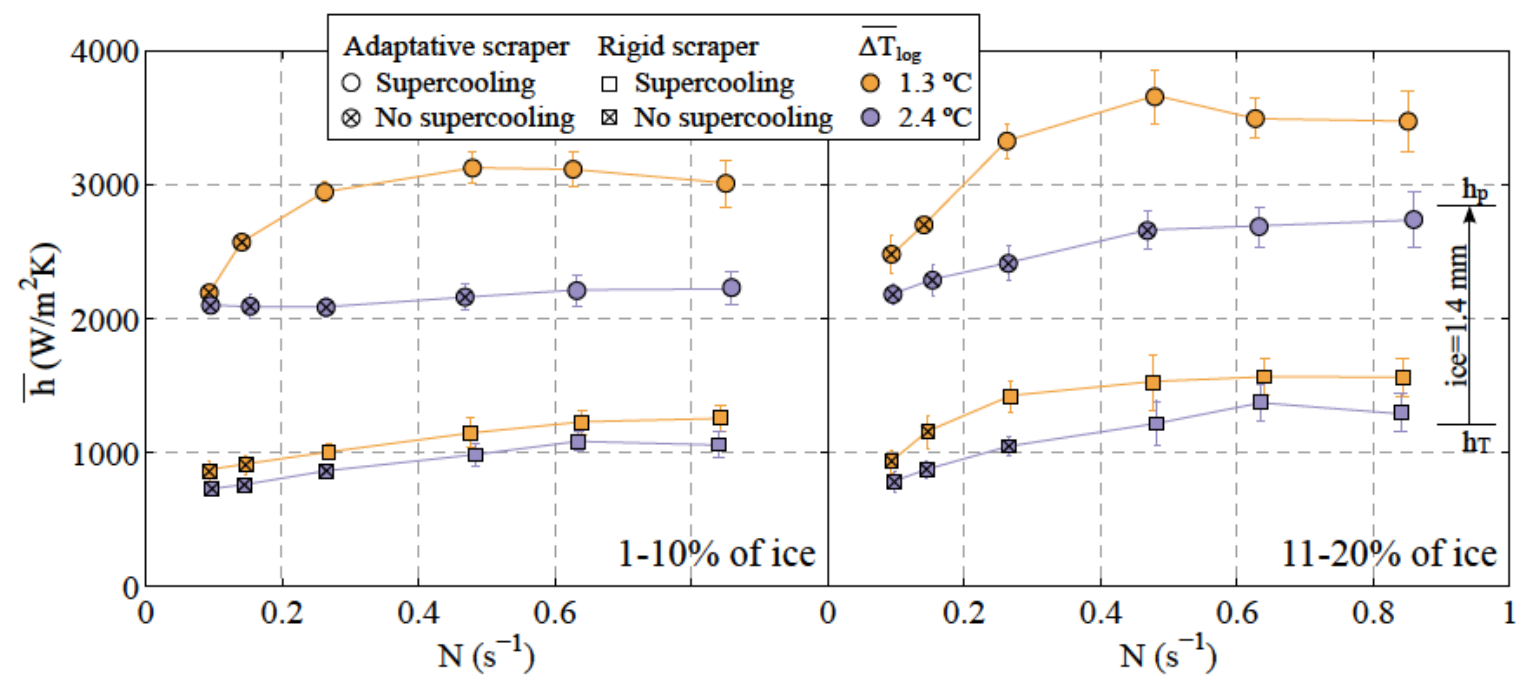

Figure 4.10: Influence of the scraping velocity on the heat transfer coefficient averaged over the freezing period for different scrapper systems and $\overline{\Delta T}_{\log }$ values

process is driven by the particle surface impacts which in turn limit the heat transfer. Therefore the trend is similar to the one shown by the rigid scrapers (where a constant ice layer is present), but with higher heat fluxes due to the effective ice removal. The presence of the thin ice layer will be reinforced by the scraping power results, as will be seen in sec. 4.6 .

The heat transfer coefficient decreases as $\overline{\Delta T}_{\text {log }}$ increases. This effect is more accentuated for the adaptable scrapers, since the increment of $\overline{\Delta T}_{\log }$ entails the aboce mentioned formation of an ice layer $\left(\overline{\Delta \mathrm{T}}_{s} \propto \overline{\Delta \mathrm{T}}_{\mathrm{log}}\right)$; this ice layer, with higher thickness, is conversely always present for the rigid scrapers. Recalling Eq. 4.2, the difference between the $\bar{h}$ values for the lower and higher $\overline{\Delta \mathrm{T}}_{\text {log }}$ can be associated to the presence of an ice layer of thickness $e_{i} \approx 0.5 \mathrm{~mm}$ (Fig. 4.8), which can be related with the ice constantly formed and removed between successive scraping actions.

From Fig. 4.7 and 4.8 it is possible to deduce that even if $\bar{h}$ decreases until a minimum value for $\overline{\Delta \mathrm{T}}_{\log }$ values as high as $2{ }^{\circ} \mathrm{C}$, at this operating condition the heat flux and therefore the ice slurry production are maximized. The decrease of $\bar{h}$ is indeed due to the higher ice production, which implies a thicker ice layer on the plate, hindering the heat transfer even if it is constantly removed. As $Q \propto h_{T} \cdot \overline{\Delta \mathrm{T}}_{\log }$ (see Eq. 4.2), it is clear that the decrease on $\bar{h}$ has a lower effect than the increase of $\frac{T_{l o g}}{\text {. As mentioned }}$ before, the level of ice formation on the plate is directly related with its supercooling degree $\overline{\Delta \mathrm{T}}_{s}$. Figure 4.11 indicates that higher $\overline{\Delta \mathrm{T}}_{\log }$ values implies higher $\overline{\Delta \mathrm{T}}_{s}$. The higher the supercooling degree is the more nucleation occurs on the plate, and therefore it represents a thicker ice layer with lower $\bar{h}$ values.

\subsubsection{Correlation for Nusselt number estimation}

Heat transfer coefficient values have been non-dimensionalized through the Nusselt number, depicted in Fig. 4.12. Both $R e_{\text {rot }}$ and $N u$ have been calculated based on the properties presented in Sec. 2.1.5. Due to the twofold effect of the ice particles (they change the rheological properties of the slurry and cause nucleation on the surface and 


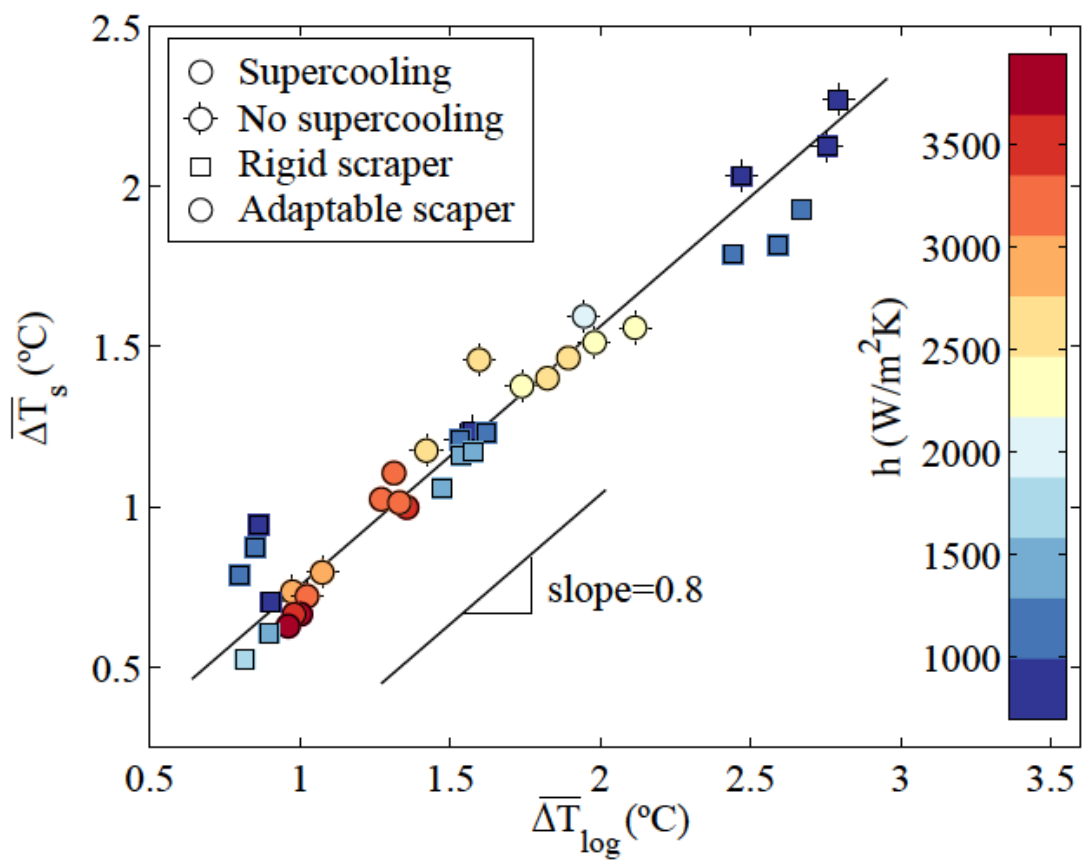

Figure 4.11: $\overline{\Delta \mathrm{T}}_{\log }$ vs. $\overline{\Delta \mathrm{T}}_{s}$ coloured by the heat transfer coefficient (adaptable scrapers)

the formation of an ice layer impacting on it), plotted results are coloured according to the product of the ice content ratio $\left(\varphi_{m}\right.$ divided by the maximum ice content of the experiment $\left.\varphi_{m, \max }\right)$ and the Froude number: $\operatorname{Fr} \cdot\left(\varphi_{m} / \varphi_{m, \max }\right)$. This new data dimension includes the effect of the phase change and considers the effect of the ice particles in the process by accounting for their quantity $\varphi_{m}$ and their distribution in the SSHPE. The particle distribution, which is in turn mainly affected by the buoyancy and the velocity induced by the scrapers, is weighted by the Froude number.

The results appear to be grouped according to the tested experimental conditions: $\overline{\Delta \mathrm{T}}_{s} \approx 0.75{ }^{\circ} \mathrm{C}$ and $\overline{\Delta T}_{s} \approx 1.5^{\circ} \mathrm{C}$. Focusing on $\overline{\Delta T}_{s} \approx 0.75^{\circ} \mathrm{C}$, the results with low ice content or high ice stratification $(\downarrow F r)$, associated with low $R e_{\text {rot }}$ values, follow a pattern that fits with the penetration theory plus a constant term $C$, that can be related with the effect of the phase change on the subcooled surface:

$$
\bar{h}=2 \sqrt{\frac{k_{t} \cdot \rho \cdot c_{p} \cdot n \cdot N}{\pi}}+C .
$$

Beyond the low effect of the ice particles in the flow (which behaves as a single phase flow), the penetration theory trend indicates that the ice particles rise after being scraped and therefore do not impact again on the subcooled surface. This absence of impacts reduce the ice formation over the surface and as a consequence the scrapers are able to remove it.

The data separate from this trend at $\operatorname{Re} e_{\text {rot }} \approx 5 \cdot 10^{4}$, when the product $F r \cdot\left(\varphi_{m} / \varphi_{m, \max }\right)$ approaches to 1: ice particles concentration is higher and they are better mixed. As a consequence the flow behaviour changes. Moreover the well mixed particles impact on the subcooled surface, generating the formation of an ice layer that worsens the heat 


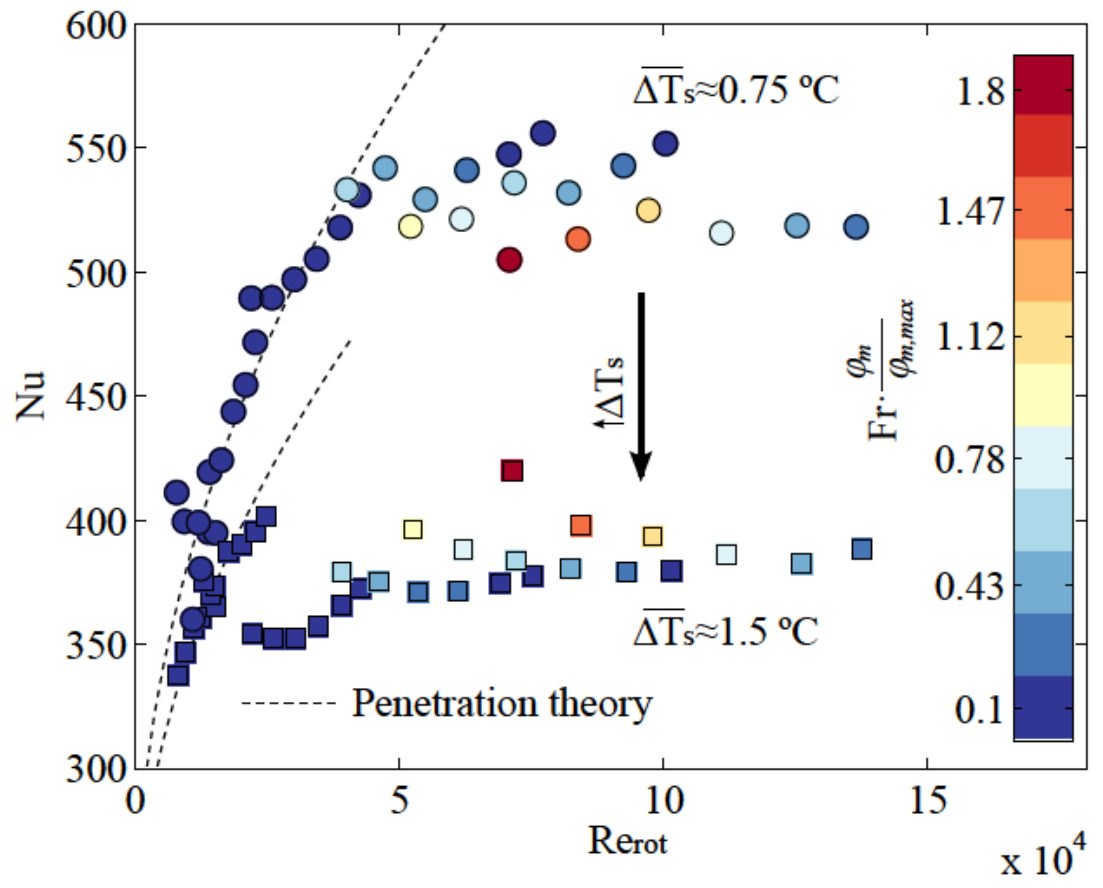

Figure 4.12: Nusselt $(\mathrm{Nu})$ number vs. rotating Reynolds $\left(R e_{r o t}\right)$ number for different experiments (adaptable scrapers)

transfer: the higher is $\mathrm{Fr} \cdot\left(\varphi_{m} / \varphi_{m, \max }\right)$ the lower is $N u$, with a low dependence with the $R e_{\text {rot }}$ number.

A composed correlation has been proposed to predict $N u$ versus $R e_{r o t}, F r$ and $\varphi_{m}$ for the $\overline{\Delta \mathrm{T}}_{s} \approx 0.75^{\circ} \mathrm{C}$ tests. For $R e_{\text {rot }}<4.7 \cdot 10^{4}$ they have been fitted to the penetration theory plus a constant therm:

$$
N u=\left(2 \sqrt{\frac{k_{t} \cdot \rho \cdot c_{p} \cdot n \cdot N}{\pi}}+1200\right) \frac{D}{k_{i}}=1.53 \operatorname{Re}_{\text {rot }}^{0.5}+229
$$

whereas for $R e_{\text {rot }}>4.7 \cdot 10^{4}$ they have been fitted performing logarithmic multiple regressions to express $\mathrm{Nu}$ versus $\operatorname{Re}_{\text {rot }}$ and the product $\mathrm{Fr} \cdot \varphi_{m}$ :

$$
N u=661.6 \operatorname{Re}_{\text {rot }}^{-0.026}\left(F r \varphi_{m}\right)^{-0.028}
$$

Influence of Prandtl number was also studied, showing to be insignificant. Fig. 4.13 presents a comparison between experimental and calculated Nusselt number values for the full range fitted, together with the regression coefficient.

The data corresponding with $\overline{\Delta T}_{s} \approx 1.5{ }^{\circ} \mathrm{C}$ show much lower $N u$ values. This decrease on $N u$ is due to the ice layer effect, as seen in Fig. 4.8. The high supercooling degree $\overline{\Delta T}_{s}$ together with the initial ice particle impacts develops an ice layer of $e_{i} \approx 0.25$ $\mathrm{mm}$, not well removed by the scrapers due to its higher level of adherence. This leads to lower values of $N u$ with an opposite behaviour of the $\overline{\Delta \mathrm{T}}_{s} \approx 1.5^{\circ} \mathrm{C}$ data: increases on $\operatorname{Re}$ rot and $\mathrm{Fr} \cdot\left(\varphi_{m} / \varphi_{m, \max }\right)$ implies a more active scraping and the positive effect of the ice removal is higher than the negative effect of the ice particles impacting on the ice layer. The certain presence of the ice layer makes impractical the proposal of 


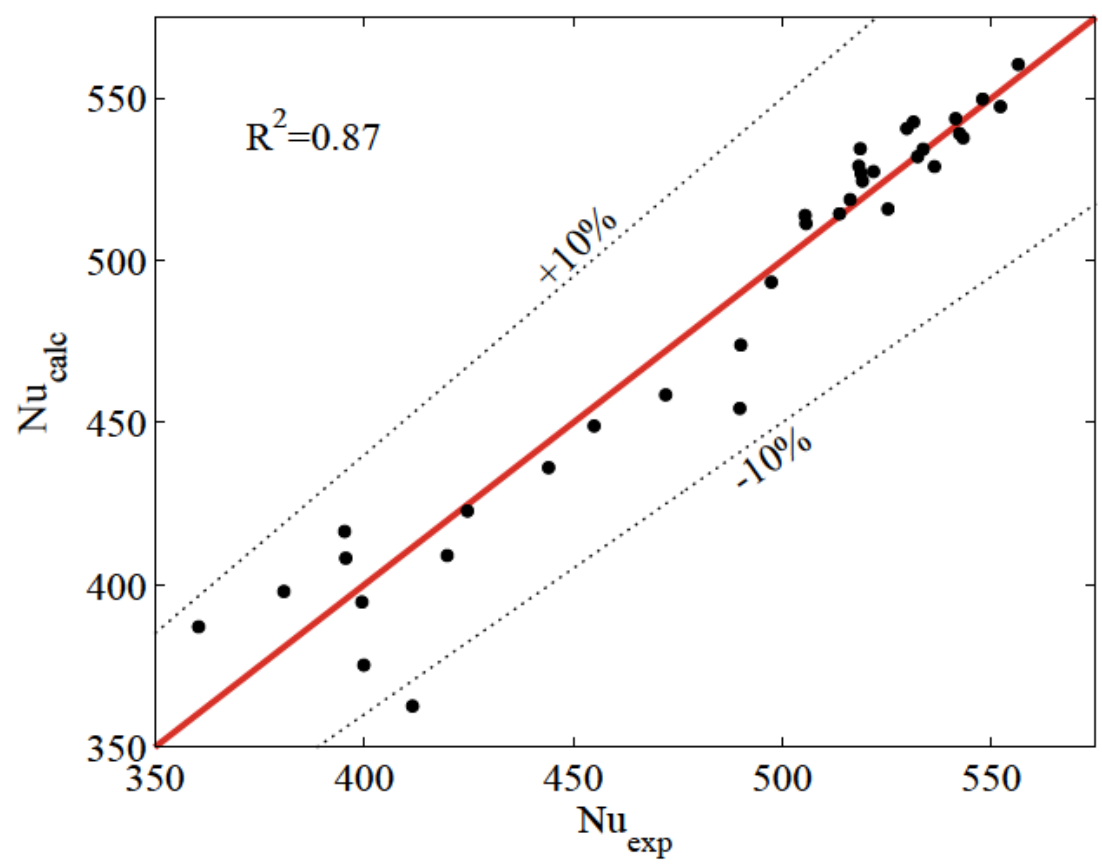

Figure 4.13: Comparison between experimental and calculated Nusselt number values

correlations for that values of $\overline{\Delta T}_{s}$.

\subsection{Ice removal evaluation}

The wall supercooling is the driving force of the ice production; once nucleation starts an ice layer appears over the steel surface. As the working fluid is not pure water but a brine, the layer consist of a lot of ice fragments, composing what is called a mushy structure [89]. The growing kinetics and morphology of the layer is related with the surface characteristics and its temperature. In general, the ice does not cover entirely and instantaneously the surface; liquid parts are retained among arms of solid parts. It means that, if the rotating velocity is high enough, the scrapers do not slide over a complete ice layer, being some areas still not covered by it. The later is true if the scraping action detaches all the formed ice. If not, the remaining ice together with the new one could lead in layer covering completely the surface.

The effectiveness of the scrapers depends on the ice-steel adhesion force. A common value to it is $0.2-0.6 \mathrm{MPa}$ [89], but it can vary significantly with the surface characteristics. In particular, the ice adhesion increases with the wettability and the roughness of the surface $[90,91]$. The roughness, directly related with the wettability, has even a higher impact on the ice adhesion values, increasing them dramatically. As a representative example, Hirata et al. [92, 93] found that by using low wettability-roughness surfaces with certain concentration and surface temperatures, the mushy layer was detached by buoyant forces, without requiring a mechanical action.

In order to determine whether the ice layer was completely removed or not by the scrapers, a comparative technique was applied to the images acquired from the top window of the SSHPE (see sec. 2.1.2). The successive images taken once the freezing 


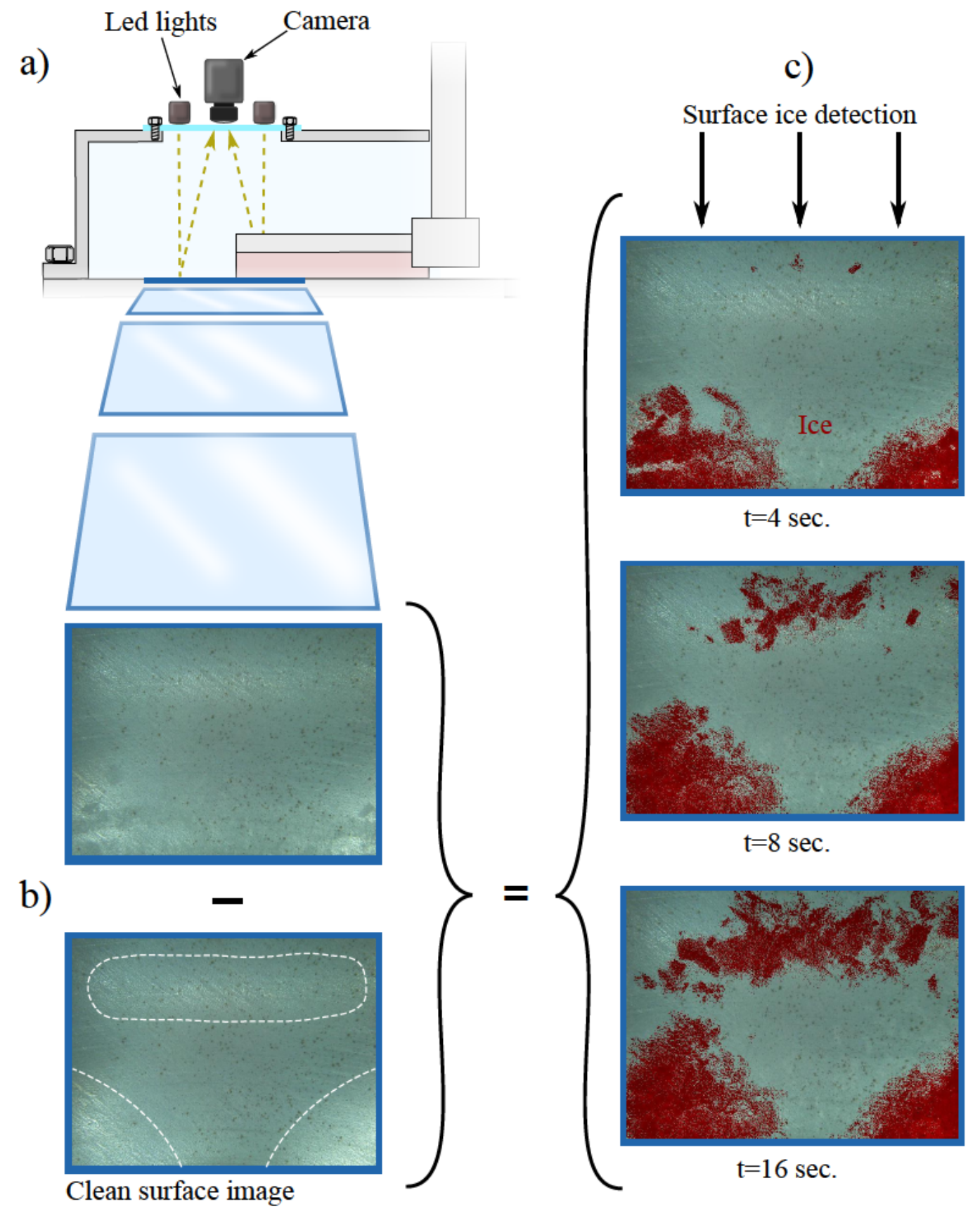

Figure 4.14: Ice detection over the plate surface with the rigid scraping system; $N=0.5$ $\mathrm{s}^{-1}$ and $\overline{\Delta \mathrm{T}}_{\log }=1.4^{\circ} \mathrm{C}$. 
period started -when the ice content of the slurry was low enough to allow visibility through it, $\approx 1 \%$ - were compared with the one taken prior to it. In that way, variations in the light reflected bye the plate form the top LED light sources could be related to the ice formation on the surface. The procedure is exposed in Fig. 4.14.

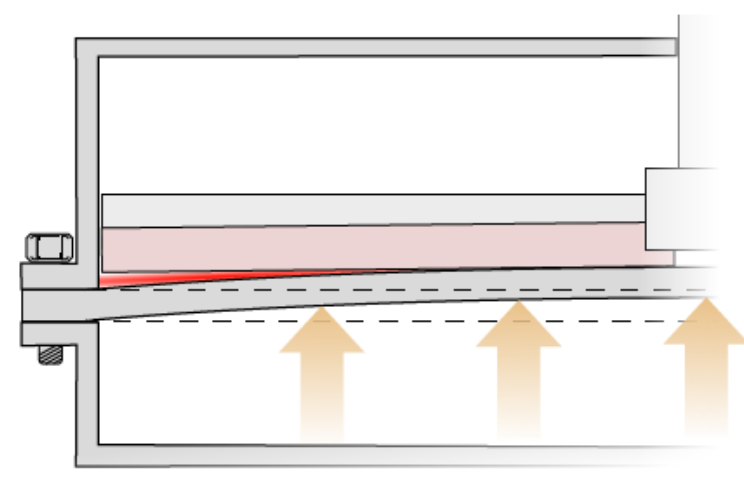

(a) Rigid scrapers

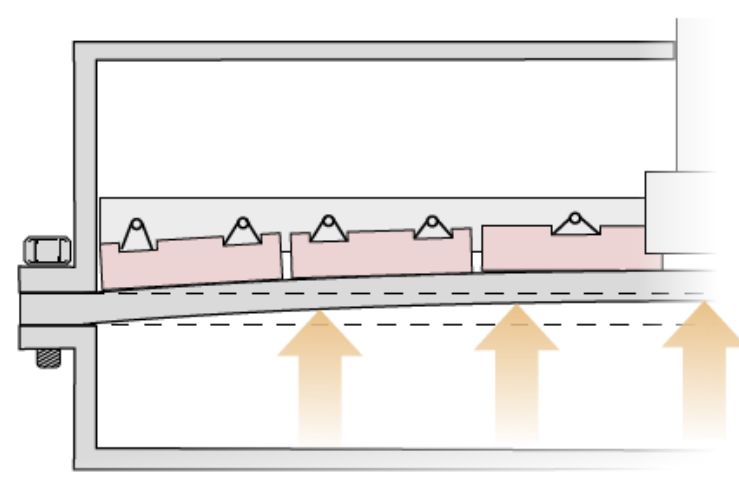

(b) Adaptable scrapers

Figure 4.15: Scraper system view.

The ice detection technique was applied to images acquired with both scraping systems, rigid and adaptable. The results shown that ice was detectable only for the rigid scraping system. The evolution of the ice layer expansion corresponds to that observed by Ishikawa et al. [89], with ice arms that evolve covering partially the surface area. The ice layer was observed for all the scraping velocities where values of $\overline{\Delta T}_{\log }>1.1$ ${ }^{\circ} \mathrm{C}$. Smaller values produced an automatic detachment of the mushy structure by buoyancy or by the flow induced by the scraper arms. The images taken when adaptable scraper system was operating did not reveal any ice layer. The absence of its detection did not mean that was not present, but that was not detectable with the sensitivity provided by the system. The layer at low ice content values could be very thin to affect the light reflections.

The absence of any observable ice layer for the adaptable scraper system is also due to the way in which each scraper system works. The pressure exerted by the coolant under the scraped plate produced a small curvature that deviates the scraper from the plate for peripheral points. This separation is avoided in the adaptable system, as is indicated schematically in Fig. 4.15.

In any case, the initial absence of ice does not mean that it was not developed later on the test. Dismounting the window cover of the SSPHE at the end of the experiments ( $20 \%$ of ice content) and checking the inside wall revealed that a thin ice layer with a smooth surface was adhering on the scraped cooling surface in some situations. It was present always when using the rigid scraper system. In the case of the adaptable scrapers, only for the tests with higher wall supercooling degrees and higher scraping velocities. It is clear that the wall supercooling favours the formation of the layer, but its relation with the scraping velocity might be less obvious. At medium ice content -i.e. $10-12 \%$ - scraping rates promotes the mixing, increasing the impact of the suspended particles over the plate surface. If the wall supercooling is high these impacts will increase the nucleation and favour the ice layer formation. This might not happen at low scraping rates, were the ice mixing is low and ice crystals are accumulated on the 
top by buoyancy. Additionally, the particles that remain in suspension adhere together forming flocculates. When the ice content is high -i.e. $15 \%$ or greater-the flocculates start to join and the working fluid becomes a uniform paste. The slurry behaves then as a non-Newtonian fluid and it does more as the ice content continues increasing. For ice concentrations near $20 \%$ the slurry behaves as a cake, rotating as a single column. At that point the convection and vortex characteristics of the previous flow disappear. The adhesive force of ice on the cooling surface gets stronger and stronger. Therefore, if the contact between scrapers and steel is not good enough, an ice layer will be formed. This may happen if a thin liquid layer exists between scrapers and plate, as will be pointed out later.

\subsection{Power consumption}

The scraper power consumption is a determinant design parameter, being its knowledge of great importance. For instance, the production process itself can be affected by the amount of energy dissipated within the fluid. Elevated power inputs can affect the ice crystals morphology or even induce their degradation.

Available power data on scraped surface crystallizers is very limited when compared to stirrers or common -without phase change- scraped surface heat exchangers. In contrast with the stirrers, in scraper surface devices the friction between scrapers and walls adds a new power demand, which could be even the most important one. Their usage in crystallizing conditions adds also the power required to break and/or detach the continuous formation of the generated solid phase.

In both stirrers and mixers the power is commonly non-dimensionalized in the form of the power number Po [94], defined as

$$
P o=\frac{P}{\rho N^{3} D^{5}},
$$

which relates the resistance force to the inertia force. However, the power number does not take into account the contribution due to the friction between solids.

In classical studies about solid-liquid mixers the power consumption was related with the density of the mixture in the stirrer zone [95]. The suspension degree of the solid particles becomes then an important factor, determining the equivalent density value of the fluid. For scraped surface heat exchangers operating without phase change, the power consumption has been assumed to be proportional to the viscosity [96], considering that the gap between scraper and walls was high enough to avoid solidsolid contact. This may not be true if the friction forces between scrapers and surface are important, as they should be under crystallizing conditions processes where the scraping action not only has to refresh the boundary layer but also pull of the crystals.

In the present work the power consumption was measured considering the one consumed in the driving electric motor by using a wattmeter (sec. 2.1.2). The acquired data corresponds therefore the total power consumed, without distinction of the different mechanisms involved -hydrodynamic friction, solid-solid friction, etc. A part of the power input was dissipated in the bearings of the rotating shaft, unavoidably linked to the vertical axial load and therefore dependent on the operating conditions. In spite of the lack of experimental evidences regarding the power consumption in the 
SSPHE, hereafter different models will be considered in order to explain the different mechanism involved and the available data.

\subsubsection{Power, torque and force}

The scraping power $S_{P}$ is equal to the product of the rotating velocity and the torque $M$ applied on the shaft

$$
S_{P}=\Theta \cdot M=2 \pi N \cdot M .
$$

The torque in turn comes from the product of the applied force $F$ in the scraper arm at a distance $r$ from the shaft,

$$
M_{T}=F \cdot r .
$$

In the case of the scrapers the force is not punctual, but instead it is distributed non-uniformly along the scraper arm, $F(r)(\mathrm{N} / \mathrm{m})$. Knowing the distributed force is a priori difficult as it depends on different aspects such as differences in the spring applied forces, the ice layer thickness and distribution over the plate, the flow inside the SSHPE, etc. The total torque is given by

$$
M_{T}=s \int_{0}^{R} F(r) d r
$$

where $s$ is the number of scrapers.

Chilling period During the chilling stage, prior to the nucleation onset, the force $F_{C}$ can be decomposed into an hydrodynamic term $F_{h}$, due to the flow resistance, and a scraper-plate friction therm $F_{f r}$

$$
F_{C}=F_{h}+F_{f r}
$$

The first one is the effect of the induced flow on the blades, where viscosity (shear stresses) and pressure gradients on the scraper arm faces oppose to their movement. The torque produced by $F_{h}$ is similar to the one in a stirrer and therefore it can be expressed as a function of the rotating Reynolds number in the SSHPE, $R e_{\text {rot }}$. Tromelen et al. proposed a formula that correlates the power number with $R e_{r o t}$ and the number of stirrer arms $s[96]$

$$
P o=L_{s} K \operatorname{Re}_{\text {rot }}^{-a} s^{0.59}
$$

where $L_{s}$ is the stirrer arm length and $a$ and $K$ are correlations constants. Later on McCabe et al. found that the Power number tends to be a constant independent of $R e_{\text {rot }}$ for values of it higher than $\sim 10^{4}$ [97]. Of course all this will be true if the impeller is also far from the tank walls.

The friction force $F_{f r}$ occurs between the PEEK blades and the plate surface of steel. It is related with the normal force applied over the scrapers $F_{N}$-mainly due to the springs action- through $F_{f r}=\gamma_{s} t F_{N}$, where $\gamma_{s} t$ is the friction coefficient between the PEEK blades and the steel surface of the plate. The friction coefficient depends highly on the surface roughness, as well as on the type of friction (i.e. dry or wet). 
A clear distinction has to be made between dry and lubricated -wet- friction. In the case of dry friction, the asperities of the two solids, PEEK and steel, are directly in contact. If a liquid is present, as in this case the brine, it will act as a lubricant between the two solid bodies reducing the friction. The role of lubricants in reducing friction and wear has widely investigated [98] and it is common to split the friction behaviour into four different dynamic regimes (see Fig. 4.16):

Pre-sliding, for very low velocities $(\sim 0)$ the contact occurs at the asperity junctions. The small displacements cause the asperity junctions to deform elastically and/or plastically.

Boundary lubrication, the velocity is not enough to create a fluid film between the two solids. As a consequence it remains a solid-solid contact resulting in a process of shearing.

Partial fluid lubrication, the lubricant is brought between the two solids through the sliding movement and a film is formed due to the viscosity of the liquid lubricant. The shear strength of a solid lubricant film at low velocities is generally higher than the shear forces of the corresponding fluid film originated at higher velocities. Thus, the friction coefficient in lubricated systems uses to decrease when the velocity increases from zero. Higher velocities imply less asperity welds and lead to lower friction forces.

Full fluid lubrication, there are no asperity contacts. The friction force increases now with the velocity due to the viscosity of the lubricant.

The resulting lubrication regime will depend on parameters like the solid roughness, the fluid viscosity and, of course, the velocity, which makes the prediction of this regime an almost impossible task.

Freezing period Once nucleation starts, an ice layer appears over the steel surface. The removal of the formed ice layer implies a pure scraping force $F_{S}$ to overcome the adhesive force on the cooling surface. Moreover, the scraping action might not remove completely the ice, existing a constant ice layer over the plate.

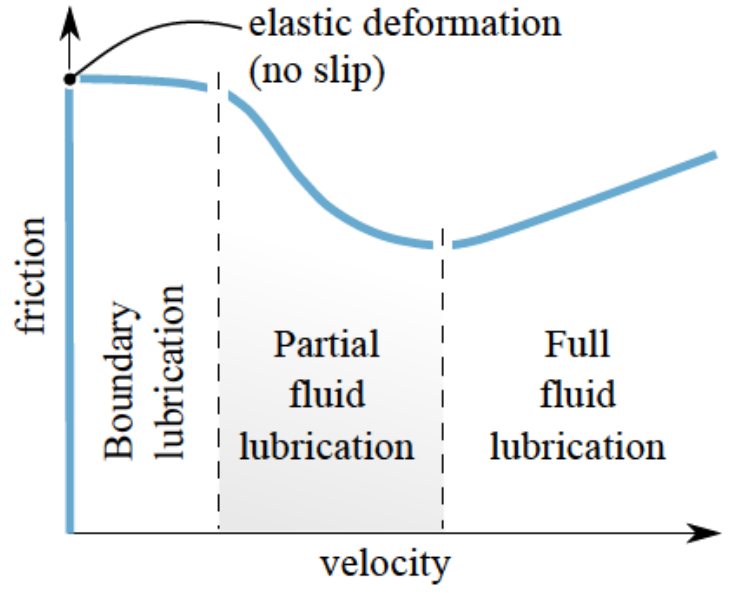

Figure 4.16: The friction force as a function of velocity for lubrication The required force in this case has to overcome the breakage resistance of the ice, as fragments of it break instead of being completely pulled off from the plate.

The non-uniform distribution of the mushy layer exposed before, plus the possibility of being not completely scraped, lead to a force composed by the friction between scrapers and steel, and the friction between scrapers and ice. Considering $\chi \in[0,1]$ to be proportional the surface covered by ice then, 


$$
F_{f}=\chi F_{f r, i}+(1-\chi) F_{f r, s t}=F_{N}\left(\chi_{\mu_{i}}+(1-\chi) \gamma_{s} t\right)
$$

where $\mu_{i}$ is the friction coefficient between the scrapers PEEK and the ice and the normal force has been assumed uniform along the scraper arms. Both friction coefficient values are difficult to determine without experimentation due to the high dependence on the surface characteristics and the existing brine layer between surfaces. Moreover, ice friction is a complex process and involves different physical and thermal mechanisms. In spite of the different studies focused on it, there is not yet a successful predictive model.

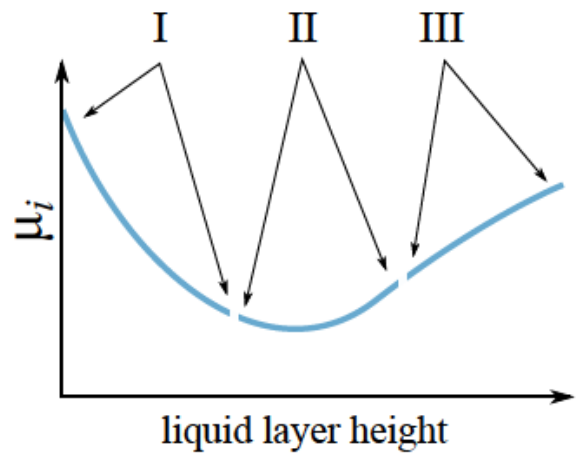

Figure 4.17: Friction regimes for ice, related to the liquid layer height [99].
It is known that the typical low friction values on ice are due to he presence of a liquidlike layer between the ice and the sliding material. This liquidlike layer is generated mostly by the heat released by the friction itself, acting as a lubricant and reducing the effective contact area between ice and slider [99]. The effect of this liquidlike layer on the friction coefficient is, nevertheless, not always favourable. As shown in Fig. 4.17 for low layer height values (few molecular layers) it acts as a lubricant, reducing $\mu_{i}$ as the solid-solid contact area is reduced (regime I). If the liquidlike layer increases in height the lubrification effect also increases, but at the same time the capillary bridges between solid surfaces acts as bonds, introducing a drag force; here is where $\mu_{i}$ reaches its minimum value (regime II). Further increments in the liquid layer thickness lead to a viscous drag (hydrodinamic friction), as it is the lubricating layer and not the solids asperities who carries the load; the value of $\mu_{i}$ increases again (regime III). The mechanism is somehow similar to that one exposed in Fig. 4.16, but the difference is that now the liquid layer can vary also due to the melting of one of the solid surfaces involved in the friction.

As both ice and scraper surfaces are immersed, a liquid layer is expected to be always present, in addition to the ice melting induced by the friction. High wall supercooling degrees will lead to ice temperatures lower than the melting point and as a consequence the layer will be more compact, reducing the layer thickness. On the contrary, lower wall supercooling degrees ensures a mushy, near melting ice layer where the liquid layer is thinner.

Variables such as the sliding velocity or the normal force applied by the sliding (scraper) surface have also an effect on the friction factor coefficient. Thus, and in general, higher sliding velocities and normal forces reduce the friction [99] until certain velocity and load values. This phenomena have also found to occur on ice sea, i.e. with water- $\mathrm{NaCl}$ solutions [100].

The total force while freezing conditions is

$$
F_{F}=F_{h}+F_{S}+F_{N}\left(\chi_{\mu_{i}}+(1-\chi) \mu_{s}\right)
$$

As the ice fraction increases in the SSPHE the slurry fluidity changes, not only due to the effect of the particles but also to the decreasing temperature of the remaining 
brine. Both cases may lead to changes in $F_{h}$. The effect of the suspended particles would be higher as their concentration increases along the test time, being negligible for the instant after the nucleation onset.

\subsubsection{Adaptable scraper system results}

As it was made with the heat transfer, the power results are presented here based in the study of their evolution with time-ice concentration and the study of the overall-time averaged values.

Time resolved values The power consumption monitored during different ice production tests are shown in Fig. 4.18: $\mathrm{N}=0.08$ and $0.80 \mathrm{~s}^{-1}$ and $\overline{\Delta \mathrm{T}}_{\log }=1.3$ and $2.4{ }^{\circ} \mathrm{C}$. The power values were smoothed by using a local regression smoothing method [101]. The plots also show the evolution of the bulk and wall temperatures. For low scraping rates (Fig. 4.18a) there is not any significant change between the chilling and the freezing periods once nucleation starts: the power trend remains equal. On the other hand in Fig. $4.18 \mathrm{~b}$ the power experiences an abrupt decreasing in that instant.

Higher scraping values require higher power inputs: $\mathrm{S}_{\mathrm{P}} \approx 180 \mathrm{~W}$ for $\mathrm{N}=0.80 \mathrm{~s}^{-1}$ whereas $\mathrm{S}_{\mathrm{P}} \approx 110 \mathrm{~W}$ for $\mathrm{N}=0.08 \mathrm{~s}^{-1}$. Moreover, the power consumption for the lower scraping velocities seems to be constant or either decreasing in time, whereas the ones for higher scraping velocities increases. As the time evolution and the ice content are related, the increasing trend of the power consumption is most probably due to the increasing level of ice content and the lost of fluidity of the slurry. Therefore, when the values of the scraping velocity are high enough the hydrodynamical force $F_{h}$ in Eq. 4.13 will increase constantly as the content in ice does. The effect of $F_{h}$ is related to the flow velocity, and therefore it is almost inappreciable for the cases with lower scraping rates. It is also worth mention that, in spite of the power consumption increasing trend with the ice content, it is never as high as the power consumed during the chilling. The low impact of the ice content in the power consumption values at low scraping velocities is also explained by the fact that at low velocities, the ice particles aggregate on the top of the SSPHE due to buoyancy and lack of mixing. This minimizes its effect on the brine properties near the scrapers. At high scraping velocities the mixing is complete and it effect is notable.

Figure 4.19 summarizes both the effect of the scraping velocity and the effect of the logarithmic mean temperature difference $\overline{\Delta T}_{\text {log }}$-related with the surface subcooling, $\overline{\Delta T}_{s}$, see Fig. 4.11. As shown before, in the cases of higher scraping velocities an abrupt decrease in power consumption of $\approx 40 \%$ takes place after the onset of nucleation. This power comes from the reduction in the friction force when passing from the chilling period (Eq. 4.10) to the freezing period (4.13). During the chilling process the friction between the scrapers PEEK and the plate steel is characterized by $\mu_{s}$. The unpolished plate surface, with a considerable level of roughness, provides a high value for the friction coefficient and the power consumption required as a consequence is high. Once the nucleation occurs, the ice appears over the surface. Independently of the scrapers ability to remove it, it is clear that part of the ice remains over the surface, which explains the decrease in the power consumed as the friction is reduced.

The idea of ice remaining over the plate surface -perhaps filling the gaps of the steel roughness- gains strength considering that the ice adhesion increases with the surface roughness as exposed in section 4.5. The value of $\mu_{s}$ is then replaced by a different one 


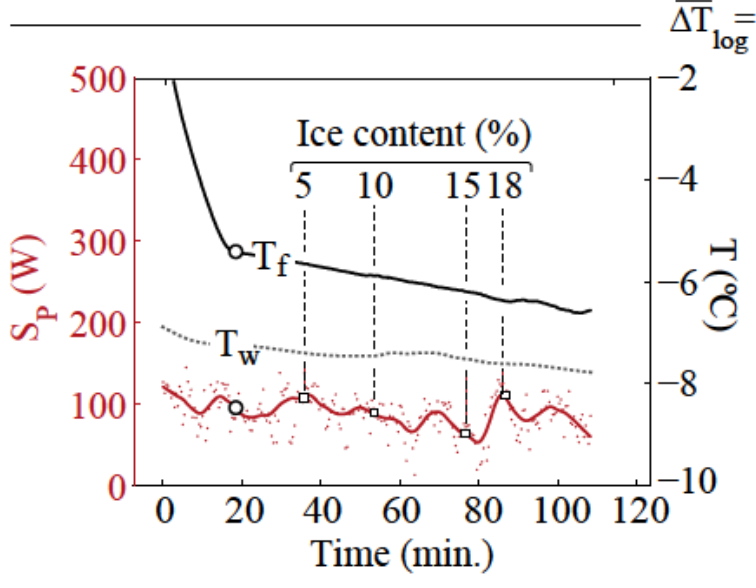

a.1) $\mathrm{N}=0.08 \mathrm{~s}^{-1}$

$\overline{\Delta \mathrm{T}}_{\log }=1.3^{\circ} \mathrm{C}$

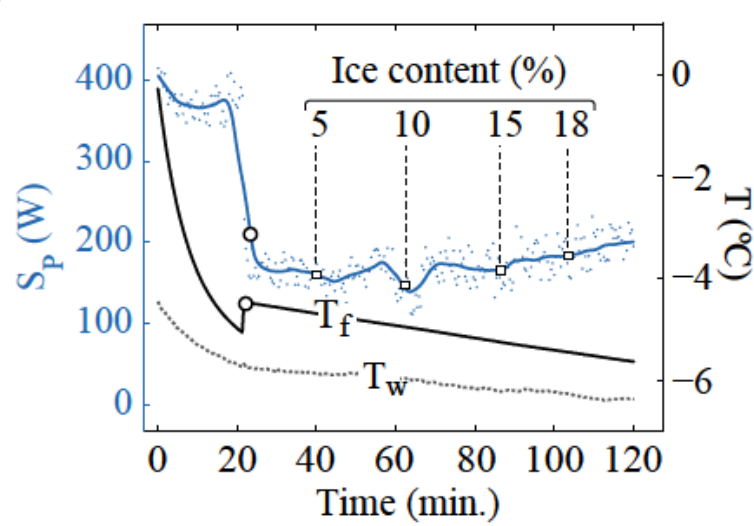

b.1) $\mathrm{N}=0.80 \mathrm{~s}^{-1}$

$$
\overline{\Delta \mathrm{T}}_{\log }=2.4^{\circ} \mathrm{C}
$$

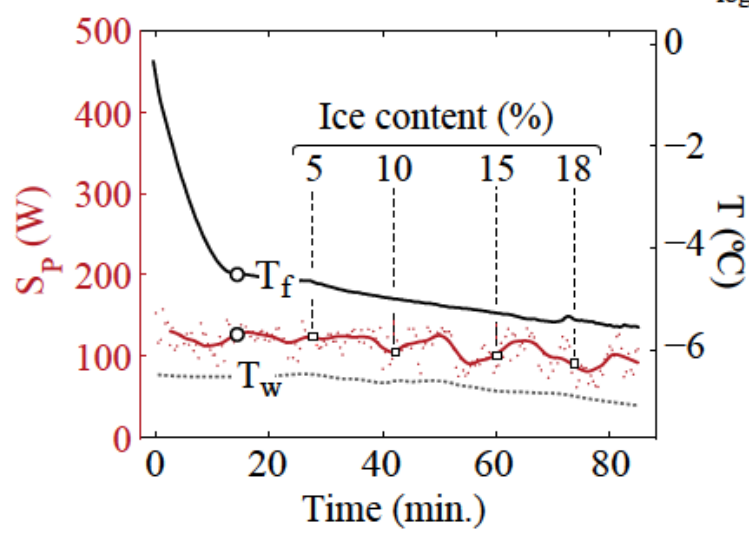

a.2) $\mathrm{N}=0.08 \mathrm{~s}^{-1}$

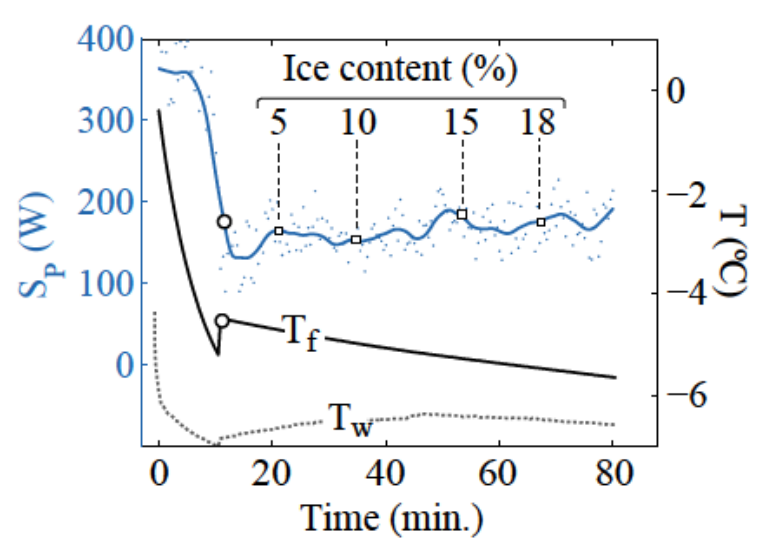

b.2) $\mathrm{N}=0.80 \mathrm{~s}^{-1}$

Figure 4.18: Scraping power consumption along time for different scraping velocities $\mathrm{N}=0.08$ and $0.8 \mathrm{~s}^{-1}$ (left to right) and mean logarithmic temperatures $\overline{\Delta \mathrm{T}}_{\log }$ of 1.3 and $2.4{ }^{\circ} \mathrm{C}$ (up to down) 


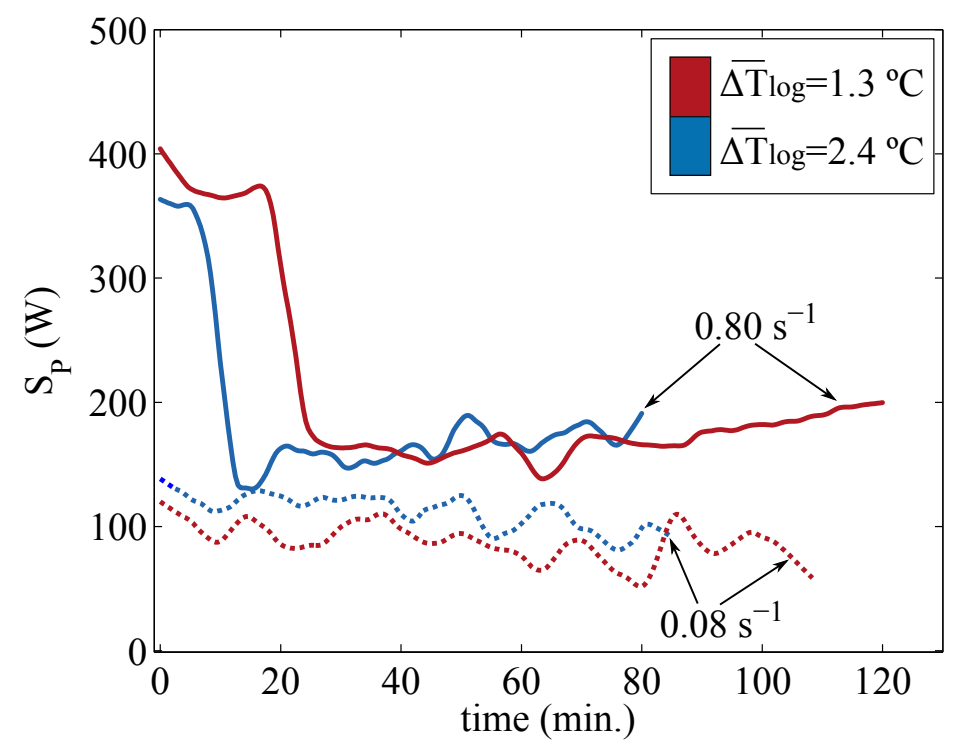

Figure 4.19: Scraping power comparative for $\Delta \mathrm{T}=1.3{ }^{\circ} \mathrm{C}$ and $\Delta \mathrm{T}=2.4{ }^{\circ} \mathrm{C}$

in between $\mu_{s}$ and $\mu_{i}$, reducing considerably the total power consumed. The ice is then acting as a lubricant or as an ice-skating rink.

In the cases with low scraping velocities (Fig. 4.19a) the lack of an instantaneous nucleation onset over all the SSPHE volume (see sec. 4.2) justifies the absence of fall in the power values. As the nucleation starts locally on the wall, the ice layer is present since almost the beginning of the experiment

The decrease in the power and in the required ice scraping force $F_{S}$ at the nucleation onset -when it was expected to increase due to the lost of fluidity in the slurry- can be then explained by two reasons:

- the negligible effect of the ice in the brine due to its low initial concentration

- and the most important, the lubricating effect of the ice layer.

The ice lubricating effect in scraped surface heat exchangers has been previously noticed in the literature. For instance Quin et al. [54] observed that the torque on the shaft did not increase and even decreased slightly after the nucleation started. The main difference with the present work is that they used a polished stainless steel surface, which can explain low descent in the torque compared with the results reported here -as here $\mu_{s}$ was much higher. What they did observe was the power consumption augmentation along time-ice content increase.

The low impact on the power data produced by the appearance of $F_{S}$ is justified by the presumable small ice thickness over the plate. Ishikawa [89] estimated the mechanical force to remove a mushy ice layer on a cooling surface, finding that the force increased with the thickness of the ice layer. Longer times between scraper actions (300 to $1000 \mathrm{~s}$.) and a lower temperature would result in a greater power demand. However, the time intervals in this study were much shorter $(3$ to $0.3 \mathrm{~s}$.). In such short times the ice layer thickness could not grow enough to generate sensible resistance to the scrapers. In contrast, the thin layer acts as a lubricant. Therefore, the presence of an 


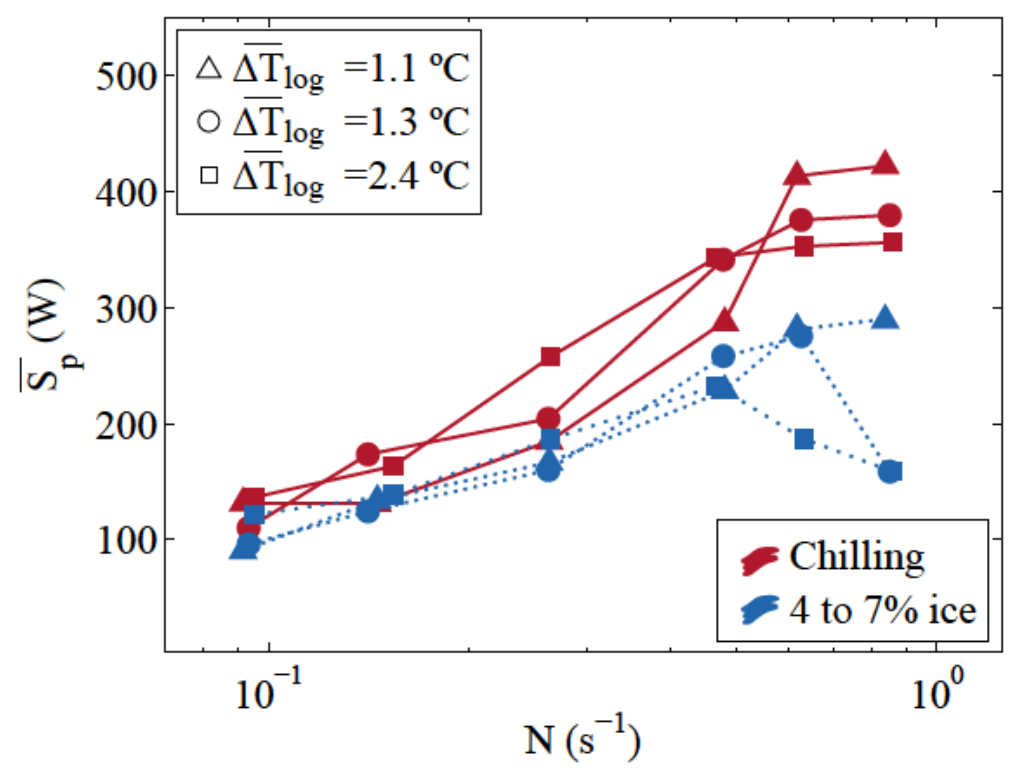

Figure 4.20: Averaged scraping power comparative for different scraping velocities and $\overline{\Delta T}_{\log }$ values before and after the nucleation onset

ice layer cannot be considered as a sign of malfunctioning of the SSPHE. Conversely, if it is small enough to avoid sensible effects on the heat transfer and $F_{S}$, its presence reduces significantly the power consumption in the scraper motor and enlarges the life time of the scraper blades, being less affected by the erosion.

Finally, a wall subcooling increase (higher $\overline{\Delta T}_{\text {log }}$ values in Fig. 4.19) implies an increase in power consumption during the freezing period, which is specially noticeable for low scraping velocities where the ice layer has more time between scraper actions to grow. However, this influence is less evident when the scraping velocity is high as the interval between scrapers action is shorter.

Time-averaged values Time-resolved values are valuable to understand the process individually. However, when comparing different working conditions, the time-averaged values can provide an overall view that allows a better comparison between them. In that way, Fig. 4.20 shows a comparative of averaged values for the power consumption before and after the nucleation onset, for and ice content range between 4 and $7 \%$. Regardless of the mean logarithmic temperature values, the power before nucleation (wet conditions) is always higher than the power consumed during ice production, as pointed out by Fig. 4.18 .

Fig. 4.21 depicts the averaged values (1 to $20 \%$ of ice cont.) and their standard deviation for different logarithmic mean temperature differences and scraping velocities. It also includes the corresponding averaged $\overline{\Delta \mathrm{T}}_{\log }$ values with their standard deviation area. For the lowest $\overline{\Delta T}_{\log }$ value the power is always increasing with $\mathrm{N}$. This trend is somehow logical, as scraping at higher velocities will imply more power demand. However, if the wall supercooling degree is increased (higher $\overline{\Delta T}_{\log }$ values) the power consumption increases until $\mathrm{N} \approx 0.55 \mathrm{~s}^{-1}$; after that value it decreases with rotating velocities. 


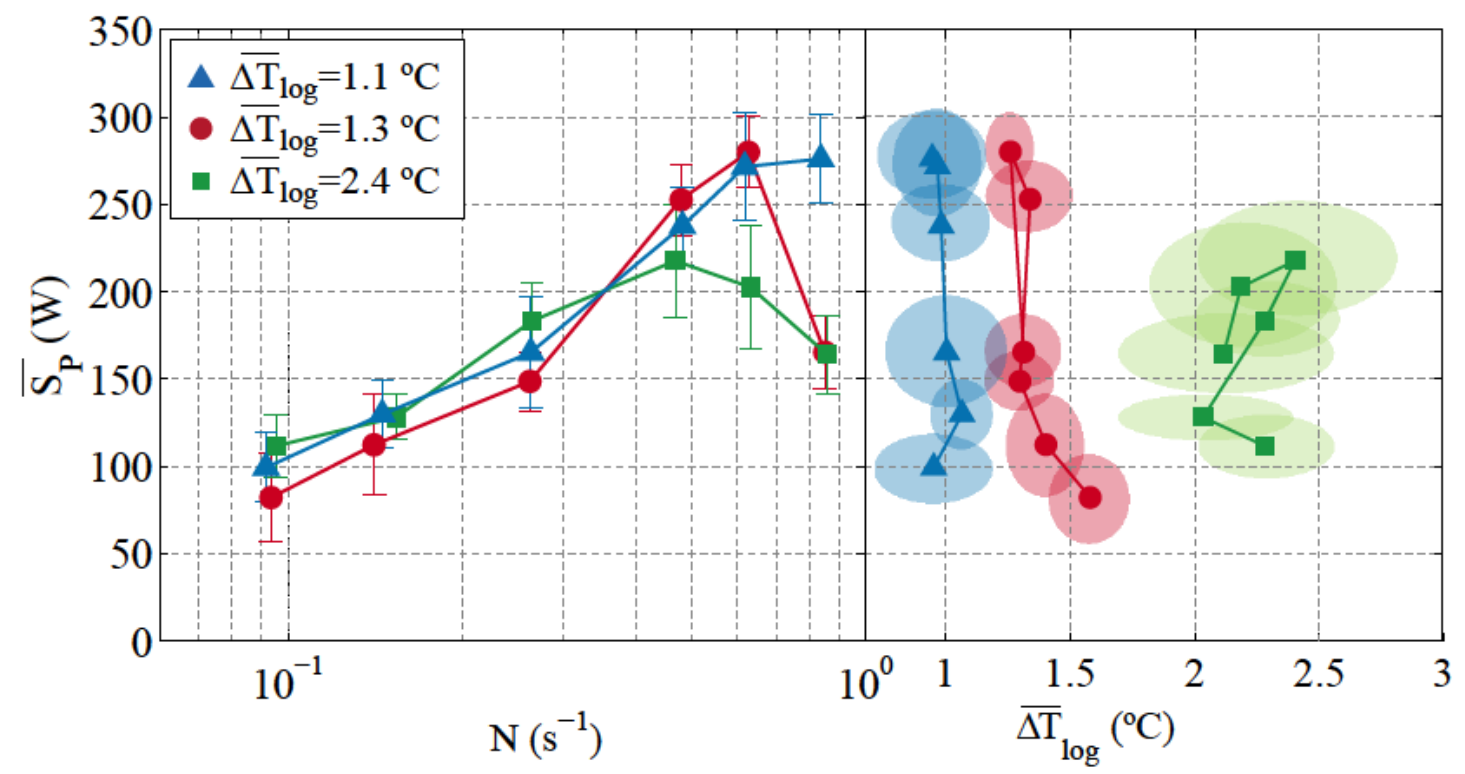

Figure 4.21: Averaged scraping power during the freezing period (ice content from 1 to $20 \%$ ) with their respective mean logarithmic temperatures for different scraping velocities

By applying Eq. 7.4, the averaged torque values can be obtained from the averaged power values. Figure 4.23 shows the torque averaged values during the chilling period and during the freezing period ( $4 \%$ to $7 \%$ of ice cont.). As expected, the torque values during freezing are lower that during chilling. In both cases the torque decreases with increasing values of the scraping velocity. This trend indicates that the friction between scrapers and surface is in a partial fluid lubrication regime (see Fig. 4.16): the brine is brought into the scraping region through the motion of the scrapers, creating a film due to the viscosity of the fluid and acting as a lubricant. As the velocity increases the fluid film thickness increases, reducing the solid-solid contact area and providing a lower shear strength that results in lower friction coefficient values.

The torque results while freezing follow a similar trend, decreasing while the velocity increases, but with lower values as was expected from the observation in Fig. 4.18. The ice is acting as an additional lubricant and therefore the torque decreases, superimposed to the decreasing trend corresponding to the partial fluid lubrication regime. Furthermore, for the higher wall supercooling degrees $\left(\overline{\Delta \mathrm{T}}_{\log }=1.3\right.$ and $\left.2.4{ }^{\circ} \mathrm{C}\right)$ in freezing conditions it is possible to observe that the torque values deviates from the trend for $\mathrm{N} \geq 0.5 \mathrm{~s}^{-1}$, corresponding with the power decrease observed in Fig. 4.21. A dashed line is plotted in Fig. 4.22 as a guide to the eye. This deviation can be related with the formation of a thick and more consistent ice layer compared with the previous mushy structure, acting as an ice skating rink. This layer is promoted by two factors: the higher wall supercooling degrees and the higher mixing of ice particles on the flow due to the higher velocities. This enhanced mixing leads to more impact of particles on the subcooled surface, which favours the ice layer formation. As the ice layer thickness increases, the contact between scraper and steel asperities is more and more reduced. In a situation where this contact is completely avoided by the ice scaling, higher scrap- 


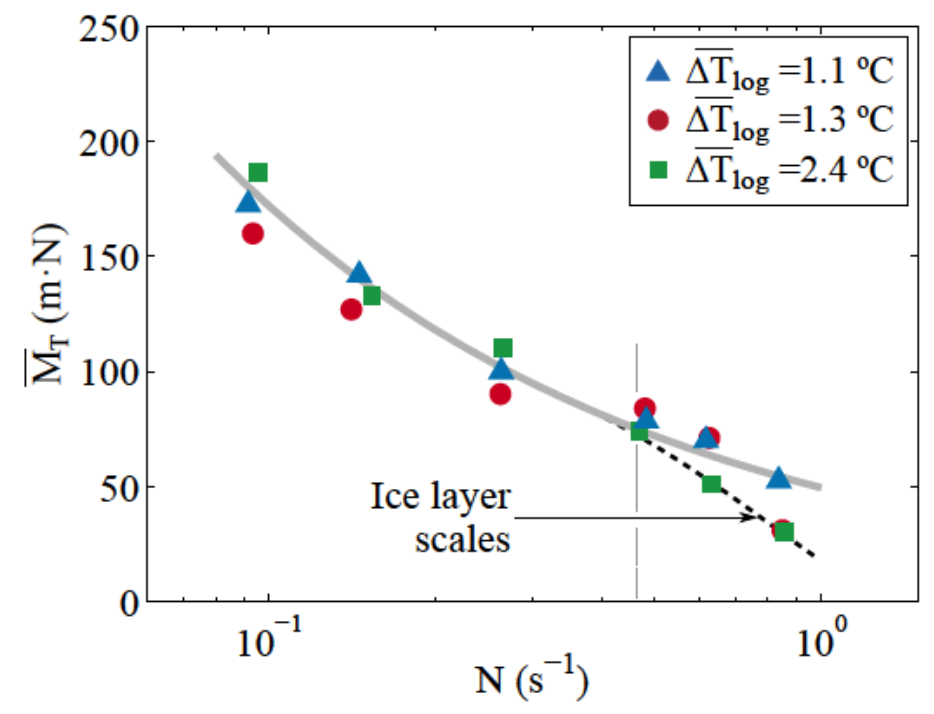

Figure 4.23: Averaged torque comparative for $\Delta \mathrm{T}=1.1{ }^{\circ} \mathrm{C}, \Delta \mathrm{T}=1.3^{\circ} \mathrm{C}$ and $\Delta \mathrm{T}=2.4$ ${ }^{\circ} \mathrm{C}$ at different scraping velocities. The dashed line is plotted as a guide to the eye

ing velocities will imply a thicker fluid layer between scrapers and ice (partial fluid lubrication regime) and according to to Fig. 4.17, for the regimes I and II, the friction coefficient will decrease.

The torque averaged during all the freezing period ( 1 to $20 \%$ of ice) is shown in Fig. 4.23. Aside from the previously noticed effect of the scraping velocity, it can be seen that the wall supercooling effect has no a clear influence on the torque for $\mathrm{N}$ values lower than $0.5 \mathrm{~s}^{-1}$. For $\mathrm{N}_{\mathrm{i}} 0.5$ values, the deviation of the torque for $\overline{\Delta \mathrm{T}}_{\mathrm{log}}=1.3$ and $2.4{ }^{\circ} \mathrm{C}$ is observed, according to the explanations above. As this behaviour is explained by the ice layer scaling, it shall have an impact in the heat flux through the plate. Recalling Fig. 4.9, it is possible to observe that the heat flux increases with the rotating velocity

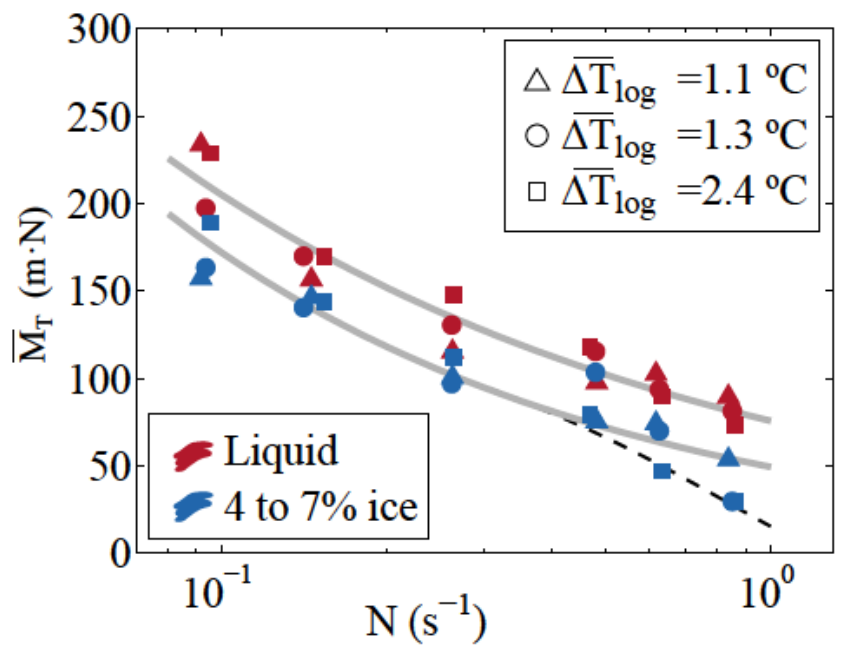

Figure 4.22: Averaged torque power comparative for different $\overline{\Delta T}_{\log }$ and $\mathrm{N}$ values $\mathrm{N} \approx 0.5 \mathrm{~s}^{-1}$. After that point, the heat flux keeps constant or even starts to decrease with further increments of the scraping velocity. The decrease in the heat flux can be then associated with the formation of an ice layer, fact also pointed out by the stagnation of the heat transfer coefficient in Fig. 4.10.

The Power number was calculated according to Eq. 4.6 by replacing $P$ by $S_{P}$. The results are plotted in Fig. 4.24 for the averaged values of the chilling period and the freezing period with different ice content (3 to $6 \%$ and 10 to $13 \%$ ), at different 
scraping velocities and $\bar{\Delta} \mathrm{T}_{\log }$ values. The first thing to be deduced is the absence of a unique relationship between the Power number and the Reynolds number. Ice content and wall supercooling play also a role. The ice content effect on the slurry fluidity is included in $\mathrm{Re}_{\mathrm{rot}}$ through the variations on viscosity and density. However, the different curves obtained at different ice content values point out the fact that the power consumption is not -at least mainly-dominated by the hydrodynamics. If that were the case, changes in the ice content will move along the same line as them will only imply changes on density and viscosity of the slurry. The different correspond to the influence of the ice content on the ice layer over the surface, highlighting that the major power consumption mechanism is the friction. Looking at Fig. 4.24, for equal or approximate $\operatorname{Re}_{\text {rot }}$ values, the Power number decreases when the ice content increases. This is due to the effect of the ice content on the ice layer scaling, which in turn reduces the friction.

The power number values for the chilling period can be fitted to an equation of the form

$$
P o=\mathrm{A} R e_{\text {rot }}^{\mathrm{B}},
$$

similar to Eq. 4.11. Whereas $\mathrm{B}=-2.44, \mathrm{~A}$ is of the order of $10^{14}$, which indicates that the friction is much higher than the hydrodynamic inertia. The high Power number values, $\mathrm{Po}>>1$, also highlight that power consumption in much more higher in this case than in the case of stirrers.

Based on Eq. 4.14 a new expression was proposed to predict the Power number for the different ice content concentrations,

$$
\stackrel{\text { Chilling }}{P o=\mathrm{ARe} e_{\text {rot }}^{\mathrm{B}}} \rightarrow \stackrel{\text { Freezing }}{\mathrm{Po}=\left(\mathrm{C} \varphi_{m}^{-1}\right) \mathrm{A} R e_{r o t}^{\mathrm{B}}}
$$

where $\mathrm{A}=1.032 \cdot 10^{14}, \mathrm{~B}=-2.48$ and $\mathrm{C}=3 / 4$. Equation 4.15 works very well for low-tomedium ice concentration values. Higher concentration values (i.e. $\varphi_{m}>17 \%$ fall out of this trend).

\subsubsection{Rigid scraper system results}

The results obtained for the rigid scraper system reproduce the same phenomena reported for the adaptable system. Figure 4.25 shows the averaged values during the whole freezing period. Compared with Fig. 4.22, power values for the rigid system are, in general, higher. This fact seems contradictory: since in the case of rigid scrapers the contact between scraper and surface is poor and the normal force of the scrapers is not guaranteed by anything, power consumption should be lower. The higher values are explained by the concept depicted in Fig. 4.15. The deformation of the plate pushes the scrapers in the central area. As they have not any mechanism to adapt to this deformation, the normal force in this small region is very high, leading then to high friction forces. The adaptable scrapers can counteract this plate deformation with the compression of the springs. Even if the normal force will increase as the spring is more compressed, it will be always lower than if the scrapers would not be able to displace vertically. 


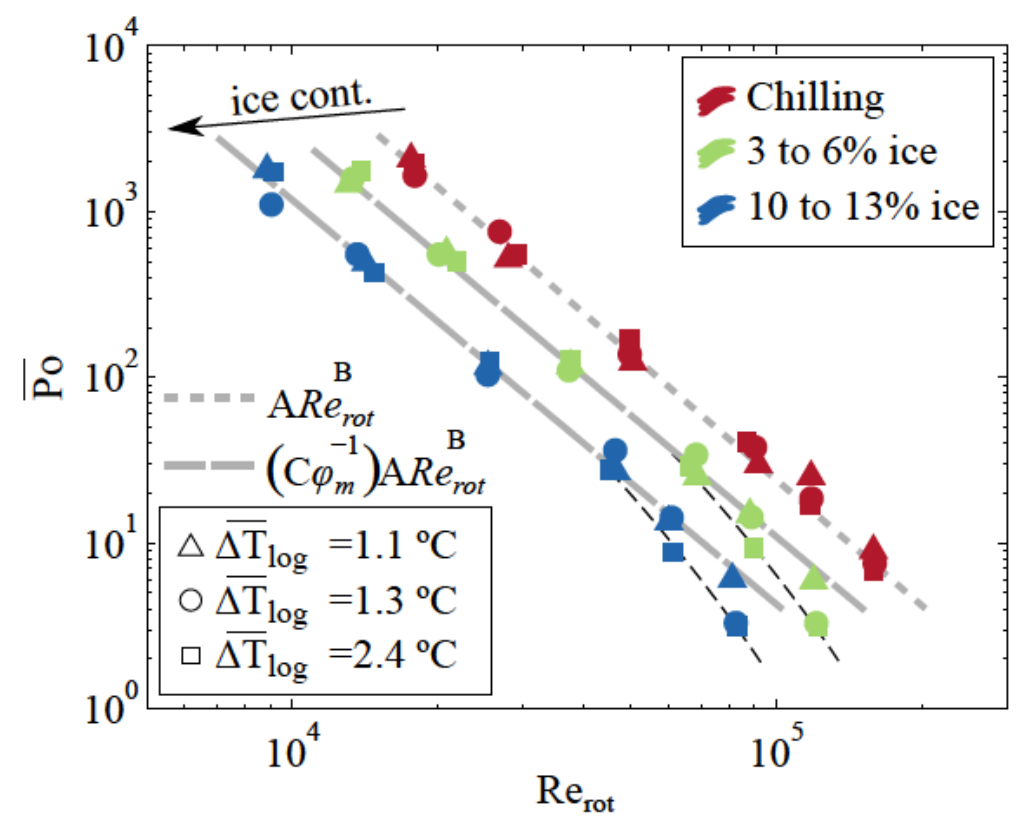

Figure 4.24: Averaged power number vs. averaged $R e_{\text {rot }}$ number comparative for different values of $\overline{\Delta T}_{\log }$ and ice concentrations.

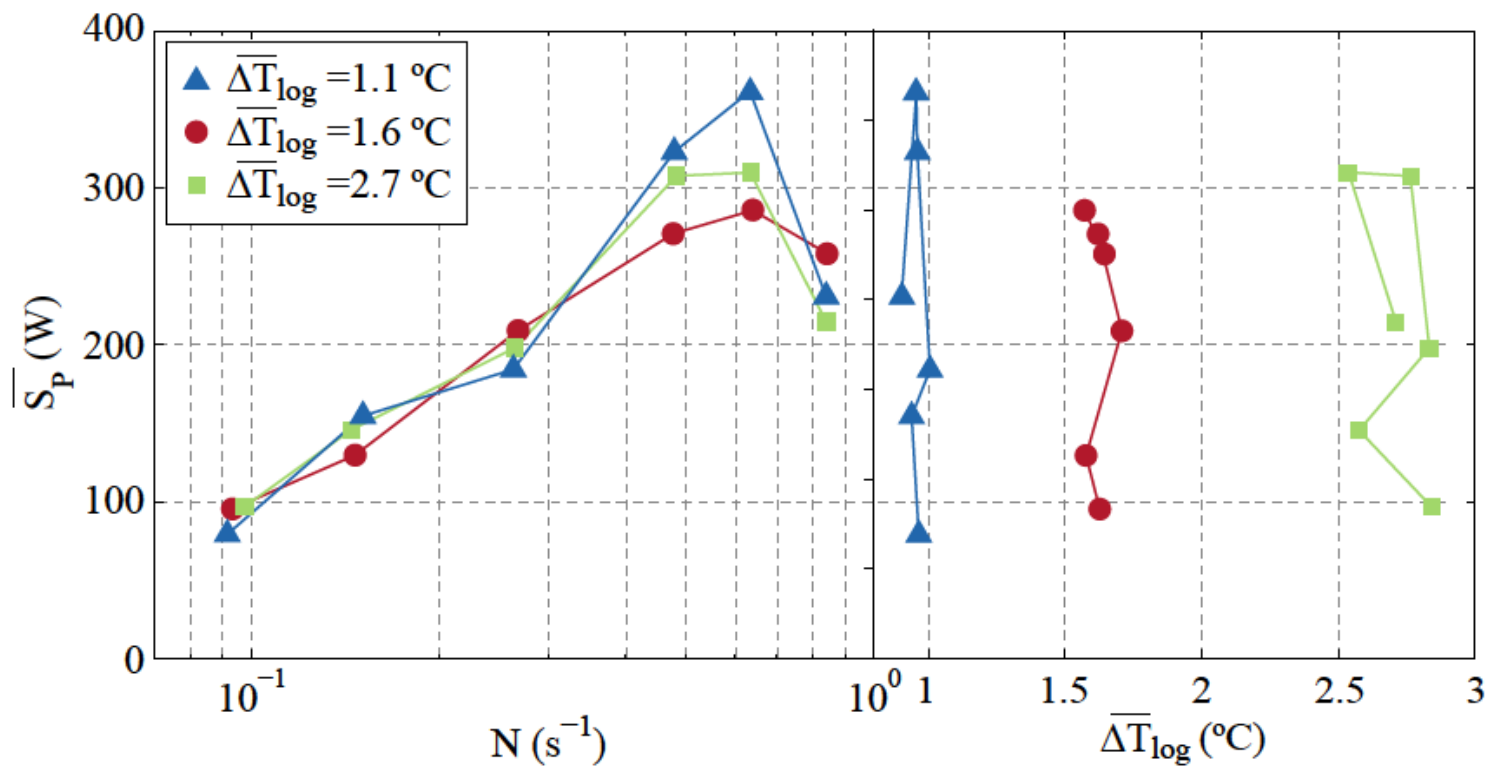

Figure 4.25: Averaged scraping power during the freezing period (ice content from 1 to $20 \%$ ) for different scraping velocities and averaged mean logarithmic temperatures: rigid scraper system 


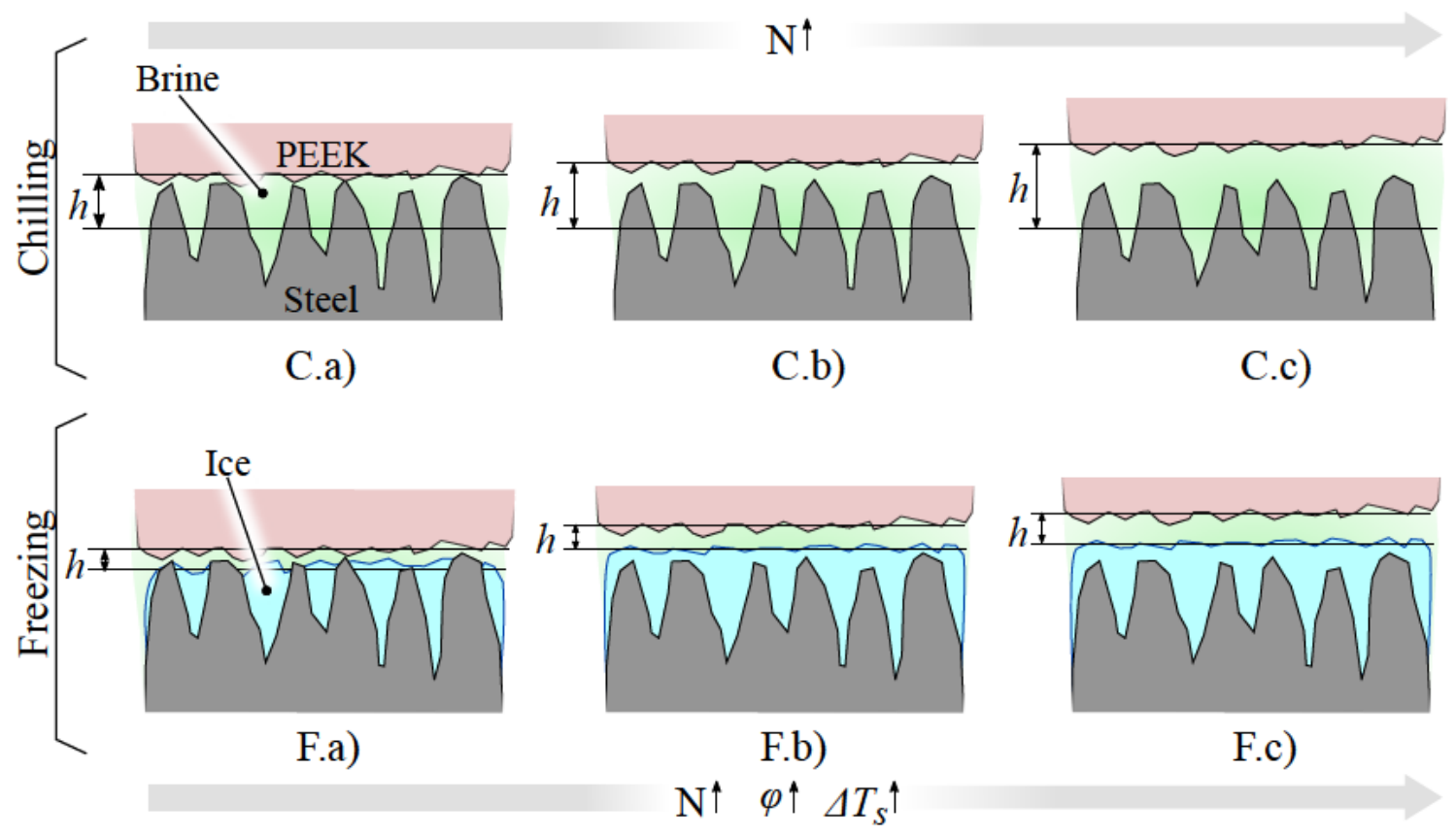

Figure 4.26: Sketch of the friction process in the SSHPE. a) Chilling b) Freezing at low $\overline{\Delta \mathrm{T}}_{\log }$ values c) Freezing at high $\overline{\Delta \mathrm{T}}_{\log }$ values

\subsection{Friction mechanism on the SSPHE}

Based on what has been shown and described in the previous sections, the friction phenomena inside the SSPHE can be summarized depending on the process conditions. A sketch is included in Fig. 4.26.

During the chilling stage, the friction depends only on the scraping velocity $\mathrm{N}$ (Fig. 4.26C.a to Fig. 4.26C.c). The brine acts as a lubricant but the normal force is high enough to ensure a solid-solid contact between scrapers and plate. As $\mathrm{N}$ increases the brine is brought into the scraping are creating thicker layers that reduce the friction (partial fluid lubrification). Therefore, higher scraping velocities reduce the contact between PEEK-steel asperities, but also reduce the scraping effectiveness.

During the freezing stage $(4.26 \mathrm{~F})$ ice appears on the surface. At low velocities the PEEK-steel contact area is high, reducing its presence to the roughness gaps of the plate (Fig.4.26F.a). Ice acts as a surface smoother, reducing the friction by reducing the direct contact between scrapers and plate. As the scraping velocity increases the brine layer separates both surfaces, allowing the ice to grow and reducing the PEEK-steel contact (Fig. 4.26F.b-F.c). Both the ice content in the slurry and the wall subcooling will promote the ice scaling. The increased lubrification for higher rotating velocities (partial fluid lubrification regime) together with the ice layer reduce considerably the friction and therefore the power consumption. 


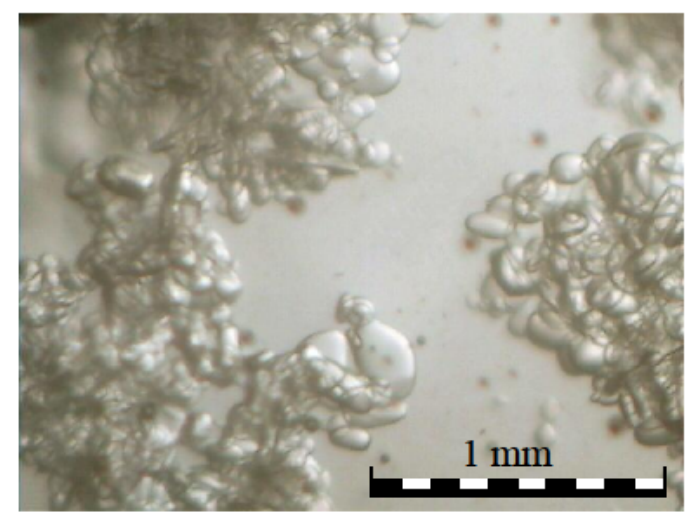

(a) $\overline{\Delta \mathrm{T}}_{\log }=1.1^{\circ} \mathrm{C}, \mathrm{N}=0.08 \mathrm{~s}^{-1}$

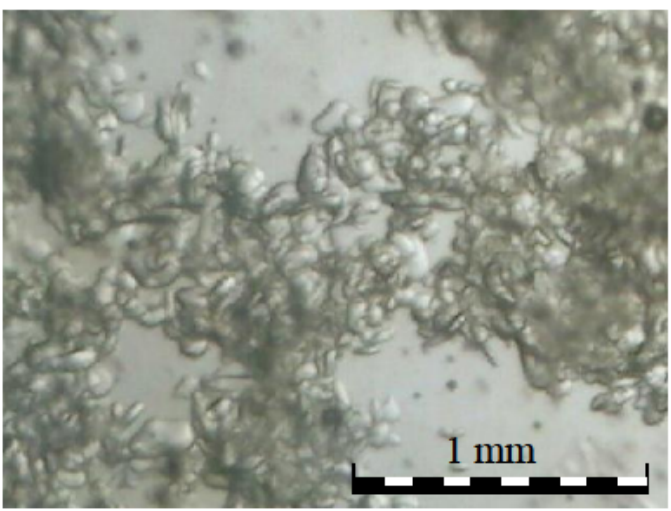

(c) $\overline{\Delta \mathrm{T}}_{\log }=2.4^{\circ} \mathrm{C}, \mathrm{N}=0.08 \mathrm{~s}^{-1}$

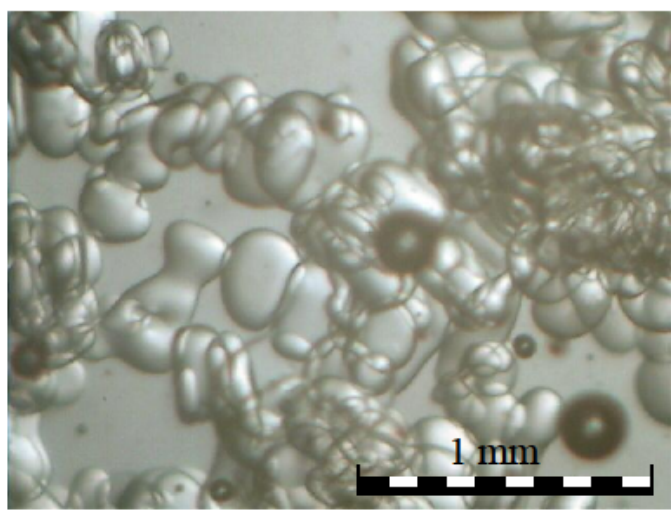

(b) $\overline{\Delta \mathrm{T}}_{\log }=1.1^{\circ} \mathrm{C}, \mathrm{N}=0.83 \mathrm{~s}^{-1}$

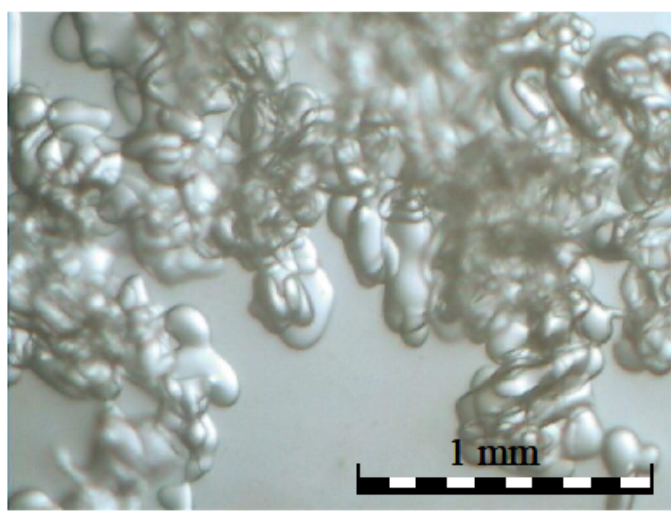

(d) $\overline{\Delta \mathrm{T}}_{\log }=2.4^{\circ} \mathrm{C}, \mathrm{N}=0.83 \mathrm{~s}^{-1}$

Figure 4.27: Ice crystals images taken with microscope at the end of each test (ice content $\approx 20 \%$ )

\subsection{Ice crystals morphology}

The morphology of the ice crystals was analysed by a microscope, making the slurry flow through a visualization section (see sec. 2.1.2). The ice crystal images where taken at the end of each test $(\approx 20 \%$ of ice content) to avoid any perturbation inside the SSPHE during the experiment -as the passage of the slurry through the visualization circuit could induce changes of its temperature due to heat losses. To investigate the evolution of the ice crystal during the test, special experiments were done for this purpose, where image of the crystals were taken at different ice concentrations inside the SSPHE.

Figure 4.27 show the obtained images for the slurry at the end of the test with different operating conditions. Going from lower to higher scraping velocities (Fig. $4.28 \mathrm{a} \rightarrow 4.28 \mathrm{~b}$ and Fig. $4.28 \mathrm{c} \rightarrow 4.28 \mathrm{~d}$ ) leads to higher and rounder ice crystals. This is explained by the fact that at higher velocities the mixing is also high and the crystals are in constant movement. Therefore the continuous impacts against the scraper arms, the wall and among themselves make them round by erosion. Moreover, the particles tend to aggregate when they impact with others, forming larger particles. The obtained ice crystals have an average diameter between $\approx 200 \mu \mathrm{m}$ and $\approx 300 \mu \mathrm{m}$.

On the other hand, when moving from lower to higher mean logarithmic temper- 


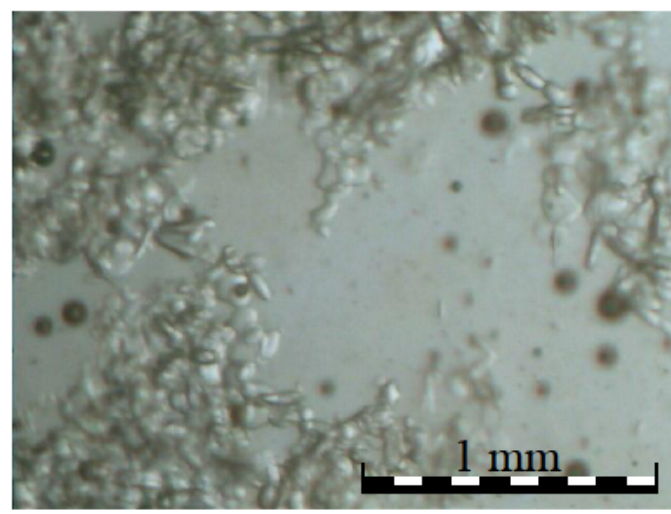

(a) $\varphi=6 \%$

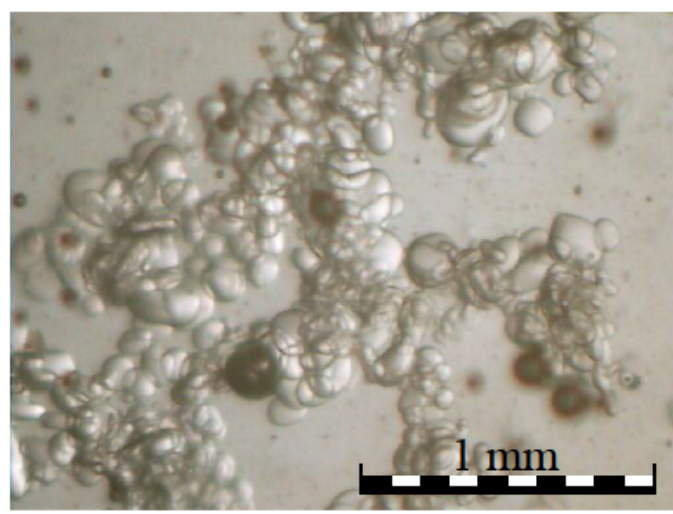

(c) $\varphi=14 \%$

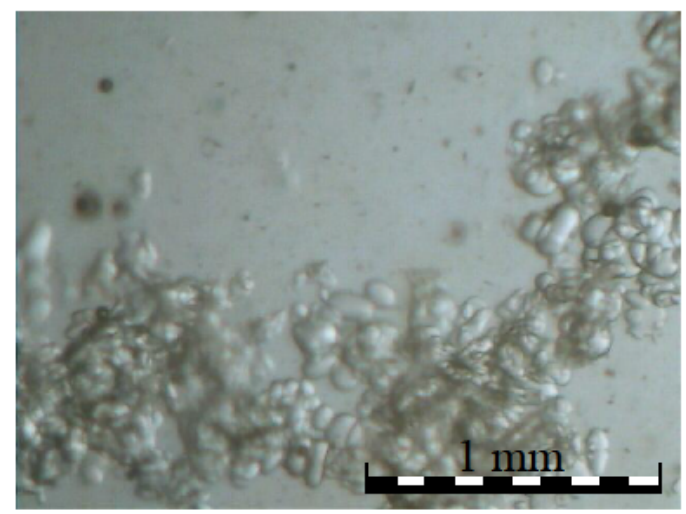

(b) $\varphi=10 \%$

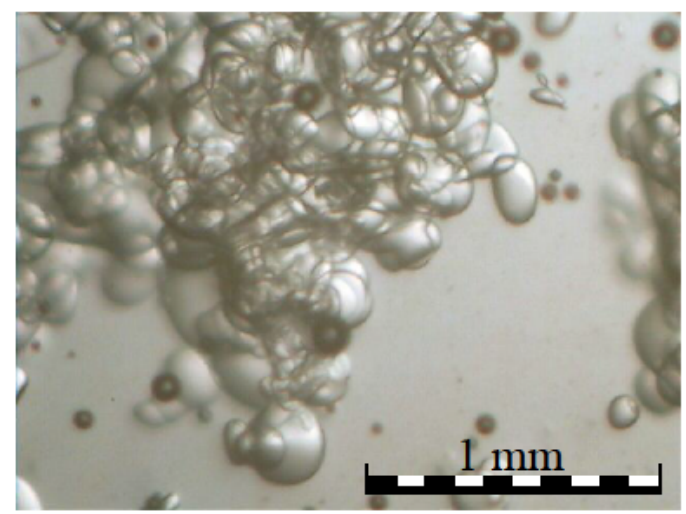

(d) $\varphi=18 \%$

Figure 4.28: Ice crystals images taken with microscope at different ice concentrations for $\overline{\Delta \mathrm{T}}_{\log }=1.1^{\circ} \mathrm{C}$ and $\mathrm{N}=0.46 \mathrm{~s}^{-1}$

atures (wall subcooling) the effect on the ice crystals is less noticeable -even if the crystals for Fig. 4.28a are slightly higher and rounded than those of Fig. 4.28c. The higher $\overline{\Delta \mathrm{T}}_{\log }$ value of Fig. 4.28c increases the heat flux and reduces the test duration. Therefore the ice crystals have less time to grow once they are scraped from the surface, being smaller and sharper. In contrast, the longer test duration of Fig. 4.28a allow them to experience small growing and erosion. The obtained ice crystals have an average value of $\approx 100 \mu \mathrm{m}$.

The degree of easiness with which the slurry was extracted by aspiration towards the visualization section revealed also information about the slurry, even if not quantifiable measured. Thus, at low scraping velocities $\left(\mathrm{N} \leq 0.25 \mathrm{~s}^{-1}\right)$ the ice particles cumulated in the top by buoyancy form a consistent layer with a more compact structure, assembling an ice-cake. This was confirmed by unbolting the top window of the SSPHE. The distribution of ice/brine in the slurry was not uniform, being more fluid in the bottom than in the top, where ice was almost "dry". As the visualization circuit inlet is placed on the side, at the higher location, this caused the pump to block often.

On the other hand, for higher scraping velocities the slurry is better mixed and the ice/brine distribution is more homogeneous in the slurry. Therefore the pump was 
easily able to drive the slurry into the visualization circuit, never getting blocked.

Finally, Fig. 4.28 includes images of the ice at different contents (equivalent to time time instants of a test). For low content (early stages of the freezing period) ice crystals are small (Fig. 4.28a): they have been recently pulled off from the surface and as consequence they have not ad time to collide and coalesce among each other, having sharp edges. Increasing the ice content/testing time the crystals become bigger and rounded, adopting the shape of a rice grain (Figs. 4.28b-4.28c) that becomes bigger and spherical as the ice content increases. The last image, Fig. 4.28d, corresponding to an ice content of $18 \%$ shows crystals very similar to those obtained at the end of the test for $\varphi=20 \%$, with round crystals of $\approx 20 \mu \mathrm{m}$. 


\subsection{Conclusions}

This chapter analysed the principal physical phenomena involved in the ice production with the SSPHE in batch mode operation. Heat transfer, scraping power consumption and ice crystal characteristics were studied by using a $7 \mathrm{wt} . \% \mathrm{NaCl}$ brine as a base solution for the ice slurry generation. Two different scraper types, rigid and surface adaptable, were compared for different rotating velocities $\left(0.1 \mathrm{~s}^{-1}\right.$ to $\left.0.8 \mathrm{~s}^{-1}\right)$ and coolant temperatures $\left(\overline{\Delta \mathrm{T}}_{\log }\right.$ from $0.5{ }^{\circ} \mathrm{C}$ to $\left.2.4{ }^{\circ} \mathrm{C}\right)$. The major findings are:

- The location of the initial nucleation in the SSPHE was found to depend on the Richardson number. The effects of buoyancy, supercooling degree and scraperarms induced velocities interact leading to an initial localized nucleation over the subcooled plate $(R i>0.02)$ or to a global fluid volume nucleation $(R i<0.02)$. The detected supercooling amplitude was found to be a good indicator of the fluid mixing inside the SSPHE upper side.

- The configurations with adaptable scrapers shown a better performance in the ice slurry generation and heat transfer, reducing the unscraped regions and avoiding ice layer scaling. Augmentations on the heat transfer coefficient of around two times were found with respect to the rigid scraper. The rotating velocity of the scrapers was found to have a low effect on the heat transfer, influenced by the supercooling degree of the surface. Maximum averaged heat fluxes of 4100 $\mathrm{W} / \mathrm{m}^{2}$ and averaged heat transfer coefficients of $4000 \mathrm{~W} / \mathrm{m}^{2} \mathrm{~K}$ were found for the adaptable scrapers with surface supercooling degrees of $\approx 0.5{ }^{\circ} \mathrm{C}$ and scraping rotating velocities of $\mathrm{N} \approx 0.46 \mathrm{~s}^{-1}$.

- Heat transfer results were non-dimensionalized by means of the Nusselt and rotating Reynolds numbers. Nusselt number for low supercooling degree values adopted the shape of the penetration theory for low $R e_{\text {rot }}$ values, whereas at high values $N u$ becomes almost independent of $R e_{\text {rot }}(N u=550)$, mostly due to the impact of the particles over the surface at higher mixing rates. Higher surface supercooling degrees led to a constant ice layer that reduced the $N u$ values to $\approx 350$. A correlation was proposed for a supercooling degree of $0.75{ }^{\circ} \mathrm{C}$ composed by the penetration theory plus a constant therm for $R e_{\text {rot }}<4.7 \cdot 10^{4}$ and a correlation based on $\operatorname{Re}_{\text {rot }}$ and $\mathrm{Fr} \cdot \varphi_{m}$ for $\operatorname{Re}_{\text {rot }}>4.7 \cdot 10^{4}$, with global $\mathrm{R}^{2}=0.87$.

- The power consumption during the chilling and freezing stages showed to be mostly related with the friction between scrapers and plate. Shaft torque was found to decrease when scraping velocity increases as consequence of the formation of a brine-lubricating layer. The onset of nucleation resulted always in important reductions of power consumption (up to 50\%) as a consequence of the appearance of ice over the surface, acting as a lubricant. Ice content increases lead to power increments as the equivalent viscosity of the slurry increased. However this effect was much lower than the friction between scrapers and plate.

- Power consumption values were non-dimensionalyzed by the Power number. The high values of $P o$ obtained revealed the nature of the process, governed by the friction. For equal $R e_{\text {rot }}$ values $P o$ was smaller as the ice content was higher 
revealing that the larger number of particles affect the ice layer over the steel surface, reducing therefore the friction.

- Higher rotating scraping velocities did not proved to be helpful in inhibiting the development of an ice fouling layer, as the higher impact of ice particles over the surface plus the higher liquid layer under the scraper promote it. On the contrary, higher rotating velocities aided to preserve a good mixing level in the slurry, keeping it homogeneous and avoiding the cumulation of ice in the top of the SSPHE. The high scraping velocity may result in heating effects, which would be undesirable. As a consequence the rotating velocity has to be balanced with the economy of the power consumption.

- The morphology of the ice crystals varied with the scraping velocity, wall subcooling and time instant of the test. Higher scraping velocity leaded to higher and rounded particles $(200-300 \mu \mathrm{m})$ as a consequence of the continuous impacts that make them fuse between each other and grow. Higher wall subcoling values reduced the test duration resulting in smaller particles. At the initial test stages (low ice content) stages particles are small $(10 \mu \mathrm{m})$ and sharp, as they are just pulled off. With time they grow in rounder shapes. 


\section{Chapter 5}

\section{Performance evaluation for different $\mathrm{NaCl}$ concentrations}

In this chapter the results of heat transfer, power consumption and ice crystals morphology obtained for different $\mathrm{NaCl}$ concentrations are presented and discussed. The ice slurry production phenomena was analysed in the previous chapter regarding concepts as heat transfer, power consumption and ice crystals morphology, being the initial $\mathrm{NaCl}$ content, $\omega_{0}$, always equal to $7.1 \%$.

However, the salt concentration can influence the process in different ways. The hardness of the ice layer is expected to decrease with the increasing salt content, as it is the responsible of the mushy structure formed over the subcooled surface. This in turn can be related with the scraping power and the heat transfer coefficient. On the other hand it has also an impact on the ice slurry usability, as the lower temperature provides a higher coolant capacity.

Aside from possible beneficial aspects, an increase in salt content may involve some negative ones, like the increase in brine viscosity (higher viscous dissipation), the higher corrosive effect on the equipment and the necessary lower temperatures of the coolant and refrigerant (see ch. 2). The descent in the evaporation temperature of the primary coolant can in turn lead to a lower process efficiency.

The aim of this chapter is, considering the above mentioned effects, to elucidate whether the salt concentration has a significant effect or not in the production of ice slurry in the SSPHE. In section 5.1 the effect of the $\mathrm{NaCl}$ concentration in the freezing point is introduced, reporting the results of the temperature evolution during the different tests. In section 5.2 the heat transfer inside the SSPHE is analysed considering five different initial $\mathrm{NaCl}$ concentrations, whereas in section 5.3 a similar analysis is done considering the effect in the scraping power consumption. Section 5.4 includes an study of the performance considering both only the SSPHE - sec. 5.4.1- and the complete facility -sec. 5.4.2. Finally, section 5.5 reports a comparison of the ice crystal images for different initial salt concentration values.

\subsection{Salt concentration and freezing point}

An increment in salt concentration results in a decrease of the freezing point. Figure 5.1 depicts the experimentally observed freezing point for the tested cases together 


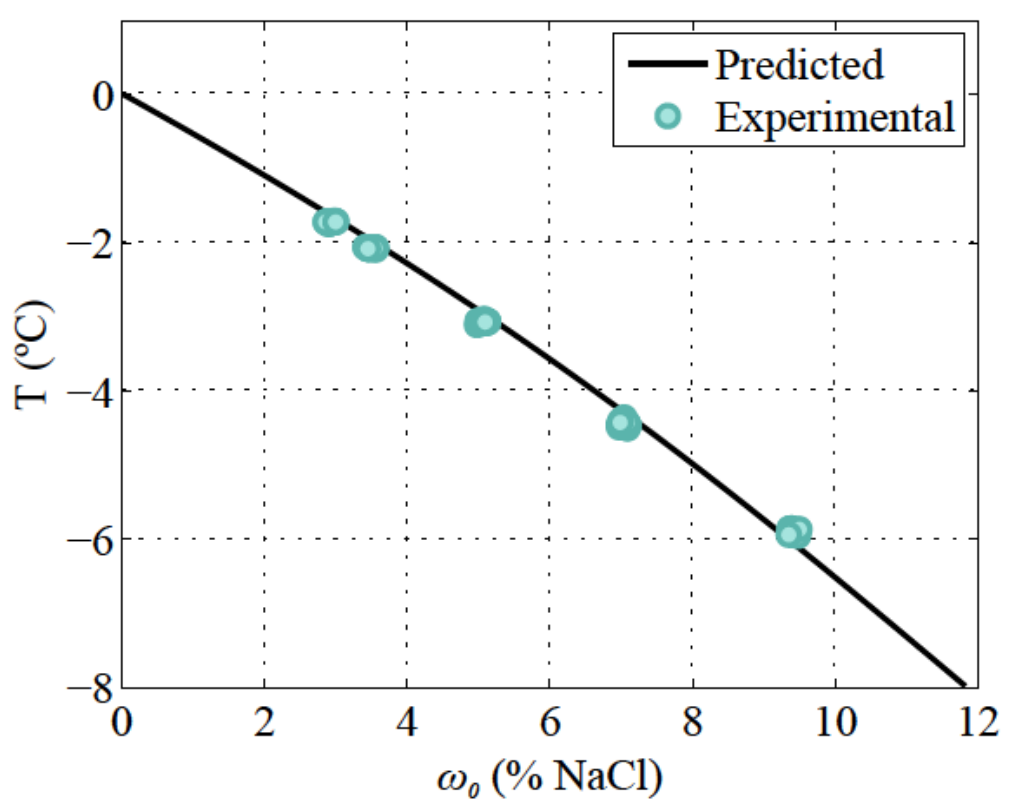

Figure 5.1: Observed vs. predicted freezing point for different $\omega_{0}$ tested values

with the curve predicted by Eq. 2.1. In general, a good agreement was found between expected and detected values, which validates not only the measurement of the $\mathrm{NaCl}$ concentration but also the brine bulk temperature measurement and the freezing point detection technique.

For the $\omega_{0}$ tested values, the freezing temperatures ranged from -2.9 to $-5.9{ }^{\circ} \mathrm{C}$. Figure 5.2 shows the temperature evolution of the brine along the test duration for different $\omega_{0}$ values (scraping velocity $\mathrm{N}=0.62 \mathrm{~s}^{-1}$ ). As it is possible to see the final slurry temperature for $20 \%$ of ice content arrives to $\approx-7{ }^{\circ} \mathrm{C}$, with $\omega_{0}=9.4 \%$.

The starting temperature of the test was decreased according to the increasing $\mathrm{NaCl}$ content, as it is possible to see in Fig. 5.2 for $t=0 \mathrm{~min}$. This was done in order to reduce the required cooling power, as in the chilling process the high heat fluxes may temporary overpass the coolant capacity of the system and exhaust the inertia of the damping tank. The cooling system was therefore used to cool down the brine till the starting temperature of the test. Once reached, the coolant circulation through the SSPHE was stopped -as the SSPHE was thermally insulated the temperature inside remained constant-in order to cool down the temperature of the coolant in the damping tank. The test started once the temperature of the coolant decreased to the desired value.

\subsection{Heat transfer}

To study the effect of the $\mathrm{NaCl}$ concentration on the heat transfer during the ice slurry production in the SSPHE, a constant logarithmic mean temperature value $\overline{\Delta \mathrm{T}}_{\log } \approx 1.3$ ${ }^{\circ} \mathrm{C}$ and three different rotating velocities $\left(0.1 \mathrm{~s}^{-1}, 0.27 \mathrm{~s}^{-1}\right.$ and $\left.0.63 \mathrm{~s}^{-1}\right)$ were chosen. The heat fluxes depicted in Fig. 5.3 are the averaged values $\overline{\mathrm{Q}}$ between the $2 \%$ and $8 \%$ of ice content instants in the slurry. According to Fig. 5.3 the heat flux increases as $\omega_{0}$ increases until $7.1 \%$. The increment is almost linear for $\mathrm{N}=0.63 \mathrm{~s}^{-1}$. 


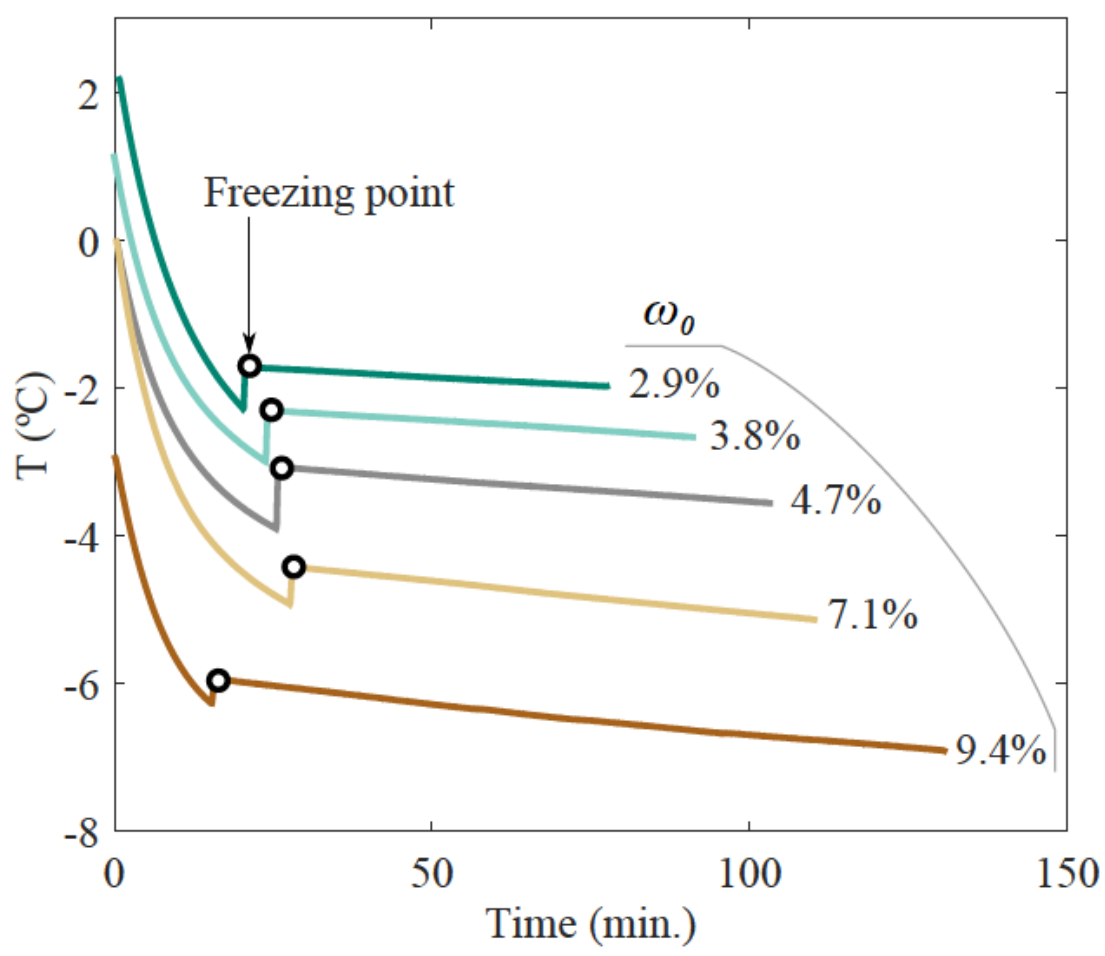

Figure 5.2: Brine/slurry temperature evolution in time during tests for different $\omega_{0}$ values $\left(\mathrm{N}=0.62 \mathrm{~s}^{-1}\right)$

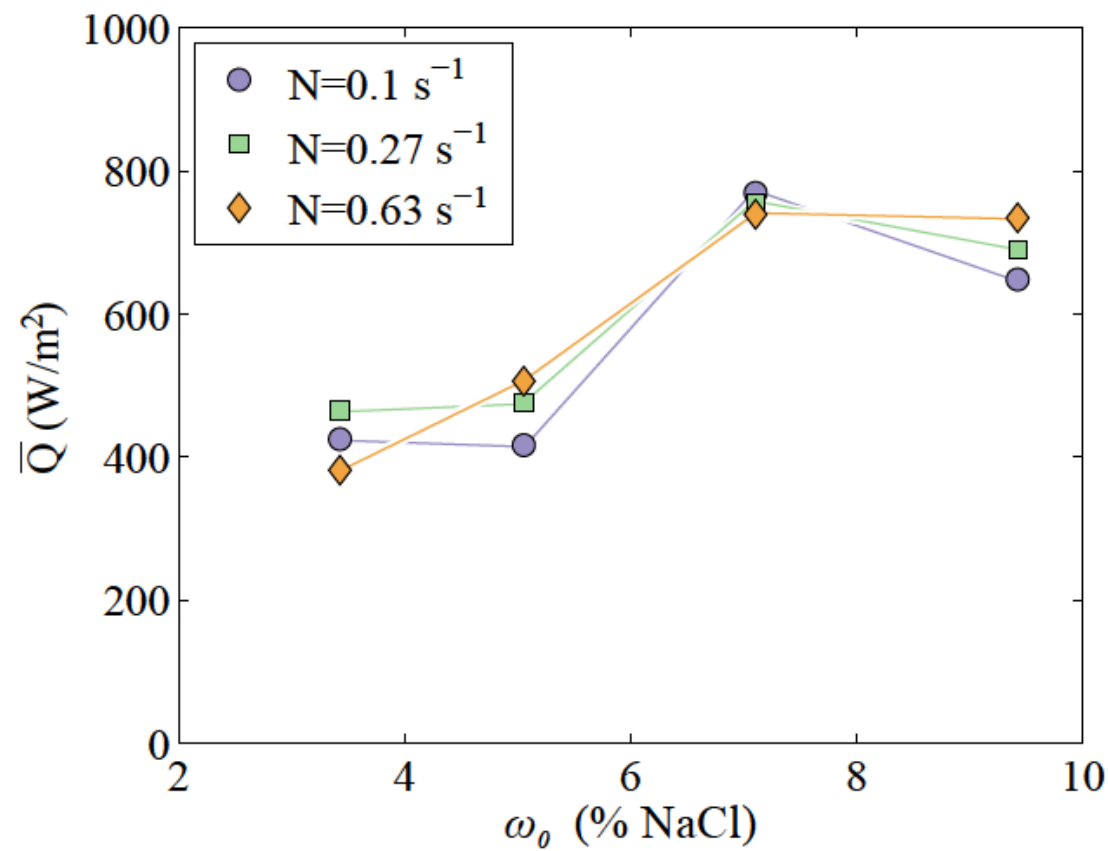

Figure 5.3: Heat flux for different $\omega_{0}$ values and scraping velocities at $\overline{\Delta \mathrm{T}}_{\text {log }} \approx 1.3^{\circ} \mathrm{C}$ 


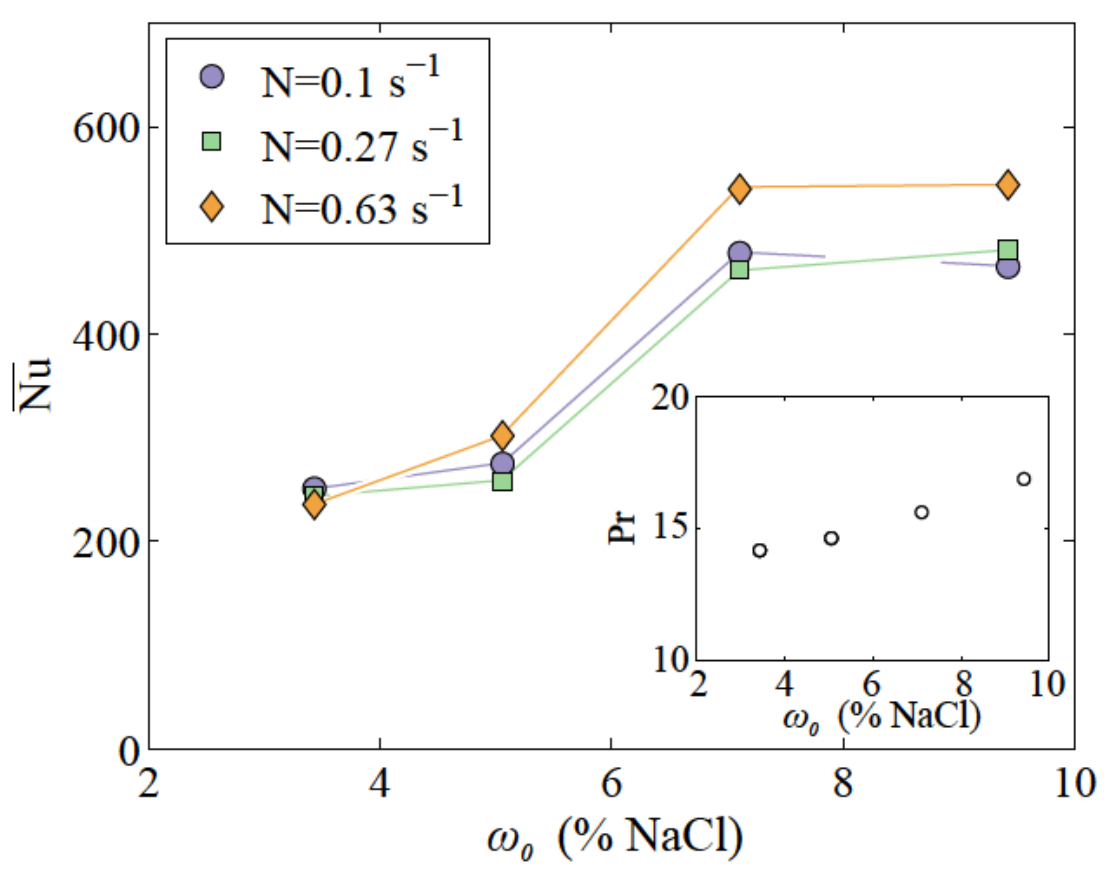

Figure 5.4: Heat transfer coefficient for different $\omega_{0}$ values and scraping velocities at $\overline{\Delta \mathrm{T}}_{\text {log }} \approx 1.3^{\circ} \mathrm{C}$

This heat flux increment can be explained by the fact that higher $\mathrm{NaCl}$ contents produce a less compact mushy structure of the ice layer formed over the plate [89, 92, 93]. As the resistance of the ice layer decreases it becomes easier to be removed by the scrapers, consequently leading higher heat fluxes. The beneficial effect of the salt in lowering the ice layer resistance seems to find a maximum for $\omega_{0}=7.1 \%$. After that value the heat flux stabilizes.

Figure 5.4 shows the averaged Nusselt number values corresponding to the cases shown in Fig. 5.3. Similar to the heat flux values, the Nusselt number increases as the $\mathrm{NaCl}$ concentration increases until $\omega_{0}=7.1 \%$, after that the Nusselt values remain equal. It is possible to observe also a difference between $\mathrm{N}=0.1 \mathrm{~s}^{-1}$ and $\mathrm{N}=0.27 \mathrm{~s}^{-1}$ scraping velocities and the higher value $\mathrm{N}=0.63 \mathrm{~s}^{-1}$, showing the last one a higher value, more evident as the concentration increases. Figure 5.4 also includes the Prandtl values of the brine -liquid phase- in correspondence to the depicted Nusselt values. Due to the effect of the $\mathrm{NaCl}$ in the water, which mostly increases the viscosity and decreases the thermal conductivity, the Prandtl number increases when the $\mathrm{NaCl}$ content increases. As a consequence, the scraping action becomes more effective not only because the mushy layer is weaker, but also because the higher Prandtl number implies thinner thermal boundary layers $[102,103]$ and therefore its removal by the scrapers has a higher heat transfer augmentation effect.

Figures 5.5a and 5.5b show respectively the heat flux and the Nusselt number values for all the performed experiments (ice content from $2 \%$ to $8 \%$ ). In Fig. 5.5a, as expected, higher $\overline{\Delta \mathrm{T}}_{\log }$ values increase the heat flux. However, according to Fig. 5.5b the Nusselt number decreases: the ice layer over the plate becomes thicker and stronger. Both figures also provide a very good map regarding where the employment of each concentration and wall subcooling are physically possible and convenient. For 
instance, the highest $\omega_{0}$ values $-9.4 \%$ and $7.1 \%$ - are only possible for $\overline{\Delta T}_{\log }<3{ }^{\circ} \mathrm{C}$ : the low freezing points require lower coolant temperatures and the facility is operating close to its limit. Still, between $\omega_{0}=9.4 \%$ and $\omega_{0}=7.1 \%$ there is a significant difference: the concentration $\omega_{0}=7.1 \%$ allows to increase the $\overline{\Delta T}_{\log }$ value from $\approx 2{ }^{\circ} \mathrm{C}$ to almost 3 ${ }^{\circ} \mathrm{C}$, with a considerably gain in the achieved heat flux of about $67 \%$. As a counterpart, the Nusselt number decreases in about $23 \%$ since the mixing is not efficient.

For the same $\overline{\Delta T}_{\text {log }}$ values, lower $\omega_{0}$ values yield in general lower heat fluxes and Nusselt numbers, as a consequence of the higher resistance of the ice layer over the plate, more difficult to remove. To obtain the same heat fluxes as for $\omega_{0}=7.1 \%$ at $\overline{\Delta \mathrm{T}}_{\log } \approx 3{ }^{\circ} \mathrm{C}$, lower concentrations have to operate at least at $\overline{\Delta \mathrm{T}}_{\log } \approx 4{ }^{\circ} \mathrm{C}$, decreasing the Nusselt number from 400 to around 200.

Figure $5.5 \mathrm{~b}$ is very representative of the process inside the SSPHE, showing that below $\omega_{0}=7.1 \%$ only maximum Nusselt numbers of around 300 can be reached. Higher $\mathrm{NaCl}$ concentrations can reach higher Nusselt number values as the ice layer becomes mushier, but they need to be operated at lower $\overline{\Delta T}_{\log }$ values due to technical limits of the rig, and this decreases considerably the heat fluxes (ice production) achieved.

The $\omega_{0}=7.1 \%$ case is, according with the exposed results, the optimum one for the ice slurry production in the SSPHE. It provides high heat fluxes without decreasing excessively the Nusselt number -avoiding the formation of thick ice layers and ensuring a good mixing.

\subsection{Power consumption}

The effect of the initial brine $\mathrm{NaCl}$ content in the scraping power consumption has been also studied. The scraping power results for several $\omega_{0}$ values and scraping velocities are included in Fig. 5.6 for $\overline{\Delta \mathrm{T}}_{\text {log }} \approx 1.3{ }^{\circ} \mathrm{C}$ and averaged for ice contents from $2 \%$ to $8 \%$. According with the depicted values, the effect of the salt concentration in the power consumption can be considered insignificant. The scraping power values remain more or less constant when increasing $\omega_{0}$.

On one hand, the addition of salt to the brine may lead to a decrease in the resistance of the ice layer adhered to the place, increasing its mushy consistence. This should in turn lead to lower power consumptions. On the other hand, the ice layer has also a lubricant effect, as discussed in the previous chapter. The invariance of the results obtained for different initial salt concentrations can be then due to a twofold effect: from one side decreases the ice resistance, but from the other one decreases also the lubricant effect of the ice.

Figure 5.7 shows the Power number values for all the scraping velocities tested, again $\overline{\Delta \mathrm{T}}_{\log } \approx 1.3{ }^{\circ} \mathrm{C}$ and $2 \%$ to $8 \%$ of ice content averaged. No clear effect of the $\mathrm{NaCl}$ initial content is observed in the $P o$ number, being almost constant for all the salt concentration values. Independently of $\omega_{0}$, the Po number decreases as $\mathrm{N}$ increases (higher $R e_{\text {rot }}$ numbers, and in comparison, lower viscous vs. inertia effects.), as seen in the previous chapter. 


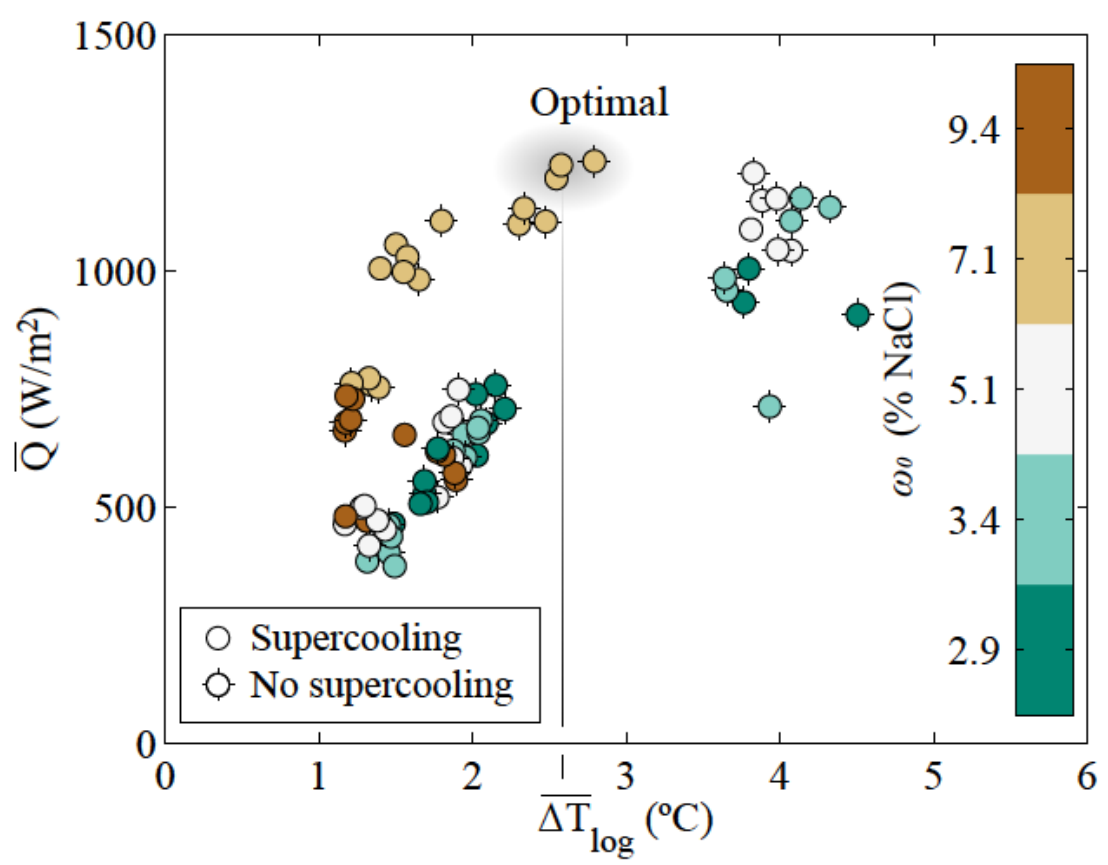

a)

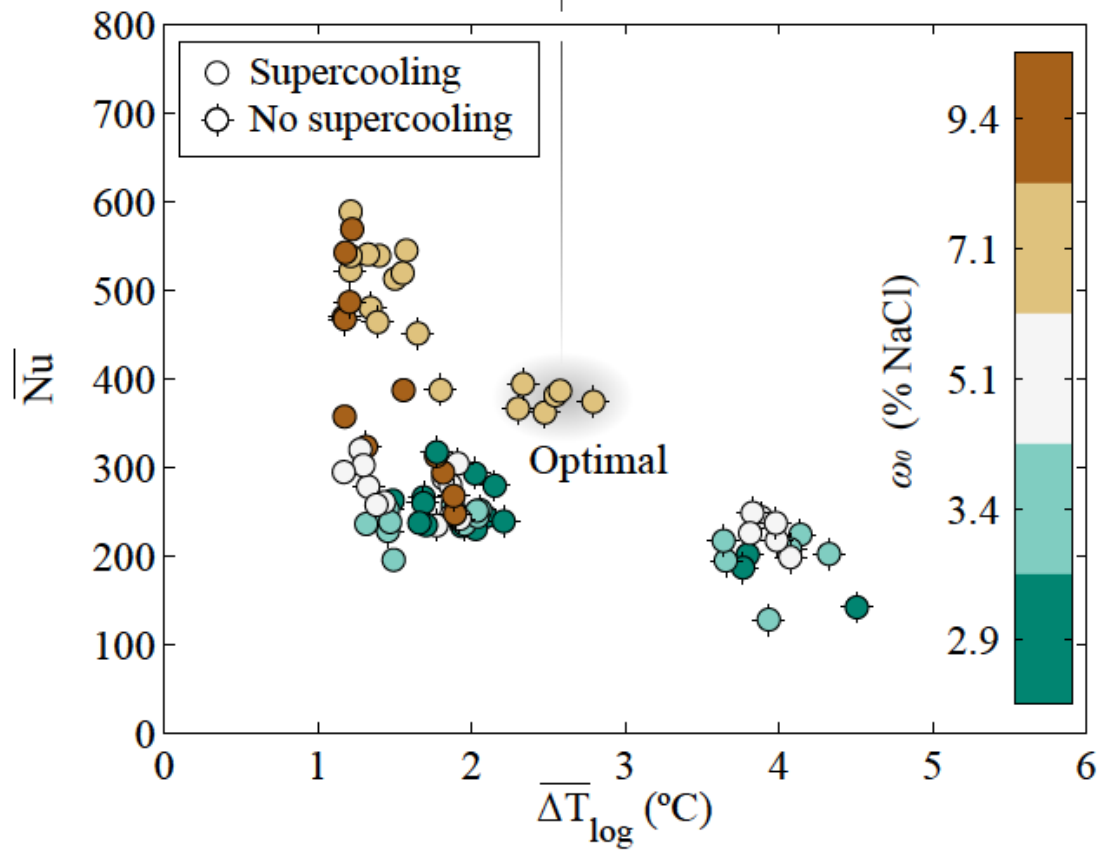

b)

Figure 5.5: Averaged, global heat flux (a) and Nusselt number (b) for all the performed experiments. Optimal operating conditions are highlighted in grey color. 


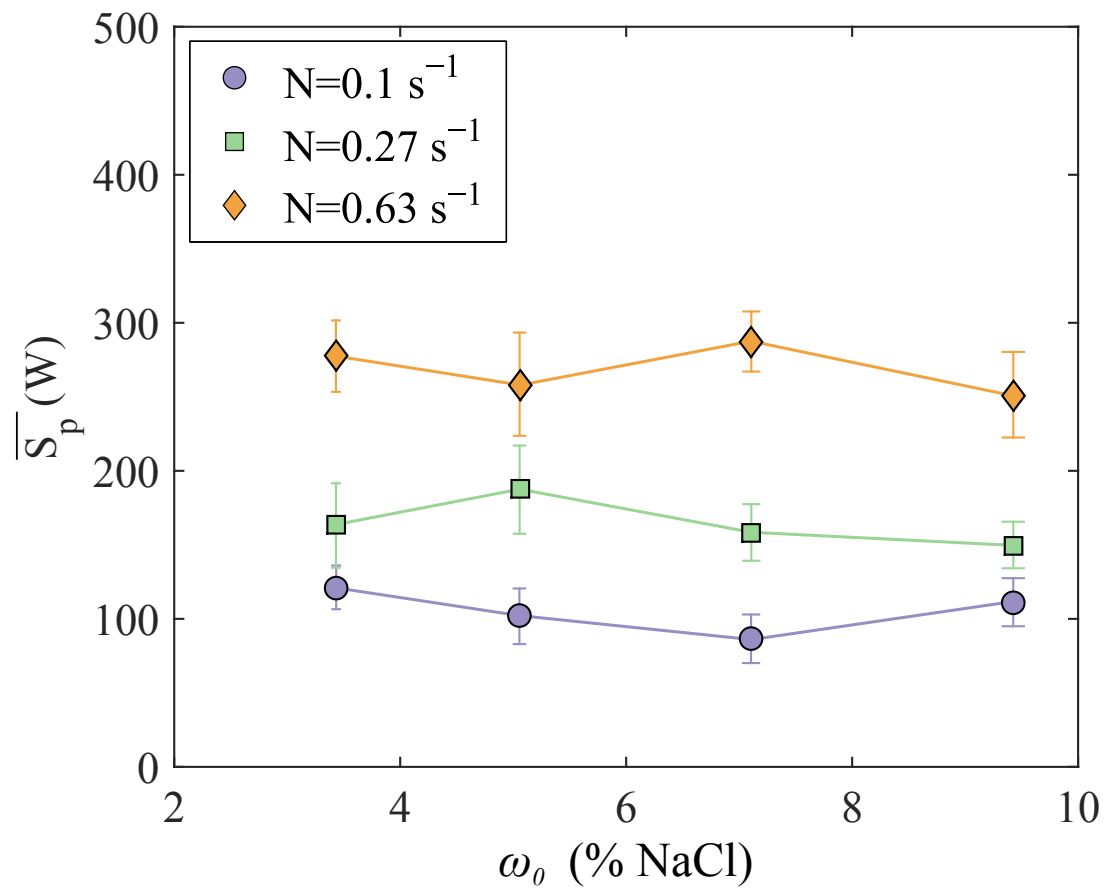

Figure 5.6: Scraping power $S_{p}$ for different $\omega_{0}$ values and scraping velocities at $\overline{\Delta \mathrm{T}}_{l o g} \approx 1.3{ }^{\circ} \mathrm{C}$, averaged between $2 \%$ and $8 \%$ of ice content

\subsection{Performance evaluation}

To establish better which is the best operating condition of the SSPHE -i.e. the condition at what the ice production is maximized with the lower energy consumptionincluding wall subcooling, scraping velocity or $\mathrm{NaCl}$ initial concentration, different aspects have to be considered. For instance, it is not enough to evaluate only which is the operating regime that provides higher heat fluxes $Q$ or lower scraping power consumptions. Moreover, the overall system energy consumption and performance have to be included.

\subsubsection{Local consideration: SSPHE}

If only the SSPHE is considered, the parameters to take into account are the heat flux $Q$ extracted from the brine -directly related with the ice being produced- and the scraping power consumption $S_{p}$. As seen in the previous chapter, higher scraping velocities increase the heat transfer coefficient and the heat flux, but they also lead to higher power consumptions. An optimal operating condition will be the one that maximizes $Q$ keeping $S_{p}$ low. Moreover, the heat $Q$ extracted from the SSPHE not only includes the brine phase change but also the value of $S_{p}$, which is transformed into heat by friction and viscous dissipation. Therefore, high $Q$ values are not good by themselves, as they can imply also high $S_{p}$ values .

In order to evaluate only the extracted heat due to the phase change (ice slurry production), the following performance number $\eta$ was defined, penalizing the elevated scraping power consumption: 


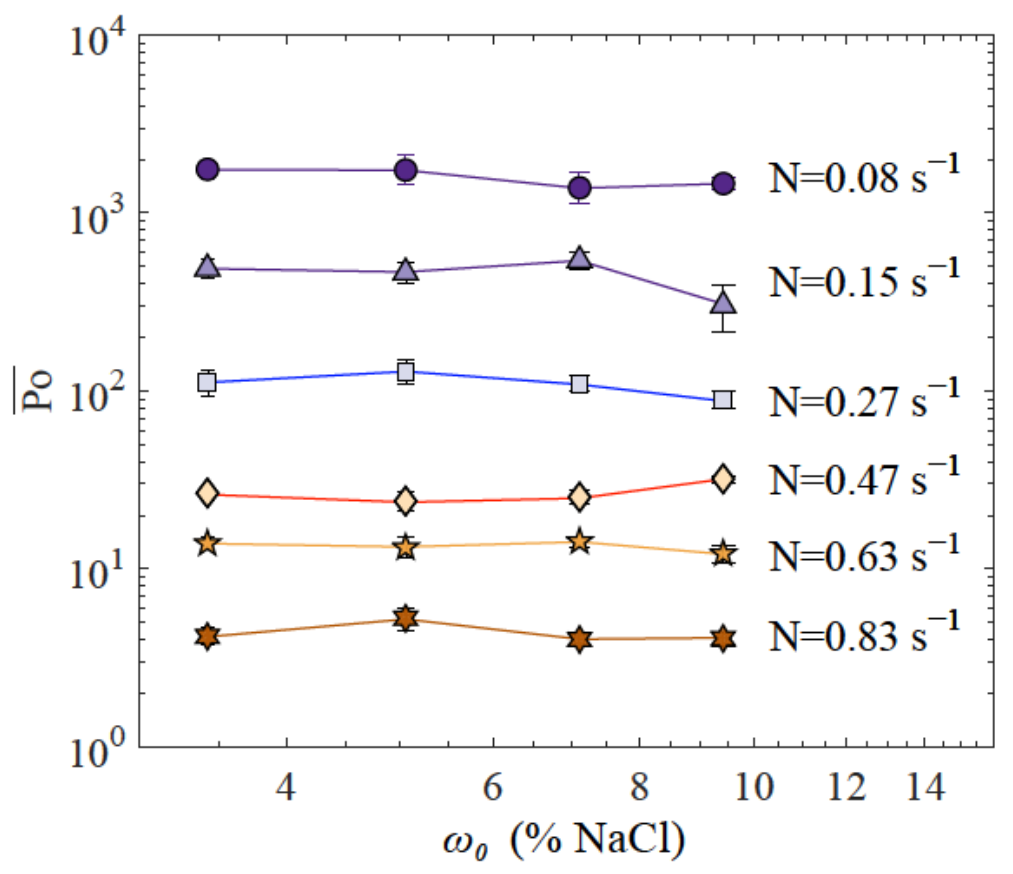

Figure 5.7: Power number $P o$ for different $\omega_{0}$ values and scraping velocities at $\overline{\Delta \mathrm{T}}_{l o g} \approx 1.3{ }^{\circ} \mathrm{C}$, averaged between $2 \%$ and $8 \%$ of ice content

$$
\eta=\frac{Q_{\text {ice }}}{S_{p}}=\frac{Q-S_{p}}{S_{p}} .
$$

The difference $Q_{i c e}=Q-S_{p}$ represents the net phase change heat. The value of $\eta$ is therefore the ratio between the scraping power and the neat heat extracted. Hence, it is desirable high values of $\eta$. Figure 5.8 shows the different results, averaged for a $2 \%$ to $8 \%$ ice content interval and including all the tests: different $\mathrm{NaCl}$ concentration, scraping velocities and $\overline{\Delta T}_{\log }$ values -all for the adaptable scrapers. A dashed line interconnects the maximum $\eta$ points.

As it is possible to see, the maximum $\eta$ values correspond to $\omega_{0}=7.1 \%$, and to an intermediate value of $\overline{\Delta T}_{\log } \approx 2.8{ }^{\circ} \mathrm{C}$. Higher $\overline{\Delta \mathrm{T}}_{\log }$ values increase the heat flux, but require higher scraping action to remove the formed ice layer, and therefore $\eta$ decreases. Lower $\overline{\Delta \mathrm{T}}_{\log }$ values provide lower heat fluxes in comparison with the heat introduced by the scrapers. Additionally, the ice layer is thinner and the friction between PEEK and plate increases. Regarding the scraping velocity, a minimum in $\eta$ is found around $\mathrm{N}=0.5 \mathrm{~s}^{-1}$. At low scraping velocities heat fluxes are low, but the $S_{p}$ implies low values also. As N increases the weight of $S_{p}$ is higher than the increasing in the phase change. Once the minimum $\eta$ value is overcame, the increase in the heat flux due to the scraper action seems to be higher than heat introduced by the scrapers themselves.

From this point of view, the best working point corresponds $\omega_{0}=7.11 \%$, at either low or high scraping velocities, and $\overline{\Delta T}_{\log }$ values around $2.8{ }^{\circ} \mathrm{C}$. The selection of low or high scraping velocities can be unbalanced considering the influence of it in the ice slurry consistence. As it will be shown in the next chapter -see chapter 6, sec. 6.2 - low scraping velocities favour the slurry stratification, leading to a slurry difficult to pump. 


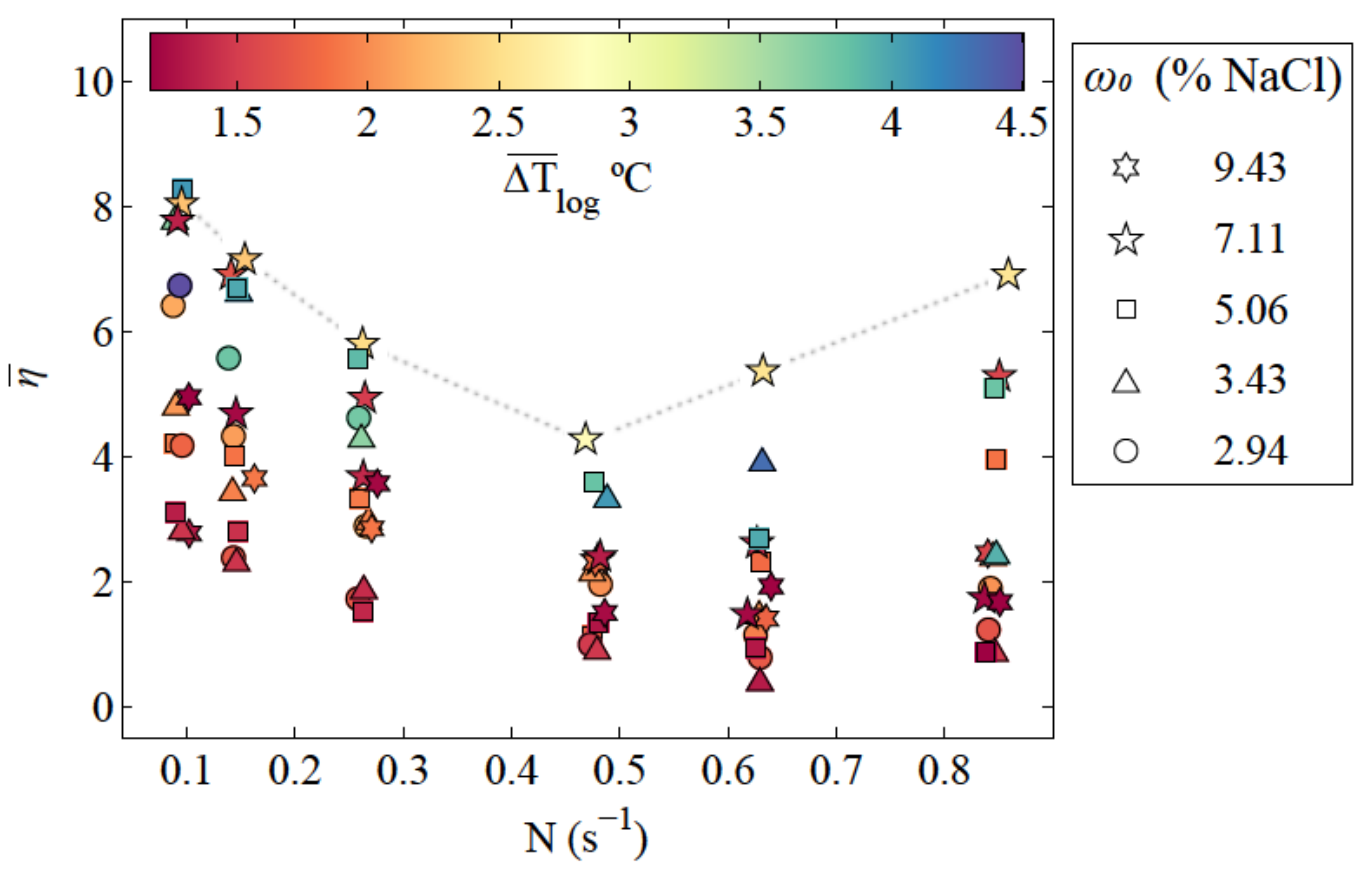

Figure 5.8: Performance number $\eta$ (Eq. 5.1) for the tested values of scraping velocity, $\omega_{0}$ values and $\overline{\Delta T}_{\log }$ (averaged for a $2-8 \%$ ice content interval.)

Higher scraping velocities ensures a good mixing avoiding the formation of solid ice blocks, ready to be pumped once the desired ice content is reached inside the SSPHE.

\subsubsection{Global consideration: SSPHE + refrigeration system}

Aside from the local analysis of the SSPHE, the determination of the optimal operating point requires also a global consideration of the system. As previously discussed, the heat flux removed from the SSPHE -and therefore capacity of ice slurry generation- increases with the wall subcooling over any other parameter. This, as a counterpart, leads to a decrement of the heat transfer coefficient, particularly pronounced for low scraping velocities and for the rigid scraper system. However, and even putting a premium on improving the ice production/time over the convection enhancement, such a decision may have consequences on the overall system performance and as a consequence on the production costs, as it will be outlined below.

The energy flow in the system, considering a quasi-steady regime, can be graphically represented by the sketch shown in Fig. 5.9. The heat $Q$ extracted from the SSHPE by the coolant corresponds to the sensible and -mostly- latent heat, $Q_{i c e}$, plus the one introduced by the scrapers (friction on the blades + viscous dissipation of the generated flow) $W_{s}=S_{p}$ (see Sec. 2.1.6). The power consumption of the two centrifugal pumps in the coolant circuit, $W_{p, 1}$ and $W_{p, 2}$ (see Fig. 2.1, can also be considered as a heat sources, since viscous dissipation will degrade the kinetic energy into thermal energy. In the evaporator-heat exchanger a total amount of heat $Q_{e}$ is absorbed by the refrigerant during its evaporation. The total heat released to the ambient in the condenser will be the latter $Q_{e}$ plus the work done by the compressor, $Q_{e}+W_{c}$.

During the freezing period (ice production) most of the heat extracted corresponds 


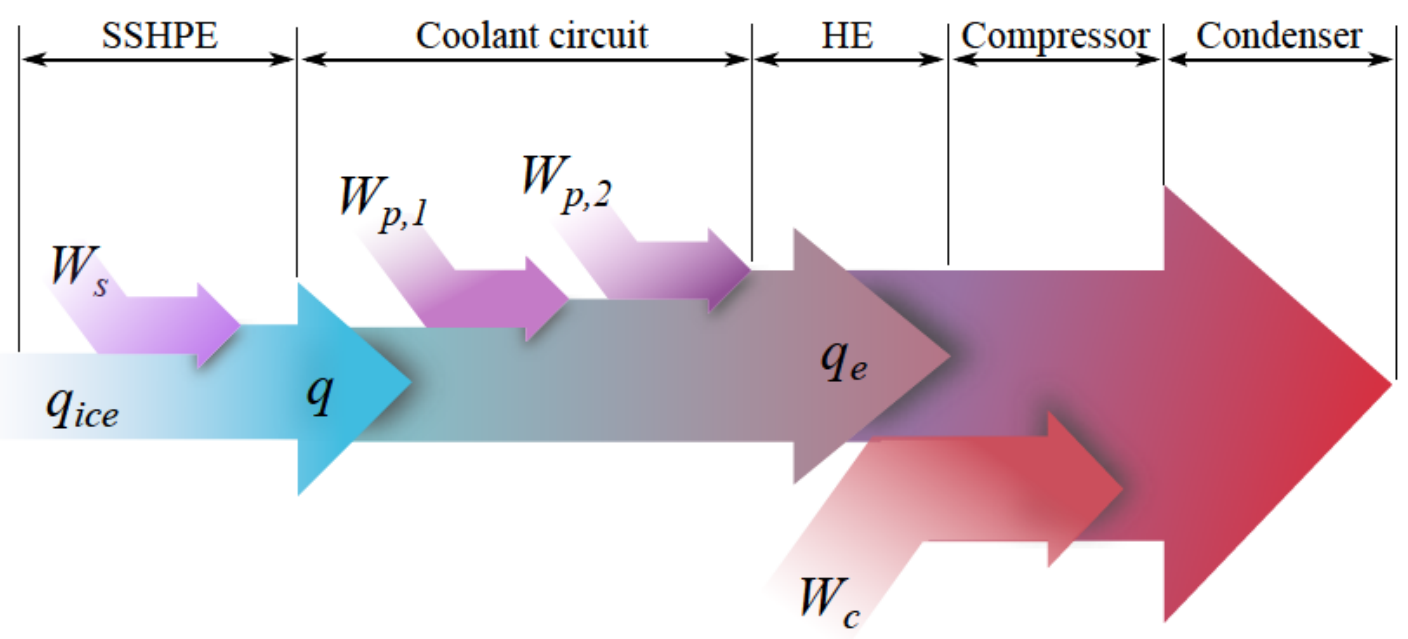

Figure 5.9: Energy flux in the global process of ice slurry production

to the phase change, whereas only a low percentage is due to the sensible heat of the liquid+ice temperature decreasing. Therefore, and for the sake of simplicity, it will be considered here that $Q_{i c e}$ will be considered here as the amount of ice generated by unit of time:

$$
\text { Ice production }(k g / s)=\frac{Q_{i c e}}{\lambda}
$$

where $L(\mathrm{~kJ} / \mathrm{kg})$ is the latent heat of fusion.

As the main power consumption of the process comes from the compressor, the efficiency of the condensing unit has to be considered when analysing the global performance. The coefficient of performance (COP) of the condensing unit is defined as the ratio between the useful heat over the invested work as [104]

$$
\mathrm{COP}=\frac{Q_{e}}{W_{c}}
$$

The above equation considers $Q_{e}$, the heat absorbed by the refrigerant in the evaporator, and not $Q_{i c e}$, which is the useful heat in the ice slurry production.

The increment of the wall subcooling, related with the mean logarithmic temperature (see Fig. 4.11), is achieved by decreasing the coolant temperature. This in turn is done by decreasing the evaporation temperature of the refrigerant at the HEevaporator. The relationship between the evaporation temperature $T_{\text {evap }}$ and the wall subcooling $\overline{\Delta T}_{s}$ is depicted in the left side of Fig. 5.10 for the case of $\omega_{0}=7.1 \%$, including the two scraper system tested -rigid and adaptable. Each point has been calculated by averaging the corresponding variables during the freezing period of the process $(1 \%$ to $20 \%$ of ice content). Moreover they have been coloured according with the averaged value of the heat flux $Q$. As expected, higher values of $\overline{\Delta T}_{s}$ imply lower evaporation temperatures. The same trend can be observed for both rigid and adaptable scrapers.

The heat flux colouring reveals again that adaptable scrapers provide higher heat fluxes with the same $\overline{\Delta T}_{s}$. The increasing of the heat flux with $\overline{\Delta T}_{s}$ can also be observed. The low effect of the scraping velocity compared with the effect of $\overline{\Delta T}_{s}$ is 


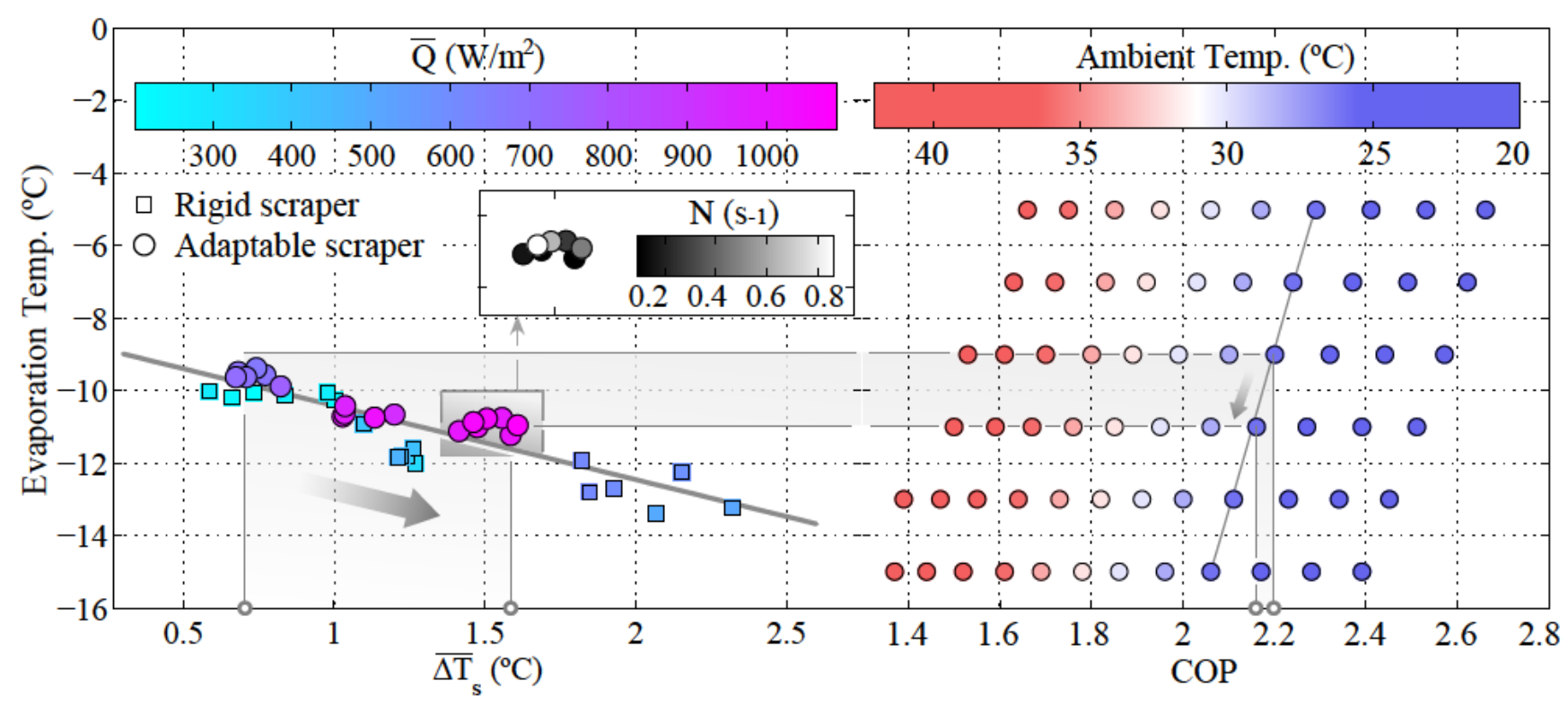

Figure 5.10: Averaged evaporation temperature vs. wall subcooling $\overline{\Delta T}_{s}\left(\omega_{0}=7.1 \%\right.$.) coloured by the averaged heat flux (left) and COP of the condensing unit for different evaporation temperatures coloured by the ambient temperature (right).

noticed by the colouring of the marked group of points, with almost the same heat flux values for different scraping velocities.

The augmentation of $Q$ with $\overline{\Delta T}_{s}$ has to be confronted with the effect of the decreasing evaporation temperature in the performance of the condensing unit. Different values of COP for different ambient and evaporation temperatures -provided by the condensing unit manufacturer [105]- are plotted in the right side of Fig. 5.10. For a constant ambient temperature, the COP value decreases when decreasing the evaporation temperature. This is expectable according to the definition of COP, and considering the basic Carnot cycle [104]

$$
\mathrm{COP}=\frac{T_{\text {cond }}}{T_{\text {cond }}-T_{\text {evap }}}
$$

where $T_{\text {cond }}$ is the condensing temperature and $T_{\text {evap }}$ the evaporation temperature, both in Kelvin. High COP values means then that the work necessary to motivate the cycle will be small for a given amount of refrigeration. Equation 5.4 shows that decreasing the condensing temperature $T_{\text {cond }}$ increases the COP. An increase in the evaporating temperature $T_{\text {evap }}$ also increases the COP due to its influence on both the numerator and the denominator. However, the evaporation temperature has a greater influence on the COP: a $1{ }^{\circ} \mathrm{C}$ increase on it exerts greater influence than a $1{ }^{\circ} \mathrm{C}$ decrease in condensing temperature. In fact, and as a general rule, raising the evaporation temperature of a refrigeration system by just two degrees it is possible to save at least $6 \%$ of the energy [106]. But raising the evaporation temperature will lead to lower ice production rates.

To evaluate the impact of decreasing the evaporation temperature in the set-up performance, $C$ will be assumed as the cost of the energy $(€ / \mathrm{kJ})$. For a certain operating 
point $a$, the ice production is equal to $Q_{e, a} / L$. If $\mathrm{COP}_{a}$ is the corresponding coefficient of performance for $a$, then the power consumed by the compressor is given by

$$
W_{c, a}=\frac{Q_{e, a}}{\operatorname{COP}_{a}}
$$

and the cost $\Upsilon$ of producing a $\mathrm{kg}$ of ice can be expressed as

$$
\Upsilon_{a}=C \frac{Q_{e, a} / \mathrm{COP}_{a}}{Q_{e, a} / L}=\frac{L \cdot C}{\mathrm{COP}_{a}}[€ / \mathrm{kg}]
$$

If a new operating point with a lower evaporation temperature is selected then the cost per $\mathrm{kg}$ of ice produced is

$$
\Upsilon_{b}=\frac{L \cdot C}{\mathrm{COP}_{b}}[€ / \mathrm{kg}] .
$$

The last expression can be rewritten as

$$
\Upsilon_{b}=C \cdot L \frac{\mathrm{COP}_{a} / \mathrm{COP}_{a}}{\mathrm{COP}_{b}}=C \cdot L \frac{\mathrm{COP}_{a} / \mathrm{COP}_{b}}{\mathrm{COP}_{a}}=\zeta \frac{C \cdot L}{\mathrm{COP}_{a}}[€ / \mathrm{kg}]
$$

where $\zeta=\mathrm{COP}_{a} / \mathrm{COP}_{b}>1$. Comparing Eq. 5.8 with Eq. 5.6 -corresponding to the initial situation $a$ - then we have that the decrease in the evaporation temperature is equivalent to increase the price of $\mathrm{kg}$ of ice produced by a factor $\zeta$.

$$
\Upsilon_{b}=\zeta \cdot \Upsilon_{a}[€ / \mathrm{kg}]
$$

The later can be applied to the experimental results of Fig. 5.10. For instance, and considering the values obtained for the adaptable scraper, going from the lower $\overline{\Delta T}_{s}$ value to the higher one corresponds to the decrease in COP indicated in the figure (considering a constant ambient temperature of $25^{\circ} \mathrm{C}$ ). Such increase in $\overline{\Delta T}_{s}$ results in an increase of the ice production $(\mathrm{kg} / \mathrm{s})$ of $60 \%$. On the other hand, according to Eq. 5.8, the $\mathrm{kg}$ of ice produced is $1.8 \%$ more expensive (referred only to the power consumption of the compressor). The same can be done for the results obtained with the rigid scrapers where going from the lower $\overline{\Delta T}_{s}$ tested to the higher the production $(\mathrm{kg} / \mathrm{s})$ increases in $156 \%$ with an increment of cost of $2.6 \%$. In spite of the high increasing of production experienced by the rigid scrapers system, it has not to be forgotten that their production rates are always lower that those ones of the adaptable scrapers. As the ice production rates employed are obtained from the real values of $Q$ measured, the previous values take into account the efficiency of the intermediary equipments (HE-evaporator, coiled circuit...).

In the light of the exposed above, the wall subcooling has a twofold effect, being responsible of the ice production rate but also of the cost per $\mathrm{kg}$ of ice produced. Decreasing the evaporation temperature (increasing $\overline{\Delta T}_{s}$ ) will increase the ice slurry production but also its cost. The operation point then has to be determined by taking into account the requirements of ice and the actual cost of the electricity. In other words, if the benefit obtained by increasing the ice slurry production are higher than the extra cost derived from it, then it is convenient to increase $\overline{\Delta \mathrm{T}}_{s}$.

Whereas the effect of the evaporation temperature in the COP is clear, the precise relationship between them depends obviously on the equipment under consideration. 


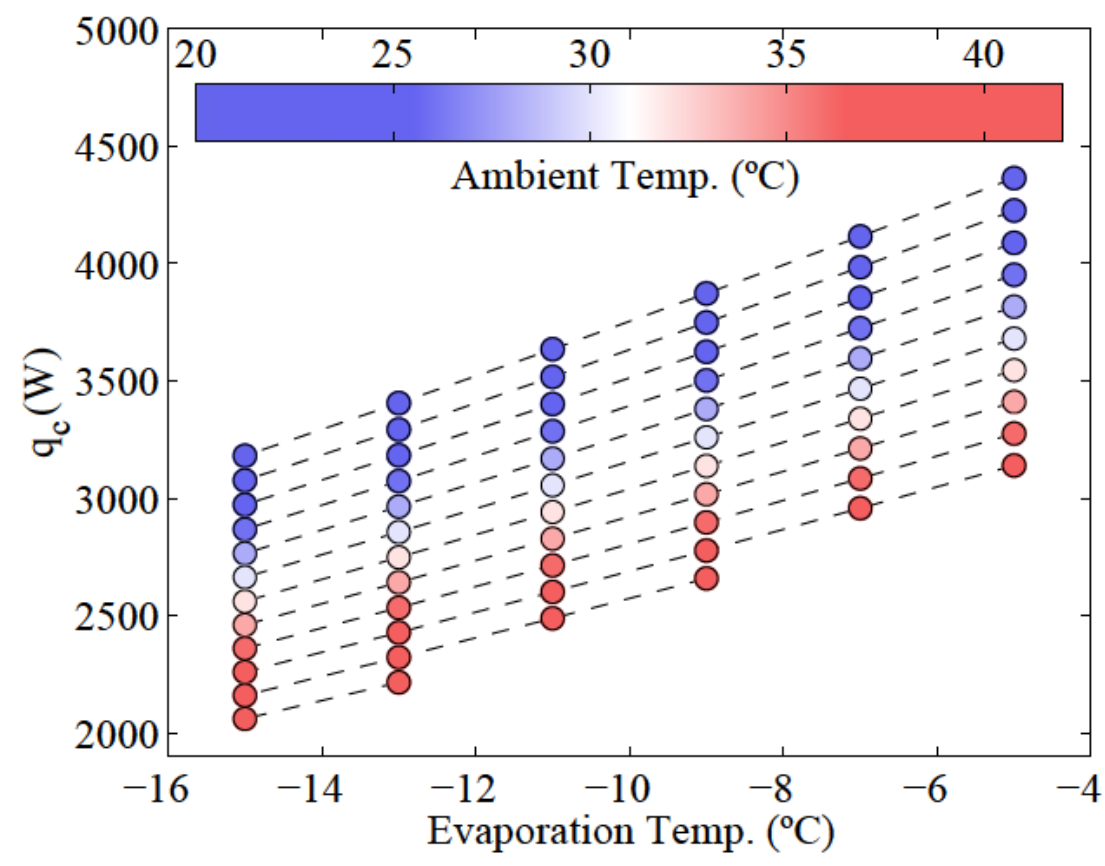

Figure 5.11: Coolant capacity $\mathrm{q}_{\mathrm{c}}$ of the condensing unit for different evaporation temperatures and coloured by the ambient temperature.

For the used condensing unit and with R507A as refrigerant the minimum evaporation temperature possible is of $-15{ }^{\circ} \mathrm{C}$. Figure 5.11 shows the relationship between evaporation temperature, ambient temperature and coolant capacity. As it is possible to see, as the evaporation temperature decreases or the ambient temperature increases the coolant capacity decreases.

The importance of the evaporation temperature on both the ice production rate and system performance makes worth to focus on the efficiency of the HE evaporator, something that is out of the scope of this work. Increasing it would lead to achieve the same or even lower coolant temperatures with higher evaporation temperatures.

\subsection{Influence in the ice crystal morphology}

In order to study the effect that the initial $\mathrm{NaCl}$ content in the brine has in the morphology of the ice crystals, a scraping velocity $\mathrm{N}=0.83 \mathrm{~s}^{-1}$ and two different $\overline{\Delta \mathrm{T}}_{\mathrm{log}}$ values, $1.3^{\circ} \mathrm{C}$ and $1.8^{\circ} \mathrm{C}$ have been chosen for comparative analysis.

Figure 5.12 shows the ice crystal microscopic images for the case of $\overline{\Delta T}_{\log }=1.3$ ${ }^{\circ} \mathrm{C}$. Ice crystals seem very similar across the different $\mathrm{NaCl}$ initial concentrations. All of them are between $200 \mu \mathrm{m}$ and $300 \mu \mathrm{m}$, without sharp edges and barely elongated (bean shape). This type of shape is mostly due to the high scraping velocity, which makes the crystals collide between themselves, rounding them and increasing their size by coalescence. The effect of increasing $\omega_{0}$ is, however, much less evident. The average crystal size seems to decrease with the increasing $\omega_{0}$ value. This can be due to the decrease of the scraped ice layer resistance, which becomes mushier and therefore the scraped ice crystals become thinner. Nevertheless this effect is not sufficiently visible 
to be quantified.

Figure 5.13 shows the ice crystal images for $\overline{\Delta T}_{\log }=1.8^{\circ} \mathrm{C}$. The higher wall subcooling is the responsible of the smaller size (compared to Fig. 5.12), resembling rice grains. As $\overline{\Delta T}_{\text {log }}$ is higher the heat flux through the surface is also higher and the ice crystals have less time to grow (see chapter 4). As in Fig. 5.12, the effect of $\omega_{0}$ is much less obvious, concluding that the ice crystals morphology is almost unaffected by the initial salt content in the brine. 


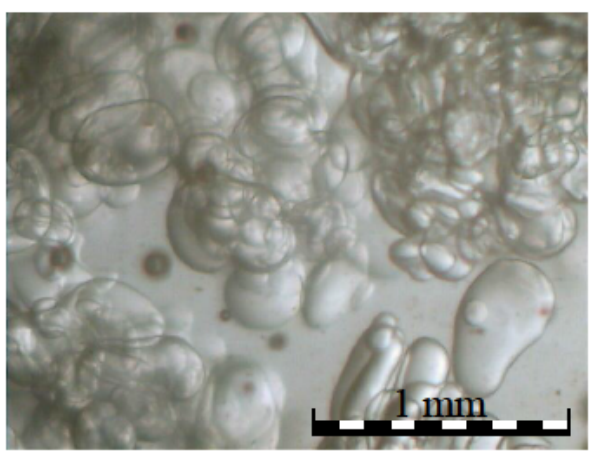

(a) $\omega_{0}=2.9 \%$

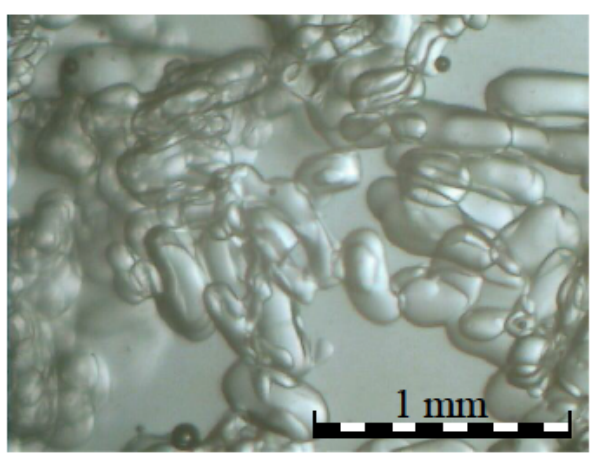

(c) $\omega_{0}=4.7 \%$

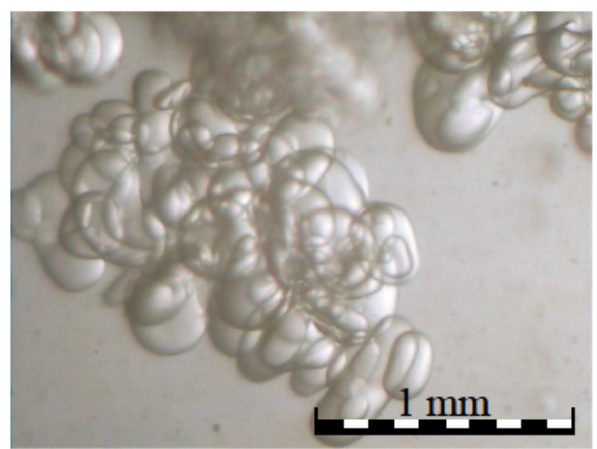

(b) $\omega_{0}=3.8 \%$

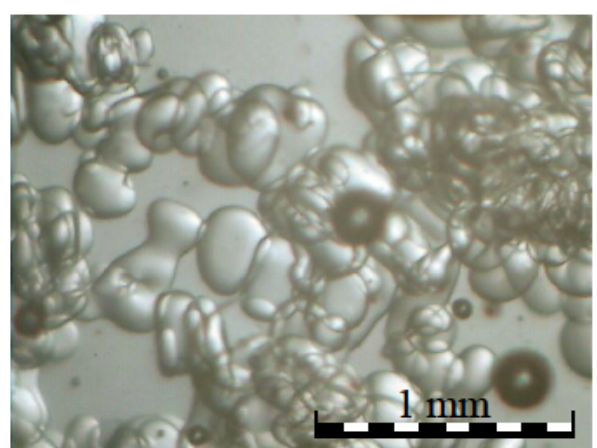

(d) $\omega_{0}=7.1 \%$

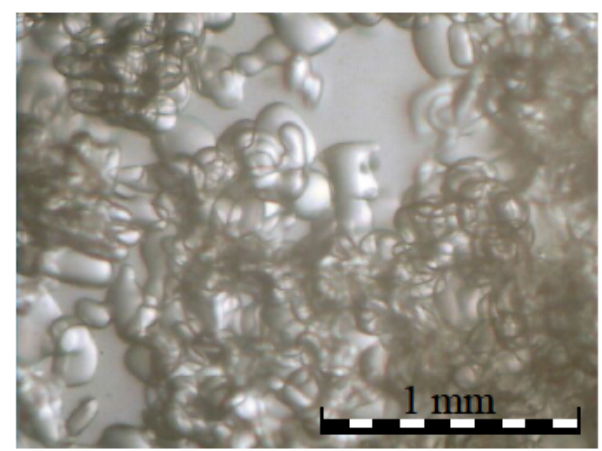

(e) $\omega_{0}=9.4 \%$

Figure 5.12: Ice crystals images taken with microscope at the end of each test (ice content $\approx 20 \%$ ): $\mathrm{N}=0.83 \mathrm{~s}^{-1}, \overline{\Delta \mathrm{T}}_{\log }=1.3{ }^{\circ} \mathrm{C}$ 


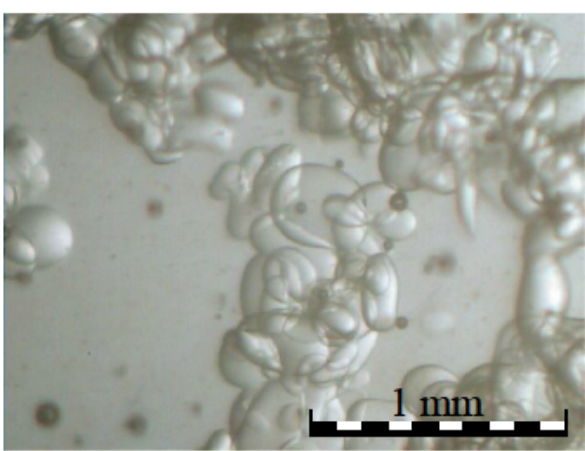

(a) $\omega_{0}=2.9 \%$

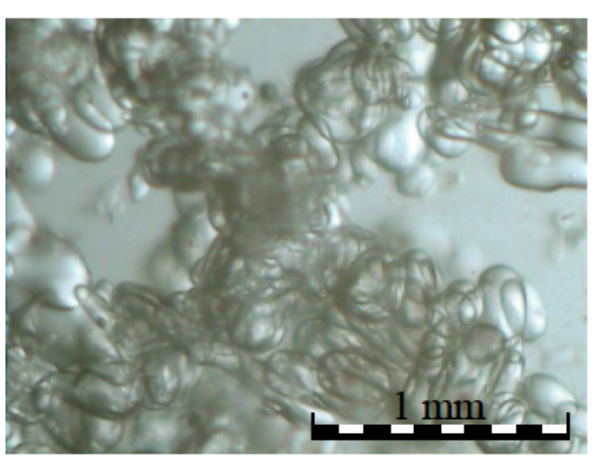

(c) $\omega_{0}=4.7 \%$

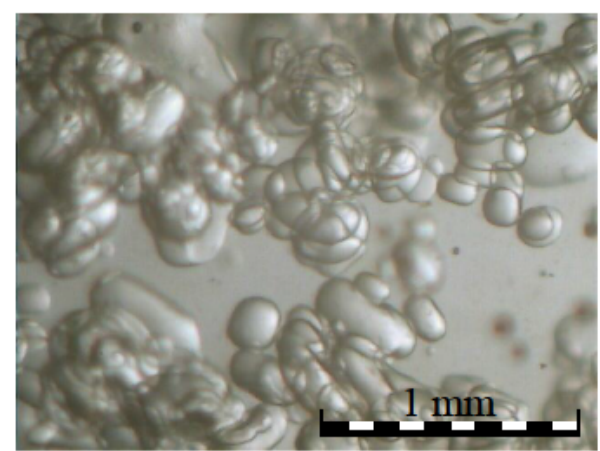

(b) $\omega_{0}=3.8 \%$

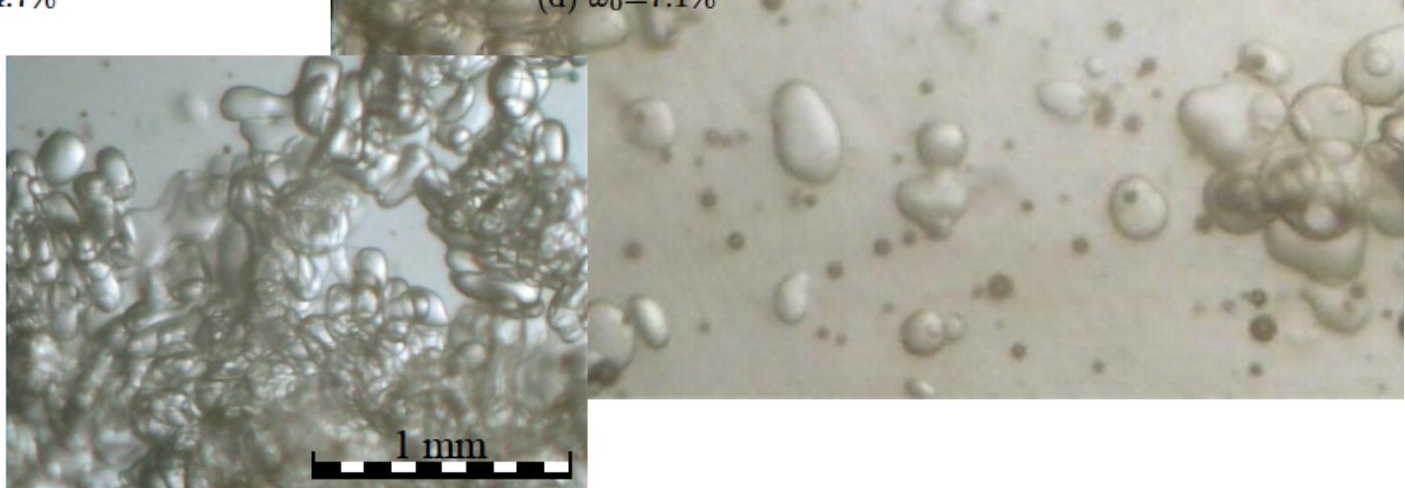

(e) $\omega_{0}=9.4 \%$

Figure 5.13: Ice crystals images taken with microscope at the end of each test (ice content $\approx 20 \%$ ): $\mathrm{N}=0.83 \mathrm{~s}^{-1}, \overline{\Delta \mathrm{T}}_{\log }=1.8{ }^{\circ} \mathrm{C}$ 


\subsection{Conclusions}

In this chapter the effect of the initial $\mathrm{NaCl}$ concentration in the brine was studied through the heat transfer, scraping power consumption and the resulting ice crystals morphology. Five different $\omega_{0}$ values were considered, testing for all of them the same range of scraping velocities. The observed freezing point agreed with the one predicted according to the salt concentration in the brine. The efficiency of the SSPHE was analysed both locally and globally, considering all the facility system. Major conclusions are as follows:

- For equal $\overline{\Delta \mathrm{T}}_{\text {log }}$ and scraping velocity values, increasing the $\mathrm{NaCl}$ resulted in increasing heat fluxes between $75 \%$ and $100 \%$, being the most significant increase for $\mathrm{N}=0.1 \mathrm{~s}^{-1}$. Such a heat flux increment was observed between $\omega_{0}=4.7 \%$ and $7.1 \%$. Higher $\omega_{0}$ values had no heat flux increasing effect.

- The Nusselt number, for equal $\bar{\Delta}_{\text {log }}$ and scraping velocity values, experience an augmentation when increasing $\omega_{0}$, in correspondence with the augmentation of the heat flux. The maximum augmentation was observed between $\omega_{0}=4.7 \%$ and $7.1 \%$, being more significant as the scraping velocity was higher. The maximum increase in $\mathrm{Nu}$ observed was of $65 \%$. This effect, related with the heat flux augmentation, was associated to effect of the $\mathrm{NaCl}$, weakening the ice strength and increasing the mushy structure of the ice layer, being easier to scrape.

- $\mathrm{A} \omega_{0}=07 \%$ and $\overline{\Delta \mathrm{T}}_{\log } \approx 2.6^{\circ} \mathrm{C}$ revealed to be the best operating condition, providing heat fluxes of $4270 \mathrm{~W} / \mathrm{m}^{2}$ and Nusselt numbers around 380 .

- Scraping power revealed to be independent of the $\omega_{0}$ value. For equal $\overline{\Delta T}_{\text {log }}$ and $\mathrm{N}$ values, scraping power remained almost constant. The same was observed for the power number. This was associated with the twofold effect of the ice layer, acting as a lubricant ans also as a resistance to the scraping blades.

- The efficiency of the SSPHE was analysed by considering the ratio $\eta$ between the effective heat extracted (due to phase change) and the scraping power. The $\omega_{0}=7 \%$ with $\overline{\Delta T}_{\log } \approx 2.6^{\circ} \mathrm{C}$ proved to be again the best operating condition, achieving a value of $\bar{\eta}=7$ for $\mathrm{N}=0.83 \mathrm{s.}^{-1}$

- The total efficiency of the ice slurry production system was evaluated through the COP of the condensing unit and the cost of producing a kilogram of ice, for the case of $\omega_{0}=7 \%$. It was concluded that, for the adaptable scraping system, increasing the ice production in $60 \%$ leaded to an increase in the cost per kilogram of ice produced of $1.8 \%$. The operating point should then be selected according to the demand and the price of the electricity.

- No clear influence of $\omega_{0}$ in the ice crystals morphology could be assessed. Only as a guess, the ice crystals size seemed to decrease when $\omega_{0}$ increased, probably due to the weakening of the ice layer, resulting in smaller crystals when scraped from the plate. 


\section{Chapter 6}

\section{Experimental flow pattern assesment}

In the previous chapters the heat transfer in the SSPHE was studied through the influence of variables like the scraping velocity, the initial $\mathrm{NaCl}$ content in the brine or the mean logarithmic temperature difference. Their influence in the scraping power consumption was also considered. Heat transfer phenomena inside the SSPHE can be then characterized by derived global parameters as the heat transfer coefficient or other dimensionless parameters as Nusselt or Reynodls numbers.

According to their name, these parameters only provide information about the global process. A better and more in deep study of the phenomena requires to understand the flow pattern inside the heat exchanger. The flow pattern may be the ultimate the responsible of variations in the heat transfer, the power consumed by the scrapers or the morphology of the ice crystals and the development of the slurry consistence. likewise, the experimental methodology to obtain the flow pattern has to reproduce the governing parameters that take place in the real SSPHE operating conditions: in particular, the flow regime and the interaction with the ice particles, which is expected to light on different aspects as the ice layer growing and its impact on the heat transfer.

The present chapter includes a wide study of the flow pattern inside the SSPHE. It considers both a single-phase flow situation -where ice particles and brine are assumed to behave as a continuous medium- and also the interaction between solid and liquid phases, determining either if the mixing is sufficiently high to consider single-phase or two-phase flow.

Two approaches are hence presented. In sections 6.1 and 6.2 a two-phase flow visualization is presented, providing qualitative data to explain the fluid-particle interaction and validate the later numerical model. In particular, sec. 6.1 includes the results of the particle injection tests, whereas sec. 6.2 includes a a stratification test for different particle concentrations $(\approx 4 \%$ wt.). In section 6.3 the PIV results -considering a continuous phase- are presented in terms of velocity fields and streamlines, providing quantitative data to characterize the single-phase flow pattern and also to validate the correspondent numerical model. Vorticity and turbulent kinetic energy distributions were also analysed. 


\subsection{Particle motion}

This section reports the results of the particle injection tests. As mentioned before, the aim of these experiments is to provide insight into the behaviour of the ice crystals immediately after they are scraped from the heat transfer surface. They can explain whether the particles follow the existent flow generated by the scraper or they are dominated by buoyancy forces due to their lower density. Three different scraping velocities $\left(0.08 \mathrm{s.}^{-1}, 0.13 \mathrm{s.}^{-1}\right.$ and $\left.0.25 \mathrm{~s}^{-1}\right)$ were tested, corresponding respectively to the rotating Reynolds numbers $1.6 \cdot 10^{4}, 2.5 \cdot 10^{4}$ and $4.8 \cdot 10^{4}$, and Froude numbers 0.42 , 0.68 and 1.3 .

Figures 6.1, 6.2 and 6.3 show respectively for $\mathrm{N}=0.08 \mathrm{s.}^{-1}, 0.15 \mathrm{s.}^{-1}$ and $0.26 \mathrm{s.}^{-1} \mathrm{a}$ sequences of images acquired after particles were released just before the wiping of the scraper. Particle clouds are enhanced in red, according to the technique described in 3.2.2. In the first case (Fig. 6.1) the particles are very dispersed, and such a dispersion increases with time. The increase in the scraping velocity makes the particle cloud smaller, being the particles more and more concentrated (Figs. 6.2 and 6.3).

The rotating motion of the scraper induces a continuous flow from its frontal side towards the rear side, bringing up the removed ice particles together with the liquid phase.

From the exposed above it appears that at very low scraping velocities the buoyancy of the particles affects their motion. Therefore, they spread and rise, most surely leading to a stratified layer of particles. The reduction of the volume occupied by them when increasing the scraping velocity responds to the increasing forced flow, which counteracts the buoyancy effect keeping the particles grouped. Indeed, between Figs. 6.2 and 6.3 is it is possible to see how the vertical distance between the particles cloud and the scraper is reduced.

Higher scraping velocities were out of the range of the recording camera and were therefore not tested. On the other side, the usage of a high speed camera would require a more powerful light source like a laser, which would not allow a uniform light condition inside the SSPHE model, hindering the visualization of the particles cloud.

The particle visualization images were processed in order to obtain the particle displacements -see sec. 3.2.2. Figure 6.4 shows the obtained displacements for $\mathrm{Re}_{\mathrm{rot}}=1.6 \cdot 10^{4} \mathrm{-Fr}=4.2 \cdot 10^{-1}$. The absolute particles displacement has been subtracted by the displacement of the scraper. After, it was increased by a factor of two (times 2 ). All the particles have a similar horizontal displacements slightly shorter than the scraper displacement (indicated with a red arrow in the figure). Some of the particle groups have an ascending vertical component, which can be due to the forced flow overpassing the scraper or to the influence of the buoyancy. Some other particle groups descend, following the fluid flow and replacing the gap left by the scraper.

The relative displacements for $\operatorname{Re}_{\text {rot }}=2.5 \cdot 10^{4}-\mathrm{Fr}=6 \cdot 8 \cdot 10^{-1}$ are shown in Fig. 6.5 with a scale factor of 1 (times 1 ). The particles cloud appears now more compact than in 6.4: the effect of the buoyant forces compared with the higher velocities decreases. The particles exhibiting a vertical displacement in the front side of the scraper are most probably drifted by the induced flow.

Finally, Fig. 6.6 shows the obtained relative displacements for the case $\mathrm{Fr}=1.3-$ $\operatorname{Re}_{\text {rot }}=4.8 \cdot 10^{4}$, now with a scale times 0.6 . The particles cloud appears now very compacted, being all of them dominated by the forced flow induced by the scraper. The 


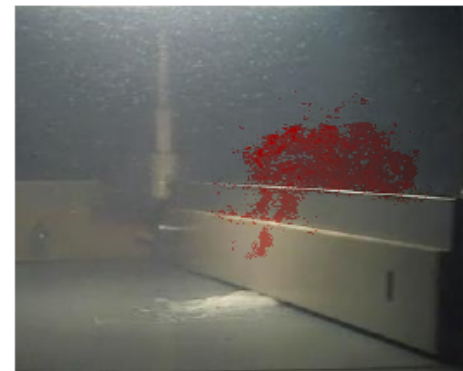

a) ts.

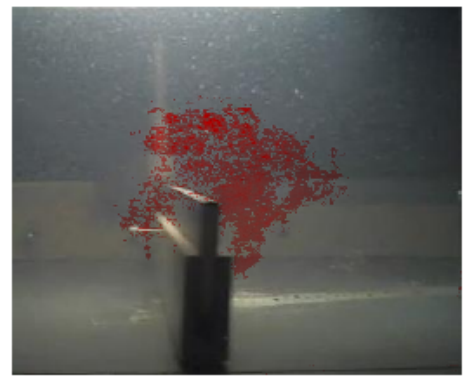

d) $\mathrm{t}+0.3 \mathrm{~s}$.

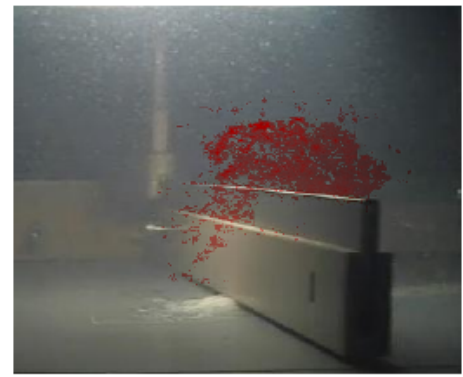

b) $\mathrm{t}+0.1 \mathrm{~s}$.

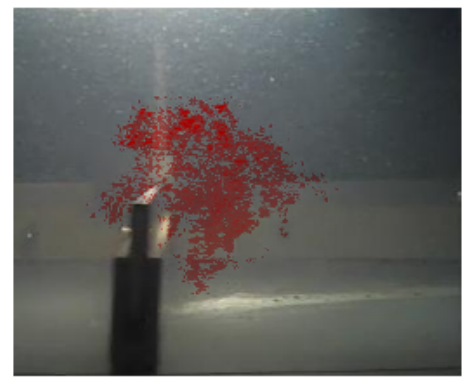

e) $\mathrm{t}+0.4 \mathrm{~s}$.

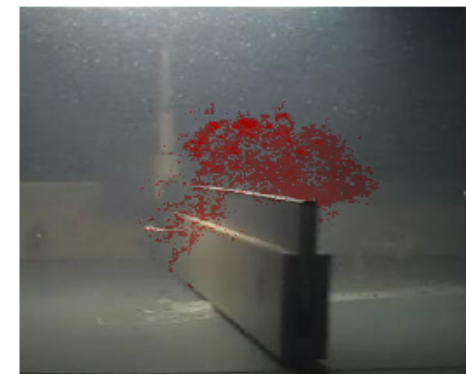

c) $\mathrm{t}+0.2 \mathrm{~s}$.

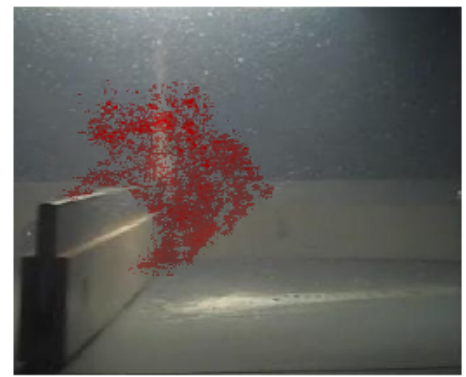

f) $\mathrm{t}+0.5 \mathrm{~s}$.

Figure 6.1: Image sequence of the particle cloud (enhanced in red) at different time instants for $\mathrm{Fr}=0.42$ and $\mathrm{Re}_{\text {rot }}=1.6 \cdot 10^{4}$.

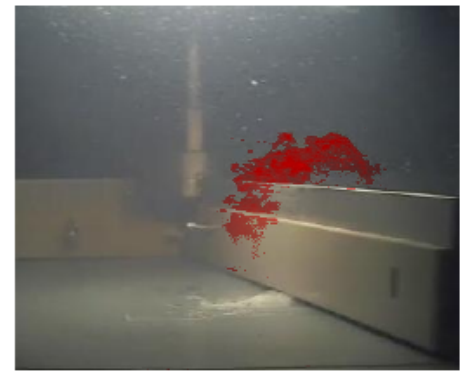

a) ts.

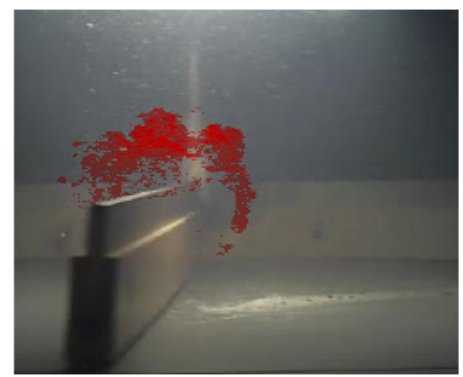

d) $\mathrm{t}+0.3 \mathrm{~s}$.

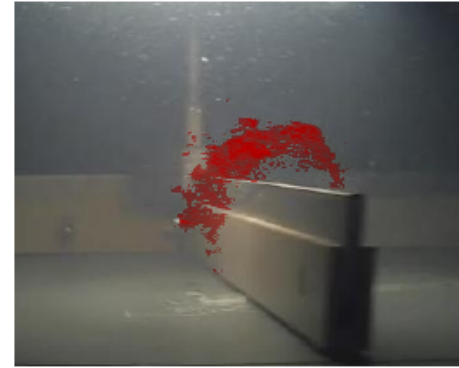

b) $\mathrm{t}+0.1 \mathrm{~s}$.

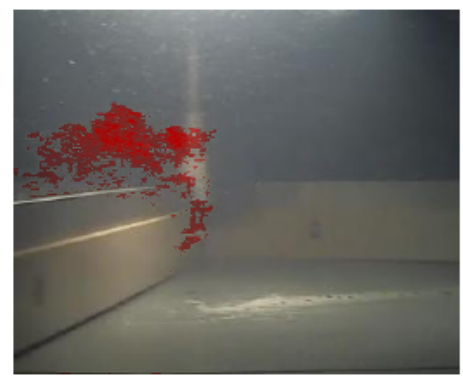

e) $\mathrm{t}+0.4 \mathrm{~s}$.

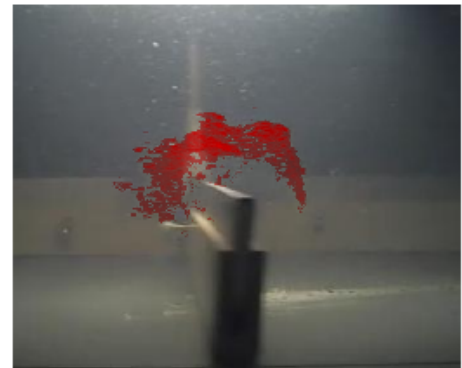

c) $\mathrm{t}+0.2 \mathrm{~s}$.

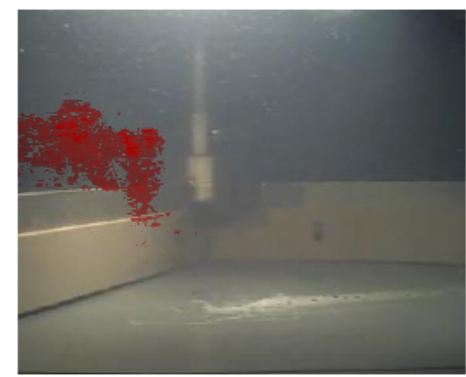

f) $\mathrm{t}+0.5 \mathrm{~s}$.

Figure 6.2: Image sequence of the particle cloud (enhanced in red) at different time instants for $\mathrm{Fr}=0.68$ and $\mathrm{Re}_{\mathrm{rot}}=2.5 \cdot 10^{4}$. 


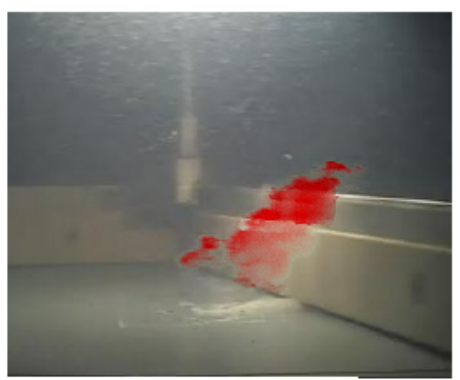

a) $\mathrm{ts}$.

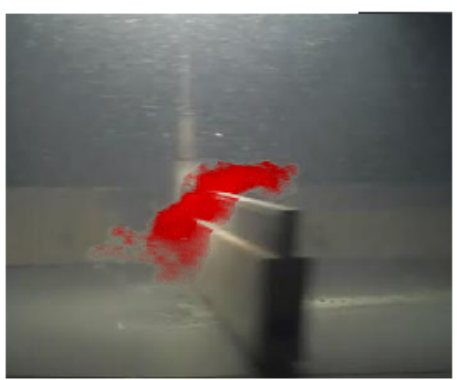

b) $\mathrm{t}+0.06 \mathrm{~s}$.

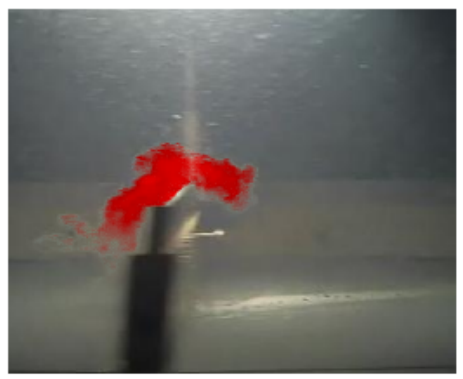

c) $\mathrm{t}+0.12 \mathrm{~s}$.

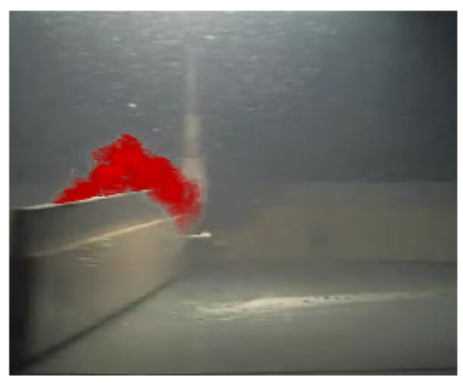

d) $\mathrm{t}+0.24 \mathrm{~s}$.

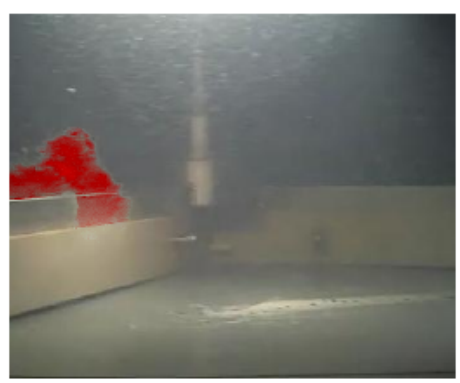

e) $\mathrm{t}+0.36 \mathrm{~s}$.

Figure 6.3: Image sequence of the particle cloud (enhanced in red) at different time instants for $\mathrm{Fr}=1.3$ and $\mathrm{Re}_{\text {rot }}=4.8 \cdot 10^{4}$.

number of vector displacement found is significantly lower. This is due to the fact that the particle cloud is more compact, and as a consequence the amount of information on each target region is lower. As a result, the size of the interrogation window has to be increased, obtaining less number of vector displacements.

Observing the three situations, the two lower scraping velocities $(\mathrm{Fr}<1)$ will probably lead to a situation of stratification during icing conditions as the particle content increases in time. Moreover, there is a low number of particles that are able to follow the scraper rear side flow and therefore the number of particle impacts on the bottom will be low or non-existent. On the other hand, in the higher scraping velocity $(\mathrm{Fr}>1)$ the inertial forces seem to dominate over the buoyant ones and the particle cloud is sent down by the rear side scraper flow. This situation will lead to a better mixed flow, avoiding the particles stratification (at least until a certain overall particles content).

\subsection{Flow stratification}

The previous section studied the motion of the particles just after the instant they are scraped from the bottom wall -without previous particle content in the fluid. However, the flow and the particles interaction inside the SSPHE may change once the particle concentration increases. Figure 6.7 shows the acquired images for different Fr-Re situations for a particle content of $4 \%$ in mass.

At low scraping velocities (Fig. 6.7a) particles are quiescent. The flow is not strong enough and particles are stacked on the top of the SSPHE, creating a stationary bed. It is then clear that at these scraping velocities the scraped ice crystals will end in 


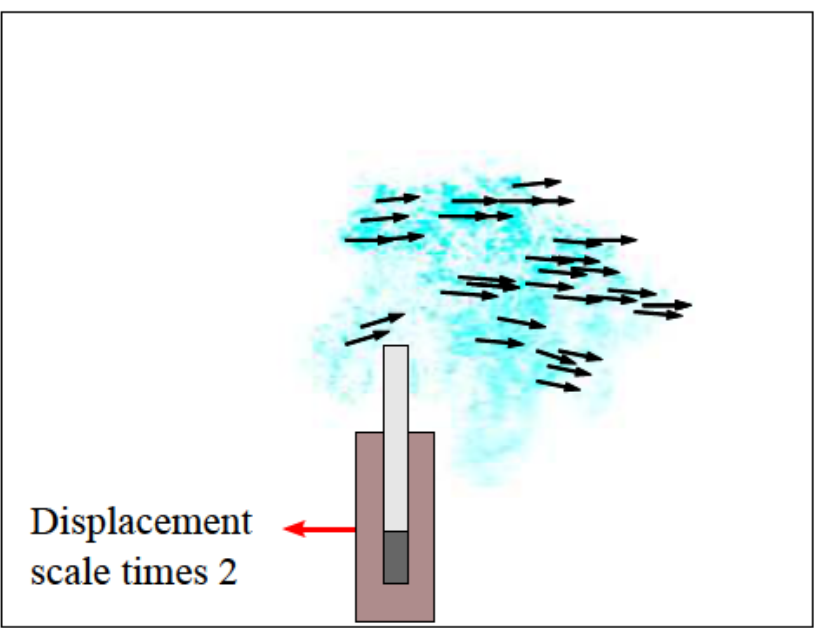

Figure 6.4: Relative displacement vectors for the injected particles cloud after $\Delta \mathrm{T}=33$ $\mathrm{ms}, \mathrm{Fr}=4.2 \cdot 10^{-1}$ and $\operatorname{Re}_{\mathrm{rot}}=1.6 \cdot 10^{4}$. Scale $\mathrm{x} 2$

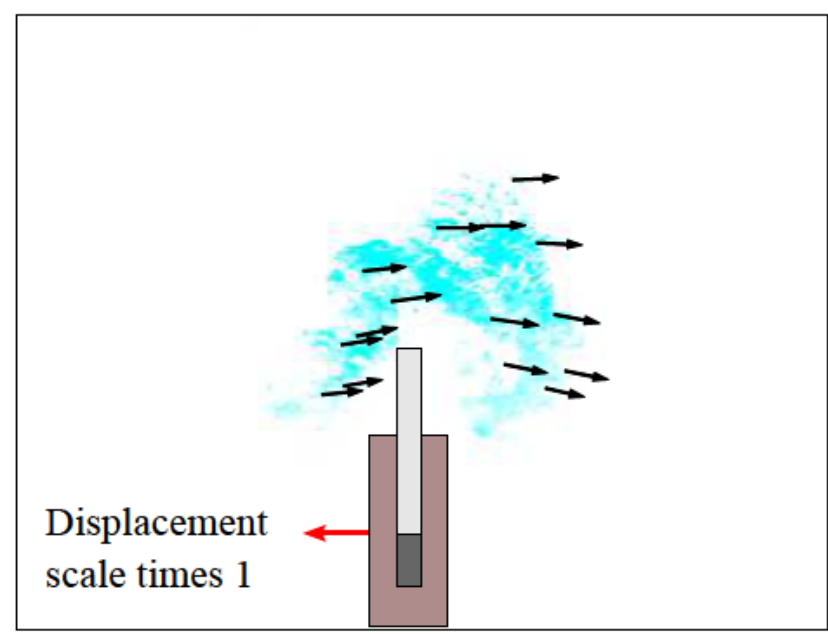

Figure 6.5: Relative displacement vectors for the injected particles cloud after $\Delta \mathrm{T}=24$ $\mathrm{ms}, \mathrm{Fr}=6.8 \cdot 10^{-1}$ and $\mathrm{Re}_{\mathrm{rot}}=2.5 \cdot 10^{4}$. Scale $\mathrm{x} 1$ 


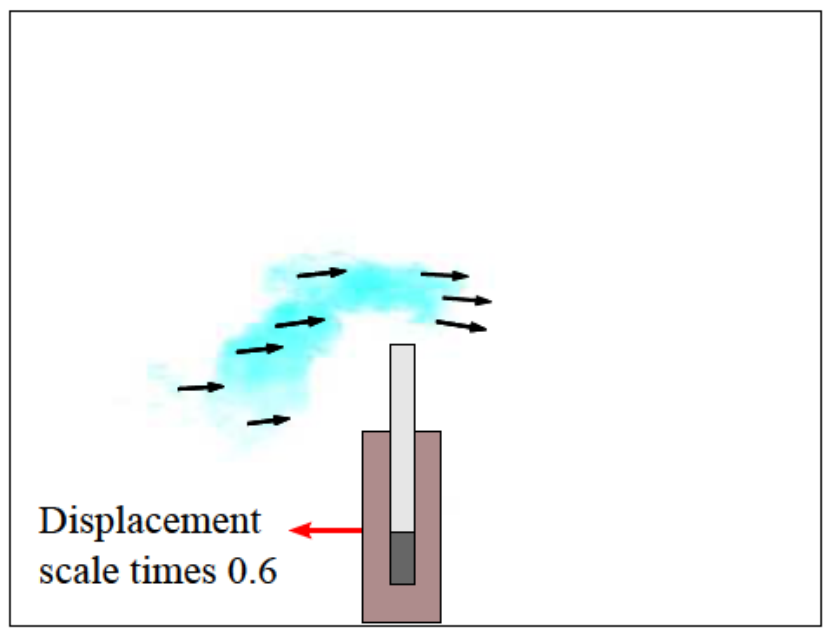

Figure 6.6: Relative displacement vectors for the injected particles cloud after $\Delta \mathrm{T}=15$ $\mathrm{ms}, \mathrm{Fr}=1.3$ and $\operatorname{Re}_{\text {rot }}=4.8 \cdot 10^{4}$. Scale $\mathrm{x} 0.6$

such a stratified layer. These situations are undesirable, as the layer may lead to and excessive ice crystal growing, being therefore difficult to pump it afterwards out of the SSPHE. The thickness of this bed grows towards the centre, as the tangential velocities are lower.

As the scraping velocity increases, a fraction of the particles starts to move. At the bottom of the stationary bed a separate moving layer appears (see Fig. 6.7b), with the formation of dune-like forms named as saltation phenomenon ([107]). Further increases on the scraping velocity leads to a moving bed that displaces under the stationary one and at $\mathrm{Fr}=0.51(6.7 \mathrm{c})$ the bed disappears near the extreme radial positions, where the flow velocity is higher. The previous stationary bed has turned into a moving bed that rotates around the shaft, whereas the particles suspended conforms an heterogeneous flow with an increasing concentration gradient towards the top.

For $\mathrm{Fr}=0.68(6.7 \mathrm{~d})$ the moving bed is practically non-existent but the particles are still on an heterogeneous flow. Higher scraping velocities $\left(\operatorname{Re}_{\text {rot }}>2.5 \cdot 10^{4}\right)$ achieve significantly higher suspension flow rates with almost an homogeneous particle flow. However, the high number of suspended particles make impossible a proper visualization. A good level of mixing is achieved for $\mathrm{N} \geq 0.25 \mathrm{~s}^{-1}$, which provides a Froude number greater than 1 , meaning that the buoyant forces are not dominant anymore. 


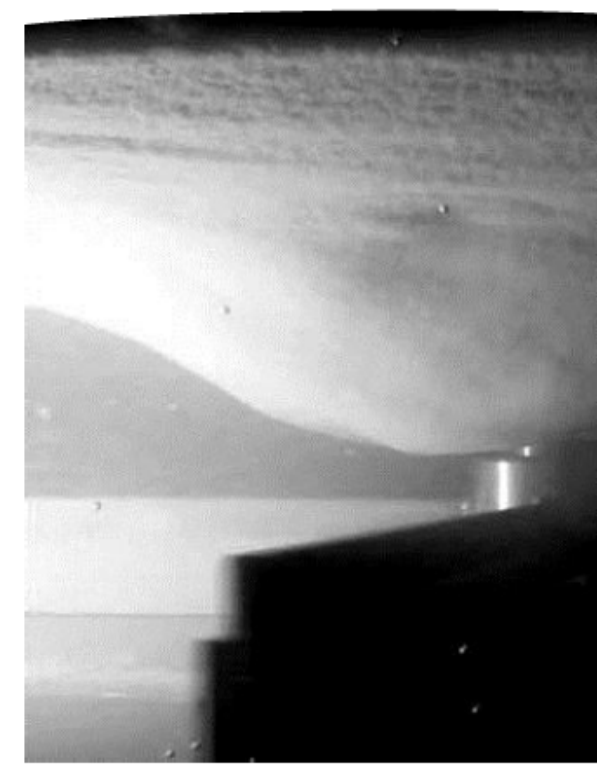

(a) $\mathrm{Fr}=2 \cdot 10^{-1}, \mathrm{Re}_{\text {rot }}=7 \cdot 6 \cdot 10^{3}$

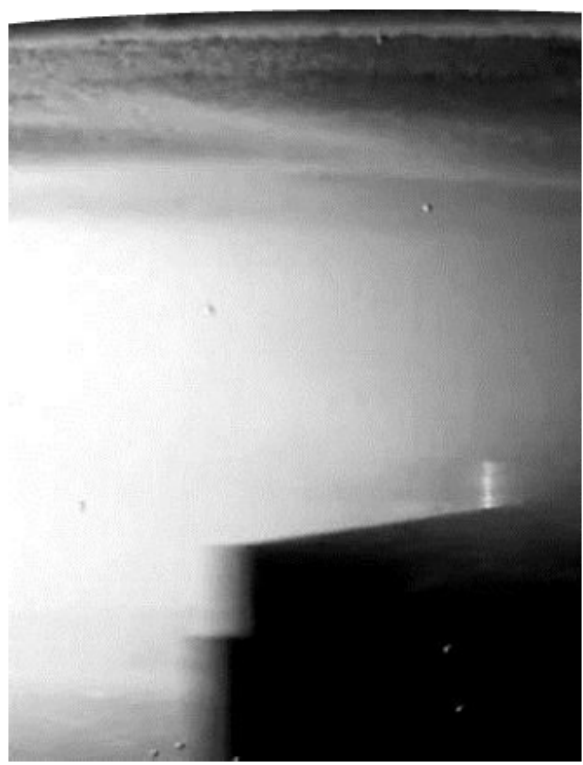

(c) $\mathrm{Fr}=5.1 \cdot 10^{-1}, \mathrm{Re}_{\mathrm{rot}}=1.9 \cdot 10^{4}$

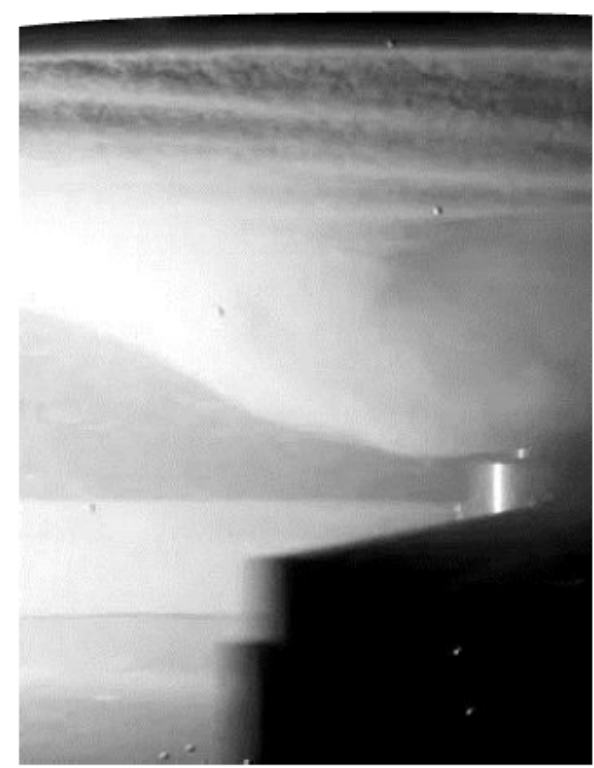

(b) $\mathrm{Fr}=4.2 \cdot 10^{-1}, \mathrm{Re}_{\text {rot }}=1.6 \cdot 10^{4}$

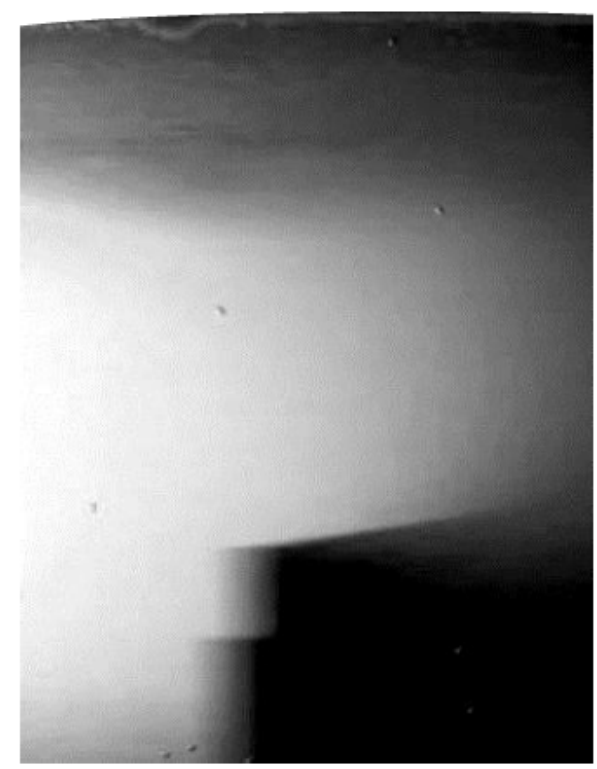

(d) $\mathrm{Fr}=6.8 \cdot 10^{-1}, \operatorname{Re}_{\text {rot }}=2.5 \cdot 10^{4}$

Figure 6.7: Stratification test images for different Froude and rotating Reynolds numbers and $4 \%$ wt. particle content 


\subsection{Particle Image Velocimetry results}

\subsubsection{Previous considerations}

\section{Single field composition procedure}

As explained in chapter 3, section 3.4, the investigated region is a plane normal to the scraper arm, located at the radial coordinate $r_{l}=0.6 R$. As the flow is only generated by the scrapers movement, the synchronization of the data acquisition with the scraper position in the so called phase-averaged technique (see. 3.4) can be used to extract the mean flow $\bar{u}$ and the turbulent velocity component $u^{\prime}$ :

$$
u=\bar{u}+u^{\prime}=U+u^{\prime}
$$

Since the width of the laser sheet is not enough to illuminate the entire region around the scraper, the image recording was done in two steps. The first step covered the left (front) side whereas the second step covered the right (rear) side. The rest of the image was masked conveniently in order to consider only the intended region. A sketched example of this procedure is shown in the top part of Fig. 6.8. The resulting regions of interest were then analysed and averaged providing the respective velocity fields.

In order to consider both fields as a single map, a unique velocity distribution was composed by merging both phase-averaged fields, re-averaging them in the overlapped region. The employed expression for the averaging is included in the central part of Fig. 6.8. It ensures a gradual change between the left and right data fields.

\section{Estimation of the out-of-plane motion}

One of the main limitations of the classical planar PIV technique is the effect of the out-of-plane motion of the particles. The out-of-plane displacements do not only imply mismatch displacement evaluations due to several reasons, like the change in the particle intensity or even the particle disappearance, which in both cases implies a loss of correlations [108].

As the camera is located perpendicular to the laser sheet, any component of the velocity parallel to the camera axis will be only detected through its projection over the laser plane. This will introduce an error over the in-plane particle displacement. The concept is well explained in [109], which also provided an expression to evaluate the relative error (called perspective error) between the in-plane displacement and the apparent one. To illustrate the problem, Fig. 6.9 shows an sketch of the PIV measurement systems inside the SSPHE. The perspective error is given by

$$
\epsilon=\frac{\Delta X_{\text {real }}-\Delta X_{\text {apparent }}}{\Delta X_{\text {real }}}=\frac{E}{\Delta X_{\text {real }}}=\frac{\Delta z}{\Delta x} \tan \theta
$$

The out-of-plane induced error will be more significant as the distance from the camera axis along the laser plane increases, as it is possible to guess from Fig. 6.9. The only way to determine the error is to know the out-of-plane velocity component. In this particular case, one can evaluate the possible out-of-plane effect by considering that the flow is purely circular, being the velocity tangential to the circular lines in each point (i,e. perpendicular to the scrapers). The concept is represented graphically in Fig. 6.9. 


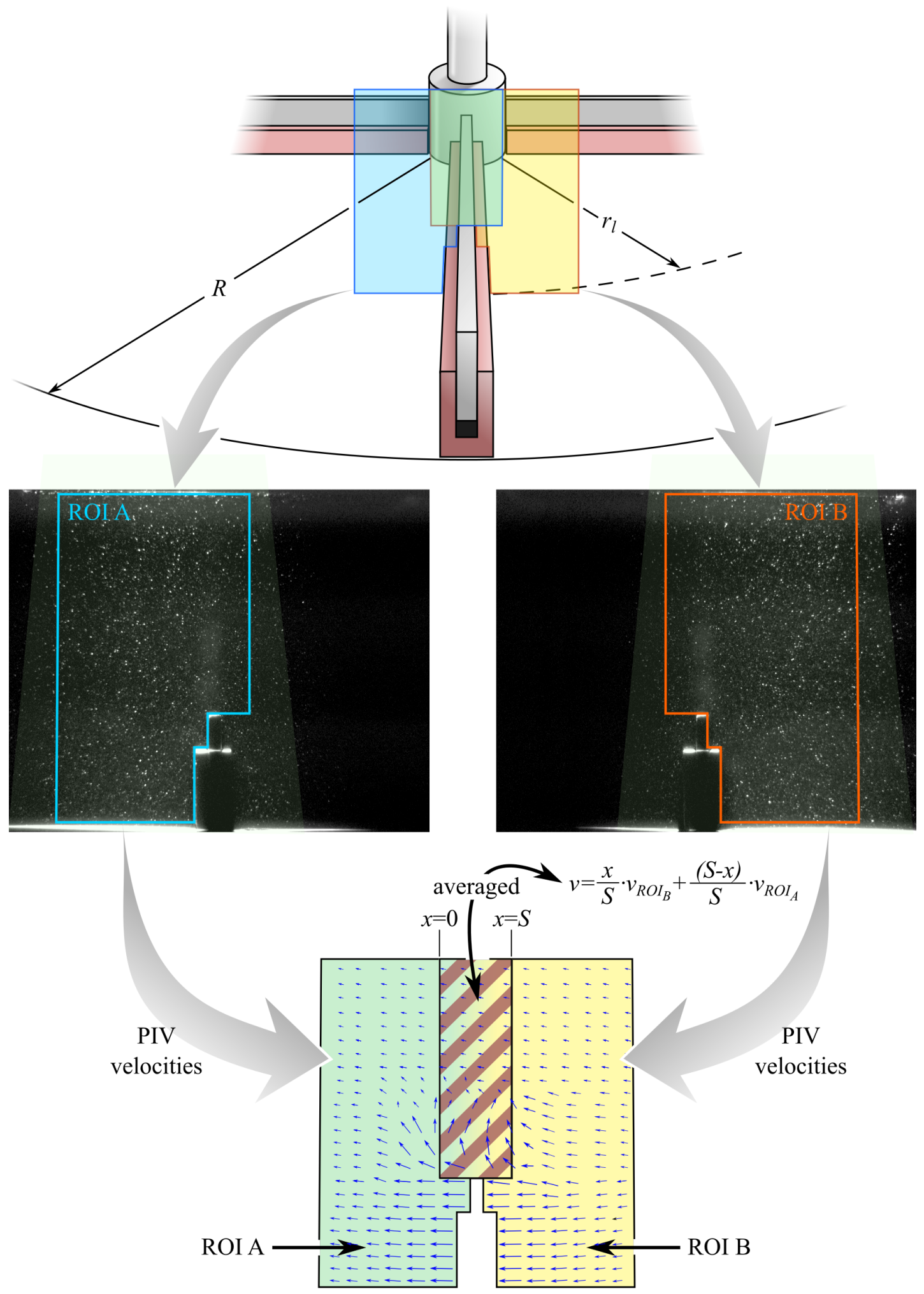

Figure 6.8: Velocity field composition process from different PIV studied regions under equivalent $\operatorname{Re}_{\text {rot }}$. 
This is a simple way to evaluate the out-of-plane motion, even if it is not completely accurate; the centrifugal forces in the flow may lead to radial velocity components.

For a point in the camera axis the velocity will be equal to $U_{0}=2 \pi N R_{0}$ where $R_{0}=$ $r_{l}$ and the particle displacement $\Delta_{0}=2 \pi N R_{0} \cdot d t$. Conversely, the most unfavourable situation will come when the particle is located at the extreme of the laser plane, where $\Delta=2 \pi N R \cdot d t$ and $R=\sqrt{R_{0}^{2}+(L / 2)^{2}}$. The displacement components along the laser plane and perpendicular to it are given by,

$$
\Delta x=2 \pi N R \cdot d t \cdot \cos \alpha, \quad \Delta z=2 \pi N R \cdot d t \cdot \sin \alpha
$$

where $\alpha=\arctan \left(L / 2 R_{0}\right), \sin \alpha=(L / 2 R)$ and $\cos \alpha=R_{0} / R$. Substituting then we have

$$
\Delta x=2 \pi N R_{0} \cdot d t, \quad \Delta z=2 \pi N L d t / 2
$$

The value of $\theta$ can by obtained by,

$$
\theta=\arctan \left[\frac{\sqrt{R^{2}+\Delta^{2}}}{d_{0}-\Delta z} \sin \left(\operatorname{arcos}\left(\frac{R_{0}+\Delta z}{\sqrt{R^{2}+\Delta^{2}}}\right)\right)\right]
$$

By replacing the values of $N, L, d_{0}$ and $d t$ in the above equations the maximum error $\epsilon$ was estimated in $\approx 3 \%$, which can be considered as acceptable.

Other source of out-of-plane motion in the SSPHE facility can be due to the centrifugal force produced by the rotating flow. This force acts in the radial direction and will induce a particle settling velocity $v_{c}$ given by [110],

$$
v_{c}=\frac{d_{p}^{2}\left(\rho_{p}-\rho_{f l}\right) r 2 \pi N}{18 \mu} .
$$

Therefore, the flow will act as a centrifugal cyclone,

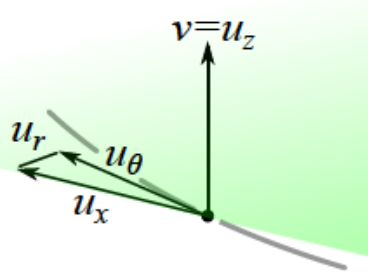

Figure 6.10: Velocity decomposition in the laser plane. cumulating gradually the particles in the periphery of the SSPHE. However, we are only concerned here of the effect that the settling velocity could have during the acquisition of the two consecutive PIV images. The settling velocity in the laser plane $\left(r=r_{l}\right)$ can be evaluated by the above expression, which considering the sampling frequency values employed gives displacements always two or three orders of magnitude lower than the laser sheet thickness. The centrifugal effect on the particles in the out-of-plane motion is hence negligible during the $\Delta \mathrm{T}$ interval.

\section{Measured velocity components}

Recalling Fig. 6.9, it can be used to determine the relationship between the PIV retrieved velocities $u, v$-along the cartesian axis $x$ and $y$ contained in the plane- and the tangential and axial velocities ( $u_{\theta}$ and $u_{z}$ respectively) corresponding to a cylindrical coordinate system centred in the scrapers rotating rod (Fig. 6.10). According to the exposed in the previous subsection, the retrieved velocity component $u$ is the projection of $u_{\theta}$ and obviously $v$ is equal to $u_{z}$. The error $\epsilon$ calculated above due to the out-of- 


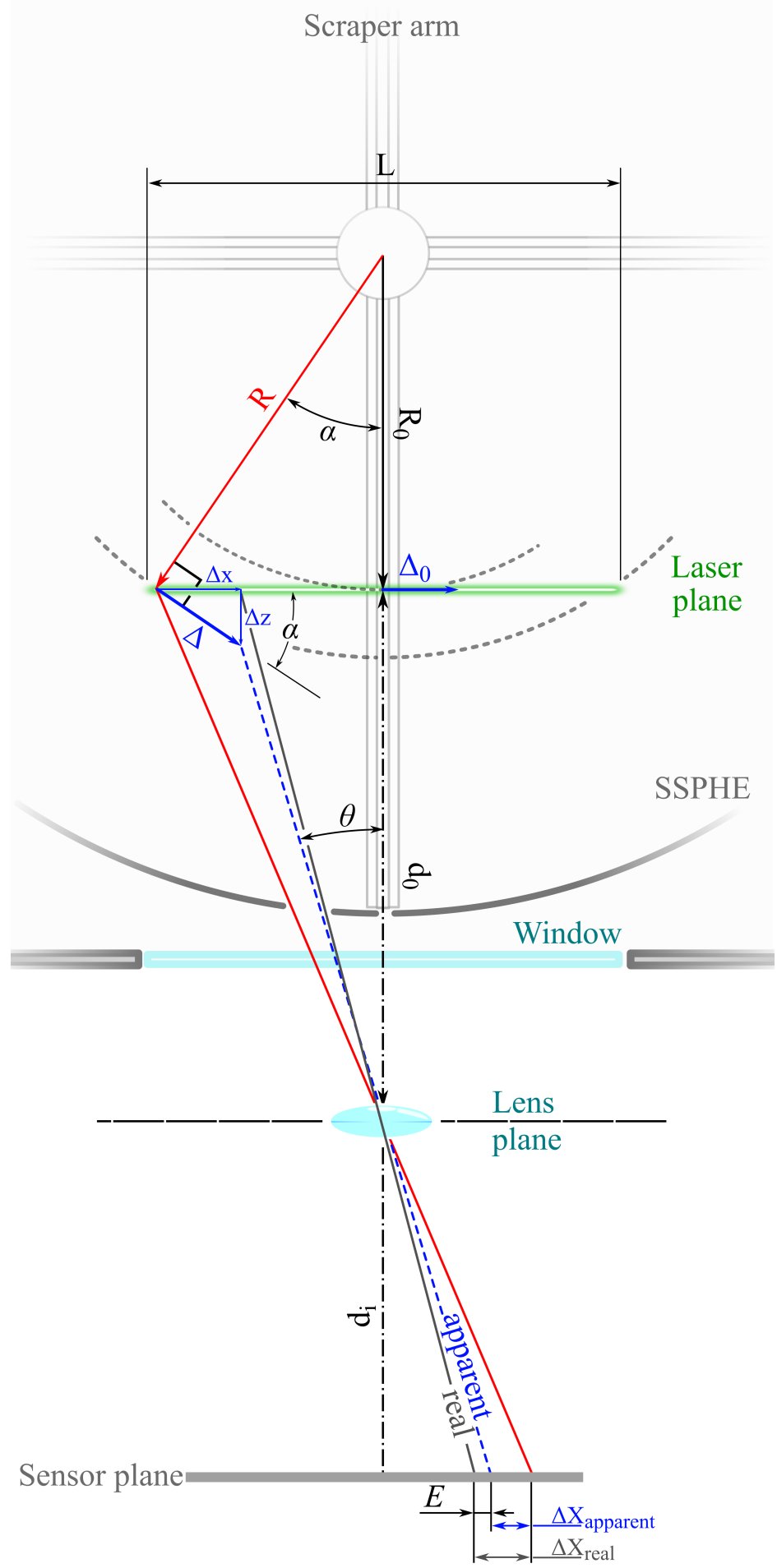

Figure 6.9: Error in the measurement of in-plane displacements due to out-of-plane motion in the SSPHE 


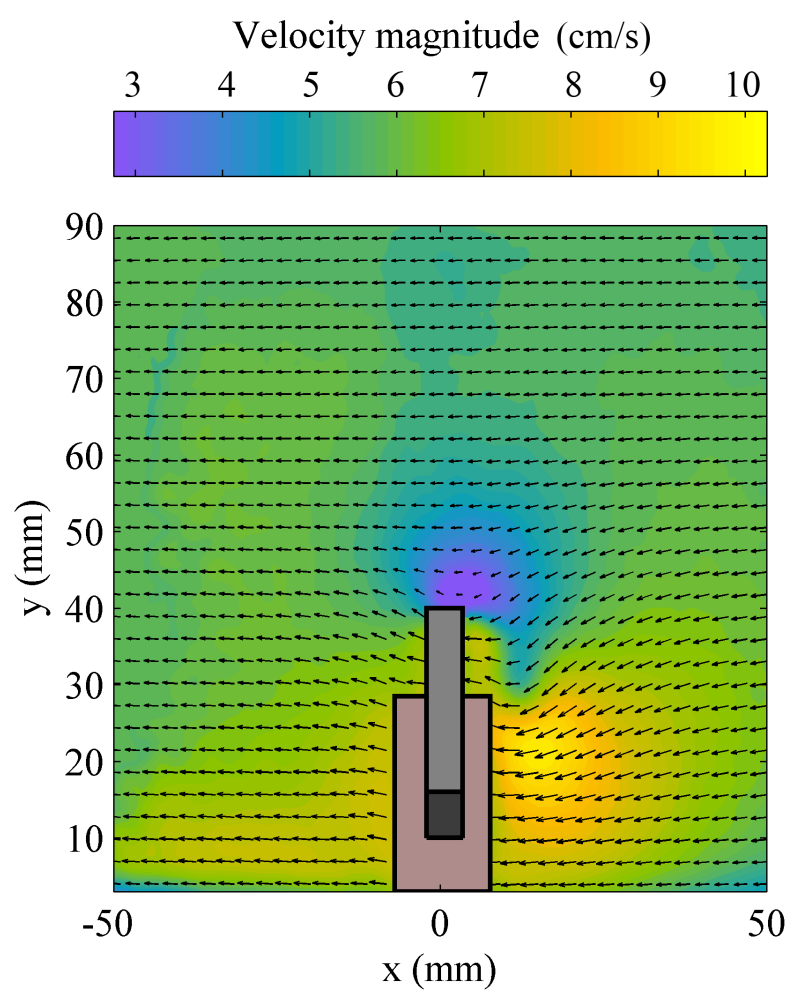

Figure 6.11: Velocity vectors and the corresponding magnitude coloured iso-contours from a stationary frame of reference (scraper moves from right to left). $R e=2.2 \cdot 10^{4}$ ).

plane motion is then associated to the mismatch between $u$ and $u_{\theta}$. As a consequence, and due to its low value, it can be assumed that $u \approx u_{\theta}$.

\subsubsection{Phase-averaged velocity field}

\section{Velocity field and flow pattern}

The velocity vector field is depicted in Fig. 6.11. It reveals a rather uniform velocity field with two distinct regions: a low velocity zone in the tip of the scraper and a high velocity zone in the rear (right) side of the scraper. However, it does not show any relevant information about the flow structure.

It results much more convenient to represent the velocity field relative to the tangential velocity of the scraper at that radial position. As a matter of fact, it is a common practice in the flow study of rotating mixers and stirrers, where the resulting relative velocity is also normalized by the blade tip velocity. The flow appears then as steady, revealing the pattern that explains the mixing process inside this type of devices [111-113]. According to that, Fig. 6.12 shows the velocity vector field relative to the scraper velocity and normalized by it, as it will be shown from now on, where

$$
\hat{u} *_{\theta}=\frac{u_{\theta}}{2 \pi N r_{l}}-1
$$




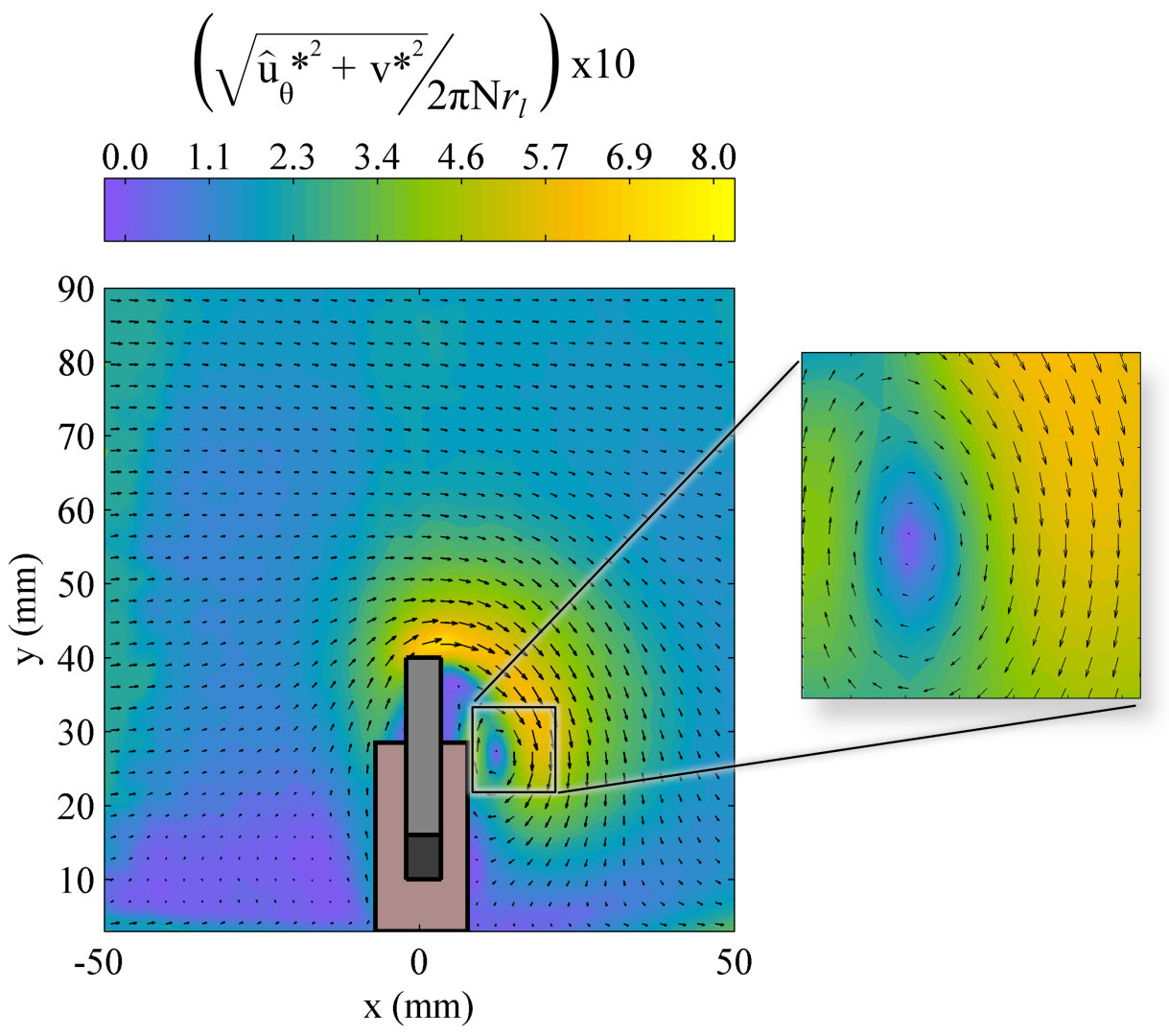

Figure 6.12: Normalized, relative (from a rotating frame of reference) velocity field, $\left.\hat{u} *_{\theta}, v * . \operatorname{Re}=2 \cdot 2 \cdot 10^{4}\right)$.

$$
v *=\frac{v}{2 \pi N r_{l}} .
$$

The relative velocity vector field reveals a recirculation on the rear side of the scraper, corresponding to a roll up vortex [114] formed from tip of the scraper. The low velocity region near the tip of Fig. 6.11 corresponds then to the high relative velocity region (backward flow) at the same location of Fig. 6.12. Inside the SSPHE the fluid motion is only due to the scraper movement, and therefore the scrapers can be seen as paddles, which sustains the rotation of the flow -more or less uniform as seen in Fig. 6.11. This flow, which tends continuously to stop due to the friction with the walls and the viscosity is continuously "pushed" by them, which creates an acceleration when the flow of the front side is deviated and forced to pass over the scraper. At the same time, the fluid on the rear side moves to replace the gap left by the moving scraper.

To compare the flow inside the SSPHE for the different tested rotating Reynolds numbers, the mean tangential and axial normalized velocities $\left\langle\hat{u} *_{\theta}, v *\right\rangle$ are shown in Figs. 6.14 to 6.17 along the lines specified in 6.13. A fast look at them reveals the existence of a common flow pattern to all of them, which tends asymptotically to a flow structure independent of the rotating Reynolds. The same phenomena has been observed in stirrers [115]. 


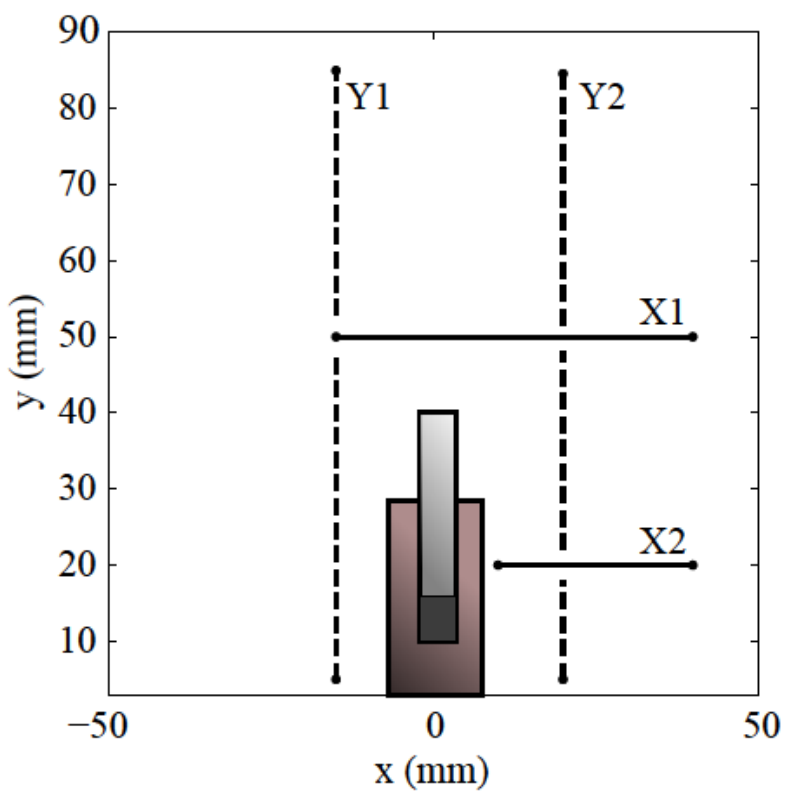

Figure 6.13: Locations of the comparative velocity profiles.

Fig. 6.14 shows the $\hat{u} *_{\theta}$ and $v *$ profiles along the horizontal $\mathrm{X} 1$ line in 6.13 . The $\hat{u} *_{\theta}$ profiles show a peak corresponding to the accelerating region slightly rearer of the tip of the scraper. As it is possible to see, the peak decreases as $\mathrm{Re}_{\text {rot }}$ increases, which means that the flow becomes more uniform. The $v *$ component shows a peak corresponding to the descending velocity due to the vortex. As occurred with $\hat{u} *_{\theta}, v *$ also tends to a $\mathrm{Re}_{\text {rot }}$ independent profile when $\mathrm{Re}_{\mathrm{rot}}$ increases.

The $\hat{u} *_{\theta}$ profiles along X2 (Fig. 6.15), in the rear side of the scraper, show an increase of the velocity as the fluid approaches to the scraper face-in therms of relative values. This can be explained by the vortex observed previously (Fig. 6.12): as the line $\mathrm{X} 2$ approaches the scraper, it enters into the vortex bottom side, increasing the absolute tangential velocity. The same is also observed for the $v *$ profiles, which increases in the same way as $\hat{u} *_{\theta}$. A significant shift between $\hat{u} *_{\theta}$ and $v *$ profiles exists however, which confirms the presence of the vortex: while $\hat{u} *_{\theta}$ increases continuously, $v *$ exhibits a final, sudden decrease, corresponding to the bottom part of the vortex, where its tangential velocity (relative to the vortex center) is parallel to the bottom wall of the SSPHE, and therefore $v *$ tends to 0 . Considering the $\mathrm{Re}_{\text {rot }}$ effect, the profiles always decrease -in absolute value- asymptotically when $\mathrm{Re}_{\text {rot }}$ increases, becoming $\mathrm{Re}_{\mathrm{rot}}$-independent. 
The vertical profiles along Y1 (in the front side of the scraper) are shown in Fig. 6.16. The $\hat{u} *_{\theta}$ velocity above the scraper tip level (dashed line) are quite uniform and $\mathrm{Re}_{\mathrm{rot}}$-independent. The values are higher compared with the part below the dashed line. This is expectable, as the relative flow to the scrapers has to pass above them. Below the dashed line the normalized tangential velocity decreases with a significant difference between the two initial values of $\mathrm{Re}_{\mathrm{rot}}$ and the last three of them. This can be associated with the increment of the turbulence in the flow, with more uniform velocity profiles in the higher $\mathrm{Re}_{\text {rot }}$ cases. The $v *$ profiles reveals a peak slightly below the scraper level, whereas they are almost equal to 0 in the other points. The peak corresponds to the flow that starts to ascend in order to overpass the scraper. As Y1 is located "upstream" of the scraper, the profiles are less affected by the scraper effect, and therefore are quite $\mathrm{Re}_{\mathrm{rot}}$-independent.

The profiles along Y2 (Fig. 6.17) correspond to the vertical coordinates of Y1, but in the rear side of the scraper, that is "downstream". The distribution of $\hat{u} *_{\theta}$ denotes a nose tip at the height of the scraper's tip (dashed line), corresponding to the roll up vortex top side. Below the dashed line, at $\mathrm{y} \approx 20 \mathrm{~mm}$ the nose appears inverted, as the vortex velocity is already turned and going towards the scraper rear side -the vortex bottom part. The $v *$ distribution shows a single peak between the two noses of $\hat{u} *_{\theta}$, corresponding to the right part of the vortex, where the velocities are parallel to the SSPHE shaft. As observed in all the previous figures, the velocity profiles tend asymptotically to $\mathrm{Re}_{\mathrm{rot}}$-independent ones as $\mathrm{Re}_{\mathrm{rot}}$ increases. The asymptotic profile corresponding to high $\mathrm{Re}_{\mathrm{rot}}$ is smother than those at lower $\mathrm{Re}_{\mathrm{rot}}$, which means that increasing the scraping velocity, the mean flow velocity increases, and the differences between high and low velocity regions is decreased. The later can be directly related with an increasing in the mixing level inside the SSPHE.

To observe more graphically the differences between the flow at low and high $\mathrm{Re}_{\text {rot }}$ values, Fig. 6.18 shows the streamlines computed from the PIV velocity vector fields for

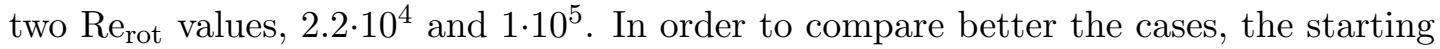
coordinate location of the streamlines in both cases is the same, equally distributed along the Y1 line of Fig. 6.13. In the lower $\mathrm{Re}_{\text {rot }}$ pattern, only the streamlines below the scraper tip level have positive inclinations, as a consequence of the deviation of the flow to avoid the scraper. Streamlines above that imaginary line are horizontal or even with negative inclination, as a consequence of the roll up vortex on the rear side of the scraper. On the contrary, in the highest $\mathrm{Re}_{\text {rot }}$ case all the streamlines exhibit a positive inclination. The higher velocity of the scraper affects now all the fluid, deviating it to the top. The streamlines will turn down presumably, out of the studied region. The increase of the scraping velocity leads then to higher mixing, where more fluid regions are forced to travel from the bottom to the top part of the SSPHE and vice versa.

As Fig. 6.18 shows, the flow deviates from the bottom part towards the top to avoid the scraper. Once it arrives to the scraper's tip, it cannot just turn around. As the Kutta condition [116] states for the viscous flows, any steady flow cannot turn around a sharp corner with a nonzero velocity. The separated layer from the scraper's tip then rolls up into an spiral, leading to a vortex, as it is shown in Fig. 6.19. What the PIV phase-averaged velocity field is able to show is only the mean vortex. However, this vortex leads to the generation of secondary small vortex along the spiral path [114]. This downward scale of vortex formation is the main responsible of mixing in stirrers $[111,117,118]$ and therefore it can be also in the SSPHE. 


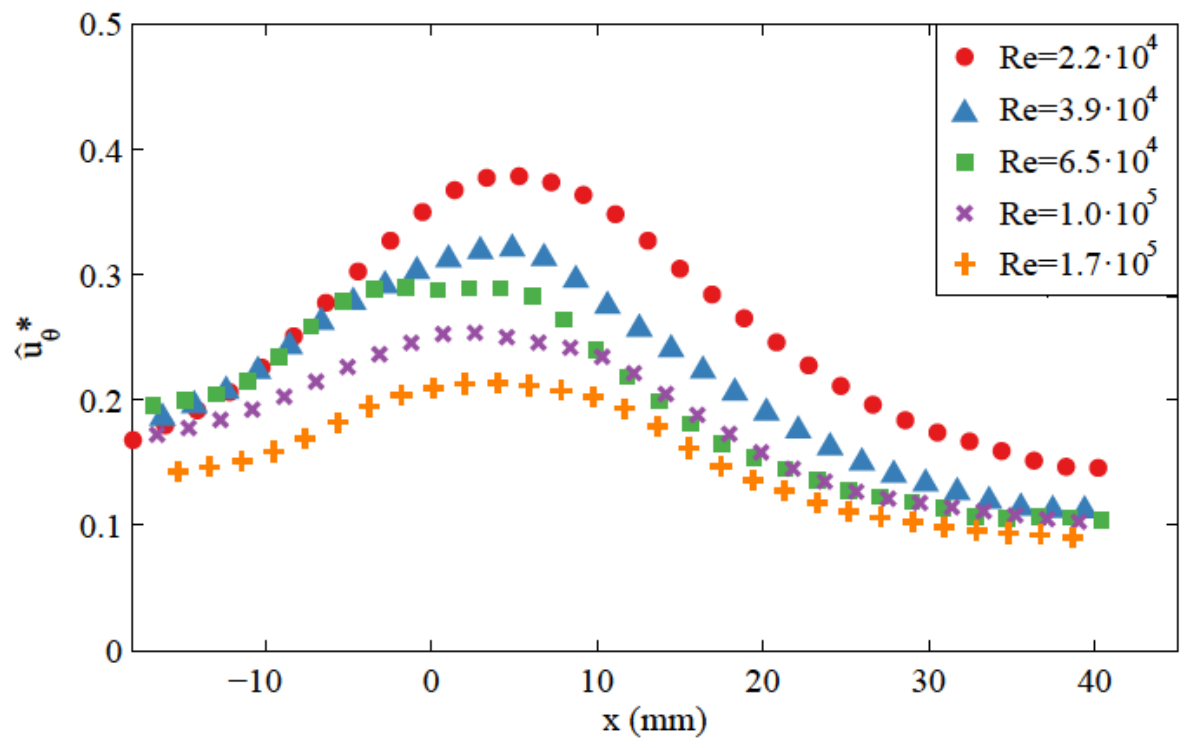

a)

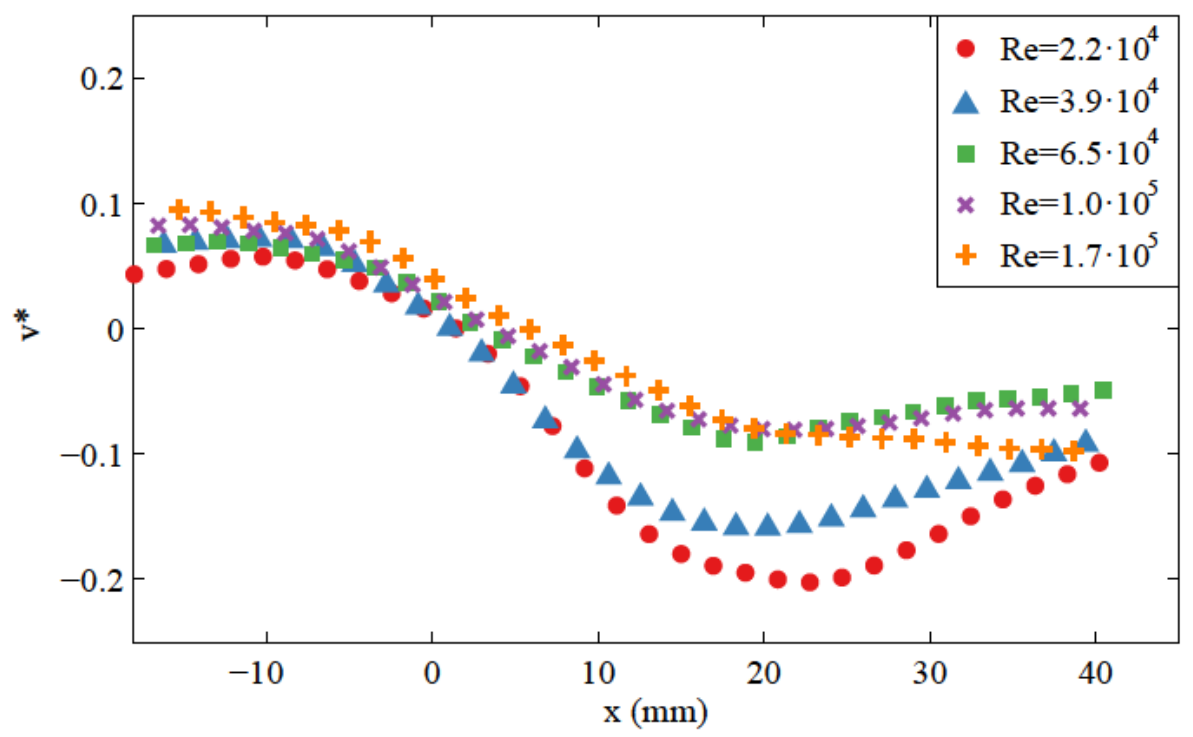

b)

Figure 6.14: Tangential and axial velocity profiles at X1. a) $\left.\hat{u} *_{\theta}, \mathbf{b}\right) v *$. 


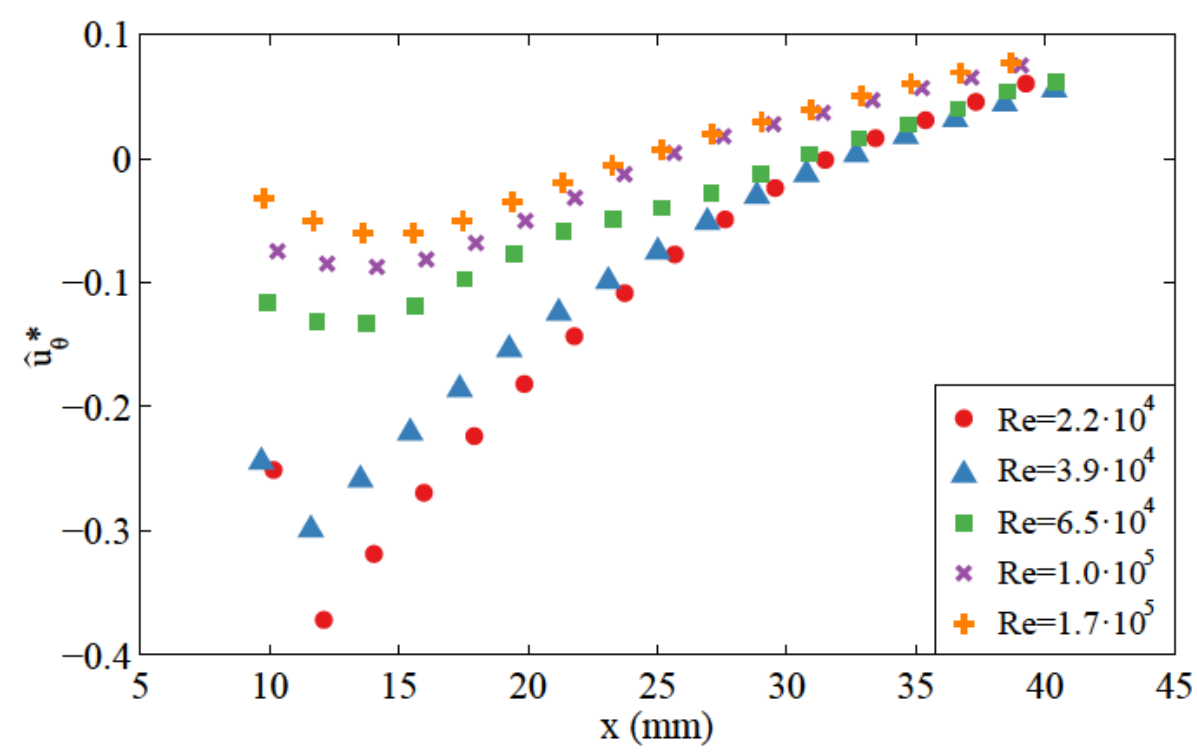

a)

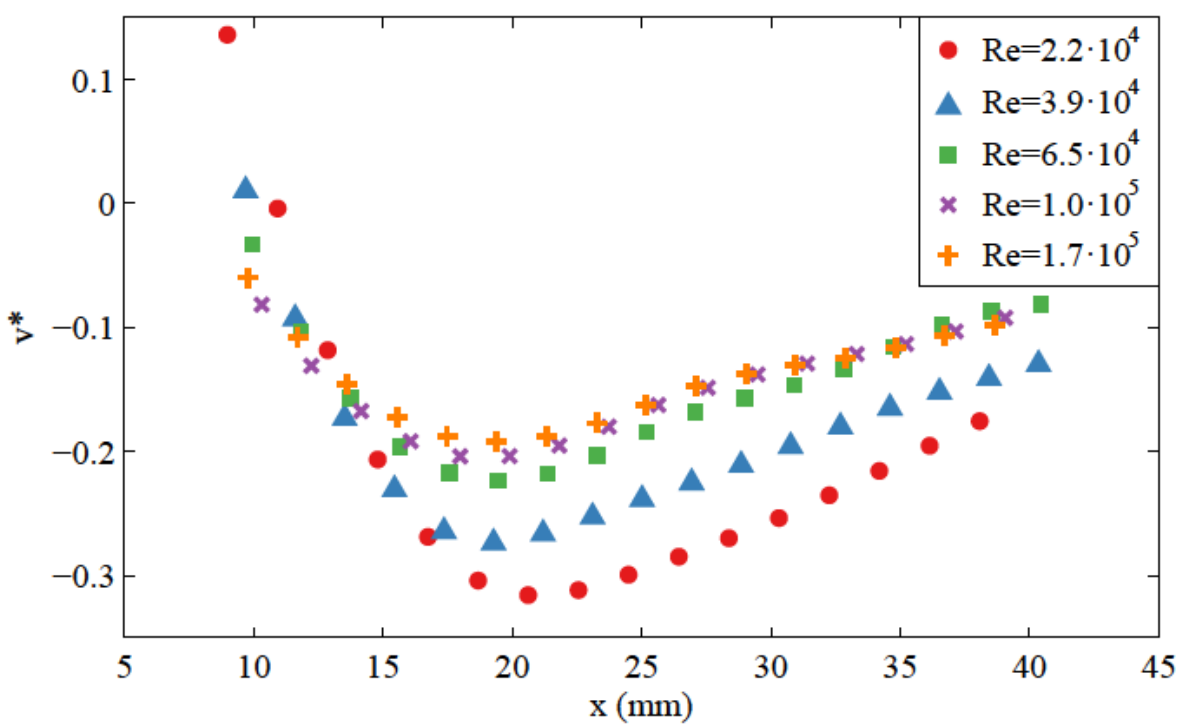

b)

Figure 6.15: Tangential and axial velocity profiles at X2. a) $\hat{u} *_{\theta}$, b) $v *$. 


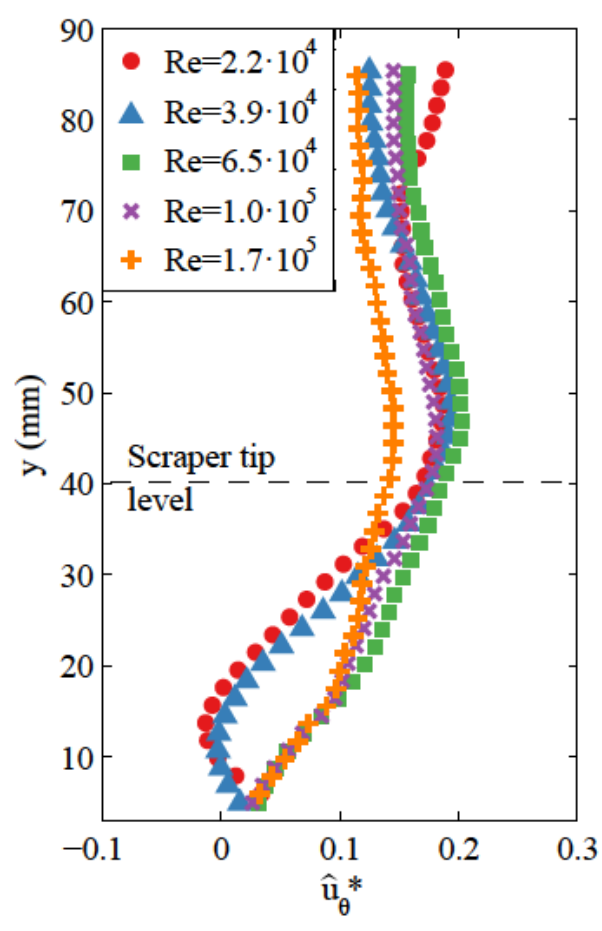

a)

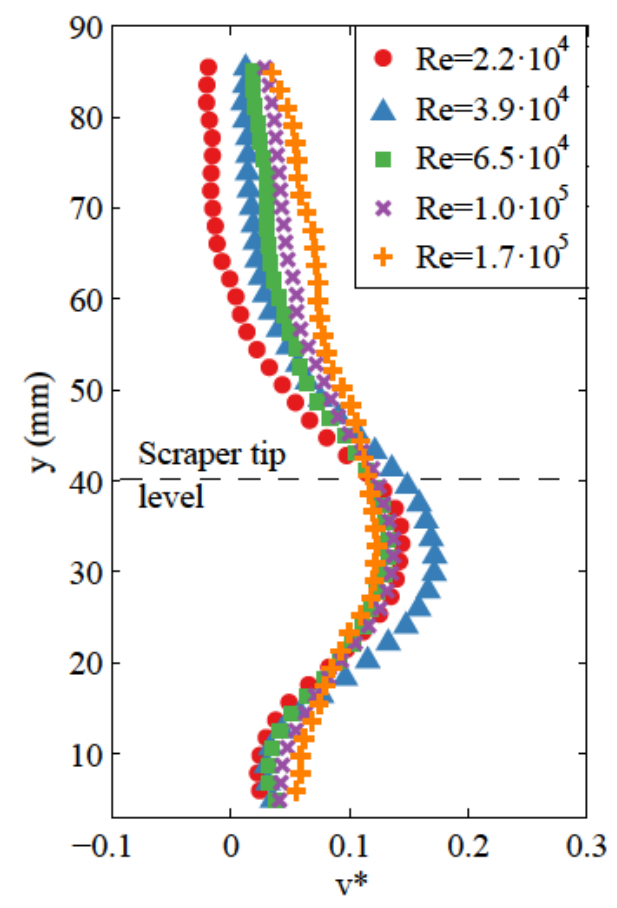

b)

Figure 6.16: Tangential and axial velocity profiles at Y1. a) $\left.\hat{u} *_{\theta}, \mathbf{b}\right) v *$.

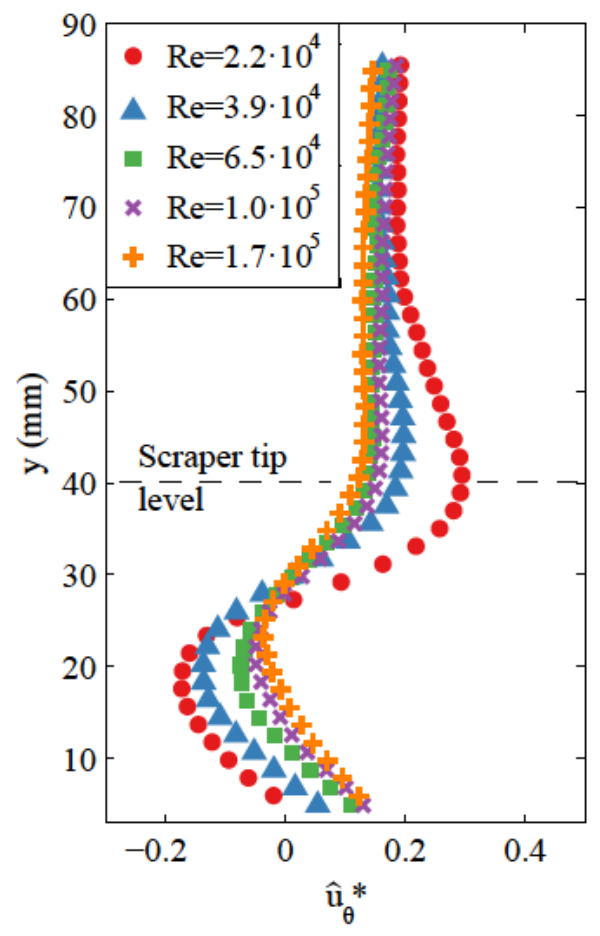

a)

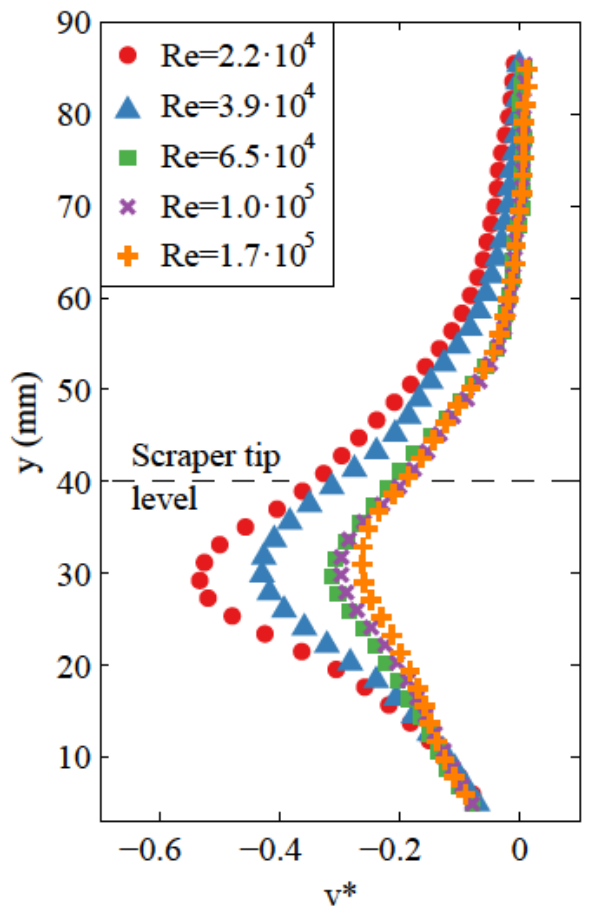

b)

Figure 6.17: Tangential and axial velocity profiles at Y2. a) $\left.\hat{u} *_{\theta}, \mathbf{b}\right) v *$. 


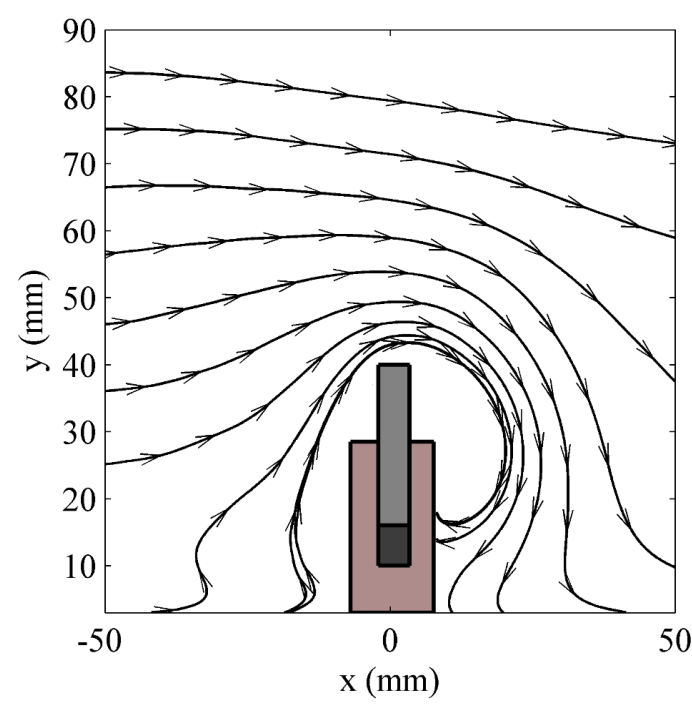

a)

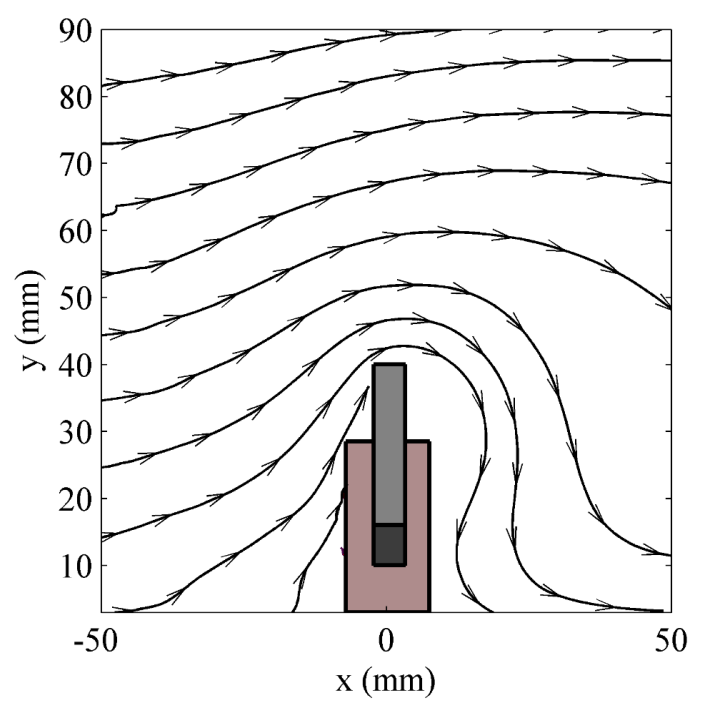

b)

Figure 6.18: Streamlines computed from the velocity relative to the scraper in the laser plane for $\mathbf{a}) \mathrm{Re}=2.2 \cdot 10^{4}$ and $\left.\mathbf{b}\right) \mathrm{Re}=1 \cdot 10^{5}$.

Fig. 6.19 shows the roll up vortex for two different $\operatorname{Re}_{\text {rot }}$ values through the streamlines and the normalized, relative velocity contours. When the scraper velocity increases the vortex elongates along the spiral vertical axis and moves its rotation center slightly upwards. At the same time its orientation changes, turning the spiral main axis towards the scraper. The velocity contours show also the minimum velocity value in the vortex core. On the left-side of the vortex core, between it and the scraper, it is possible to see a small region where there is a slight velocity increase due to the acceleration of the vortex velocity as the space is narrowed.

In order to study the roll up vortex, Fig. 6.21 shows, for each $\mathrm{Re}_{\text {rot }}$ tested, the vortex tangential velocity profiles from its core along a radial line at $45^{\circ}$. The vortex tangential velocity $u_{\theta, v}$-referred to the vortex rotation center- has been normalized with the scraper velocity $2 \pi \mathrm{N} r_{l}$, whereas the corresponding radial coordinate has been normalized with $r_{l}$, that is $r *=r_{v} / r_{l}$. In all the cases the velocity increases from the vortex core till a maximum, decreasing after with an absolute slope that is slower than the previous rising part. It is also clear that, as the scrapers rotating velocity increases $\left(\mathrm{Re}_{\mathrm{rot}}\right.$ increases), the vortex velocity tends to one asymptotic profile where the peak ratio is smaller; the mean velocity of the flow is higher and the flow field more uniform.

The vortex tangential velocity can be also represented normalized by its maximum value $u_{\theta, v} / \max \left(u_{\theta, v}\right)$, and normalizing the vortex radial coordinate $r_{v}$ by the location $\delta_{v}$ where the maximum velocity takes place. Fig. 6.21 a compares the different tested cases by using this procedure. All the velocity profiles tends clearly to a unique one, with a not evident effect of the $\mathrm{Re}_{\text {rot }}$ number. In Fig. $6.21 \mathrm{~b}$ it has been included the solution provided by different classical models. The continuous line corresponds to the Rankine model [119], where: 


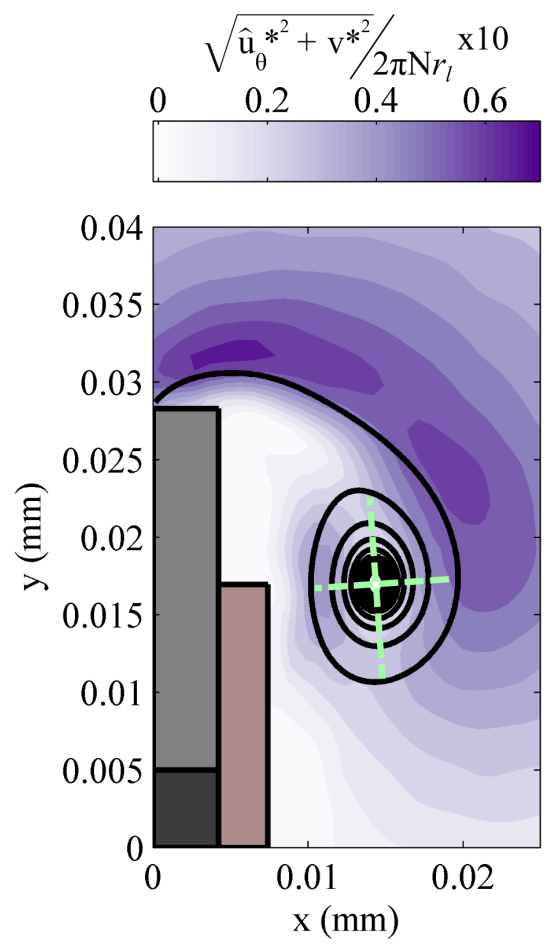

a)

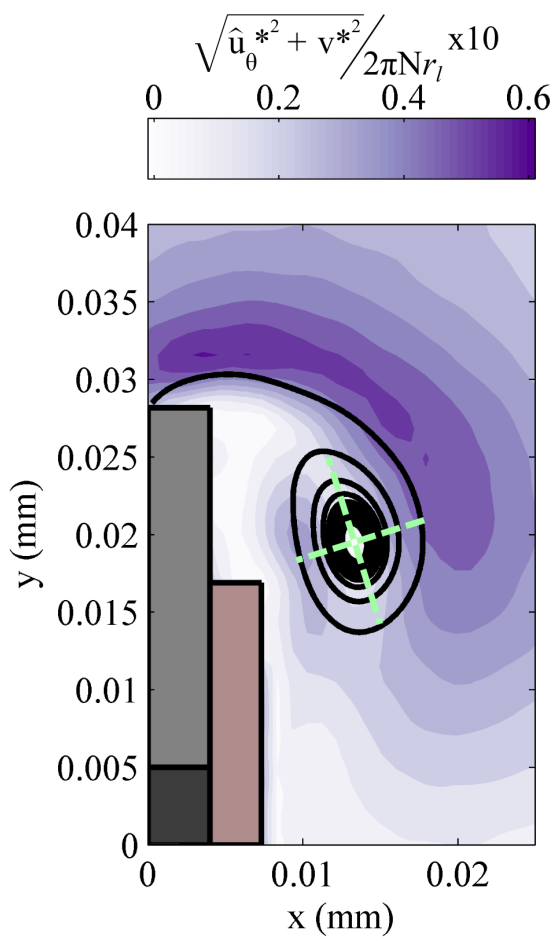

b)

Figure 6.19: Roll up vortex for $\mathbf{a}) \operatorname{Re}=2.2 \cdot 10^{4}$ and $\left.\mathbf{b}\right) \mathrm{Re}=3.9 \cdot 10^{4}$.

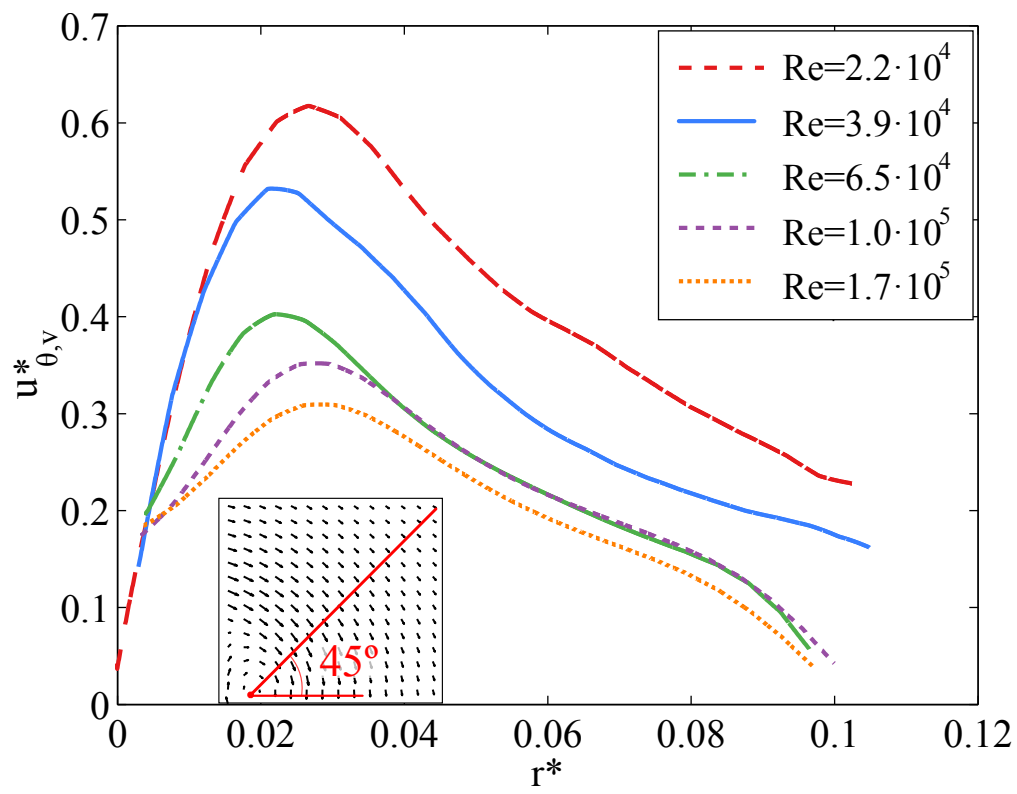

Figure 6.20: Vortex dimensionless tangential velocity along its radius $\left(r_{v} / r_{l}\right)$ for the different $\mathrm{Re}_{\text {rot }}$ tested. 


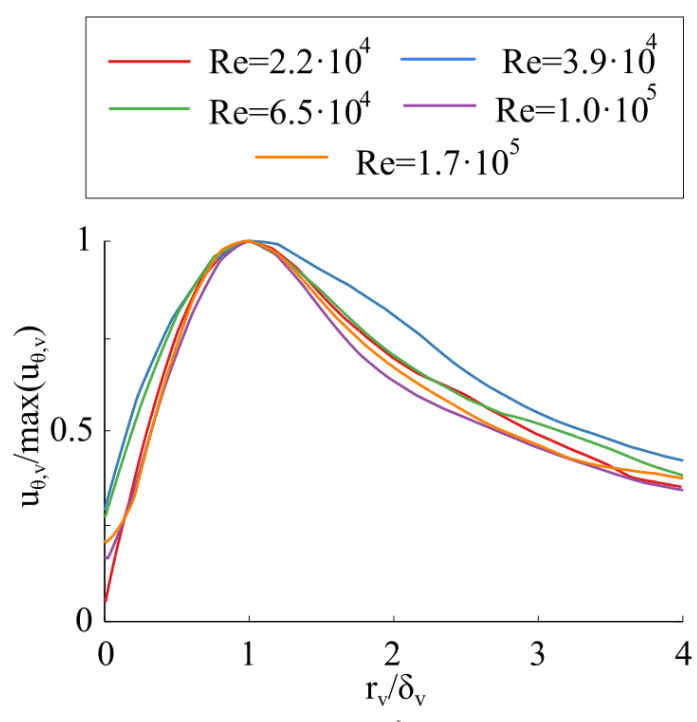

a)

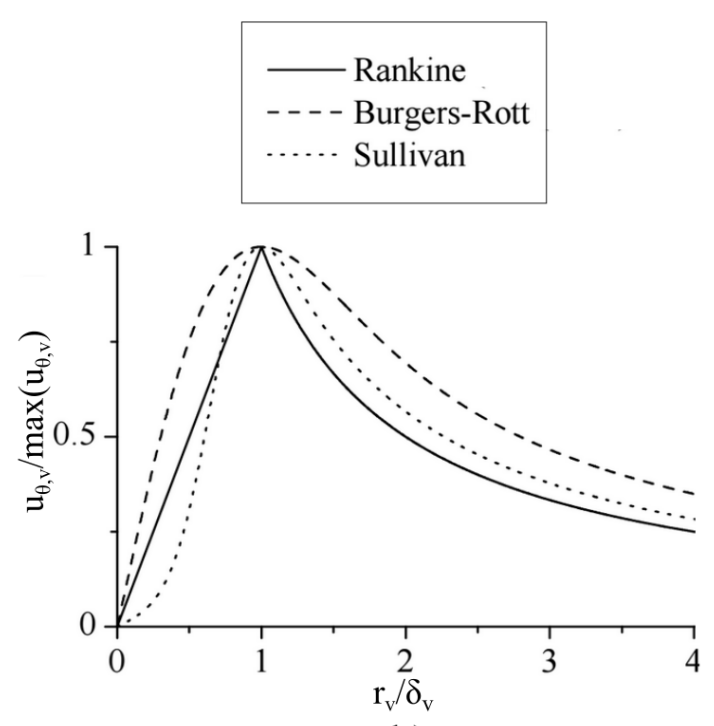

b)

Figure 6.21: Vortex normalized swirl velocity for the different tested cases (a) compared with different classical solutions (b) from [121].

$$
\frac{u_{\theta, v}}{\max \left(u_{\theta, v}\right)}= \begin{cases}\frac{r_{v}}{\delta_{v}}, & r_{v} \leq \delta_{v} \\ \frac{\delta_{v}}{r_{v}}, & r_{v}>\delta_{v}\end{cases}
$$

As established by Rankine, on 6.21a for $r_{v} / \delta_{v} \leq 1$ the velocity increases approximately proportional to the radius, as it would occur in a solid rotating cylinder. The outer part where the velocity decreases corresponds to an irrationally vortex, where the velocity is proportional to the inverse of the radius. In spite of the Rankine's model simplicity, it provides an acceptable description of the velocity inside the vortex. The more complex Burgers-Rott model [120] is however the one that predicts better the vortex velocity field.

The roll up vortex is the responsible of an important secondary flow in the SSPHE compared with common stirrers with vertical paddles, where the mixing blades are far enough of the bottom of the agitated tank. In such situations, as when a plate moves in a normal direction, two symmetric roll up vortices appears in each of the blade ends $[112,113]$. The symmetric, counter-rotating vortices, counteract any vertical flow beyond the symmetry plane. However, in the SSPHE case the vortex only appears in the top side of the scraper, which creates a net axial flow towards the SSPHE bottom plate (see Fig. 6.18). This axial flow, which enhances the mixing, has also a negative aspect. If it is strong enough, it can transport the scraped ice particles to impact against the bottom plate, which will increase the nucleation and promote the ice layer.

The flow pattern in the SSPHE retrieved by the PIV technique can be represented by the sketch of Fig. 6.22. The sketched flow pattern considers only the in-plane flow and, as a consequence of the technique limitation, does not reproduce any radial flow component. Three different regions can be distinguished. On the top there is an 


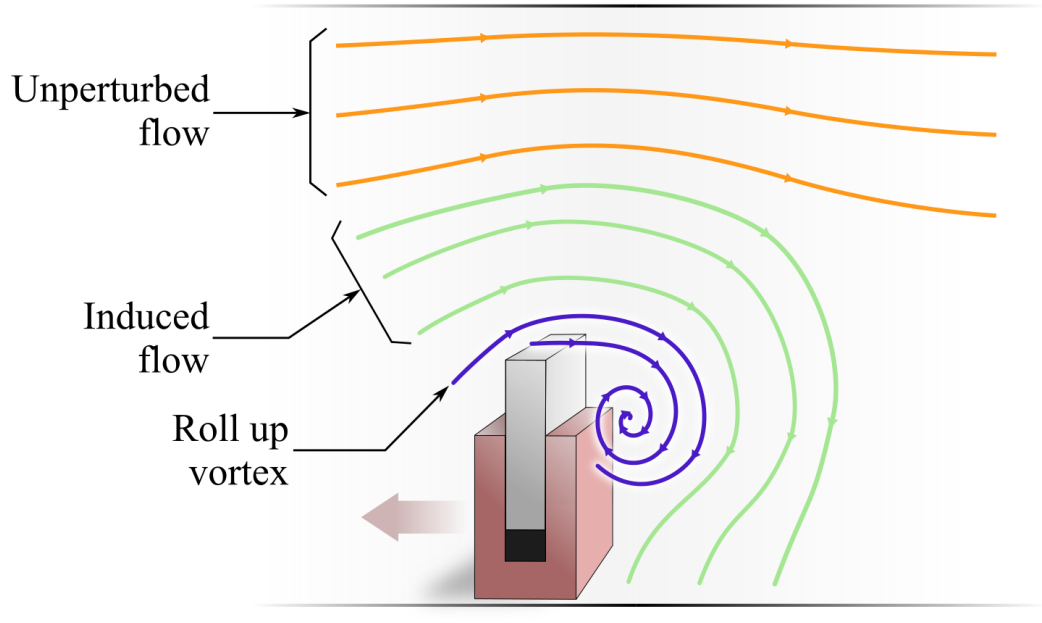

Figure 6.22: Sketch of flow pattern inside the SSPHE retrieved from PIV experiements. Radial components are not considered.

unperturbed flow. This flow is parallel to the top wall, without mixing with the rest of the fluid volume. In the rear side of the scraper a roll up vortex is formed. Between the roll up vortex and the top, unperturbed flow, a deviated flow region appears without entering completely in the vortex. It results in an induced vertical -or axial- flow towards the bottom. The spatial limit between these regions varies with the rotating Reynolds number, increasing the mixing as it increases.

Comparing the described flow structure with the flow patterns that can be found in mixers or stirrers, Winardi et al. [122] found that when employing vertical paddles the flow pattern can be divided in turn into different ones that can exist independently or coexist. One of the more probable flow patterns matches the one observed here, with the formation of an starting vortex backwards the impeller. However, this flow pattern implies also a strong radial component, something that due to the technique used here could not be studied. The similarities of the flow pattern between the one observed for the SSPHE and those corresponding to stirrers are a support for the obtained data but at the same time confirm the requirement of numerical simulations that can provide additional information about the three dimensional flow. 


\section{Vorticity}

In order to investigate the structure of the roll up vortex and the separating shear layer on the scraper's tip, vorticity has been calculated from the phase-averaged, relative velocity fields. It was normalized with the angular velocity of the scrapers as

$$
\Omega *=\frac{\left(\frac{\partial v}{\partial x}-\frac{\partial \hat{u}_{\theta}}{\partial y}\right)}{2 \pi N}
$$

Fig. 6.23 shows the contour maps for $\Omega *$. The minimum contour level has been chosen as 4 , which is twice the one of the rigid body rotation of the scrapers.

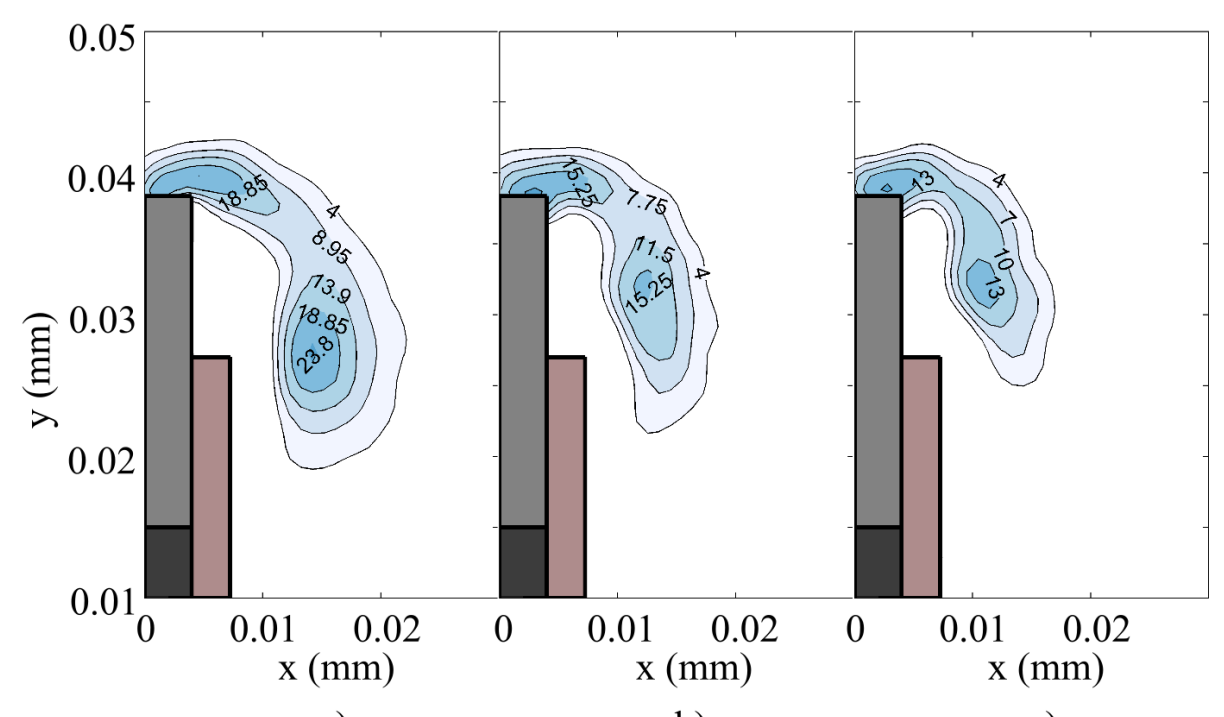

a)

b)

c)

Figure 6.23: Dimensionless vorticity contours for a) $\operatorname{Re}=2.2 \cdot 10^{4}$, b) $\operatorname{Re}=6.5 \cdot 10^{4}$ and c) $\operatorname{Re}=1.7 \cdot 10^{5}$.

Vorticity is concentrated in two locations: in the scraper's tip -the area corresponding to the separation shear layer- and in the roll up vortex core. The dimensionless vorticity peaks in the second location are higher than in the first one. If the $\mathrm{Re}_{\mathrm{rot}}$ value increases the vortex core rises its location, whereas $\Omega *$ decreases. The vorticity increases with $\mathrm{Re}_{\mathrm{rot}}$, but the relative values do not behave equally, which means that the increasing in the scrapers rotating velocity is not proportional to the swirling of the vortex.

The later is more noticeable in Fig. 6.24, which includes the variations of the peak dimensionless vorticity value for the two maximum locations, the scraper's tip and the roll up vortex. Both decreases as $\mathrm{Re}_{\text {rot }}$ increases, but the dimensionless vorticity separation shear layer does faster, being always the one of the vortex higher, even if for the lowest $\operatorname{Re}_{\text {rot }}$ both were almost equal. 


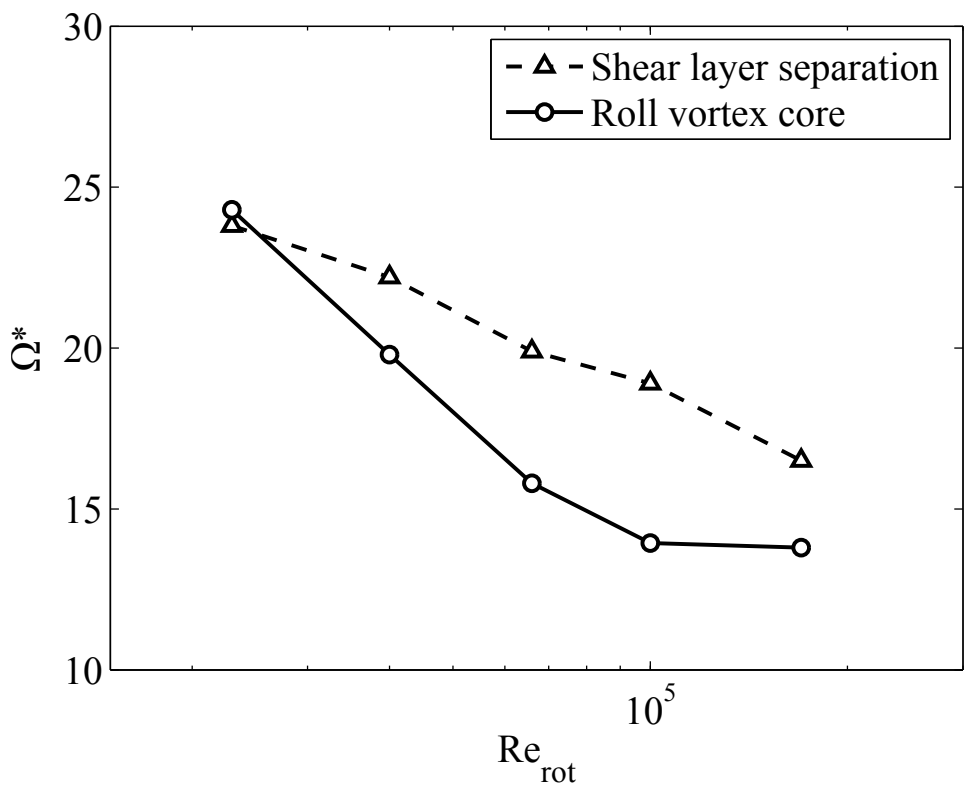

Figure 6.24: Dimensionless vorticity peak values $\Omega *$ variations for the tested rotative Reynolds number.

\section{Turbulent kinetic energy}

In the previous sections it has be shown the existence of a mean rotating flow induced by the scrapers. It has been also shown that the scrapers create local perturbations in the form of roll up vortices, which are quasi-steady from a rotating reference frame, or fluctuating from an steady reference frame. As it can be deduced from the Re $\mathrm{rot}_{\mathrm{r}}$ values, turbulent fluctuations have to be also present, which finally dissipate the kinetic energy.

According to the decomposition of the velocity into a mean value plus the turbulent fluctuations,

$$
u=\bar{u}+u^{\prime}=U+u^{\prime},
$$

the turbulent kinetic energy $k$ is calculated from three RMS velocity components by

$$
k=\frac{1}{2}\left(u^{\prime 2}+v^{\prime 2}+w^{\prime 2}\right)
$$

However, as there is no knowledge of the third RMS velocity -the radial component$k$ can be estimated by using a pseudo-isotropic assumption:

$$
k=\frac{3}{4}\left(u^{\prime 2}+v^{\prime 2}\right)=\frac{3}{4}\left(u_{\theta}^{\prime 2}+v^{\prime 2}\right)
$$

Even if the above formula has been successfully considered by different authors, finding only small differences when comparing with 3D PIV values [123, 124], the study of the turbulence flow from planar PIV in "strong" 3D flows has to be taken with care. The out-of-plane motion is proved to have a considerable impact in the turbulence analysis [125]. Even if the out-of-plane motion was considered negligible in 
the previous section, such assumption was done only based in the consideration that the out of plane motion was produced by a circular flow. However, and due to the similarities that the flow pattern observed in the SSPHE shares with the flow pattern reported in similar works regarding mixers and stirrers, a considerably radial flow may exists.

The turbulent kinetic energy, computed as indicated in 6.13, was nondimensionalized by the square of the scraper tangential velocity at $r=r_{l}$ :

$$
k *=\frac{k}{\left(2 \pi N r_{l}\right)^{2}} .
$$

Figure 6.25 depicts the dimensionless turbulent kinetic energy, $k *$, contours for the case $\operatorname{Re}_{\text {rot }}=2.2 \cdot 10^{4}$. The contours shows the higher values of $k *$ in the rear side of the scraper, with an almost uniform value elsewhere. The maximum value of $k *$ is located in the position of the roll up vortex. This peak value in the vortex core is connected with the scraper tip through a bridge of high $k *$ values, which can be associated to the high shear of the separated flow.

In order to compare the effect of the Reynolds number, Fig. 6.26 zooms the scraper's rear side region for three different $\operatorname{Re}_{\text {rot }}$ values. The peak value of $k *$ decreases with $\mathrm{Re}_{\mathrm{rot}}$, but at the same time

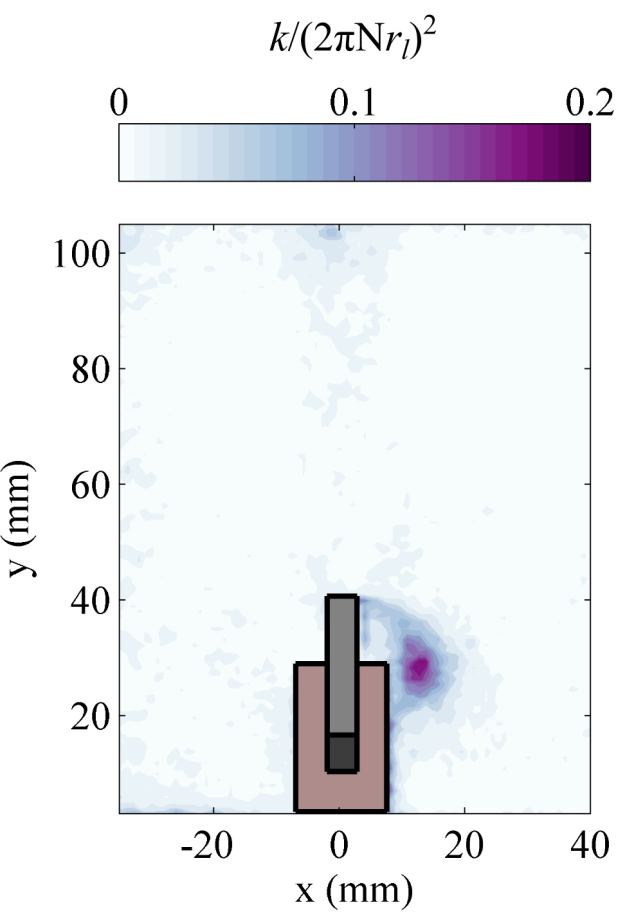

Figure 6.25: Dimensionless turbulent kinetic energy $k *$ contours in the investigated area for $\mathrm{Re}=2.2 \cdot 10^{4}$. the turbulent kinetic energy increases in the path between the peak ant the scraper's tip. These results are in agreement with those observed in stirred vessels, where several authors noticed the link that exists between turbulence and roll up and trailing vortices [111]. In fact, they observed that the maximum turbulent kinetic energy is higher in the vortex region than elsewhere. Fig. 6.26 also shows that increasing the rotating velocity of the scrapers also increases the area with high turbulent kinetic energy. It can be then deduced that the roll up vortex is the main responsible of the energy transfer from the rotation of the blades to the creation of turbulent kinetic energy.

The relationship between the $\mathrm{Re}_{\text {rot }}$ number and the peak $k *$ value obtained for each case is shown Figure 6.27. According to that, there is a clear link between them of the type $k * \propto \operatorname{Re}_{\text {rot }}^{1 / 2}$.

\subsubsection{Instantaneous velocity field}

The phase-averaging of the velocity field provided reliable information about the flow in the SSPHE. However, the averaging can hide some of the phenomena inside. Individual observation of each vector field revealed different patterns in each frame, having all in common the structure described in the previous section and sketched in Fig. 6.22. For 
instance, Fig. 6.28 shows different vector fields processed from a single pair of images each. In each one it is possible to observe the presence of the roll up vortex, but its precise location and extension varies. Moreover, secondary structures appear on the right bottom area. Some of the variations can be attributed to the turbulent flow, but can also denote the presence of other type of flow oscillations.

The study of this pattern modes is complex, as the vector field retrieved from a single pair of images posses an elevated uncertainty. Only certain numerical approaches, like Large Eddy Simulations or Direct Numerical Simulations, can provide clear and valid information. However, it provides an idea of the complexity of the flow inside of the SSPHE.

\subsection{Flow pattern influence in the heat transfer results}

The results presented here by the different visualization techniques can provide insight into the heat transfer process already studied in chapter 4. Recalling here Figs. 4.9 and 4.10 , the effect of increasing the scraping velocity was a slow augmentation in the heat transfer coefficient. This augmentation, more significant in the lower scraping velocities, is in general favoured by the presence of adaptable scraping systems, with respect to the rigid scrapers design.

The PIV results presented in this chapter can support this behaviour. Figures from Fig. 6.14 to Fig. 6.17 show that the flow pattern in the SSPHE exhibits small variations for the range of the scraping velocities studied. The characteristic flow pattern described in Fig. 6.12 reveals the roll up vortex as the main mixing agent. The higher scraping velocity values compared to the lowest one provide higher fluid velocities and a turbulence increment, but no significant variations in the flow pattern. This can justify the absence of significant augmentations in heat transfer, as it occurs in other situations where the flow pattern experiences significant changes. For instance, Solano [69] found a clear effect in the heat transfer when increasing the scraping velocity in alternative scraping surface tubular heat exchangers, finding at the same time flow pattern variations with the formation of vortices and recirculations responsible of the enhancement in heat transfer.

Moreover, the stratification tests have revealed that the stratification of the ice crystals, strong at low scraping velocities, decreased to almost disappear when the scraping velocity increased (see Fig. 6.7). When the flow is less stratified the content of ice particles on it increases. Due to the higher velocities the turbulence increases too. Even if it has not been quantitatively demonstrated by the results in this chapter, the impact of the particles over the plate can play an important role in the ice layer formation, and then it can also explain the low effect of the higher scraping values, expected to remove faster the formed ice layer: the ice layer is being formed at higher rates.

Finally, and reinforcing the conclusion formulated in chapter 4, it can be then assumed that the main resistance to heat transfer is due to the ice layer formation and removal, and not to the flow mixing, clearly visible in Figs. 4.9 and 4.10. 


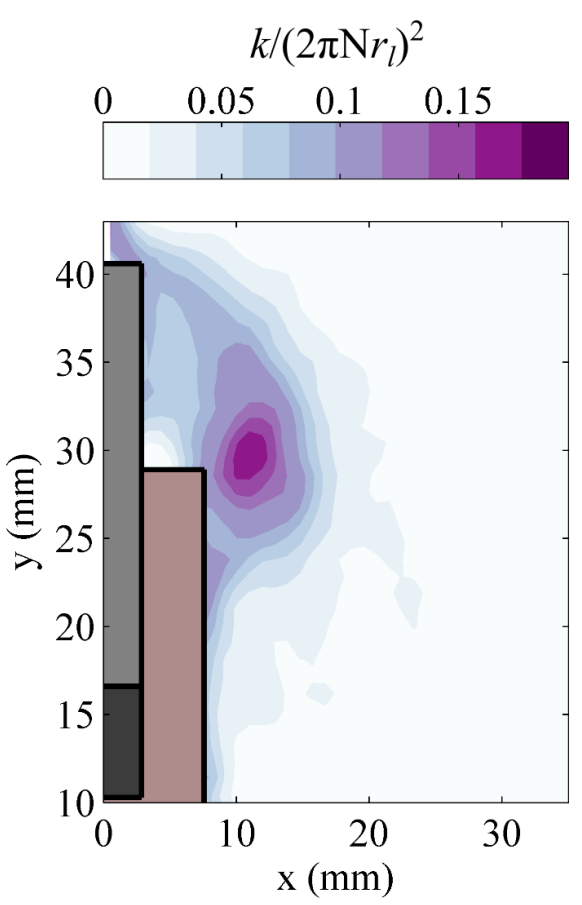

a)

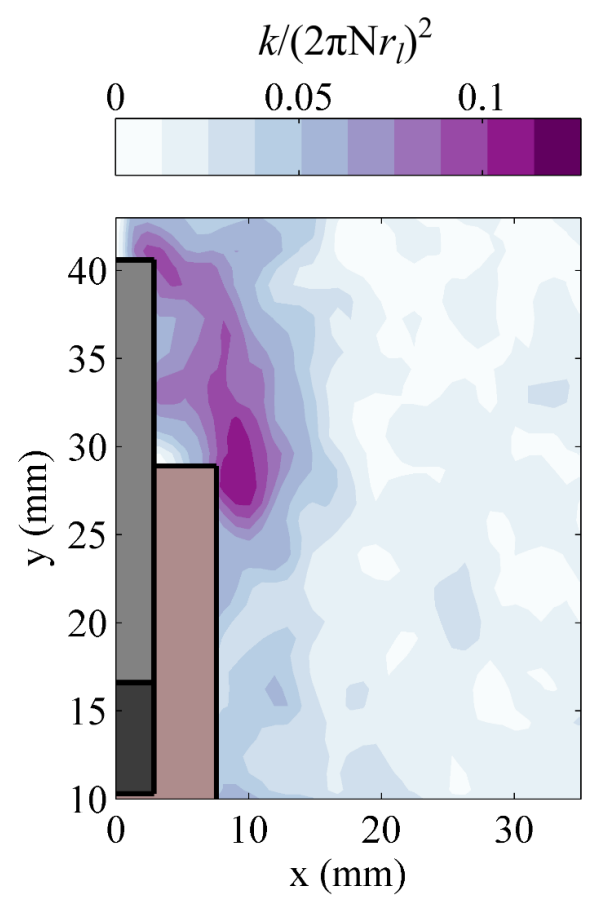

c)

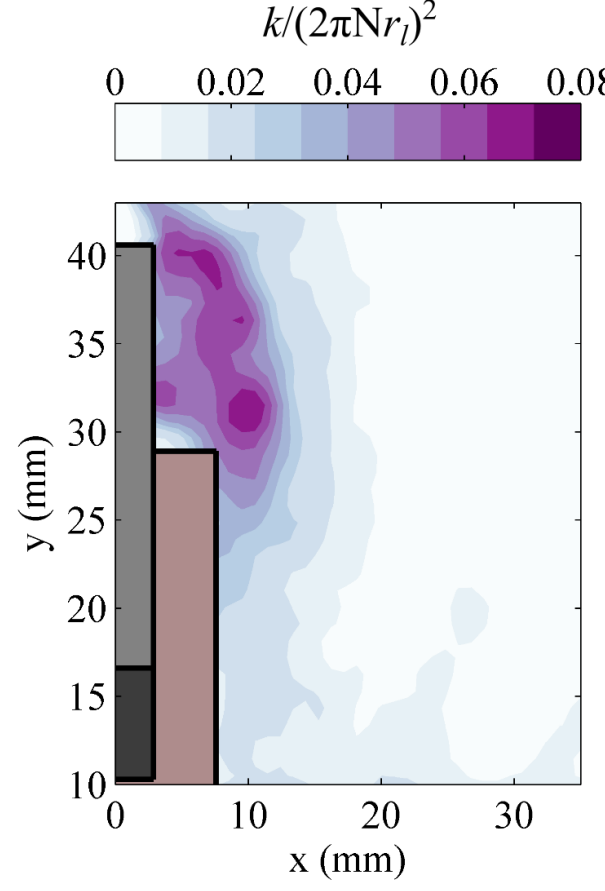

b)

Figure 6.26: Dimensionless turbulent kinetic energy $k *$ contours for a) $\operatorname{Re}=2.2 \cdot 10^{4}, \mathbf{b}$ ) $\mathrm{Re}=6.5 \cdot 10^{4}$ and $\left.\mathbf{c}\right) \mathrm{Re}=1.7 \cdot 10^{5}$. 


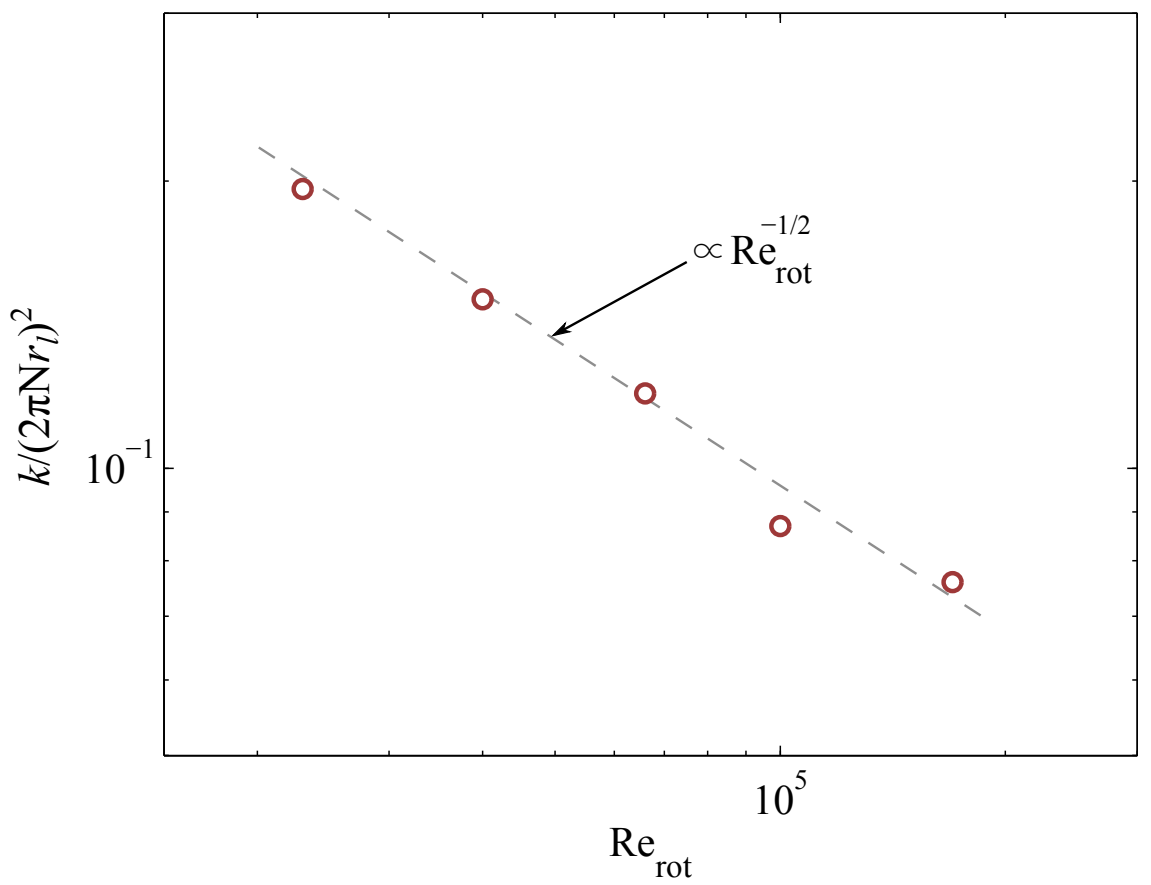

Figure 6.27: Dimensionless turbulent kinetic energy $k *$ peak values for the different Re $_{\text {rot }}$ values tested. 

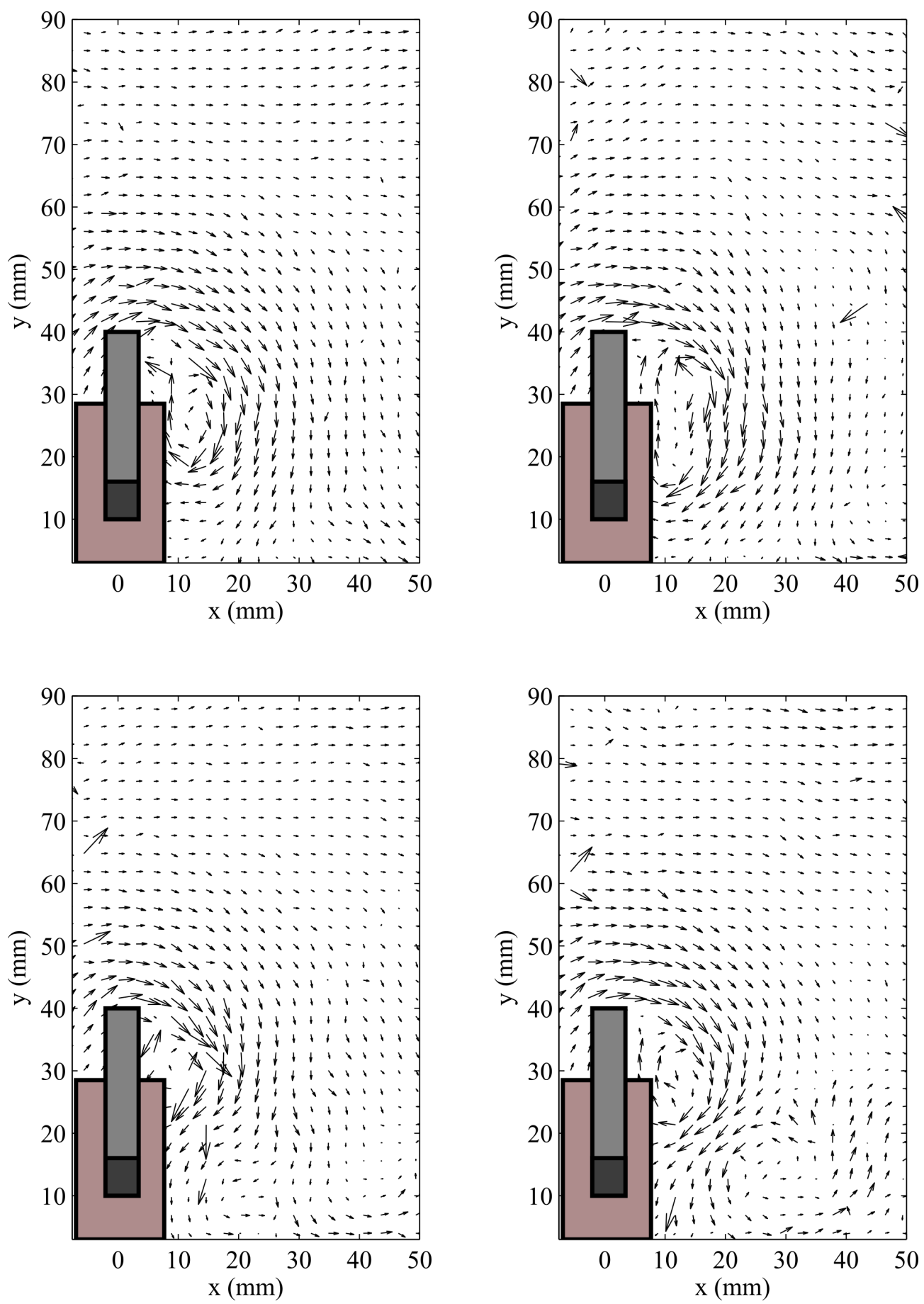

Figure 6.28: Different instantaneous velocity vector fields for $\operatorname{Re}_{\mathrm{rot}}=3.9 \cdot 10^{4}$. 


\subsection{Conclusions}

This chapter analysed both the motion of ice-like particles and the flow pattern inside the SSPHE. Medium density polyethylene (MDPE) particles were used, matching the same conditions that take place inside the SSPHE during the ice slurry production through the rotating Reynolds number and the Froude number. Particles were injected from the bottom, once the flow was established. Particle stratification tests were also carried out, with a concentration of the MDPE particles equal to $4 \%$ in weight. The flow pattern was studied by the planar PIV technique, in a plane perpendicular to the scraper blade located at $r=0.6 R$. A phase average procedure was followed in order to obtain the mean velocity field and the turbulence kinetic energy. Major conclusion are as follows:

- The particles injected at a low scraping velocity $\left(\operatorname{Re}_{\mathrm{rot}}=1.6 \cdot 10^{4}\right)$ showed higher dispersion of the particles cloud that those at higher velocities $\left(\operatorname{Re}_{\mathrm{rot}}=4.8 \cdot 10^{4}\right)$. The dispersion of the particles was associated to their buoyancy, which makes them unable to follow the fluid flow generated by the scrapers. At the higher tested velocity $\left(\mathrm{Re}_{\mathrm{rot}}=4.8 \cdot 10^{4}\right)$, the particles cloud appeared more compacted and no particle groups where observed detached from the main group. The value of the Fr $>1$ most probably indicated that the buoyant forces were not any more significant.

- The particle stratification revealed that for $\operatorname{Re}_{\text {rot }} \approx 7.6 \cdot 10^{3}$, the particles are quiescent, stacked on the top in a stationary bed. As the scraping velocity increases, part of the particles start to move, appearing a separate moving layer at the bottom of the stationary bed. At $\mathrm{Fr}=5 \cdot 1 \cdot 10^{-1}$ the bed disappears near the extreme radial positions, with a moving, turning bed around the shaft. For $\mathrm{Fr}=6.8 \cdot 10^{-1}$ the moving bed disappears leading to an heterogeneous flow, that becomes almost homogeneous at faster scraping velocities $\left(\operatorname{Re}_{\text {rot }}>2.5 \cdot 10^{4}\right)$.

- The flow pattern inside the SSPHE could be described by the PIV study for the range $2.2 \cdot 10^{4} \geq \operatorname{Re}_{\text {rot }} \geq 1.7 \cdot 10^{5}$. Absolute velocities were transformed into velocities relative to the scraper. The observed pattern consisted in three different regions: 1) an unperturbed, horizontal flow on the top of the SSPHE, 2) a roll up vortex on the rear side of the scraper and 3) a net downwards flow induced by the vortex. As $\mathrm{Re}_{\text {rot }}$ increases the unperturbed region is reduced, and the downwards flow increases. The flow deviated by the scraper upwards increases with the rotating velocity. The combination of upwards and downwards flow can increase the mixing, being the roll up vortex the main mixing agent.

- The normalized velocity field was normalized by the scrapers tangential velocity at the location of the laser sheet. As the rotating velocity of the scrapers increases the normalized velocity profiles evolved to a $\operatorname{Re}_{\text {rot }}$-independent profile where, compared with the low rotating velocity situations, velocity in the fluid is more uniform and low velocity regions tend to disappear.

- The roll up vortex is formed from the flow separated form the scraper. The high shear, separated flow rolls up into a spiral shape. Vorticity values revealed the location of the vortex core, which ascends slightly as the rotating velocity 
increases. They also revealed the high shear fluid layer on the scraper's tip. The analysis of the vortex tangential velocity (referred to the vortex center) showed a good agreement with the different classical modelling methods.

- Turbulent kinetic energy was higher in the roll up vortex, achieving a peak in the vortex core. The scraper's tip also showed high turbulent kinetic values as a consequence of the high shear. Increasing the rotating velocity of the scrapers leaded to the extension of the turbulence generated by the vortex. The peak turbulence kinetic ratio could be related with the rotating Reynolds number, and the roll up vortex appeared to be the main mechanism of energy transfer and turbulence generation in the SSPHE flow. 


\section{Chapter 7}

\section{Numerical modelling}

The previous chapter provided a description of the single-phase and two-phase flow pattern in the SSPHE by using flow visualization techniques. The results revealed a characteristic structure, consisting of a tangential flow and the presence of a roll up vortex in the rear side of the scrapers. The two-phase experiments confirmed also the importance of the buoyancy effect of the ice particles for the tested rotating velocities.

The experimental results, even enlightening, have several limitations. The presence of any radial velocity component could only be sensed and the high particle movement in the two-phase experiments limited the accuracy of the results. Furthermore, the PIV technique, when encountering strong out-of-plane motions and transitory patterns, is not the most suitable for the turbulence analysis.

In order to extend the scope of the experimental results, numerical simulations were conducted by using the finite volume method and different turbulence modellings. A Lagrangian study of ice-like particle motion was combined with the flow simulation to provide quantitative information of the velocity effect on the particles distribution after being scraped.

Section 7.2 contains a brief description of the numerical solution procedure, including also the different turbulence models employed. Sections 7.5 and 7.6 explain respectively the two different model approaches to the SSPHE problem: the 2D-Lagrangian particles and the complete 3D flow.

\subsection{Previous considerations}

Before describing the numerical model, a thought has to be made about what should it include. As it was shown in chapters 4 and 5 , the heat transfer phenomena inside the SSPHE is strongly dominated by the phase change. The later is linked with the scrapers actions and other physicals aspects like the ice breakage and the surface roughness.

To include such a number of variables and their physical connections in a numerical model results therefore highly complex and falls out of the scope of the present work. On the other hand, a simpler model which considers only the flow inside the SSPHE induced by the scrapers is by far more reachable, and can provide valuable information. Moreover, the existence of lagrangian models allows to simulate with a certain level of accuracy the motion of particles which the same size and density of the ice, and this motion can be extrapolated to predict stratification situations. 
Hence, the numerical model presented in this chapter attempts only to study the fluid flow and the ice particles motion, being the modelling of the heat transfer out of reach.

\subsection{Governing equations}

The governing equations of the CFD method are introduced here and the finite volume method (FVM) is briefly described.

The fluid flow is described by the conservation of mass, momentum and energy. In its general form, the conservation equation for a flow quantity $\phi$ is [126]

$$
\frac{\partial \rho \phi}{\partial t}+\nabla \cdot(\rho \mathbf{u} \phi)-\nabla \cdot\left(\rho \Gamma_{\phi} \nabla \phi\right)=S_{\phi}
$$

where $\rho$ is the density, $t$ the time, $\mathbf{u}$ the velocity, $\Gamma_{\phi}$ the diffusivity and $S_{\phi}$ is a source term.

Applying the previous equation to the mass conservation (continuity equation) leads to $\phi=1$, and if $S_{\phi}=0$ then

$$
\frac{\partial \rho}{\partial t}+\nabla \cdot \rho \mathbf{u}=0
$$

which in an incompressible fluid is reduced to

$$
\nabla \cdot \mathbf{u}=0
$$

The momentum equation can be written as [127]:

$$
\frac{\partial \rho \mathbf{u}}{\partial t}+\nabla \cdot(\rho \mathbf{u u})=-\nabla p+\nabla \cdot \tau+\rho \mathbf{g}
$$

where $\mathbf{g}$ is the gravity acceleration and $\tau$ is the viscous stress tensor

$$
\tau=-\mu\left(\nabla \mathbf{u}+(\nabla \mathbf{u})^{T}\right)
$$

being $\mu$ the viscosity of the fluid and, assuming it Newtonian and incompressible, $\nabla \cdot \tau=\mu \nabla^{2} \mathbf{u}$.

\subsection{Turbulence modelling}

The numerical modelling of a turbulent flow is based on the conservation of mass and momentum Navier-Stokes equations [128]. The most straight-forward approach is the Direct Numerical Simulation (DNS), which directly solves the equations by discretisation and numerical algorithms. DNS attempts to solve all scales of motion in the fluid, from the largest coherent structures to the smallest dissipating eddies, without any averaging or smoothing of the solution field. The main drawback is that the grid should be detailed enough to capture the Kolmogorov scales, and the domain should be large enough to capture the largest turbulence scales. The later means that the cost of the simulation is proportional to the Reynolds number cubed. DNS is then limited to simple geometries at relative low Reynolds numbers. 
To reduce the amount of scales to be resolved, an averaging operator may be applied to the Navier-Stokes equations. The classical averaging method is the ensemble average, which produced the Reynolds Average Navier-Stokes equations (RANS). This is equivalent to an infinite set of experiments being sampled at the same time. The RANS equations introduce new unknowns into the equations inf the form of the Reynolds stress tensor. This stress tensor represents the effects of all turbulent fluctuations and has to be modelled to close the system. RANS can be employed to solve flows in complex geometries and at high Reynolds numbers. The the approach has a main drawback: the turbulence model must represent a very wide range of scales. The small scales depend mainly of the viscosity but the larger structures depend on the flow, and therefore the RANS performs poorly when separation or free shear flows are present.

The final approach to model turbulence is the Large Eddy Simulation (LES). It does not resolve the full range of turbulent scales as DNS, but captures a larger range of scales than the RANS approach. LES is like to apply DNS to the large scales and RANS to the small scales. Therefore LES is more suitable for flows where effect of the large structures is important, as there can be agitated tanks and in particular the SSPHE.

\subsubsection{Reynolds Averaging}

In the Reynolds-Averaged Navier Stokes (RANS), the formulation is simplified by separating the flow variables into a mean and a fluctuating component, making then turbulence stationary [129]. If $\mathbf{u}=(u, v, w)=\left(u_{1}, u_{2}, u_{3}\right)$, the decomposition of any velocity component can be made as:

$$
u(x, t)=\bar{u}(x)+u^{\prime}(x, t)=U+u^{\prime}
$$

The following properties can be established for the fluctuating values around the mean:

$$
\begin{gathered}
\overline{\phi^{\prime}}=0 \quad \overline{\phi+\varphi}=\bar{\phi}+\bar{\varphi} \\
\overline{\phi \varphi}=\overline{\phi \varphi}+\overline{\phi^{\prime} \varphi^{\prime}} \quad \frac{\overline{\partial \phi}}{\partial t}=\frac{\partial \bar{\phi}}{\partial t}
\end{gathered}
$$

where the time average is calculated by:

$$
U=\lim _{T \rightarrow \infty} \frac{1}{T} \int u(x, t) d t
$$

If the Navier-Stokes momentum equation (Eq. 7.4) is averaged at both sides, 6 more unknowns appear corresponding to the products $u_{i}^{\prime} u_{j}^{\prime}$. They are added to the viscous term on the right hand side, as a symmetric Reynolds-Stress Tensor $\tau_{\text {turb }, i, j}$. These new unknowns require additional equations to close the problem, which is known as turbulence modelling.

Prandtl introduced the concept of turbulent kinetic energy in 1945, representing the kinetic energy contained in the velocity fluctuations,

$$
k=\frac{1}{2} \overline{u_{i}^{\prime} u_{i}^{\prime}}
$$


and being a basic variable in the turbulence modelling. From the above equation, it is possible to see that $k$ is proportional to the tensor $\tau_{\text {turb }, i, j}$. If $k$ is calculated then the number of unknowns will be reduced to 3 , as $\tau_{\text {turb }, i, i}=-\overline{u_{i}^{\prime} u_{i}^{\prime}}=-2 k$.

The production, transport and dissipation of the turbulent kinetic energy can be expressed into an equation [129]:

$$
\frac{\partial k}{\partial t}+U_{j} \frac{\partial k}{\partial k_{j}}=\tau_{\text {turb }, i, j} \frac{\partial U_{i}}{\partial x_{j}}-\epsilon+\frac{\partial}{\partial x_{j}}\left[\nu \frac{\partial k}{\partial t}-\frac{1}{2} \overline{u_{i}^{\prime} u_{i}^{\prime} u_{j}^{\prime}}-\frac{1}{\rho} \overline{p^{\prime} u_{j}^{\prime}}\right]
$$

where $\epsilon$ is called dissipation rate: the rate at which turbulent kinetic energy is converted into thermal internal energy

$$
\nu \overline{\frac{\partial u_{i}^{\prime}}{\partial x_{k}} \frac{\partial u_{i}^{\prime}}{\partial x_{k}}}
$$

The specific dissipation rate of energy in unit volume and time can also be defined as:

$$
\omega=c \frac{k^{1 / 2}}{l}
$$

where $l$ is the turbulent length scale and $c$ is a dimensionless constant.

The closure of the problem requires of two approximations. The first one comes from Boussinesq, who stated that the tensor $\tau_{\text {turb }, i, j}$ can be approximated to the product of the turbulent eddy viscosity $\mu_{t}$ and the mean strain-rate tensor. The momentum transfer by turbulent eddies, the turbulent eddy kinematic viscosity $\nu_{t}$ can be written as

$$
\nu_{t}=C_{\mu} \frac{k^{1 / 2}}{l}
$$

where $C_{\mu}$ is a dimensionless constant.

The second approximation is that the turbulent transport term -the turbulent energy transported by turbulent fluctuations- and the pressure diffusion term -the transport of turbulent energy by pressure fluctuations- are approximated to [129]:

$$
\frac{1}{2} \overline{u_{i}^{\prime} u_{i}^{\prime} u_{j}^{\prime}}+\frac{1}{\rho} \overline{p^{\prime} u_{j}^{\prime}}=\frac{\nu_{t}}{\sigma_{k}} \frac{\partial k}{\partial x_{j}}
$$

where $\sigma_{k}$ is a closure coefficient.

A turbulent model that has $\epsilon$ as a variable in the $k$ equation is known as $k$-Epsilon turbulent model, whereas if $\epsilon$ is replaced by $\omega$ it is called $k$-Omega.

The standard $k$-Epsilon model [130] has two model equations, one for $k$ and one for $\epsilon$-developed similarly as the transport equation for $k$. It performs reasonably well for $2 \mathrm{D}$ and free shear flows, but the results are quite inaccurate when dealing with 3D flows which involve separation [131]. The $k$-Omega model [129] can be directly used to treat the near wall viscous sub-layer, providing a better accuracy for free shear and separated flows. However, it does not work well at free stream conditions and is sensible to changes in the inlet $\omega$ value.

The $k$-Omega SST model, which is the one used in the present work, appeared in 1994 [132]. The shear stress transport (SST) formulation performs a combination 
of the $k-\epsilon$ and $k-\omega$ model strengths. The model works as $k$-Omega near the wall and as $k$-Epsilon in the free shear flow. It can be used in the viscous sub-layer as the $k$-Omega model. The switch to the $k$-Epsilon behaviour in the free stream avoids the above mentioned $k$-Omega problem of the sensitivity to the $\omega$ inlet value.

\section{Near wall treatment}

The turbulent models described in the previous section can only be applied at fully turbulent conditions. For instance, the low Reynolds effect near the wall will not be considered. To correct this there are two different approaches, the so called wall functions or the Low-Reynolds models.

Wall functions When a wall function is applied the law of the wall is used in the fully developed turbulent boundary layer $\left(30<y^{+}<200\right)$ with a suitable value of $C$ to relate the velocity field to the surface shear stress and to ensure a proper matching between the viscous and logarithmic velocity profiles [129]:

$$
\begin{gathered}
U^{+}=y^{+} \\
U^{+}=\frac{1}{k} \ln \left(y^{+}\right)+C
\end{gathered}
$$

where $U^{+}=U / u_{\tau}$ and $y^{+}=u_{\tau} y / \nu$. Therefore, it is necessary that the first node is located at $y^{+} \geq 30$. The values of $k \omega$ and $\epsilon$ used as a boundary conditions are given by

$$
k=\frac{u_{\tau}^{2}}{\sqrt{\beta^{*}}}, \quad \omega=\frac{k^{1 / 2}}{\beta^{* 1 / 4} k y}, \quad \epsilon=\frac{\beta^{* 3 / 4} k^{3 / 2}}{k y}
$$

As wall functions are approximations, they work better when applied to internal flows where the effect of the boundary layer in the main flow is low. Moreover, they are quite sensitive to the location of the first node near the wall.

Low Reynolds models In the Low Reynolds model the boundary layer for $y^{+}>1$ is resolved, which means that the Low Reynolds effect are taken into account. More details can be found at [129]. From the turbulence modelling methods introduced, the only one that can be used in the viscous sub-layer is the $k$-Omega SST, which is the only one among them suitable to be used as a Low Reynolds mode.

\subsubsection{LES modelling}

As explained before, in the LES turbulence modelling the large eddies are simulated, and only the small ones are modelled. This makes LES more powerful than RANS, but necessary with a high computational cost.

The division between the scales simulated and those modelled is done by a filtering operation, which filters out scales smaller than the mesh size. The filtering decomposes the velocity into a sum of resolvable-scale filtered velocity $\bar{u}_{i}$ and a sub-grid scale (SGS) velocity $u_{i}^{\prime}$, with a filter with $\Delta$ (see [129]): 


$$
u_{i}=\bar{u}_{i}+u_{i}^{\prime} \text { and } \Delta=(\Delta x \Delta y \Delta z)^{1 / 3}
$$

A generalized filter is defined by the convolution integral

$$
\bar{u}_{i}(x, t)=\iiint G(x-\chi ; \Delta) u_{i}(\chi, t) d^{3} \chi
$$

where $G$ is the filtering function

$$
G(x-\chi ; \Delta)= \begin{cases}1 / \Delta^{3} & \left|x_{i}-\chi_{i}\right|<\Delta x_{i} / 2 \\ 0, & \text { otherwise }\end{cases}
$$

Different filters have been proposed. Either if they are isotropic or not, homogeneous or not, in all the cases the filter introduces a scale $\Delta$ that represents the smallest turbulence scale allowed by the filter.

The fundamental problem of LES is to establish a satisfactory model for the stresses coming from the SGS component. Indeed, the sub-grid scales constitute a significant portion of the turbulence spectrum. Smagorinsky [133] proposed the first model for the SGS stresses, assuming that they follow a gradient-diffusion process, similar to molecular motion ([129]):

$$
\tau_{i, j} 2 \mu_{T} S_{i, j}, \quad S_{i, j}=\frac{1}{2}\left(\frac{\partial \bar{u}_{i}}{\partial x_{j}}+\frac{\partial \bar{u}_{j}}{\partial x_{i}}\right)
$$

where the Smagorinsky eddy viscosity is

$$
\mu_{T}=\rho\left(C_{S} \Delta\right)^{2} \sqrt{S_{i, j} S_{i, j}}
$$

and $C_{S}$ is the Smagorinsky coefficient, calibrated to $0.10<C_{S}<0.24$.

According to Pope ([131]), LES should simulate the eddies that contain $80 \%$ of the energy and model the $20 \%$ remaining. The length scales are calculated as:

Large eddies $l_{0}=\left(\frac{\nu^{3}}{\epsilon}\right)^{1 / 4} R e^{3 / 4}$

Small eddies $\nu=\left(\frac{\nu^{3}}{\epsilon}\right)^{1 / 4}$

where $\epsilon$-the dissipation rate- in the $k$-Omega model can be computed as $(9 / 100) k \omega$

\subsection{Finite Volume Method}

The OpenFOAM software package [134] was used. OpenFOAM is an open source CFD software written in $\mathrm{C}++$, which uses the finite volume discretization schemes to solve the Navier-Stokes equations.

The Finite Volume Method subdivides the fluid domain into a finite number of smaller control volumes. The transport equations are then integrated over each one of these control volumes by approximating the variation of the flow properties between the mesh points with differencing schemes. For a better explanation to the method refer to Ferziger and Peric [126] or Versteeg andMalalasekera [135]. 


\subsubsection{Domain discretization}

The solution domain is divided or discretized into a number of cells or control volumes. The control volumes are do not overlap one another, filling completely and continuously the domain. The variables are stored at the cell centroid. Each cell is bounded by a set of flat faces, aligned in an structured or unstructured way. Two neighbour cells can only share one face. When a face belongs only to a cell it is called boundary cell. Roughly, a mesh is composed of:

- Points defined by their location in the space

- Faces defined by a list of points

- Cells defined by a list of faces

- Boundary patches defined by a list of boundary faces that belong only to a boundary patch

\subsubsection{Discretised equations}

The transport partial differential equation can be transformed into an algebraic expression that can be expressed as

$$
\mathbf{M x}=\mathbf{b}
$$

being $\mathbf{M}$ a square matrix, $\mathbf{x}$ the dependent variable vector and $\mathbf{b}$ the source vector. In the FVM the discretization of each term is done by first integrating the term over a cell volume $V$. Spatial derivatives can be converted to integrals over the cell surface $S$ bounding the volume by using the Gauss's theorem:

$$
\int_{V} \nabla \phi=\int_{S} d \mathbf{S}_{\phi}
$$

where $\mathbf{S}$ is the surface are vector and $\phi$ can represent any variable. The matrix $\mathbf{M}$ is then the implicit terms and the source vector $\mathbf{b}$ constitute the explicit terms.

\section{Diffusion term}

The diffusion term is integrated over a control volume and linearised as

$$
\int_{V} \nabla \cdot(\Gamma \nabla \phi) d \mathbf{V}=i n t_{S} d(\mathbf{S} \cdot(\Gamma \nabla \phi))=\sum_{f} \Gamma_{f} \mathbf{S}_{f} \cdot(\nabla \phi)_{f}
$$

The above can be discretisized when the length vector $\mathbf{d}$ between the centre of the cell of interest $\boldsymbol{P}$ and the centre of a neighbouring cell $\boldsymbol{N}$ is orthogonal to the face:

$$
\mathbf{S}_{f} \cdot(\nabla \phi)_{f}=\left|\mathbf{S}_{f}\right| \frac{\phi_{N}-\phi_{P}}{|\mathbf{d}|}
$$

Non-orthogonal meshes require of an additional explicit term as it is explicated in [136] 


\section{Convection term}

The convection term is integrated over a control volume and linearised as

$$
\int_{V} \nabla \cdot(\rho \mathbf{U} \phi) d \mathbf{V}=\int_{S} d(\rho \mathbf{U} \phi)=\sum_{f} \Gamma_{f} \mathbf{S}_{f} \cdot(\rho \mathbf{U})_{f} \phi_{f}=\sum_{f} F \phi_{f}
$$

The face field $\phi_{f}$ can be evaluated using a variety of schemes, being the two more common:

- Central differencing, a second-order accurate but unbounded. The error of discreatisation reduces with the square of the grid spacing and the limits of $\phi$ are not necessarily preserved

$$
\phi_{f}=f_{x} \phi_{p}+\left(1-f_{x}\right) \phi_{N}
$$

where $f_{x}=\overline{f N} / \overline{P N}$.

- Upwind differencing, a first-order accurate and bounded. Determines $\phi_{f}$ from the direction of the flow

$$
\phi_{f}= \begin{cases}\phi_{P} & \text { for } F>0 \\ 0, & \text { for } F<0\end{cases}
$$

The schemes above can be combined in order to preserve boundedness with an acceptable accuracy. Moreover, there are several more schemes aside from them, developed for specific purposes.

\section{Gradient term}

The gradient term is an explicit term. It is evaluated by the Gauss integration applying the Gauss's theorem to the volume integral:

$$
\int_{V} \nabla \phi d \mathbf{V}=\int_{S} d(\mathbf{S} \phi)=\sum_{f} S_{f} \phi_{f}
$$

More ways to evaluate the gradient term can be found in [136]

\section{Time derivative term}

The time derivative term $\partial / \partial t$ is integrated over the control volume as

$$
\frac{\partial}{\partial t} \int_{V} \rho \phi d \mathbf{V}
$$

which is discretised by

- $\phi^{n}=\phi(t+\Delta \mathrm{T})$ for the new values at the time step being solved

- $\phi^{0}=\phi(t)$ for the old values that were stored from the previous time step 
In the present work the scheme used was the Euler implicit, which is a first order scheme accurate in time -the discretisation error reduces with smaller time steps. Is defined as:

$$
\frac{\partial}{\partial t} \int_{V} \rho \phi d \mathbf{V}=\frac{\left(\rho_{p} \phi_{p} \mathbf{V}\right)^{n}-\left(\rho_{p} \phi_{p} \mathbf{V}\right)^{0}}{\Delta \mathrm{T}}
$$

\subsubsection{Temporal discretisation}

The spatial derivatives in a transient problem need to be considered as $\phi$ is a function of space and time. The spatial and temporal derivatives are averaged over one or more time steps. Denoting by $\Lambda \phi$ the spatial terms, where $\Lambda$ is any spatial operator, then a transient partial differential equation can be expressed as

$$
\int_{t}^{t+\Delta \mathrm{T}}\left[\frac{\partial}{\partial t} \int_{V} \rho \phi d \mathbf{V}+\int_{V} \Lambda \phi d \mathbf{V}\right] d t=0
$$

According to the Euler implicit method, the first term can be written as

$$
\int_{t}^{t+\Delta \mathrm{T}}\left[\int_{V} \Lambda \phi d \mathbf{V}\right] d t=\frac{\left(\rho_{P} \phi_{P} \mathbf{V}\right)^{n}-\left(\rho_{P} \phi_{P} \mathbf{V}\right)^{0}}{\Delta \mathrm{T}} \Delta \mathrm{T}
$$

and the second term as

$$
\int_{t}^{t+\Delta \mathrm{T}}\left[\int_{V} \Lambda \phi d \mathbf{V}\right] d t=\int_{t}^{t+\Delta \mathrm{T}} \Lambda^{*} \phi d t
$$

where $\Lambda^{*}$ is the spatial discretisation of $\Lambda$. The previous integral can be discretised in different ways:

- Euler implicit, takes only current values $\phi^{n}$. It is first order accurate in time, guaranteeing boundedness and unconditional stability

- Explicit, takes only values $\phi^{0}$ from the previous time step. It guarantees boundedness and is first order accurate in time. It will be unstable if the Courant number Co is greater thatn one

$$
C o=\frac{\mathbf{U}_{f} \cdot \mathbf{d}}{\left|\mathbf{d}^{2}\right|} \Delta \mathrm{T}
$$

being $\mathbf{U}_{f}$ the velocity of the flow and $\mathbf{d}$ the length between to neighbouring cell centres.

- Crank Nicholson, take a mean of current values $\phi^{n}$ and old values $\phi^{o}$. It is second order accurate in time, unconditionally stable, but does not guarantee boundedness.

Among the above schemes, the Euler implicit is the most stable one, being the one used in the present work. 


\subsubsection{Pressure-Velocity coupling}

The linear dependence between pressure and velocity in the continuity and the momentum equations (Eqs. 7.3 and 7.4) can be treated in two ways: by simultaneous algorithms or by segregated approach. In the first one the complete system of equations is solved simultaneously over the entire domain, which has a high computational cost. In the second approach the equations are solved in sequence. The PISO [137] and SIMPLE [138] and their derivatives as the PIMPLE (merged PISO-SIMPLE) are the most popular methods of solving the pressure-velocity coupling. As they are iterative, they can handle the non-linearity in the velocity equation with low storage requirements.

\section{PISO algorithm}

The PISO (Pressure-Implicit with Splitting of Operators) algorithm was developed for solving transient flow calculations described by the discretised Navier-Stokes system for incompressible flow. The loop consists of an implicit momentum predictor followed by a series of pressure solutions and explicit velocity corrections. The loop is repeated until a pre-set tolerance is reached.

\section{SIMPLE algorithm}

As when solving a steady-state problem iteratively the non-linearity of the system becomes more important -the effective time-step is much larger-, a different algorithm is required. The SIMPLE (Semi-Implicit Method for Pressure-Linked Equations) algorithm starts from a guessed pressure field, from which the velocity is solved. The momentum equation is under-relaxed with an under-relaxation factor. Once the velocity is known, the pressure equation is then solved and a new set of fluxes is calculated, where the pressure field includes both the pressure error and the convection-diffusion error. The pressure solution is under-relaxed in order to include the velocity part of the error. The values recommended for the relaxation factor are 0.2 for the pressure and 0.8 for the velocity.

\subsubsection{Boundary conditions}

The boundary conditions delimit the computational domain and emulate the physical problem as close as possible. They have to be defined for each variable and can be divided into two types, Dirichlet and Neumann. Dirichlet prescribes the value of the dependent variable on the boundary, whereas Neumann prescribes the gradient of the variable normal to the boundary.

In problems where the flow is periodic, they can be studied only by a period of the geometry and using what is called cyclic or periodic boundary condition. This is not really a boundary condition, as the only thing it does is to "connect" the boundary with the values of the periodic side -in the direction normal to the periodic boundary which goes into the domain. 


\subsection{The two-dimensional model: lagrangian ice particles tracking}

The purpose of this model is to study the movement of solid particles with density and size similar to those scraped from the surface of the SSPHE. In order to have a representative result, the model must have the capability of performing a lagrangian particle tracking, including the turbulent dispersion effect. Moreover, not only the drag force has to be considered but also the buoyancy.

These requirements, added to the flow simulation in a geometrically accurate model of the SSPHE, would lead to a high numerical cost. A reasonable way to simplify the model without an excessive way of accuracy cost can be to consider only the flow in the SSPHE as circular, neglecting any possible radial component. This is obviously not a good simplification if the full flow field has to be studied, but can be a good approximation in terms of flow-particle interaction if the idea is to study the mixing and stratification. Notwithstanding this approach have been already considered for similar purposes - and validated experimentally- by [63]. The idea here is to follow their work, but adding the buoyant effect and the turbulence interaction to the particles.

Therefore, the model here consists of an infinite number of scrapers, of infinite length, which move normal to themselves in an infinite channel, as sketched in Fig. 7.1a. The velocity of the scrapers is equal to the tangential velocity of the rotating scraper in the SSPHE at $r=0.6 R$, the plane investigated experimentally by PIV.

\subsubsection{Translating reference frame}

As explained in chapter 6, the flow inside the SSPHE can be considered steady from a rotating frame of view whose rotating axis is aligned with the rotating shaft. The same occurs if the system is the one described above, shown in Fig. 7.1a, but now the relative reference system is translating linearly together with the scraper. As the scrapers are moving with a linear, constant velocity, the relative reference system does the same. Hence, and since it does not have any acceleration, no inertial forces have to be considered in the momentum equation applied to the translating reference frame, an all the velocities on it (relative velocities $u_{r}$ ) can be transformed into absolute velocities $u$ simply by adding the velocity of the scrapers $U_{s}$ :

$$
u=u_{r}+U_{s}
$$

The idea is graphically depicted in Fig. 7.1. As the flow is periodic between scrapers, a domain can be selected as shown in Fig. 7.1b, where the scrapers move normal to it. Using the translating reference frame the problem of Fig. $7.1 \mathrm{~b}$ can be translated to the one of Fig. $7.1 \mathrm{c}$ where the walls are moving at $U_{s}$ tangential to them, in the opposite direction in what the scraper -now steady- was moving inFig. 7.1b. Moreover, not only the top and bottom walls need to be moved: the whole fluid has to move horizontally at $U_{s}$ in the same direction of the walls as initial condition.

The translating frame methodology allows to solve the problem without employing more complex methodologies such as dynamic mesh deformation, which would increase considerably the complexity of the model. 


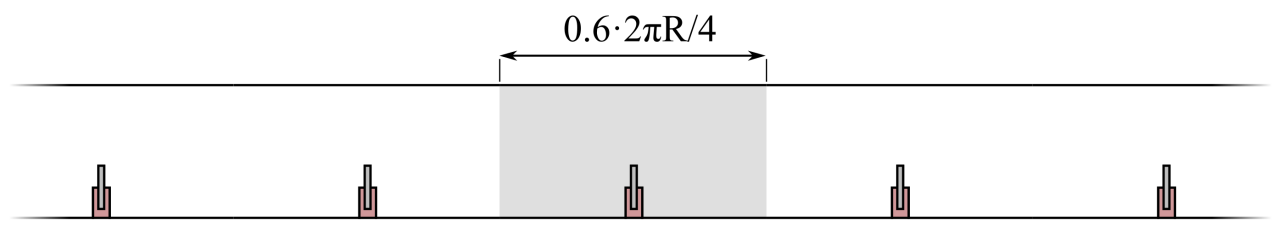

a)

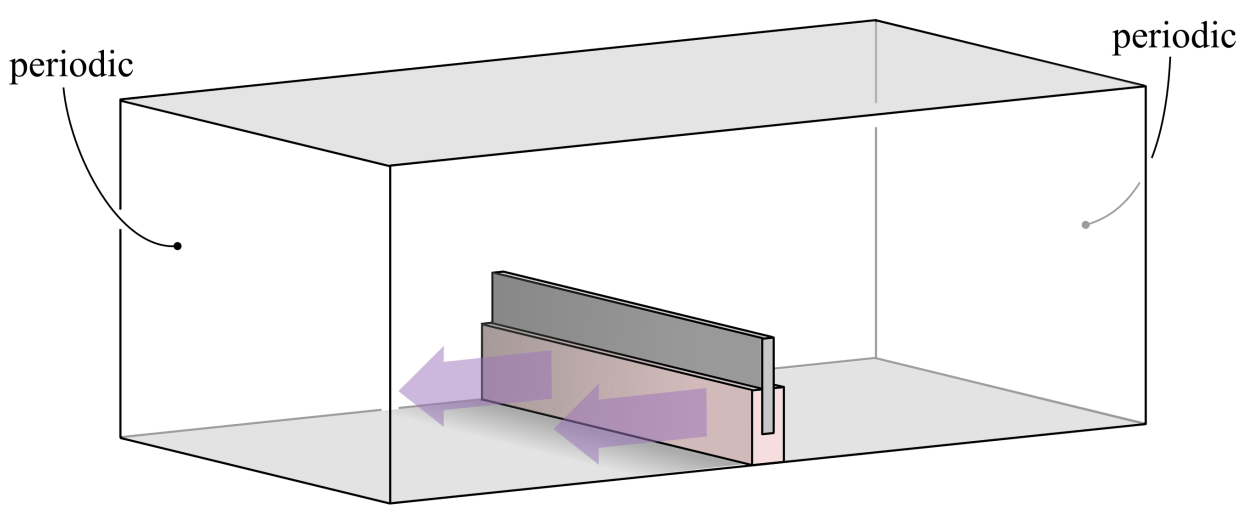

b)

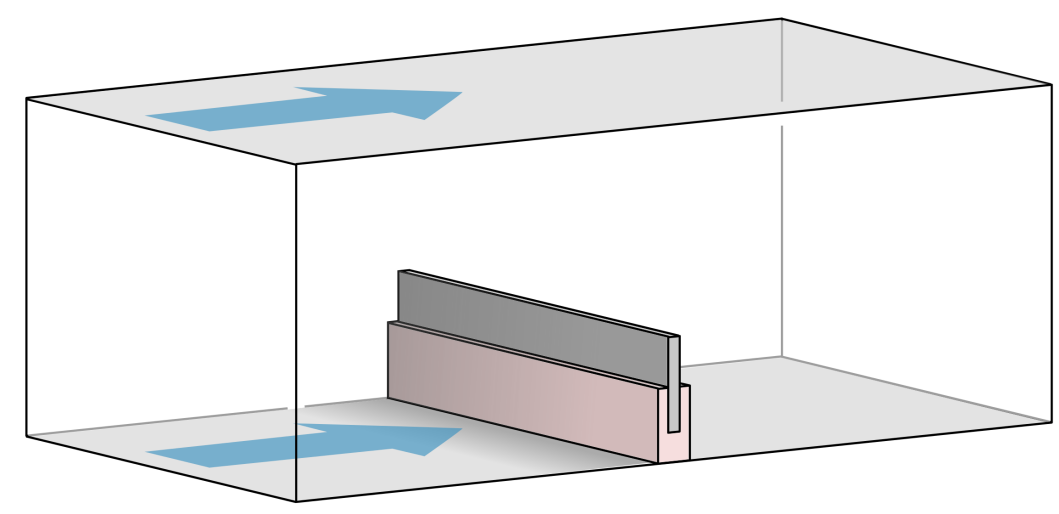

c)

Figure 7.1: a) Bidimensional approach to the SSPHE problem b) periodic domain with absolute velocities c) periodic domains using the translating reference frame (relative velocities) 


\subsubsection{Computational domain and mesh}

The studied computational domain is the one shown in Fig. 7.1c. Two different meshes where created in order to test two different turbulence models: $k$-Omega SST with Low Reynolds and LES. Both meshes had a regular structure.

For the $k-\omega$ SST Low Reynolds model, a 2D mesh was used, as the problem was two-dimensional. The mesh is shown in Fig. 7.2. The mesh was refined near the walls in order to have $y^{+} \leq 1$. For the LES simulations a 3D mesh was used, extruded from the one shown in Fig. 7.2. The extrusion span was equal to $3 \mathrm{~cm}$, equal to the height of the scraper. As centrifugal forces are not present in this model, the flow in the direction along the scraper (radial direction in the real SSPHE) can only be due to turbulence. Hence, a span wise thickness equal to the eight of the scraper was considered sufficient. This assumption will be later confirmed by the residence of the particles inside the domain, without leaving it through the side boundaries. The mesh was refined in all the directions by a factor of 2 in order to increase the resolution.

\subsubsection{The lagrangian frame: injected particles}

In the lagrangian frame a particle is defined by the location of its center $x_{\mathrm{p}}$, its diameter $\mathrm{d}_{\mathrm{p}}$, its velocity $U_{\mathrm{p}}$ and its density $\mu_{\mathrm{p}}$. Each particle location is calculated from

$$
\frac{d x_{\mathrm{p}}}{d t}=U_{\mathrm{p}}
$$

being the motion of the particle governed by the Newton's equation

$$
m_{\mathrm{p}} \frac{d U_{\mathrm{p}}}{d t}=\sum \mathrm{F}
$$

with $m_{\mathrm{p}}$ the mass of the particle $\left(1 / 6 \cdot \rho_{\mathrm{p}} \pi \mathrm{d}_{\mathrm{p}}^{3}\right)$. Assuming that the flow is dilute (we consider low ice concentrations) the dominant force is the drag $F_{D}$, and then

$$
\sum \mathrm{F}=\mathrm{F}_{\mathrm{D}}+\mathrm{F}_{\mathrm{B}}
$$

where $\mathrm{F}_{\mathrm{B}}=\left(\rho_{f}-\rho_{\mathrm{p}}\right) \cdot 1 / 6 \cdot \pi \mathrm{d}_{\mathrm{p}}^{3}$ is the buoyancy force. The drag force can be expressed as

$$
\mathrm{F}_{\mathrm{D}}=-m_{\mathrm{p}} \frac{U_{\mathrm{p}}-U}{\tau_{\mathrm{p}}}
$$

where $U$ is the velocity of the fluid and $\tau_{\mathrm{p}}$ is the relaxation time of the particles, i.e., the time it takes for a particle to respond to changes in the local flow velocity:

$$
\tau_{\mathrm{p}}=\frac{3}{4} \frac{\rho_{\mathrm{p}} \mathrm{d}_{\mathrm{p}}}{\rho_{f} C_{\mathrm{D}}\left|U-U_{\mathrm{p}}\right|}
$$

where $C_{\mathrm{D}}$ is the drag coefficient, evaluated analytically by the Stokes law [139].

Hence, the fluid velocity $U$ calculated in the Eulerian frame is needed from the calculation of the drag force in the Lagrangian frame. It has to be interpolated at the position of the particle from the neighbour grid points, $U_{\mathrm{f}, \mathrm{p}}$. The velocity of the particle is finally calculated by: 


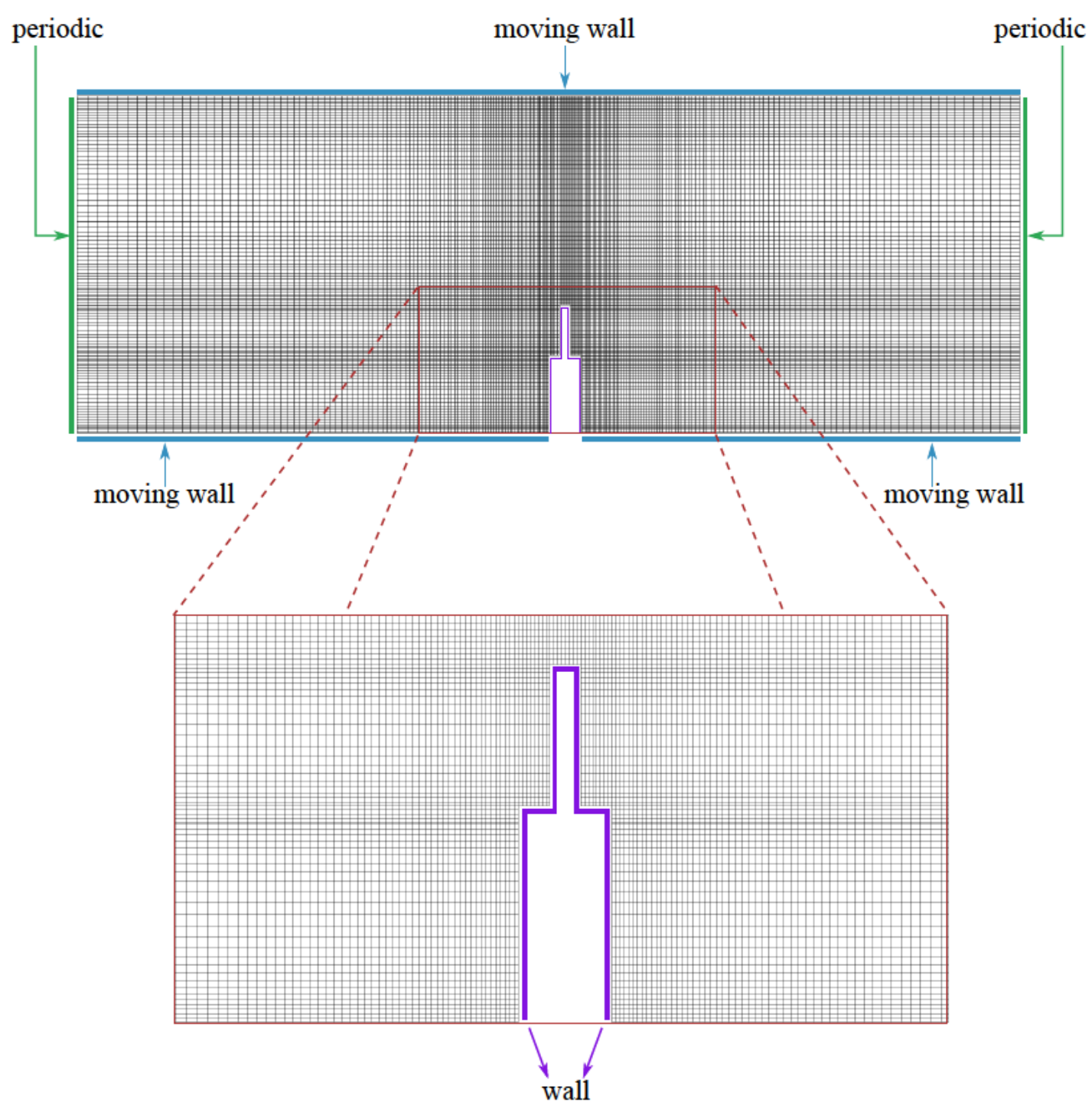

Figure 7.2: Mesh used for the particle tracking simulations with the $k$-Omega SST Low Reynolds model. 


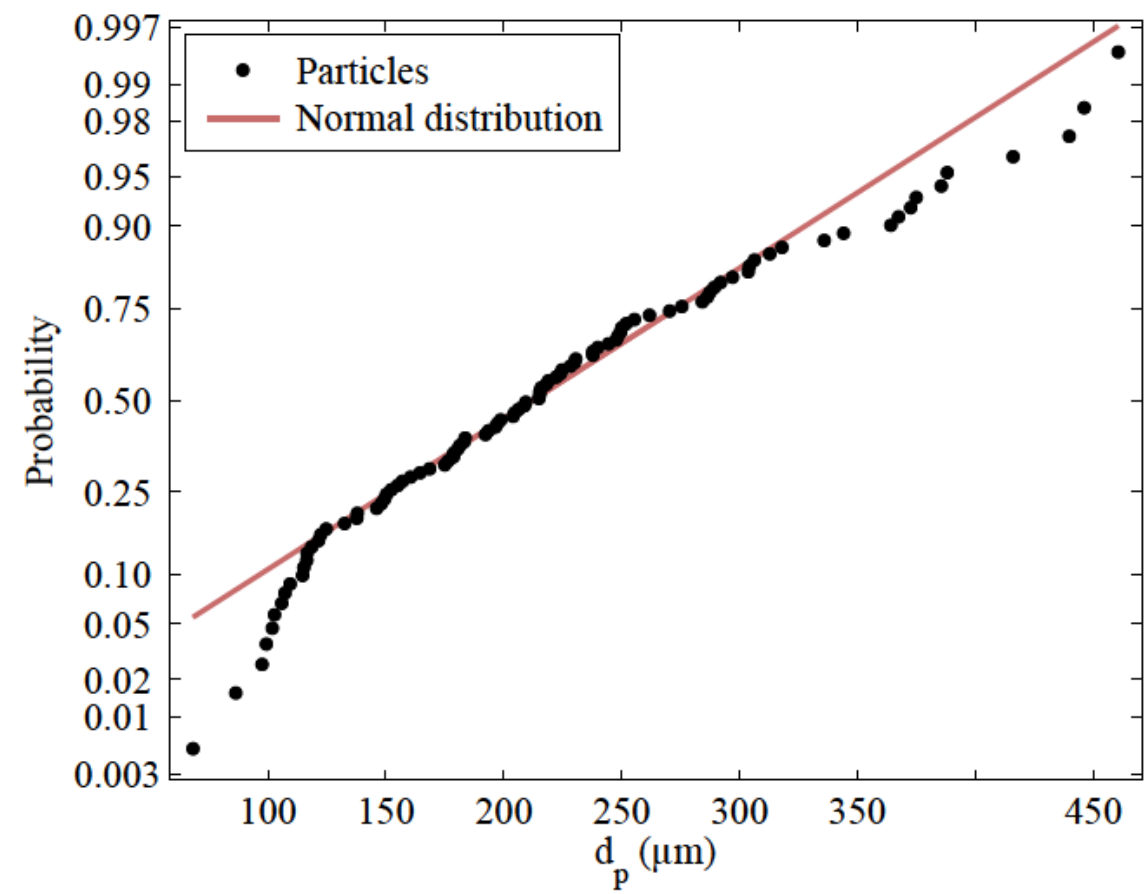

Figure 7.3: Distribution of the injected particles by their diameter, very close to a normal distribution.

$$
m_{\mathrm{p}} \frac{U_{\mathrm{p}}^{t+\Delta \mathrm{T}}-U_{\mathrm{p}}^{t}}{\Delta \mathrm{T}}=-m_{\mathrm{p}} \frac{U_{\mathrm{p}}^{t+\Delta \mathrm{T}}-U_{\mathrm{f}, \mathrm{p}}^{t}}{\tau_{\mathrm{p}}}+\mathrm{F}_{\mathrm{B}}
$$

The position of the particle is evaluated by Eq. 7.36

A 4-way coupling model was employed, where interaction between fluid an particles, as well as particle collision with the walls and among themselves is considered.

Injected particles A total number of 100 particles with a density $\mu_{\mathrm{p}}=935 \mathrm{~kg} / \mathrm{m}^{3}$ were injected with an initial horizontal velocity equal to that of the walls. Translated to an absolute reference frame where the walls are steady and the scrapers are moving, the particles were initially steady. The injected particles have spherical shape and a wide number of diameters from $d_{p}=100 \mu \mathrm{m}$ to $d_{p}=460 \mu \mathrm{m}$, following a normal distribution as it is possible to see in Fig. 7.3. The particles distribution intended to match the diameters observed experimentally by microscope (see chapter 4 sec. 4.8).

\subsubsection{Initial and boundary conditions}

For both RANS and LES turbulence modelling simulations, an incompressible, transient solver was used. The particles were injected once the flow was developed. A PIMPLE algorithm (mix of PISO and SIMPLE) was used to solve the coupling between pressure and velocity.

In order to achieve a developed flow prior to the injection of the particles, two different strategies were followed depending on the turbulence modelling: 
Table 7.1: Velocity and pressure boundary condition for the two-dimensional model

\begin{tabular}{lllll}
\hline & \multicolumn{2}{c}{$\mathrm{U}\left(\mathrm{m} \mathrm{s}^{-1}\right)$} & \multicolumn{2}{c}{$\mathrm{p} / \rho\left(\mathrm{m} \mathrm{s}^{-2}\right)$} \\
\cline { 2 - 5 } Boundary & type & value & type & value \\
\hline \hline top & moving wall & $2 \pi \mathrm{N} \cdot 0.6 \mathrm{R}$ & fixed gradient & 0 \\
bottom & moving wall & $2 \pi \mathrm{N} \cdot 0.6 \mathrm{R}$ & fixed gradient & 0 \\
scraper & wall & - & fixed gradient & 0 \\
inlet/outlet & periodic & - & periodic & - \\
\hline
\end{tabular}

$k-\omega$ SST Low Reynolds: the flow field was obtained trough steady simulations, using the SIMPLE algorithm. The obtained velocity field was used then as initial one for the particle injection simulations.

LES: Transient simulations using the PIMPLE algorithm were performed until a simulated time of $20 \mathrm{sec}$. The particle were then injected.

The velocity and pressure conditions were the same for both RANS and LES simulations, summarized in table 7.2. The velocity boundary conditions are shown also in Fig. 7.2. The wall or moving wall boundary conditions are introduced in OpenFOAM through the fixedValue entry, specifying either $\left(\begin{array}{lll}0 & 0 & 0\end{array}\right)$ or $\left(\begin{array}{lll}-U & 0 & 0\end{array}\right)$ as values, where $\mathrm{U}$ is the moving velocity of the wall. The periodic condition is introduced by the cyclic condition.

Pressure is set to zero gradient in all the walls and periodic in the inlet/outlet boundaries. The cell reference pressure is in all the cases set to 0 .

The boundary conditions for the turbulence values depend obviously on the turbulence model:

RANS For the $k-\omega$, as a Low Reynolds mesh is used $\left(y^{+} \leq 1\right)$ no wall functions have to be employed for the turbulent kinetic energy. However, in OpenFOAM the value of $k-\omega$ cannot be equal to zero -it will lead to a $1 / 0$ term. Instead, a very low value $\approx 0$ is used through the fixedValue entry. In the case of $\omega$, the omegaWallFunction automatically sets it to the correct near wall value. Since at the wall $\omega$ depends on the wall grid spacing, is the function omegaWallFunction who does the calculation. The asymptotic value is fixed according to [132]

$$
\omega_{w}=\frac{10 \cdot 6 \nu}{0.075 y_{n}^{2}} \approx 5300
$$

where $y_{n}$ is the distance from the first node to the wall. The boundary conditions types and values are summarized in table 7.2.

LES The LES model does not use the $k, \omega$ and $\mu_{t}$ properties. The Smagorinsky model calculates the SGS stress tensor from the sub-grid eddy viscosity, which sets gradient equal to 0 in all the walls and periodicity in the inlet/outlet. A value of $c_{S}=0.12$ was used for the Smagorinsky constant [64]. 
Table 7.2: Turbulence variables boundary conditions for RANS

\begin{tabular}{lllll}
\hline \multirow{2}{*}{ Boundary } & \multicolumn{2}{c}{$k \mathrm{~m}^{2} \mathrm{~s}^{-2}$} & \multicolumn{2}{c}{$\omega \mathrm{s}^{-1}$} \\
\cline { 2 - 5 } & type & value & type & value \\
\hline \hline top & fixedValue & $1 \cdot 10^{-10}$ & omegaWallFunction & $\omega_{w}$ \\
bottom & fixedValue & $1 \cdot 10^{-10}$ & omegaWallFunction & $\omega_{w}$ \\
scraper & fixedValue & $1 \cdot 10^{-10}$ & omegaWallFunction & $\omega_{w}$ \\
inlet/outlet & periodic & - & periodic & - \\
\hline
\end{tabular}

Table 7.2: Turbulence variables boundary conditions for RANS (continuation)

\begin{tabular}{lll}
\hline & \multicolumn{2}{c}{$\mu_{t} \mathrm{~m}^{2} \mathrm{~s}^{-1}$} \\
\cline { 2 - 3 } Boundary & type & value \\
\hline \hline top & mutLowReWallFunction & $1 \cdot 10^{-10}$ \\
bottom & mutLowReWallFunction & $1 \cdot 10^{-10}$ \\
scraper & mutLowReWallFunction & $1 \cdot 10^{-10}$ \\
inlet/outlet & periodic & - \\
\hline
\end{tabular}

\subsection{The three-dimensional model}

The objective of the three-dimensional model is to study the flow pattern inside the SSPHE. Hence, the possible radial velocity models need to be considered through the inclusion of the Coriolis and centrifugal forces in the Navier-Stokes momentum equation. To model the turbulence, the $k$-Omega SST with Low Reynolds wall treatment is used.

\subsubsection{Rotating reference frame}

The underlying idea of the Rotating Reference Frame methodology is similar to that of the Translating Reference Frame: to move the walls and fluid instead of the scraper, so the problem is steady. Figure 7.4 shows graphically the MRF concept. However, in the case of the rotating frame, acceleration exists due to the rotation, and two additional terms need to be included in the Navier-Stokes momentum equation: the Coriolis and the Centrifugal forces. Hence, the steady version of Eq. 7.4 to the rotating frame with the relative $u_{r}$ velocities is:

$$
\begin{array}{r}
\nabla \cdot\left(\rho \mathbf{u}_{r} \mathbf{u}_{r}\right)=\overbrace{2 \Omega \times \mathbf{u}_{r}}^{\text {Coriolis force }}+\overbrace{\omega \times(\Omega \times r)}^{\text {Centrifugal force }} \\
-\nabla p+\nabla \cdot\left(-\mu\left(\nabla \mathbf{u}_{r}+\left(\nabla \mathbf{u}_{r}\right)^{T}\right)\right)
\end{array}
$$

where the relative velocity is:

$$
\mathbf{u}=\mathbf{u}_{r}+\Omega \times r
$$



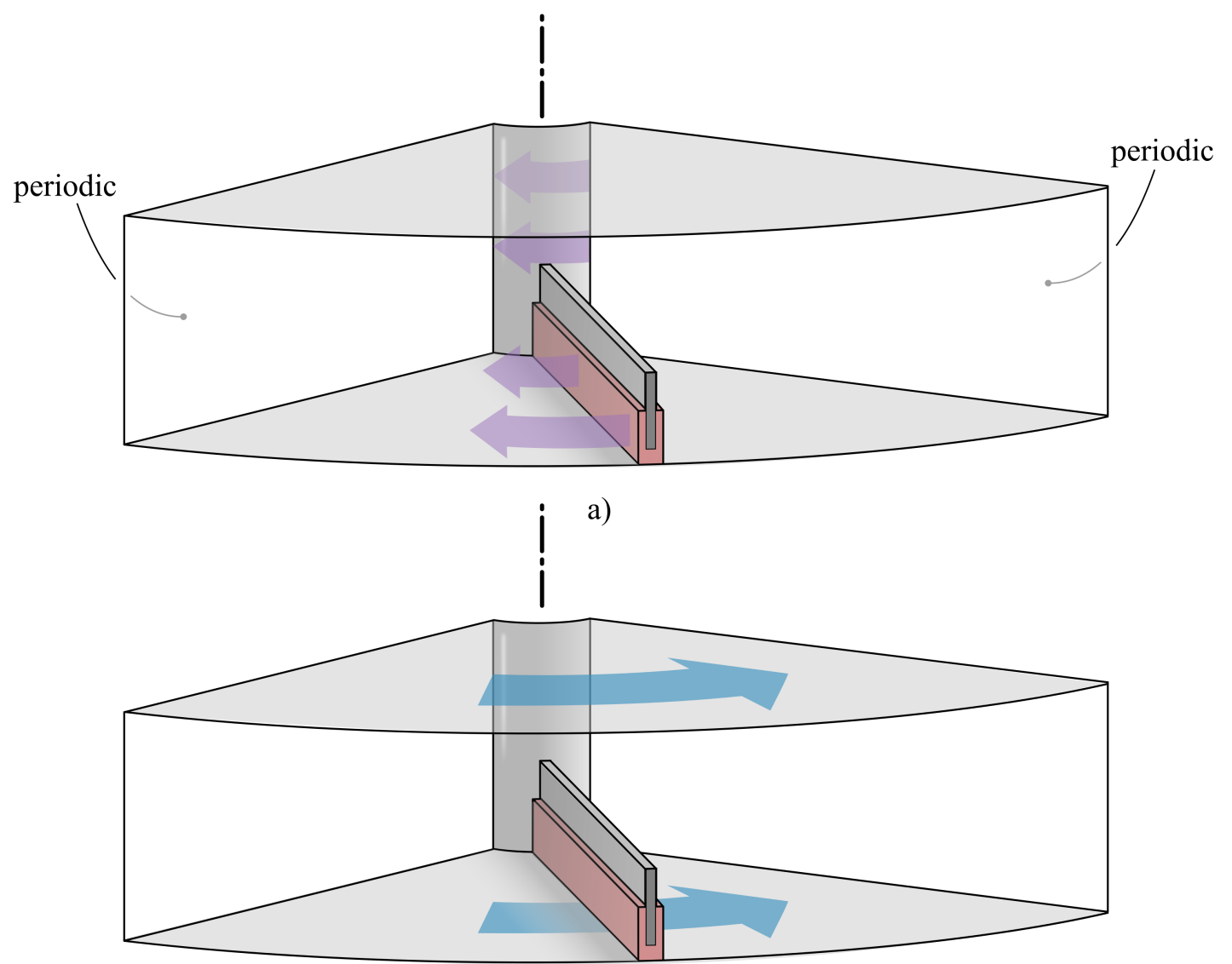

b)

Figure 7.4: Moving reference frame applied to the SSPHE.

\subsubsection{Computational domain and mesh}

The relative flow inside the SSPHE is geometrically periodic, with four planes that divide the rotating volume in periodic sub-volumes. Each sub-volume contains one scraper inside, as it is shown in Fig. 7.5a. The computational domain can be therefore only one of these sub-domains with periodic conditions in the dividing planes (7.5b). The computational domain is the fluid volume left by the scraper, as it is depicted in Fig. 7.5c.

To properly mesh the volume, reducing the non-orthogonality to the minimum, the geometry was divided into different blocks according to the sketch of Fig. 7.6. The division allowed to use hexahedral elements in all the blocks except 3 and 4 , which required tetrahedral meshing. The mesh was more refined in the blocks 5 to 14, which correspond to the region near the scraper. Figure 7.7.

Figure 7.7 shows the mesh at different vertical and horizontal sections across the domain. In all the wall boundaries $y^{+}$was lower than 1 . 


\subsubsection{Boundary conditions}

As the solver is steady, initial conditions are not relevant here. Boundary conditions are similar to those employed for the two-dimensional model: the scraper and shaft remain steady, whereas the top and bottom walls rotate at $2 \pi N$. The inlet and outlet sections are linked through the periodic boundary condition (cyclic in OpenFOAM). Pressure was set to gradient equal to 0 in all the boundaries except the periodic, and the cell reference pressure was set equal to 0 .

The values of $k, \omega$ and $\mu_{t}$ are equal to those shown in table 7.2 , with the value of $\omega_{w}$ was calculated also according to 7.42. The boundary corresponding to the shaft had the same boundary conditions as the other walls.

\subsubsection{Important remarks}

The 3D model presents difficulties associated with solving flows in rotating reference frames. Continuity and momentum equations have a high degree of coupling as the influence of the rotating term increases -higher rotating velocities. The later may lead to instabilities in the solver which requires a particular way of address the problem. In particular:

- Simulations where started at a very low rotating velocity $-10 \%$ of the minimum case studied- using a laminar model. Velocity was increased then by a factor of 1.5 , introducing the turbulent model once $\operatorname{Re}_{\text {rot }}>10^{\text {text }}$. The increasing of the rotating velocity was always done by a 1.5 factor, and increased only when the simulation reached convergence.

- Under-relaxation factor were always kept between 0.3-0.5 for both velocity and turbulent magnitudes. 


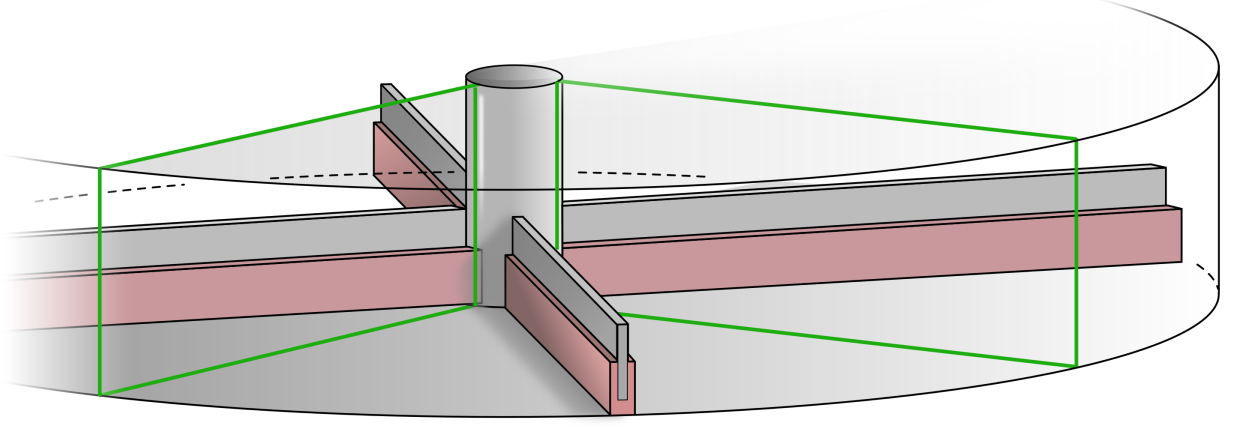

a)

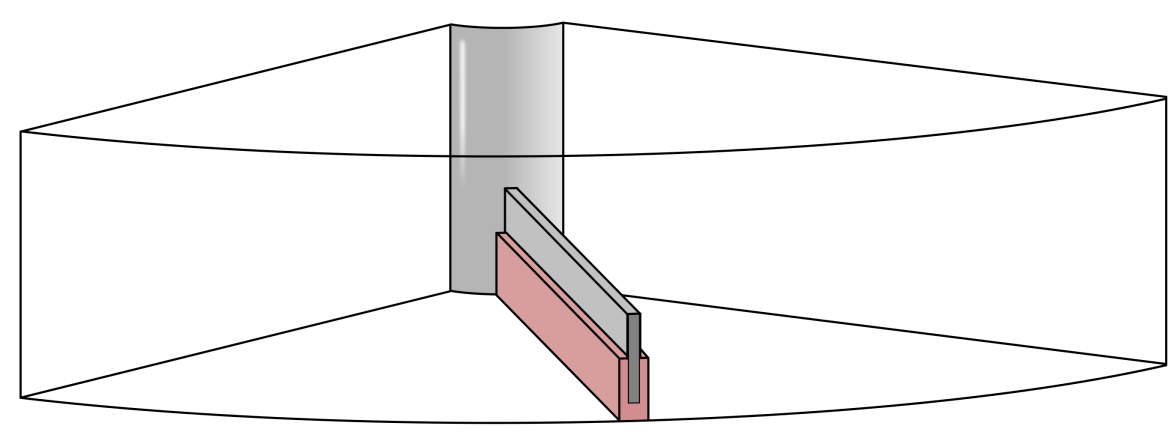

b)

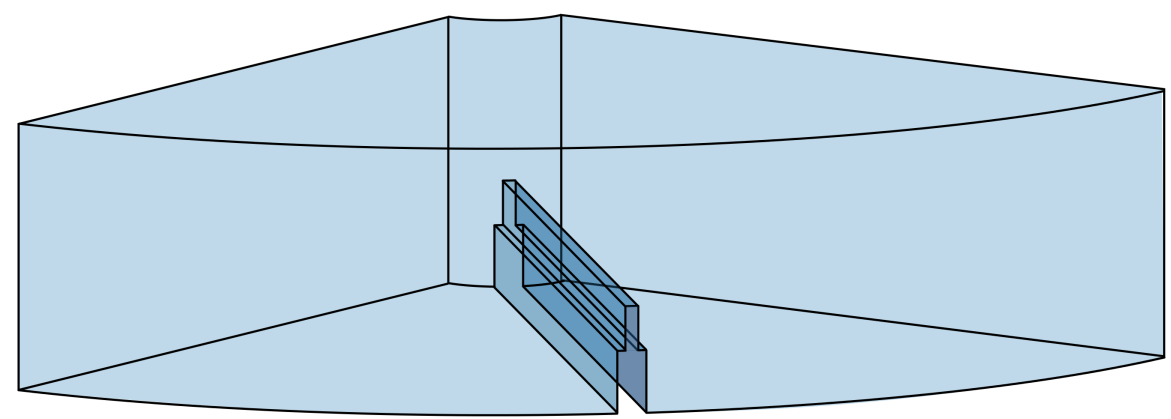

c)

Figure 7.5: 3D computational domain. 

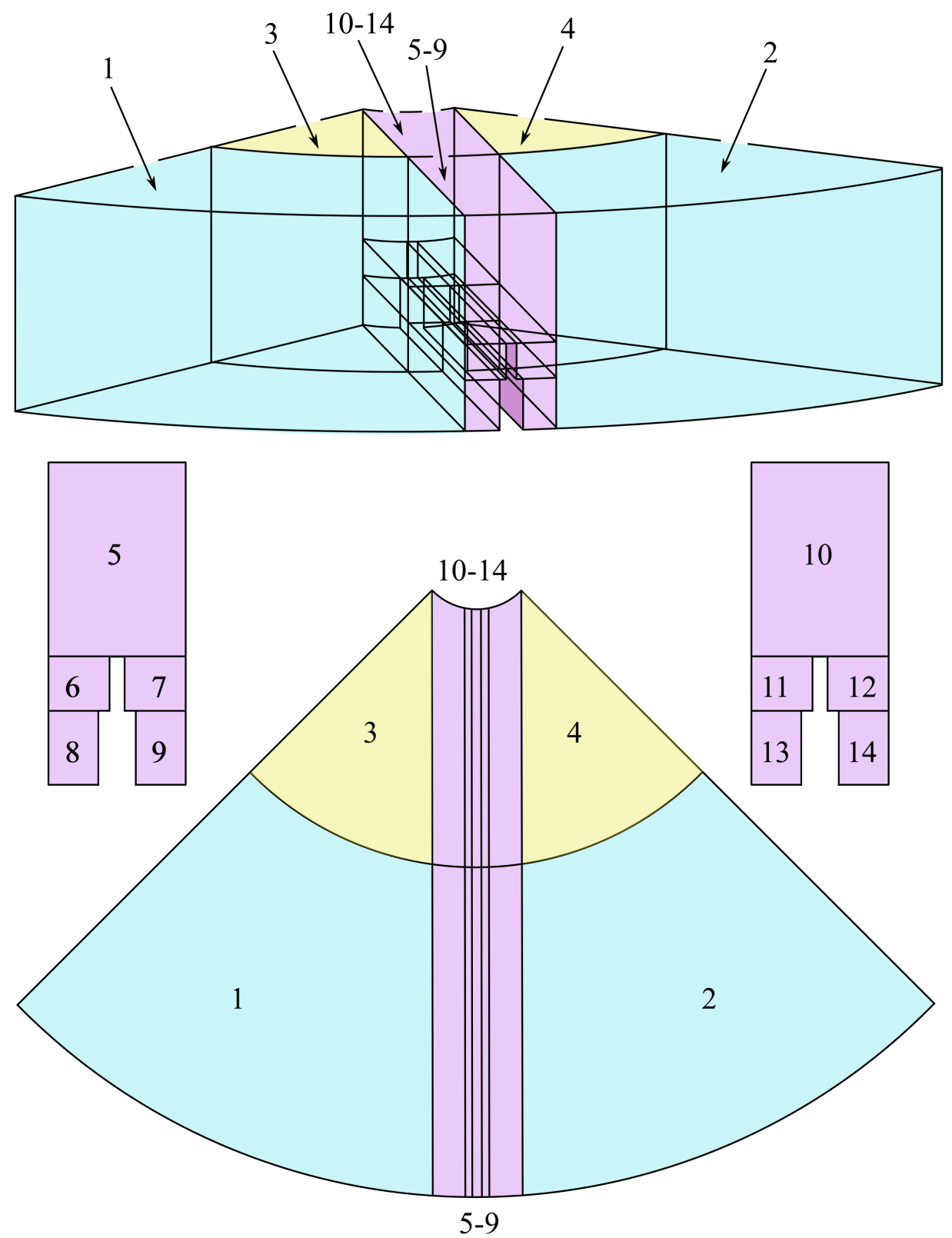

Figure 7.6: Fluid domain volume subdivision for meshing. 

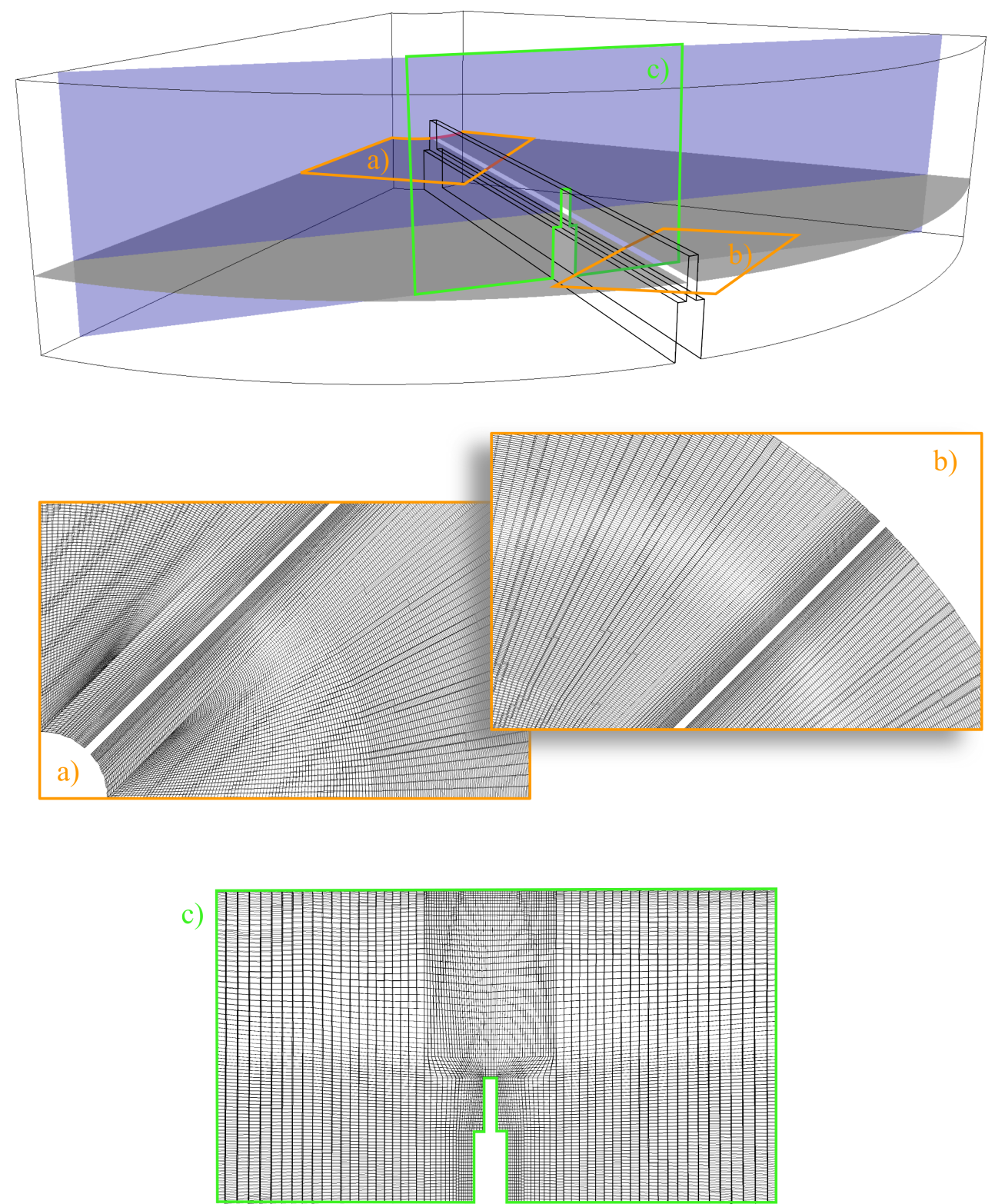

Figure 7.7: Mesh detail at different cross sections of the domain. 


\section{Chapter 8}

\section{Numerical flow pattern assesment}

This chapter presents a numerical flow pattern particle tracking assessment provided by the finite volume method methodology described in chapter 7 . The chapter is divided into two main sections. The first one, section 8.1, includes the results of two-dimensional simulations with lagrangian particle tracking. The second one, section 8.2, includes the results of the three-dimensional simulations of the SSPHE considering single-phase flow.

The first section is divided in turn into two subsections. The $k-\omega$ analysis is included in the first one (sec. 8.1.1): the velocity field (sec. 8.1.1) data are reported and compared within each case. A more extended analysis is done for the lagrangian particle tracking results, including the effect of the scraping velocity in the instantaneous particle distribution after 15 seconds of the injection (sec. 8.1.1), the spatial particle tracking (sec. 8.1.1) and the time-dependent particle tracking (sec. 8.1.1) of different representative particle sizes. The second part of the two-dimensional approach includes the results of the three-dimensional LES turbulence modelling (sec. 8.1.2), both the velocity field (sec. 8.1.2) and the particle tracking (sec. 8.1.2).

The second section includes the results of the three-dimensional simulations, reporting the velocity field and the flow pattern description (sec. 8.2). The pressure distribution over the scraper blade is also analysed.

\subsection{Results for the two-dimensional approach}

\subsubsection{The $k$ - $\omega$ turbulence modelling}

\section{Velocity field}

The numerical velocities were non-dimensionalized by the velocity of the scraper -in this case linear- as it was done in chapter 6 . Hence, we have

$$
\hat{u} *=\frac{u-U_{s}}{U_{s}}, \quad v *=\frac{v}{U_{s}}
$$

where $U_{s}$ is the velocity of the scraper. The dimensionless velocity magnitude is $U *=\sqrt{\hat{u} *^{2}+v *^{2}}$. Figure 8.1 shows the $U *$ vector field and iso-contours for the case corresponding to $\mathrm{Re}_{\mathrm{rot}}=6.5 \cdot 10^{4}$. The velocity relative to the scraper reaches the maximum on the top, corresponding to the non-slip fluid near the stationary wall. This high velocity layer was not observed in the PIV experiments (see Fig. 6.12) as the 


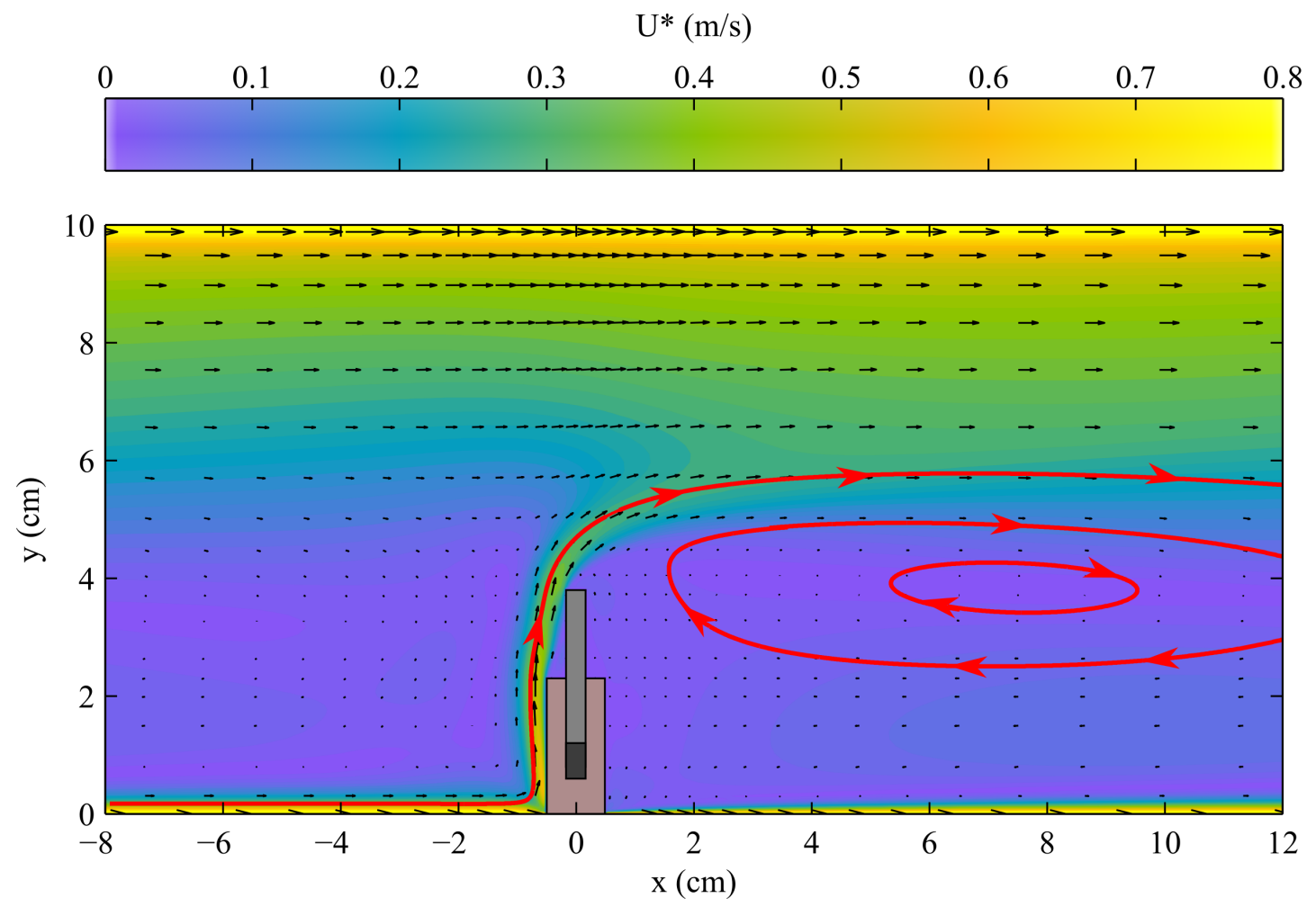

Figure 8.1: Vector field and iso-contours for $U *$ using the $k-\omega$ turbulence modelling, $\mathrm{Re}_{\mathrm{rot}}=6.5 A \cdot 10^{4}$. Scraper moves from right to left.

technique is not reliable near the walls and in particular in the top one, where high laser reflections were present. The contours of Fig. 8.1 show clearly, highlighted by the red streamlines, the flow deviated by the scraper from the bottom of the SSPHE including the boundary layer- and the recirculation in the rear (right) side. The results are similar in terms of dimensionless velocity value and flow structure compared with the results obtained by the PIV experiments -for instance see Fig. 6.12. However, the extension of the vortex is more elongated towards the horizontal direction. This can be due to the two-dimensional approach, which simplifies the flow neglecting any possible effect of the centrifugal forces that would lead to radial velocity components. Moreover, the distances between scrapers was taken as the circumferential length between them $\left(2 \pi r_{l} / 4\right)$, which may or may be not the best approximation for the separation of the scrapers in a $2 \mathrm{D}$ simplification.

The same flow pattern was always reproduced independently of the Reynolds number value simulated. Therefore, and for a better comparison, only the selected dimensionless velocity profiles were represented for each case. Fig. 8.2 shows the $\hat{u} *$ and $v *$ velocity profiles along two vertical lines located at $1 \mathrm{~cm}$ in front and $2 \mathrm{~cm}$ in the rear side of the scraper. As expected, relative horizontal velocities were higher on the top of the volume, far from the scraper. Below the level of the tip of the scraper the horizontal velocities are near 0 . This difference is increased in the rear side of the scraper, as the flow has passed through the top of it and has been accelerated. Unlike the profiles in the section prior to the scraper, the velocities in the bottom part after the scraper are 
slightly negative, corresponding to the reverse flow in the recirculation.

Considering now the vertical velocities, before the scraper the flow turns towards the top to avoid it, with a peak at $y \approx 2 \mathrm{~cm}$. After the scraper -right side of the image-, the vertical velocity shows positive and negative values as the profile line goes across the recirculation. Regarding the variations between different Reynolds situations, the effect is the same as the observed in the PIV results i.e., increasing the scraping velocity leads to more uniform profiles, whereas decreasing it accentuates the peaks in the profiles -always referred to the velocity of the scraper.

\section{Particle distribution}

As explained in the previous chapter, particles were released once the flow was developed. In the case of the $k-\omega$ turbulence modelling, the developed flow was achieved by using transient simulations. The location of the particles after 15 seconds of the injection for all the simulated cases can be observed in Fig. 8.3. Two observations can be made. The first is that bigger particles tend to stratify more than the smaller ones, which are better mixed. The second one is that the velocity of the scraper has a clear influence on the mixing of the particles. In Fig. 8.3a, almost all the particles are on the top, out of the influence of the recirculation generated by the scraper. As the scraping velocity increases the particles are more distributed along the fluid volume, and the effect of the particles size in their distribution decreases. In the last case (Fig. 8.3e all the particles are concentrated along an horizontal line, captured by the recirculation.

In order to do a more qualitative comparison between the different cases, the cumulative distribution along the vertical coordinate of the particles considering all the domain is depicted in Fig. 8.4. The effect of the scraping velocity on the particles distribution is clearly visible. For the slower scraping velocity case case all the particles are located at maximum $2 \mathrm{~cm}$ from the top wall. This distribution would lead clearly to a complete stratified situation, where the particles remain steady on the top of the SSPHE without any interaction with the fluid flow. In this situation the ice particles can form large blocks, which will reduce the fluidity of the slurry, or directly make it not pumpable. As the scraping velocity increases the particles have a wider distribution. In the higher scraping velocity case there are particles at only $2 \mathrm{~cm}$ of the scraped wall.

From the cumulative particles distribution $C_{P}$ is possible to calculate the concentration of particles per vertical unit length as

$$
\left.C_{P}=\frac{d(\% \text { of particles }) / 100}{d y} \text { [particles } / \mathrm{cm}\right] .
$$

Figure 8.5 shows the concentration of particles for each case simulated. The first case confirms the stratification of the particles, whereas the last denotes that all the particles are closed in the vortex. The cases in between exhibit a more distributed concentration of particles, with different situations in each one. The second case $\left(\operatorname{Re}_{\mathrm{rot}}=3.9 \cdot 10^{4}\right)$ still has stratified particles while the third and fourth cases $\left(\operatorname{Re}_{\text {rot }}=1.0 \cdot 10^{4}\right.$ and $\left.\operatorname{Re}_{\text {rot }}=1.7 \cdot 10^{5}\right)$ have more concentration of particles towards the vortex. 

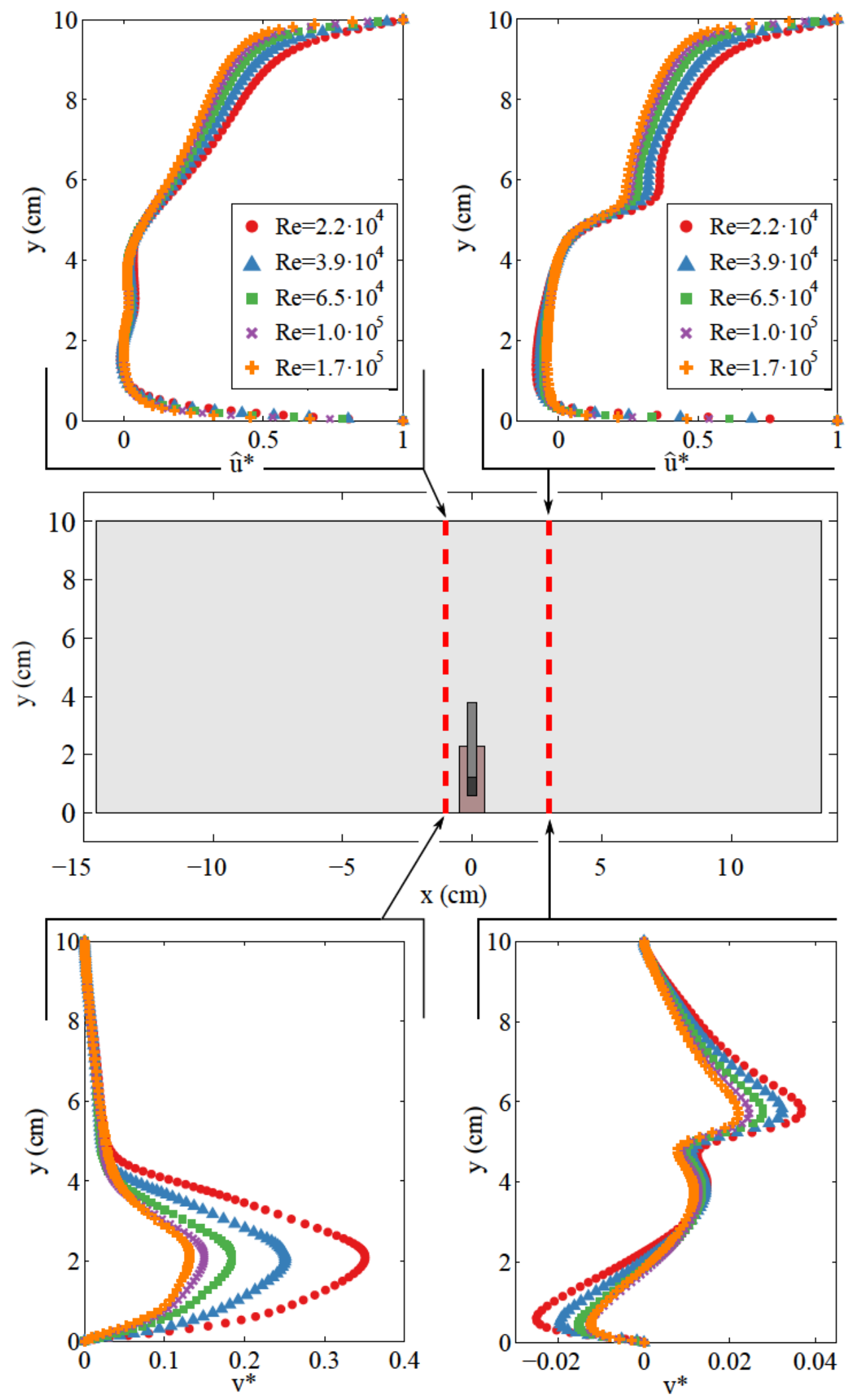

Figure 8.2: Comparison of dimensionless velocity profiles for the different simulated cases. Rotating Reynolds number are in correspondence with those tested in the visualization experiments (see chapter 6). 


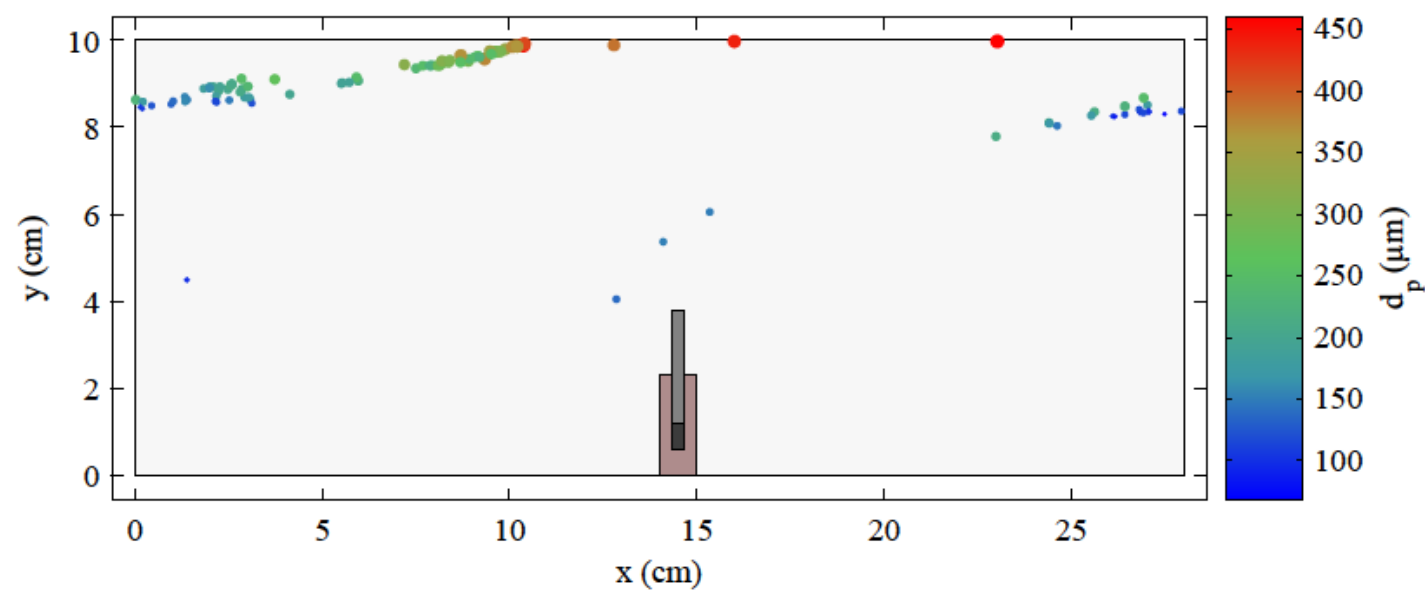

(a)

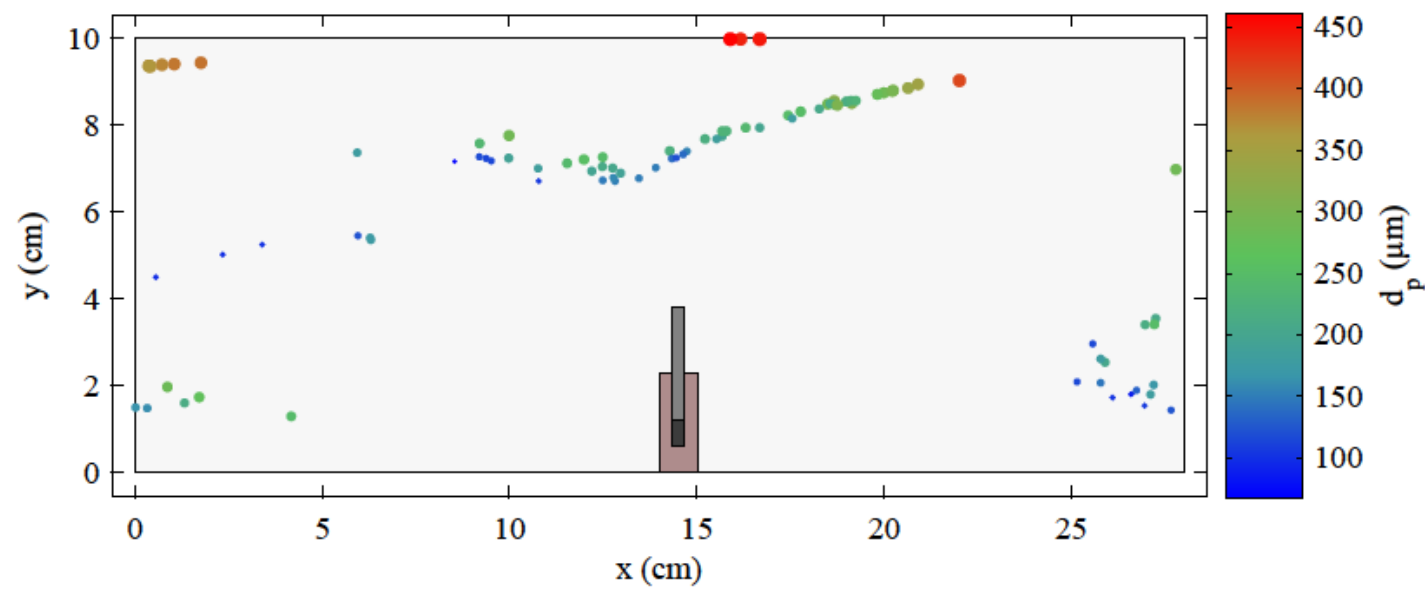

(b)

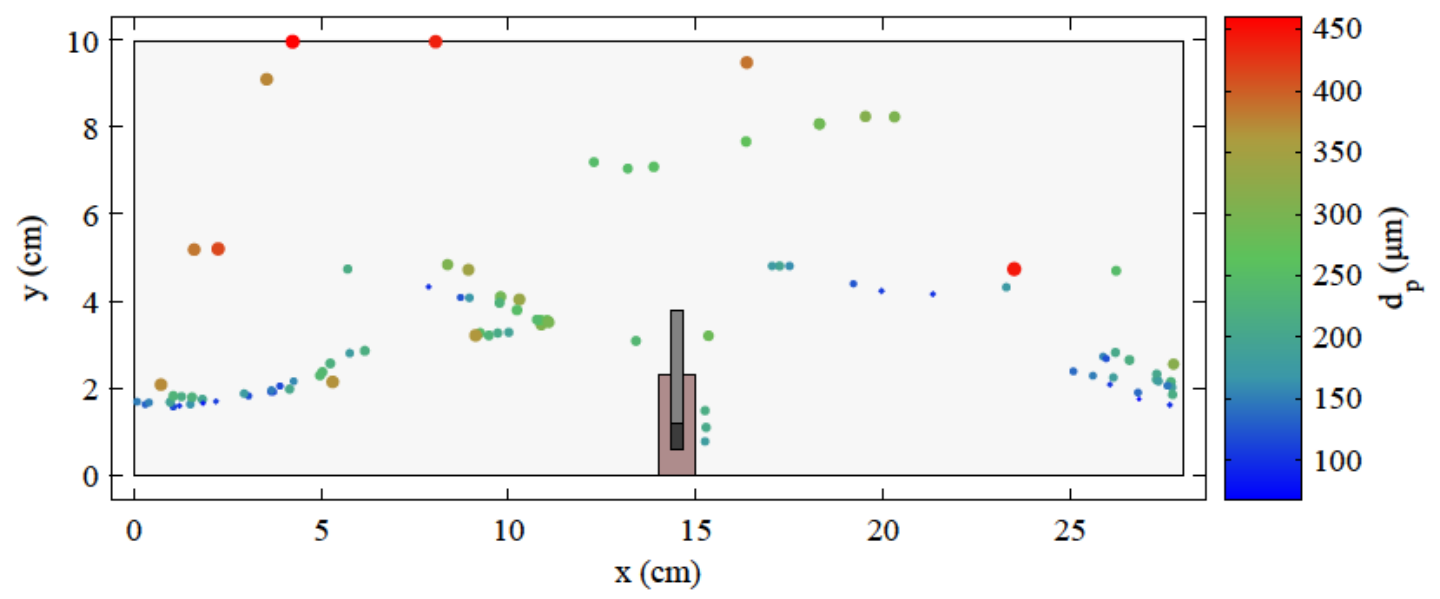

(c)

Figure 8.3: Particles location for the different tested cases after 15 seconds of the injection. a) $\operatorname{Re}_{\text {rot }}=2.2 \cdot 10^{4}$, b) $\operatorname{Re}_{\text {rot }}=3.9 \cdot 10^{4}$, c) $\operatorname{Re}_{\text {rot }}=6.5 \cdot 10^{4}$. Scraper motion from right to left. 


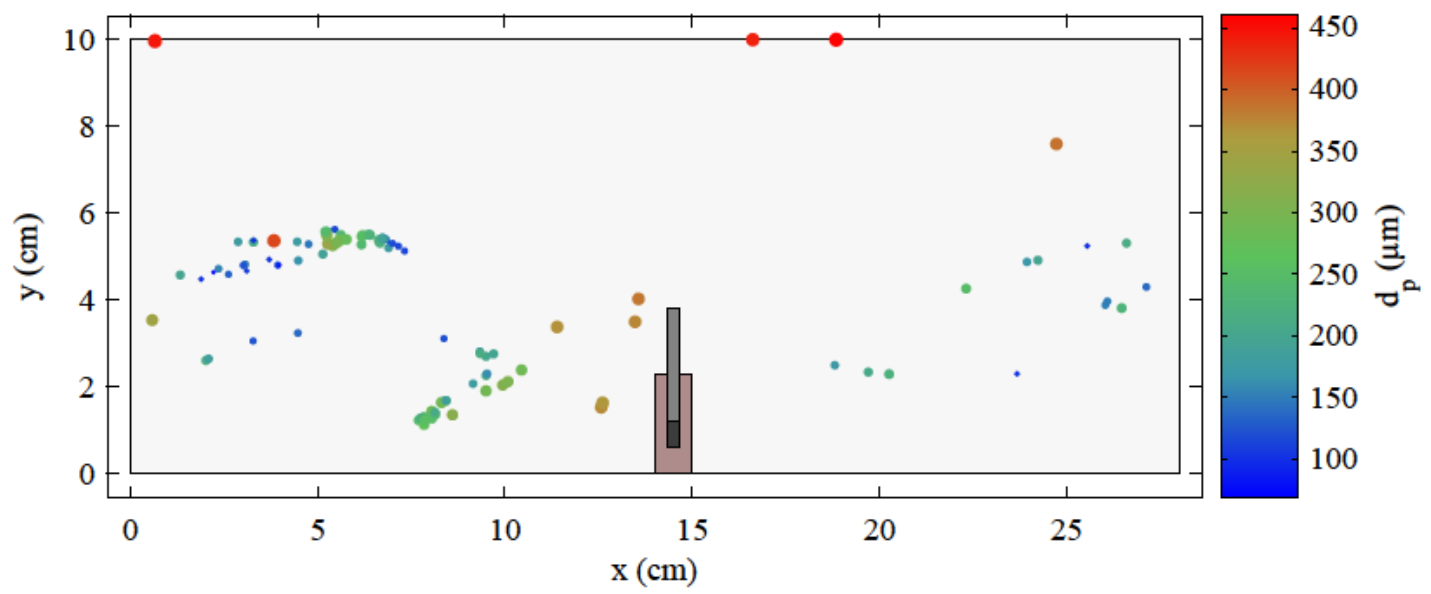

(d)

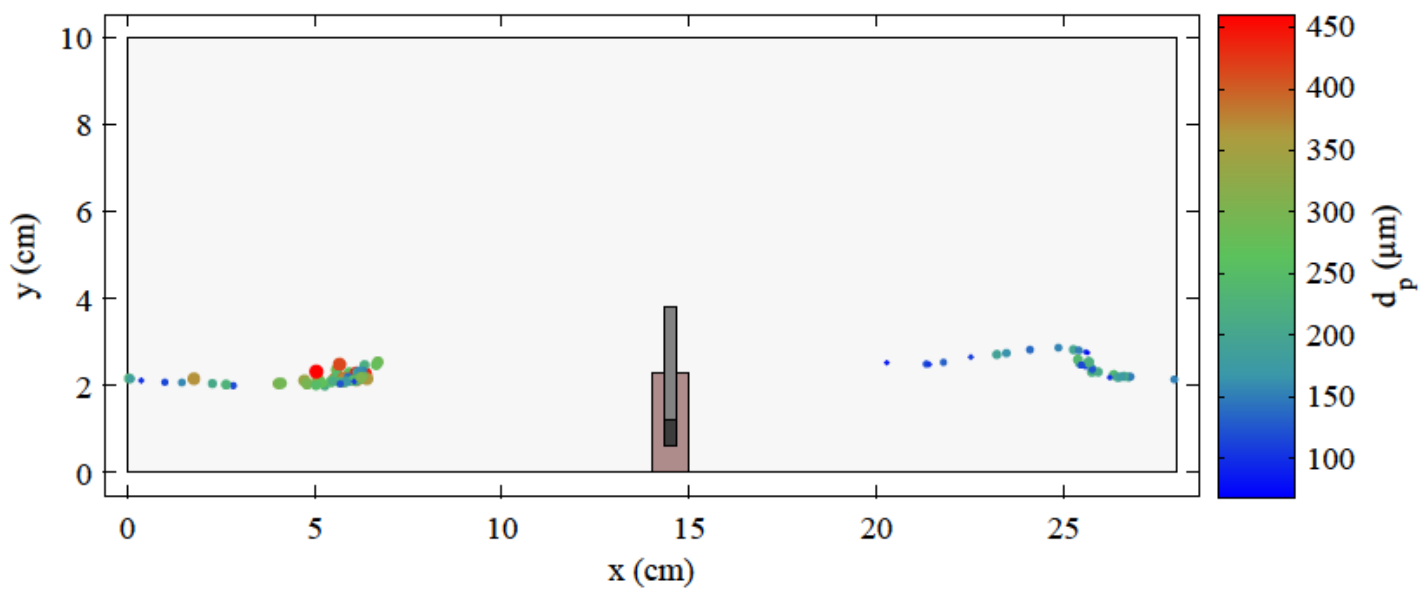

(e)

Figure 8.3: Location of the particles for the different tested cases after 15 seconds of the injection. d) $\left.\operatorname{Re}_{\text {rot }}=1.0 \cdot 10^{4}, \mathrm{e}\right) \mathrm{Re}_{\mathrm{rot}}=1.7 \cdot 10^{5}$. Scraper motion from right to left. 


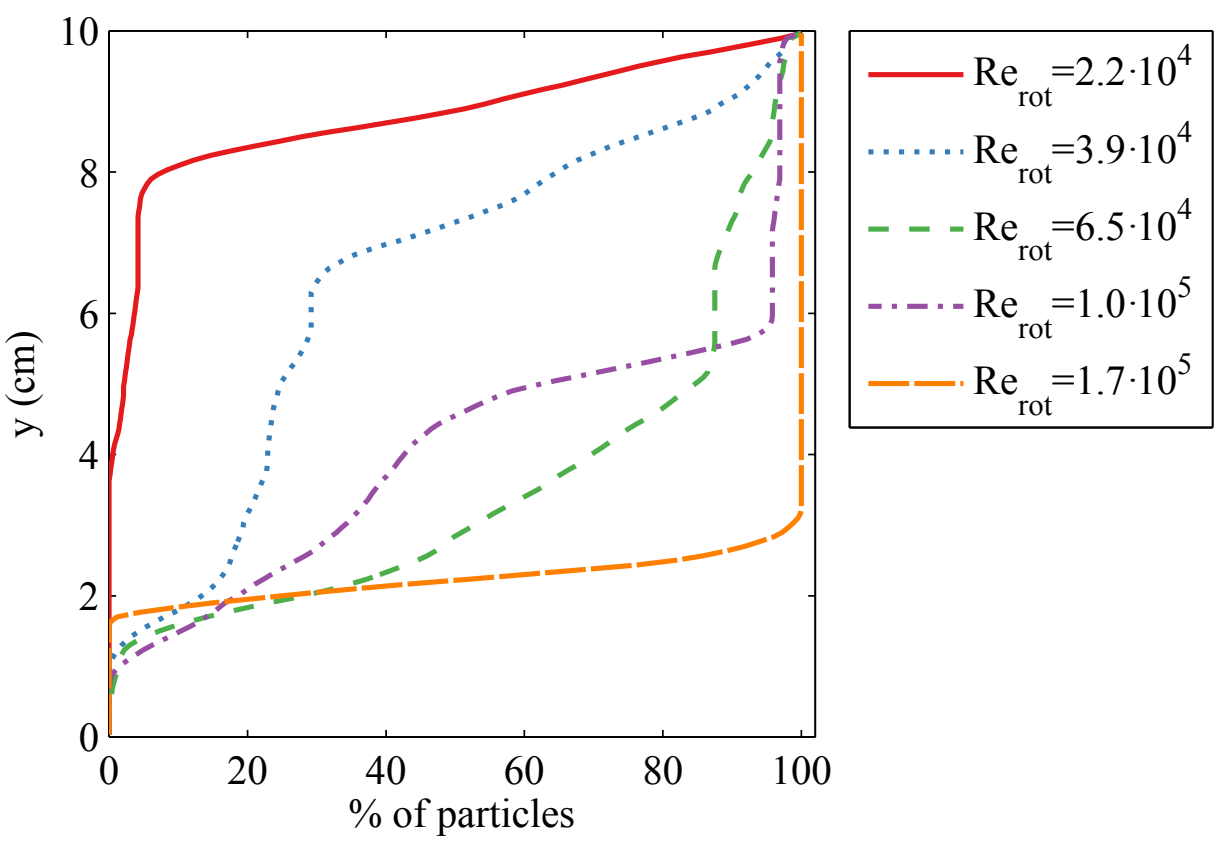

Figure 8.4: Cumulative distribution of particles (\%) along the vertical distance. 


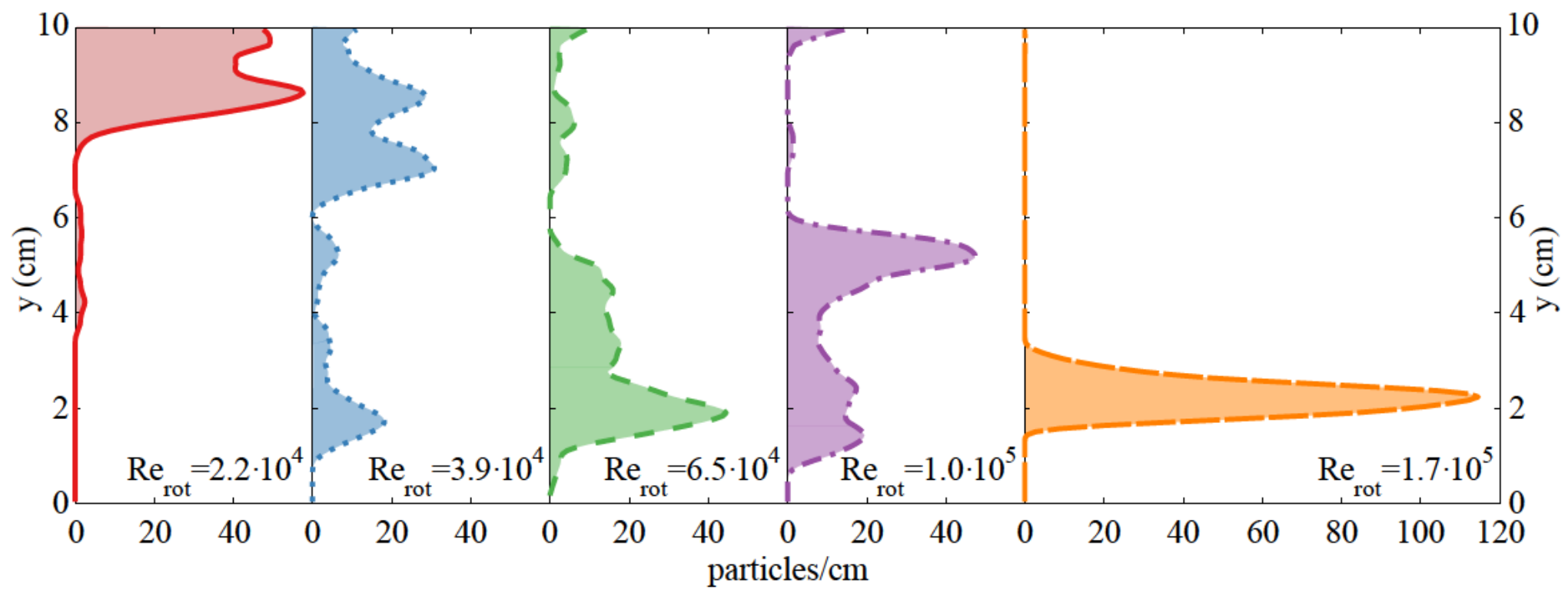

Figure 8.5: Concentration of particles $C_{P}$ [particles $\left./ \mathrm{cm}\right]$ for the different cases simulated. 


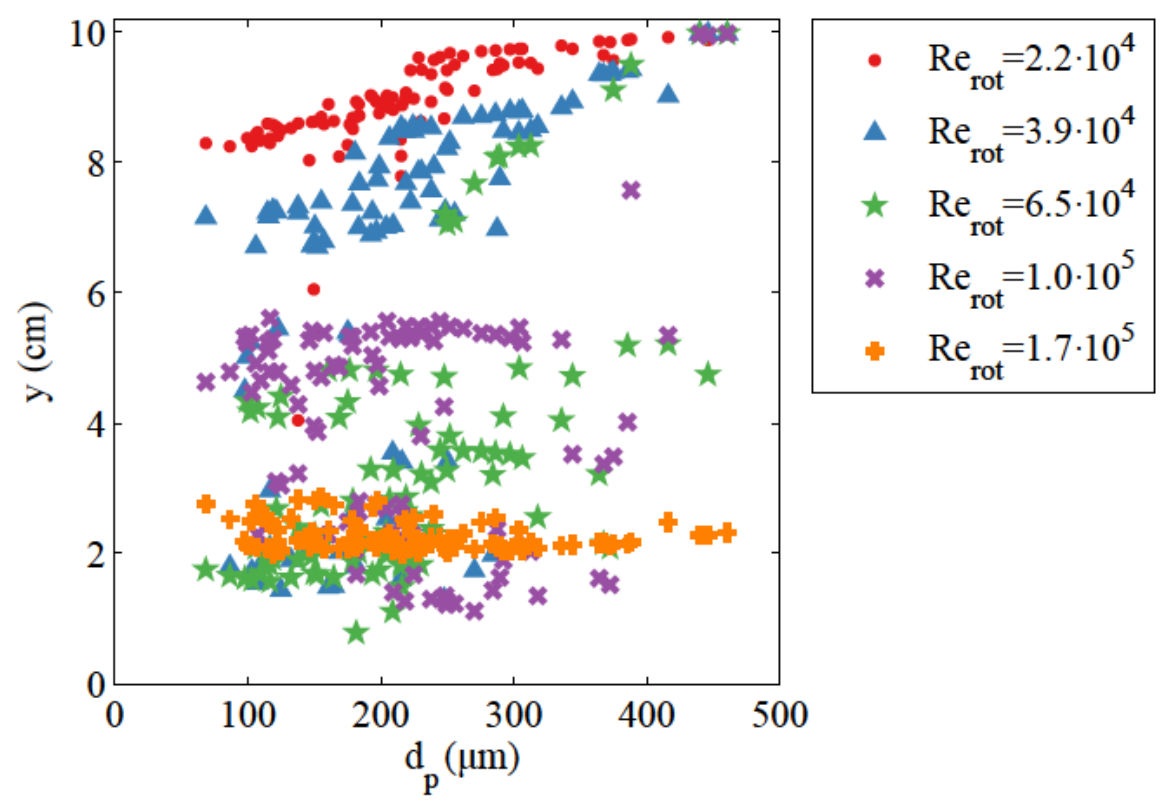

Figure 8.6: Vertical location of the particles after 15 seconds of the injection as a function of the operating regime $\left(\operatorname{Re}_{\text {rot }}\right)$ and particle diameter $\left(d_{p}\right)$.

To observe the effect that the size of the particles has on the distribution, Fig. 8.6 represents the vertical location of each particle versus its diameter, including all the simulated cases. For the lower scraping velocity cases there is a clear influence of the particles diameter on their vertical location: the bigger they are the more on the top are located. However, as the scraping velocity increases this effect vanishes, and for the higher velocity case the effect of the size is completely nonexistent.

\section{Spatial particle tracking}

For a more graphical interpretation of the simulation results, three different particles, with three different representative sizes were tracked for plotting their trajectory in each of the simulated cases. The particles sizes are respectively $100 \mu \mathrm{m} 250 \mu \mathrm{m}$ and $460 \mu \mathrm{m}$. As the domain represents only one scraper with periodic boundary conditions, once a particle leaves the domain from one of the periodic boundaries it enters the domain from the other periodic boundary. If this occurs it means that the particle has abandoned this "scraper domain" to enter the domain of the following scraper.

To take this into account, when representing the trajectory of the particle, if a particle leaves the volume the $x$ coordinate of its location is modified by adding or subtracting the length of the domain, depending on which periodic side is crossed. For instance, if a particle leaves the domain from the right periodic boundary, its $x$ location coordinate when plotting the trajectory is incremented by $28 \mathrm{~cm}$, which is the length of the periodic domain. In the same way, if the particle leaves the domain from the left boundary the $x$ coordinate is reduced by $28 \mathrm{~cm}$.

Figure 8.7 plots the trajectories of each one of the mentioned particles for each one of the simulated cases. For the smaller particle $\left(d_{p}=100 \mu \mathrm{m}\right.$, Fig. 8.7a) it is possible to see that only for the lower velocity case $\left(\operatorname{Re}_{\text {rot }}=2.2 \cdot 10^{4}\right)$ it escapes from the 
recirculation. The velocity of the vortex was then not high enough to trap the particle, and then it rose continuously till the top wall. For all the other cases the particle was captured by the vortex, and remained swirling inside it.

Regarding the middle size particle $\left(\mathrm{d}_{\mathrm{p}}=250 \mu \mathrm{m}\right.$, Fig. 8.7b) it escapes the vortex also for the second velocity case $\left(\operatorname{Re}_{\text {rot }}=3.9 \cdot 10^{4}\right)$. Buoyancy force obviously increases as the diameter of the particle does. The larger particle $\left(d_{p}=460 \mu \mathrm{m}\right.$, Fig. 8.7c $)$ escapes the recirculation in all the cases except the last one $\left(\operatorname{Re}_{\text {rot }}=1.7 \cdot 10^{5}\right)$, which is the only one with strong enough velocities to capture the particle into the recirculation vortex. All the other cases would lead to a more or less stratified flow, where a portion of the solid phase is stacked on the top.

It is then possible to conclude that when a particle is not captured by the vortex there is no chance for it to be captured anymore, and it will rise irretrievably due to the buoyancy force. The time that the particle will take to reach the top wall depends on the velocity of the horizontal flow that drags it.

\section{Time-dependent particle tracking}

To complement the previous spatial tracking, the vertical location of the above mentioned particles $\left(\mathrm{d}_{\mathrm{p}}=100 \mu \mathrm{m} \mathrm{d}_{\mathrm{p}}=250 \mu \mathrm{m}\right.$ and $\left.\mathrm{d}_{\mathrm{p}}=460 \mu \mathrm{m}\right)$ is here plotted versus time after their injection. The dots that appear in each time-lines correspond to the moment the particle leaves the domain. If the point is filled, it means that the particle exited through the right boundary, if it is empty the particle exited the domain through the left boundary.

In Fig. 8.8a $\left(d_{p}=100 \mu m\right)$, only for the lower scraping velocity $\left(\operatorname{Re}_{\text {rot }}=2.2 \cdot 10^{4}\right)$ the particle is stratified in the top in less than 15 sec. after being injected. In all the other cases the particle goes up and down as it is captured inside the recirculation. It is possible to see that the oscillations have a different period depending on the case: higher scraping velocity implies higher frequencies. On the other hand, the amplitude is not constant, which denotes the instability of the flow.

In Fig. $8.8 \mathrm{~b}\left(\mathrm{~d}_{\mathrm{p}}=250 \mu \mathrm{m}\right)$, the same situation of Fig. $8.8 \mathrm{~b}$ is reproduced, but now the particle rises to the top for the two lower scraping velocity cases $\left(\operatorname{Re}_{\mathrm{rot}}=2.2 \cdot 10^{4}\right.$ and $\left.\operatorname{Re}_{\text {rot }}=3.9 \cdot 10^{4}\right)$. Comparing Fig. $8.8 \mathrm{a}$ with Fig. $8.8 \mathrm{~b}$, it is possible to see that as the scraping velocity increases the time-lines becomes more similar between different particles. For instance, time-lines for $\operatorname{Re}_{\text {rot }}=1.7 \cdot 10^{5}$ are completely equal for both $\mathrm{d}_{\mathrm{p}}=100$ $\mu \mathrm{m}$ and $\mathrm{d}_{\mathrm{p}}=250 \mu \mathrm{m}$. However, as the scraping velocity decreases the oscillations are different between different particles. This can be explained by the drag force of the flow over the particle: when the vortex velocity is high $\left(\operatorname{Re}_{\text {rot }}=1.7 \cdot 10^{5}\right)$ the drag force is also high, and the influence of the change in size of the particle is negligible, but when the velocity decreases the drag force is more sensible to the size of the particle. Finally, in Fig. 8.8c $\left(d_{p}=460 \mu \mathrm{m}\right)$ the particle reaches the top in all the cases except in the last one. Moreover, in all the cases the particle reaches the top in almost the same time, near 15 seconds after its injection.

To conclude, Fig. 8.9 compares the residence time -the time that a particle stays inside the domain before leaving it- normalized by the scraping period $\mathrm{T}$-the time that the scrapers takes to scrap all the domain length- for two different particles: $d_{p}=100$ $\mu \mathrm{m}$ and $\mathrm{d}_{\mathrm{p}}=460 \mu \mathrm{m}$. Looking to the lower scraping velocity, it is possible to see how the particles rise to the top and remains there steady: the normalized residence time 


$$
\left[\begin{array}{cc}
-\mathrm{Re}_{\text {rot }}=2.2 \cdot 10^{4} & ---\mathrm{Re}_{\text {rot }}=6.5 \cdot 10^{4} \\
-\cdots-\cdot-\mathrm{Re}_{\text {rot }}=1.0 \cdot 10^{5} & -\mathrm{Re}_{\text {rot }}=1.7 \cdot 10^{5}
\end{array}\right]
$$

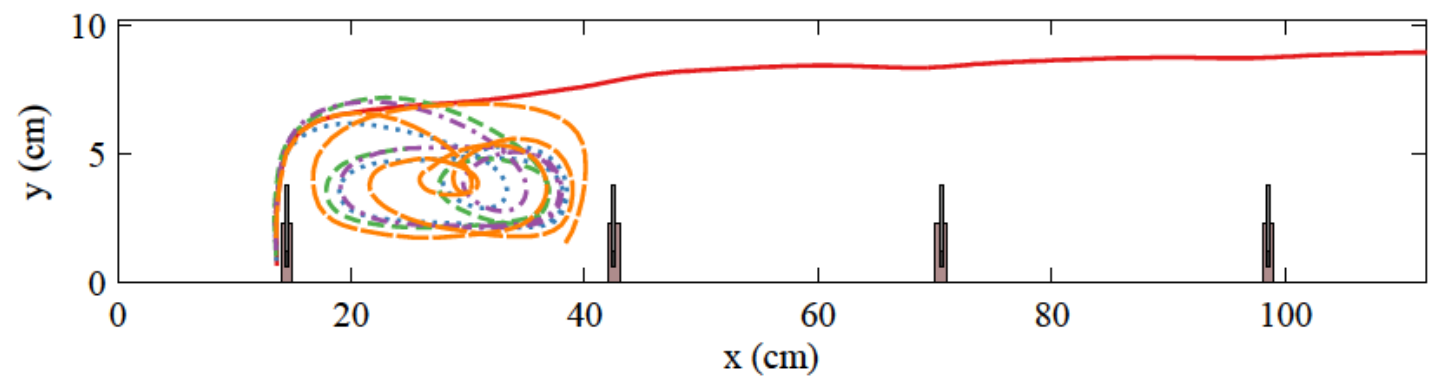

a)

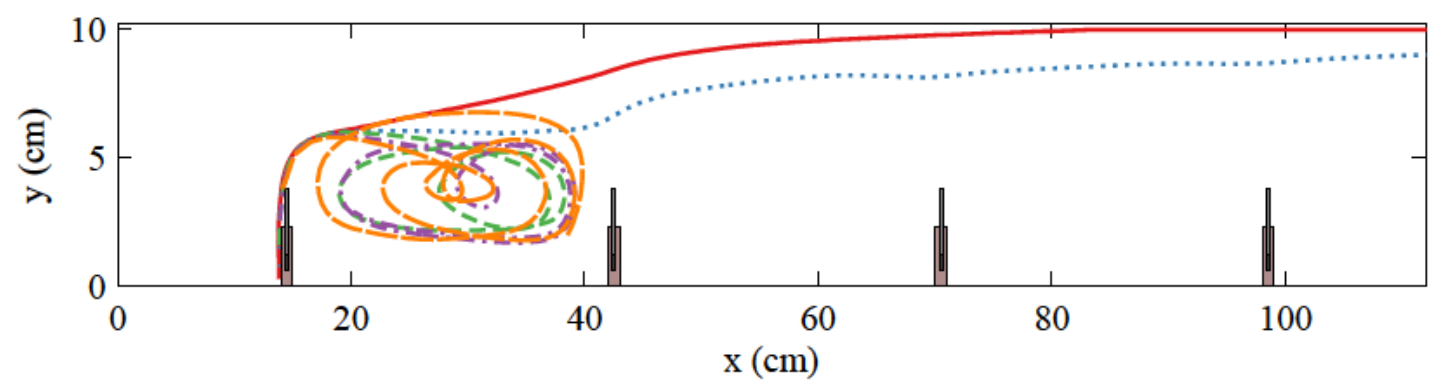

b)

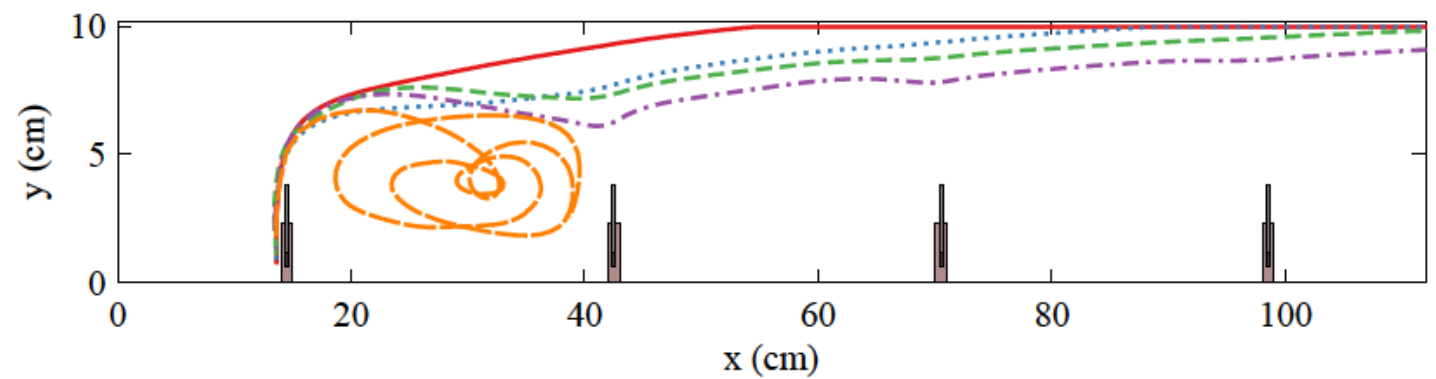

c)

Figure 8.7: Trajectories of the different particles for eeach one of the cases $\left(\operatorname{Re}_{\mathrm{rot}}\right)$ simulated. a) $\left.d_{p}=100 \mu \mathrm{m} \mathrm{b}\right) d_{p}=250 \mu \mathrm{m}$ and c) $d_{p}=460 \mu \mathrm{m}$. 


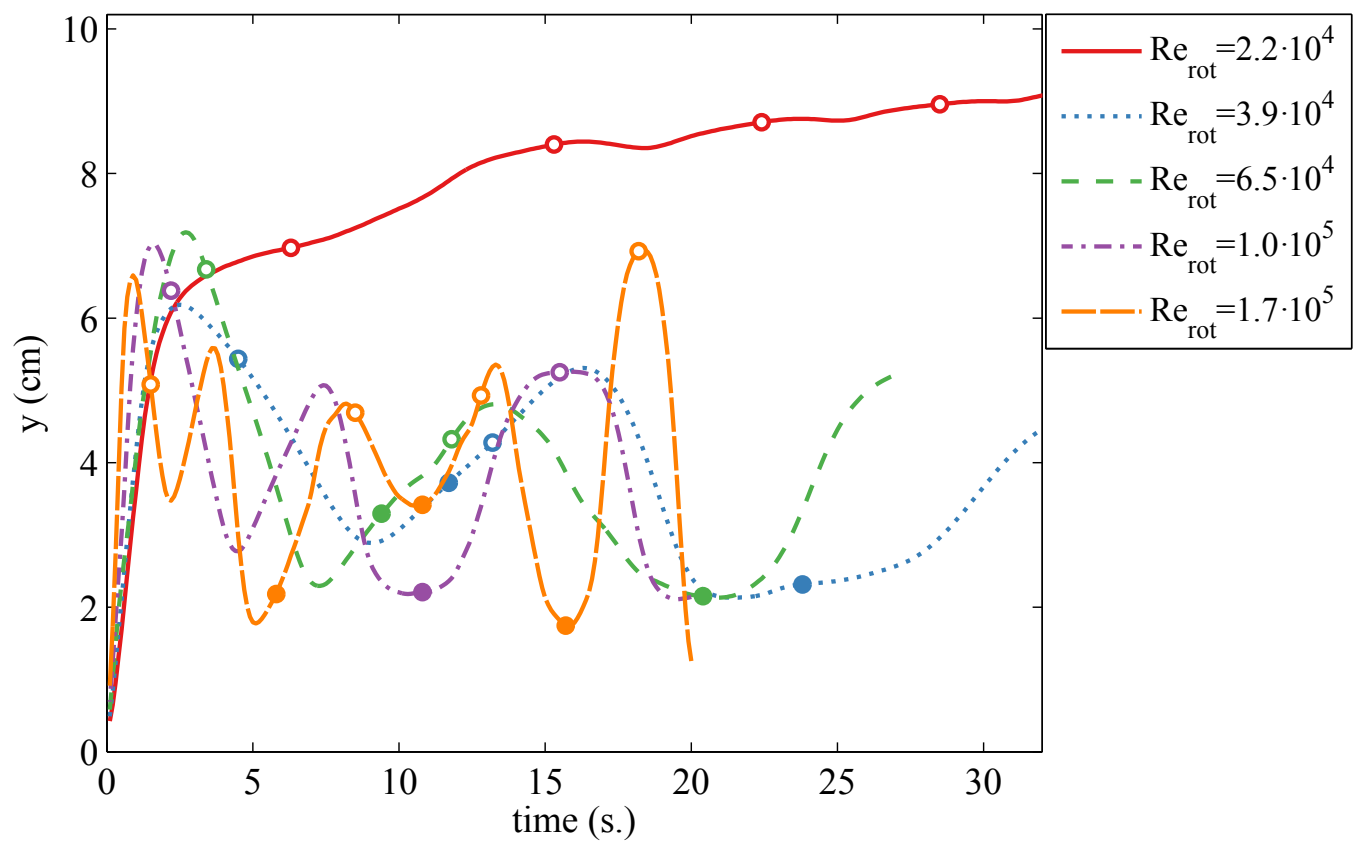

(a)

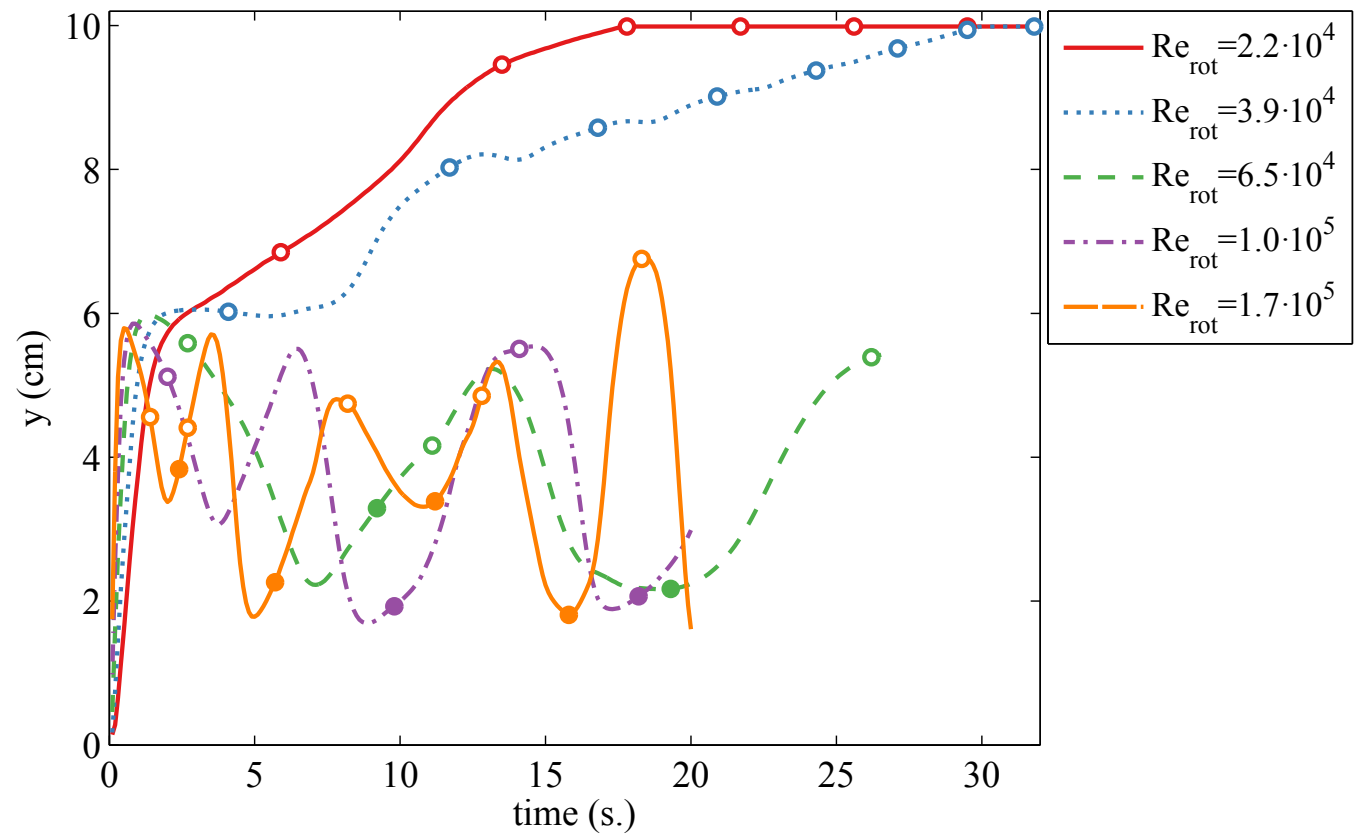

(b)

Figure 8.8: Location of the particle for the different tested cases $\left(\mathrm{Re}_{\mathrm{rot}}\right)$ after 15 seconds of its injection. a) $\left.d_{p}=100 \mu \mathrm{m}, \mathbf{b}\right) d_{p}=250 \mu \mathrm{m}$ 


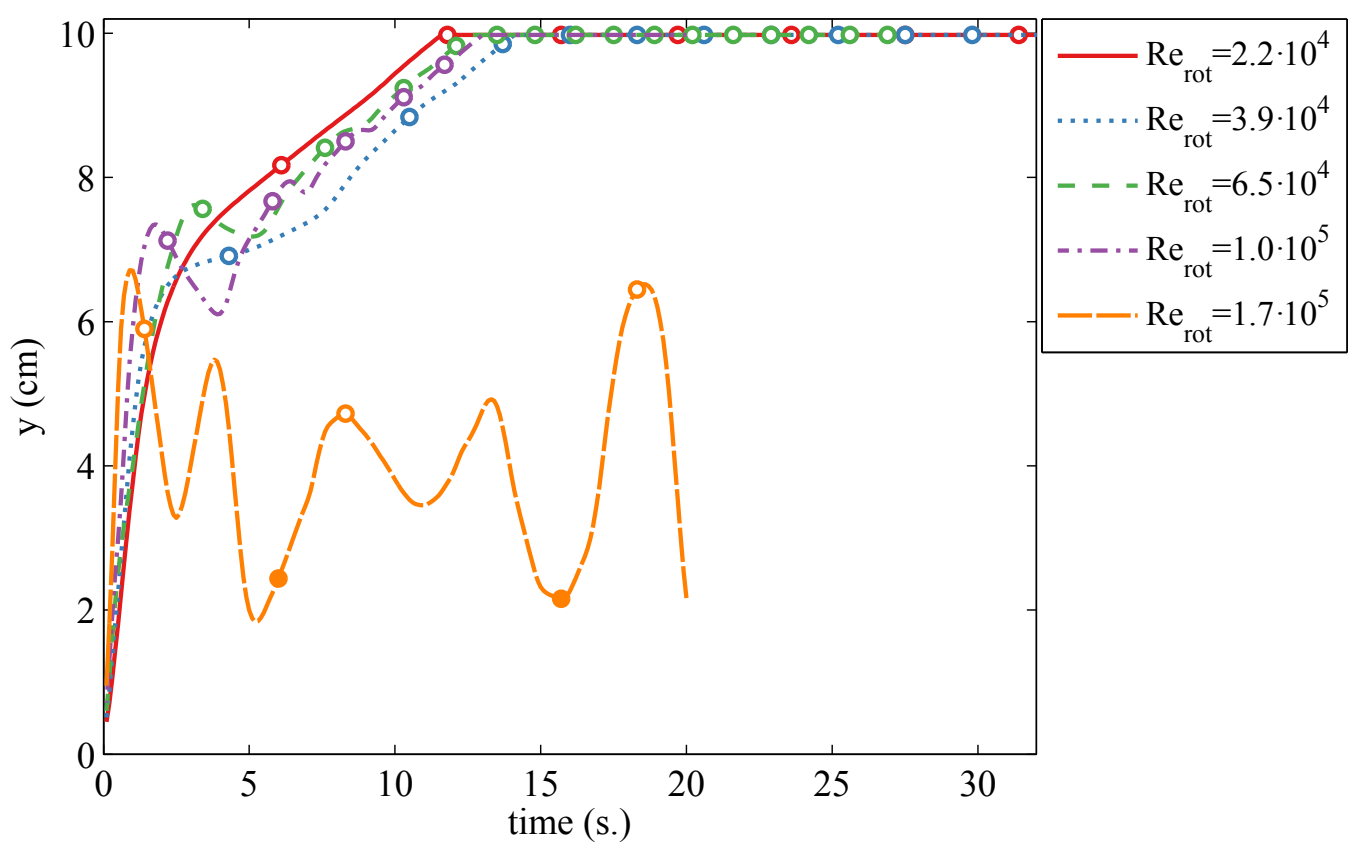

(c)

Figure 8.8: Location of the particle for the different tested cases $\left(\operatorname{Re}_{\mathrm{rot}}\right)$ after 15 seconds of its injection. c) $d_{p}=460 \mu \mathrm{m}$ (continued)

is equal to 1 , which means that the particle has no relative movement respect to the top wall. As the scraping velocity increases $\left(\uparrow \mathrm{Re}_{\mathrm{rot}}\right)$ the normalized residence time increases since the particle is captured by the flow. For instance, in the lower particle size (8.9a) all the other scraping velocities make the normalized residence time differ from one, whereas in the higher particle size $(8.9 \mathrm{~b})$ only the higher scraping velocity has a different normalized residence time as in all the lower velocities it tends to one (particles get stratified, remaining on the top wall without movement).

\subsubsection{The LES turbulence modelling}

\section{Velocity field}

As in 8.1.1, the numerical LES velocities where non-dimensionalized by the velocity of the scraper (see Eq. 8.1). Fig. 8.10 shows the time averaged iso-contours for the relative, normalized velocity field $U *$ obtained by using the LES turbulence modelling. It also includes the most representative stream lines. Comparing it with Fig. 8.1, Fig. 8.10 reveals a similar flow pattern but in this case the vortex length -along the horizontal dimension- is shorter, and the perturbation introduced by it arrives to higher locations of the fluid domain. The LES turbulence modelling is providing then some differences in the flow pattern not reproduced by the RANS simulations with the $k-\omega$ model previously reported.

The difference between LES and RANS models obey to the expectations according to existent works in the literature. It has been demonstrated that when studying the flow around sharp or rounded objects the RANS model predict too large separated 


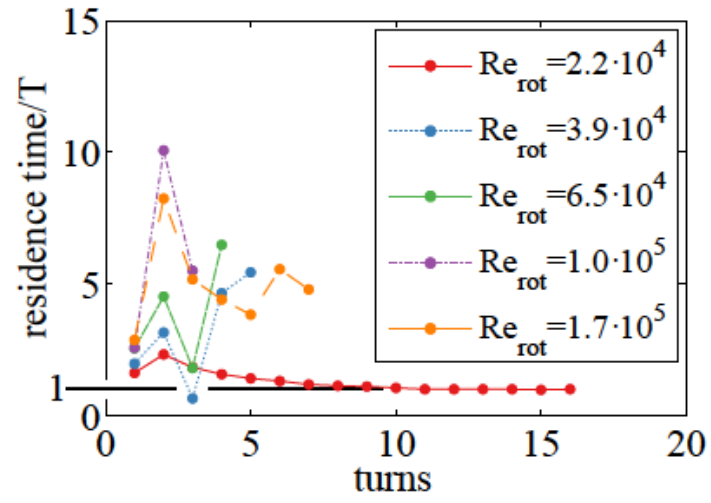

a)

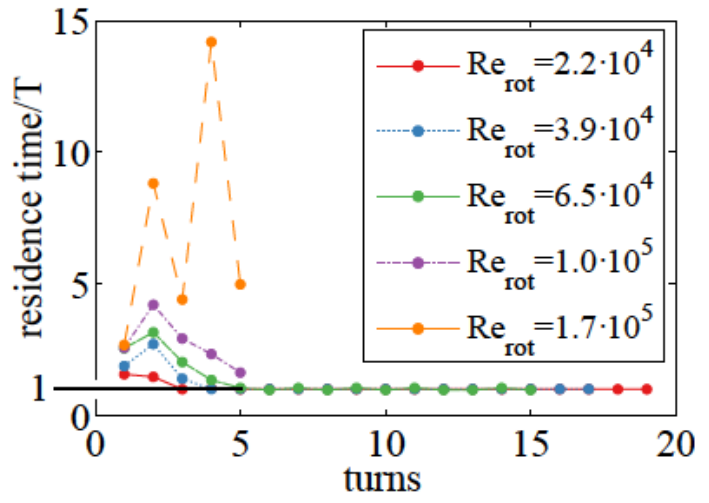

b)

Figure 8.9: Normalized particle residence time vs. number of turn of the particle over the domain. a) $d_{p}=100 \mu \mathrm{m}$, b) $d_{p}=460 \mu \mathrm{m}$.

regions compared to LES models, that in general are more accurate to predict the flow $[140,141]$.

The effect of the large eddies can be better appreciated with iso-contours of the instantaneous $U *$ field. For instance, Figs. 8.11a and 8.11b include them for $\operatorname{Re}_{\text {rot }}=3.9 \cdot 10^{4}$ and $\operatorname{Re}_{\text {rot }}=1.7 \cdot 10^{5}$ once the flow was developed -i.e. the time averaged flow does not change with increasing time. The velocity field shows alternations with regions of high and low velocities, revealing the presence of sub-structures, far from the uniformity shown in Figs. 8.1 and 8.10. These substructures correspond to the presence of the large eddies that increase the mixing. Comparing Fig. 8.11a with Fig. 8.11b, the last one shows a larger area with high velocities $(U * \geq 0.5)$. This is normal, as higher scraping velocity increases the level of turbulence and mixing.

\section{Spatial particle tracking}

The particle tracking shown in Fig. 8.12 includes the trajectories for the three above considered particles in the cloud $\left(d_{p}=100 \mu \mathrm{m}, d_{p}=250 \mu \mathrm{m}\right.$ and $\left.d_{p}=460 \mu \mathrm{m}\right)$ and two different cases $\left(\operatorname{Re}_{\text {rot }}=3.9 \cdot 10^{4}\right.$ and $\left.\operatorname{Re}_{\text {rot }}=1.7 \cdot 10^{5}\right)$.

For the first -lower Reynolds- case (Fig. 8.12a) the particles rise fast to the top region of the domain. The larger particle oscillates few times an then stratify on the top, whereas the other two smaller particles are carried by the flow oscillations. The trajectories of those two particles do not allow to predict whether or not stratification takes place. It may occur at any time that particles escape the main flow and enter in the low velocity region near the top.

The second case (Fig. 8.12b) shows a completely different situation. In this case all the particles are carried by the flow and the vortices are strong enough to make the particles impact the bottom wall. The diameter of the particle is in this case not playing a significant role, as the one that escapes the first vortex was the medium size of the three. Once the impact takes place the particle can by driven again by the flow or induce additional nucleation, being in this case attached to the wall. Any of the two possibilities are impossible to predict.

Comparing both RANS and LES results, only the last turbulence model one was 


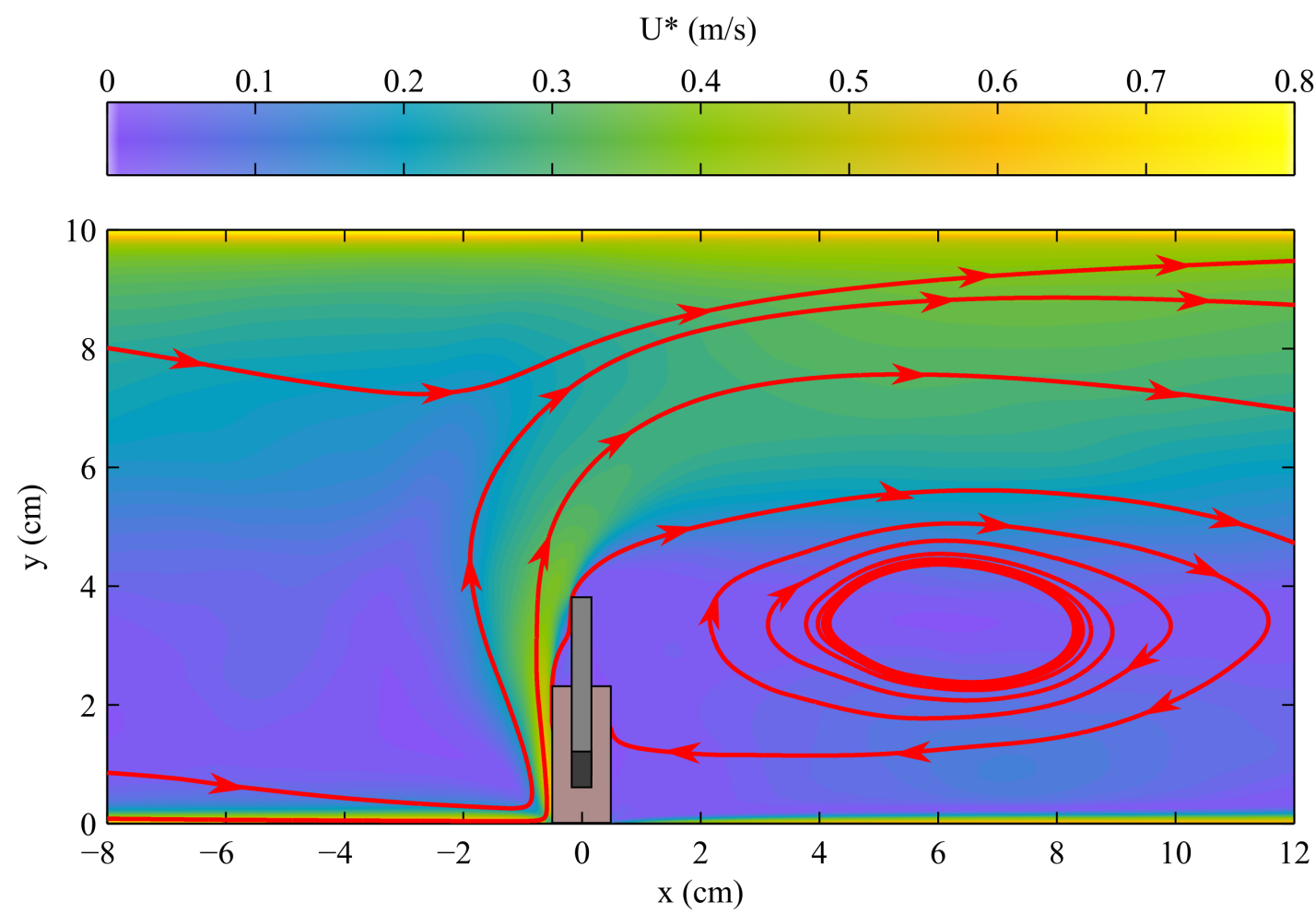

Figure 8.10: Time averaged iso-contours and streamlines for $U *$ using LES turbulence modelling, $\operatorname{Re}_{\mathrm{rot}}=1.7 \cdot 10^{5}$. Scraper moves from right to left. 


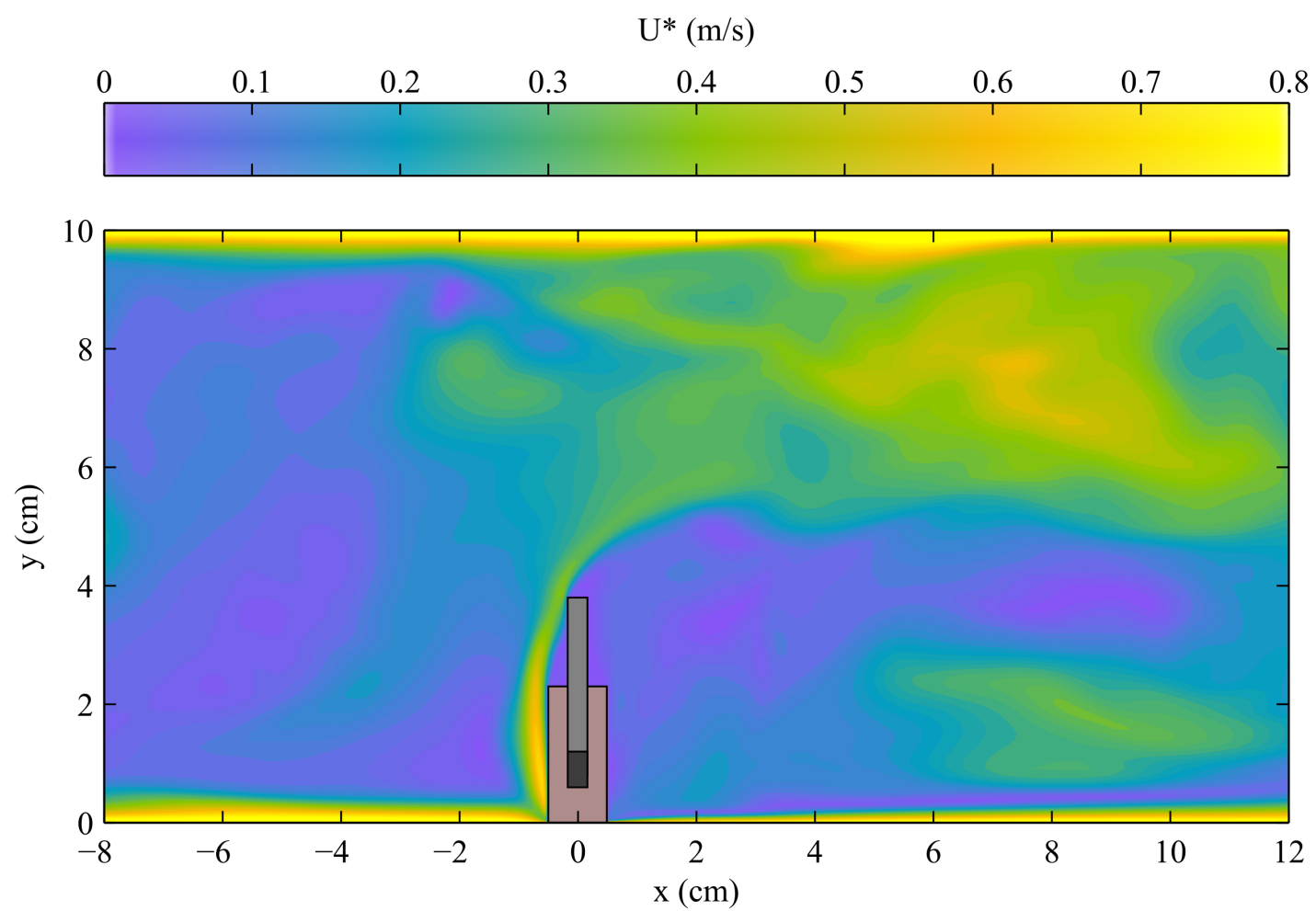

(a)

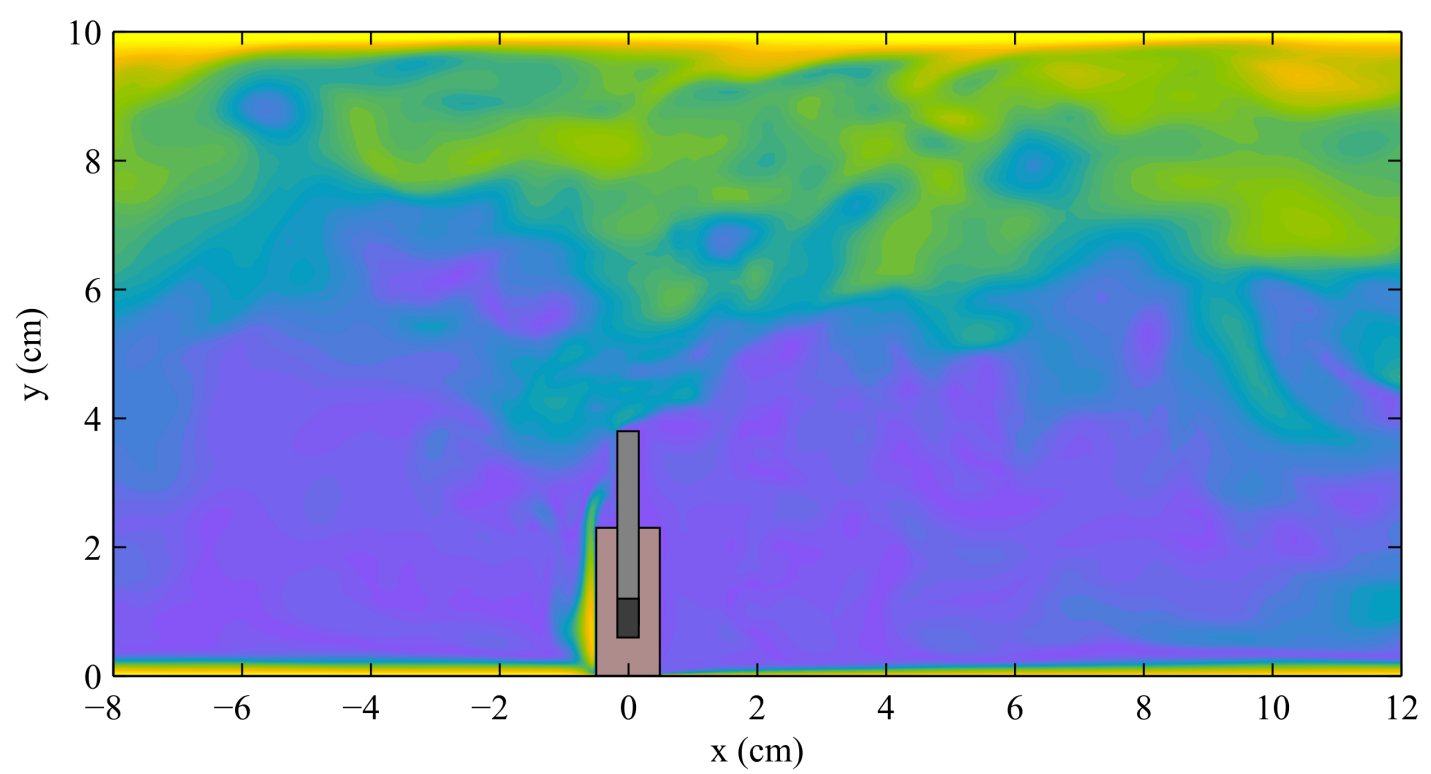

(b)

Figure 8.11: Instantaneous iso-contours for $U *$ using LES turbulence modelling. Scraper move from right to left. a) $\left.\operatorname{Re}_{\mathrm{rot}}=3.9 \cdot 10^{4} \mathbf{b}\right) \operatorname{Re}_{\mathrm{rot}}=1.7 \cdot 10^{5}$ 


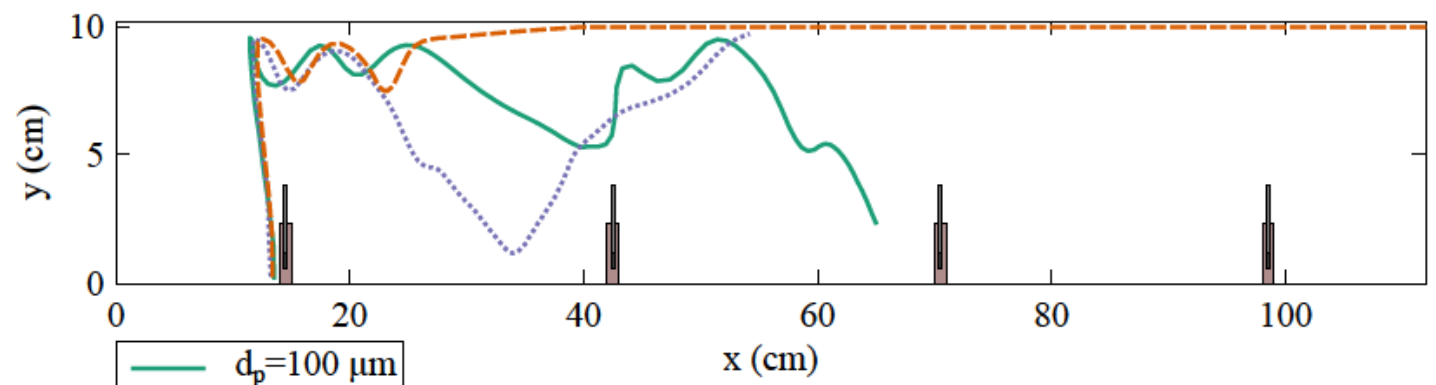

a)

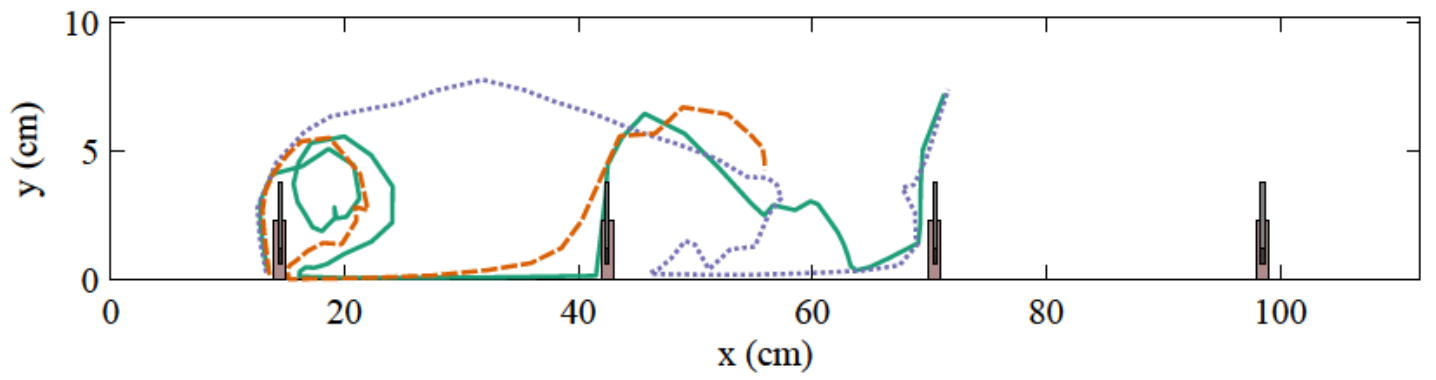

b)

Figure 8.12: Particle trajectories for each one of the cases simulated. a) $\operatorname{Re}_{\mathrm{rot}}=3.9 \cdot 10^{4}$, b) $\operatorname{Re}_{\text {rot }}=1.7 \cdot 10^{5}$.

able to predict the impact of particles over the plate surface. The RANS model however provided results on the particle stratification that where in a good agreement with the experimental evidences. Considering the high cost of the LES simulations versus the RANS ones the data provided by the RANS modelling can be of great value, as they can determine if a good mixing is present in the SSPHE. The main drawback is the failure in predicting the impact of the particles over the plate. This impact could explain the fact that the heat transfer does not increases at high scraping rates -the impacts lead to the ice layer scaling- as may be expected by the higher mixing.

It can be then concluded that even if both turbulence modelling approaches can provide insight into two different phenomena -particle stratification and impact over the plate- none of them has provided good enough results for considering it as fully successful. Moreover, the flow pattern obtained by each of them differed significantly from the one observed experimentally in chapter 5: the roll up vortex was in both cases more distant from the scraper rear side. The two-dimensional approach is therefore a low computational resource able to predict some global parameters of the flow, but cannot be considered as an accurate model in the problem study.

\subsection{Results for the three-dimensional approach}

Figure 8.13 shows the dimensionless velocity iso-contours and the parallel -in-planevector field in a plane perpendicular to the scraper. Such plane corresponds to the 
plane of the laser sheet in the PIV experiments, $r=r_{l}=0.6 R$, and the velocities are non-dimensionalized with the scraper tangential velocity at that point, $2 \pi \mathrm{N}=r_{l}$. In Fig. 8.13a the roll-up vortex is present, as observed in the PIV experiments. Figure 8.13b shows a detailed view of the plane. Comparing Fig. 8.13b with Fig. 6.12 of chapter 6 , both of them agree on the flow pattern and on the magnitude of the velocity. The numerical results reproduce indeed very well the experimental measured values in terms of vortex diameter. The presence of the roll-up vortex with the same velocity magnitude and dimensions confirms the fact that the lack of agreement with the $2 \mathrm{D}$ simulations was due to the absence of rotation.

There is, however, a considerable difference between Fig. 8.13b (numerical) and Fig. 6.12 (experimental): whereas the vortex core shows low velocity values in Fig. 6.12, in Fig. 8.13b it occurs the opposite, higher velocity values are found inside it. This, as will be seen later, is due to the strong radial flow -parallel to the scraper- that takes place in the vortex core. This radial flow was impossible to detect by the PIV measurements, as it was perpendicular to the laser sheet.

The magnitude of the radial flow can be better appreciated in Fig. 8.14, which shows the velocity vector field along a tangential plane. As it is possible to notice, the highest relative velocities - excepting the walls- are found in the vortex core, and have a high radial component.

This radial flow has been observed in mixer and stirrers with a similar configuration of agitators, as was mentioned in chapter 6 . The numerical results reveal hence a similar radial flow also in the SSPHE. Recalling the PIV experiments, the presence of this outof-plane components can explain the low number of velocity vectors in the vortex core: particles there move out of the plane, and hence the cross correlation failed in retrieving any valid displacement.

Figure 8.15 provides a more illustrative view of the velocity field inside the SSPHE. In particular, it depicts the iso-contours of $\mathrm{U}^{*}$ and the corresponding in-plane (radial) vector field over different radial planes. In the periodic section (Fig. 8.15a) and the section at $\theta=45^{\circ}$ (Fig. 8.15b), the relative velocity is considerably uniform, with a value of $\mathrm{U}^{*} \approx 0.4$. A near zero velocity region is located in the proximity of the actuator shaft, where the tangential velocity of the scraper is lower. The velocity is mainly tangential (perpendicular to the plane). The radial component of the velocity is directed out of the SSPHE in almost all the plane except near the top and bottom walls, where the radial velocity is directed to the rotating shaft with slightly higher values.

The flow pattern far from the scraper is then characterized by a main tangential (circular) flow with a small radial component originated by the centrifugal forces that make the flow drift from the circumferential path. The flow that drifts out is balanced with a radial flow towards the shaft that takes place near the walls, where the centrifugal forces are lower due to the slip effect of the wall.

In the plane on the top of the scraper (Fig. 8.15c) the velocity reaches its highest value near the external wall. The radial flow generated by the scraper impacts there and is deviated. The in-plane vector field shows the vertical flow generated by the scraper. It increases almost linearly along the scraper along its radial coordinate, as the tangential velocity of the scraper is higher. In all the plane the radial component is directed mainly to the shaft: as the main radial velocity takes place in the rear side of the scraper (see Fig. 8.14), this strong centrifugal flow needs to be balanced with a flow in the opposite direction. 


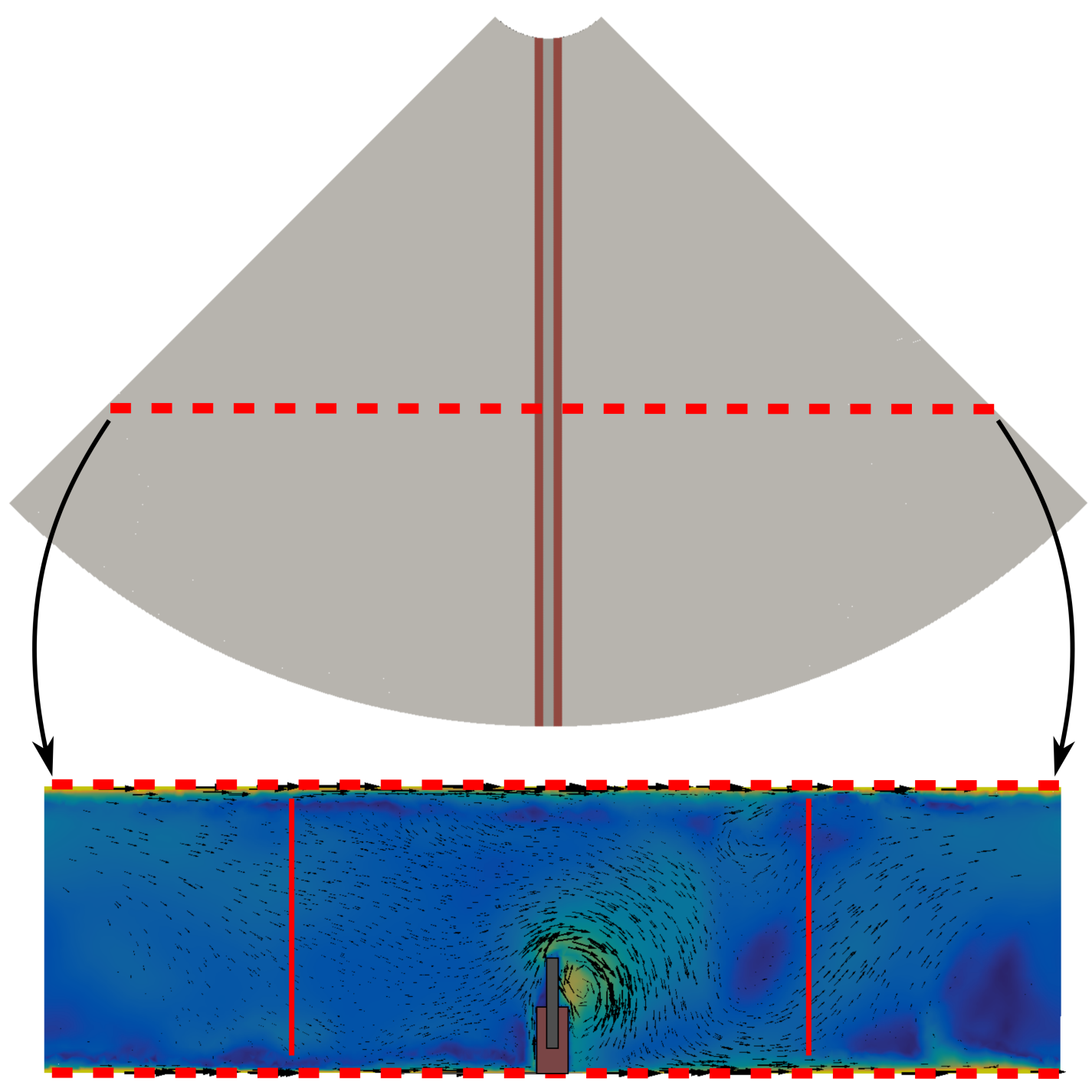

a)

Figure 8.13: Iso-contours and vector field for $\mathrm{U}^{*}$ obtained by the 3D numerical model at the location corresponding with the laser sheet of the PIV experiments $\left(\operatorname{Re}_{\mathrm{rot}}=6.5 \cdot 10^{4}\right)$. a) plane location sketch and velocities 


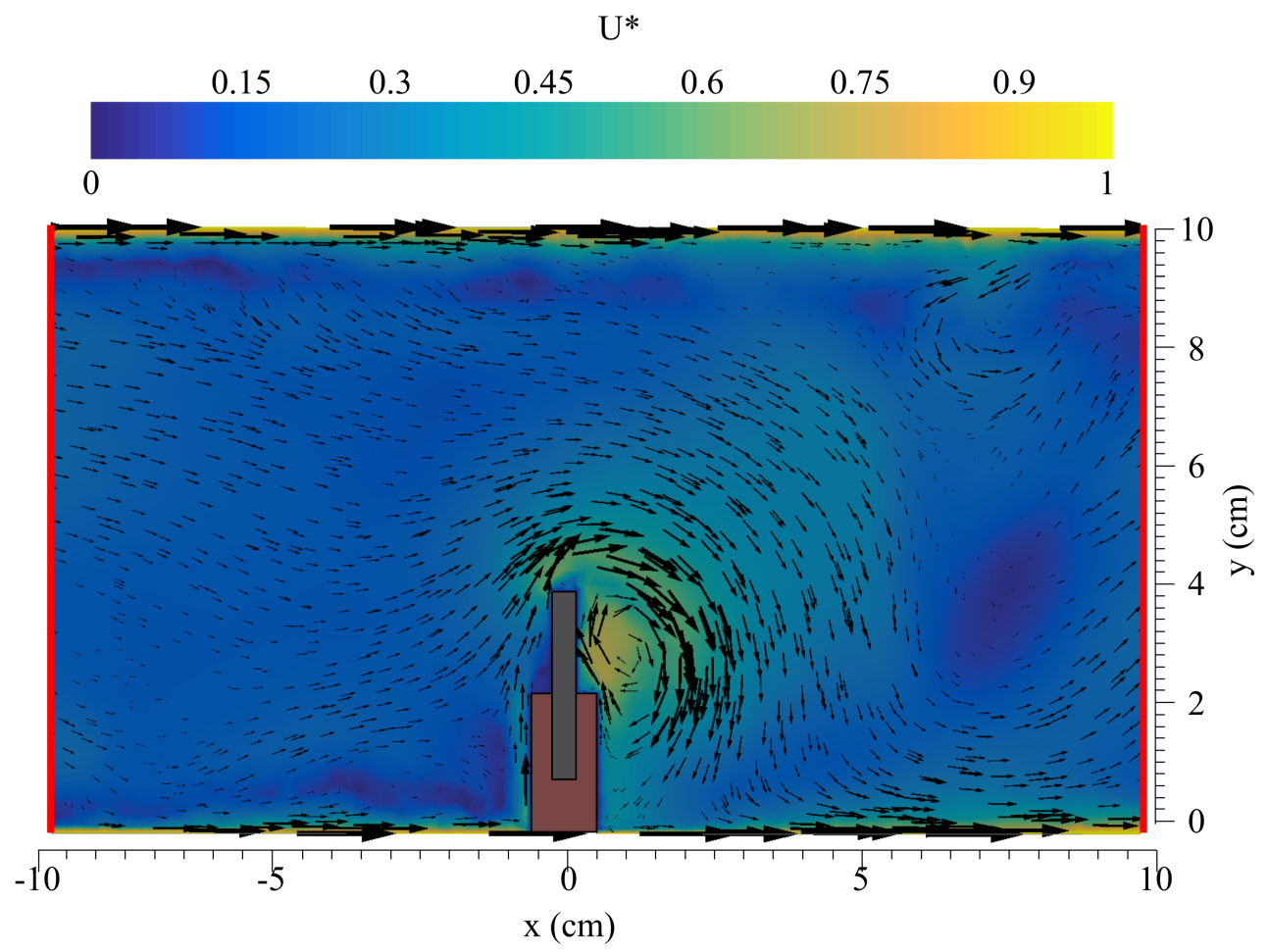

b)

Figure 8.13: Iso-contours and vector field for $\mathrm{U}^{*}$ obtained by the 3D numerical model at the location corresponding with the laser sheet of the PIV experiments $\left(\operatorname{Re}_{\mathrm{rot}}=6.5 \cdot 10^{4}\right)$. b) detail of extracted velocities (continued) 


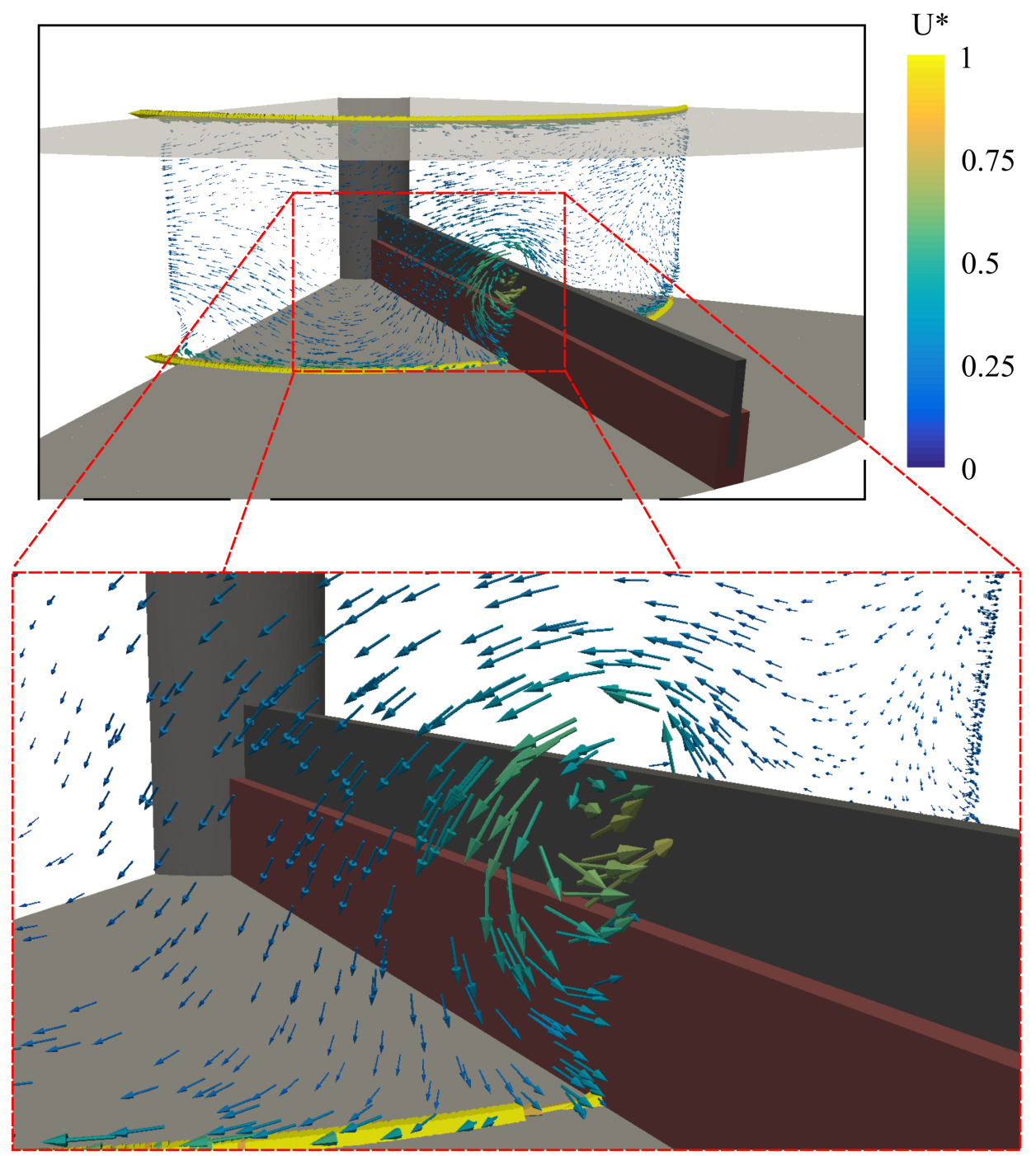

Figure 8.14: Three-dimensional vector field for $\mathrm{U}^{*}$ in a tangential plane $r=\mathrm{R} / 2$ $\left(\operatorname{Re}_{\mathrm{rot}}=6.5 \cdot 10^{4}\right)$. 
Fig. $8.15 \mathrm{~d}$, with the plane located at $45^{\circ}$ after the scraper, shows a velocity field very different from that of Fig. $8.15 \mathrm{c}, 45^{\circ}$ before the scraper. The flow is very influenced by the scraper, and the radial flow is strong and irregular, appearing different in-plane vortical structures. This complex flow pattern provides a good approximation of the nature of the flow, but also points to the limited validity of the RANS simulations. The observed structures are most probably changing in time, as was also observed with the roll-up vortex in the instantaneous PIV measurements (see Fig. 6.28, in chapter 6 ), and therefore only a LES turbulence modelling would be able to provide a more significant data in this aspect. Therefore, the RANS simulation helps understand the global pattern, but not the transient structures that take place in the SSPHE.

Figure 8.16 shows the iso-contours for the radial, dimensionless velocity $\left(\mathrm{u}_{r}{ }^{*}\right)$ in two different tangential planes, together with the in plane (tangential) velocity vector field. Comparing the two planes, it is worth to mention the increasing of the roll-up vortex size as the radial coordinate increases -from the inside to the outside. The higher tangential velocity of the scraper originates therefore a bigger vortex. From the iso-countours it is also possible to see that the highest radial velocity takes place in the vortex and the scraper rear region (as was seen in Fig. 8.14). Figure 8.16,as Fig. 8.15, allows to see the different flow in the two regions, before and after the scraper. The flow before the scraper is more or less uniform, with the velocity almost horizontal. It start to change very near to the scraper, with the flow being deviated over it. On the rear side of the scraper the pattern is completely different. The flow is irregular, with a high radial velocity region (roll-up vortex) and secondary vortexes that appear near the top wall. This is a proof of the instability of the flow.

The flow stream lines starting on a vertical line located in the periodic plane are depicted in Fig. 8.17. The stream lines are coloured by the magnitude of $\mathrm{U}^{*}$. it can be observed that the initial rake of streamlines are separated into two different groups (Fig. 8.17a). One group follows a mainly tangential (circular) flow, with almost no variance in its radial coordinate. The other group however enters into the vortex and experiences a radial displacement while turning into it, arriving even to the lateral wall. Looking to Fig. 8.17b it is possible to see that whether the streamlines enter the vortex or keep in a circular path depends mostly on the vertical (here axial, $z$ ) coordinate. Thereby, streamlines starting at $z / \mathrm{H}>0.7$ are able to escape the roll up vortex and to continue a circular trajectory. Below that level stream lines enters the vortex and follow a rolling, radial flow.

To clarify this pattern, Fig. 8.18 includes two stream lines of each group, coloured in different colors. This distinction of flows can be understood as a mixing frontier, dividing the fluid that is highly mixed and the one that remains more or less unperturbed. As has been seen in the PIV results (see chapter 6) and more recently in the $2 \mathrm{D}$ numerical simulation results, this frontier can be moved by increasing or decreasing the scraping velocity.

It can be then concluded that the $3 \mathrm{D}$ numerical simulations were able to reproduce the more significant pattern observed in the PIV experiments: the roll-up vortex, providing also similar values in terms of relative velocities. Moreover, the numerical results provided a valuable information about the global flow pattern and in particular about the presence and importance of the radial flow inside the SSPHE. 
a)

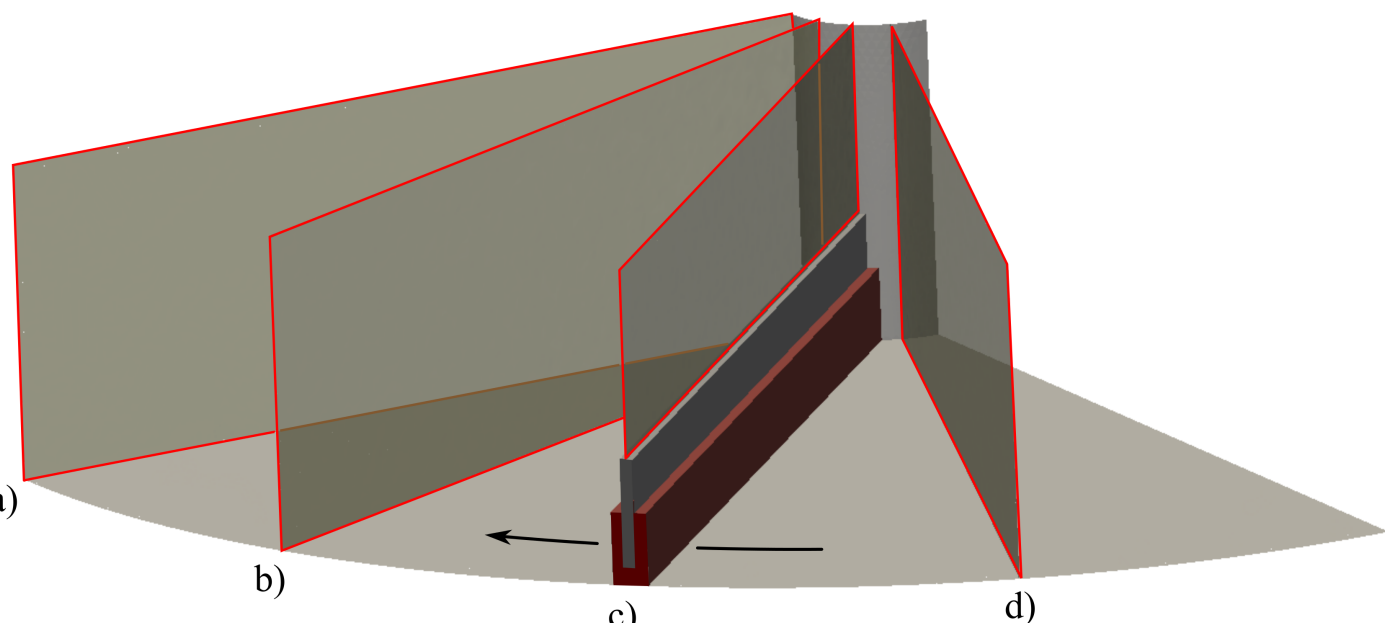

c)

d)

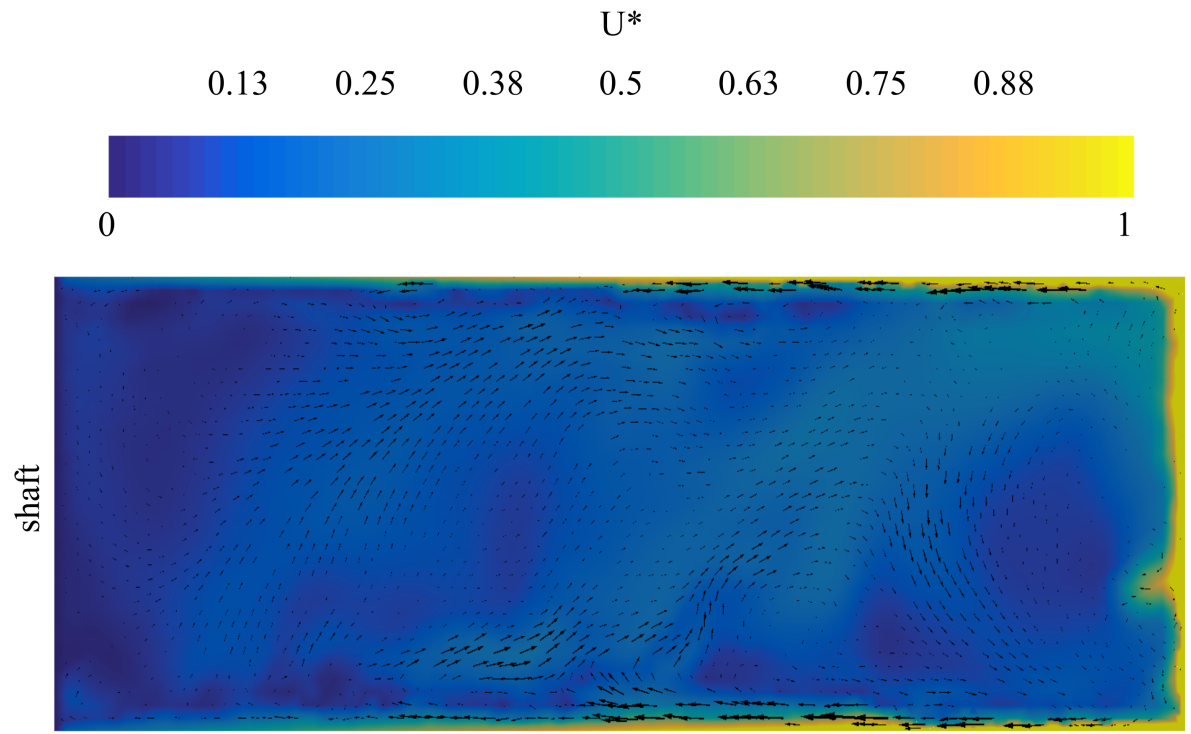

a)

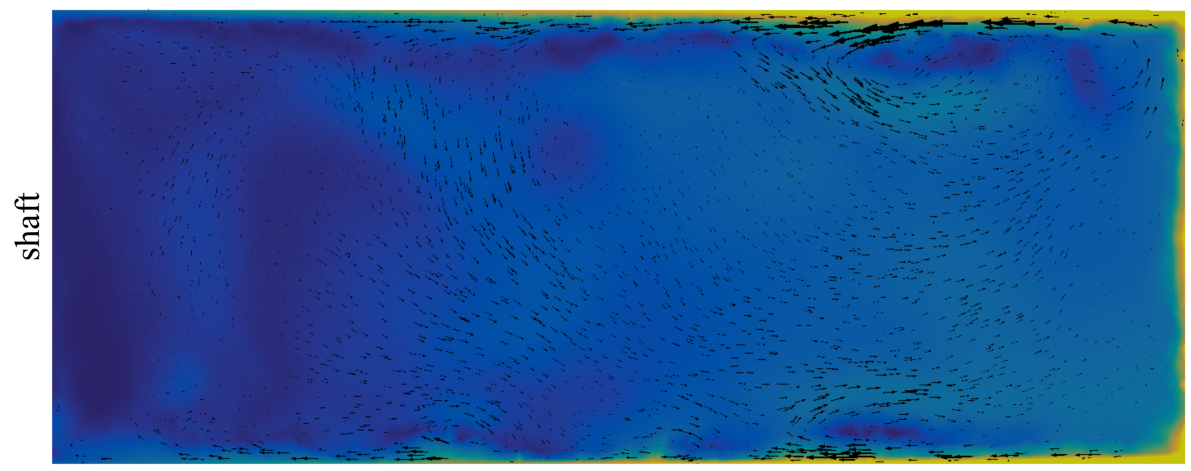

b)

Figure 8.15: Iso-contours and vector field for $\mathrm{U}^{*}$ in different radial planes $\left(\operatorname{Re}_{\text {rot }}=6.5 \cdot 10^{4}\right)$ : a) periodic plane $\theta=0^{\circ}$, b) $\theta=45^{\circ}$. 


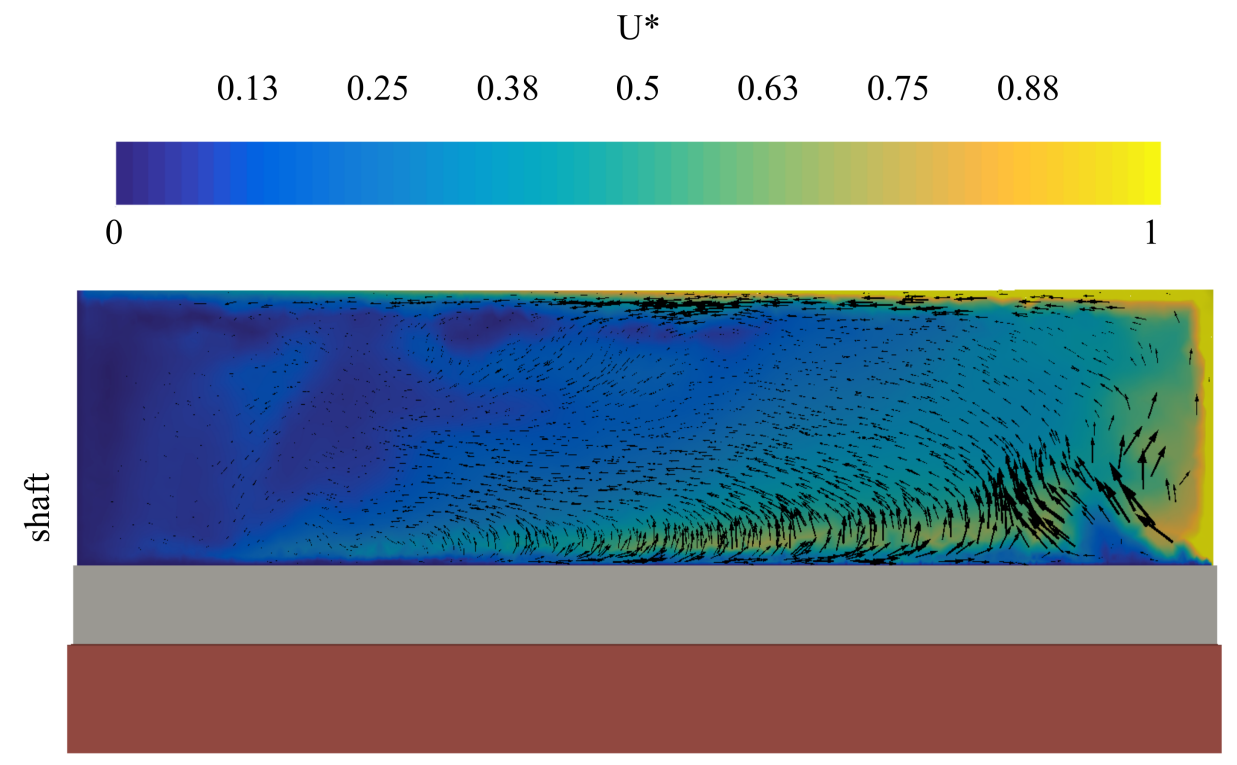

c)

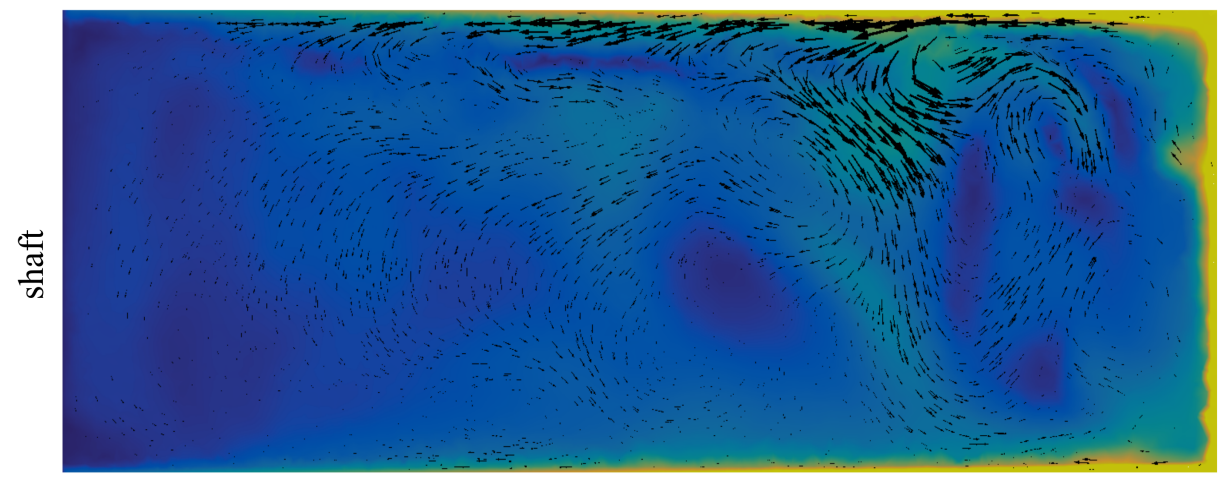

d)

Figure 8.15: Iso-contours and vector field for $\mathrm{U}^{*}$ in different radial planes $\left(\operatorname{Re}_{\text {rot }}=6.5 \cdot 10^{4}\right)$ : c) scraper plane $\theta=90^{\circ}$, d) $\theta=135^{\circ}$ (continued). 


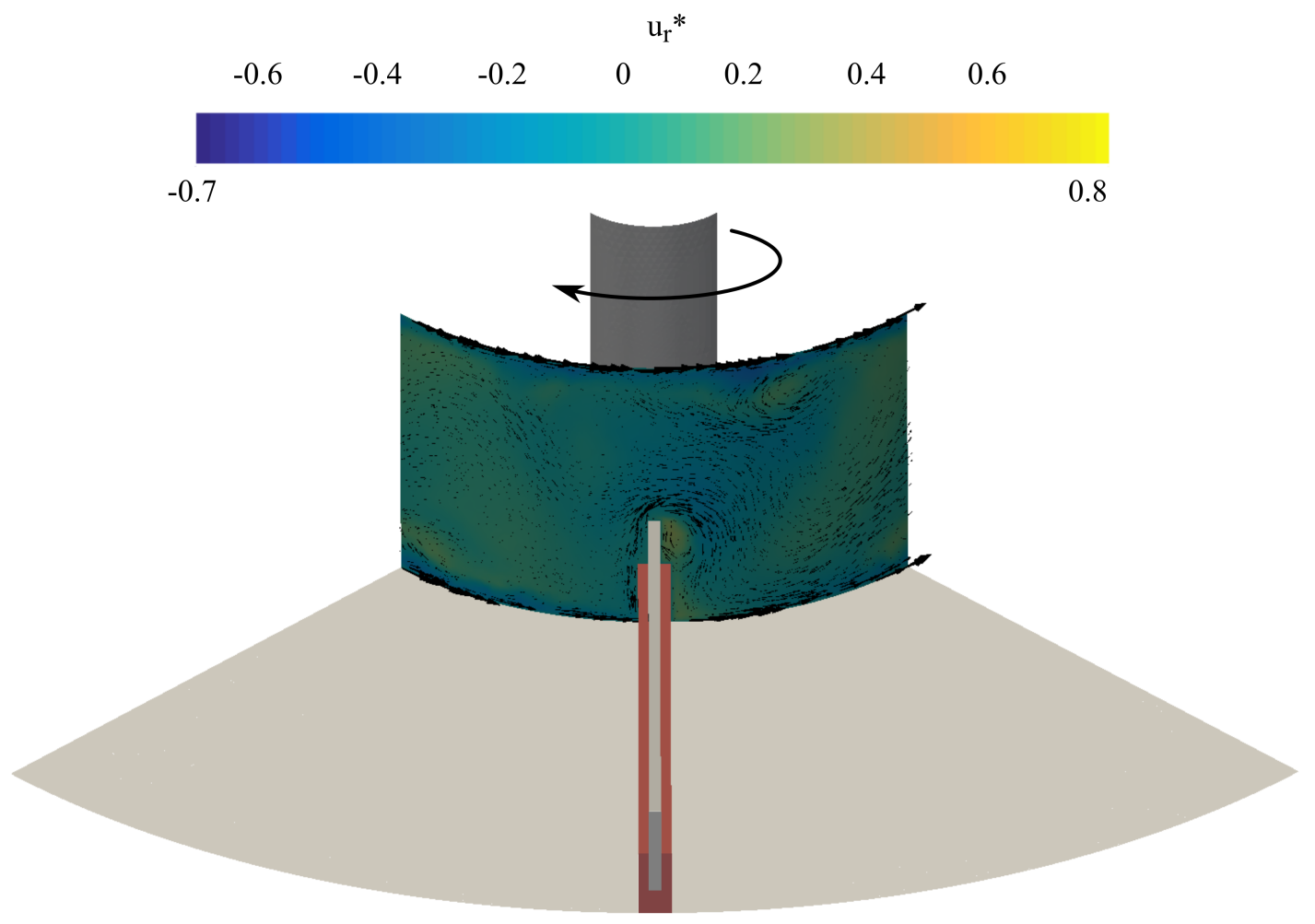

a)

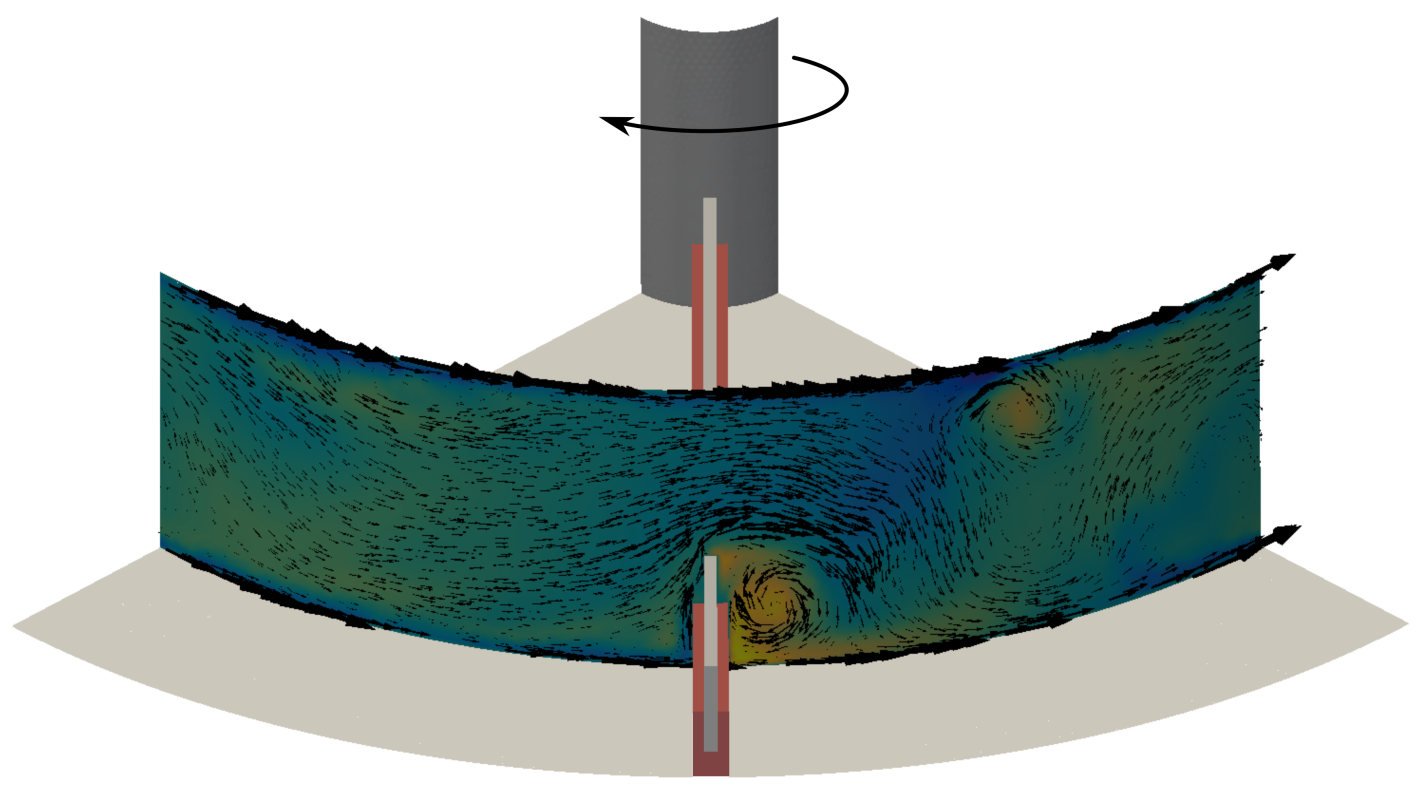

b)

Figure 8.16: Iso-contours and vector field for $\mathrm{U}^{*}$ in different tangential planes planes $\left(\operatorname{Re}_{\text {rot }}=6.5 \cdot 10^{4}\right):$ a) $\left.r / \mathrm{R}=0.4, \mathbf{b}\right) r / \mathrm{R}=0.8$. 


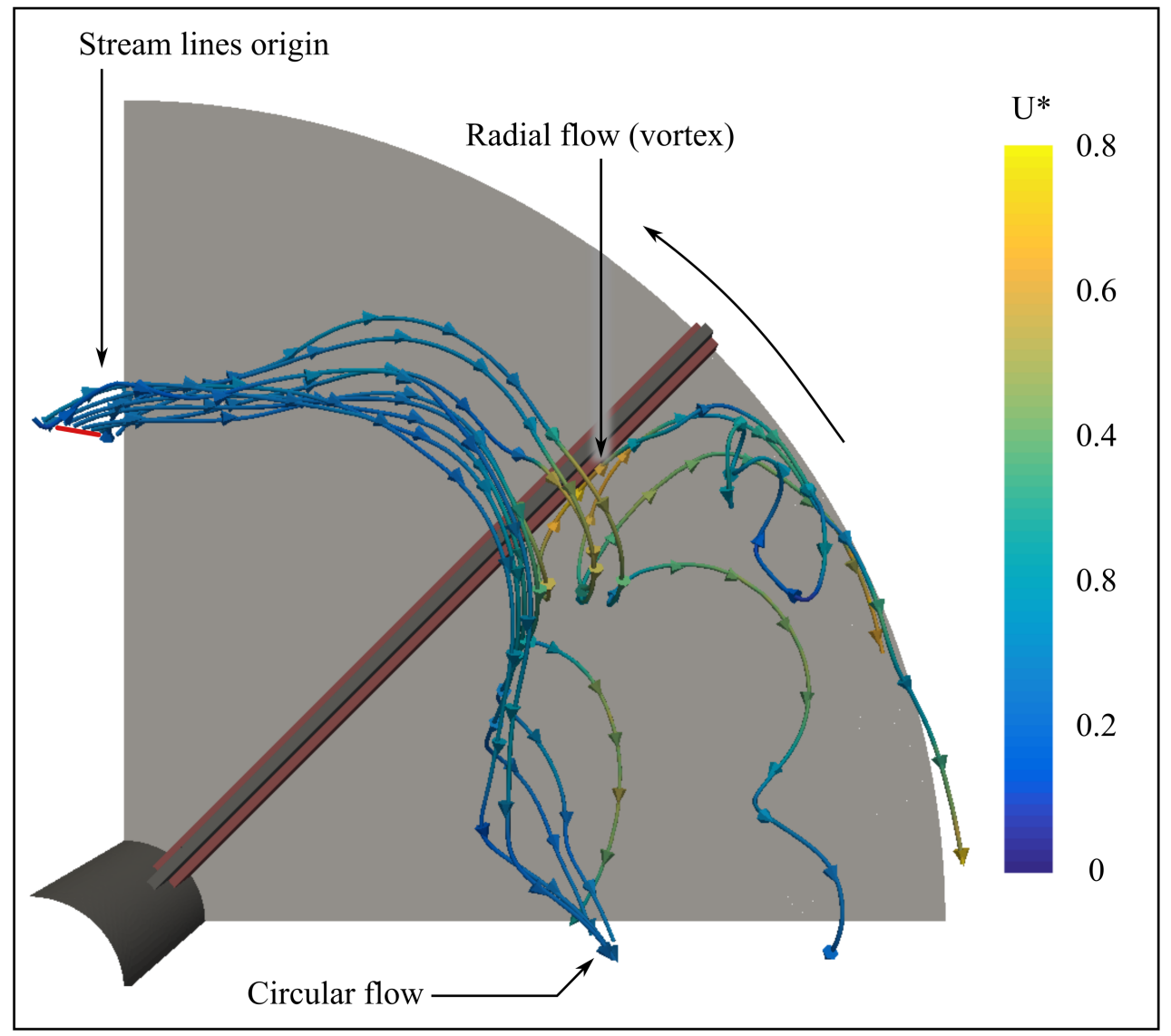

a)

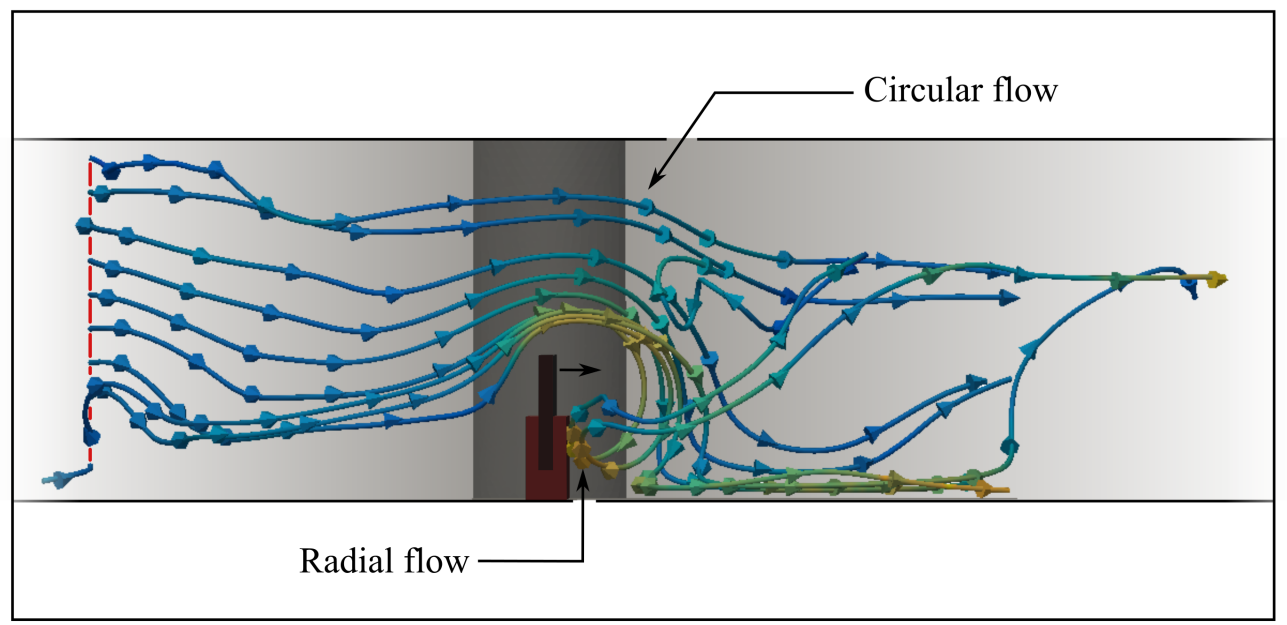

b)

Figure 8.17: Stream lines rake (origin is indicated in the figure) colored by the $\mathrm{U}^{*}$ values $\left(\operatorname{Re}_{\mathrm{rot}}=6 \cdot 5 \cdot 10^{4}\right)$. $\left.\mathbf{a}\right)$ top view $\left.\mathbf{b}\right)$ front view. 

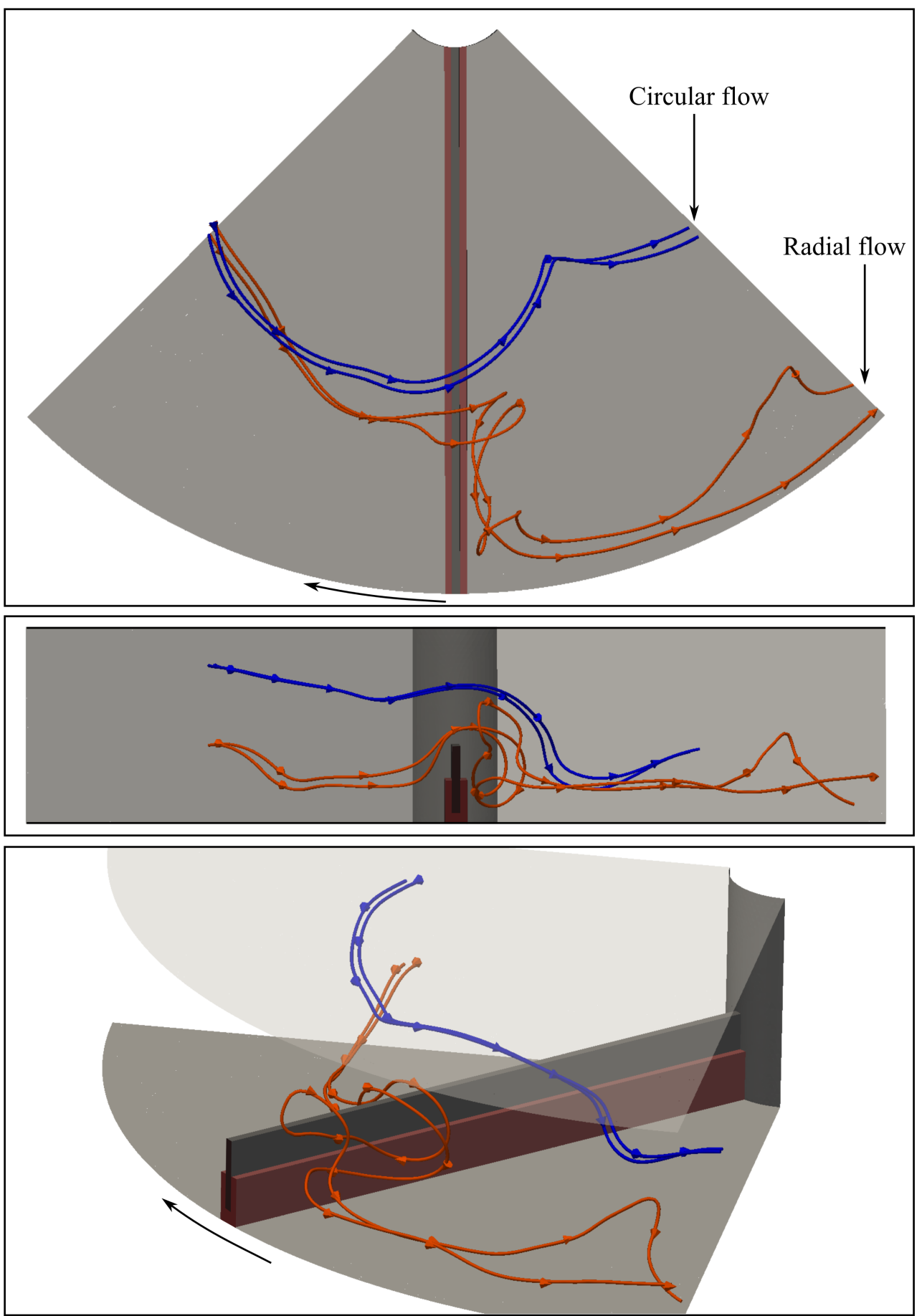

Figure 8.18: Different views of the stream lines coloured by the type of flow encountered in the SPHEE $\left(\operatorname{Re}_{\mathrm{rot}}=6.5 \cdot 10^{4}\right)$ : radial flow (vortex) $\mathbf{a}$ circular flow (tangential). 


\subsection{Conclusions}

This chapter presented and analysed the different results obtained from the numerical simulations. The results are divided into two main groups, those provided by the lagrangian particle tracking of $2 \mathrm{D}$ simulations and those provided by the single-phase $3 \mathrm{D}$ simulations. The 2D simulations were performed considering two approaches for the turbulence modelling: RANS low Reyndols $k-\omega$ and LES, which required of a third dimension normal to the plane. The 3D simulations were performed using a low Reynolds $k-\omega$ turbulence model. Major conclusion are as follows:

- The velocity field obtained by the $2 \mathrm{D}$ simulations with the $k-\omega$ turbulence modelling resulted in very uniform velocity field with a large recirculation after the scraper. The velocity values were similar to those obtained experimentally by PIV but the model failed in predicting the roll-up vortex swirl and dimensions. The maximum velocity values where found very near to the scraper tip, in the vertically deviated flow. The most probably reason for the $2 \mathrm{D}$ model inaccuracy in the flow pattern respect to the PIV data is the absence of centrifugal forces and hence of radial flow.

- The lagrangian particle tracking based on the $2 \mathrm{D}, k-\omega$ simulations revealed a critical relationship between the scraping velocity, the particle size and the mixing of the particles in the flow. For instance, it was found that only the highest scraping rate $\left(\mathrm{Re}=1.7 \cdot 10^{5}\right)$ ensured a complete mixing of all the particles. For the middle simulated scraping rate $\left(\mathrm{Re}=6.5 \cdot 10^{4}\right)$ only the largest particles $\left(\mathrm{d}_{\mathrm{p}}=460 \mu \mathrm{m}\right)$ stratified whereas the rest of them remained in the flow. The lowest scraping rate $\left(\operatorname{Re}=2.2 \cdot 10^{4}\right)$ was unable to mix the particles and all of them ended stratified on the top. According to the results found with during the particle visualization tests and those found during the ice slurry production test, the value of $R e=6 \cdot 5 \cdot 10^{4}$ as a critical value between an acceptable mixing and a stratified ice layer was well predicted by the simulations.

- The time-averaged velocity field obtained by the simulations of the two-dimensional approach with the 3D LES turbulence modelling showed a very similar result to that obtained by the RANS simulations, with a slightly shorter recirculation, but still far from the PIV results. The instantaneous velocity field however resulted to be very different, with the presence of large and small vortices in a fluctuating flow pattern. Comparing the two scraping velocities tested $\left(\mathrm{Re}=3.9 \cdot 10^{4}\right.$ and $\operatorname{Re}=1.7 \cdot 10^{5}$ ), the highest one showed a more active flow, with larger high velocity regions.

- LES simulations also revealed a critical relationship with the scraping velocity, whereas the effect of the particle size appear to be less important compared to the effect of the flow oscillations. At the lower tested scraping velocity $\left(\operatorname{Re}=3.9 \cdot 10^{4}\right)$ only the higher particles $\left(\mathrm{d}_{\mathrm{p}}=460 \mu \mathrm{m}\right)$ stratified, whereas the smaller ones remained in the flow. At the higher velocity $\left(\mathrm{Re}=1.7 \cdot 10^{5}\right)$ the simulations reproduced the impact of the particles with the scraped surface due to a vortex recirculation. The particle size does not play any role in this case. The two-dimensional approach can provide some representative results specially in terms of particle 
stratification, but cannot be considered as a successful model as fails in the prediction of the flow patter.

- The 3D, single-phase flow simulations were able to reproduce the roll-up vortex observed experimentally either in size an in velocity magnitude values. This fact, compared with the $2 \mathrm{D}$ results confirmed that the $3 \mathrm{D}$ flow and the roll-up vortex are closely related. The simulations revealed the existence of a high radial flow in the core of the vortex, being this the undoubted mixing mechanism in the SSPHE. The radial appeared to be stronger as the radius of the scraper was larger. In general, the radial flow is always towards the outer vertical walls, where only near the top and bottom walls have a radial flow towards the shaft.

- The numerically obtained flow pattern consisted in to different regions. One that remains more or less unperturbed, with a predominant tangential (circular flow) and one that contains the roll-up vortex, with a strong radial component that displaces from the radially inside to the outside region of the SSPHE. The separation between to this regions is defined as an axial (vertical) coordinate level, and can be displaced up or down with the scraping velocity. 


\section{Chapter 9}

\section{Conclusions and final remarks}

The present Doctoral Thesis provided insight into the ice slurry production in scraped surface plate heat exchangers. Different operating conditions have been tested in order to characterize the production rate and performance of the device.

The applied methodology was mainly experimental, performing a global analysis of the problem. The influence of the scraping action and scraping velocity, wall subcooling degree and initial salt concentration was studied by considering their effect on the heat transfer, power consumption and the morphology of the ice crystals. The flow pattern and the effect of the solid phase in the flow was also analysed. The experimental study was complemented with numerical simulations, which attempted to provide a complete description of the flow structure.

The employed approach in the problem analysis, combining different techniques to provide a global and complete description, make of this work one of the unique in its kind. The major conclusions are presented hereafter.

\subsection{Experimental methodology}

\subsubsection{Ice slurry production facility}

1. An experimental facility was designed and built up to carry out experiments on the ice slurry production in the scraped surface plate heat exchanger. The facility allowed to investigate the effect of operating parameters as the scraping rotating velocity, the wall subcooling degree and the initial salt concentration on the brine.

2. A method to measure the wall temperature was developed, based in thermocouples embedded in the scraped surface. The accuracy of the method combined with the accuracy in the slurry bulk temperature measurement allowed to determine the Nusselt number for different operating conditions. The facility also permitted the measurement of the power consumed by the scrapers driving motor and microscopic observation of the ice crystals.

3. Two scraping systems were tested. The first one, rigid, consisted of single-piece blades mounted rigidly on the rotating arms. The second one, adaptable, consisted of smaller blades mounted to the arms through pusher springs which ensured the contact between blade and scraped surface. 


\subsubsection{Flow visualization facility}

1. An experimental facility was designed and built up to carry out flow visualization experiments. The facility was able to reproduce the same conditions in terms of rotating Reynolds number as the ice slurry production facility.

2. Flow visualization test with two-phase flow were performed to determine the trend to the solid particles either to mix or stratify. Particles were injected from the bottom, prior to the scraper passage.

3. Particle Image Velocimetry tests, with a high speed camera and a laser optics, were performed to analyse the flow pattern of a single phase-flow. The image acquisitions was synchronized with the scraper location by means of an optical trigger system

\subsection{Findings in the study and modelling of the ice slurry production in the SSHPE}

1. The location of the initial nucleation in the SSPHE was found to depend on the Richardson number. The effects of buoyancy, supercooling degree and scraperarms induced velocities interact leading to an initial localized nucleation over the subcooled plate $(R i>0.02)$ or to a global fluid volume nucleation $(R i<0.02)$. The detected supercooling amplitude was found to be a good indicator of the fluid mixing inside the SSPHE upper side.

2. The configurations with adaptable scrapers shown a better performance in the ice slurry generation and heat transfer, reducing the unscraped regions and avoiding ice layer scaling. Augmentations on the heat transfer coefficient of around two times were found with respect to the rigid scraper. The rotating velocity of the scrapers was found to have a low effect on the heat transfer, influenced by the supercooling degree of the surface. Maximum averaged heat fluxes of 4100 $\mathrm{W} / \mathrm{m}^{2}$ and averaged heat transfer coefficients of $4000 \mathrm{~W} / \mathrm{m}^{2} \mathrm{~K}$ were found for the adaptable scrapers with surface supercooling degrees of $\approx 0.5{ }^{\circ} \mathrm{C}$ and scraping rotating velocities of $\mathrm{N} \approx 0.46 \mathrm{~s}^{-1}$.

3. Heat transfer results were non-dimensionalized by means of the Nusselt and rotating Reynolds numbers. Nusselt number for low supercooling degree values adopted the shape of the penetration theory for low $R e_{\text {rot }}$ values, whereas at high values $N u$ becomes almost independent of $R e_{r o t}(N u=550)$, mostly due to the impact of the particles over the surface at higher mixing rates. Higher surface supercooling degrees led to a constant ice layer that reduced the $N u$ values to $\approx 350$. A correlation was proposed for a supercooling degree of $0.75^{\circ} \mathrm{C}$ composed by the penetration theory plus a constant therm for $R e_{\text {rot }}<4.7 \cdot 10^{4}$ and a correlation based on $\operatorname{Re}_{\text {rot }}$ and $\mathrm{Fr} \cdot \varphi_{m}$ for $\operatorname{Re}_{\text {rot }}>4.7 \cdot 10^{4}$, with global $\mathrm{R}^{2}=0.87$.

4. The power consumption during the chilling and freezing stages showed to be mostly related with the friction between scrapers and plate. Shaft torque was found to decrease when scraping velocity increases as consequence of the formation of a brine-lubricating layer. The onset of nucleation resulted always in 
important reductions of power consumption (up to 50\%) as a consequence of the appearance of ice over the surface, acting as a lubricant. Ice content increases lead to power increments as the equivalent viscosity of the slurry increased. However this effect was much lower than the friction between scrapers and plate.

5. Power consumption values were non-dimensionalyzed by the Power number. The high values of $P o$ obtained revealed the nature of the process, governed by the friction. For equal $R e_{r o t}$ values $P o$ was smaller as the ice content was higher revealing that the larger number of particles affect the ice layer over the steel surface, reducing therefore the friction.

6. Higher rotating scraping velocities did not proved to be helpful in inhibiting the development of an ice fouling layer, as the higher impact of ice particles over the surface plus the higher liquid layer under the scraper promote it. On the contrary, higher rotating velocities aided to preserve a good mixing level in the slurry, keeping it homogeneous and avoiding the cumulation of ice in the top of the SSPHE. The high scraping velocity may result in heating effects, which would be undesirable. As a consequence the rotating velocity has to be balanced with the economy of the power consumption.

7. The morphology of the ice crystals varied with the scraping velocity, wall subcooling and time instant of the test. Higher scraping velocity leaded to higher and rounded particles (200-300 $\mu \mathrm{m})$ as a consequence of the continuous impacts that make them fuse between each other and grow. Higher wall subcoling values reduced the test duration resulting in smaller particles. At the initial test stages (low ice content) stages particles are small $(10 \mu \mathrm{m})$ and sharp, as they are just pulled off. With time they grow in rounder shapes.

\subsection{Findings in the performance evaluation for different $\mathrm{NaCl}$ concentrations}

1. For equal $\overline{\Delta T}_{\text {log }}$ and scraping velocity values, increasing the $\mathrm{NaCl}$ resulted in increasing heat fluxes between $75 \%$ and $100 \%$, being the most significant increase for $\mathrm{N}=0.1 \mathrm{s.}^{-1}$. Such a heat flux increment was observed between $\omega_{0}=4.7 \%$ and 7.1\%. Higher $\omega_{0}$ values had no heat flux increasing effect.

2. The Nusselt number, for equal $\overline{\Delta T}_{\text {log }}$ and scraping velocity values, experience an augmentation when increasing $\omega_{0}$, in correspondence with the augmentation of the heat flux. The maximum augmentation was observed between $\omega_{0}=4.7 \%$ and $7.1 \%$, being more significant as the scraping velocity was higher. The maximum increase in $\mathrm{Nu}$ observed was of $65 \%$. This effect, related with the heat flux augmentation, was associated to effect of the $\mathrm{NaCl}$, weakening the ice strength and increasing the mushy structure of the ice layer, being easier to scrape.

3. A $\omega_{0}=07 \%$ and $\overline{\Delta \mathrm{T}}_{\log } \approx 2.6^{\circ} \mathrm{C}$ revealed to be the best operating condition, providing heat fluxes of $4270 \mathrm{~W} / \mathrm{m}^{2}$ and Nusselt numbers around 380 .

4. Scraping power revealed to be independent of the $\omega_{0}$ value. For equal $\overline{\Delta T}_{\text {log }}$ and $\mathrm{N}$ values, scraping power remained almost constant. The same was observed for 
the power number. This was associated with the twofold effect of the ice layer, acting as a lubricant ans also as a resistance to the scraping blades.

5. The efficiency of the SSPHE was analysed by considering the ratio $\eta$ between the effective heat extracted (due to phase change) and the scraping power. The $\omega_{0}=7 \%$ with $\overline{\Delta T}_{\log } \approx 2.6^{\circ} \mathrm{C}$ proved to be again the best operating condition, achieving a value of $\bar{\eta}=7$ for $\mathrm{N}=0.83 \mathrm{s.}^{-1}$

6. The total efficiency of the ice slurry production system was evaluated through the COP of the condensing unit and the cost of producing a kilogram of ice, for the case of $\omega_{0}=7 \%$. It was concluded that, for the adaptable scraping system, increasing the ice production in $60 \%$ leaded to an increase in the cost per kilogram of ice produced of $1.8 \%$. The operating point should then be selected according to the demand and the price of the electricity.

7. No clear influence of $\omega_{0}$ in the ice crystals morphology could be assessed. Only as a guess, the ice crystals size seemed to decrease when $\omega_{0}$ increased, probably due to the weakening of the ice layer, resulting in smaller crystals when scraped from the plate.

\subsection{Findings in the experimental flow pattern assessment}

1. The particles injected at a low scraping velocity $\left(\operatorname{Re}_{\mathrm{rot}}=1.6 \cdot 10^{4}\right)$ showed higher dispersion of the particles cloud that those at higher velocities $\left(\operatorname{Re}_{\mathrm{rot}}=4.8 \cdot 10^{4}\right)$. The dispersion of the particles was associated to their buoyancy, which makes them unable to follow the fluid flow generated by the scrapers. At the higher tested velocity $\left(\operatorname{Re}_{\mathrm{rot}}=4.8 \cdot 10^{4}\right)$, the particles cloud appeared more compacted and no particle groups where observed detached from the main group. The value of the $\mathrm{Fr}>1$ most probably indicated that the buoyant forces were not any more significant.

2. The particle stratification revealed that for $\operatorname{Re}_{\text {rot }} \approx 7.6 \cdot 10^{3}$, the particles are quiescent, stacked on the top in a stationary bed. As the scraping velocity increases, part of the particles start to move, appearing a separate moving layer at the bottom of the stationary bed. At $\mathrm{Fr}=5 \cdot 1 \cdot 10^{-1}$ the bed disappears near the extreme radial positions, with a moving, turning bed around the shaft. For $\mathrm{Fr}=6.8 \cdot 10^{-1}$ the moving bed disappears leading to an heterogeneous flow, that becomes almost homogeneous at faster scraping velocities $\left(\operatorname{Re}_{\text {rot }}>2.5 \cdot 10^{4}\right)$.

3. The flow pattern inside the SSPHE could be described by the PIV study for the range $2.2 \cdot 10^{4} \geq \operatorname{Re}_{\text {rot }} \geq 1.7 \cdot 10^{5}$. Absolute velocities were transformed into velocities relative to the scraper. The observed pattern consisted in three different regions: 1) an unperturbed, horizontal flow on the top of the SSPHE, 2) a roll up vortex on the rear side of the scraper and 3) a net downwards flow induced by the vortex. As $\mathrm{Re}_{\text {rot }}$ increases the unperturbed region is reduced, and the downwards flow increases. The flow deviated by the scraper upwards increases with the rotating velocity. The combination of upwards and downwards flow can increase the mixing, being the roll up vortex the main mixing agent. 
4. The normalized velocity field was normalized by the scrapers tangential velocity at the location of the laser sheet. As the rotating velocity of the scrapers increases the normalized velocity profiles evolved to a $\mathrm{Re}_{\mathrm{rot}}$-independent profile where, compared with the low rotating velocity situations, velocity in the fluid is more uniform and low velocity regions tend to disappear.

5. The roll up vortex is formed from the flow separated form the scraper. The high shear, separated flow rolls up into a spiral shape. Vorticity values revealed the location of the vortex core, which ascends slightly as the rotating velocity increases. They also revealed the high shear fluid layer on the scraper's tip. The analysis of the vortex tangential velocity (referred to the vortex center) showed a good agreement with the different classical modelling methods.

6. Turbulent kinetic energy was higher in the roll up vortex, achieving a peak in the vortex core. The scraper's tip also showed high turbulent kinetic values as a consequence of the high shear. Increasing the rotating velocity of the scrapers leaded to the extension of the turbulence generated by the vortex. The peak turbulence kinetic ratio could be related with the rotating Reynolds number, and the roll up vortex appeared to be the main mechanism of energy transfer and turbulence generation in the SSPHE flow.

\subsection{Numerical methodology}

\subsection{Findings in the numerical flow pattern and particle tracking assessment}

1. The velocity field obtained by the $2 \mathrm{D}$ simulations with the $k-\omega$ turbulence modelling resulted in very uniform velocity field with a large recirculation after the scraper. The velocity values were similar to those obtained experimentally by PIV but the model failed in predicting the roll-up vortex swirl and dimensions. The maximum velocity values where found very near to the scraper tip, in the vertically deviated flow. The most probably reason for the $2 \mathrm{D}$ model inaccuracy in the flow pattern respect to the PIV data is the absence of centrifugal forces and hence of radial flow.

2. The lagrangian particle tracking based on the $2 \mathrm{D}, k-\omega$ simulations revealed a critical relationship between the scraping velocity, the particle size and the mixing of the particles in the flow. For instance, it was found that only the highest scraping rate $\left(\mathrm{Re}=1.7 \cdot 10^{5}\right)$ ensured a complete mixing of all the particles. For the middle simulated scraping rate $\left(\operatorname{Re}=6.5 \cdot 10^{4}\right)$ only the largest particles $\left(\mathrm{d}_{\mathrm{p}}=460 \mu \mathrm{m}\right)$ stratified whereas the rest of them remained in the flow. The lowest scraping rate $\left(\operatorname{Re}=2.2 \cdot 10^{4}\right)$ was unable to mix the particles and all of them ended stratified on the top. According to the results found with during the particle visualization tests and those found during the ice slurry production test, the value of $\operatorname{Re}=6 \cdot 5 \cdot 10^{4}$ as a critical value between an acceptable mixing and a stratified ice layer was well predicted by the simulations. 
3. The time-averaged velocity field obtained by the simulations of the two-dimensional approach with the 3D LES turbulence modelling showed a very similar result to that obtained by the RANS simulations, with a slightly shorter recirculation, but still far from the PIV results. The instantaneous velocity field however resulted to be very different, with the presence of large and small vortices in a fluctuating flow pattern. Comparing the two scraping velocities tested $\left(\mathrm{Re}=3.9 \cdot 10^{4}\right.$ and $\operatorname{Re}=1.7 \cdot 10^{5}$ ), the highest one showed a more active flow, with larger high velocity regions.

4. LES simulations also revealed a critical relationship with the scraping velocity, whereas the effect of the particle size appear to be less important compared to the effect of the flow oscillations. At the lower tested scraping velocity $\left(\operatorname{Re}=3.9 \cdot 10^{4}\right)$ only the higher particles $\left(d_{p}=460 \mu \mathrm{m}\right)$ stratified, whereas the smaller ones remained in the flow. At the higher velocity $\left(\mathrm{Re}=1.7 \cdot 10^{5}\right)$ the simulations reproduced the impact of the particles with the scraped surface due to a vortex recirculation. The particle size does not play any role in this case. The two-dimensional approach can provide some representative results specially in terms of particle stratification, but cannot be considered as a successful model as fails in the prediction of the flow patter.

5. The 3D, single-phase flow simulations were able to reproduce the roll-up vortex observed experimentally either in size an in velocity magnitude values. This fact, compared with the $2 \mathrm{D}$ results confirmed that the $3 \mathrm{D}$ flow and the roll-up vortex are closely related. The simulations revealed the existence of a high radial flow in the core of the vortex, being this the undoubted mixing mechanism in the SSPHE. The radial appeared to be stronger as the radius of the scraper was larger. In general, the radial flow is always towards the outer vertical walls, where only near the top and bottom walls have a radial flow towards the shaft.

6. The numerically obtained flow pattern consisted in to different regions. One that remains more or less unperturbed, with a predominant tangential (circular flow) and one that contains the roll-up vortex, with a strong radial component that displaces from the radially inside to the outside region of the SSPHE. The separation between to this regions is defined as an axial (vertical) coordinate level, and can be displaced up or down with the scraping velocity.

\subsection{Publications}

During the development of the Doctoral Thesis (including the research stage at York University) different contributions were made to either International Conferences, National Conferences or Peer Review Journals:

1. D.S. Martńez, J.P. Solano, F. Illán, A. Viedma, "Production of ice slurry in a scraped-surface plate heat exchanger", in Seminar Eurotherm 96 on Convective Heat Transfer Brussels (Belgium), September, 2013

2. D.S. Martínez, A. García, J.P. Solano, A. Viedma, "Heat transfer enhancement of laminar and transitional Newtonian and non-Newtonian flows in tubes with 
wire coil inserts", International Journal of Heat and Mass Transfer. Vol. 76 pp. 5440-548 (2014).

3. D.S. Martínez, J.P. Solano, F. Illán, A. Viedma, "Analysis of heat transfer phenomena during ice slurry production in scraped surface plate heat exchangers", International Journal of Refrigeration. Vol. 48 pp. 221-232 (2014).

4. D.S. Martítenez, M.A. Cabrerizo-Vilchez, A. Viedma, A. Amirfazli. "Morphological dynamics of a falling drop in a magnetic field". 67th Annual Meeting of the APS Division of Fluid Dynamics. November 23-25, 2014. San Francisco, California (United States)

5. D.S. Martínez, J.P Solano, F. Illán, A. Viedma. "Medida de la temperatura de pared en intercambiadores de calor de superficie rascada mediante termopares embebidos". IX Congreso Nacional de Ingeniera Termodinmica: libro de actas. Cartagena: Universidad Politécnica de Cartagena, Servicio de Documentación, 2015. 1432 p. ISBN: 978-84-606-8931-7 


\section{Appendix A}

\section{Adaptable scraping system desing}

The torsion springs were specifically designed and manufactured for this application, provided by Muelles CROM(C). The objective was to maximize the force over the scrapers taking up a minimum of space. They are made of a 302 AISI stainless steel wire $(d=1.6 \mathrm{~mm})$ with $\mathrm{N}=3.81$ turns. The dimensions are depicted in Fig. A.1. The spring rate is, according to [142],

$$
\kappa=\frac{d^{4} E}{64 D N} \approx 10 \mathrm{Nmm} / \mathrm{deg}^{\circ}
$$

where $E=185000 \mathrm{~N} / \mathrm{mm}^{2}$ is the Young modulus. The vertical force $\mathrm{F}$ exerted by a spring over the scraper is related to the torque $M$ in the spring (Fig. A.2) and, neglecting frictional forces between spring and scraper, can be calculated as

$$
M=\mathrm{F} \sin (\theta / 2) \cdot r=\kappa \theta
$$

For the two operating positions of Fig. A.1 $\left(\theta_{\min }=113^{\circ}\right.$ and $\left.\theta_{\max }=141^{\circ}\right)$ the corresponding vertical displacement of the scraper blades is of $\approx 4.8 \mathrm{~mm}$ (Fig. A.3). The spring torque for these two positions is respectively of $M_{\min }=50 \mathrm{Nmm}$ and $M_{\max }=342$ Nmm respectively. The maximum force is then $\mathrm{F}=19.5 \mathrm{~N}$, which means $39 \mathrm{~N}$ for each scraper blade with two springs mounted on it. The resistance $M_{r}$ of the spring, giving
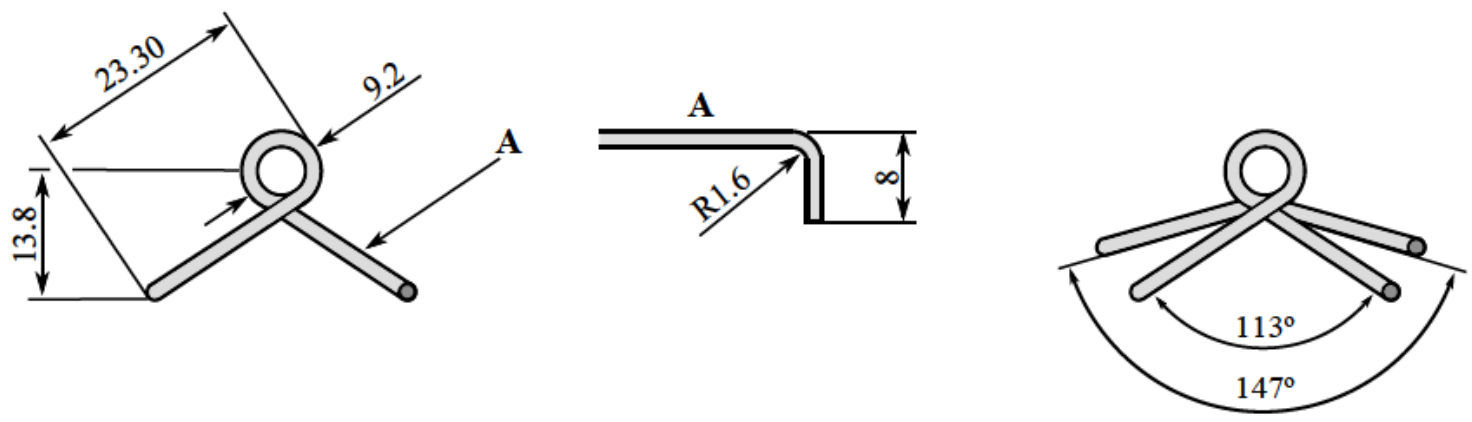

Figure A.1: Torsion spring. From left to right: spring dimensions (mm), scraper-push tail and working position 


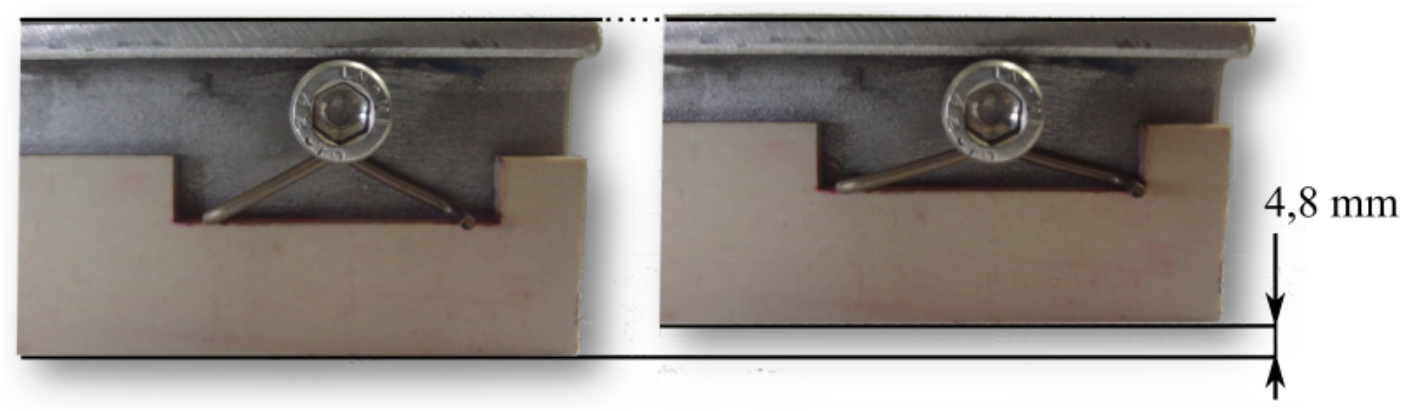

Figure A.3: Working angle (left) and maximum scraper blade vertical displacement (right), corresponding for the maximum spring torsion

by the equation [142]:

$$
M_{r}=\frac{S_{y} \pi d^{3}}{32 K}=361 \mathrm{Nmm}>342 \mathrm{Nmm}
$$

where $S_{y}=545 \mathrm{MPa}$ is the maximum allowed effort on the spring and $K$ is an stress concentration factor [142] that for this case is $K=1.21$. From the above equation is clear that the maximum force exerted by the springs is not enough to break the arms.

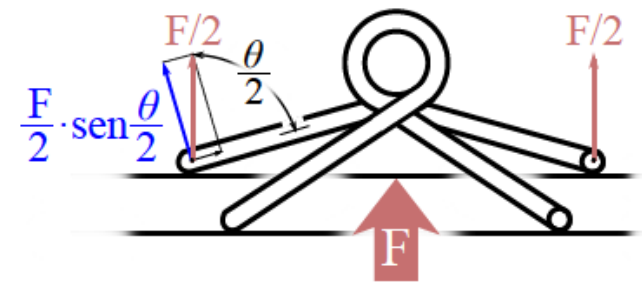

Figure A.2: Force diagram acting on the spring.

On the other hand the resistance of the screw to shear stresses can be evaluated through [143]:

$$
\mathrm{F}_{r}=\frac{0.5 f_{u b} A}{1.25}=431 \mathrm{~N}>>19.5 \mathrm{~N}
$$

where $f_{u b}=700 \mathrm{~N} / \mathrm{mm}^{2}$ is the ultimate tensile strength corresponding to the used screws and $A$ their cross sectional area where the shear stress is applied. The momentum on the screw is, due to the low load and the short distance between the spring (force application point) and the threaded section, negligible compared to its resistance. The resistance of the arm to flexing momentum in the most unfavourable point -near the shaft- is also ensured, as the total momentum generated by the vertical spring forces is lower than the resistant momentum of the arm

$$
\sum_{i=1}^{5} F_{i} \cdot l_{i}=21 \mathrm{Nm}<<f_{u} \frac{I_{x}}{y_{\max }}=343 \mathrm{Nm}
$$

where $l_{i}$ are the respective distances from each spring till the shaft, $f_{u}=70 \mathrm{~kg} / \mathrm{mm}^{2}$ is the material ultimate tensile strength, $I_{s}$ is the momentum of inertia of the arm cross section and $y_{\max }$ the farthest point from the center of gravity.

To balance any non-vertical forces, each scraper blade is mounted with two opposed springs (see Fig. 2.7). Only the scraper blade near to the rod, due to its shorter length, has a single torsion spring. A $3 \mathrm{~mm}$ gap was intentionally left between near side scraper blades to allow unequal spring torsions. To avoid any possible uncleaned track on the 
plate which may lead to ice scaling, scraper blades have unequal length - shifted by $\mathrm{S}=7 \mathrm{~mm}$ - within successive arms. In this way, any plate area unscraped by an arm will be scraped by the following one. An sketch of the scraper blades disposition for two consecutive arms is shown in Fig. A.4.

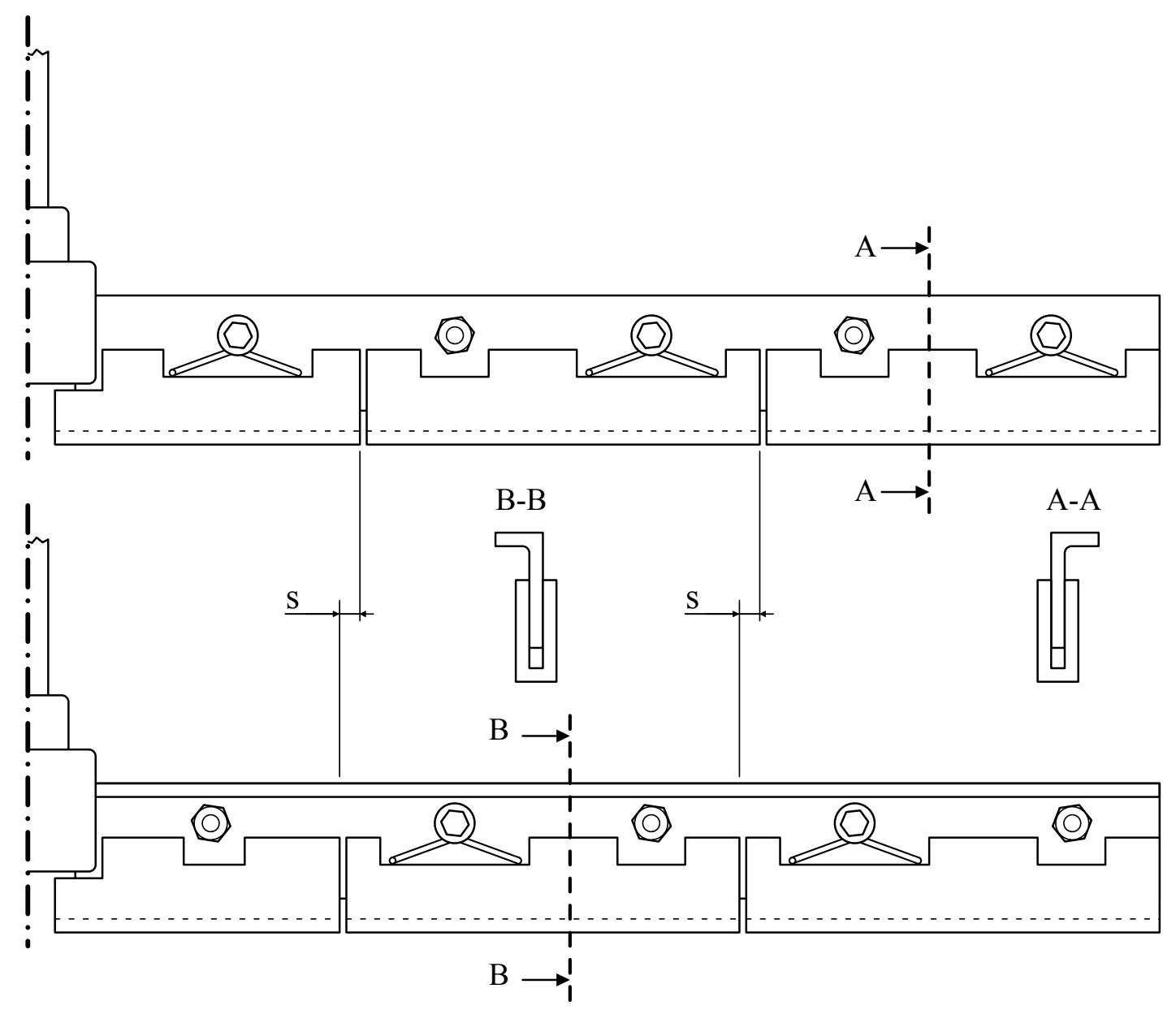

Figure A.4: Scraper blades misalignment for two consecutive arms. $\mathrm{S}=7 \mathrm{~mm}$ 


\section{Appendix B}

\section{Embedded thermocouples: calibration and uncertainty calculation}

For the calibration of the embedded thermocouples a commercial freezer cabinet was used. In order to avoid any temperature oscillation inside the freezer no thermostat or any other temperature control system was used. With the plate inside, it run continuously up to 24 hours until the temperature inside was stabilized (by thermal equilibrium with the outside). To achieve different temperatures inside the freezer several fans were introduced inside, acting as heat sources and avoiding any thermal stratification. By changing the power and number of the fans inside the freezer a curve with 9 calibration points was made. As a reference temperature, the average of two RTD PT100 class 1/10-DIN were used. The reference probes were located separately into the freezer, verifying that the temperature was uniform. Once the temperature was stable, values were acquired during at least 3 hours at 1 sample/minute.

The HP Agilent data logger counts with internal calibration curves for the different thermocouple types, including the T. Thus and for simplicity, instead of calibrating the thermocouples by their voltages the calibration was done based on the temperature provided by the readings of the data logger. Figure B.1 shows the time evolution of the reference temperature inside the freezer together with the temperatures measured by the thermocouples. The values considered for the calibration were those corresponding to the stable period. The Chauvenet's criteria was used for the discarding of outlier points [144]. Figure B.1 shows the averaged temperature reference values and the averaged temperature measurements for each calibration point. A straight line regression was used for the calibration of the thermocouple temperatures [144],

$$
\bar{T}_{r f}=A \cdot \bar{T}_{t c}+B
$$

where $A$ is the slope of the line, $B$ is the abscissa intercept and $\bar{T}_{r f}$ and $\bar{T}_{t c}$ are respectively the reference and the thermocouple temperatures,

$$
\bar{T}_{t c}=\frac{1}{N} \sum_{i=1}^{N} T_{t c, i} \quad \bar{T}_{r f}=\frac{1}{N} \sum_{i=1}^{N} T_{r f, i}
$$



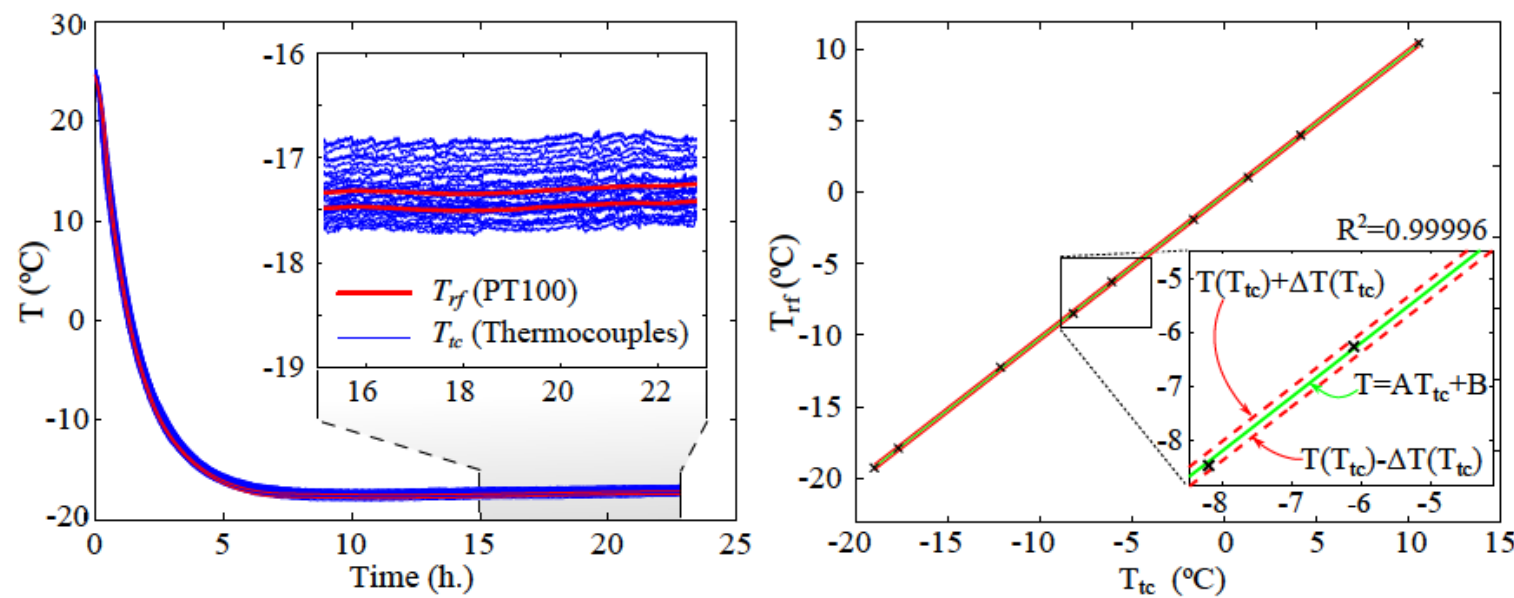

Figure B.1: Time evolution of the temperature measured by thermocouples and reference probes inside the freezing cabinet; detail of the stable region from where calibration point was averaged (left), calibration curve with uncertainty intervals for one of the thermocouples (right)

$A$ and $B$ are obtained from,

$$
\begin{gathered}
A=\frac{N \sum_{i=1}^{n} \bar{T}_{t c, i} \bar{T}_{r f, i}-\sum_{i=1}^{n} \bar{T}_{t c, i} \sum_{i=1}^{n} \bar{T}_{r f, i}}{N \sum_{i=1}^{n} \bar{T}_{t c, i}^{2}-\left(\sum_{i=1}^{n} \bar{T}_{t c, i}\right)^{2}} \\
B=\frac{\sum_{i=1}^{n} \bar{T}_{t c, i}^{2} \sum_{i=1}^{n} \bar{T}_{t c, i} \bar{T}_{r f, i}-\sum_{i=1}^{n} \bar{T}_{t c, i} \sum_{i=1}^{n}\left(\bar{T}_{t c, i} \bar{T}_{r f, i}\right)}{N \sum_{i=1}^{n} \bar{T}_{t c, i}^{2}-\left(\sum_{i=1}^{n} \bar{T}_{t c, i}\right)^{2}}
\end{gathered}
$$

Once the calibration is done, the corrected temperature $T$ can be obtained through the one measured by the thermocouple, $T_{\text {new }}$, by

$$
T=A \cdot T_{\text {new }}+B
$$

\section{Uncertainty calculation}

The uncertainty in $T$ of Eq. B.5 should include the uncertainty in the calibration curve as well as the uncertainty in the measurement system (HP data logger). The uncertainty was calculated using a numerical approximation of the Taylor series method for uncertainty propagation [145], where the partial derivatives are approached by using a forward-differencing finite-difference scheme. The total uncertainty $E(T)$ can be expressed as a sum of the uncertainties that contribute to the $T$ value.

$$
E(T)^{2}=\sum_{i=1}^{J}\left(\frac{\partial T}{\partial X_{i}}\right)^{2} E\left(X_{i}\right)^{2} \approx \underbrace{\sum_{i=1}^{J}\left(\frac{\Delta \mathrm{T}}{\Delta X_{i}}\right)^{2} E\left(X_{i}\right)^{2}}_{\text {numerical approx. }}
$$

where $X_{i}=1, \ldots X_{J}$ are the $J$ variables interfering in the measurement of $T$ and $E\left(X_{i}\right)$ their corresponding uncertainty. The sources of uncertainty in $\Delta \mathrm{T}$ for a new temperature measurement are: 
1) Uncertainty in the reference PT100 probes

2) Uncertainty in the data averaging obtaining the calibration points

3) Uncertainty due to the linear regression

4) Uncertainty due to the voltage measurement

The first three uncertainty sources will affect to the uncertainty of a new measurement through their effect in the calibration. The uncertainty introduced through the voltage measurement will affect to the calibration and to the new measurement.

Taking into account the uncertainty sources 1)-2) by the classical Taylor method would require to differentiate Eq. B.5 respect to the calibration reference temperature, which in turn will require to differentiate Eqs. B.3 and B.4. Instead the numerical approach allow us to approximate the derivatives as small changes in the output due to changes in the input equal to the uncertainty in the variable, $\delta X$.

The uncertainty in the reference probes $E\left(T_{r f}\right)$ is the sum of the uncertainty of the probe itself plus the uncertainty of the data logger, that is, $0.03{ }^{\circ} \mathrm{C}+0.06{ }^{\circ} \mathrm{C}=$ $0.09{ }^{\circ} \mathrm{C}$. On the other hand, the uncertainty due to the temperature averaging $\bar{T}_{r f}$ can by calculated as $1.96 \sigma$ with a $95 \%$ confidence level, where $\sigma$ is the corresponding standard deviation. The effect of these two uncertainties in the determination of the regression coefficients $A$ and $B$ is then evaluated by getting a new coefficients $A_{0}$ and $B_{0}$ obtained from adding to the $T_{r f}$ temperatures a $\Delta \mathrm{T}_{r f}$ equal to its uncertainty $E\left(T_{r f}\right)=$ $E\left(T_{r f}\right)+1.6 \sigma$. The uncertainty in the calibration curve due to the uncertainty in the reference probes, $E\left(T_{p r b}\right)$ can then be evaluated through Eq. B.7, where the interval along the sum is evaluated goes from the first to the last calibration temperature.

$$
\begin{aligned}
& \left.\begin{array}{c}
T_{t c} \\
T_{r f}
\end{array}\right\} \underset{\text { regression }}{\stackrel{\text { linear }}{T=A \cdot T_{\text {new }}+B}} \\
& E\left(T_{p r b}\right)=\lim _{N \rightarrow \infty}\left[\frac{1.96}{N} \sum_{i=1}^{N}\left(T_{i}-T_{i}^{\prime}\right)^{2}\right]^{1 / 2} \\
& \overbrace{T^{\prime}=A_{0} \cdot T_{\text {new }}+B_{0}}^{\uparrow} \stackrel{\text { linear }}{\text { regression }}\left\{\begin{array}{l}
T_{t c} \\
T_{r f}+\Delta \mathrm{T}_{r f}
\end{array}\right.
\end{aligned}
$$

A similar procedure can be followed to evaluate the uncertainty due to the thermocouple averaging in the calibration points $\left(\bar{T}_{t c}\right)$ and to the voltage measurement. As before, the uncertainty associated to the averaging can be calculated as $1.96 \sigma$. The uncertainty due to the voltage measurement by the data logger is[146]

$$
E\left(T_{V o l t}\right)=\frac{E(V)}{S_{b}}
$$


where $E(V)=0.008 \mathrm{mV}$ is the uncertainty of the voltage measurement in the data logger and $S_{b}$ the Seebeck coefficient for the type T thermocouples $\left(\approx 43 \mu \mathrm{V} /{ }^{\circ} \mathrm{C}\right)$. The new values of $T_{t c}$ obtained from adding to them the total uncertainty $E\left(T_{V o l t}\right)+1.6 \sigma$ are used to get the uncertainty due to the thermocouples during the calibration, $E\left(T_{t c}\right)$, in a similar way than in Eq. B.7 for the reference PT100 probes.

The uncertainty for a new measurement, with a $95 \%$ level of confidence can be expressed as [144]

$$
E\left(T_{N}\right)=2\left[s_{T}^{2}\left(\frac{1}{N}+\frac{\left(T_{n e w}-\bar{T}_{t c}\right)^{2}}{s_{T T}}\right)\right]^{1 / 2}
$$

where $N$ is the number of calibration data pairs, $9, \bar{T}_{t c}$ is defined in Eq. B.2, $s_{T}$ is the standard error of the regression,

$$
s_{T}=\left[\frac{\sum_{i=1}^{N}\left(T_{r f, i}-A \cdot T_{t c, i}-B\right)}{N-2}\right]^{1 / 2}
$$

and

$$
s_{T T}=\sum_{i=1}^{N} T_{t c, i}^{2}-\frac{\left(\sum_{i=1}^{N} T_{t c, i}\right)^{2}}{N} .
$$

The uncertainty of the voltage reading $E\left(T_{V, n e w}\right)$ should be reconsidered for a new measurements. Following the Taylor series for the propagation of uncertainties and applying Eq. B.8,

$$
E\left(T_{V, \text { new }}\right)=\frac{\partial T}{\partial V}=\frac{\partial T}{\partial T_{\text {new }}} \frac{\partial T_{\text {new }}}{\partial V}=A \cdot E\left(T_{V \text { olt }}\right)
$$

Once the different uncertainties are known, the total uncertainty can then obtained by the sum of squares,

$$
E(T)^{2}=E\left(T_{p r b}\right)^{2}+E\left(T_{t c}\right)^{2}+E\left(T_{N}\right)^{2}+E\left(T_{V o l t}\right)^{2}
$$

Therefore the calibrated temperature given by each thermocouple, as a function of the one measured by the data logger can be expressed as $T\left(T_{\text {new }}\right)=A \cdot T_{\text {new }}+B \pm$ $E\left(T\left(T_{\text {new }}\right)\right)$. The uncertainties obtained for the different thermocouples are plotted, as a function of the temperature, in Fig. B.2 (left). As the coolant temperature will never be higher than $-5{ }^{\circ} \mathrm{C}$ where the uncertainty is minimum, the most unfavourable point will occur for the lowest coolant temperature tested, $\approx 12{ }^{\circ} \mathrm{C}$. The right part of Fig. B.2 shows the histogram of uncertainties for that temperature, where the maximum uncertainty if of $\approx 0.26{ }^{\circ} \mathrm{C}$ for one of the thermocouples and the lower is $\approx 0.19{ }^{\circ} \mathrm{C}$ for five of them, being the rest between these two values. 


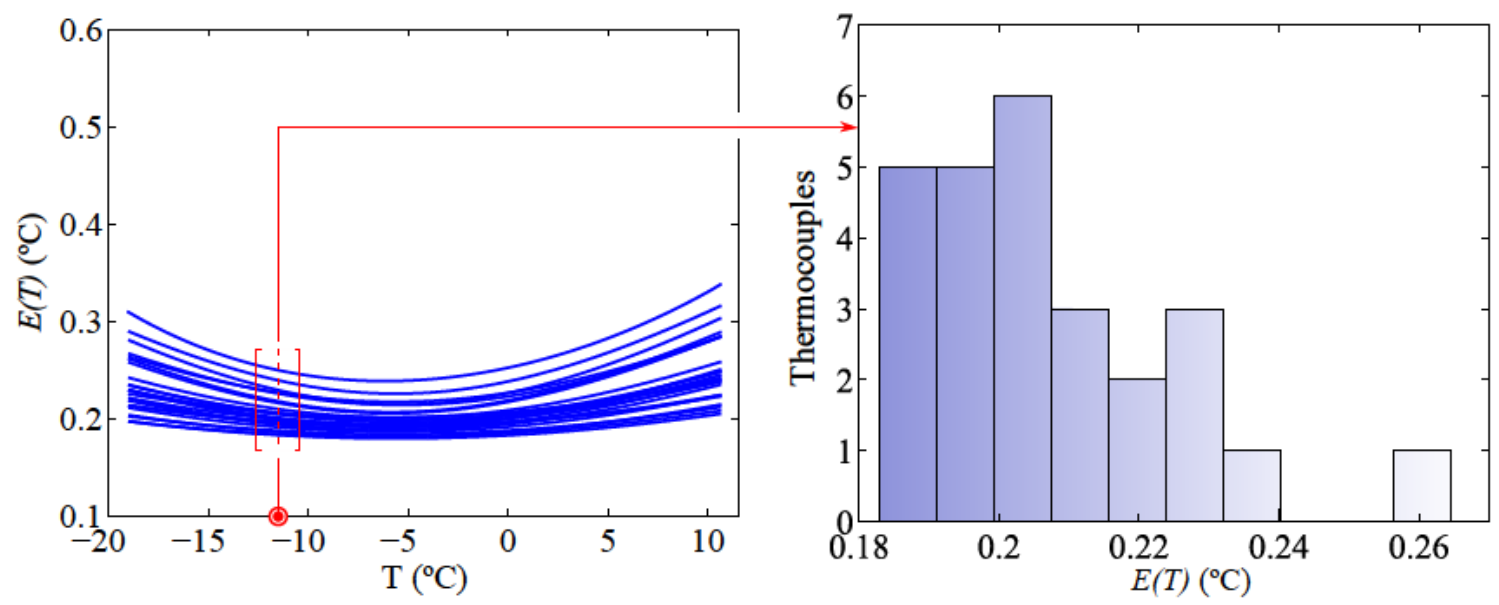

Figure B.2: Uncertainty curves for all the thermocouples (left) and uncertainty histogram at $-11.5^{\circ} \mathrm{C}$ (right). 


\section{Bibliography}

[1] State of the art on phase change material slurries. Energy Conversion and Management, 65:120-132, 2013. Global Conference on Renewable energy and Energy Efficiency for Desert Regions 2011.

[2] M. Kauffeld, M.J. Wang, V. Goldstein, and K.E. Kasza. Ice slurry applications. International Journal of Refrigeration, 33(8):1491-1505, 2010.

[3] Choi U. Kasza, K. Reducing the high costs of district heating and cooling. Specif. Eng, 56:39-42, 1986.

[4] J. Kaminsky K. Kasza, U. Choi. Advanced energy transmission fluids for heating and cooling systems. page 93. ASHRAE Trans., 1988.

[5] Choi U. Kasza, K. Advanced energy transmission fluids for district heating and cooling.

[6] Peter W. Egolf and Michael Kauffeld. From physical properties of ice slurries to industrial ice slurry applications. International Journal of Refrigeration, 28(1):4 $-12,2005$.

[7] S. Lund T.M. Hansen M. Kauffeld, K.G. Christensen. Experience with ice slurry. Yverdon-les-Bains, Switzerland, May. 27-28 1999. Proceedings of the First Workshop on Ice Slurries of the International Institute of Refrigeration.

[8] M. Yamada M. Tanino S. Fukusako, Y. Kozawa. Research and development activities on ice slurries in japan. Yverdon-les-Bains, Switzerland, May 1999. Proceedings of the First Workshop on Ice Slurries of the International Institute of Refrigeration.

[9] Hayashi K. Kasza, K. A method for measuring ice particle slurry agglomeration in storage tanks. ASHRAE Trans, 106(1):117-123, 2000.

[10] O. Bel. Contribution a l'étude du comportement thermohydraulique d'un mélange diphasique dans une boucle frigorifique a stockage d'énergie. Ph.D thesis. L'Institute National des Sciences Appliqueés de Lyon, France, 1996.

[11] P.W. Egolf B. Frei. Viscometry applied to the bingham substance ice slurry. Paris, France, May 2000. Proceedings of the Second Workshop on Ice Slurries of the International Institute of Refrigeration. 
[12] M. Kauffeld T.M. Hansen, M. Radosevic. Behaviour of ice slurry in thermal storage. ASHRAE research project RP 1166. Final Report, February 2002.

[13] T. Hozumi H. Kumano S. Okawa, A. Saito. Effect of ice/water storage on the permeability of the mixtures. pages 49-54. Proceedings of the International Conference on Fundamental Research on Thermal Energy Storage to Preserve Environment, January 2002.

[14] V. Ayel, O. Lottin, and H. Peerhossaini. Rheology, flow behaviour and heat transfer of ice slurries: a review of the state of the art. International Journal of Refrigeration, 26(1):95 - 107, 2003.

[15] A. Kitanovski, D. Vuarnoz, D. Ata-Caesar, P. W. Egolf, T. M. Hansen, and Christian Doetsch. The fluid dynamics of ice slurry. International Journal of Refrigeration, 28(1):37-50, 2005.

[16] Christensen K.G. Kauffeld, M. Heat transfer measurements with ice slurry. pages 127-1141, Washington, D.C., 1997. IIR Conference: Heat Transfer Issues in Natural Refrigerants.

[17] F. Meili P.W. Egolf. O. Sari, D. Vuarnoz. Visualization of ice slurries and ice slurry flows. 25.

[18] A. Melinder. Acurrate thermophysical property values of water solutions are important for ice slurry modelling and calculations. pages 11-18, Horw/Lucerne, Switzerland, 16-18 May, 2001. Proceedings of the Third Workshop on Ice Slurries of the International Institute of Refrigeration.

[19] A. Melinder. Using property values of water solutions and ice to estimate ice concentration and enthalpy values of ice slurries. pages 19-26, Horw/Lucerne, Switzerland, 1618 May, 2001. Proceedings of the Third Workshop on Ice Slurries of the International Institute of Refrigeration.

[20] A. Melinder. Properties and other aspects of aqueous solutions used for single phase and ice slurry applications. International Journal of Refrigeration, 33(8):1506-1512, 2010. Phase Change Materials and Slurries for Refrigeration and Air Conditioning.

[21] F. Illán and A. Viedma. Experimental study on pressure drop and heat transfer in pipelines for brine based ice slurry part ii: Dimensional analysis and rheological model. International Journal of Refrigeration, 32(5):1024 - 1031, 2009.

[22] F. Illán and A. Viedma. Experimental study on pressure drop and heat transfer in pipelines for brine based ice slurry. part i: Operational parameters correlations. International Journal of Refrigeration, 32(5):1015 - 1023, 2009.

[23] Ashley C.S. Monteiro and Pradeep K. Bansal. Pressure drop characteristics and rheological modeling of ice slurry flow in pipes. International Journal of Refrigeration, 33(8):1523 - 1532, 2010. 
[24] Jean-Pierre Bédécarrats, Franoise Strub, and Christophe Peuvrel. Thermal and hydrodynamic considerations of ice slurry in heat exchangers. International Journal of Refrigeration, 32(7):1791 - 1800, 2009.

[25] J. Bellas, I. Chaer, and S.A. Tassou. Heat transfer and pressure drop of ice slurries in plate heat exchangers. Applied Thermal Engineering, 22(7):721 - 732, 2002 .

[26] Fernando Illán Gómez. Caracterización experimental del comportamiento termohidraúlico del hielo liquido generado a partir de disoluciones de NACl. Aplicación a cambiadores de calor, apr 2008.

[27] Andrej Kitanovski and Alojz Poredos. Concentration distribution and viscosity of ice-slurry in heterogeneous flow. International Journal of Refrigeration, 25(6):827 $-835,2002$.

[28] Reghem P Stutz B. Friction losses of two-phase flow liquid-solid. 18.

[29] Beata Niezgoda-elasko. Heat transfer of ice slurry flows in tubes. International Journal of Refrigeration, 29(3):437 - 450, 2006.

[30] B.D. Knodel, D.M. France, U.S. Choi, and M.W. Wambsganss. Heat transfer and pressure drop in ice-water slurries. Applied Thermal Engineering, 20(7):671 $-685,2000$.

[31] B. Lee V. Goldstein E. Stamatiou, M. Kawaji. Experimental investigations of ice-slurry flow and heat transfer in a plate-type heat exchanger. 16.

[32] Ralph L. Webb. Principles of enhanced heat transfer. Taylor \& Francis, 2005.

[33] Kawaji M. Egolf P.W. Kauffeld, M. Handbook on Ice Slurries e Fundamentals and Engineering. (Eds.). IIF/IIR, Paris, 2005.

[34] P. Zhang and Z.W. Ma. An overview of fundamental studies and applications of phase change material slurries to secondary loop refrigeration and air conditioning systems. Renewable and Sustainable Energy Reviews, 16(7):5021 - 5058, 2012.

[35] I. Bellas and S.A. Tassou. Present and future applications of ice slurries. International Journal of Refrigeration, 28(1):115 - 121, 2005.

[36] Qiqi Tian, Guogeng He, Hong Wang, and Dehua Cai. Simulation on transportation safety of ice slurry in ice cooling system of buildings. Energy and Buildings, $72: 262-270,2014$.

[37] Y.H. Yau and S.K. Lee. Feasibility study of an ice slurry-cooling coil for $\{$ HVAC and r systems in a tropical building. Applied Energy, 87(8):2699 - 2711, 2010.

[38] M. J. Wang and N. Kusumoto. Ice slurry based thermal storage in multifunctional buildings. Heat and Mass Transfer, 37(6):597-604, 2001.

[39] E. Stamatiou, J.W. Meewisse, and M. Kawaji. Ice slurry generation involving moving parts. International Journal of Refrigeration, 28(1):60 - 72, 2005. 
[40] O'Hanlon JE Joseph-B Yundt B. Gladis SP, Marciniak MJ. Ice crystal slurry tes system using the orbital rod evaporator. 1996.

[41] Infante Ferreira CA Pronk P, Meewisse JW. Heat transfer model for a fluidised bed ice slurry generator. 2001.

[42] Gakuo F Masayuki I. Method for discharging supercooled water in supercooling ice making system., 1996. Patent JP8285418.

[43] T Kiatsiriroat, S Vithayasai, N Vorayos, A Nuntaphan, and N Vorayos. Heat transfer prediction for a direct contact ice thermal energy storage. Energy Conversion and Management, 44(4):497 - 508, 2003.

[44] I.d.t technologies ltd. vacuum ice maker (vim) for thermal energy storage (tes), 2010.

[45] Chetan S Rao and Richard W Hartel. Scraped surface heat exchangers. Critical reviews in food science and nutrition, 46(3):207-19, 2006.

[46] M. B. Lakhdar, R. Cerecero, G. Alvarez, J. Guilpart, D. Flick, and A. Lallemand. Heat transfer with freezing in a scraped surface heat exchanger. Applied Thermal Engineering, 25(1):45-60, 2005.

[47] Frank G.F. Qin, Xiao Dong Chen, Shashini Ramachandra, and Kevin Free. Heat transfer and power consumption in a scraped-surface heat exchanger while freezing aqueous solutions. Separation and Purification Technology, 48(2):150 - 158, 2006.

[48] Chetan S. Rao and Richard W. Hartel. Scraped surface heat exchangers. Critical Reviews in Food Science and Nutrition, 46(3):207-219, 2006.

[49] H. Abichandani, S.C. Sharma, and D.R. Heldman. Hydrodynamics and heat transfer in liquid full scraped surface heat exchanger -a review. Journal of Food Process Engineering, 9:121-141, 1987.

[50] R. Cuevas and M. Cheryan. Heat transfer in a vertical, liquid-full scrapedsurface heat exchanger. application of the penetration theory and wilson plots models [relevant to food processing]. Journal of Food Process Engineering, 5:1-21, 1982.

[51] P. Harriot. Heat transfer in scraped surface heat exchangers. Chem. Eng. Prog. Symp. Ser, 29:137-139, 1959.

[52] A. H. Skelland, D. R. Oliver, and S. Tooke. Heat transfer in a water-cooled scraped-surface heat exchanger. Brit. Chem. Eng, 7:346-353, 1962.

[53] R. Degoede. Crystallization of paraxylene with scraped surface heat exchangers. Technical report, September 1988.

[54] Frank G. F. Qin, Shashini Premathilaka, Xiao Dong Chen, and Kevin W. Free. The shaft torque change in a laboratory scraped surface heat exchanger used for making ice slurries. Asia-Pacific Journal of Chemical Engineering, 2(6):618-630, 2007. 
[55] Mourad Yataghene, Fayolle Francine, and Legrand Jack. Flow patterns analysis using experimental $\{\mathrm{PIV}\}$ technique inside scraped surface heat exchanger in continuous flow condition. Applied Thermal Engineering, 31(1415):2855 - 2868, 2011.

[56] R. J. C. Vaessen, M. M. Seckler, and G. J. Witkmap. Heat transfer in scraped eutectic crystallizers. International Journal of Heat and Mass Transfer, 47(4):717$728,2004$.

[57] F. van der Ham, G.J. Witkamp, J. de Graauw, and G.M. van Rosmalen. Eutectic freeze crystallization: Application to process streams and waste water purification. Chemical Engineering and Processing: Process Intensification, 37(2):207 213, 1998.

[58] F van der Ham, G.J Witkamp, J de Graauw, and G.M van Rosmalen. Eutectic freeze crystallization simultaneous formation and separation of two solid phases. Journal of Crystal Growth, 198199, Part 1:744 - 748, 1999.

[59] R.J.C. Vaessen, C. Himawan, and G.J. Witkamp. Scale formation of ice from electrolyte solutions on a scraped surface heat exchanger plate. Journal of Crystal Growth, 237-239, Part 3(0):2172-2177, 2002.

[60] R.J.C. Vaessen, B.J.H. Janse, M.M. Seckler, and G.J. Witkamp. Evaluation of the performance of a newly developed eutectic freeze crystallizer: Scraped cooled wall crystaliizer. Chemical Engineering Research and Design, 81(10):1363 - 1372, 2003. Separation Processes.

[61] Infante-Ferreira C.A. Rodriguez-Pascual-M. Witkamp G.J. Pronk, P. Maximum temperature difference without ice-scaling in scraped surface crystallizers during eutectic freeze crystallization. 11.

[62] Ravelet F. Delfos-R.-Witkamp G.J. Rodriguez-Pascual, M. Measurement of flow field and wall temperature distribution in a scraped heat exchanger crystallizer. 2008 .

[63] M. Rodriguez Pascual, J.J. Derksen, G.M. Van Rosmalen, and G.J. Witkamp. Flow and particle motion in scraped heat exchanger crystallizers. Chemical Engineering Science, 64(24):5153 - 5161, 2009.

[64] M. Rodriguez Pascual, F. Ravelet, R. Delfos, J.J. Derksen, and G.J. Witkamp. Large eddy simulations and stereoscopic particle image velocimetry measurements in a scraped heat exchanger crystallizer geometry. Chemical Engineering Science, 64(9):2127-2135, 2009.

[65] Frank G.F. Qin, X. D. Chen, and A. B. Russell. Heat transfer at the subcooledscraped surface with/without phase change. AIChE Journal, 49(8):1947-1955, 2003.

[66] Frank G.F. Qin, Jian Chao Zhao, Andrew B. Russell, Xiao Dong Chen, John J. Chen, and Lindsay Robertson. Simulation and experiment of the unsteady heat 
transport in the onset time of nucleation and crystallization of ice from the subcooled solution. International Journal of Heat and Mass Transfer, 46(17):32213231, 2003.

[67] Frank G.F. Qin, A. B. Russell, X. D. Chen, and L. Robertson. Ice fouling on a subcooled metal surface examined by thermo-response and electrical conductivity. Journal of Food Engineering, 59(4):421-429, 2003.

[68] Frank G.F. Qin, X. D. Chen, and K. Free. Freezing on subcooled surfaces, phenomena, modeling and applications. International Journal of Heat and Mass Transfer, 52(5-6):1245-1253, 2009.

[69] Juan Pedro Solano Fernández. Análisis del flujo y de la transmisión de calor en intercambiadores de superficie rascada por movimiento lineal alternativo, apr 2009.

[70] Damián CresipĹlorens. Comportamiento de fluidos no newtonianos en intercambiadores de calor tubulares con rascador alternativo, apr 2015 .

[71] Thomas W. Kerlin. Practical thermocouple thermometry. Research Triangle Park, NC: Instrument Society of America, 1990.

[72] A. Melinder. Thermophysical properties of liquid secondary refrigerants. International Institute of Refrigeration, 1997.

[73] R.H. Perry, D. Green. Perry's Chemical Engineer's Handbook. fifth edition, McGraw-Hill, New York, 1973.

[74] D.G. Thomas. Transport characteristics of suspension. J. Colloid. Sci., 20:267$277,1965$.

[75] Warren M. Rohsenow; James P. Hartnett; Young I. Cho. Natural convection. McGraw-Hill Professional, 1998.

[76] R. J. Moffat. Describing the uncertainties in experimental results. Experimental Thermal and Fluid Science, 1(1):3-17, 1988.

[77] ISO, Guide to the Expression for Uncertainty Measurement, first ed. International Organization for Standarization, Switzerland, 1995.

[78] A. Giachetti. Matching techniques to compute image motion. Image Vision and Computing, 18(3):247-260, 2000.

[79] JP Lewis. Fast normalized cross-correlation. Vision interface, 10(1):120-123, 1995.

[80] Willert C.E. wereley s. Kompenhans J. Raffel, M. Particle Image Velocimetry. A Practical Guide. Springer-Verlag Berlin Heidelberg, 2007.

[81] VKI Faculty. Measurement Techniques in Fluid Dynamics. An introduction. 3rd revised edition, 2010. 
[82] Khaled J. Hammad and George Papadopoulos. Phase-resolved piv measurements in a stirred tank. Los Angeles, CA, USA, 2000. AIChE.

[83] E.J. Thielicke, W. \& Stamhuis. Affordable and accurate digital particle image velocimetry in matlab. Journal of Open Research Software, 2(1):e30, 2014.

[84] Matteo Frigo and Steven G. Johnson. The design and implementation of FFTW3. Proceedings of the IEEE, 93(2):216-231, 2005. Special issue on "Program Generation, Optimization, and Platform Adaptation".

[85] Recommended procedures and guidelines for uncertainty analysis in particle imaging velocimetry. International Towing Tank Conference., pages 453-455, 2008.

[86] F. Scarano. Particle Image Velocimetry. Development and Applications. Von Karman Institute for Fluid Dynamics, 2000.

[87] E.F.J. Overmars, N.G.W. Warncke, C. Poelma, and J. Westerweel. Bias errors in piv: the pixel locking effect revisited. 15th Int Symp on Applications of Laser Techniques to Fluid Mechanics. Lisbon, Portugal, 2010.

[88] V.W. Uhl. Mixing Vol. III, Theory and applications. Elsevier Science Accademic Press, 2012.

[89] Masaaki Ishikawa, Tetsuo Hirata, and Toru Fujii. Force estimation of mechanical removing processes of mushy structure in an aqueous solution. International Journal of Refrigeration, 25(2):208 - 217, 2002.

[90] M. Zou, S. Beckford, R. Wei, C. Ellis, G. Hatton, and M.A. Miller. Effects of surface roughness and energy on ice adhesion strength. Applied Surface Science, 257(8):3786 - 3792, 2011.

[91] Anne-Marie Kietzig, Savvas G. Hatzikiriakos, and Peter Englezos. Ice friction: The effects of surface roughness, structure, and hydrophobicity. Journal of Applied Physics, 106(2), 2009.

[92] Tetsuo Hirata, Koji Nagasaka, and Masaaki Ishikawa. Crystal ice formation of solution and its removal phenomena at cooled horizontal solid surface: Part i: ice removal phenomena. International Journal of Heat and Mass Transfer, 43(3):333 $-339,2000$.

[93] Tetsuo Hirata, Mitsutoshi Kato, Koji Nagasaka, and Masaaki Ishikawa. Crystal ice formation of solution and its removal phenomena at cooled horizontal solid surface: Part ii: onset of ice removal condition. International Journal of Heat and Mass Transfer, 43(5):757-765, 2000.

[94] Y.T. Shah. Design parameters for mechanically agitated reactors. volume 17 of Advances in Chemical Engineering, pages 1 - 206. Academic Press, 1991.

[95] A.T.-C. Mak. Doctoral thesis, University of London, 1992.

[96] A.M. Trommelen. Doctoral thesis, Delft University of Technology, 1970. 
[97] Harriott P. McCabe WL, Smith JC. McGraw-Hill, Inc.: Boston, MA, 2001.

[98] Brian Armstrong-Hlouvry, Pierre Dupont, and Carlos Canudas De Wit. A survey of models, analysis tools and compensation methods for the control of machines with friction. Automatica, 30(7):1083 - 1138, 1994.

[99] Anne-Marie Kietzig, Savvas G. Hatzikiriakos, and Peter Englezos. Physics of ice friction. Journal of Applied Physics, 107(8), 2010.

[100] Ben Lishman, Peter Sammonds, and Danny Feltham. A rate and state friction law for saline ice. Journal of Geophysical Research: Oceans, 116(C5), 2011.

[101] Catherine Loader. Smoothing: Local Regression Techniques. Springer Handbooks of Computational Statistics. Springer Berlin Heidelberg, 2012.

[102] J.P. Solano, A. Garca, P.G. Vicente, and A. Viedma. Flow field and heat transfer investigation in tubes of heat exchangers with motionless scrapers. Applied Thermal Engineering, 31(1112):2013 - 2024, 2011.

[103] J.P. Solano, A. Garca, P.G. Vicente, and A. Viedma. Performance evaluation of a zero-fouling reciprocating scraped-surface heat exchanger. Heat Transfer Engineering, 32(3-4):331-338, 2011.

[104] W. F. Stoecker. Industrial Refrigeration Handbook. New York: McGraw-Hill, 1998.

[105] Khlmaschinenbau GmbH BITZER. Air-cooled condensing units, 2013.

[106] Summerer, F. Energy savings on the low pressure side of a refrigeration plant. Güntner technical article, Güntner AG \& CO. KG, 2007.

[107] Sumer M. Peker, Serif S. Helvaci, H. Banu Yener, Berrin Íkizler, and Alp Alparslan. Solid-Liquid Two Phase Flow. Elsevier, 2008.

[108] Holger Nobach. The Particle Image Velocimetry - Characteristics, Limits and Possible Applications. InTech, 2012.

[109] A.K. Prasad and R.J. Adrian. Stereoscopic particle image velocimetry applied to liquid flows. Experiments in Fluids, 15(1):49-60, 1993.

[110] Richard Holdich. Fundamentals of Particle Technology. Midland Information Technology and Publishing, 2002.

[111] Renaud Escudi and Alain Lin. Experimental analysis of hydrodynamics in a radially agitated tank. AIChE Journal, 49(3):585-603, 2003.

[112] Kazumi Suzukawa, Keisuke Kato, Shinsuke Mochizuki, and Hideo Oaka. Vortex structures around a flat paddle impeller in a stirred vessel. JSME International Journal Series B Fluids and Thermal Engineering, 49(2):426-433, 2006.

[113] K. Suzukawa, S. Mochizuki, and H. Osaka. Effect of the attack angle on the roll and trailing vortex structures in an agitated vessel with a paddle impeller. Chemical Engineering Science, 61(9):2791 - 2798, 2006. 
[114] Q.-X. Lian and Z. Huang. Starting flow and structures of the starting vortex behind bluff bodies with sharp edges. Experiments in Fluids, 8(1-2):95-103, 1989.

[115] H.S. Yoon, D.F. Hill, S. Balachandar, R.J. Adrian, and M.Y. Ha. Reynolds number scaling of flow in a rushton turbine stirred thank. part i - mean flow, circular jet and tip vortex scaling. Chemical Engineering Science, 60(12):3169 3183, 2005.

[116] Ma Hui-Yang Zhou Ming-De Wu, Jie-Zhi. Vortical Flows. Springer, 2015.

[117] Renaud Escudi, Denis Bouyer, and Alain Lin. Characterization of trailing vortices generated by a rushton turbine. AIChE Journal, 50(1):75-86, 2004.

[118] J.M.M Sousa and J.C.F Pereira. Rollup region of a turbulent trailing vortex issued from a blade with flow separation. Experimental Thermal and Fluid Science, 20(34):150 - 161, 2000.

[119] W.J.M. Rankine and W.J. Millar. A Manual of Applied Mechanics. C. Griffin and Co., London, UK, 1858.

[120] J.M. Burgers. A mathematical model illustrating the theory of turbulence. 1:171 $-199,1948$.

[121] Brian A. Maicke and Joseph Majdalani. The Particle Image Velocimetry - Characteristics, Limits and Possible Applications. InTech, 2012.

[122] Nakao S. Winardi S. and Nagase Y. Pattern recognition in flow visualization around a paddle impeller. Journal of Chemical Engineering of Japan, 21(5):503$508,1988$.

[123] F.R. Khan, C.D. Rielly, and D.A.R. Brown. Angle-resolved stereo-piv measurements close to a down-pumping pitched-blade turbine. Chemical Engineering Science, 61(9):2799 - 2806, 2006.

[124] K.H.K. Chung, M. Barigou, and M.J.H. Simmons. Reconstruction of 3-d flow field inside miniature stirred vessels using a 2-d $\{\mathrm{PIV}\}$ technique. Chemical Engineering Research and Design, 85(5):560 - 567, 2007.

[125] B. Lecordier and M. Trinité. Advanced piv algorithms with image distortion validation and comparison using synthetic images of turbulent flow. In Particle Image Velocimetry: Recent Improvements, pages 115-132. Springer Berlin Heidelberg, 2004 .

[126] J. Ferziger and M. Peric. Computational Methods for Fluid Dynamics. third ed. Springer, Berlin, 2002.

[127] Stewart W. Bird, R. and E. Lightfoot. Transport Phenomena. second ed. John Wiley and Sons, Inc., 2002.

[128] E. De Villiers. The potential of large eddy simulation for wall bounded flows. Ph.D Thesis, Imperial College of Science, Technology and Medicine., 2006. 
[129] C. Wilcox, D. Turbulent modelling for cfd. 2006.

[130] B. E. Launder and D. B. Spalding. The numerical computation of turbulent flows. Computer Methods in Applied Mechanics and Engineering, 3, 1974.

[131] Stephen B. Pope. Turbulent flows. Cambridge University Press, 2000.

[132] F. R. Menter. Two-equation eddy-viscosity turbulence models for engineering applications. AIAA Journal, 32:1598-1605, 1994.

[133] J. Smagorinsky. General circulation experiments with the primitive equations. Mon. Wea. Rev., 91(3):99-164, 1963.

[134] H. Weller. OpenCFD Limited. www.opencfd.co.uk, 2011.

[135] H. K. Versteeg and W. Malalasekera. An Introduction to Computational Fluid Dynamics. The Finite Volume Method. Longman Group Ltd., London, 1995.

[136] H. Weller. OpenFOAM Programmer's Guide. OpenCFD Limited www.opencfd.co.uk, 2011.

[137] I. Issa, R. Solution of the implicitly discretised fluid flow equations by operatorsplitting. J. Comput. Phys., 62(1):40-65, 1986.

[138] Suhas V. Patankar. Numerical heat transfer and fluid flow. Series in computational methods in mechanics and thermal sciences. Hemisphere Pub. Corp. New York, 1980.

[139] G. G. Stokes. On the Effect of the Internal Friction of Fluids on the Motion of Pendulums. Transactions of the Cambridge Philosophical Society, 9:8, 1851.

[140] W. Rodi. Comparison of les and rans calculations of the flow around bluff bodies. Journal of Wind Engineering and Industrial Aerodynamics, 6971:55 - 75, 1997.

[141] T. Nishino, G.T. Roberts, and X. Zhang. Unsteady rans and detached-eddy simulations of flow around a circular cylinder in ground effect. Journal of Fluids and Structures, 24(1):18 - 33.

[142] J. Keith Nisbett Budynas, Richard G. and Joseph Edward Shigley. Shigley's Mechanical Engineering Design. New York: McGraw-Hill, 2011.

[143] Euro Inox/SCI. Design Manual For Structural Stainless Steel. Third ed. Euro Inox and The Steel Construction Institute, 2006.

[144] Hugh W. Coleman and W. Glenn Steele. Data Analysis, Regression, and Reporting of Results. John Wiley \& Sons, Inc., 2009.

[145] Hugh W. Coleman and W. Glenn Steele. Appendix B. John Wiley \& Sons, Inc., 2009.

[146] G. W. Burns and M. G. Scroger. Natl. Inst. Stand. Technol. Spec. Publ. 250-35, 1989. 